WALTER RIED

\title{
INFORMATION \\ UND NUTZEN DER \\ MEDIZINISCHEN \\ DIAGNOSTIK
}




\section{WALTER RIED}

\section{INFORMATION UND NUTZEN DER MEDIZINISCHEN DIAGNOSTIK}

Im Mittelpunkt der vorliegenden Arbeit steht die theoretische Analyse des Wohlfahrtseffekts, den der Einsatz der medizinischen Diagnostik beim Patienten auslösen kann. Die Abbildung dieses Nutzeneffekts erfolgt in einem entscheidungstheoretischen Ansatz, der es zugleich gestattet, die Bedeutung wichtiger Einflußgrößen wie z.B. der medizinischen Ausgangslage oder der diagnostischen Information zu diskutieren. Darüber hinaus möchte die Arbeit einen Beitrag zur Kosten-Nutzen-Analyse diagnostischer Maßnahmen leisten, indem der Nutzen für den Patienten mit Hilfe eines speziellen Zahlungsbereitschaftskonzepts dargestellt und eingehend untersucht wird.

Walter Ried wurde 1959 in Mainz geboren. Nach Studienaufenthalten in Buckingham/England studierte er Volkswirtschaftslehre von 1980 bis 1986 an der Universität Mannheim. Von 1986 bis 1991 war er wissenschaftlicher Mitarbeiter am Lehrstuhl für Volkswirtschaftslehre, insbesondere Planung und Verwaltung öffentlicher Wirtschaft. 
Information und Nutzen der medizinischen Diagnostik 


\section{ALLOKATION IM \\ MARKTWIRTSCHAFTLICHEN SYSTEM}

Herausgegeben von

Heinz König, Hans-Heinrich Nachtkamp, Ulrich Schlieper, Eberhard Wille

\section{Band 33}

\section{(3) \\ PETER LANG}

Frankfurt am Main - Berlin - Bern - New York · Paris · Wien 


\section{WALTER RIED}

\section{INFORMATION UND NUTZEN DER MEDIZINISCHEN DIAGNOSTIK}

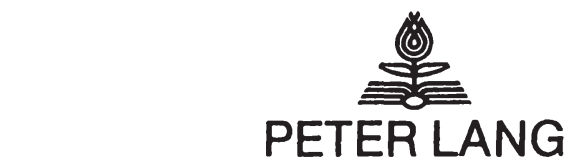

Frankfurt am Main · Berlin - Bern · New York - Paris · Wien 
Die Deutsche Bibliothek - CIP-Einheitsaufnahme

Ried, Walter:

Information und Nutzen der medizinischen Diagnostik / Walter

Ried. - Frankfurt am Main ; Berlin ; Bern ; Now York ; Paris ;

Wien : Lang, 1992

(Allokation im marktwirtschaftlichen System ; Bd. 33)

Zugl.: Mannheim, Univ., Diss., 1992

ISBN 3-631-45199-7

NE: GT

Open Access: The online version of this publication is published on www.peterlang.com and www.econstor.eu under the international Creative Commons License CC-BY 4.0. Learn more on how you can use and share this work: http://creativecommons. org/licenses/by/4.0.

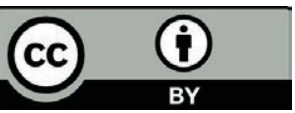

This book is available Open Access thanks to the kind support of ZBW - Leibniz-Informationszentrum Wirtschaft.

\author{
D 180 \\ ISSN 0939-7728 \\ ISBN 3-631-45199-7 \\ ISBN 978-3-631-75573-0 (eBook) \\ (c) Verlag Peter Lang GmbH, Frankfurt am Main 1992 \\ Alle Rechte vorbehalten.
}

Das Werk einschließlich aller seiner Teile ist urheberrechtlich geschūtzt. Jede Verwertung außerhalb der engen Grenzen des Urheberrechtsgesetzes ist ohne Zustimmung des Verlages unzulässig und strafbar. Das gilt insbesondere fūr Vervielfältigungen, Übersetzungen, Mikroverfilmungen und die Einspeicherung und Verarbeitung in elektronischen Systemen.

Printed in Germany 124567 
meinen Eltern

Walter Ried - 978-3-631-75573-0

Downloaded from PubFactory at 01/11/2019 03:26:15AM

via free access 
Walter Ried - 978-3-631-75573-0

Downloaded from PubFactory at 01/11/2019 03:26:15AM

via free access 


\section{Vorwort}

Während die Untersuchung einzelner therapeutischer Leistungen aus gesamtwirtschaftlicher Perspektive schon früh einen wesentlichen Bestandteil der Gesundheitsökonomik bildete, erfuhr die medizinische Diagnostik lange Zeit eine vergleichsweise geringe Beachtung. Die vorliegende Arbeit versucht, einen Beitrag zur KostenNutzen-Analyse diagnostischer Maßnahmen zu leisten, indem mit der Ändenung der Wohlfahrtsposition des Patienten eine zentrale Komponente in einem entscheidungstheoretischen Ansatz dargestellt wird. Dabei steht mit dem Nutzen des Patienten zunächst die monetäre Bewertung dieser Wohlfahrtsänderung im Vordergrund, deren Ableitung mit Hilfe eines speziellen Zahlungsbereitschaftskonzepts erfolgt. Ein zweiter Schwerpunkt liegt auf der Diskussion des Einflusses, den typische Elemente der medizinischen Entscheidungssituation auf die Existenz und den Umfang dieses Nutzeneffekts ausüben.

Die Arbeit entstand während meiner Tätigkeit am Lehrstuhl für Volkswirtschaftslehre, insbesondere Planung und Verwaltung öffentlicher Wirtschaft, der Universität Mannheim. Sie wurde im Wintersemester 1991/92 von der Fakultät für Volkswirtschaftslehre und Statistik der Universität Mannheim als Dissertation angenommen.

Mein Dank gebührt in erster Linie meinem Doktorvater, Herrn Professor Dr. Eberhard Wille, der nicht nur die Anregung zu dieser Arbeit gab, sondern sie zugleich in allen Phasen ihrer Entstehung in vielfältiger Weise unterstützte. Besonders hervorheben möchte ich seine immerwährende Bereitschaft zu Diskussionen, in denen er durch seine stets konstruktive Kritik den Fortgang der Arbeit maßgeblich gefördert hat. Ferner danke ich dem Korreferenten, Herrn Professor Dr. Horst Stenger, für einige wertvolle Hinweise, die das vierte Kapitel betreffen. Gerne nutze ich die Gelegenheit, den Herren Dr. Manfred Erbsland, Dr. Martin Hornberger und Dr. Volker Ulrich für ihre zahlreichen Anmerkungen und Verbesserungsvorschläge zu danken. Schließlich gilt mein Dank den Teilnehmern der volkswirtschaftlichen Mittelbau-Seminare an den Universitäten Mannheim und Saarbrücken, von deren Diskussionsbeiträgen ich ebenfalls profitieren durfte. Last, but certainly not least danke ich meiner Verlobten, Frau Diplom-Volkswirt Christine Hohenhinnebusch, die insbesondere in der "Endphase" nicht nur die Opportunitätskosten dieser Arbeit in ruhiger Gelassenheit mitgetragen hat, sondern zugleich durch ihren aufmunternden Zuspruch einen wichtigen positiven Beitrag leistete. 
Ich widme diese Arbeit meinen Eltern, die mir in großzügiger Weise meine Ausbildung ermöglicht haben und damit bei hinreichend weiter Betrachtung in erheblichem Umfang an der Vollendung dieser Schrift beteiligt sind.

Mannheim, den 6. Mai 1992

Walter Ried 


\section{Inhaltsverzeichnis}

1. Einleitung 5

1.1 Die These einer exzessiven medizinischen Diagnostik 5

$\begin{array}{lll}1.2 & \text { Anliegen der Arbeit und Disposition } & 9\end{array}$

2. Grundlagen 15

2.1 Kosten- und Nutzeneffekte im Gesundheitswesen 15

2.1.1 Darstellung und Kategorisierung 15

2.1.2 Die Bedeutung direkter intangibler Effekte 22

2.2 Die Bewertung individueller Wohlfahrtsänderungen 25

2.2.1 Individuelle Zahlungsbereitschaften bei Sicherheit 25

2.2.2 Individuelle Zahlungsbereitschaften bei Unsicherheit 36

2.3 Medizinische Diagnostik 44

2.3.1 Der Diagnosebegriff in der Medizin 44

2.3.2 Diagnostische Maßnahmen $\quad 50$

2.4 Entscheidungstheoretische Voraussetzungen 56

2.4.1 Krankheitszustände 56

2.4.2 Bedingte Wahrscheinlichkeiten und das Theorem von Bayes 59

2.4.3 Diagnostische Maßnahmen als Informationsinstrumente 63

3. Die Information diagnostischer Maßnahmen 69

$3.1 \quad$ Binäre und binarisierte Tests $\quad 69$

$\begin{array}{lll}3.2 & \text { Sensitivität und Spezifität } & 71\end{array}$

$\begin{array}{lll}3.2 .1 & \text { Definition und Erläuterung } & 71\end{array}$

3.2.2 Die Schätzung der Kennziffern eines Diagnosetests 77

$\begin{array}{lll}3.2 .3 & \text { ROC-Analyse } & 82\end{array}$

$\begin{array}{lll}3.3 & \text { Prädiktive Werte } & 92\end{array}$

3.3.1 Positiver und negativer prädiktiver Wert 92

$\begin{array}{lll}\text { 3.3.2 Graphische Analyse } & 99\end{array}$

3.4 Die Informationsleistung diagnostischer Maßnahmen im allgemeinen Fall 
3.5 Zur Verwendung des Bayes-Theorems in der medizinischen Diagnostik 109

3.6 Der Informationsgewinn diagnostischer Maßnahmen 119

3.6.1 Exkurs: Informationstheoretische Grundlagen 119

3.6.1.1 Zum Begriff der Information $\quad 119$

3.6.1.2 Die Entropie als Informationsmaß 122

$\begin{array}{ll}\text { 3.6.1.3 Meßgrößen für den Informationsgewinn } & 130\end{array}$

$\begin{array}{ll}\text { 3.6.2 Anwendung auf diagnostische Maßnahmen } & 134\end{array}$

4. Der Nutzen diagnostischer Maßnahmen für den Patienten 139

4.1 Die Rolle der Diagnostik 139

4.2 Die Entscheidungssituation des Arztes 142

4.2.1 Mögliche ärztliche Entscheidungen 142

4.2.2 Handlungskonsequenzen und ihre Bewertung 147

4.2.3 Die Bewertung ärztlicher Entscheidungen 156

4.2.4 Der Arzt als Agent des Patienten 163

4.3 Der Optionspreis diagnostischer Maßnahmen 168

$\begin{array}{lll}\text { 4.3.1 Vorüberlegung } & 168\end{array}$

4.3.2 Der Optionspreis diagnostischer Maßnahmen im allgemeinen Fall 175

4.3.2.1 Der Optionspreis der vollkommenen diagnostischen Information 175

4.3.2.2 Der Optionspreis unvollkommener diagnostischer Maßnahmen 183

4.3.3 Der Optionspreis diagnostischer Maßnahmen bei "linearem Risikonutzen" 186

4.3.3.1 Einführung einer monetären Verlustfunktion 186

4.3.3.2 Der Optionspreis der diagnostischen Information 193

$\begin{array}{lll}\text { 4.3.3.3 Graphische Analyse } & 197\end{array}$

4.3.3.4 Zur Bedeutung der Annahme eines "linearen Risikonutzens" 208

4.3.4 Der Optionspreis diagnostischer Maßnahmen aus gesamtwirtschaftlicher Perspektive 
4.4 Die Analyse des diagnostischen Nutzeneffekts 211

4.4.1 Differentielle Nutzeneffekte diagnostischer Maßnahmen 211

4.4.1.1 Zum Outputcharakter der diagnostischen Information 212

4.4.1.2 Die Beurteilung des diagnostischen Nutzeneffekts 215

4.4.2 Der Einfluß einzelner Komponenten der medizinischen Entscheidungssituation $\quad 224$

4.4.3 Der Informationsgrad diagnostischer Maßnahmen 245

4.5 Die Berücksichtigung intangibler Kosteneffekte 252

5. Zusammenfassung und Ausblick 257

$\begin{array}{ll}\text { Literaturverzeichnis } & 271\end{array}$ 


\section{Abbildungsverzeichnis}

Abb. 1: Zur Klassifikation von Effekten im Gesundheitswesen

Abb. 2: Die Ermittlung von $\mathrm{KV}(0 \rightarrow 1)$ und $\ddot{A} \mathrm{~V}(0 \rightarrow 1)$

Abb. 3: Der diagnostische Prozeß nach Gross

Abb. 4: Zum Begriff der diagnostischen Strategie

Abb. 5: Bedingte Wahrscheinlichkeiten der Testergebnisse von $T_{j}$

Abb. 6: Zur Charakterisierung eines binarisierten Diagnosetests $T_{j}$

Abb. 7: Die Menge der möglichen Kennzifferkombinationen eines binarisierten Diagnosetests

Abb. 8: Der Vergleich der Kennziffern binarisierter Diagnosetests

Abb. 9: Die Abhängigkeit des positiven prädiktiven Werts von $\mathrm{P}\left(\mathrm{K}_{1}\right)$

Abb. 10: Die Abhängigkeit des negativen prädiktiven Werts von $\mathrm{P}\left(\overline{\mathrm{K}}_{1}\right)$

Abb. 11: Die Informationsleistung binarisierter diagnostischer Maßnahmen

Abb. 12: Die Konsequenzen ärztlicher Aktionen

Abb. 13: Nutzenallokationen aufgrund nicht-randomisierter Aktionen

Abb. 14: Die Entscheidungssituation ohne weitere Diagnostik

Abb. 15: Der Optionspreis der unvollkommenenen diagnostischen Maßnahme $T_{j}$ 
Abb. 16: Der Optionspreis von $T_{j}$ bei veränderten prioren

Wahrscheinlichkeiten

Abb. 17: Der Optionspreis der vollkommenen diagnostischen Information

Abb. 18: Der Optionspreis der vollkommenen diagnostischen Information bei Existenz einer gleichmäßig besten Aktion

Abb. 19: Der Nutzeneffekt einer Vorsorgeuntersuchung $T_{j}$

Abb. 20: Diagnostische Kennziffern und prädiktive Werte von $T_{1}$ und $T_{2}$

Abb. 21: Graphische Darstellung von dreielementigen Wahrscheinlichkeitsverteilungen

Abb. 22: Die Struktur der medizinischen Entscheidungssituation

Abb. 23: Der differentielle Nutzeneffekt von $T_{1}$ in der Ausgangslage $P_{0}$

Abb. 24: Der differentielle Nutzeneffekt von $T_{1}$ in der Ausgangslage $P_{0}{ }^{\prime}$

Abb. 25: Der differentielle Nutzeneffekt von $T_{2}$

Abb. 26: Die Präferenzen des Patienten für diagnostische Kennziffern

Abb. 27: Die Präferenzen des Patienten für diagnostische Maßnahmen in der Entscheidungssituation des "screening"

Abb. 28: Die Präferenzen des Patienten für diagnostische Maßnahmen in der differentialdiagnostischen Entscheidungssituation

Abb. 29: Vergleich des Informationsgrads zweier Diagnosetests 
Walter Ried - 978-3-631-75573-0

Downloaded from PubFactory at 01/11/2019 03:26:15AM

via free access 


\section{EINLEITUNG}

\subsection{Die These einer exzessiven medizinischen Diagnostik}

In den letzten Jahrzehnten hat der technische Fortschritt die Entwicklung der medizinischen Diagnostik maßgeblich beeinflußt. Dadurch verfügt der einzelne Arzt heute über eine Vielzahl von Möglichkeiten, therapierelevante Informationen auch in solchen Fällen zu beschaffen, in denen früher eine genaue diagnostische Abklärung nicht zu erreichen war. Beispielsweise gelingt es mit Hilfe der vor knapp zwanzig Jahren eingeführten Computertomographie ${ }^{1}$, sowohl das Gehirn als auch andere Körperteile im Vergleich zu den herkömmlichen Verfahren wesentlich genauer darzustellen. Ähnlich eröffnet ein Kernspintomograph ${ }^{2}$ die Möglichkeit, durch den Einsatz von Magnetfeldern unter anderem eine nochmals verbesserte Visualisierung einzelner Gewebeschichten des menschlichen Körpers zu gewinnen. Beide Diagnosemethoden gehören zu den bildgebenden Verfahren, deren Einsatzbereich zunächst primär im Krankenhaus lag, da es sich um kostspielige medizinische Großgeräte handelt.

Beinahe ebenso drastisch hat sich das Spektrum der diagnostischen Möglichkeiten im ambulanten Bereich verändert. So ist die Computertomographie heute vielerorts auch ambulant verfügbar. Daneben treten schon seit geraumer Zeit an die Seite der traditionell vom Arzt erhobenen Anamnese und der körperlichen Untersuchung in zunehmendem Maße sogenannte Laborleistungen, die über bestimmte Parameterwerte informieren. Häufig handelt es sich dabei um Leistungen, die der Arzt vornehmlich aus Kostengründen nicht (mehr) unmittelbar selbst erbringt, sondern an Laborgemeinschaften delegiert ${ }^{3}$. Im Gegensatz dazu führen neuartige Testsysteme ${ }^{4}$ zumindest einen Teil der Labordiagnostik in die ärztliche Praxis zurück: im Sinne einer patientennahen Analytik bietet beispielsweise die Trockenchemie $^{5}$ die Möglichkeit, innerhalb weniger Minuten wichtige Parameter zu bestimmen.

Diese Erweiterung der diagnostischen Kapazitäten zeitigt allerdings keineswegs ausschließlich positive Folgen. Nicht wenige Kritiker auch aus den Reihen der Ärzte-

1: Zur Computertomographie vgl. WAGNER, J.L. (1981) sowie SCHICKE, R.K. (1984).

2: Vgl. GUYATT, G./DRUMMOND, M.F. (1986).

3: Unter gewissen Voraussetzungen handelt es sich dabei dennoch um selbst erbrachte Leistungen, für die der Arzt eine Vergütung berechnen darf; vgl. NARR, H. (1984), S. 74f.

4: Einen Überblick geben BELSEY, R./BAER, D./SEWELL, D. (1986) und HLTON, S. (1990).

5: Zur Trockenchemie vgl. SELECTA SUPPLEMENT (1987) und WILLE, E. (1987). 
schaft monieren, es werde eine "exzessive Diagnostik"6 betrieben, da ein beträchtlicher Teil der von den Ärzten veranlaßten Diagnosetests aus medizinischer Sicht unnötig sei $^{7}$. Dieser These zufolge betreiben die Ärzte häufig eine "Schrotschußdiagnostik"8, indem sie eine Vielzahl indiskriminierter, d.h. durch die speziellen Beschwerden des Patienten nicht gerechtfertigter, Untersuchungen durchführen ${ }^{9}$. In diesem Zusammenhang zeichnen plakative Schlagworte, die von einem "diagnostischen Rundumschlag"10, einem "diagnostischen Rauschzustand"11 oder gar einem "diagnostic overkill"12 sprechen, ein sicherlich übertriebenes Bild der tatsächlichen Situation, das in der Tendenz gleichwohl zutreffend erscheint.

In der Bundesrepublik Deutschland vertritt auch der Sachverständigenrat für die Konzertierte Aktion im Gesundheitswesen die These einer übermäßigen Inanspruchnahme diagnostischer Leistungen und begründet diese damit, daß die Ärzte in zu geringem Umfang gezielte Diagnostik betreiben ${ }^{13}$. Die starke Entwicklung der Mengenkomponente insbesondere bei den Laborleistungen spiegeln im übrigen auch die Honorarvereinbarungen wider, die die Verbände der Ersatzkassen mit der Kassenärztlichen Bundesvereinigung in den letzten Jahren getroffen haben: Nachdem in den Jahren 1981 und 1983 in zwei Schritten die Vergütung der wichtigsten Laborleistungen teilweise massiv herabgesetzt worden war, gingen die Ersatzkassen ab Oktober 1983 dazu über, für etwa drei Viertel der im Laborbereich abgerechneten Leistungen eine Pauschale zu vereinbaren ${ }^{14}$. Entgegen ihrer ursprünglichen Zielsetzung gelang den Vertragspartnern in den folgenden Jahren die Rückkehr zur

6: ANSCHÜTZ, F. (1988).

7: Vgl. z.B. FINEBERG, H.V. (1979); WILLIAMS, S.V. et al. (1982); MORGAN, P.P. (1984); MEISTER, H. (1985); LUDBROOK, A./MOONEY, G.H. (1986); STEIN, R. (1986); o.V. (1987).

8: $\quad$ MEISTER, H. (1985), S. 682.

9: Vgl. MEISTER, H. (1985), S. 682f. sowie o.V. (1987). Diese Art der Diagnostik diskutieren auch Wong und Lincoln, wie schon der bezeichnende Titel "Ready! Fire!...Aim!" ihres Artikels verrät; vgl. WONG, E.T/LINCOLN, T.L. (1983).

10: ANSCHÜTZ, F. (1985), S. 6.

11: So der 1. Vorsizzende des Vorstands der KV Niedersachsen, Dr. Weinhold, in einem Leserbrief der Medical Tribune; zitiert nach FISCHER, N. (1984), S. 281.

12: HUTTEN, H. (1985).

13: Vgl. SACHVERSTÄNDIGENRAT FÜR DIE KONZERTIERTE AKTION IM GESUNDHEITSWESEN (1989), Tz. 142, 143.

14: Vgl. FISCHER, N. (1983). 
Einzelleistungsvergütung in diesem Bereich nicht, da die Mengenzunahme nicht eingedämmt werden konnte ${ }^{15}$.

Zur Begründung einer exzessiven Nutzung vorhandener diagnostischer Möglichkeiten lassen sich mehrere Faktoren anführen, die als mögliche Ursachen in Betracht kommen ${ }^{16}$. So besteht insbesondere für den niedergelassenen Arzt unter Umständen aufgrund der speziellen Form der Vergütung seiner Leistungen ein finanzieller Anreiz, Diagnostik über das medizinisch notwendige $\mathrm{MaB}$ hinaus zu betreiben ${ }^{17}$. Dies gilt beispielsweise in der Bundesrepublik für die Laborleistungen, deren starke Mengenausweitung zuvor schon angesprochen wurde ${ }^{18}$. Ein ähnliches Argument betrifft diejenigen diagnostischen Leistungen, die mit Hilfe von medizintechnischen Großgeräten - etwa dem Computertomographen - erbracht werden. In diesem Fall kann es für den einzelnen Arzt wirtschaftlich günstig sein, einen möglichst hohen Auslastungsgrad bei diesen Geräten zu erzielen.

Als weiterer möglicher Faktor wird in der Literatur das mangelnde Wissen bzw. die mangelnde Erfahrung der Ärzte genannt ${ }^{19}$. Diese Einschätzung betrifft zunächst speziell die jüngeren Ärzte, die im Umgang mit Patienten noch wenig Erfahrung besitzen. Aus diesem Grund vermögen sie die Bedeutung insbesondere der anamnestischen Daten nicht vollständig zu erkennen und benötigen demzufolge ein Übermaß an weiteren Untersuchungen, um zu einer befriedigenden diagnostischen Abklärung eines Krankheitsfalls zu gelangen. Andererseits dürften eher dienstältere niedergelassene Ärzte mit neuartigen Diagnosetests nicht in ausreichendem Maß vertraut sein, so daß diese Ärztegruppe die entsprechenden Testergebnisse auch in Situationen anfordert, in denen ihnen keine diagnostische Aussagekraft zukommt. A priori bleibt unklar, welcher dieser beiden Effekte dominiert. Insofern überrascht es kaum, wenn empirische Studien zu keinem eindeutigen Ergebnis hinsichtlich des Zusammenhangs

15: Vgl. FISCHER, N. (1985). Allerdings ergab sich diese Pauschalienung ab Juli 1986 auch als Folge der Umstrukturierung des Einheitlichen Bewertungsmaßstabs für die ärztlichen Leistungen (EBM). Die im Vergleich zu den übrigen Leistungen unvermindert starke Mengenentwicklung im Laborbereich kommt in diesem Fall darin zum Ausdruck, daß für Laborleistungen ein eigener Punktwert vereinbart wurde.

16: Vgl. dazu auch FINEBERG, H.V. (1979), S. $147 \mathrm{f}$.

17: Vgl. DANIELS, M./SCHROEDER, S.A. (1977), S. 487; KÖBBERLING, J.TRAMPISCH, H.-J./WINDELER, J. (1989), S. 4.

18: Vgl. WILLE, E. (1987), S. 325ff. sowie MOSS, J.M. (1987) zu einer ähnlichen Einschätzung für die USA.

19: Vgl. z.B. MEISTER, H. (1985), S. 682; WONG, E.T./LINCOLN, T.L. (1983), S. 2511. 
kommen, der zwischen dem Alter der Ärzte und der Anzahl der von ihnen durchgeführten Diagnosetests besteht ${ }^{20}$.

Darüber hinaus spielt die Anspruchshaltung der Patienten offenbar bei manchen Testanforderungen eine Rolle. Dieser Einfluß liegt vornehmlich in denjenigen Situationen vor, in denen der Arzt eigentlich schon aufgrund der qualitativen Befunde der Anamnese und der körperlichen Untersuchung die Diagnose stellen könnte. Zusätzlich veranlaßte Labortests dienen in diesem Kontext dazu, den Wunsch des Patienten nach einer angemessenen "diagnostischen Grundversorgung" bzw. sein Verlangen nach quantitativen Befunden zu befriedigen ${ }^{21}$.

Ähnlich können die Resultate quantitativer Analysetests, die in besonderer Weise intersubjektiv überprüfbar sind, die Aufgabe erfüllen, den Arzt für den Fall abzusichern, daß der Patient wegen unerwünschter Behandlungsfolgen einen Rechtsstreit anstrengt. Die durch den Arzt zusätzlich betriebene Diagnostik gehört zur sogenannten Defensivmedizin und wird als "Absicherungsdiagnostik"22 bezeichnet. Dieser Effekt dürfte vor allem in den USA anzutreffen sein, da dort die Haftungsregeln für das ärztliche Handeln und die darauf aufbauende Rechtsprechung in den letzten Jahren erheblich verschärft wurden ${ }^{23}$.

Schließlich vertritt insbesondere Wieland die Auffassung, dem diagnostischen Fortschritt seien keine entsprechenden Innovationen bei den therapeutischen Möglichkeiten gefolgt ${ }^{24}$. Falls dieses Argument zutrifft, bleibt ein Teil der augenblicklich verfügbaren diagnostischen Information notwendigerweise ohne therapeutische Konsequenz ${ }^{25}$. Die verbesserte Diagnostik repräsentiert aus dieser Perspektive eine Inve-

20: Vgl. SCHROEDER, S.A. et al. (1973); DANIELS,M./SCHROEDER, S.A. (1977), S. 486f.; GREENLAND, P./MUSHLIN, A.I./GRINER, P.F. (1979), S. 867 sowie DONALDSON, R.M./FEINSTEIN, A.R./JOYCE, C.M. (1984).

21: Vgl. MEISTER, H. (1985), S. 683. In einer empirischen Studie konnte allerdings ein interessanter "Placebo-Effekt" dieser aus medizinischer Sicht unnötigen Diagnosetests nachgewiesen werden; vgl. SOX, H.C./MARGULIES, I./SOX, C.H. (1981).

22: HASINGER, A. (1985), S. 110.

23: Vgl. MANUEL, B.M. (1990), S. 628.

24: Vgl. WIELAND, W. (1975), der zudem die Bedeutung der Diagnose generell für überschätzt hält.

25: Vgl. in diesem Kontext auch das Beispiel bei HASINGER, A. (1985), S. $107 \mathrm{f}$. 
stition, deren künftige Erträge maßgeblich von der Weiterentwicklung des dazu komplementären therapeutischen Wissens abhängen ${ }^{26}$.

Mit dieser keineswegs vollständigen Aufzählung sind einige wichtige Einflußfaktoren genannt, welche die starke Ausweitung diagnostischer Leistungen zu begründen vermögen. Es wäre indessen voreilig anzunehmen, die These einer Übernutzung vorhandener diagnostischer Kapazitäten sei erst in neuerer Zeit entstanden. Dies belegt ein älteres medizinisches Lehrbuch, in dem das "diagnostische Chaos"27 beschrieben wird, mit dem sich der Arzt angesichts der Vielzahl neuer diagnostischer Methoden konfrontiert sieht. Ähnlich bemängelten angesehene amerikanische Internisten schon vor über fünfzig Jahren den Mißbrauch, der im oftmals unkritischen Einsatz der Labordiagnostik besteht ${ }^{28}$.

\subsection{Anliegen der Arbeit und Disposition}

Aus ökonomischer Sicht impliziert die These einer exzessiven medizinischen Diagnostik, daß insgesamt zu viele Ressourcen in die Erstellung diagnostischer Leistungen fließen. Unabhängig von der Gültigkeit dieser Behauptung im allgemeinen läßt sich eine derartige Fehlallokation im Einzelfall nur belegen, wenn man die interessierende Leistung einer gesamtwirtschaftlichen Vorteilhaftigkeitsanalyse unterzieht. Die vorliegende Arbeit versucht, hierzu einen Beitrag in konzeptioneller Hinsicht zu leisten, indem mit dem Nutzen des Patienten eine zentrale Komponente in einem entscheidungstheoretischen Ansatz abgebildet und analysiert wird.

In diesem Zusammenhang geht es vor allem um die Erfassung und die Bewertung derjenigen Gesundheitseffekte, die der Arzt dem Patienten aufgrund der diagnostischen Information verschaffen kann. Obwohl beispielsweise Kosten-Nutzen-Analysen auf dem Gebiet der medizinischen Diagnostik schon vorliegen ${ }^{29}$, fehlt bislang eine umfassende Darstellung der allgemeinen Kennzeichen diagnostischer Leistungen, die zugleich die damit verbundenen Auswirkungen auf die Wohlfahrt des Patienten genauer abbildet. Die vorliegende Arbeit versucht, diese Lücke zu schließen, indem die Information und der Nutzen, den die medizinischen Diagnostik dem Pati-

26: Vgl. BANTA, H.D./McNEIL, B.J. (1978), S. 17; WARNER, K.E.JLUCE, B.R. (1982), S. 113.

27: SCHULTEN, H. (1961), S. 47.

28: Vgl. MILLER, S.R. (1932), S. 348. 
enten stiften kann, in einem allgemeinen entscheidungstheoretischen Modellrahmen analysiert werden.

Mit der Entscheidung, bei einem Patienten weitere Befunde zu erheben, entschließt sich der Arzt dazu, zusätzliche Informationen zu beschaffen, die ihm Aufschluß über den Krankheitszustand des Patienten zu geben versprechen. In produktionstheoretischer Hinsicht repräsentieren diagnostische Leistungen daher Informationsgüter, deren unmittelbare Auswirkung in einer Veränderung der Informationslage des Arztes besteht. Unter bestimmten Voraussetzungen läßt sich dieser Effekt durch die Revision derjenigen Wahrscheinlichkeiten beschreiben, die der Arzt den möglichen Krankheitszuständen beimißt. Dieser Tatbestand bildet die Grundlage für die Definition der Informationsleistung, die eine diagnostische Leistung in einer gegebenen Situation dem Arzt erbringt. Ein wesentliches Anliegen der vorliegenden Arbeit besteht darin, die in einer diagnostischen Leistung enthaltene neue Information anhand dieser Informationsleistung darzustellen und in einem geeigneten Modellrahmen zu analysieren.

Die in der Literatur bereits vorhandenen Arbeiten zur Ermittlung des Nutzeneffekts, den die Information einer diagnostischen Leistung beim Patienten auslöst, vermögen nicht vollständig zu befriedigen. Eine Gruppe von Autoren vertritt einen zu allgemeinen Ansatz, der von den spezifischen Charakteristika einer medizinischen Entscheidungssituation weitgehend abstrahiert und daher eine genaue Beschreibung des interessierenden Nutzeneffekts nicht zuläßt ${ }^{30}$. Im Gegensatz dazu untersuchen die übrigen Autoren einzelne Diagnosetests und verharren damit auf der Ebene speziell ausgewählter Beispiele ${ }^{31}$. Die vorliegende Arbeit entwickelt aus diesem Grund einen eigenen Modellrahmen, der die gewünschte Beschreibung des patientenbezogenen Nutzeneffekts der medizinisch-diagnostischen Information in allgemeiner Form leisten soll. Die darauf aufbauende, detaillierte Analyse dieses Nutzeneffekts stellt das zentrale Anliegen der Arbeit dar. Bei dem zu diesem Zweck verwendeten entscheidungstheoretischen Ansatz handelt es sich im Vergleich zu den oben angesprochenen Arbeiten um einen Ansatz mit mittlerem Abstraktionsgrad, mit dessen

29: Einen Überblick über Kosten-Nutzen-Analysen diagnostischer Leistungen geben DRUMMOND, M.F. et al. (1986), S. 114ff.

30: Vgl. CHU, J.T. (1967); FLAGLE, C.D. (1967); ATTCHISON, J. (1970); PATRICK, E.A. (1977); PATRICK, E.A. (1979); HABBEMA, J.D.F./HILDEN, J. (1981); GROSS, R. (1982) und BÜTTNER, J. (1985). 
Hilfe unter anderem die Zahlungsbereitschaft eines Patienten für den Vorteil ermittelt werden kann, der ihm aus einer diagnostischen Leistung erwächst.

Die Bestimmung individueller Zahlungsbereitschaften für diagnostische Leistungen erscheint aus mehreren Gründen bedeutsam. Zunächst existieren für diese Leistungen in der Regel keine Marktpreise. Dies hat zur Folge, daß vorhandene Preisrelationen nicht unmittelbar in eine gesamtwirtschaftliche Vorteilhaftigkeitsanalyse eingehen können. Weiterhin besteht in vielen Ländern die Möglichkeit oder sogar der Zwang zu einem umfassenden Krankenversicherungsschutz, der den Preis, den der Patient für die von ihm in Anspruch genommenen medizinischen Leistungen zu zahlen hat, auf (nahezu) Null reduziert. Schließlich besitzt der Arzt einen erheblichen Einfluß auf die Festlegung der individuellen Nachfrageentscheidung. Unter diesen Voraussetzungen läßt sich von der Inanspruchnahme etwa einer diagnostischen Leistung nicht auf deren Wertschätzung durch den Patienten schließen ${ }^{32}$. In diesem Fall können Zahlungsbereitschaften dazu dienen, die individuelle Wertschätzung dieser Leistung in theoretischer Hinsicht exakt anzugeben.

Am Beginn des zweiten Kapitels, das die Grundlagen der Analyse diagnostischer Leistungen behandelt, steht eine Erläuterung verschiedener Kosten- und Nutzeneffekte, die grundsätzlich im Rahmen einer gesamtwirtschaftlichen Vorteilhaftigkeitsanalyse medizinischer Leistungen auftreten können. Besondere Aufmerksamkeit verdienen in diesem Zusammenhang direkte intangible Effekte, die im wesentlichen die gesundheitlichen Auswirkungen beim Patienten beschreiben. Im Anschluß daran folgt eine Darstellung individueller Zahlungsbereitschaften, die in theoretischer Hinsicht das geeignete Konzept zur Bewertung von Kosten- und Nutzeneffekten bzw. der damit verbundenen individuellen Wohlfahrtsänderungen repräsentieren. Da die beim Patienten aufgrund einer diagnostischen Leistung mögliche Wohlfahrtsänderung im allgemeinen vom Zufall abhängt, ist es notwendig, individuelle Zahlungsbereitschaften bei Unsicherheit abzuleiten.

Mit der medizinischen Diagnostik wird in Abschnitt 2.3 das zentrale Erkenntnisobjekt der vorliegenden Arbeit vorgestellt. Von besonderer Bedeutung erweisen sich dabei diagnostische Maßnahmen, die als Teil des diagnostischen Prozesses den Ge-

31: Vgl. z.B. GINSBERG, A.S./OFFENSEND, F.L. (1968); KEELER, E.B. (1976) sowie insbesondere WEINSTEIN, M.C. et al. (1980).

32: Vgl. dazu ausführlicher WILLE, E. (1986), S. $103 \mathrm{f}$. 
genstand der gesamten weiteren Analyse darstellen. Den Abschluß des zweiten Kapitels bildet die Erläuterung einiger Bestandteile des entscheidungstheoretischen Modellrahmens, der später zur Untersuchung des Nutzeneffekts dient, den eine diagnostische Maßnahme beim Patienten auslöst. Die Ausführungen beschränken sich an dieser Stelle auf diejenigen Voraussetzungen, die zur Analyse der Information diagnostischer Maßnahmen benötigt werden.

Das dritte Kapitel behandelt schwergewichtig die Ansätze zur Kennzeichnung der Information, die der Arzt durch den Einsatz einer diagnostischen Maßnahme zusätzlich erhält. Dabei konzentriert sich die Darstellung zunächst auf den Spezialfall binärer bzw. binarisierter Diagnosetests, dem in diesem Zusammenhang auch die Literatur ihr Hauptaugenmerk schenkt. Aufgrund ihrer einfachen Struktur erscheint diese spezielle Gruppe diagnostischer Maßnahmen in besonderem Maße geeignet, das grundsätzliche Vorgehen zur Bestimmung der Informationsleistung in dem gewählten Modellrahmen zu veranschaulichen. Dieses Argument gilt gleichermaßen für die Analyse der Abhängigkeit, welche die Informationsleistung einer derartigen diagnostischen Maßnahme von der in der Ausgangslage - d.h. vor ihrem Einsatz - vorhandenen Information aufweist.

Daran anknüpfend läßt sich die Informationsleistung ganz allgemein für sämtliche diagnostischen Maßnahmen angeben, die in dieser Arbeit betrachtet werden. In diesem Kontext erfolgt eine Charakterisienung von zwei besonders interessanten Kategorien, die das Spektrum möglicher Informationsleistungen eingrenzen. Diese beiden polaren Fälle repräsentieren diejenigen diagnostischen Maßnahmen, die dem Arzt entweder vollkommene oder überhaupt keine Information in Bezug auf den Krankheitszustand des Patienten liefern. Am Ende des dritten Kapitels werden diagnostische Maßnahmen aus der Perspektive der Informationstheorie analysiert. Dies geschieht mit der doppelten Zielsetzung, sowohl den Begriff der diagnostischen Information im Sinne eines Informationsgewinns zu präzisieren als auch die Verbindung zur bisher untersuchten Informationsleistung herauszuarbeiten.

Im Mittelpunkt des vierten Kapitels steht die Analyse des Nutzeneffekts, den der Arzt dem Patienten aufgrund der Information einer diagnostischen Maßnahme verschaffen kann. Dazu sind zunächst weitere Voraussetzungen anzugeben, welche die Beschreibung der zugrundeliegenden medizinischen Entscheidungssituation vervollständigen. Mit ihrer Hilfe erfolgt dann die Ableitung der Zahlungsbereitschaft, die ein Patient für die optimale Verwertung der diagnostischen Information durch den 
Arzt besitzt. Damit liegt eine individuelle Bewertung eines wesentlichen Nutzeneffekts der diagnostischen Information vor, die unmittelbar in eine gesamtwirtschaftliche Vorteilhaftigkeitsanalyse eingehen kann. Dabei vereinfachen sich die Ermittlung und die Untersuchung dieses Optionspreises einer diagnostischen Maßnahme beträchtlich, wenn die Präferenzen des Patienten zusätzlichen Restriktionen genügen. Diese günstige Eigenschaft legt es nahe, den Spezialfall des Optionspreises einer diagnostischen Maßnahme bei "linearem Risikonutzen" des Patienten einer gesonderten Analyse zu unterziehen.

Im Anschluß daran wird der entscheidungstheoretische Ansatz dieser Arbeit weiter ausgewertet, um unter anderem die dabei gewonnenen Resultate mit den Ergebnissen anderer Studien zu vergleichen. In diesem Zusammenhang geht es zunächst um die Frage, inwieweit der hier verwendete Modellrahmen denjenigen Nutzeneffekt vollständig erfaßt, der sich beim Patienten aufgrund der diagnostischen Information einstellt. Der Schwerpunkt der Ausführungen liegt indessen auf der Analyse des Einflusses, den die einzelnen Komponenten der medizinischen Entscheidungssituation auf die Existenz und den Umfang des patientenbezogenen Nutzeneffekts einer diagnostischen Maßnahme besitzen. Dabei eröffnet sich zugleich die Möglichkeit, die in der Literatur zu diesem Thema verwendeten Ansätze aus einer einheitlichen Perspektive zu untersuchen. Schließlich wird mit den intangiblen Kosteneffekten, die der Einsatz einer diagnostischen Maßnahme beim Patienten verursachen kann, eine wichtige Erweiterung des entscheidungstheoretischen Modellrahmens diskutiert.

Das letzte Kapitel faßt die wichtigsten Ergebnisse der Arbeit zusammen und schließt mit einem Ausblick, der im Hinblick auf die medizinische Diagnostik Ansatzpunkte für weitere Forschungsprojekte skizziert. 
Walter Ried - 978-3-631-75573-0

Downloaded from PubFactory at 01/11/2019 03:26:15AM

via free access 


\section{GRUNDLAGEN}

\subsection{Kosten- und Nutzeneffekte im Gesundheitswesen}

\subsubsection{Darstellung und Kategorisierung}

Jede Entscheidung, die ein Arzt im Hinblick auf die weitere Vorgehensweise bei einem Patienten trifft, hat Auswirkungen auf die Allokation im Gesundheitswesen. Dies gilt unabhängig davon, ob diese Entscheidung den Einsatz diagnostischer oder therapeutischer Leistungen oder eine andere ärztliche Handlung vorsieht ${ }^{1}$. Zur Beurteilung der dadurch bewirkten Veränderung der Allokation stehen grundsätzlich verschiedene Perspektiven zur Auswahl. Das Ergebnis einer derartigen Untersuchung hängt im allgemeinen davon ab, ob diese z.B. aus dem Blickwinkel eines einzelnen Arztes oder eines Krankenversicherers erfolgt ${ }^{2}$.

Aus volkswirtschaftlicher Sicht liegt es indessen nahe, Entscheidungen über die Allokation im Gesundheitswesen einer wohlfahrtstheoretischen Analyse zu unterziehen. Im Rahmen der damit verbundenen gesamtwirtschaftlichen Betrachtungsweise geht es im wesentlichen darum festzustellen, ob der Übergang von einer Allokation zu einer anderen für eine Gesellschaft insgesamt als vorteilhaft einzustufen ist. Die wohlfahrtsökonomische Beurteilung einer medizinischen Maßnahme läuft daher auf eine Vorteilhaftigkeitsanalyse der dadurch induzierten Veränderung der Allokation hinaus ${ }^{3}$. Eine notwendige Grundlage dieser Analyse bildet die Bestandsaufnahme - d.h. die quantitative Erfassung und die monetäre Bewertung - derjenigen Wirkungen, welche die zu untersuchende Allokationsentscheidung auf die individuellen Wohlfahrtspositionen zeitigt.

Im allgemeinen wird die Wohlfahrt einiger Individuen durch eine Allokationsentscheidung negativ beeinflußt, während sich gleichzeitig die Wohlfahrtspositionen anderer Individuen verbessern. In diesem Zusammenhang repräsentiert eine Verringerung der individuellen Wohlfahrt einen Kosteneffekt. Entsprechend zählt eine

1: In die zuletzt genannte Kategorie fällt etwa die Einweisung des Patienten in ein Krankenhaus; vgl. Abschnitt 4.2.1.

2: Vgl. dazu allgemein LUFT, H.S. (1976); WEINSTEIN, M.C. (1990) sowie zur Analyse von Vorsorgeuntersuchungen aus der Perspektive eines Krankenversicherers LUCE, B.R. (1981), S. 3f. und GRAVELLE, H.S.E./SIMPSON, P.R./CHAMBERLAIN, J. (1982).

3: In diesem Zusammenhang sind keineswegs nur diejenigen allokativen Effekte zu berücksichtigen, die im Gesundheitswesen auftreten. 
Erhöhung der individuellen Wohlfahrt zu den Nutzeneffekten, sofern sie auf die Veränderung der Allokation zurückgeht. Diese Terminologie entstammt der KostenNutzen-Analyse, die eine wichtige Methode der gesamtwirtschaftlichen Vorteilhaftigkeitsanalyse darstellt ${ }^{4}$. Neben dieser Methode existieren noch andere Verfahren, die eine gesamtwirtschaftliche Beurteilung von Allokationsentscheidungen leisten können. Weil diese Verfahren in ihrem Anspruch weniger umfassend sind und daher nur unter restriktiveren Voraussetzungen eine Aussage über die gesamtwirtschaftliche Vorteilhaftigkeit einer Maßnahme liefern, vermeiden sie zugleich einige Bewertungsprobleme, welche die Kosten-Nutzen-Analyse in ihrer praktischen Anwendung kennzeichnen 5 .

In der Wohlfahrtstheorie wird gezeigt, da $B$ individuelle Wohlfahrtsänderungen grundsätzlich in monetären Einheiten gemessen werden können. Es handelt sich dabei um maximale Zahlungsbereitschaften, die ein Individuum aufgrund der bei ihm auftretenden Folgen einer Allokationsentscheidung entfaltet ${ }^{6}$. Diese individuellen Zahlungsbereitschaften repräsentieren monetäre Nutzenäquivalente, die als Kosten

4: Grundlegend zur Kosten-Nutzen-Analyse sind z.B. ANDERSON, L.G./SETTLE, R.F. (1977); PEARCE, D.W./NASH, C.A. (1981); PEARCE, D.W. (1983) sowie MISHAN, E.J. (1988). Die Kosten-Nutzen-Analyse stützt sich wesentlich auf das Konzept der potentiellen ParetoVerbesserung, das auf Kaldor und Hicks zurückgeht; vgl. KALDOR, N. (1939) und HICKS, J.R. (1940). Indessen besitzt dieses Konzept Schwächen, die seine Tauglichkeit für den Vergleich verschiedener Allokationen einschränken; vgl. dazu SCITOVSKY, T. (1941); GORMAN, W.M. (1955); BOADWAY, R.W. (1974) sowie BOADWAY, R.W./BRUCE, N. (1984), S. 96ff. Im übrigen besteht keine Äquivalenz zwischen einer potentiellen Pareto-Verbesserung und den in der Kosten-Nutzen-Analyse üblicherweise verwendeten Entscheidungskriterien; vgl. BOADWAY, R.W./BRUCE, N. (1984), S. 262ff. und BLACKORBY, C./DONALDSON, D. (1990).

Nicht zuletzt aufgrund dieser Probleme versucht man neuerdings, die Kosten-Nutzen-Analyse direkt in eine allgemeine Gleichgewichtsanalyse einzubetten; vgl. dazu HAMMOND, P.J. (1980) und DREZE, J/STERN, N. (1987).

5: Da Bewertungsprobleme im Gesundheitswesen häufig auftreten, gelangen diese im Vergleich zur Kosten-Nutzen-Analyse weniger umfassenden Methoden dort verstärkt zum Einsatz; vgl. DRUMMOND, M.F./STODDART, G.L./TORRANCE, G.W. (1987) sowie JOHANNESSON, M./JÖNSSON, B. (1990), S. $5 \mathrm{ff}$.

6: Diese Formulierung deutet schon an, daß verschiedene Konzepte zur Messung individueller Zahlungsbereitschaften existieren; vgl. dazu ausführlich Abschnitt 2.2. 
(Nutzen) bezeichnet werden, falls sie Kosteneffekte (Nutzeneffekte) erfassen ${ }^{7}$. Demnach ist eine Allokationsentscheidung durch die vollständige Angabe aller individuellen Kosten und Nutzen charakterisiert. Die einzelnen Kosten- und Nutzengrößen bilden daher den zentralen Bestandteil einer gesamtwirtschaftlichen Vorteilhaftigkeitsanalyse, die auf dem individualistischen Ansatz der Paretianischen Wohlfahrtsökonomik beruht.

In der Regel liegen in einer Ökonomie für die mit einer Veränderung der Allokation verbundenen Kosten- und Nutzeneffekte zumindest teilweise Bewertungen durch Marktpreise oder in anderer Form vor. Dabei sind grundsätzlich drei verschiedene Konstellationen zu unterscheiden, die im Hinblick auf das Verhältnis zwischen derartigen Bewertungen und den individuellen Kosten und Nutzen auftreten können. Zunächst folgt aus den zuvor angestellten Überlegungen unmittelbar, wann eine schon vorhandene Bewertung unmodifiziert in die wohlfahrtsökonomische Analyse einer Allokationsentscheidung übernommen werden kann. Insoweit nämlich eine Bewertung einen Kosteneffekt (Nutzeneffekt) exakt widerspiegelt, handelt es sich bei den von einem Individuum empfangenen (geleisteten) Zahlungen um Kosten (Nutzen) im Sinne der Kosten-Nutzen-Analyse. In diesem günstigen Fall wird die Wohlfahrtsposition des betrachteten Individuums durch die untersuchte Allokationsentscheidung nicht berührt. Eine gesamtwirtschaftliche Vorteilhaftigkeitsanalyse kann daher diese Zahlungen direkt auf der Kosten- bzw. Nutzenseite verbuchen.

Indessen brauchen schon vorhandene Bewertungen die individuellen Wohlfahrtsänderungen nicht mit Notwendigkeit adäquat auszudrücken. Besteht eine derartige Diskrepanz, dann entsprechen die von einem Individuum geleisteten bzw. empfangenen Zahlungen nicht den tatsächlichen Nutzen bzw. Kosten, die aufgrund der Allokationsentscheidung bei ihm anfallen. Die vorhandenen Bewertungen sind ent-

7: Die individuellen Kosten einer Allokationsentscheidung messen entgangene Nutzeneffekte in monetären Einheiten und entsprechen daher gerade ihren Opportunitätskosten; vgl. dazu ausführlich BUCHANAN, J.M. (1969); FISHER, G.H. (1971), S. $24 f f$.

Im übrigen erscheint die im deutschen Sprachgebrauch übliche Terminologie insofern unglücklich gewählt, als der Begriff des "Nutzens" in der Theorie individueller Präferenzen noch eine andere Bedeutung trägt. Davon sind die im Text angesprochenen Nutzen - etwa im Sinne der Kosten-Nutzen-Analyse - strikt zu unterscheiden. Die englische Terminologie vollzieht diesen Unterschied auch semantisch, indem mit "utility" und "benefit" zwei verschiedene Begriffe zur Abgrenzung dienen. Die frühe deutschsprachige Literatur zur Gesundheitsökonomie trug diesem Sachverhalt Rechnung und übersetzte "benefits" als "Erträge"; vgl. RAHNER, E. (1965) sowie BILLERBECK, K. (1968). 
sprechend zu korrigieren, um zu den richtigen Kosten- bzw. Nutzengrößen zu gelangen ${ }^{8}$. Schließlich kann es vorkommen, daß für einzelne Kosten- oder Nutzeneffekte noch keinerlei Bewertung vorliegt. Einer gesamtwirtschaftlichen Vorteilhaftigkeitsanalyse fällt unter dieser Voraussetzung die schwierige Aufgabe zu, anhand eigener Bewertungen die damit verbundenen individuellen Kosten und Nutzen zu ermitteln.

Typischerweise tritt bei Allokationsentscheidungen, die z.B. einer Kosten-NutzenAnalyse unterzogen werden, jede dieser drei Konstellationen auf. Obwohl alle Kosten- und Nutzeneffekte in individuellen Wohlfahrtsänderungen letztlich dieselbe Ursache besitzen, wird man daher im Rahmen einer gesamtwirtschaftlichen Vorteilhaftigkeitsanalyse dennoch ganz unterschiedlich vorgehen müssen, um die dazugehörigen Kosten und Nutzen zu ermitteln. Es liegt nahe, je nach der erforderlichen Vorgehensweise einzelne Kosteneffekte (Nutzeneffekte) in Gruppen zusammenzufassen, wobei sämtliche Effekte innerhalb einer Gruppe gerade eine Kostenart (Nutzenart) repräsentieren. Eine derartige Gruppierung in verschiedene Kosten- und Nutzenarten hilft, die zur Beurteilung einer Allokationsentscheidung benötigte Information in geeigneter Form zu strukturieren. Insgesamt existiert eine Vielfalt unterschiedlicher Klassifikationen in Kosten- und Nutzenarten, da neben dem oben besprochenen Kriterium der Existenz von Bewertungen noch andere Kriterien zur Einteilung der möglichen Kosten- und Nutzeneffekte dienen können ${ }^{9}$.

In der Gesundheitsökonomik findet man häufig eine Unterscheidung zwischen tangiblen und intangiblen Effekten auf der einen und zwischen direkten und indirekten Effekten auf der anderen Seite ${ }^{10}$. Wie die nachfolgende Erläuterung zeigt, beruht diese Unterscheidung auf zwei Kriterien, die gemeinsam bei konsistenter Anwendung eine Einteilung in jeweils vier verschiedene Kosten- und Nutzenarten ermöglichen. Um das jeweils zugrundeliegende Gliederungsprinzip zu verdeutlichen, kommen die beiden Kriterien zunächst einzeln zur Sprache. Die Abgrenzung intan-

8: Vgl. dazu ANDEL, N. (1977), S. 488ff.; HESSE, H. (1980), S. 371ff.; ARNOLD, V. (1980), S. 383ff sowie SCHMID, A.A. (1989), S. 59ff.

9: Vgl. dazu WEISBROD, B.A. (1968); ANDERSON, L.G./SETTLE, R.F. (1977), S. 19ff.; MUSGRA VE, R.A./MUSGRAVE, P.B./KULLMER, L. (1987), S. 202ff.

10: Eine weitere Unterscheidung, die intermediäre und finale Effekte abgrenzt, spielt insbesondere in der Analyse der medizinischen Diagnostik eine wichtige Rolle; vgl. dazu Abschnitt 4.4.1.1. 
gibler von tangiblen Kosten- bzw. Nutzeneffekten stützt sich wesentlich auf die Existenz einer entsprechenden Bewertung in der betrachteten Ökonomie ${ }^{11}$. Wenn für eine individuelle Wohlfahrtsänderung eine monetäre Bewertung durch Marktpreise oder in anderer Form vorliegt, läßt sich der dazugehörige Kosten- bzw. Nutzeneffekt als tangibel einstufen. Dagegen handelt es sich um einen intangiblen Effekt, falls eine gesamtwirtschaftliche Vorteilhaftigkeitsanalyse für eine individuelle Wohlfahrtsänderung eine von Grund auf eigene Bewertung entwickeln muß. Die Klassifikation in tangible und intangible Effekte nimmt Bezug auf das System der in einer Ökonomie vorhandenen Bewertungen und erweist sich insofern als zeitabhängig ${ }^{12}$.

Im Gesundheitswesen repräsentiert beispielsweise der Verbrauch von Medikamenten einen tangiblen Effekt, da hierfür Preise existieren ${ }^{13}$. In diese Kategorie fallen grundsätzlich auch ambulante ärztliche Leistungen, für die eine Bewertung in Gestalt des dazugehörigen Honorars vorliegt. Allerdings erfolgt die Bewertung in diesem Fall zumindest in der Bundesrepublik nicht über Marktpreise, sondern im Rahmen einer Gebührenordnung ${ }^{14}$. Einen weiteren tangiblen Effekt stellt schließlich der Produktionsausfall dar, der durch die Krankheit erwerbstätiger Personen entsteht ${ }^{15}$. Im Gegensatz dazu sind die Schmerzen eines Patienten als intangibler Effekt

11: Vgl. WEISBROD, B.A. (1968), S. 261; ANDERSON, L.G./SETTLE, R.F. (1977), S. 23 sowie MISHAN, E.J. (1988), S. 203.

12: Diese Abhängigkeit betonen z.B. WEISBROD, B.A. (1968), S. 261f. und HANUSCH, H. (1987), S. 10.

Es wäre indessen unzweckmäßig, intangible Effekte als grundsätzlich nicht meß- bzw. bewertbar einzustufen, da die Wohlfahrtstheorie die allgemeine MeBbarkeit individueller Wohlfahrtsänderungen in monetären Einheiten nachweist. Die Einordnung eines Effekts als intangibel hängt wesentlich von der Spezifikation und Zuordnung von Eigentumsrechten und damit der Entwicklung des Marktsystems in einer Ökonomie ab. Beispielsweise handelt es sich bei den durch die Luftverschmutzung verursachten individuellen Wohlfahrtseinbußen zunächst um intangible Kosteneffekte, die bei Verwirklichung einer Zertifikatelösung tangibel würden.

13: Es hängt von der betrachteten Allokationsentscheidung ab, ob dieser Effekt auf der Kostenoder der Nutzenseite zu verbuchen ist: induziert eine medizinische Maßnahme einen erhöhten (reduzierten) Verbrauch von Medikamenten, repräsentiert die dazugehörige Bewertung Kosten (Nutzen). Eine analoge Argumentation gilt für die anderen Effekte, die im Text angesprochen werden.

14: Zur Preisbildung bei ambulanten ärztlichen Leistungen in der Bundesrepublik vgl. ZOHLNHÖFER, W./SCHMIDT, P.-G. (1985).

15: Dieser Effekt spielt insbesondere bei Krankheitskostenstudien eine wichtige Rolle; vgl. HODGSON, T.A./MEINERS, M.R. (1982); TOLPIN, H.G./BENTKOVER, J.D. (1983) sowie HENKE, K.-D. (1986). 
einzuordnen, da für sie keine Bewertung in monetären Einheiten vorliegt. In dieselbe Kategorie gehören die Folgen einer Umstellung des Lebenswandels, die Angehörige bei einem schweren Krankheitsfall in der Familie zu tragen haben.

Die zweite, in der Gesundheitsökonomik ebenfalls häufig anzutreffende Unterscheidung zwischen direkten und indirekten Effekten erweist sich insofern als problematisch, als die entsprechende Abgrenzung in der Literatur sehr unterschiedlich erfolgt $^{16}$. Dies beeinträchtigt die Nützlichkeit der darauf beruhenden Klassifikation erheblich, da die Vielfalt der vorhandenen Varianten ihre Ordnungsleistung schmälert. In dieser Arbeit werden diejenigen Effekte als direkte Effekte eingestuft, die bei Individuen anfallen, die in einem bestimmten Sinn unmittelbar von der Veränderung der Allokation betroffen sind. Direkte Effekte treten bei Individuen auf, die an der Produktion oder am Konsum derjenigen Leistungen teilnehmen, welche die zu untersuchende Allokationsentscheidung beinhaltet. Demzufolge können bei Individuen, die nicht in diesem Sinn unmittelbar von der Veränderung der Allokation betroffen sind, ausschließlich indirekte Effekte auftreten ${ }^{17}$. Dieses formale Kriterium hat unter anderem zur Folge, daß beim Patienten eintretende Wohlfahrtsänderungen grundsätzlich als direkte Effekte medizinischer Maßnahmen rangieren.

Die Abgrenzung direkter von indirekten Effekten kann anhand derselben Beispiele veranschaulicht werden, die zuvor zur Illustration der Einteilung in tangible und intangible Kosten- bzw. Nutzeneffekte dienten. In diesem Zusammenhang zählen sowohl der Verbrauch an Medikamenten als auch die von einem Arzt erbrachten Leistungen zu den direkten Effekten einer Allokationsentscheidung im Gesundheitswesen. Diese Zuordnung gilt ebenfalls für die Schmerzen, die ein Patient erlei-

16: Vgl. dazu die Diskrepanzen in den Klassifikationen von WEINSTEIN, M.C./FINEBERG, H.V. (1978); WEINSTEIN, M.C. (1981), S. 318; STODDART, G.L. (1982), S. 400f.; HANUSCH, H. (1987), S. 9; SCHMID, A.A. (1989), S. 59ff. sowie STRAUB, W.H./GUR, D. (1990).

17: Neben ihrer einfachen Anwendbarkeit dürfte ein weiterer Vorteil dieser Abgrenzung darin bestehen, daß sie explizit auf die Notwendigkeit verweist, im Rahmen einer gesamtwirtschaftlichen Vorteilhaftigkeitsanalyse auch Wohlfahrtsänderungen zu berücksichtigen, die außerhalb des von einer Allokationsentscheidung unmittelbar betroffenen Personenkreises auftreten.

Indessen weist auch diese Klassifikation von Kosten- bzw. Nutzeneffekten eine Abhängigkeit von der Entwicklung des Marktsystems in einer Ökonomie ab: ein (technologischer) externer Effekt, der ursprünglich als indirekter Effekt einzustufen war, rangiert nach erfolgter Internalisierung als direkter Effekt. 
det. Dagegen sind die Effekte, die bei seinen Angehörigen auftreten, grundsätzlich der Kategorie der indirekten Effekte zuzuschlagen. Schließlich handelt es sich bei den morbiditätsbedingten Produktionsausfällen zumindest dann um indirekte Effekte, falls das betrachtete Individuum unselbständig beschäftigt ist ${ }^{18}$.

Abb. 1: Zur Klassifikation von Effekten im Gesundheitswesen

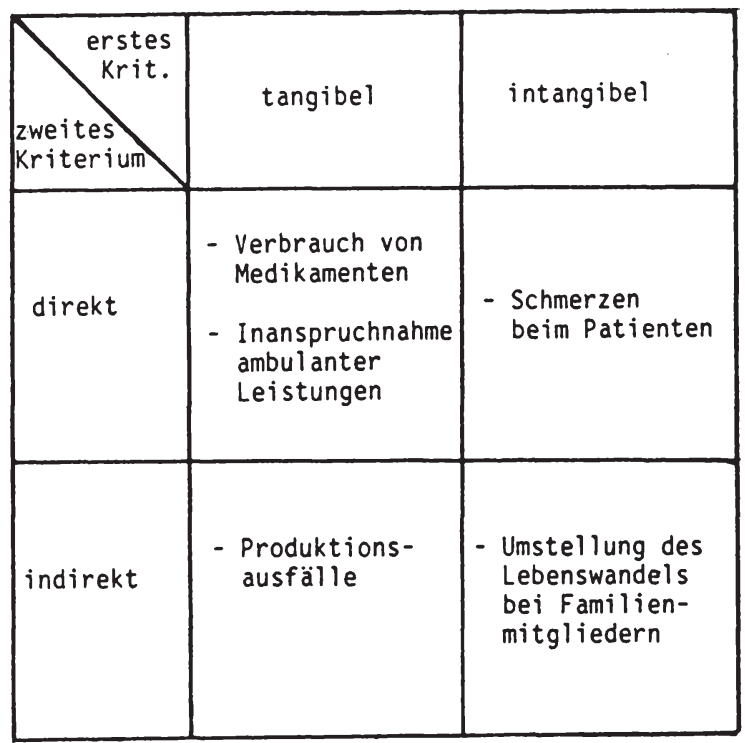

Wendet man die beiden Unterscheidungsmerkmale gemeinsam an, um zu einer verfeinerten Klassifikation von Kosten- und Nutzeneffekten im Gesundheitswesen zu

18: Sofern sie die Folgen eines Produktionsausfalls selbst zu tragen haben, müßte dieser Effekt gemä $B$ der hier verwendeten Abgrenzung bei selbständig Beschäftigten als direkter Effekt eingestuft werden. 
gelangen, ergeben sich jeweils vier verschiedene Kategorien. Wie Abbildung 1 zeigt, werden diese durch tangible direkte, tangible indirekte, intangible direkte sowie intangible indirekte Effekte repräsentiert ${ }^{19}$.

Mit diesen vier Kosten- bzw. Nutzenarten ergibt sich eine nützliche Gliederung derjenigen Information, die für eine gesamtwirtschaftliche Vorteilhaftigkeitsanalyse benötigt wird. Dies gilt nicht zuletzt deshalb, weil das Vorgehen zur Erfassung und Bewertung eines Effekts im Rahmen einer derartigen Analyse wesentlich davon abhängt, welcher Kategorie dieser Effekt angehört. Zunächst strukturiert das Kriterium zur Abgrenzung direkter und indirekter Effekte das Erfassungsproblem, indem zwei grundsätzlich verschiedene Gruppen von Individuen identifiziert werden, bei denen die Effekte einer Allokationsentscheidung auftreten können. Sodann weist die Unterscheidung einer tangiblen und einer intangiblen Kosten- bzw. Nutzenart insbesondere auf jene Effekte hin, deren Bewertung Probleme bereitet.

\subsubsection{Die Bedeutung direkter intangibler Effekte}

Grundsätzlich können bei medizinischen Leistungen sämtliche Kosten- und Nutzenarten auftreten, die im vorangegangenen Abschnitt angesprochen wurden. Die Aufgabe einer gesamtwirtschaftlichen Vorteilhaftigkeitsanalyse dieser Leistungen besteht deshalb darin, in jeder Kosten- und Nutzenkategorie die dazugehörigen Effekte möglichst vollständig zu erfassen und korrekt zu bewerten. Jedoch beschränken sich die in der gesundheitsökonomischen Literatur vorliegenden Kosten-Nutzen-Analysen häufig darauf, lediglich die tangiblen Effekte in diesem umfassenden Sinn zu berück-

19: Vgl. CULliS, J.G./WEST, P.A. (1979), S. 172; MUSGRAVE, R.A./MUSGRAVE, P.B./KULLMER, L. (1987), S. 203 sowie WILLE, E. (1989), S. 334ff.

Im übrigen findet man in der gesundheitsökonomischen Literatur noch andere Klassifikationen der Kosten- und Nutzenarten, wobei die wohl am häufigsten gebrauchte Einteilung direkte, indirekte und intangible Effekte unterscheidet; vgl. KLARMAN, H.E. (1965); GLASS, N.J. (1973); ALTER, UJKLAUSING, M. (1974); KLARMAN, H.E. (1974); HODGSON, T.A./MEINERS, M.R. (1982); ADAM, H. (1985) sowie HENKE, K.-D. (1986). Indessen handelt es sich um eine inkonsistente Klassifikation, die aus der unvollständigen Verwendung zweier verschiedener Abgrenzungskriterien resultiert; vgl. dazu ebenfalls kritisch WILLE, E. (1989), S. 334.

Ähnlich problematisch erscheint der Versuch, sämtliche intangiblen Effekte der Kategorie der indirekten Effekte zuzurechnen; vgl. dazu SIEBERT, C.D./ZAIDI, M.A. (1975), S. 207; TOLPIN, H.G. (1980), S. 219f. sowie TOLPIN, H.G./BENTKOVER, J.D. (1983), S. 184. 
sichtigen $^{20}$. Es überrascht insofern nicht, daß die Ansätze zur Quantifizierung und Bewertung tangibler Kosten- und Nutzeneffekte besonders weit entwickelt sind ${ }^{21}$. Da Marktpreise im Gesundheitswesen weitgehend fehlen, repräsentieren vorhandene Bewertungen häufig kein adäquates monetäres Äquivalent der mit einer Allokationsentscheidung verbundenen tangiblen Effekte und sind aus diesem Grund zu korrigieren 22 . Ein typisches Beispiel stellen die Ausgabeneinsparungen dar, die aus der frühzeitigen Entlassung eines Patienten aus dem Krankenhaus resultieren. Sofern das Krankenhaus sämtliche Leistungen unspezifisch über einen einheitlichen Pflegekostentagessatz abrechnet, überschätzt diese Ausgabengröße die tatsächlich erreichte Kostenreduktion unter Umständen beträchtlich ${ }^{23}$.

Die exakte Erfassung und Bewertung intangibler Effekte bereitet in der Regel im Vergleich zu den tangiblen Effekten größere Schwierigkeiten. Aus diesem Grund enthalten Kosten-Nutzen-Analysen im Gesundheitswesen nur selten eine monetäre Bewertung dieser Effekte ${ }^{24}$. Man begnügt sich stattdessen damit, intangible Effekte nachrichtlich - d.h. ohne Quantifizierung und Bewertung - zu erfassen, um ihre Existenz zumindest auf diese Weise zu dokumentieren. Allgemein liefert dieses Vorgehen im Rahmen einer gesamtwirtschaftlichen Vorteilhaftigkeitsanalyse zunächst nur dann korrekte Ergebnisse, wenn die untersuchte Allokationsentscheidung keine oder als vernachlässigbar einzustufende intangible Effekte hervorruft ${ }^{25}$. Daneben gibt es Fälle, in denen intangible Effekte ausschließlich oder zumindest hauptsächlich auf je-

20: Vgl. zu dieser Einschätzung z.B. DRUMMOND, M.F./STODDART, G.L. (1985), S. 358.

Zur Kosten-Nutzen-Analyse im Gesundheitswesen vgl. WARNER, K.E./LUCE, B.R. (1982); HORISBERGER, B./VAN EIMEREN, W. (1986) sowie DRUMMOND, M.F. et al. (1986).

21: Auf diesem Gebiet liegen im übrigen die wesentlichen Verdienste der Krankheitskostenstudien; vgl. z.B. HODGSON, T.A. (1983).

22: Vgl. dazu WEISBROD, B.A. (1981), S. 531ff.; CULYER, A.J.MAYNARD, A.K. (1981), S. 6; SCITOVSKY, A.A. (1982) sowie LINDENEG, K. (1985).

23: Vgl. z.B. JÖNSSON, B./LINDGREN, B. (1980), S. $28 \mathrm{f}$.

24: Dies gilt nicht für die Pionierarbeit von KLARMAN, H.E. (1965); vgl. dazu auch ABT, C.C. (1977). Mitunter werden intangible Effekte völlig vernachlässigt, wie z.B. die Arbeiten von STEINER, K.C.SMITH, H.A. (1973); HAGARD, S./CARTER, F./MILNE, R.G. (1976) und GOSS, S. (1983) zeigen.

25: Weil die im Gesundheitswesen typischerweise untersuchten Allokationsentscheidungen medizinische Leistungen repräsentieren, bei denen intangible Effekte vornehmlich auf der Nutzenseite anfallen, betrifft dies im wesentlichen diejenigen Situationen, in denen eine reine Kostenanalyse ausreicht; vgl. dazu CULYER, A.J./MAYNARD, A.K. (1981) sowie allgemein DRUMMOND, M.F./STODDART, G.L./TORRANCE, G.W. (1987), S. $39 \mathrm{ff.}$ 
ner Seite auftreten, die zugleich ein Übergewicht bei den tangiblen Effekten verzeichnet ${ }^{26}$. Unter dieser günstigen Voraussetzung zeigt schon die Analyse der tangiblen Effekte zuverlässig an, ob eine Veränderung der Allokation vorteilhaft im Sinne der Kosten-Nutzen-Analyse ist.

Vielfach sind jedoch die Voraussetzungen einer gesamtwirtschaftlichen Vorteilhaftigkeitsanalyse medizinischer Leistungen weniger günstig, da ihre tangiblen und ihre intangiblen Effekte hauptsächlich auf verschiedenen Seiten der Kosten-Nutzen-Bilanz anfallen ${ }^{27}$. Empirisch dominiert in diesem Zusammenhang diejenige Konstellation, bei der die interessierende Allokationsentscheidung tangible Effekte schwergewichtig auf der Kostenseite aufweist, während ihre intangiblen Effekte vornehmlich die Nutzenseite besetzen ${ }^{28}$. In diesen Fällen stellen Verbesserungen des individuellen Gesundheitszustands ${ }^{29}$ der Patienten einen, wenn nicht den wesentlichen Nutzeneffekt der entsprechenden medizinischen Leistung dar ${ }^{30}$. Dieser direkte intangible Effekt läßt sich als primäre Nutzenkomponente der betrachteten Allokationsentscheidung einstufen, aus der unter Umständen noch weitere Nutzeneffekte abgeleitet werden können ${ }^{31}$. Als Beispiel einer medizinischen Maßnahme, bei der die

26: Dies trifft offenbar auf die Analysen von HORISBERGER, B. (1984) und DAGENAIS, D.L./COURVILLE, L./DAGENAIS, M. (1985) zu.

27: Bei einer ausschließlich nachrichtlichen Berücksichtigung intangibler Effekte kann die Analyse daher keine zuverlässige Aussage über die Vorteilhaftigkeit einer Leistung treffen. Als bescheideneres Ergebnis läßt sich in diesem Fall angeben, für welche monetären Bewertungen der intangiblen Effekte die betrachtete medizinische Leistung insgesamt vorteilhaft erscheint. Wenn z.B. tangible Effekte vornehmlich auf der Kostenseite auftreten, repräsentiert ihr Saldo die "net measurable effects", die als Preisuntergrenze für den Saldo der intangiblen Effekte zu interpretieren sind; vgl. zu diesem Ansatz BUXTON, M.J./WEST, R.R. (1975), S. 378; WEISBROD, B.A. (1981), S. 546; WARNER, K.E./LUCE, B.R. (1982), S. 145 ff. sowie GILLESPIE, K.N. et al. (1986).

28: Aufgrund ihrer größeren empirischen Relevanz konzentrieren sich die weiteren Ausführungen auf diese Konstellation. Indessen gelten analoge Argumente für diejenigen medizinischen Leistungen, die ihre Nutzen in Gestalt von Kosteneinsparungen hauptsächlich durch intangible Kosteneffekte "erkaufen".

29: Der Begriff des Gesundheitszustands umfaßt sowohl subjektive als auch objektive Komponenten; vgl. z.B. BOWLING, A. (1991), S. 3ff.

30: Vgl. WILLIAMS, A. (1974), S. 254; DUNLOP, D.W. (1975), S. 135; MARGOLIS, J. (1977), S. 216; BODEN, L.I. (1979), S. 1210 sowie HODGSON, T.A./MEINERS, M.R. (1982), S. $446 \mathrm{ff}$.

31: Vgl. WEISBROD, B.A. (1981), S. 534; STODDART, G.L. (1982), S. 402. 
zuvor angesprochene Konstellation im Hinblick auf tangible und intangible Effekte vorliegt, läßt sich die Einführung einer neuen, kostspieligen Therapie nennen, die den Gesundheitszustand bestimmter Patienten zu verbessern verspricht.

Die besondere Bedeutung der direkten intangiblen Effekte im Rahmen einer gesamtwirtschaftlichen Vorteilhaftigkeitsanalyse medizinischer Leistungen ergibt sich daher zunächst aus ihrem potentiell großen Umfang, der das Ergebnis einer derartigen Analyse beeinflussen kann. Daneben erscheint eine Untersuchung dieser Effekte im Gesundheitswesen auch deshalb notwendig, weil ihre monetäre Bewertung vergleichsweise wenig entwickelt ist. Beide Gründe sprechen dafür, der Berücksichtigung von Veränderungen des individuellen Gesundheitszustands und ihrer Bewertung durch den Patienten besondere Aufmerksamkeit zu schenken. Dies gilt speziell für die Analyse der medizinischen Diagnostik, da bei Allokationsentscheidungen in diesem Bereich direkte intangible Effekte ebenfalls eine wesentliche Rolle spielen können.

\subsection{Die Bewertung individueller Wohlfahrtsänderungen}

\subsubsection{Individuelle Zahlungsbereitschaften bei Sicherheit}

Das Grundproblem einer gesamtwirtschaftlichen Vorteilhaftigkeitsanalyse von Allokationsentscheidungen besteht in der Erfassung und Messung der damit verbundenen individuellen Wohlfahrtsänderungen. Wie lassen sich diese Kosten und Nutzen ermitteln? Die Pionierarbeit auf diesem Gebiet stammt von Jules Dupuit, der insbesondere die Messung der Nutzeneffekte öffentlicher Projekte untersuchte und zu diesem Zweck das Konzept der maximalen Zahlungsbereitschaft entwickelte ${ }^{32}$. Dupuit versuchte, eine Wertgröße zu bestimmen, die zutreffend den Nutzenzuwachs widerspiegelt, der einem Individuum aus der Verfügbarkeit eines Guts zu einem bestimmten Preis erwächst ${ }^{33}$. In diesem Zusammenhang erkannte er, daß zwischen der maximalen Zahlungsbereitschaft, die ein Individuum für die von ihm nachgefragte Menge eines Guts besitzt, und der von ihm tatsächlich geleisteten Zahlung im allgemeinen ein Unterschied besteht. Die Zahlung bildet lediglich eine monetäre Untergrenze der Zahlungsbereitschaft, die das Individuum maximal für die Möglichkeit entfalten kann, sämtliche Einheiten des betrachteten Guts zu einem einheitlichen

32: Vgl. DUPUIT, J. (1844), S. $261 \mathrm{ff}$.

33: Obwohl aus dieser Formulierung der kardinale nutzentheoretische Ansatz von Dupuit hervorgeht, erweist sich seine Analyse insgesamt in vielerlei Hinsicht als erstaunlich modern. 
Preis zu erwerben. Insoweit die maximale individuelle Zahlungsbereitschaft höher ausfällt als die erfolgte Zahlung, entsteht dem Individuum ein Nutzeneffekt, der in konzeptioneller Hinsicht einer Faktorrente entspricht ${ }^{34}$. Da diese Rente jedoch dem Individuum in seiner Eigenschaft als Konsument zufließt, handelt es sich um eine Konsumentenrente.

Das Konzept der maximalen Zahlungsbereitschaft kann nicht nur in der von Dupuit speziell untersuchten Situation, sondern ganz allgemein dazu dienen, monetäre Äquivalente individueller Wohlfahrtsänderungen abzuleiten. Insofern hat Dupuit die theoretische Grundlage zur Bewertung von Kosten- und Nutzeneffekten geschaffen. Allerdings zeigte die weitere Entwicklung, daß es nicht möglich ist, diese Effekte durch eine einzige, theoretisch vollständig befriedigende Meßgröße zu erfassen. Aus diesem Grund existieren heute verschiedene Ansätze, die jeweils die Veränderung einer individuellen Wohlfahrtsposition anhand von Zahlungsbereitschaften auszudrücken suchen ${ }^{35}$. Die darauf aufbauenden Variationsmaße repräsentieren eine wichtige Klasse individueller Wohlfahrtsmaße $\mathrm{e}^{36}$. Im folgenden werden mit der Kompensations- und der Äquivalenzvariation zwei auf Sir John Hicks zurückgehende Variationsmaße vorgestellt ${ }^{37}$. Diese bilden die in der theoretischen Literatur zur KostenNutzen-Analyse vornehmlich diskutierten Wohlfahrtsmaße, die zugleich geeignet sind, die grundsätzliche Vorgehensweise bei der Messung individueller Wohlfahrtsänderungen zu veranschaulichen.

34: Als Faktorrente bezeichnet man den Überschuß des tatsächlichen Faktorentgelts über diejenige Zahlung, die der Eigentümer des Faktors für diese spezielle Beschäftigung mindestens akzeptieren würde; vgl. z.B. MISHAN, E.J. (1988), S. 54.

35: Die Arbeit von Dupuit wurde zunächst kaum beachtet, bis Alfred Marshall die Analyse von Konsumentenrenten wieder aufnahm; vgl. zur historischen Entwicklung CURRIE, J.M./MURPHY, J.A./SCHMITZ, A. (1971) sowie EKELUND, R.B.JHEBERT, R.F. (1985).

36: Vgl. AHLHEIM, M/WAGENHALS, G. (1988).

Neben den Variationsmaßen bilden die sogenannten Indexmaße eine zweite wesentliche Kategorie individueller Wohlfahrtsmaße; vgl. dazu AHLHEIM, M./ROSE, M. (1989), S. $137 \mathrm{ff}$.

37: Strenggenommen geht lediglich die Idee, individuelle Wohlfahrtsänderungen mit Hilfe einkommenskompensierter ("Hicks'scher") Nachfragen zu messen, auf Hicks zurück, der damit für die entscheidende Verbesserung gegenüber der problembehafteten Marshall'schen Analyse von Konsumentenrenten sorgte. Im Anschluß daran zeigte Henderson, daß dieser Ansatz zu insgesamt vier verschiedenen individuellen Wohlfahrtsmaßen führt; vgl. HENDERSON, A. (1941) sowie HICKS, J.R. (1981). Neben den beiden im Text erläuterten Variationsmaßen handelt es sich dabei noch um die kompensierende und die äquivalente Rente; vgl. dazu NG, Y.-K. (1979), S. 85ff. sowie ausführlich CORNWALL, R.C. (1984), S. $587 \mathrm{ff}$. 
In der weiteren Analyse wird die Konsumentscheidung eines Individuums betrachtet, dem ein Pauscheinkommen $Y$ zur Verfügung steht ${ }^{38}$. Dieser Konsument kann zwischen $\mathrm{N}$ verschiedenen Gütern wählen, die in beliebigen nichtnegativen Mengen erhältlich und deren Preise fest vorgegeben, d.h. durch ihn nicht zu beeinflussen sind ${ }^{39}$. Die Präferenzen des Konsumenten lassen sich durch eine stetige, vollständige und transitive binäre Relation beschreiben, die auf der Konsummenge $\mathrm{X}$, einer geeigneten Teilmenge des $R^{N}$, definiert ist ${ }^{40}$. Unterstellt man ferner strenge Konvexität sowie Nichtsättigung, dann ergibt sich die Möglichkeit, die individuellen Präferenzen vermöge einer streng quasi-konkaven und monotonen Nutzenfunktion zu repräsentieren, deren Definitionsbereich die Konsummenge bildet. Schließlich wird die Nutzenfunktion als zweimal stetig differenzierbar angenommen.

Unter diesen Voraussetzungen maximiert der Konsument seine Präferenzen bei gegebenem Preisvektor $p$ und Pauscheinkommen Y, indem er ein Güterbündel mit folgender Eigenschaft wählt ${ }^{41}$ :

$$
\mathbf{x}^{*}=\arg \max |\mathrm{u}(\mathrm{x})| \mathrm{x} \in \mathrm{X} ; \sum_{\mathrm{i}=1}^{\mathrm{N}} \mathrm{p}_{\mathrm{i}} \cdot \mathrm{x}_{\mathrm{i}}=\mathrm{Y} \mid \text {. }
$$

Dieser Vektor der Marshall'schen Nachfragen ist eindeutig bestimmt und hängt lediglich von den Parametern $p$ und $Y$ ab. Aus diesem Grund läßt sich die allgemeine Lösung des Entscheidungsproblems durch Funktionen $x_{i}(p, Y)$ angeben, die als Marshall'sche Nachfragefunktionen bezeichnet werden. Mit Hilfe dieser Nachfragefunktionen erhält man die indirekte Nutzenfunktion $v(p, Y)$ des Konsumenten, die einer durch $\mathrm{p}$ und $\mathrm{Y}$ gekennzeichneten Situation das maximal erreichbare Nutzenniveau zuordnet ${ }^{42}$ :

$$
\mathrm{v}(\mathrm{p}, \mathrm{y})=\max |\mathrm{u}(\mathrm{x})| \mathrm{x} \in \mathrm{X} ; \sum_{\mathrm{i}=1}^{\mathrm{N}} \mathrm{p}_{\mathrm{i}} \cdot \mathrm{x}_{\mathrm{i}}=\mathrm{Y} \mid .
$$

38: Sowohl die Kompensations- als auch die Äquivalenzvariation nehmen Bezug auf das typische Entscheidungsproblem eines Konsumenten; vgl. dazu ausführlich VARIAN, H.R. (1984), S. 115ff.; AHLHEIM, M./ROSE, M. (1989), S. $248 f f$.

39: Dies impliziert ein Preisnehmerverhalten des Konsumenten.

40: Dabei impliziert die Vollständigkeit der Präferenzrelation ihre Reflexivität. Aufgrund dieser Eigenschaft handelt es sich um eine Relation, welche die schwache Präferenz beschreibt.

41: Der Operator "arg max" ordnet einer Menge von Funktionswerten die Urbilder ihrer maximalen Elemente zu.

42: Um die Funktionen $u$ und $v$ auch begrifflich zu unterscheiden, bezeichnet man $u$ als direkte Nutzenfunktion. 
Ähnlich führt die Untersuchung des zur Nutzenmaximierung dualen Problems der Ausgabenminimierung auf die einkommenskompensierten bzw. Hicks'schen Nachfragefunktionen $h_{i}(p, u)$, die von den Marshall'schen Nachfragefunktionen zu unterscheiden sind ${ }^{43}$. Der Vektor $h(p, u)$ der Hicks'schen Nachfragen gibt das vom Konsumenten nachgefragte Güterbündel an, wenn er sich bei Gültigkeit des Preisvektors $\mathrm{p}$ mindestens das Nutzenniveau u sichern möchte. Mit Hilfe dieser Nachfragefunktionen gewinnt man in einfacher Weise die Ausgabenfunktion e(p,u) des Konsumenten:

$$
e(p, u)=\sum_{i=1}^{N} p_{i} \cdot h_{i}(p, u) .
$$

Die Ausgabenfunktion e(p,u) gibt die Ausgaben an, die der Konsument bei vorgegebenen Güterpreisen $p$ mindestens tätigen muß, um das Nutzenniveau u zu erreichen. Ebenso wie die indirekte Nutzenfunktion besitzt auch die Ausgabenfunktion unter den getroffenen Annahmen die Eigenschaft der zweimaligen stetigen Differenzierbarkeit ${ }^{44}$.

Wie man anhand der indirekten Nutzenfunktion erkennt, determinieren in dem betrachteten Entscheidungsproblem die Güterpreise $\mathrm{p}$ und das individuelle Pauscheinkommen $Y$ den Nutzen und damit die Wohlfahrt des Konsumenten. Es liegt daher nahe, individuelle Wohlfahrtsänderungen als Folge einer Veränderung dieser Parameter zu modellieren. Die beiden zu diskutierenden individuellen Wohlfahrtsmaße versuchen dementsprechend, den aus dem Übergang von einer Ausgangslage $\left(\mathrm{p}^{0}, \mathrm{Y}^{0}\right)$ in eine neue Situation $\left(\mathrm{p}^{1}, \mathrm{Y}^{1}\right)$ resultierenden Wohlfahrtseffekt in monetären Einheiten auszudrücken. In diesem Zusammenhang repräsentiert die Kompensationsvariation denjenigen Geldbetrag KV, den der Konsument für das Eintreten der neuen Situation maximal zu zahlen bereit ist. Die Kompensationsvariation gibt demnach gerade die maximale Zahlungsbereitschaft an, die der Konsument in der neuen Situation $\left(\mathrm{p}^{1}, \mathrm{Y}^{1}\right)$ entfaltet, ohne die durch $\left(\mathrm{p}^{0}, \mathrm{Y}^{0}\right)$ charakterisierte Ausgangslage (strikt) zu präferieren. Aus dieser Definition ergibt sich zunächst in

43: Vgl. zur Verwendung des Dualitätsbegriffs in der mikroökonomischen Haushaltstheorie kritisch NEWMAN, P. (1987) sowie ausführlich AHLHEIM, M. (1985).

44: Zu weiteren Eigenschaften dieser Funktionen vgl. VARIAN, H.R. (1984), S. 121ff. sowie AHLHEIM, M./ROSE, M. (1989), S. 263ff. 
formaler Hinsicht eine implizite Darstellung der Kompensationsvariation über die indirekte Nutzenfunktion:

$$
\mathrm{v}\left(\mathrm{p}^{1}, \mathrm{Y}^{1}-\mathrm{KV}\right)=\mathrm{v}\left(\mathrm{p}^{0}, \mathrm{Y}^{0}\right)
$$

Bei fest vorgegebenen, strikt positiven Güterpreisen bildet das für den Konsumenten maximal erreichbare Nutzenniveau eine streng monotone Funktion seines Einkommens, d.h. es existiert ein eineindeutiger Zusammenhang zwischen den Werten der Funktion $\mathrm{v}$ und ihrem Einkommensargument ${ }^{45}$. Bezeichne $\mathrm{v}^{-1}$ die unter dieser Voraussetzung wohldefinierte Umkehrfunktion von $\mathrm{v}$, dann gilt:

$$
\begin{gathered}
\mathrm{Y}^{1}=\mathrm{v}^{-1}\left(\mathrm{p}^{1}, \mathrm{u}^{1}\right), \\
\mathrm{Y}^{1}-\mathrm{KV}(0 \rightarrow 1)=\mathrm{v}^{-1}\left(\mathrm{p}^{1}, \mathrm{u}^{0}\right),
\end{gathered}
$$

wobei $\mathrm{u}^{1}$ bzw. $\mathrm{u}^{0}$ das Nutzenniveau des Konsumenten in der neuen Situation, $\mathrm{v}\left(\mathrm{p}^{1}, \mathrm{Y}^{1}\right)$, bzw. in der Ausgangslage, $\mathrm{v}\left(\mathrm{p}^{0}, \mathrm{Y}^{0}\right)$, bezeichnet. Weiter weist der bei dem Geldbetrag KV angefügte Klammerausdruck darauf hin, daß es sich um die Kompensationsvariation für den Übergang von einer durch die Superskripte " 0 " in eine durch die Superskripte "1" indizierte Situation handelt. Daraus erhält man als explizite Darstellung der individuellen Zahlungsbereitschaft KV:

$$
\mathrm{KV}(0 \rightarrow 1)=\mathrm{v}^{-1}\left(\mathrm{p}^{1}, \mathrm{u}^{1}\right)-\mathrm{v}^{-1}\left(\mathrm{p}^{1}, \mathrm{u}^{0}\right)
$$

Aufgrund des dualen Zusammenhangs zwischen der indirekten Nutzenfunktion $\mathbf{v}$ und der Ausgabenfunktion e gilt ${ }^{46}$ :

$$
\mathrm{KV}(0 \rightarrow 1)=e\left(\mathrm{p}^{1}, \mathrm{u}^{1}\right)-e\left(\mathrm{p}^{1}, \mathrm{u}^{0}\right)
$$

45: Daraus folgt unmittelbar ein positives (negatives) Vorzeichen der Kompensationsvariation, falls der Konsument in der neuen Situation einen höheren (niedrigeren) Nutzen erzielt. Diese Vorzeicheneigenschaft entspricht der Interpretation der Kompensationsvariation als einer individuellen Zahlungsbereitschaft.

46: Vgl. z.B. VARIAN, H.R. (1984), S. 122. 
Aus dieser Gleichung geht hervor, $\mathrm{da} B$ die Kompensationsvariation einen monetären Ausdruck für die Diskrepanz der beiden Nutzenniveaus $\mathrm{u}^{1}$ und $\mathrm{u}^{0}$ darstellt, wobei der in der neuen Situation gültige Preisvektor $\mathrm{p}^{1}$ zugrundegelegt wird. Da weiterhin $\mathrm{Y}^{1}=$ $e\left(p^{1}, u^{1}\right)$ und $Y^{0}=e\left(p^{0}, u^{0}\right)$ gilt, erhält man durch Erweitern:

$$
\begin{aligned}
\mathrm{KV}(0 \rightarrow 1) & =\mathrm{Y}^{1}-\mathrm{e}\left(\mathrm{p}^{1}, \mathrm{u}^{0}\right)+\mathrm{Y}^{0}-\mathrm{Y}^{0} \\
& =\mathrm{Y}^{1}-\mathrm{Y}^{0}+\mathrm{e}\left(\mathrm{p}^{0}, \mathrm{u}^{0}\right)-\mathrm{e}\left(\mathrm{p}^{1}, \mathrm{u}^{0}\right) .
\end{aligned}
$$

Aufgrund der stetigen Differenzierbarkeit der Ausgabenfunktion läßt sich dieser Ausdruck umformulieren zu:

$$
\mathrm{KV}\left(0_{\mapsto \rightarrow 1}\right)=\Delta \mathrm{Y}+\int_{\mathrm{p}^{1}}^{\mathrm{p}^{0}} \sum_{\mathrm{i}=1}^{\mathrm{N}} \frac{\partial \mathrm{e}\left(\mathrm{p}, \mathrm{u}^{0}\right)}{\partial \mathrm{pi}} \mathrm{dpi}
$$

wobei der Integrand entlang eines stückweise stetig differenzierbaren Weges, der $\mathrm{p}^{1}$ mit $\mathrm{p}^{0}$ verbindet, integriert wird ${ }^{47}$. Mit Hilfe von Shepard's Lemma ergibt sich schließlich:

$$
K V\left(0_{\mapsto} \rightarrow 1\right)=\Delta Y+\int_{p^{1}}^{p^{0}} \sum_{i=1}^{N} h_{i}\left(p, u^{0}\right) d p_{i}
$$

Diese Darstellung zeigt, in welcher Weise eine auf Veränderungen der Güterpreise $\mathrm{p}$ und des Pauscheinkommens $\mathrm{Y}$ beruhende individuelle Wohlfahrtsänderung im Sinne der Kompensationsvariation in eine reine Einkommensänderung umgerechnet werden kann. Die mit dem Übergang von der Ausgangslage in die neue Situation verbundene Veränderung des individuellen Pauscheinkommens $\mathrm{Y}$ geht dabei unmittelbar in die Berechnung von KV ein, während der Preiseffekt durch das in Gleichung (2.11) auf der rechten Seite stehende Integral erfaßt wird. Bei diesem zu-

47: Vgl. HEUSER, H. (1988), S. 381. 
letzt genannten Summanden handelt es sich um ein Wegintegral, dessen Wert nicht vom speziell gewählten Pfad der Preisänderung von $\mathrm{p}^{1}$ nach $\mathrm{p}^{0}$ abhängt, da der Integrand ein Gradientenfeld darstellt ${ }^{48}$.

Im Gegensatz dazu bezeichnet die Äquivalenzvariation denjenigen Geldbetrag $\ddot{A} \mathrm{~V}$, den der Konsument im Falle einer Beibehaltung der Ausgangslage mindestens erhalten muß, damit er die neue Situation nicht (strikt) präferiert. Konzeptionell repräsentiert die Äquivalenzvariation somit das Gegenteil einer maximalen Zahlungsbereitschaft, da sie die zur Kompensation einer Wohlfahrtsänderung minimal notwendigen Zahlungen an den Konsumenten angibt. Man könnte die Äquivalenzvariation insofern als eine minimale Zahlungsakzeptanz bezeichnen ${ }^{49}$. Formal führt die Definition der Äquivalenzvariation zunächst auf die folgende implizite Darstellung:

$$
\mathrm{v}\left(\mathrm{p}^{0}, \mathrm{Y}^{0}+\ddot{\mathrm{A}} \mathrm{V}\right)=\mathrm{v}\left(\mathrm{p}^{1}, \mathrm{Y}^{1}\right)
$$

Mit Hilfe derselben Vorgehensweise, die zuvor bei der Ableitung der Kompensationsvariation eingesetzt wurde, ergibt sich als explizite Darstellung für die Äquivalenzvariation:

$$
\begin{aligned}
\ddot{A} V(0 \rightarrow 1) & =v^{-1}\left(p^{0}, u^{1}\right)-v^{-1}\left(p^{0}, u^{0}\right) \\
& =e\left(p^{0}, u^{1}\right)-e\left(p^{0}, u^{0}\right) .
\end{aligned}
$$

Aus einem Vergleich dieser letzten Gleichung mit Gleichung (2.8) läßt sich die strukturelle Ähnlichkeit der beiden individuellen Wohlfahrtsmaße erkennen: auch die Äquivalenzvariation liefert einen monetären Ausdruck für die Diskrepanz der beiden

48: Dies bedeutet, daß der Integrand den Gradienten einer stetig differenzierbaren Funktion bildet. Wie der Übergang von Gleichung (2.9) nach Gleichung (2.10) zeigt, besitzt das in der Darstellung der Kompensationsvariation auftretende Integral bei parametrisch vorgegebenem Nutzenniveau (hier: $u^{0}$ ) gerade den Gradienten der Ausgabenfunktion als Integrand. Daraus folgt die Eigenschaft der Pfadunabhängigkeit dieses Integrals; vgl. dazu ausführlich HEUSER, H. (1988), S. 383ff.

49: Wie auch die Erläuterungen des Vorzeichens dieses individuellen Wohlfahrtsmaßes weiter unten zeigen, läßt sich die negative Äquivalenzvariation (- ̈̈V) als maximale Zahlungsbereitschaft interpretieren. 
Nutzenniveaus $u^{1}$ und $u^{0}$, wobei im Unterschied zur Kompensationsvariation nun der in der Ausgangslage gültige Preisvektor $\mathrm{p}^{0}$ zugrundegelegt wird. Dabei weist die Äquivalenzvariation ein positives Vorzeichen auf, wenn der Konsument die neue Situation $\left(\mathrm{p}^{1}, \mathrm{Y}^{1}\right)$ gegenüber der durch $\left(\mathrm{p}^{0}, \mathrm{Y}^{0}\right)$ charakterisierten Ausgangslage (strikt) präferiert ${ }^{50}$. Über ganz ähnliche Umformungen wie zuvor im Falle der Kompensationsvariation erhält man schließlich als Darstellung der Äquivalenzvariation:

$$
\ddot{A} V\left(0_{r} \rightarrow 1\right)=\Delta Y+\int_{p^{1}}^{p^{0}} \sum_{i=1}^{N} h_{i}\left(p, u^{1}\right) d p_{i}
$$

Falls beim Übergang von der Ausgangslage in die neue Situation lediglich ein Güterpreis variiert, lassen sich die beiden besprochenen individuellen Wohlfahrtsmaße graphisch veranschaulichen. Abbildung 2 zeigt die Bestimmung von $\mathrm{KV}(0 \rightarrow 1)$ und $\ddot{\mathrm{A} V}(0 \rightarrow 1)$ in diesem einfachen Fall, wobei ohne Beschränkung der Allgemeinheit eine Preisänderung beim ersten Gut untersucht wird. Die Mengen dieses Guts sind auf der Abszisse abgetragen, während auf der Ordinate die Ausgaben für die restlichen N-1 Güter gemessen werden, die zu einer Hicks'schen "composite commodity" zusammengefaßt sind ${ }^{51}$.

In der Ausgangslage konsumiert das Individuum im Punkt A. In der neuen Situation, in der sich der Preis des ersten Guts verringert und das individuelle Pauscheinkommen $\mathrm{Y}$ erhöht hat, beschreibt B die nutzenmaximierende Wahl des Kon-

50: Entsprechend besitzt die Äquivalenzvariation bei einer Wohlfahrtsverringerung ein negatives Vorzeichen und gibt die maximal mögliche Zahlung des Konsumenten in der Ausgangslage an, damit er die neue Situation nicht (strikt) präferiert. Aus diesen Eigenschaften folgt unmittelbar, daß die negative Äquivalenzvariation gerade diejenige Zahlungsbereitschaft angibt, die der Konsument maximal für die Beibehaltung der Ausgangslage entfalten kann. Diese Interpretation bestätigt die folgende Gleichung, welche die negative Äquivalenzvariation als Kompensationsvariation ausweist:

$$
-\ddot{\mathrm{AV}}(1 \rightarrow 0)=\mathrm{KV}(0 \rightarrow 1)
$$

Diese Beziehung ergibt sich aus den Gleichungen (2.4) und (2.12).

51: Vgl. zu dieser Darstellung z.B. CORNWALL, R.C. (1984), S. 588. Einen einfachen Beweis für die allgemeine Zulässigkeit dieser Vorgehensweise liefert CARTER, M. (1990). 
sumenten $^{52}$. Im allgemeinen - auch die Abbildung zeigt eine derartige Konstellation unterscheiden sich die beiden individuellen Wohlfahrtsmaße $\mathrm{KV}(0 \rightarrow 1)$ und $\ddot{A} V(0 \rightarrow 1)$. Sie stimmen genau dann überein, wenn bei dem betrachteten Gut kein Einkommenseffekt auftritt 53 .

Abb. 2: Die Ermittlung von $\mathrm{KV}(0 \rightarrow 1)$ und $\mathrm{A} V(0 \rightarrow 1)$

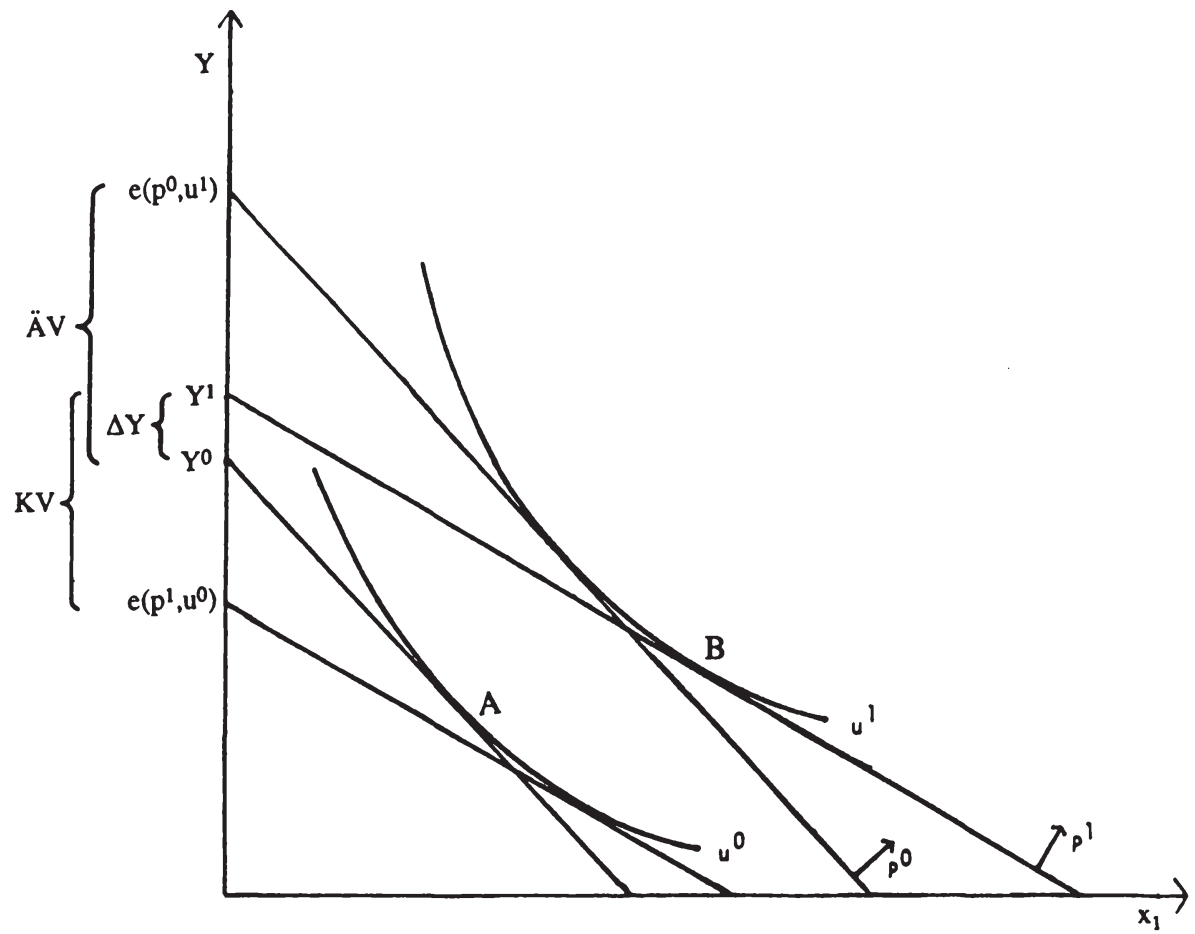

52: Die zu A und B gehörenden Ordinatenwerte geben die Ausgaben für alle anderen Güter an, deren Preise gemäß Voraussetzung unverändert bleiben.

53: In diesem Fall stimmen die beiden Hicks-Nachfragen $h_{1}\left(p, u^{0}\right)$ und $h_{1}\left(p, u^{1}\right)$ überein und die Gleichheit beider Wohlfahrtsmaße folgt aus den Gleichungen (2.11) und (2.14). 
Es läßt sich zeigen, daß die Kompensations- und die Äquivalenzvariation als Spezialfälle eines allgemeineren Konzepts zur Messung individueller Wohlfahrtsänderungen interpretiert werden können. Dies geschieht mit Hilfe einer speziellen Ausgabenfunktion, die im Ansatz auf McKenzie zurückgeht und insbesondere von Samuelson einige Zeit später wieder verwendet wurde ${ }^{54}$. McKenzie untersuchte die minimal notwendigen Ausgaben, die der Konsument tätigen muß, um bei vorgegebenen Preisen $\mathrm{p}^{\mathrm{R}}$ ein Güterbündel nachfragen zu können, das ihm keinen geringeren Nutzen stiftet als ein Referenzbündel $x^{R}$. Mit dieser Fragestellung eng verwandt ist die Analyse desjenigen Einkommens, das ein Konsument bei festen Güterpreisen $p^{R}$ mindestens benötigt, um denselben Nutzen wie in einer durch $(p, Y)$ charakterisierten Situation zu erzielen. Als Lösung dieses Problems erhält man eine Funktion, die jedem Tripel $\left(\mathrm{p}^{\mathrm{R}} ; \mathrm{p}, \mathrm{Y}\right)$ ein spezielles Pauscheinkommen des Konsumenten zuordnet. Für diese Funktion $\mathrm{m}$ gilt ${ }^{55}$ :

$$
\begin{aligned}
\mathrm{m}\left(\mathrm{p}^{\mathrm{R}} ; \mathrm{p}, \mathrm{Y}\right) & =\min \left\{\sum_{\mathrm{i}=1}^{N} \mathrm{p}_{\mathrm{i}}^{\mathrm{R}} \cdot \mathrm{x}_{\mathrm{i}} \mid \mathrm{x} \in \mathrm{X}_{\mathrm{i}} ; \mathrm{u}(\mathrm{x}) \geq \mathrm{v}(\mathrm{p}, \mathrm{Y})\right\} \\
& =\sum_{\mathrm{i}=1}^{N} \mathrm{p}_{\mathrm{i}}^{\mathrm{R}} \cdot \mathrm{h}_{\mathrm{i}}\left[\mathrm{p}^{\mathrm{R}}, \mathrm{v}(\mathrm{p}, \mathrm{Y})\right] \\
& =\mathrm{e}\left[\mathrm{p}^{\mathrm{R}} ; \mathrm{v}(\mathrm{p}, \mathrm{Y})\right]
\end{aligned}
$$

Die Funktion $m$ wird als äquivalente Einkommensfunktion bezeichnet ${ }^{56}$. Die Namensgebung reflektiert die Eigenschaft dieser Funktion, zu den Güterpreisen $\mathrm{p}^{\mathrm{R}}$ ge-

54: Vgl. McKENZIE, L. (1957); SAMUELSON, P.A. (1974), S. 1272ff.

55: Vgl. BOADWAY, R.W./BRUCE, N. (1984), S. 38. Dabei wird der Güterpreisvektor $\mathrm{p}^{\mathbf{R}}$ aus Gründen, die weiter unten offensichtlich werden, durch einen Strichpunkt von den übrigen Argumenten dieser Funktion getrennt.

56: Vgl. BOADWAY, R.W./BRUCE, N. (1984), S.38; LAISNEY, F./SCHMACHTENBERG, R. (1989), S. 2.

Allerdings sind in der Literatur verschiedene Bezeichnungen für diese Funktion gebräuchlich. Während Kaiser ebenso wie Hanusch von einer "geldmetrischen Nutzenfunktion" spricht, bezeichnen Varian und Ahlheim/Wagenhals diese Funktion als "indirekte Kompensationsfunktion" und Blackorby/Donaldson schließlich verwenden den Terminus "indirekte geldmetrische Nutzenfunktion"; vgl. KAISER, H. (1989), S. 10f.; HANUSCH, H. (1987), S. 50ff.; VARIAN, H.R. (1984), S. 124f.; AHLHEIM, M./WAGENHALS, G. (1988), S. 171; BLACKORBY, C./DONALDSON, D. (1988), S. 122. 
rade jenes Einkommen anzugeben, das der Konsument (mindestens) benötigt, um die Nutzenposition $\mathrm{v}(\mathrm{p}, \mathrm{Y}) \mathrm{zu}$ erreichen. Es gilt speziell:

$$
\mathrm{v}\left[\mathrm{p}^{\mathrm{R}}, \mathrm{m}\left(\mathrm{p}^{\mathrm{R}} ; \mathrm{p}, \mathrm{Y}\right)\right]=\mathrm{v}(\mathrm{p}, \mathrm{Y}) .
$$

Weiterhin ergibt sich aufgrund des dualen Zusammenhangs zwischen der indirekten Nutzenfunktion und der Ausgabenfunktion:

$$
\mathrm{m}\left(\mathrm{p}^{\mathrm{R}} ; \mathrm{p}^{\mathrm{R}}, \mathrm{Y}\right)=\mathrm{e}\left[\mathrm{p}^{\mathrm{R}}, \mathrm{v}\left(\mathrm{p}^{\mathrm{R}}, \mathrm{Y}\right)\right]=\mathrm{Y} .
$$

Für die Analyse individueller Wohlfahrtsänderungen besitzt die folgende Eigenschaft der äquivalenten Einkommensfunktion eine herausragende Bedeutung: bleibt der Güterpreisvektor $\mathrm{p}^{\mathrm{R}}$ konstant - man bezeichnet in diesem Fall $\mathrm{p}^{\mathrm{R}}$ als Referenzpreisvektor -, dann repräsentiert $m$ eine (streng) monotone Transformation der indirekten Nutzenfunktion des Konsumenten ${ }^{57}$. Unter dieser Voraussetzung stellt $\mathrm{m}$ selbst eine indirekte Nutzenfunktion dar, die sich für die individuelle Wohlfahrtsanalyse als besonders nützlich erweist. Mit ihrer Hilfe gelingt es, die individuelle Wohlfahrtsänderung beim Übergang von einer als Ausgangslage bezeichneten PreisEinkommens-Konstellation $\left(\mathrm{p}^{0}, \mathrm{Y}^{0}\right)$ in eine neue, durch $\left(\mathrm{p}^{1}, \mathrm{Y}^{1}\right)$ charakterisierte Situation in monetären Einheiten zu messen, wobei der Referenzpreisvektor $\mathrm{p}^{\mathrm{R}}$ als Grundlage dient. Die Messung erfolgt über einen Vergleich der äquivalenten Einkommen, die der Konsument bei Gültigkeit des Preisvektors $\mathrm{p}^{\mathrm{R}}$ benötigen würde, um die in den beiden betrachteten Situationen jeweils realisierte Nutzenposition zu erreichen. Man erhält auf diese Weise ein individuelles Wohlfahrtsmaß I, das lediglich vom Referenzpreisvektor $\mathrm{p}^{\mathrm{R}}$ abhängt:

$$
I\left(p^{R}, 0 \rightarrow 1\right)=m\left(p^{R} ; p^{1}, Y^{1}\right)-m\left(p^{R} ; p^{0}, Y^{0}\right)
$$

Wählt man die in der neuen Situation vorliegenden Preise $\mathrm{p}^{1}$ als Referenzpreisvektor, dann ergibt sich gerade die Kompensationsvariation ${ }^{58}$ :

$$
\mathrm{I}\left(\mathrm{p}^{1}, 0 \rightarrow 1\right)=\mathrm{KV}(0 \rightarrow 1)=\mathrm{m}\left(\mathrm{p}^{1} ; \mathrm{p}^{1}, \mathrm{Y}^{1}\right)-\mathrm{m}\left(\mathrm{p}^{1} ; \mathrm{p}^{0}, \mathrm{Y}^{0}\right)
$$

57: Vgl. AHLHEIM, M./WAGENHALS, G. (1988), S. 172.

58: Vgl. Gleichung (2.8). 
Hingegen erhält man die Äquivalenzvariation, wenn man den in der Ausgangslage gültigen Preisvektor $\mathrm{p}^{0}$ als Vergleichsbasis der in den beiden Situationen realisierten Nutzenpositionen heranzieht ${ }^{59}$ :

$$
\mathrm{I}\left(\mathrm{p}^{0}, 0 \rightarrow 1\right)=\ddot{\mathrm{A} V}(0 \rightarrow 1)=\mathrm{m}\left(\mathrm{p}^{0} ; \mathrm{p}^{1}, \mathrm{Y}^{1}\right)-\mathrm{m}\left(\mathrm{p}^{0} ; \mathrm{p}^{0}, \mathrm{Y}^{0}\right)
$$

Die Kompensations- und die Äquivalenzvariation erweisen sich damit als spezielle Elemente einer unendlichen Menge individueller Wohlfahrtsmaße, deren bevorzugte Diskussion in der Literatur auf den engen Zusammenhang zurückzuführen sein dürfte, der zwischen den bei ihnen verwendeten Referenzpreisvektoren und den analysierten Preis-Einkommens-Situationen besteht. Allen individuellen Wohlfahrtsmaßen $I\left(p^{R}, 0 \rightarrow 1\right)$ ist jedoch gemeinsam, daß sie letztlich die Diskrepanz zweier Nutzenpositionen eines Individuums in monetären Größen auszudrücken versuchen 60 . Diese Messungen stehen im übrigen völlig im Einklang mit der ordinalen Nutzentheorie, da jedes dieser Wohlfahrtsmaße invariant gegenüber verschiedenen (zulässigen) Repräsentationen der individuellen Präferenzen is $t^{61}$.

\subsubsection{Individuelle Zahlungsbereitschaften bei Unsicherheit}

Die Auswirkungen einer medizinischen Leistung auf die individuelle Gesundheit und damit zugleich die individuelle Wohlfahrt hängen wesentlich davon $\mathrm{ab}$, in welchem Krankheitszustand sich der Patient befindet ${ }^{62}$. Da im weiteren Verlauf dieser Arbeit von der Voraussetzung ausgegangen wird, daß der tatsächliche Krankheitszustand des Patienten unbekannt ist, überträgt sich diese Unsicherheit auf die mit dem Einsatz einer medizinischen Leistung verbundenen wohlfahrtsrelevanten Konsequenzen. Diese Aussage gilt insbesondere für diagnostische Leistungen, die mit der Unsi-

59: Vgl. Gleichung (2.13).

60: Vgl. dazu auch AHLHEIM, M./ROSE, M. (1989), S. $59 \mathrm{ff}$.

61: Diese Aussage gilt, weil die Hicks'schen Nachfragefunktionen durch die spezielle Wahl des Nutzenindexes nicht beeinflußt werden, d.h. man hat

$$
h_{i}(p, u)=h_{i}[p, f(u)] \quad i=1, \ldots, N
$$

wobei f eine streng monoton steigende Funktion repräsentiert.

62: Zum Begriff des Krankheitszustands vgl. ausführlich Abschnitt 2.4.1. 
cherheit hinsichtlich des sich einstellenden Testergebnisses ein eigenes stochastisches Element in die Analyse einführen ${ }^{63}$. Allgemein konfrontiert die Anwendung einer medizinischen Leistung den Patienten mit einem Prospekt, d.h. mit einer Menge möglicher Konsequenzen, die paarweise unvereinbar sind und deren Eintrittswahrscheinlichkeiten sich zu Eins summieren. Die dazugehörige individuelle Wohlfahrtsänderung resultiert aus einem Vergleich dieses Prospekts mit demjenigen Prospekt, der sich für den Patienten ohne die Inanspruchnahme der betrachteten Leistung ergeben hätte. Um diese Wohlfahrtsänderung in monetären Einheiten messen zu können, benötigt man daher individuelle Zahlungsbereitschaften, die der Unsicherheit bezüglich der beim Patienten entstehenden Konsequenzen Rechnung tragen. Diese Zahlungsbereitschaften dienen grundsätzlich dazu, den zwischen zwei Prospekten bestehenden Unterschied in der individuellen Wohlfahrt mittels eines monetären Maßes zu erfassen.

Vor der Ermittlung individueller Zahlungsbereitschaften bei Unsicherheit erweist es sich deshalb als notwendig, die anhand dieser Maße zu vergleichenden Prospekte näher zu beschreiben. $\mathrm{Zu}$ diesem $\mathrm{Zweck}$ wird ein Individuum betrachtet, das sich in genau einem aus $n$ möglichen Zuständen $K_{i}(i=1, \ldots, n)$ befinden $k a n n{ }^{64}$. Weiterhin gilt die Annahme, daß die Eintrittswahrscheinlichkeiten $P_{i}=P\left(K_{i}\right)$ der verschiedenen Zustände bekannt sind. In jedem dieser Zustände erhält das Individuum ein strikt positives Pauscheinkommen $Y_{\mathrm{i}}$, dessen Höhe zustandsabhängig sein kann. Das n-Tupel aller $\mathrm{Y}_{\mathrm{i}}$ bezeichnet die individuelle Anfangsausstattung mit bedingten, d.h. zustandsabhängigen, Einkommensforderungen. Daneben wird eine zustandsunabhängige Variable a betrachtet, die entweder den Wert $a_{1}$ oder den Wert $a_{0}$ annimmt. In diesem Zusammenhang wird vorausgesetzt, daß die Variable a keinen Einfluß auf das individuelle Pauscheinkommen besitzt ${ }^{65}$. Die Ausprägungen $a_{1}$ bzw. $a_{0}$ kennzeichnen dann die beiden Situationen - z.B. zwei verschiedene medizinische Leistungen -, die anhand eines monetären Maßes in ihrem Einfluß auf die indi-

63: Diese Aussage gilt allerdings nur, falls die betrachtete diagnostische Leistung keine vollkommene Information liefert; vgl. dazu Abschnitt 3.4.

64: Diese Zustände lassen sich beispielsweise als Krankheitszustände interpretieren.

65: Diese Annahme ist nicht essentiell, d.h. die weiter unten abgeleiteten individuellen Zahlungsbereitschaften lassen sich ebenso bestimmen, wenn die Variable a die Höhe der zustandsabhängigen Einkommensforderungen beeinflußt; vgl. dazu auch Abschnitt 4.3. 
viduelle Wohlfahrt zu vergleichen sind. Das Individuum steht damit vor der Aufgabe, Prospekte $\left[\mathrm{Y}_{\mathrm{i}}, \mathrm{a}\right] \mathrm{zu}$ bewerten, die wie folgt definiert sind:

$$
\left[Y_{i}, a\right]=\left[\begin{array}{ccc}
\left(Y_{1}, a\right) & \ldots & \left(Y_{n}, a\right) \\
& & \\
P_{1} & \ldots & P_{n}
\end{array}\right] .
$$

Dabei wird angenommen, das Individuum besitze Präferenzen auf der Menge aller derartigen Prospekte, die eine Repräsentation mit Hilfe von zustandsabhängigen von Neumann-Morgenstern Nutzenfunktionen $u_{i}\left(Y_{i}, a\right)$ erlauben 66 :

$$
V\left(\left[Y_{i}, a\right]\right)=\sum_{i=1}^{n} P_{i} \cdot u_{i}\left(Y_{i}, a\right) \text {. }
$$

Die Funktionen $u_{i}\left(Y_{i}, a\right)$ stellen zustandsabhängige bzw. bedingte indirekte Nutzenfunktionen dar, in die der Wert der diskreten Variable a parametrisch eingeht ${ }^{67}$. Das Fehlen eines Preisarguments bei diesen Funktionen weist darauf hin, daß die Güterpreise in der folgenden Analyse als konstant vorausgesetzt sind. Wie Gleichung (2.22) zeigt, hängt der Nutzeneffekt eines bestimmten Einkommens bei gegebener Ausprägung der Variablen a vom Zustand ab, in dem sich das Individuum befindet. Diese Eigenschaft dürfte in vielen Fällen als plausibel einzuschätzen sein 68 .

66: Zur Darstellung zustandsabhängiger individueller Präferenzen auf einer Menge von Prospekten vgl. HIRSHLEIFER, J. (1965), S. 523ff.; ARROW, K.J. (1974), S. 3ff.; HIRSHLEIFER, J./RILEY, J.G. (1979), S. 1387ff. sowie DEHEZ, P./DREZE, J.H. (1982), S. $41 \mathrm{ff.}$

67: Vgl. z.B. SCHMALENSEE, R. (1972), S. 814 sowie GRAHAM, D.A. (1981), S. 716.

68: Dies gilt nicht zuletzt für Anwendungen im Gesundheitswesen; vgl. ARROW, K.J. (1974), S. 5.

Im übrigen ergibt sich die Notwendigkeit einer zustandsabhängigen Modellierung der individuellen Präferenzen immer dann, wenn die Argumente eines Prospekts die aus der Sicht des Individuums relevanten Konsequenzen in ihrer Abhängigkeit vom jeweils vorliegenden $\mathrm{Zu}$ stand nicht vollständig beschreiben; vgl. ARROW, K.J. (1974), S. 5f.; EISEN, R. (1979), S. 36 sowie GALLAGHER, D.R.SMITH, V.K. (1985), S. 135, Fußnote 8.

Umgekehrt gelingt in dieser Arbeit eine Beschreibung der individuellen Präferenzen für medizinische Leistungen durch eine zustandsunabhängige von Neumann-Morgenstern Nutzenfunktion, da alle wohlfahrtsrelevanten Effekte über eine Konsequenzenfunktion explizit berücksichtigt werden; vgl. dazu ausführlich Kapitel 4.2. 
Eine letzte Voraussetzung betrifft schließlich die Möglichkeiten, bedingte Einkommensforderungen zu tauschen. Mit Hilfe eines derartigen Handels kann das betrachtete Individuum seine Anfangsausstattung gegen eine Reallokation seiner zustandsabhängigen Einkommen tauschen, um eine günstigere Anpassung an das vorhandene Risiko zu erreichen ${ }^{69}$. Allgemein hängen individuelle Zahlungsbereitschaften bei Unsicherheit wesentlich davon ab, ob und zu welchen Preisen die Möglichkeit zur Reallokation bedingter Einkommensforderungen besteht. Im folgenden wird zunächst jeder Einkommenstransfer zwischen einzelnen Zuständen vollständig ausgeschlossen ${ }^{70}$. Interpretiert man die möglichen Zustände $K_{i}$ als Krankheitszustände, dann scheint die Existenz einer Krankenversicherung dieser Annahme zu widersprechen. Allerdings eröffnet die Krankenversicherung nur in sehr beschränktem Ausmaß die Möglichkeit, bedingte Einkommensforderungen zu tauschen ${ }^{71}$. Insbesondere im Rahmen der Ableitung individueller Zahlungsbereitschaften für einzelne medizinische Leistungen erscheint es sinnvoll, von der oben angesprochenen Voraussetzung auszugehen, da die Bewertung dieser Leistungen im allgemeinen im Kontext einer parametrisch vorgegebenen Versicherungsentscheidung erfolgt.

Wie aus den Voraussetzungen hervorgeht, charakterisieren die Werte $a_{0}$ bzw. $a_{1}$ gerade die jeweils vorliegende Situation. Bezeichne an die Ausgangslage, dann erfolgt die individuelle Bewertung der neuen Situation $a_{1}$ durch einen Vergleich der beiden Prospekte $\left[\mathrm{Y}_{\mathrm{i}}, \mathrm{a}_{1}\right]$ und $\left[\mathrm{Y}_{\mathrm{i}}, \mathrm{a}_{0}\right]$. Als kompensierende maximale Zahlungsbereitschaft soll jeder Vektor von - im allgemeinen zustandsabhängigen Zahlungen in der neuen Situation bezeichnet werden, der das Individuum gerade indifferent zu dem in der Ausgangslage vorliegenden Prospekt $\left[\mathrm{Y}_{\mathrm{i}}, \mathrm{a}_{0}\right]$ werden läßt ${ }^{72}$.

69: Diese Reallokation bedingter Einkommensforderungen kann z.B. über spezielle Wertpapiere erfolgen, die auch als Arrow-Zertifikate bezeichnet werden; vgl. ARROW, K.J. (1964), S. 92ff.; EISEN, R. (1979), S. $71 \mathrm{ff}$.

70: Es existieren also keine "contingent claims markets" für die untersuchten Zustände; vgl. zu dieser Annahme SCHMALENSEE, R. (1972), S. 822f.; GRAHAM, D.A. (1981), S. 722 sowie GALLAGHER, D.R./SMITH, V.K. (1985), S. 137, insbesondere Fußnote 11.

71: Üblicherweise bestehen die Leistungen einer Krankenversicherung in der (teilweisen) Finanzienung einer weitgehend durch Ärzte festgelegten, zustandsabhängigen Inanspruchnahme medizinischer Leistungen.

72: Die Namensgebung steht im Einklang mit der Tatsache, daß es sich um eine Übertragung des Konzepts der Kompensationsvariation auf Risikosituationen handelt. 
Ein derartiger Vektor $\mathrm{z}$ besitzt $\mathrm{n}$ Komponenten $\mathrm{z}_{\mathrm{i}}$, die die folgende Gleichung erfüllen:

$$
\begin{aligned}
& V\left(\left[Y_{i}-z_{i}, a_{1}\right]\right)=V\left(\left[Y_{i}, a_{0}\right]\right) . \\
& \text { bzw. } \sum_{i=1}^{n} P_{i} \cdot u_{i}\left(Y_{i}-z_{i}, a_{1}\right)=\sum_{i=1}^{n} P_{i} \cdot u_{i}\left(Y_{i}, a_{0}\right) .
\end{aligned}
$$

Wie Graham am Beispiel zweier Zustände anschaulich gezeigt hat, existieren im allgemeinen unendlich viele kompensierende maximale Zahlungsbereitschaften ${ }^{73}$. Jeder Vektor $z$, der Gleichung (2.23a) genügt, repräsentiert eine spezielle Kombination von in der neuen Situation zu leistenden, zustandsabhängigen Zahlungen, die das betrachtete Individuum zwischen den Prospekten der neuen Situation und der Ausgangslage indifferent werden lassen ${ }^{74}$. Insofern bleibt zunächst unklar, was unter der individuellen Zahlungsbereitschaft für $a_{1}$ genau zu verstehen ist.

In der Literatur haben zwei spezielle Konzepte Bedeutung erlangt, die aus der Menge aller kompensierenden maximalen Zahlungsbereitschaften gerade zwei Elemente herausgreifen. Es handelt sich dabei zunächst um den kompensierenden Optionspreis $\mathrm{z}^{\mathrm{OP}}$, der als vom Zustand unabhängige individuelle Zahlungsbereitschaft

73: Vgl. GRAHAM, D.A. (1981), S. $716 \mathrm{ff}$.

74: Dabei kann die Menge der z, die Gleichung (2.23a) erfüllen, beispielsweise durch eine Nichtnegativitätsbedingung folgender Art eingeschränkt werden, um die Teilmenge der zulässigen kompensierenden maximalen Zahlungsbereitschaften zu erhalten:

$$
Y_{i}-z_{i} \geq 0 ; i=1, \ldots, n \text {. }
$$

Wie man anhand von Gleichung (2.23a) nachprüft, stellt jeder zulässige Vektor $\mathbf{z}$ in folgendem Sinn eine maximale Zahlungsbereitschaft dar:

aus $\mathrm{z}^{\prime} \geq \mathrm{z}$ und $\mathrm{z}^{\prime} \neq \mathrm{z}$ folgt: $\mathrm{V}\left(\left[\mathrm{Y}_{\mathrm{i}}-\mathrm{z}_{\mathrm{i}}, \mathrm{a}_{1}\right]\right)<\mathrm{V}\left(\left[\mathrm{Y}_{\mathrm{i}}, \mathrm{a}_{0}\right]\right)$,

wenn die übliche Annahme einer strengen Monotonie aller zustandsabhängigen Nutzenfunktionen $u_{i}$ in ihrem Einkommensargument gilt. 
für $\mathrm{a}_{1}$ definiert ist ${ }^{75}$. Es gilt also:

$$
z_{k} O P=z_{j} O P ; \quad k=1, \ldots, n ; \quad j=1, \ldots, n .
$$

Aus der charakterisierenden Eigenschaft der Zustandsunabhängigkeit ergibt sich die Möglichkeit, diesen Optionspreis als ex-ante-Konzept einer kompensierenden maximalen Zahlungsbereitschaft zu interpretieren. Der kompensierende Optionspreis repräsentiert gerade jene Zahlungsbereitschaft, die ein Individuum für $a_{1} z u$ entfalten bereit ist, bevor der tatsächlich vorliegende Zustand bekannt wird ${ }^{76}$.

Im Gegensatz dazu stellt die kompensierende Konsumentenrente $\mathrm{z}^{\mathrm{KR}}$ denjenigen Vektor von Zahlungen in der neuen Situation dar, der die individuelle Nutzenallokation im Vergleich zur Ausgangslage nicht beeinflußt ${ }^{77}$. Es handelt sich um ein ex-post-Konzept, da die vom Individuum zu leistenden Zahlungen im allgemeinen vom eingetretenen Zustand abhängen. Formal erhält man die kompensierende Konsumentenrente aus den Gleichungen:

$$
u_{i}\left(Y_{i}-z_{i}^{K R}, a_{1}\right)=u_{i}\left(Y_{i}, a_{0}\right) ; \quad i=1, \ldots, n .
$$

Bei der Zahlungsbereitschaft $\mathrm{z}^{\mathrm{KR}}$ realisiert das betrachtete Individuum immer, d.h. unabhängig vom vorliegenden Zustand, gerade die in der Ausgangslage erreichte Nutzenposition. Daraus ergibt sich als charakterisierende Eigenschaft der kompensierenden Konsumentenrente, die ein Individuum für eine neue Situation besitzt, ein gegenüber der Ausgangslage unverändertes Risiko.

75: Vgl. zu dieser Namensgebung sowie zum Konzept des Optionspreises allgemein SCHMALENSEE, R. (1972), S. 817ff.; SMITH, V.K.(1987), S. $287 f$.

Der Optionspreis ist im übrigen streng vom Konzept des Optionswerts zu unterscheiden, das in der Literatur im AnschluB an WEISBROD, B.A. (1964) eine intensive Diskussion entfacht hat; vgl. dazu z.B. GRAHAM, D.A. (1981); BISHOP, R.C. (1982) und SMITH, V.K. (1990).

76: Vgl. zu dieser Interpretation SMITH, V.K. (1987), S. 289.

77: Vgl. SCHMALENSEE, R. (1972), S. 817; GRAHAM, D.A. (1981), S. 716. 
Selbstverständlich lassen sich neben den kompensierenden maximalen Zahlungsbereitschaften noch andere Maße konstruieren, um individuelle Wohlfahrtsänderungen bei Unsicherheit zu messen ${ }^{78}$. Indessen reichen die soeben dargestellten Maße aus, um die grundsätzliche Vorgehensweise zu veranschaulichen, wenn keine Märkte für bedingte Einkommensforderungen existieren. Darüber hinaus gelangt im weiteren Verlauf dieser Arbeit lediglich das Konzept des kompensierenden Optionspreises zur Anwendung, um individuelle Zahlungsbereitschaften für diagnostische Leistungen abzuleiten. Dies läßt sich erstens damit begründen, $\mathrm{da} ß$ die kompensierenden maximalen Zahlungsbereitschaften ganz allgemein konzeptionell am ehesten die Aufgabe erfüllen, individuelle Zahlungsbereitschaften für den Übergang in eine neue Situation anzugeben. Zweitens sprechen Praktikabilitätserwägungen zugunsten eines zustandsunabhängigen Zahlungsbereitschafts-Konzepts, weil häufig keine Möglichkeit besteht, zustandsabhängige Zahlungen von einem Individuum einzufordern ${ }^{79}$. Allerdings darf nicht übersehen werden, daß der kompensierende Optionspreis im allgemeinen nur eine zweitbeste Lösung darstellt, da zustandsabhängige Zahlungen dem Individuum eine bessere Risikoanpassung ermöglichen würden ${ }^{80}$. Aufgrund fehlender Tauschmöglichkeiten für bedingte Einkommensforderungen kann die Allokation sowohl in der Ausgangslage als auch in der neuen Situation unter Risikoaspekten ineffizient sein. Insgesamt mißt der kompensierende Optionspreis daher nicht allein den auf $a_{1}$ zurückgehenden Nutzeneffekt, sondern reflektiert zusätzlich die individuelle Bewertung des in der neuen Situation veränderten Risikos ${ }^{81}$.

78: An erster Stelle wäre hier an die äquivalenten minimalen Zahlungsakzeptanzen zu denken, die in konzeptioneller Hinsicht der (im Sicherheitsfall untersuchten) Äquivalenzvariation entsprechen. Eine äquivalente minimale Zahlungsakzeptanz repräsentiert einen Vektor, dessen n Komponenten jene in der Regel zustandsabhängigen Zahlungen enthalten, die das Individuum in der Ausgangslage mindestens benötigt, um die neue Situation nicht (strikt) zu präferieren. Wie zuvor im Fall der kompensierenden maximalen Zahlungsbereitschaft existieren im allgemeinen unendlich viele Maße für die individuelle Zahlungsakzeptanz. Das Interesse konzentriert sich daher auf den äquivalenten Optionspreis und die äquivalente Konsumentenrente, die in naheliegender Weise definiert sind; vgl. dazu SCHMALENSEE, R. (1972), S. 817.

79: Vgl. zu diesem Argument BOHM, P. (1975), S. 736, ähnlich MENDELSOHN, R./STRANG, W.J. (1984), S. 1098.

Diese Tatsache mag auch erklären, warum Versicherer ihre typischerweise zustandsabhängigen Leistungen zu einem zustandsunabhängigen Preis - der Prämie - anbieten.

80: Vgl. SMITH, V.K. (1990), S. 198.

81: Falls die neue Situation eine gleichmäßigere Nutzenallokation auf die einzelnen Zustände kennzeichnet, werden risiko-averse Individuen diesen Effekt positiv bewerten; vgl. dazu das Beispiel bei ZECKHAUSER, R. (1969), S. 547ff. 
Welche individuellen Zahlungsbereitschaften ergeben sich, wenn man die zuvor getroffene Annahme des Fehlens von Märkten für bedingte Einkommensforderungen aufhebt? Wenn derartige Märkte vorhanden sind, dann besteht für das Individuum die Möglichkeit, zu festen Preisen zustandsabhängige Einkommensforderungen zu tauschen und auf diese Weise eine bestmögliche Anpassung an das vorhandene Risiko $\mathrm{zu}$ erreichen ${ }^{82}$. Auch unter dieser Voraussetzung existieren im allgemeinen unendlich viele kompensierende maximale Zahlungsbereitschaften für den Übergang in eine neue Situation. Wie zuvor wird jede dieser Zahlungsbereitschaften durch einen Vektor repräsentiert, dessen Komponenten die zu den möglichen Zuständen gehörenden individuellen Zahlungen enthalten. Das gemeinsame Merkmal aller Zahlungsbereitschaften besteht darin, daß sie bei den herrschenden Preisen für bedingte Einkommensforderungen denselben Marktwert besitzen ${ }^{83}$. Diese charakteristische Identität beruht letztlich auf der Möglichkeit des Individuums, durch eine Reallokation seiner bedingten Einkommensforderungen jenen negativen Effekt zu kompensieren, den eine spezielle Zahlungsbereitschaft in ihrer Aufteilung auf die einzelnen Zustände unter Risikoaspekten mit sich bringt.

Falls die Preise der bedingten Einkommensfordenungen "fair" sind, entspricht der Marktwert einer Einkommensallokation immer ihrem - unter Berïcksichtigung der Wahrscheinlichkeiten der möglichen Zustände berechneten - erwarteten Wert ${ }^{84}$. In diesem Spezialfall stimmt demzufolge der Marktwert jeder individuellen Zahlungsbereitschaft mit ihrem erwarteten Wert überein. Unter der Voraussetzung fairer Preise kann sich ein Individuum in jeder Situation optimal an das vorhandene Risiko anpassen. Kompensierende maximale Zahlungsbereitschaften messen dann den "reinen" Nutzeneffekt, den der Übergang in eine neue Situation für ein Individuum erzeugt, da in den beiden zu vergleichenden Situationen jeweils eine effiziente Risikoallokation vorliegt ${ }^{85}$.

82: Vgl. zur Darstellung des individuellen Nutzenmaximierungsproblems im Falle der Existenz von "contingent claims markets" SCHMALENSEE, R. (1972), S. 819ff. sowie GALLAGHER, D.R./SMITH, V.K. (1985), S. 137.

83: Vgl. dazu ausführlich SCHMALENSEE, R. (1972), S. 819f. sowie die Argumentation von COOK, P.J./GRAHAM, D.A. (1977), S. 152, im Kontext "fairer" Preise für bedingte Einkommensforderungen.

84: Vgl. dazu genauer COOK, P.J./GRAHAM, D.A. (1977), S. 148ff.; GALLAGHER, D.R./SMITH, V.K. (1985), S. 135ff.

85: Vgl. dazu die Kategorisierung möglicher Nutzeneffekte bei COOK, P.J./GRAHAM, D.A. (1977), S. 151f., insbesondere Fußnote 18, sowie allgemein SMITH, V.K. (1990), S. $200 f$. 


\subsection{Medizinische Diagnostik}

\subsubsection{Der Diagnosebegriff in der Medizin}

Etymologisch geht das Wort "Diagnose" auf den griechischen Ausdruck "diagnosis" bzw. das dazugehörige Verb zurück und läßt sich mit "Unterscheidung" bzw. "unterscheiden" übersetzen ${ }^{86}$. In dieser Bedeutung findet der Begriff heute in den meisten Wissenschaften Anwendung. Ganz allgemein, d.h. ohne medizin-spezifische Ausrichtung, bezeichnet etwa der Brockhaus mit "Diagnose" die "methodische Erforschung der Merkmale eines Gegenstandes oder einer Person, um ihn mit bereits bekannten Begriffen erfassen zu können". In dieser Definition lassen sich schon zwei unterschiedliche Bedeutungen des Wortes Diagnose feststellen: sowohl der Prozeß der Merkmalsbestimmung einerseits, als auch die (sich daran anschließende) Zuordnung eines bekannten Begriffs andererseits tragen offenbar die Bezeichnung "Diagnose".

Diese Ambivalenz kennzeichnet auch die Verwendung des Diagnosebegriffs in der Medizin; unter Diagnose versteht man hier die "Allokation eines Patienten zu einer oder mehreren (beschriebenen) Krankheitsentitäten"87, wobei darunter sowohl alle Vorgänge fallen, die dem Erkennen dieser Krankheit(en) aus verschiedenen Informationsquellen dienen ${ }^{88}$, als auch die Benennung des Krankheitsbildes selbst mit Hilfe von Beschreibungen bereits bekannter Krankheitsentitäten (Nosologie).

Um diese beiden Bedeutungen begrifflich voneinander zu trennen, werden im folgenden jene (ärztlichen) Handlungen, die auf die Ermittlung des Krankheitsbildes gerichtet sind, zunächst als Komponenten des diagnostischen Prozesses bzw. in ihrer Gesamtheit als Diagnostik bezeichnet ${ }^{89}$. Abstrakt formuliert ${ }^{90}$, erschließt und nutzt der Arzt im Verlauf dieses Prozesses eine Reihe von Informationsquellen, die ihm für die (anschließende) Diagnosestellung wichtig erscheinen. Daraus ergibt sich eine Diagnose, deren Sicherheitsgrad von der Güte der vorliegenden Information abhängt.

86: Vgl. OSBORNE, C.A. (1983), S. 891; PARRINO, T.A./MITCHELL, R. (1989), S. 18.

87: KELLER, H. (1986), S. 153.

88: Vgl. ENGLE, R.L./DAVIS, B.J. (1963), S. 513.

89: Vgl. ZIMMERMANN, S./QUIETZSCH, D. (1983), S. 8.

90: Vgl. dazu konkreter Abschnitt 2.3.2. 
Diese diagnostische Hypothese ${ }^{91}$ ist im Anschluß daran im Lichte neuer Informationen zu überprüfen und gegebenenfalls zu modifizieren oder auch zu verwerfen, falls andere Hypothesen besser im Einklang mit den vorhandenen Daten stehen. Der damit skizzierte Kreislauf der schrittweisen Hypothesenbildung und -überprüfung, der je nach Bedarf mehrmals durchlaufen werden kann bzw. sogar muß, stellt somit das wesentliche Strukturmerkmal des diagnostischen Prozesses dar ${ }^{92}$, an dessen Ende eine durch die zur Verfügung stehenden Fakten möglichst gut gestützte Hypothese steht ${ }^{93}$. In dieser Hinsicht ähnelt das ärztliche Vorgehen dem Procedere eines Kriminalisten ebenso wie dem "piecemeal engineering" des Wissenschaftlers. Es überrascht daher nicht, daß der diagnostische Entscheidungsproze $B$ nicht selten als typischer Anwendungsfall des deduktiv-nomologischen Modells herangezogen wird ${ }^{94}$.

Diese allgemeine Charakterisierung des diagnostischen Prozesses deckt im übrigen ganz verschiedene konkrete Vorgehensweisen des Arztes ab ${ }^{95}$. Eine mögliche Form der Diagnostik besteht darin, diagnostische Untersuchungen nacheinander durchzuführen und damit den Prozeß der Informationssuche in eine Vielzahl von Schritten zu untergliedern. In diesem Fall entscheidet der Arzt jeweils aufgrund der bereits vorliegenden diagnostischen Information, welche Untersuchungen zusätzlich notwendig sind. Neben diesem stufendiagnostischen Vorgehen kann der Arzt alternativ einzelne Schritte innerhalb des diagnostischen Prozesses zusammenfassen, indem er beispielsweise verschiedene Laborwerte parallel erhebt. Im Extremfall führt diese Vorgehensweise dazu, daß der Arzt sämtliche diagnostischen Informationen zeitgleich ermittelt. Eine Möglichkeit, den diagnostischen Prozeß zu veranschaulichen, zeigt Abbildung 3.

91: Vgl. ALBERT, D.A. (1977), S. $213 f$.

92: Da sich im Normalfall die zur Verfügung stehenden Informationen ständig erweitern, sprechen manche Autoren präziser von einer "diagnostischen Spirale"; vgl. GROSS, R. (1973), S. 784 oder auch ZIMMERMANN, S.QUIETZSCH, D. (1983), S. 10.

93: Vgl. dazu ausführlich DAHMER, J. (1980).

94: Vgl. BUNGE, M. (1967), S. 224; MEDAWAR, P.B. (1969), S. 42; JENNETT, B. (1986), S. 10 sowie kritisch PARRINO, T.A./MITCHELL, R. (1989), S. $25 \mathrm{ff}$.

95: Vgl. VOGT, W. (1982), S. $177 f$. 
Abb. 3: Der diagnostische Prozeß nach Gross ${ }^{96}$

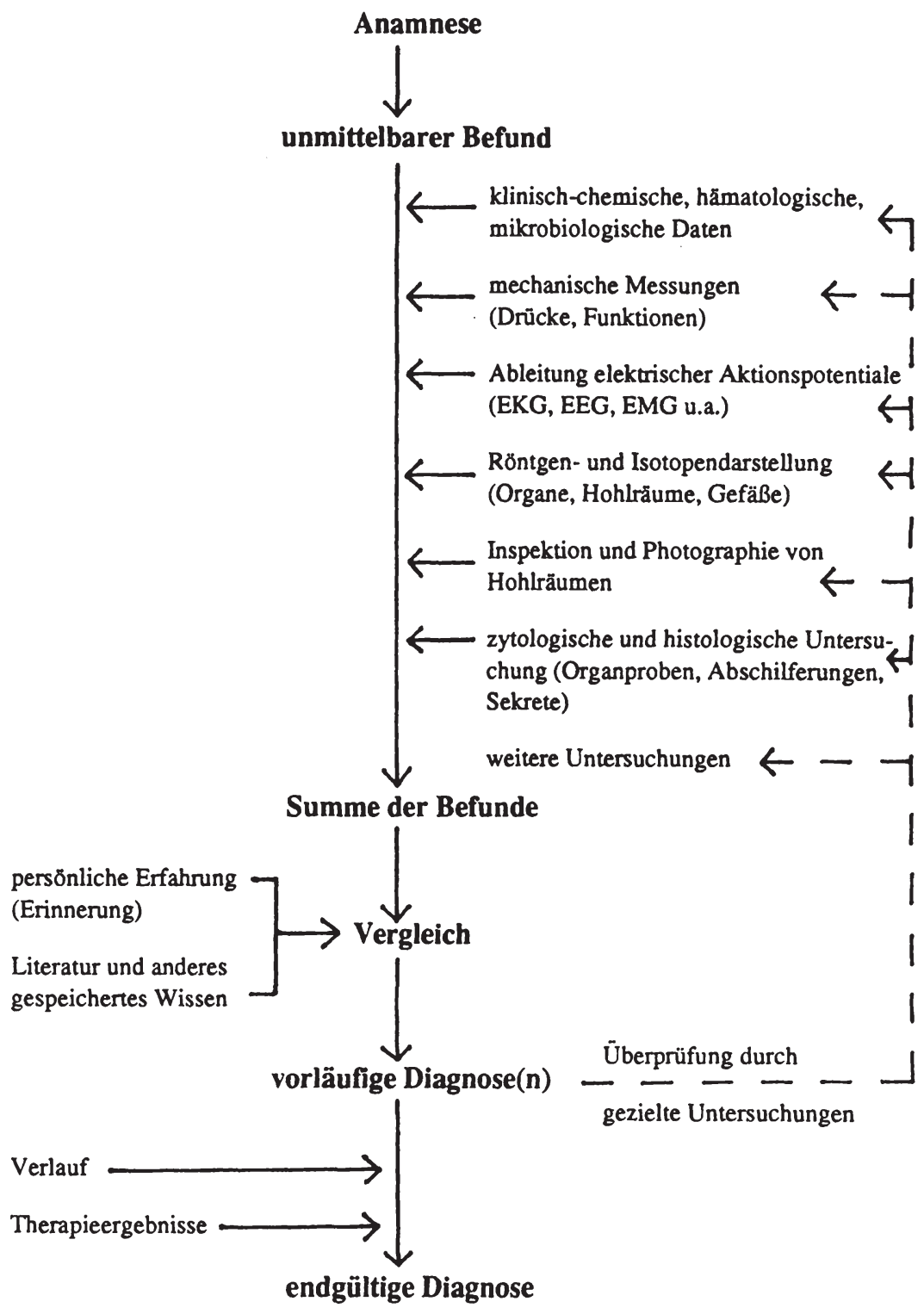

96: GROSS, R. (1973), S. 784. 
In manchen Fällen faßt man den Begriff der medizinischen Diagnostik eng und ordnet darunter ausschließlich jene Handlungen ein, die der Arzt zum Zwecke der Diagnosestellung durchführt. Indessen spricht viel dafür, dabei auch Maßnahmen im Rahmen der sekundären Prävention, d.h. Vorsorgeuntersuchungen ("screening"), zu berücksichtigen 97 . Während der Anstoß zur "normalen" Diagnostik vom Patienten ausgeht, der aufgrund seiner Beschwerden ${ }^{98}$ den Arzt aufsucht, handelt es sich bei den Vorsorgeuntersuchungen um ein Angebot an einen gefährdeten, aber beschwerdefreien Kreis von Personen ${ }^{99}$. Grundsätzlich erhebt der Arzt auch in diesem Fall Informationen, mit deren Hilfe er über das weitere Vorgehen zu entscheiden hat. Daher werden im folgenden Maßnahmen des "screening" ebenfalls als zur Diagnostik zugehörig eingestuft, die damit in der hier verwendeten Charakterisierung einen breiteren Objektbereich als im medizinischen Sprachgebrauch teilweise üblich umfaßt.

Häufig läßt sich die Diagnose aufgrund der lediglich probabilistischen Aussagekraft diagnostischer Informationen nicht mit letzter Sicherheit ermitteln ${ }^{100}$. In diesen Fällen repräsentiert die Diagnose eine "Wahrscheinlichkeitsaussage über das Vorhandensein einer Krankheit"101. Insofern kann umgekehrt jede im Verlauf des diagnostischen Prozesses gebildete Hypothese auch als Diagnose aufgefaßt werden; genauer: als Diagnose, die einen ganz bestimmten Wissensstand des Arztes reflektiert (vgl. dazu Abbildung 3). Je nach Art und Umfang der berücksichtigten Informationen ergeben sich somit unterschiedlich gut abgesicherte bzw. verschiedene Diagnosen, die gegeneinander abzugrenzen sind. Um diese Verbindung zwischen Diagnose und diagnostischem Prozeß auch begrifflich zu verdeutlichen, unterscheidet man mehrere Arten der Diagnose:

97: Zur Definition der sekundären Prävention vgl. z.B. VON STACKELBERG, H.H. (1980), S. 2.; WAGNER, G. (1981), S. 54.

98: Beschwerden können als vom Patienten wahrgenommene Symptome bezeichnet werden. Zur Abgrenzung zwischen Beschwerden, Befunden und Symptomen siehe ZÖLLNER, N. (1986).

99: Zu den Besonderheiten des "screening" im Vergleich zur normalen Diagnostik siehe ausführlich HABBEMA, J.D.F./VAN OORTMARSSEN, G.J. (1982) sowie BLUMBERG, M.S. (1957), S. 351.

100: Vgl. dazu ausführlich Kapitel 3 dieser Arbeit.

101: RICHTER, K. et al. (1988), S. 655. 
- die Blickdiagnose, bei der der Arzt dem Patienten die Beschwerden im Sinne des Wortes "ansieht" oder bestenfalls noch unmittelbare "sonstige sensorische Wahrnehmungen"102 zu Rate zieht;

- die Symptom- oder Anhiebsdiagnose ${ }^{103}$, für die schon wenige anamnestische Daten sowie eine kurze körperliche Untersuchung ausreichen;

- die Vermutungsdiagnose, die im Vergleich zur Symptomdiagnose auf einer eingehenden Anamnese und einer ausführlichen körperlichen Untersuchung aufbaut;

- die vorläufige Diagnose, die zusätzlich noch andere Untersuchungsverfahren (z.B. Röntgen) sowie die Ergebnisse von Labortests berücksichtigt, und schließlich

- die Abschlußdiagnose, in die als weitere Information das Ergebnis der Verlaufskontrolle eingeht.

Diese Aufzählung erhebt keinerlei Anspruch auf Vollständigkeit, da sich je nach verwendeter Einteilung des diagnostischen Prozesses weitere spezielle Diagnosebegriffe ergeben ${ }^{104}$. Überdies findet sich die hier referierte begriffliche Unterteilung nicht einheitlich in der medizinischen Literatur: beispielsweise bezeichnet Meister die Vermutungsdiagnose (wie oben definiert) als Verdachtsdiagnose ${ }^{105}$, während Zimmermann/Quietzsch von der vorläufigen als einer Vermutungsdiagnose sprechen ${ }^{106}$ und Dahmer den Begriff der Arbeitsdiagnose verwendet ${ }^{107}$, damit aber ebenfalls die vorläufige Diagnose in der oben angesprochenen Bedeutung meint.

Diese Vielfalt diagnosebezogener Termini, deren uneinheitliche Verwendung man bedauern mag, belegt in empirischer Hinsicht die zuvor angestellten Überlegungen in

102: VOGT, W. (1982), S. 177.

103: $\mathrm{Zu}$ diesem und den folgenden speziellen Diagnosebegriffen vgl. ANSCHÜTZ, F. (1985), S. $5 f$.

104: So erwähnt z.B. GROSS noch die Einweisungs- und die Entlassungsdiagnose; vgl. GROSS, R. (1969), S. 7.

105: Vgl. MEISTER, H. (1985), S. 681. Aufgrund des hypothetischen Charakters jeder Diagnose erscheint die Verwendung des Begriffs "Vermutungsdiagnose" zur Kennzeichnung einer speziellen Gruppe von Diagnosen ohnehin unglücklich gewählt.

106: Vgl. ZIMMERMANN, S./QUIETZSCH, D. (1983), S. 10.

107: Vgl. DAHMER, J. (1980), S. 33. 
Bezug auf den Zusammenhang zwischen Diagnose und diagnostischem Prozeß. Je nach ihrer Position im diagnostischen ProzeB, also nach Art und Umfang der berücksichtigten Informationen, ergeben sich qualitativ verschiedene Diagnosen ${ }^{108}$. Diese Eigenschaft begründet nach Gross eine von insgesamt drei Arten der zeitlichen Abhängigkeit des medizinischen Diagnosebegriffs ${ }^{109}$. Ein weiterer, unmittelbar einsichtiger, Einfluß der Zeit auf die ärztliche Diagnose folgt aus der Veränderung einer Krankheit im Zeitablauf, also der Existenz eines Krankheitsverlaufs: je nach dem Zeitpunkt innerhalb dieses Krankheitsverlaufs wird bei der (korrekten) Diagnose ein modifiziertes Krankheitsbild festgestellt. Schließlich ergibt sich noch eine dritte zeitliche Abhängigkeit der medizinischen Diagnose, da die diagnostischen Möglichkeiten zu jedem beliebigen Zeitpunkt begrenzt werden durch den Stand des medizinischen Fachwissens, das seinerseits eine Funktion der Zeit ist. Engle/Davis unterscheiden in diesem Zusammenhang fünf Sicherheitsgrade, die eine Diagnose aufgrund der vorhandenen ätiologischen Kenntnisse aufweisen kann ${ }^{110}$.

Neben diesen primär zeitbedingten Einflüssen wirken noch andere Faktoren auf den individuellen Krankheitsverlauf und damit auf die ärztliche Diagnose ein. Es handelt sich hierbei um sogenannte diagnostische Parameter ${ }^{111}$, die zusammengefaßt werden können in der Gruppe der Umweltfaktoren einerseits ${ }^{112}$ und der Gruppe der individuell-spezifischen Parameter andererseits (z.B. individuelle Gewohnheiten, dispositive Faktoren), die die Persönlichkeit eines Patienten ausmachen. Schon allein diese Vielfalt der diagnostischen Parameter, die gewissermaßen als Störgrößen im Sinne individueller Abweichungen vom typischen Krankheitsmuster und -verlauf auftreten, deutet auf das Spannungsverhältnis hin, in dem sich die ärztliche Diagnostik befindet: nämlich die Notwendigkeit, sowohl das "Allgemeine" oder "Typische" eines Krankheitsfalls zu erfassen (und damit die Verbindung zur Nosologie herzustellen), als auch in erforderlichem Umfang auf die individuellen Gegebenheiten, das "Besondere", einzugehen; insofern sind Diagnosen immer "Kompromisse aus den Erfordernissen: Typisierung und Individualisierung."113.

108: Diese müssen allerdings nicht unterschiedlichen Inhalts sein.

109: Vgl. GROSS, R. (1969), S. $6 \mathrm{ff}$.

110: Vgl. ENGLE, R.L./DAVIS, B.J. (1963), S. $517 f f$.

111: Vgl. GROSS, R. (1969), S. 20ff; vgl. auch GUDER, W.G. (1980), der bei klinisch-chemischen Untersuchungen zwischen Einflußgrößen und Störgrößen unterscheidet.

112: KELLER unterscheidet drei Umweltfaktoren; vgl. KELLER, H. (1986), S. 152.

113: GROSS, R. (1969), S. 9; ähnlich ENGLE, R.L. (1964), S. 12. 
Es reicht also bei einer medizinischen Diagnose nicht aus, lediglich allgemein die (vermutete) Krankheit eines Patienten zu benennen. Vielmehr müssen weitere Angaben hinzukommen, um die fallspezifischen Besonderheiten zu erfassen. Eine Diagnose, die diesen Erfordernissen Rechnung trägt, sollte demnach Auskunft geben über:

- die Art der Erkrankung,

- deren Lokalisation und Dauer,

- (soweit möglich) deren Ursache und

- die näheren Kennzeichen des Patienten ${ }^{114}$.

Erst in dieser erweiterten Form stellt der Inhalt der medizinischen Diagnose diejenigen Informationen bereit, die ein weiteres, Erfolg versprechendes ärztliches Handeln ermöglichen.

\subsubsection{Diagnostische Maßnahmen}

Um beispielsweise Kosten-Nutzen-Betrachtungen anstellen zu können, benötigt man sowohl eine Beschreibung der ärztlichen Vorgehensweise auf dem Weg zur Diagnose als auch genaue Angaben über die Therapie und deren Auswirkungen auf den Gesundheitszustand des Patienten. Beide Komponenten zusammen ergeben eine vollständige Darstellung der Entscheidung des Arztes. Im folgenden geht es zunächst darum, den diagnostischen Prozeß näher zu analysieren. $\mathrm{Zu}$ diesem $\mathrm{Zweck}$ wird jedes mögliche diagnostische Vorgehen des Arztes bei einem Krankheitsfall als diagnostische Strategie bezeichnet ${ }^{115}$. Eine diagnostische Strategie umfaßt also sämtliche Untersuchungen, die ein Arzt fallbezogen zur Erstellung der Diagnose entweder selbst durchführt oder an andere Personen delegiert. Die Information, die im Verlauf der Diagnostik erhoben wird, kann im allgemeinen sowohl die Abfolge weiterer diagnosebezogener Handlungen als auch den Endpunkt des diagnostischen Prozesses selbst beeinflussen. Damit läßt sich eine diagnostische Strategie als auf einen Krank-

\section{4: Vgl. GROSS, R. (1982), S. 58.}

115: Eine diagnostische Strategie stellt allerdings keine Strategie im entscheidungstheoretischen Sinn dar, da sie lediglich das Vorgehen des Arztes bei der Informationsbeschaffung spezifiziert. 
heitsfall bezogenes Vorgehen interpretieren, das in Abhängigkeit von der vorliegenden diagnostischen Information eindeutig entweder die nächste Untersuchung oder auch den $A b s c h l u B$ der Diagnostik angibt.

Ein Beispiel ${ }^{116}$ möge zur Veranschaulichung dieser Terminologie dienen: angenommen, ein Allgemeinarzt werde in seiner Praxis von einem Mann aufgesucht, der auf den ersten Blick unter Rückenschmerzen zu leiden scheint ${ }^{117}$. Das weitere Vorgehen des Arztes könnte beispielsweise darin bestehen, zunächst in knapper Form die aktuellen Beschwerden des Patienten zu ermitteln. Aus dieser krankheitsbezogenen Anamnese erfährt der Arzt Näheres über die heftigen Schmerzen, die der Patient in der Kreuzgegend verspürt und prüft im Anschluß daran, ob sich aus der biographischen und der Familienanamnese zusätzliche diagnoserelevante Informationen gewinnen lassen. Aufgrund der Untersuchung 118 derjenigen Körperregionen, in denen die Symptome aufgetreten sind, und der Ergebnisse einiger Labortests kann der Arzt die Diagnose so weit einengen, $\mathrm{da} B$ er sich zur Einleitung der Therapie entschlie $\beta \mathrm{t}^{119}$. Damit ist in diesem Beispiel der diagnostische Prozeß vorläufig abgeschlossen.

Im Sinne der oben eingeführten Terminologie stellt die Gesamtheit der Handlungen, die der Arzt zur (vorläufigen) diagnostischen Abklärung dieses Falls in der geschilderten Reihenfolge gewählt hat, einen Teil seiner diagnostischen Strategie dar. Es handelt sich dabei um jenen Bestandteil, der für diese spezielle diagnostische Information zur Anwendung kommt. Wäre beispielsweise das Ergebnis der Anamnese unterschiedlich ausgefallen, hätte der Arzt im Rahmen derselben diagnostischen Strategie möglicherweise ein anderes Vorgehen gewählt.

116: Vgl. ausführlich DAHMER, J. (1980).

117: Mit dieser Blickdiagnose, die der Arzt aus dem Verhalten des Patienten (leicht gebückter Gang o.ä.) unmittelbar ableiten kann, hat der diagnostische Prozeß schon begonnen.

118: Vgl. zur körperlichen Untersuchung und zur Anamnese ausführlich: GROSS, R./SPECHTMEYER, H. (1982).

119: Das bedeutet nicht, daß mit der Therapie grundsätzlich erst nach Abschluß der Diagnostik begonnen werden kann. 


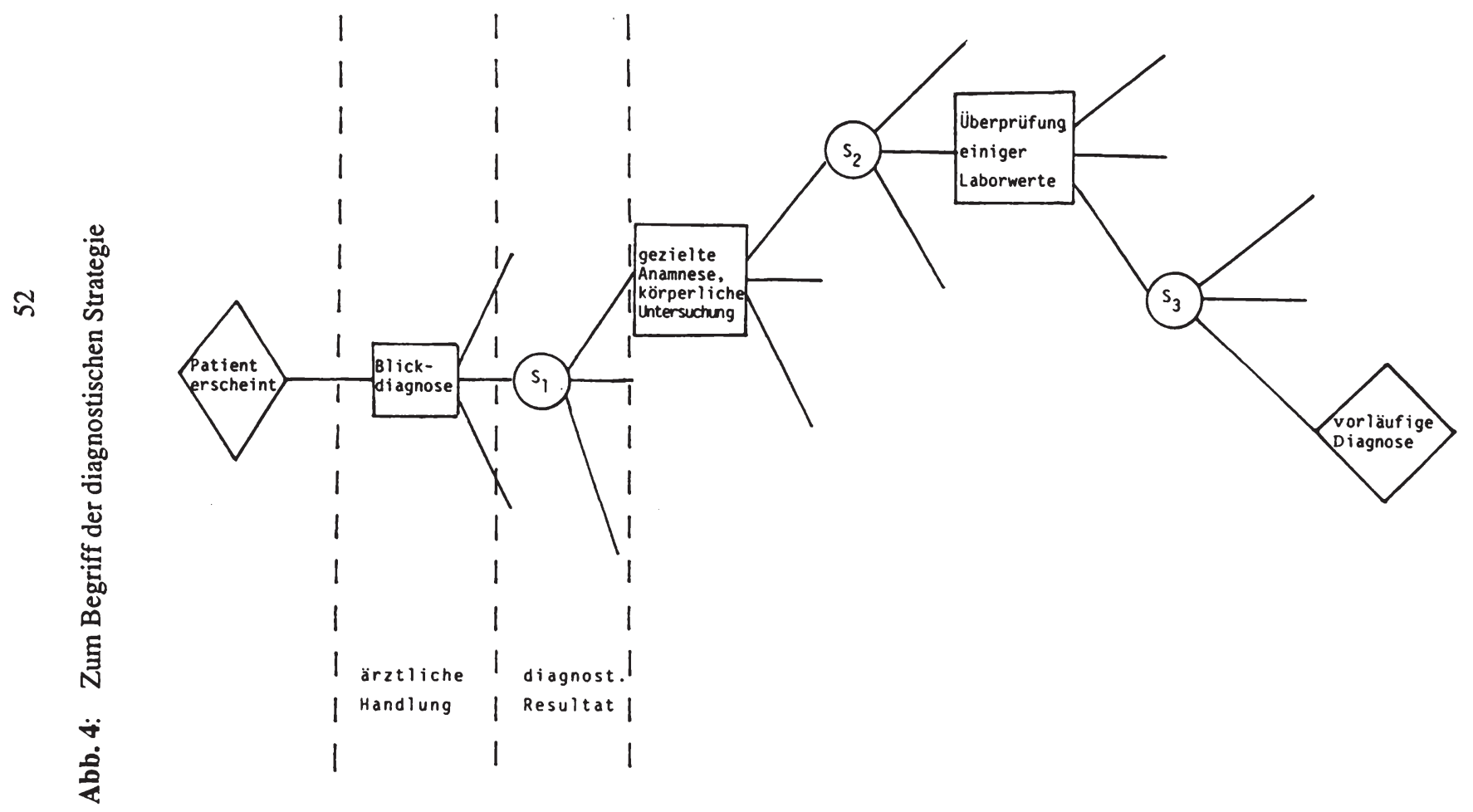

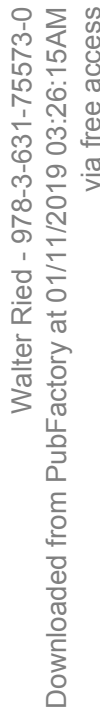


Diese Abhängigkeit der einzelnen Schritte einer diagnostischen Strategie von den jeweils zur Verfügung stehenden Informationen versucht Abbildung $4 \mathrm{zu}$ veranschaulichen. Die rechteckig umrandeten Felder kennzeichnen diagnosebezogene Handlungen, während innerhalb der Kreise die Größen $S_{i}$ Symptome bzw. Befunde darstellen, also jene diagnostischen Informationen, die auch als diagnostische Resultate bezeichnet werden. Sowohl auf der Ebene der ärztlichen Handlungen als auch bei den diagnostischen Informationen existieren im allgemeinen mehrere $\mathrm{Al}$ ternativen ${ }^{120}$. Entscheidet jedoch der Arzt über den nächsten diagnostischen Schritt gerade diese Selektion kennzeichnet eine diagnostische Strategie -, so bestimmt im Gegensatz dazu der Zufall, welches diagnostische Resultat im Anschluß daran auftritt $^{121}$. Nicht zuletzt der besseren Übersichtlichkeit wegen zeigt Abbildung 4 daher nur jenen Bestandteil der vom Arzt ausgewählten diagnostischen Strategie, der sich auf die Symptome $S_{1}, S_{2}$ und $S_{3}$ bezieht.

Gegeben eine bestimmte Abfolge diagnosebezogener Informationen, die im Verlauf der Diagnostik auftreten, liefert eine diagnostische Strategie eine genaue Abfolge einzelner ärztlicher Handlungen auf dem Weg zur Diagnose. Es kann beispielsweise sinnvoll sein, innerhalb der Anamnese und eventuell auch innerhalb der körperlichen Untersuchung noch weitere Teilschritte gegeneinander abzugrenzen, wenn das weitere diagnostische Vorgehen des Arztes von den Ergebnissen dieser Teilschritte abhängt. Umgekehrt besteht vielfach für den Arzt die Möglichkeit, einen Teil der Anamnese zeitlich parallel zur körperlichen Untersuchung zu erheben ${ }^{122}$; in diesem Fall sind beide Vorgänge als ein gemeinsamer Schritt innerhalb der diagnostischen Strategie anzusehen.

Wie zuvor erwähnt, steht dem Arzt eine ganze Reihe alternativer Vorgehensweisen zur diagnostischen Abklärung eines Krankheitsfalls zur Verfügung. So hätte in dem oben angesprochenen Beispiel anstelle der ausführlichen auch eine gezielte Anamnese erfolgen können, ebenso wäre eine eingehendere körperliche Untersuchung, eine Röntgenanalyse, die Überprüfung einer größeren Anzahl von Laborwerten oder eine Kombination dieser Alternativen denkbar gewesen. Jede dieser Modifikationen des ursprünglichen Vorgehens - bei gleicher zur Verfügung stehender

120: Dies verdeutlicht Abbildung 4, indem sowohl von jeder diagnosebezogenen Handlung als auch von jedem $S_{i}$ mehrere Pfade ausgehen.

121: Zur Erläuterung dieser Zufallsabhängigkeit diagnostischer Informationen vgl. 2.4.3.

122: Vgl. GROSS, R. (1969), S. 36ff. 
diagnostischer Information - führt zu einer anderen diagnostischen Strategie. Wenn man alle möglichen diagnostischen Strategien im Zusammenhang mit einem bestimmten Krankheitsfall - bzw. einer homogenen Gruppe von Krankheitsfällen - auflistet, erhält man eine vollständige Beschreibung des damit verbundenen diagnostischen Prozesses. Die Erstellung einer solchen Liste bildet eine unverzichtbare Voraussetzung für die ökonomische Analyse der medizinischen Diagnostik, denn ohne deren Kenntnis ließe sich nur eine isolierte Betrachtung einer diagnostischen Strategie durchführen ${ }^{123}$. Die ökonomische Analyse zeichnet sich im Gegensatz dazu jedoch dadurch aus, daß auch bei der Untersuchung einer einzelnen Strategie die Kosten und Nutzen alternativer (hier: diagnostischer) Vorgehensweisen $\mathrm{zu}$ berücksichtigen sind.

Die vorangegangenen Ausführungen brachten den zusammengesetzten Charakter diagnostischer Strategien zum Ausdruck, von denen jede einzelne in der Regel aus mehreren ärztlichen Handlungen besteht. Diese einzelnen Komponenten diagnostischer Strategien sollen im folgenden als diagnostische Maßnahmen bezeichnet werden. Als diagnostische Maßnahme gilt mithin jede Maßnahme, durch die ein Arzt oder eine mit dessen Kompetenzen ausgestattete Person medizinische Informationen zum Zwecke der Feststellung des Krankheitszustands eines Individuums erhebt ${ }^{124}$. An dieser Stelle wird bewußt nicht von diagnostischen Leistungen gesprochen, um in der weiteren Analyse nicht auf einen Begriffsinhalt festgelegt zu sein, den etwa die Gebührenordnung für ärztliche Leistungen bestimmt ${ }^{125}$.

Die Definition einer diagnostischen Maßnahme umfaßt erstens auch jene Maßnahmen, die nicht von einem Arzt getroffen werden. Im folgenden wird allerdings davon ausgegangen, daß zumindest mittelbar ein Arzt für die Durchführung diagnostischer Maßnahmen verantwortlich zeichnet (z.B. Tests in einer Laborgemeinschaft). Zweitens muß eine diagnostische Maßnahme nicht notwendigerweise zusätzliche medizinische Informationen erbringen; im Extremfall ergibt sich keine Veränderung der vorhandenen Informationsbasis ${ }^{126}$. Schließlich muß die Person, die sich

123: Vgl. dazu ausführlich Kapitel 4, in dem allerdings lediglich ein Teil des diagnostischen Prozesses analysiert wird.

124: Vgl. GROSS, R./SPECHTMEYER, H. (1982), S. 3. Zum Begriff des Krankheitszustands vgl. Abschnitt 2.4.1.

125: Vgl. ähnlich KÖBBERLING, J./TRAMPISCH, H.-J./WINDELER, J. (1989), S. 4.

126: Dies betrifft die diagnostischen Maßnahmen ohne Informationsleistung; vgl. dazu Abschnitt 3.4 . 
einer diagnostischen Maßnahme unterzieht, nicht unbedingt krank sein, d.h. es fallen unter die oben angegebene Definition auch Maßnahmen im Rahmen der sekundären Prävention (Früherkennung bzw. "screening"), desgleichen die Anwendung von Diagnosetests bei als gesund vermuteten Personen, um das Vorliegen bestimmter Krankheiten auszuschließen ${ }^{127}$.

In theoretischer Hinsicht erweist sich die oben gegebene Definition zunächst als problematisch im Hinblick auf die Abgrenzung einzelner diagnostischer Maßnahmen untereinander. Es bleibt nämlich unklar, wie stark eine diagnostische Strategie disaggregiert werden muß, um zu den diagnostischen Maßnahmen zu gelangen. Bei der Überprüfung von Laborwerten bietet es sich an, jeden einzelnen Test bzw. jede Order als diagnostische Maßnahme anzusehen. Anders sieht es im Fall der Anamnese aus: es wäre prinzipiell mit der Definition vereinbar, z. B. jede einzelne Frage, die der Arzt im Rahmen der Anamnese an den Patienten richtet, als eigene diagnostische Maßnahme aufzufassen mit der Folge, daß sich für eine diagnostische Strategie eine unüberschaubare Anzahl diagnostischer $\mathrm{Maßnahmen} \mathrm{ergäbe.} \mathrm{Verständlicherweise}$ geht diese detaillierte Unterteilung für die Zwecke der ökonomischen Analyse zu weit. Eine sinnvolle Unterteilung läßt sich jedoch mit Hilfe der Entscheidungstheorie finden, indem man die konkret vorliegende medizinische Entscheidungssituation berücksichtigt ${ }^{128}$. Daher dürften allfällige Abgrenzungsprobleme bei der ökonomischen Untersuchung eines speziellen diagnostischen Problems nicht auftauchen.

Die weiteren Ausführungen nehmen vornehmlich Bezug auf die Analyse klinischchemischer Labortests, die auch die Literatur durchgängig als Referenzfall auswählt. Schon an dieser Stelle muß allerdings darauf hingewiesen werden, daß die dabei gewonnenen Erkenntnisse grundsätzlich auch für Maßnahmen der Anamnese und der körperlichen Untersuchung gelten ${ }^{129}$. Diese Übertragbarkeit der Resultate beruht auf einer grundlegenden strukturellen Ähnlichkeit aller diagnostischen Maßnahmen, die

127: Vgl. KELLER, H. (1986), S. 151.

128: Häufig dürfte es zum Beispiel völlig ausreichen, zwischen einer gezielten und einer umfassenden Anamnese zu unterscheiden.

129: Vgl. HABBEMA, J.D.F./HILDEN, J/BJERREGAARD, B. (1978), S. 219; WEINSTEIN, M.C. et al. (1980), S. 79 sowie DEPARTMENT OF CLINICAL EPIDEMIOLOGY AND BIOSTATISTICS (1983), S. 429. Ein Unterschied zwischen diesen häufig unterschiedenen Klassen diagnostischer Maßnahmen besteht insofem, als die Ergebnisse sowohl der Anamnese als auch der körperlichen Untersuchung schwieriger zu quantifizieren sind; vgl. LUSTED, L.B.STAHL, W.R. (1964), S. 158. 
sich wie folgt beschreiben läßt. Vor der Durchführung einer solchen Maßnahme besitzt der Arzt eine Vermutung über das beim Patienten vorhandene Krankheitsbild. Diese Vermutung kann zum Beispiel auf den Ergebnissen zuvor veranlaßter diagnostischer Maßnahmen beruhen. Aufgrund der $\mathrm{zu}$ analysierenden diagnostischen Maßnahme erhält der Arzt neue Informationen, die seine Wahl des weiteren diagnostischen und therapeutischen Vorgehens beeinflussen. Dieser Sachverhalt kennzeichnet sowohl die Anamnese als auch die körperliche Untersuchung, die klinisch-chemischen Labortests und die bildgebenden Verfahren (z.B. Computer- und Kernspintomographie). Insofern erscheint es sinnvoll, diese diagnostischen Maßnahmen sämtlich in einem weiteren Sinne als Diagnosetests aufzufassen ${ }^{130}$.

\subsection{Entscheidungstheoretische Voraussetzungen}

\subsubsection{Krankheitszustände}

Die für die weitere Analyse unterstellte medizinische Entscheidungssituation läßt sich wie folgt skizzieren ${ }^{131}$ : der Arzt hat bei einem Patienten bestimmte Symptome festgestellt, möglicherweise auch schon Labortests durchgeführt. Nun steht er vor dem Problem, über die weitere Diagnostik entscheiden zu müssen. Zur genauen Kennzeichnung dieser-im folgenden als Ausgangslage bezeichneten-Situation benötigt man insbesondere eine vollständige Liste aller Krankheitszustände, die aus der Sicht des Arztes bei dem betreffenden Patienten in Betracht zu ziehen sind. Dabei wird bewußt von Krankheitszuständen anstelle von Krankheiten gesprochen, um nicht auf die Übernahme bereits existierender Krankheits-Klassifikationen angewiesen zu sein ${ }^{132}$.

130: Siehe dazu DEPARTMENT OF CLINICAL EPIDEMIOLOGY AND BIOSTATISTICS (1983), S. 562 (insbesondere Tabelle 4).

131: Es handelt sich lediglich um diejenigen Aspekte, die für die Analyse der Information diagnostischer Maßnahmen in Kapitel 3 von Bedeutung sind. Eine vollständige Beschreibung findet sich in Abschnitt 4.2.

132: Diese terminologische Konvention reflektiert die weiter unten angesprochenen Forderungen, welche die Menge der Krankheitszustände erfüllen muß, um einen Zustandsraum im Sinne der Entscheidungstheorie zu bilden. So weist beispielsweise Ginsberg auf die Unzulänglichkeit des Schemas der WHO zur Klassifikation von Krankheiten (ICD) für die Analyse spezieller medizinischer Entscheidungssituationen hin; vgl. GINSBERG, A.S. (1972), S. $215 \mathrm{f}$. Zum Problem der Definition und Abgrenzung von Krankheiten vgl. CROFT, D.J./MACHOL, R.E. (1974), S. 69; CARD, W.I. (1975), S. 194 sowie ausführlich WULFF, H.R. (1979) und WULFF, H.R. (1981), S. 46ff. 
Im weiteren Verlauf dieser Arbeit wird unterstellt, die Menge $\mathrm{K}$ der Krankheitszustände, die der Arzt in der Ausgangslage bei einem Patienten in Betracht zu ziehen hat, sei endlich ${ }^{133}$. In der entscheidungstheoretischen Literatur bezeichnet man $\mathrm{K}$ als Zustandsraum, wobei die einzelnen Zustände so definiert sind, daß in jeder möglichen Situation genau ein Element von $\mathrm{K}$ den "wahren" Zustand angibt ${ }^{134}$. Die einzelnen Krankheitszustände $\mathrm{K}_{\mathrm{i}}(\mathrm{i}=1, \ldots, \mathrm{n})$ bilden demzufolge eine Partition der Menge $\mathrm{K}$. Dabei braucht nicht immer jedes Element $\mathrm{K}_{\mathrm{i}}$ dieser Menge einen Krankheitszustand im eigentlichen Sinn zu repräsentieren, da in einigen Fällen auch die Möglichkeit völliger Gesundheit des Patienten nicht auszuschließen sein wird. Die Annahme der Endlichkeit mag für manche medizinische Entscheidungssituation restriktiv erscheinen, da unter Umständen schon innerhalb einer Krankheit die Übergänge zwischen einzelnen Zuständen fließend sind und eine vollständige Berücksichtigung ein Kontinuum von Möglichkeiten nahelegt. Andererseits ist es durchaus denkbar, daß eine überabzählbare Zustandsmenge $\Omega$ mit Hilfe einer geeigneten Äquivalenzrelation in eine endliche Anzahl "payoff-relevanter" Zustandsklassen $\Omega_{\mathrm{i}}$ zerlegt werden kann, die für die entscheidungstheoretische Analyse allein von Belang sind und den oben angegebenen $\mathrm{K}_{\mathrm{i}}$ entsprechen ${ }^{135}$. Eine Zustandsklasse $\Omega_{\mathrm{i}}$ enthält dann sämtliche Krankheitszustände, für die eine beliebige ärztliche Handlung jeweils denselben Nutzen für den Patienten erzeugt ${ }^{136}$.

133: Formal stellen die Krankheitszustände spezielle Ereignisse (Elementarereignisse) einer Ereignisalgebra dar. Da sich zu jeder Ereignisalgebra eine isomorphe Mengenalgebra finden läßt, bedeutet die Betrachtung der entsprechenden Mengen keine Einschränkung; vgl. RENYI, A. (1966), S. 12ff. sowie RENYI, A. (1970), S. $5 \mathrm{ff}$.

134: Vgl. z.B. BAMBERG, G./COENENBERG, A.G. (1989), S. 16.

135: Zum Konzept des "payoff-relevanten" Zustands vgl. MARSCHAK, J./MIYASAWA, K. (1968), S. 202f.

136: Im Hinblick auf die Nutzenposition des Patienten ergibt es in diesem Fall keinen Sinn, zwischen den einzelnen Elementen einer Zustandsklasse zu unterscheiden.

Aus der Perspektive der Entscheidungstheorie besitzt die Menge der Krankheitszustände zumindest dann eine genügend feine Struktur, wenn sie die Angabe einer deterministischen Konsequenzenfunktion erlaubt. Vgl. dazu BAMBERG, G./COENENBERG, A.G. (1989), S. 22f. sowie im medizinisch-diagnostischen Kontext HABBEMA, J.D.F./HILDEN, J. (1981), S. 81 und die in Abschnitt 4.2.2 der vorliegenden Arbeit eingeführte Konsequenzenfunktion. 
Weiterhin wird angenommen, den einzelnen Krankheitszuständen $\mathrm{K}_{\mathrm{i}}$ lassen sich Wahrscheinlichkeiten $\mathrm{P}\left(\mathrm{K}_{\mathrm{i}}\right)$ in einer Weise zuordnen, die es erlaubt, $\mathrm{P}$ als Wahrscheinlichkeitsverteilung auf $\mathrm{K}$ zu interpretieren. In diesem Fall bezeichnet man $\mathrm{P}$ als priore Wahrscheinlichkeitsverteilung auf der Menge K der Krankheitszustände ${ }^{137}$. Dieser Annahme liegt die Vorstellung zugrunde, der Arzt sei in der Lage, sein gesamtes Wissen in Bezug auf die als möglich erachteten Krankheitszustände in der Form einer Wahrscheinlichkeitsverteilung zu quantifizieren ${ }^{138}$. Aufgrund ihrer Herkunft bezeichnet man die Größen $\mathrm{P}\left(\mathrm{K}_{\mathrm{i}}\right)$ auch als subjektive Wahrscheinlichkeiten. Unter dieser Voraussetzung läßt sich der Krankheitszustand eines Patienten als Zufallsvariable $\mathrm{K}$ veranschaulichen, deren Ausprägungen in der Ausgangslage die prioren Wahrscheinlichkeiten zukommen ${ }^{139}$.

Obwohl die Annahme der Existenz einer prioren Wahrscheinlichkeitsverteilung in der Entscheidungstheorie sehr häufig verwendet wird, ist es nicht immer einfach, eine geeignete theoretische Begründung zu finden. Schließlich kann man mit einigem Recht argumentieren, es sei objektiv schon entschieden und damit keineswegs zufallsabhängig, in welchem Krankheitszustand ein Patient sich befindet. Ohne auf die Kontroverse um die Bedeutung und die Nützlichkeit subjektiver Wahrscheinlichkeiten näher einzugehen, läßt sich diesem Argument mit Bezug auf die hier interessierende medizinische Entscheidungssituation die Möglichkeit einer häufigkeitstheoretischen Interpretation entgegenhalten ${ }^{140}$. Vielfach weiß der Arzt aus früheren, ähnlich gelagerten Entscheidungssituationen, mit welchen Anteilen die einzelnen Krankheitszustände $\mathrm{K}_{\mathrm{i}}$ aufgetreten sind. Es liegt dann nahe, diese Anteile als Schätzungen für die gesuchten Wahrscheinlichkeiten heranzuziehen ${ }^{141}$.

137: Zum Begriff und zur Interpretation der prioren Verteilung siehe BERGER, J.O. (1985), S. 74ff. Das Adjektiv "prior" weist darauf hin, daß diese Wahrscheinlichkeiten vor der Beschaffung von weiteren (hier: diagnostischen) Informationen als gültig erachtet werden.

138: Vgl. dazu LINDLEY, D.V. (1974), S. $11 \mathrm{ff}$.

139: Der Begriff der Zufallsvariable wird hier - wie allgemein in der Entscheidungstheorie üblich weit gefaßt. Als Zufallsvariable gilt jede (meßbare) Funktion auf einem Ereignisraum; vgl. BLACKWELL, D./GIRSHICK, M.A. (1954), S. 79; FERGUSON, T.S. (1967), S. 31; FERSCHL, F. (1975), S. 97 sowie BREMAUD, P. (1988), S. 33f.

140: Vgl. dazu BERGER, J.O. (1985), S. 20ff. sowie die dort angegebene Literatur.

141: Vgl. dazu SINN, H.-W. (1980), S. $16 \mathrm{ff}$. 


\subsubsection{Bedingte Wahrscheinlichkeiten und das Theorem von Bayes}

Sei $(\Omega, I, P)$ ein Wahrscheinlichkeitsraum, der den Axiomen von Kolmogoroff genügt ${ }^{142}$. Die Menge $\Omega$ repräsentiert den Ergebnisraum, $\mathcal{I}$ bezeichnet eine geeignete $\sigma$-Algebra von Teilmengen von $\Omega$ und $P$ stellt ein auf $\mathcal{I}$ definiertes Wahrscheinlichkeitsmaß dar ${ }^{143}$. Wie schon in Abschnitt 2.4.1 angesprochen, lassen sich die Elemente von $\mathfrak{I}$ als Ereignisse interpretieren. Im folgenden interessiert speziell die Frage, in welcher Weise das Eintreten eines Ereignisses $A \in \mathfrak{I}$ die Wahrscheinlichkeiten der in $\mathfrak{I}$ enthaltenen Ereignisse beeinflußt. Dazu muß zunächst der Begriff der bedingten Wahrscheinlichkeit eingeführt werden.

Unter der bedingten Wahrscheinlichkeit eines Ereignisses $B$ gegeben ein (anderes) Ereignis A - Schreibweise $\mathrm{P}(\mathrm{B} \mid \mathrm{A})$ - versteht man die Wahrscheinlichkeit, mit der B eintritt, falls das Ereignis A realisiert wurde. Um diese Größe sinnvoll definieren zu können, müssen sowohl $A$ als auch B Elemente der gewählten $\sigma$-Algebra sein. Wenn man mit $\mathrm{P}(\mathrm{A})$ bzw. $\mathrm{P}(\mathrm{A} \cap \mathrm{B})$ die unbedingten Wahrscheinlichkeiten des Eintretens von $A$ bzw. von $A$ und $B$ zusammen bezeichnet, läßt sich die bedingte Wahrscheinlichkeit wie folgt definieren ${ }^{144}$ :

$$
\begin{aligned}
& P(B \mid A)=\frac{P(A \cap B)}{P(A)}, \\
& P(A)>0 ; \quad A, B \in \mathfrak{I} .
\end{aligned}
$$

Dabei ist $\mathrm{P}(\mathrm{A})>0$ vorauszusetzen, um einen wohldefinierten Ausdruck zu erhalten: $P(A)=0$ impliziert aufgrund der Monotonie des Wahrscheinlichkeitsmaßes $\mathrm{P}(\mathrm{A} \cap \mathrm{B})=0$ und man erhält für die bedingte Wahrscheinlichkeit $\mathrm{P}(\mathrm{B} \mid \mathrm{A})$ einen unbestimmten Ausdruck ${ }^{145}$.

142: Vgl. z.B. RENYI, A. (1966), S. 35f. sowie PFANZAGL, J. (1988), Kapitel 1.

143: Bekanntlich gibt es bei überabzählbarem $\Omega$ o-Algebren - wie z.B. die Potenzmenge von $\Omega$-, die "zu groß" sind, um ein Maß zuzulassen; vgl. BILLINGSLEY, P. (1986), S. 41.

144: Exakter wäre es, anstelle von unbedingten Wahrscheinlichkeiten von Wahrscheinlichkeiten zu sprechen, die unter denjenigen Bedingungen Gültigkeit besitzen, die das ursprüngliche $\mathrm{Zu}$ fallsexperiment $(\Omega, \Im)$ kennzeichnen. Vgl. dazu sowie zum Begriff des Experiments RENYI, A. (1970), Kapitel 1.

145: Dennoch kann die bedingte Wahrscheinlichkeit in einigen Fällen auch für $P(A)=0$ sinnvoll definiert werden; vgl. RENYI, A. (1966), S. 219. 
Falls A ein Ereignis mit den in der Definition geforderten Eigenschaften ist, stellt $\mathrm{P}(\cdot \mid \mathrm{A})$ selbst ein Wahrscheinlichkeitsmaß dar, d.h. die bedingte Wahrscheinlichkeit ist nichtnegativ, volladditiv und erfüllt die Bedingung $P(\Omega \mid A)=1$. Vielfach bezeichnet man $\mathrm{P}(\mathrm{B})$ als priore, $\mathrm{P}(\mathrm{B} \mid \mathrm{A})$ hingegen als posteriore Wahrscheinlichkeit des Ereignisses B. Mit dieser Terminologie wird jedoch nichts über die Reihenfolge ausgesagt, in der die Ereignisse A bzw. B eintreten. Eine zeitliche Struktur besteht lediglich im Hinblick auf die zur Verfügung stehenden Informationen ${ }^{146}$.

Der hier eingeschlagene Weg, bedingte mit Hilfe von unbedingten Wahrscheinlichkeiten zu definieren, entspricht einer in der Literatur weitgehend üblichen Vorgehensweise. Im Gegensatz dazu argumentiert Renyi, alle Wahrscheinlichkeiten von Ereignissen seien als bedingt anzusehen, da sie sich ebenfalls auf bestimmte - häufig als selbstverständlich angenommene - Bedingungen beziehen ${ }^{147}$. Um dies zu untermauern, konstruiert er bedingte Wahrscheinlichkeitsräume, in denen ausschließlich bedingte Wahrscheinlichkeiten auftreten. Er kann dann zeigen, daß es möglich ist, daraus einen gewöhnlichen, mit einem "unbedingten" Wahrscheinlichkeitsmaß ausgestatteten Wahrscheinlichkeitsraum abzuleiten, der somit einen Spezialfall eines bedingten Wahrscheinlichkeitsraums repräsentiert. In dieser Sichtweise unterscheidet unbedingte und bedingte Wahrscheinlichkeiten lediglich, da $B$ erstere Bezug nehmen auf Umstände, die in den Ergebnisraum $\Omega$ bzw. das darauf definierte Wahrscheinlichkeitsmaß eingegangen sind, während letztere eine zusätzliche Bedingung in Gestalt eines Ereignisses berücksichtigen ${ }^{148}$.

Um das Theorem von Bayes ableiten zu können, wird nun eine Menge von Ereignissen $B_{i} \in \mathfrak{I}(i=1, \ldots, n)$ betrachtet, die eine Partition des Ergebnisraums $\Omega$ darstellen sollen, d.h. die $B_{i}$ sind paarweise disjunkte Ereignisse, deren Vereinigung ge-

146: Es kann durchaus vorkommen - wie schon in Abschnitt 2.4.1 angedeutet, trifft dies typischerweise auf die medizinische Diagnostik zu -, daß objektiv die Frage des Eintretens von B gar nicht mehr zufallsbehaftet, sondern schon entschieden ist. Dennoch erweist sich die Verwendung bedingter Wahrscheinlichkeiten als sinnvoll, wenn die Information über die Realisation von B nicht unmittelbar zugänglich ist.

147: Vgl. RENYI, A. (1970), S. 33ff.

148: Renyi führt als Beispiel das Experiment "Einmaliges Ausspielen eines Würfels", bei dessen Beschreibung etwa die Annahme (d.h. Bedingung) eines "fairen" Würfels verwendet wird; vgl. RENYI, A. (1970), S. 35. 
rade $\Omega$ ergibt. Unter dieser Voraussetzung läßt sich die Wahrscheinlichkeit eines beliebigen Ereignisses $\mathrm{A} \in \mathfrak{I}$ mit Hilfe des Satzes von der totalen Wahrscheinlichkeit bestimmen $^{149}$ :

$$
P(A)=\sum_{i=1}^{n} P\left(A \cap B_{i}\right)
$$

Die einzelnen Ereignisse $A \cap B_{i}$ stellen Teilmengen von $\mathfrak{I}$ dar, die eine Partition des Ereignisses A bilden, so daß Beziehung (2.27) aus der Additivität von P folgt. Gemäß der Definition bedingter Wahrscheinlichkeiten gilt ferner:

$$
P\left(A \cap B_{i}\right)=P\left(A \mid B_{i}\right) \cdot P\left(B_{i}\right) ; \quad i=1, \ldots, n
$$

Damit ergibt sich für die Wahrscheinlichkeit des Ereignisses A:

$$
P(A)=\sum_{i=1}^{n} P\left(A \mid B_{i}\right) \cdot P\left(B_{i}\right)
$$

Interessiert man sich für die Wahrscheinlichkeit eines Ereignisses $B_{k}$ gegeben das Ereignis A, dann folgt dafür aus der Definition bedingter Wahrscheinlichkeiten:

$$
P\left(B_{k} \mid A\right)=\frac{P\left(B_{k} \cap A\right)}{P(A)} \text {. }
$$

Ersetzt man die im Zähler bzw. im Nenner stehenden Größen durch die in Beziehung (2.28) bzw. (2.27a) angegebenen Ausdrücke, so erhält man schließlich:

$$
P\left(B_{k} \mid A\right)=\frac{P\left(A \mid B_{k}\right) \cdot P\left(B_{k}\right)}{\sum_{i=1}^{n} P\left(A \mid B_{i}\right) \cdot P\left(B_{i}\right)} ; k=1, \ldots, n
$$

149: Vgl. z.B. GOLBERG, M.A. (1984), S. 160. 
Gleichung (2.30) repräsentiert eine Variante des Bayes-Theorems ${ }^{150}$. Mit Hilfe dieser Beziehung läßt sich eine a priori, d.h. ohne Kenntnis der Realisation von $A$ vorliegende Wahrscheinlichkeit [hier: $\mathrm{P}\left(\mathrm{B}_{\mathrm{k}}\right)$ ] in die entsprechende posteriore Wahrscheinlichkeit überführen, die bedingte Wahrscheinlichkeit $P\left(B_{k} \mid A\right)$. Da dies für jedes Ereignis in der betrachteten Partition zutrifft, gibt das Bayes-Theorem an, in welcher Weise die priore Wahrscheinlichkeitsverteilung für die Ereignisse $B_{i}(i=1, \ldots, n)$ durch das Wissen bezüglich $\mathrm{A}$ in eine posteriore Verteilung zu transformieren ist.

Tritt das Ereignis A hingegen nicht ein, so kann auch diese Information zur Bestimmung der posterioren Wahrscheinlichkeiten der Ereignisse $B_{i}$ herangezogen werden. Besitzt das zu A bezüglich $\Omega$ komplementäre Ereignis $\bar{A}$ positive Wahrscheinlichkeit, dann liefert Gleichung (2.30) die gesuchten Größen $P\left(B_{k} \mid \bar{A}\right)$, wenn jeweils A durch $\bar{A}$ ersetzt wird. In diesem Fall stellt $\bar{A}$ die Bedingung dar, unter welcher die posteriore Wahrscheinlichkeitsverteilung der Ereignisse $\mathrm{B}_{\mathrm{i}}$ abgeleitet wird.

Die beiden Ereignismengen $\left\{B_{1}, \ldots, B_{n}\right\}$ und $\{A, \bar{A}\}$ stellen Partitionen desselben Ergebnisraums $\Omega$ dar. Jede dieser Partitionen läßt sich als Menge der möglichen Ausgänge eines geeignet gewählten Zufallsexperiments deuten. Angenommen, man interessiere sich für dasjenige Zufallsexperiment, dessen Ausgänge die $B_{i}$ sind und das aus diesem Grund als das ursprüngliche Zufallsexperiment bezeichnet werden soll. Für dieses Experiment gelten die - als bekannt vorausgesetzten - prioren Wahrscheinlichkeiten $\mathrm{P}\left(\mathrm{B}_{\mathrm{i}}\right)$. Dieses ursprüngliche Experiment kann in folgender Weise als zusammengesetztes Experiment interpretiert werden: man führt zunächst ein "Startexperiment" durch, das entweder A oder $\bar{A}$ als Ausgang liefert. Im AnschluB daran folgt ein weiteres Experiment, das in Abhängigkeit vom Ergebnis des Startexperiments die eigentlich interessierenden Ereignisse $B_{i}$ mit den dazugehörigen bedingten Wahrscheinlichkeiten erzeugt. In dieser Sichtweise ist das ursprüngliche Zufallsexperiment äquivalent zu einer Kombination aus einem Startexperiment und

150: Eine Erweiterung auf eine abzählbare Partition ist unproblematisch; vgl. GOLBERG, M.A. (1984), S. 164. Eine allgemeinere Darstellung gibt FISZ, M. (1980).

Im übrigen kann man geteilter Auffassung darüber sein, ob die Bezeichnung "Theorem" in diesem Fall überhaupt angebracht ist, da die Aussage aus der Definition der bedingten Wahrscheinlichkeit beinahe direkt folgt; vgl. dazu kritisch GOTTINGER, H.W. (1980), S. 14 sowie BREMAUD, P. (1988), S. 25. 
zwei bedingten Folgeexperimenten. Falls das Startexperiment allgemeiner die Ausgänge $A_{j}(j=1, \ldots, m)$ aufweisen würde, müßte man entsprechend $m$ Folgeexperimente berücksichtigen ${ }^{151}$.

Im Hinblick auf die Wahrscheinlichkeiten der Ereignisse $B_{i}$ entscheidet somit der Ausgang des Startexperiments darüber, welche bedingte bzw. posteriore Verteilung gezogen wird. Die unmittelbare Auswirkung des Startexperiments besteht demnach in einer Veränderung der Wahrscheinlichkeitsverteilung für die interessierenden Ereignisse. Diese Feststellung führt auf die Vermutung, der Ausgang eines derartigen Experiments enthalte umso mehr Information über das eigentlich interessierende Zufallsexperiment, je stärker die priore und die posteriore Verteilung differieren ${ }^{152}$. In Abschnitt 3.6 wird gezeigt, daß diese Vermutung zutrifft, wenn man die Diskrepanz zwischen je zwei Wahrscheinlichkeitsverteilungen in geeigneter Weise definiert.

\subsubsection{Diagnostische Maßnahmen als Informationsinstrumente}

Im folgenden werden ausschließlich Situationen betrachtet, in denen dem Arzt eine endliche Anzahl diagnostischer Maßnahmen $T_{j}\left(j=1, \ldots, m^{\prime}\right)$ zur Verfügung steht. Dabei handelt es sich einerseits um diagnostische Maßnahmen gemäß der in Abschnitt 2.3.2 angegebenen Definition, die präziser als einfache diagnostische Maßnahmen bezeichnet werden. Andererseits wird grundsätzlich auch die Möglichkeit zugelassen, eine feste Anzahl einfacher diagnostischer Maßnahmen gemeinsam durchzuführen. Derartige Vorgehensweisen können als zusammengesetzte diagnostische Maßnahmen bezeichnet werden. Ein naheliegendes Beispiel stellt die Anforderung mehrerer Laborwerte durch den Arzt dar; jeder einzelne Laborwert repräsentiert eine einfache diagnostische Maßnahme, während das gesamte "Paket" eine zusammengesetzte dia-

151: Die Ereignisse $A_{j}$ bilden eine Partition des betrachteten Ergebnisraums.

152: Zwischen der prioren und der posterioren Wahrscheinlichkeitsverteilung besteht ein $\mathrm{Zu}$ sammenhang, denn die Wahrscheinlichkeit eines Ereignisses läßt sich als Erwartungswert einer geeignet gewählten Zufallsvariable darstellen. In Analogie dazu ergibt sich die bedingte Wahrscheinlichkeit als bedingter Erwartungswert. Die bedingte Wahrscheinlichkeit eines Ereignisses stellt in dieser Sicht eine Zufallsvariable dar, deren mathematische Erwartung durch die unbedingte Wahrscheinlichkeit des Ereignisses gegeben ist. Insgesamt bedeutet dies, da $B$ die unbedingte Wahrscheinlichkeit eines Ereignisses gerade der mathematischen Erwartung seiner bedingten Wahrscheinlichkeit entspricht; vgl. BLACKWELL, D./GIRSHICK, M.A. (1954), S. 80ff. 
gnostische Maßnahme bildet. Mit dieser Erweiterung ändert sich an der Endlichkeit der Menge durchführbarer Diagnosetests $T_{j}(j=1, \ldots, m)$ nichts, lediglich die Anzahl ihrer Elemente hat sich vergrößert ${ }^{153}$ (es gilt: $m>m^{\prime}$ ).

Typischerweise kennt der Arzt vor dem Einsatz einer diagnostischen Maßnahme deren Ergebnis nicht, da sich mehrere Resultate einstellen können. Die Menge aller möglichen Testergebnisse der diagnostischen Maßnahme $T_{j}$ sei mit $Z_{j}$ bezeichnet. In dieser Arbeit wird unterstellt, diese Menge sei für alle in Betracht zu ziehenden Diagnosetests endlich; ihre Elemente sind die diagnostischen Resultate $z_{j h}\left(h=1, \ldots, k_{j}\right)$. Wie aus der Angabe der Indexmenge hervorgeht, hängt die Anzahl der möglichen Testergebnisse im allgemeinen von der jeweils betrachteten diagnostischen $\mathrm{MaB}$ nahme ab.

Das Resultat eines Diagnosetests läßt sich damit als Zufallsvariable $Z_{j}$ interpretieren, deren mögliche Ausprägungen durch die $z_{j h}$ gegeben sind ${ }^{154}$. Die Annahme einer endlichen Ergebnismenge erscheint auf den ersten Blick problematisch, denn die Resultate vieler diagnostischer Maßnahmen lassen sich durch metrisch skalierte Variablen beschreiben. Dies gilt z.B. für die Ergebnisse einer Reihe klinisch-chemischer Tests, aber auch von Blutdruckmessungen, EKG und EEG. Hier böte es sich an, ein Kontinuum von Möglichkeiten zu berücksichtigen. Andererseits gibt man in praxi die Resultate dieser Tests in diskreter Form an ${ }^{155}$. Weiterhin dürfte in vielen medizinischen Entscheidungssituationen sehr kleinen Veränderungen diagnostischer Te-

153: Diese Erweiterung bietet sich an, weil die ökonomische Analyse einfacher und zusammengesetzter diagnostischer Maßnahmen keine grundsătzlichen Unterschiede aufweist.

154: Wie schon die Variable $\mathrm{K}$ trägt auch die Variable $\mathrm{Z}_{\mathrm{j}}$ zwei Bedeutungen. Einerseits bezeichnet sie einen Ergebnisraum, andererseits eine Zufallsvariable, deren Ausprägungen gerade die Elemente dieses Raums sind. Da aus dem Zusammenhang hervorgeht, in welcher Bedeutung die Variablen jeweils verwendet werden, wird diese Doppelbelegung in Kauf genommen, um die Notation übersichtlicher zu halten.

155: Dies ergibt sich auch aus der begrenzten Meßgenauigkeit der verwendeten Instrumente, die dazu zwingt, den Elementen eines (u.U. sehr kleinen) Intervalles denselben Meßwert zuzuweisen. 
stergebnisse eine geringe Bedeutung zukommen ${ }^{156}$. Dies rechtfertigt die Annahme einer endlichen Ergebnismenge bei den hier betrachteten diagnostischen Maßnahmen $^{157}$.

In der Regel hängen die Wahrscheinlichkeiten der einzelnen Testergebnisse davon $\mathrm{ab}$, welcher Krankheitszustand bei einem Patienten vorliegt ${ }^{158}$. Diese $\mathrm{Zu}-$ standsabhängigkeit bedeutet, daß für jedes Element der Menge $\mathrm{K}$ eine spezielle Wahrscheinlichkeitsverteilung auf der Menge der möglichen Testergebnisse existiert. Es handelt sich dabei um bedingte Verteilungen, deren Bedingung jeweils durch einen Krankheitszustand gegeben ist. Diesen Sachverhalt veranschaulicht die nachfolgende Abbildung, die ganz allgemein für eine diagnostische $M a ß n a h m e ~ T_{j}$ deren zu einer bestimmten, durch die Menge K gekennzeichneten Ausgangslage gehörenden - bedingte Wahrscheinlichkeitsverteilungen spaltenweise zusammenstellt. Analog stehen in einer Zeile die Likelihood-Werte jeweils eines Testergebnisses für sämtliche Krankheitszustände ${ }^{159}$.

Die Matrix $P_{j}$ dieser bedingten Wahrscheinlichkeiten charakterisiert eine diagnostische Maßnahme $T_{j}$. In diesem Zusammenhang stellt $P_{j}$ immer eine spaltenstochastische Matrix dar, d.h. $P_{j}$ enthält ausschließlich nichtnegative Elemente und die Spaltensummen betragen jeweils Eins. Wie in Kapitel 3 ausführlich gezeigt wird, spielen die durch die möglichen Krankheitszustände des Patienten bedingten Wahrscheinlichkeitsverteilungen der Testergebnisse im Rahmen der Bestimmung der diagnostischen Information eine wichtige Rolle.

156: Auch deshalb wird die Annahme der Endlichkeit häufig verwendet; vgl. z.B. TEATHER, D. (1974a), S. 37.

157: Überdies hilft die Annahme, einige technische Probleme zu vermeiden; dies betrifft etwa die Konstruktion geeigneter Wahrscheinlichkeitsräume oder auch die Meßbarkeit der verwendeten Funktionen.

158: Ohne eine derartige Abhängigkeit liefert die betreffende diagnostische Maßnahme keine neue Information; vgl. dazu auch Abschnitt 3.6.2.

159: Vgl. zum Begriff der Likelihood-Funktion Abschnitt 3.3.1. 
Abb. 5: Bedingte Wahrscheinlichkeiten der Testergebnisse von $T_{j}$

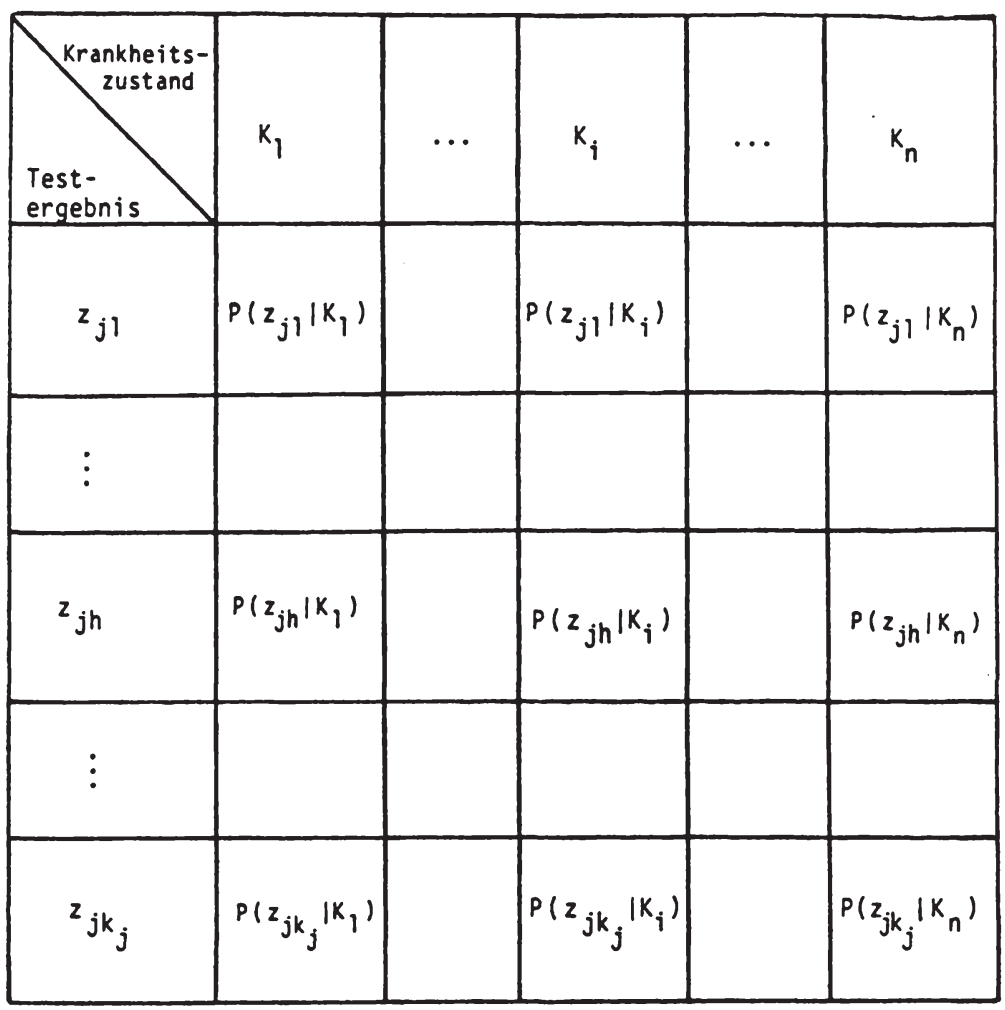

Die priore Wahrscheinlichkeitsverteilung der Krankheitszustände und die bedingten Wahrscheinlichkeiten der Testresultate bilden wichtige Voraussetzungen für die Analyse der Information diagnostischer Maßnahmen. Aus der Sicht des Arztes stellen die einzelnen Krankheitszustände diejenigen Ereignisse dar, auf die sich sein Interesse konzentriert. Weiterhin sind diagnostische Maßnahmen als Zufallsexperimente anzusehen, die aufgrund der Abhängigkeit ihrer Resultate vom Krankheitszustand des Patienten Information liefern. Es liegt nun nahe, mit Hilfe des Bayes-Theo- 
rems zu ermitteln, in welcher Weise ein Testergebnis die prioren Wahrscheinlichkeiten der Krankheitszustände modifiziert: diagnostische Maßnahmen scheinen den im vorangegangenen Abschnitt erläuterten Startexperimenten zu entsprechen, deren Ausgang Aufschluß über das ursprüngliche Zufallsexperiment mit den Ausgängen $\mathrm{K}_{1}, \ldots, \mathrm{K}_{\mathrm{n}}$ liefert.

Dennoch gelingt die Übertragung nicht unmittelbar, da das einer diagnostischen Maßnahme entsprechende Zufallsexperiment nicht mit dem eigentlich interessierenden Zufallsexperiment verbunden ist. Einerseits kann das durch die Partition der Menge $\mathrm{K}$ in Krankheitszustände $\mathrm{K}_{\mathrm{i}}$ repräsentierte Zufallsexperiment ohne die betreffende diagnostische Maßnahme "durchgeführt" werden; andererseits vermag ein Diagnosetest Information in ganz verschiedenen medizinischen Ausgangslagen zu liefern. Im allgemeinen sind deshalb die Resultate einer diagnostischen Maßnahme nicht auf der Menge $\mathrm{K}$ zu definieren und bilden auch keine Partition dieser Menge ${ }^{160}$.

Aus diesem Grund muß zusätzlich zu den oben genannten Voraussetzungen die "Anwendbarkeit der Rechengesetze für bedingte Wahrscheinlichkeiten"161 gefordert werden. Formal läßt sich diese Annahme als Übergang zu einem erweiterten Wahrscheinlichkeitsraum auffassen. Sie impliziert die Berücksichtigung des Produktraums $\mathrm{K} \times \mathrm{Z}_{\mathrm{j}}$ als Menge der möglichen Kombinationen aus Krankheitszustand und Resultat der diagnostischen $M a ß n a h m e T_{j}$ in einer bestimmten medizinischen Ausgangslage. Weiterhin beinhaltet diese Annahme die Existenz einer auf $\mathrm{K} \times \mathrm{Z}_{\mathrm{j}}$ definierten Funktion Q mit folgenden Eigenschaften ${ }^{162}$ :

$$
\begin{aligned}
\mathrm{Q}\left(\mathrm{K}_{\mathrm{i}} \times Z_{\mathrm{j}}\right) & =\mathrm{P}\left(\mathrm{K}_{\mathrm{j}}\right) ; \mathrm{i}=1, \ldots, \mathrm{n} ; \\
\mathrm{Q}\left(\mathrm{K} \times \mathrm{z}_{\mathrm{jh}} \mid \mathrm{K}_{\mathrm{i}} \times Z_{\mathrm{j}}\right) & =\mathrm{P}\left(\mathrm{z}_{\mathrm{jh}} \mid \mathrm{K}_{\mathrm{i}}\right) ; \mathrm{h}=1, \ldots, \mathrm{k}_{\mathrm{j}} ; \mathrm{i}=1, \ldots, \mathrm{n} .
\end{aligned}
$$

160: Vgl. FIRCHAU, V. (1980), S. 9. Im Gegensatz dazu trifft Marschak in seinen frühen Arbeiten genau diese Annahme, vgl. z.B. MARSCHAK, J. (1959), S. 96.

161: FERSCHL, F. (1975), S. 97.

162: Vgl. BLACKWELL, D./GIRSHICK, M.A. (1954), S. 85f. sowie RAIFFA, H./SCHLAIFER, R. (1961), S. 1 ff. 
Diese Erweiterung des Ergebnisraums schafft die Voraussetzungen für eine Anwendung des Bayes-Theorems. Die den einzelnen Krankheitszuständen entsprechenden Ereignisse $K_{i} \times Z_{j}(i=1, \ldots, n)$ bilden ebenso eine Partition dieses Ergebnisraums wie die Mengen $K \times z_{j h}\left(h=1, \ldots, k_{j}\right)$, die die möglichen Testresultate repräsentieren. Die Gleichungen (2.31) und (2.32) geben an, in welcher Weise die bisher verwendeten Wahrscheinlichkeiten das Wahrscheinlichkeitsmaß $Q$ festlegen. In dieser Form entspricht eine diagnostische Maßnahme vollständig dem in Abschnitt 2.4.2 beschriebenen Startexperiment. Daher können einzelne Testergebnisse zur Bestimmung bedingter Wahrscheinlichkeiten für die in Betracht gezogenen Krankheitszustände dienen ${ }^{163}$. Um die Notation übersichtlich zu halten, werden im folgenden weiterhin die Wahrscheinlichkeiten $\mathrm{P}\left(\mathrm{K}_{\mathrm{i}}\right)$ bzw. $\mathrm{P}\left(\mathrm{z}_{\mathrm{jh}} \mid \mathrm{K}_{\mathrm{i}}\right)$ verwendet, wobei die oben zusätzlich eingeführte Annahme ihre Gültigkeit behält.

Die Kennzeichnung des Zusammenhangs zwischen den möglichen Krankheitszuständen des Patienten und den Testergebnissen einer diagnostischen Maßnahme mit Hilfe einer Menge bedingter Wahrscheinlichkeitsverteilungen schafft eine direkte Verbindung zur Entscheidungstheorie. Insbesondere kann die dort übliche Terminologie übernommen werden. Man bezeichnet $T_{j}$ als Informationsinstrument bzw. Informationssystem bezüglich der Menge $\mathrm{K}$ der Krankheitszustände ${ }^{164}$. In Analogie dazu werden die möglichen Testergebnisse $z_{j h}$ als Nachrichten angesehen, welche das Informationsinstrument $T_{j}$ über $K$ liefern kann. Entsprechend stellt $Z_{j}$ die dazugehörige Nachrichtenmenge dar.

163: Vgl. dazu Kapitel 3.

164: Vgl. BAMBERG, G./COENENBERG, A.G. (1989), S. 18. Der Begriff geht vermutlich auf Marschak zurück; vgl. MARSCHAK, J. (1959) sowie MARSCHAK, J./MIYASAWA, K. (1968), S. 205ff.

Der Ansatz, diagnostische Maßnahmen als Informationsinstrumente zu sehen, die durch eine Matrix $P_{j}$ charakterisiert werden kōnnen, findet sich schon bei BÜTTNER, J. (1977), S. 7. 


\section{DIE INFORMATION DIAGNOSTISCHER MASSNAHMEN}

\subsection{Binäre und binarisierte Tests}

Die folgenden Ausführungen stellen auf eine medizinische Entscheidungssituation ab, die im Vergleich zu der in Abschnitt 2.4 skizzierten Situation eine besonders einfache Struktur aufweist. Die Analyse der Informationsleistung diagnostischer Maßnahmen in den folgenden Abschnitten 3.2 und $3.3 \mathrm{nimmt}$ ausschließlich auf diesen einfachen Fall Bezug. Erst die Abschnitte 3.4 bis 3.6 behandeln die Beschreibung und die Ermittlung der diagnostischen Information im allgemeinen Fall, d.h. in einer beliebigen medizinischen Entscheidungssituation, die den in Abschnitt 2.4 genannten Voraussetzungen genügt. Zur Begründung dieses stufenweisen Vorgehens lassen sich zwei Argumente anführen. Erstens erscheint die detaillierte Untersuchung einfacher medizinischer Entscheidungssituationen in besonderem $\mathrm{MaB}$ geeignet, auf die allgemeine Analyse der Informationsleistung einer diagnostischen Maßnahme vorzubereiten. Weiterhin läßt sie sich damit rechtfertigen, daß es sich um den in der Literatur bevorzugt diskutierten Fall handelt.

Zunächst äußert sich die Vereinfachung der medizinischen Entscheidungssituation in der Annahme, lediglich zwei mögliche Krankheitszustände des Patienten seien zu berücksichtigen. Dabei spielt ein Krankheitszustand - es handle sich um $\mathrm{K}_{1}$ - aus der Sicht des Arztes eine wichtigere Rolle, z.B. weil $\mathrm{K}_{1}$ eine höhere priore Wahrscheinlichkeit besitzt. In einigen Situationen reicht es aus, tatsächlich nur einen weiteren Krankheitszustand als Alternative in die Analyse aufzunehmen. In allen anderen Fällen jedoch berücksichtigt man ganz allgemein das $\mathrm{zu} \mathrm{K}_{1}$ komplementäre Ereignis $\overline{\mathrm{K}}_{1}$, das sämtliche, in der betrachteten Situation mit $\mathrm{K}_{1}$ konkurrierenden Krankheitshypothesen $\left(K_{2}, \ldots, K_{n}\right)$ zusammenfaßt.

Darüber hinaus unterscheidet man bei den diagnostischen Maßnahmen jeweils lediglich zwei Testergebnisse, die als positive bzw. negative Resultate klassifiziert werden. Wie zuvor bei der Annahme bezüglich der Krankheitszustände kann auch diese Voraussetzung sozusagen in natürlicher Weise erfüllt sein, wenn der zu untersuchende Diagnosetest tatsächlich nur zwei mögliche Resultate aufweist. In diesem Spezialfall handelt es sich um einen binären Test. Trifft dies hingegen nicht zu, so muß die Menge $Z_{j}$ der möglichen Testergebnisse in geeigneter Form partitioniert 
werden. Formal läßt sich dies bei der diagnostischen $M a ß n a h m e T_{j}$ erreichen, indem man auf $Z_{j}$ eine Zufallsvariable $Y_{j}$ definiert, die lediglich zwei Werte annehmen kann $^{1}$ :

$$
Y_{j}: Z_{j} \rightarrow\left\{y_{j 1}, y_{j 2}\right\}
$$

Mit dieser Zuordnung entscheidet man sich dafür, anstelle der ursprünglichen Testergebnisse die Realisationen der Zufallsvariablen $Y_{j}$ zu beobachten bzw. zu berücksichtigen. Jene Testergebnisse, die $z u y_{j 1}$ führen, stellen beispielsweise die positiven Resultate dar, während alle übrigen die negativen Resultate repräsentieren. In dieser Form bezeichnet man $T_{j}$ als binarisierten Test ${ }^{2}$. Binäre bzw. binarisierte Tests werden vornehmlich in denjenigen medizinischen Entscheidungssituationen untersucht, in denen lediglich $\mathrm{K}_{1}$ und $\overline{\mathrm{K}}_{1}$ als mögliche Krankheitszustände des Patienten zu unterscheiden sind ${ }^{3}$.

Grundsätzlich kann jede diagnostische Maßnahme als binarisierter Test dargestellt werden. Häufig bietet sich zu diesem Zweck eine Partitionierung der Ergebnismenge in zwei zusammenhängende Teilbereiche an. Beispielsweise rangieren bei manchen klinisch-chemischen Tests diejenigen Testergebnisse als positive (negative) Resultate, die oberhalb (unterhalb) einer bestimmten Ausprägung des gemessenen Merkmals liegen. Dieser kritische Wert stellt einen Schwellenwert ${ }^{4}$ dar, der zur Binarisierung der diagnostischen Resultate dient.

1: Die zuvor angesprochene Partition der Menge $Z_{j}$ ergibt sich durch die dazugehörigen Urbildmengen $Y_{j}^{-1}\left(y_{j 1}\right), Y_{j}^{-1}\left(y_{j 2}\right)$.

2: In der angelsächsischen Literatur findet sich der Begriff Test mit dichotomisierten Resultaten; vgl. z.B. WEINSTEIN, M.C. et al. (1980), S. 83; ALBERT, A. (1982), S. 1113.

3: Richter et al. sprechen in diesem Fall aus naheliegenden Gründen von einem einfachen Diagnosetest; vgl. RICHTER, K. et al. (1988), S. 656.

4: In der englischsprachigen Literatur wird dieser Wert als "cutoff value" bezeichnet; vgl. McNEIL, B.J.JADELSTEIN, S.J. (1976) sowie METZ, C.E. (1978).

Je nach Test und medizinischer Ausgangslage können auch Ergebnisse unterhalb des Schwellenwerts als positiv einzustufen sein; dies trifft etwa auf Blutdruckmessungen bei Patienten mit Hypotonieverdacht zu. 


\subsection{Sensitivität und Spezifität}

\subsubsection{Definition und Erläuterung}

Mit der Sensitivität und der Spezifität existieren zwei verschiedene diagnostische Kenngrößen, die gemeinsam häufig zur Charakterisierung klinisch-chemischer Tests dienen, gemäß der Argumentation in Abschnitt 2.3.2 jedoch auch auf diagnostische Maßnahmen im allgemeinen anwendbar sind ${ }^{5}$. Unter der Sensitivität eines Diagnosetests versteht man die Wahrscheinlichkeit, mit der eine "kranke" Person ein positives Testergebnis erzielt. Als "krank" im Sinne dieser Definition gelten ausschließlich diejenigen Personen, die mit der durch den Test zu überprüfenden bzw. zu erkennenden Krankheit, der "target disorder"6, belastet sind 7 . Mit anderen Worten: die Sensitivität stellt den zu erwartenden Anteil der korrekt als positiv eingestuften Fälle dar ${ }^{8}$. Im Gegensatz dazu bezeichnet die Spezifität jene Wahrscheinlichkeit, mit der ein Diagnosetest zu einem negativen Ergebnis führt bei Personen ohne die betreffende Krankheit ${ }^{9}$. Die Spezifität einer diagnostischen Maßnahme gibt damit für eine geeignet ausgewählte Untersuchungsgruppe den zu erwartenden Anteil der wahr negativen Fälle an ${ }^{10}$.

Beide Kenngrößen, die Sensitivität wie auch die Spezifität, lassen sich somit als bedingte Wahrscheinlichkeiten auffassen: die Sensitivität als Wahrscheinlichkeit für ein positives Testresultat gegeben die Präsenz der "target disorder", und entsprechend die Spezifität als die Wahrscheinlichkeit eines negativen Resultats unter der Bedingung der Abwesenheit dieser Krankheit. Bezeichne $y_{j 1}$ ein positives Resultat bei der diagnostischen Maßnahme $T_{j}$, dann stellt $P\left(y_{j 1} \mid K_{1}\right)$ die Sensitivität dieses Tests dar. Analog gibt die Wahrscheinlichkeit $\mathrm{P}\left(\mathrm{y}_{\mathrm{j} 2} \mid \overline{\mathrm{K}}_{1}\right)$ dessen Spezifität an.

5: Diese Begriffe wurden offenbar von Yerushalmy eingeführt; vgl. YERUSHALMY, J. (1947), S. 1435 .

6: Es handelt sich dabei nach der in Abschnitt 3.1 getroffenen Verabredung um die Krankheit $\mathrm{K}_{1}$.

7: Diese Personen werden im folgenden auch als positive Fälle bezeichnet.

8: $\quad$ Vgl. RICHTER, K. et al. (1988), S. 656.

9: Dies sind die negativen Fälle. Bei diesen Personen trifft das Ereignis $\bar{K}_{1}$ zu, es kann sich dabei sowohl um völlig gesunde Personen (z.B. beim "screening") handeln als auch um Patienten, die an anderen Erkrankungen leiden.

10: Zur Auswahl der Untersuchungsgruppe vgl. Abschnitt 3.2.2. 
Bei einem Diagnosetest, dessen Sensitivität und Spezifität unvollkommen sind, also unter Eins liegen, kann es zu Fehleinstufungen sowohl eines Teils der positiven als auch einiger negativer Fälle kommen. Dementsprechend lassen sich noch zwei weitere Merkmalskombinationen aus Testresultat und tatsächlich vorliegendem Krankheitszustand unterscheiden:

- einerseits diejenigen Fälle, in denen Personen durch den Test unzutreffend als positiv ausgewiesen werden; es handelt sich dabei um die falsch positiven Fälle. Die Wahrscheinlichkeit $\mathrm{P}\left(\mathrm{y}_{\mathrm{j} 1} \mid \overline{\mathrm{K}}_{1}\right)$ dieses Ereignisses beträgt Eins abzüglich der Spezifität.

- andererseits die zu Unrecht durch den betrachteten Test mit einem negativen Resultat belegten Personen; diese werden als falsch negative Fälle bezeichnet. Die entsprechende Wahrscheinlichkeit $P\left(y_{j 2} \mid K_{1}\right)$ addiert sich mit der Sensitivität zu Eins.

In einer medizinischen Entscheidungssituation, in der mit $\mathrm{K}_{1}$ und $\overline{\mathrm{K}}_{1}$ lediglich zwei mögliche Krankheitszustände des Patienten unterschieden werden, läßt sich ein binarisierter Diagnosetest mit Hilfe von zwei bedingten Wahrscheinlichkeitsverteilungen charakterisieren, wie die nachstehende Abbildung 6 veranschaulicht.

In jeder Spalte ist aufgrund der Additivitätsbedingung jeweils eine bedingte Wahrscheinlichkeit redundant, so daß zur Charakterisierung eines binarisierten Diagnosetests in diesem Fall die Angabe von Sensitivität und Spezifität ausreicht. Wenn eine diagnostische Maßnahme bei beiden Kennziffern den Wert Eins erzielt, erlauben ihre Testergebnisse immer einen fehlerfreien Rückschluß auf den Krankheitszustand des Patienten. In diesem besonderen Fall spricht man von einer perfekten diagnostischen Maßnahme ${ }^{11}$; aus entscheidungstheoretischer Sicht handelt es sich dabei um ein vollkommenes Informationsinstrument ${ }^{12}$.

11: Vgl. z.B. SOX, H.C. (1986), S. 61.

12: Vgl. dazu näher Abschnitt 3.4. 
Abb. 6: Zur Charakterisierung eines binarisierten Diagnosetests $T_{j}$

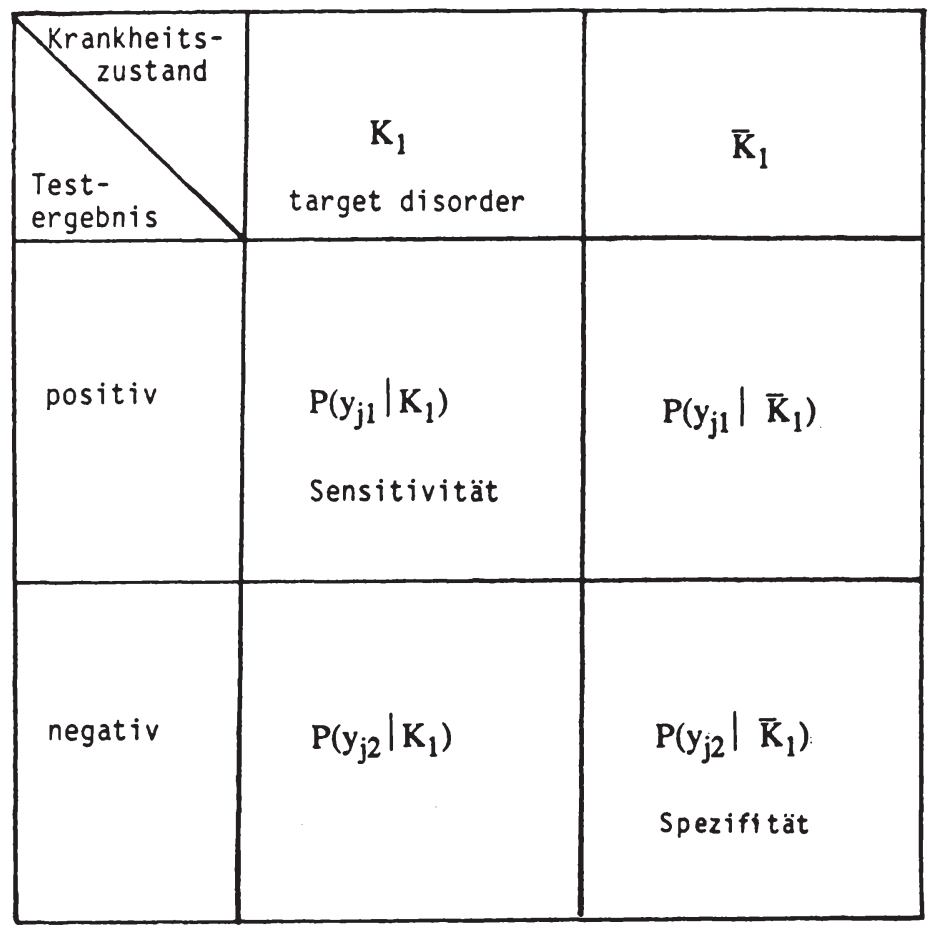

Man bezeichnet Sensitivität und Spezifität als Kennziffern eines Diagnosetests, da sie dessen Fähigkeit zur Unterscheidung positiver und negativer Fälle beschreiben. Bei einer binarisierten diagnostischen Maßnahme gibt es mehrere Möglichkeiten, die Menge der möglichen Testergebnisse in einen positiven und einen negativen Bereich zu partitionieren. Jede spezielle Aufteilung repräsentiert eine bestimmte Binarisierung bzw. Testeinstellung. Im allgemeinen beeinflußt die Testeinstellung die exakten Werte der Kennziffern Sensitivität und Spezifität, die somit von der jeweils 
verwendeten Definition positiver und negativer Testergebnisse abhängen. Eine einfache Konstellation liegt vor, wenn ein Test lediglich die Ausprägungen eines metrisch skalierten Merkmals (z.B. Blutdruck) mißt. Es gelingt dann vielfach, einen Schwellenwert festzulegen, der die Menge der möglichen Testergebnisse in einen positiven und einen negativen Bereich unterteilt ${ }^{13}$. Bei anderen diagnostischen MaBnahmen wie etwa den bildgebenden Verfahren (Röntgen, Computer-, Kernspintomographie) gestaltet sich diese Aufgabe schwieriger ${ }^{14}$. Dennoch benötigt man auch bzw. gerade in diesen Fällen eine genaue Vorstellung davon, wann ein Testresultat als positiv gilt. Die möglichst präzise Definition positiver und negativer Testresultate stellt eine unabdingbare Voraussetzung für die Bestimmung von Sensitivität und Spezifität dar, weil diese Festlegung die "wahren" Werte dieser beiden Kenngrößen unmittelbar beeinflußt.

Vielfach wird davon ausgegangen, daß bei fest vorgegebener Testeinstellung Sensitivität und Spezifität unveränderliche Kenngrößen einer diagnostischen Maßnahme darstellen ${ }^{15}$. Indessen handelt es sich dabei um eine Annahme, deren Gültigkeit keineswegs als gesichert angesehen werden kann ${ }^{16}$. Zur Veranschaulichung genügt es, die beiden in der Literatur verwendeten Modellvorstellungen $\mathrm{zu}$ betrachten, aus denen Sensitivität und Spezifität abgeleitet werden. Beide nehmen Bezug auf die relevanten Grundgesamtheiten derjenigen Individuen, die sich entweder im Krankheitszustand $\mathrm{K}_{1}$ befinden, oder bei denen das Vorliegen von $\mathrm{K}_{1}$ auszuschließen ist ${ }^{17}$. Einer Modellvorstellung zufolge sind die individuellen Testergebnisse auch dann zufallsabhängig, wenn keine Meßfehler auftreten ${ }^{18}$. Unter dieser Voraussetzung läßt sich das Testergebnis eines Individuums durch eine Bernoulli-Verteilung beschreiben. Die Verteilungen des Parameters dieser Verteilung in den beiden Grundgesamt-

13: Ob eine derart einfache Aufteilung in zwei zusammenhängende Bereiche als sinnvoll anzusehen ist, hängt von den Verteilungen des Merkmals in den Grundgesamtheiten der Individuen in Krankheitszustand $\mathrm{K}_{1}$ bzw. $\overline{\mathrm{K}}_{1}$ ab.

14: Vgl. GILLESPIE, K.N. et al. (1986), S. 541.

15: Vgl. LEDLEY, R.S./LUSTED, L.B. (1959), S. 14; DEPARTMENT OF CLINICAL EPIDEMIOLOGY AND BIOSTATISTICS (1981), S. 705; GRINER, P.F. et al. (1981), S. 560; DIAMOND, G.A. (1984), S. 48 sowie PHILLIPS, W.C./SCOTT, J.A.BLASCZCYNSKI, G. (1983), S. 1267.

16: Vgl. DAWID, A.P. (1976), S. 651.; BEGG, C.B. (1987), S. 411 f. sowie RICHTER, K. et al. (1988), S. 657.

17: Hierbei sind zweckmäßigerweise nur diejenigen Individuen zu berücksichtigen, bei denen eine Anwendung der betreffenden diagnostischen Maßnahme in Frage kommt.

18: Vgl. NEYMAN, J. (1947); NISSEN-MEYER, S. (1964), S. 733. 
heiten legen die Testkennziffern fest: die Sensitivität ergibt sich als mittlere Wahrscheinlichkeit eines positiven Testergebnisses bei Personen mit der interessierenden Krankheit, während die Spezifität die mittlere Wahrscheinlichkeit eines negativen Resultats bei Personen ohne diese Krankheit angibt ${ }^{19}$.

Andererseits kann man von der These ausgehen, daß zu einem bestimmten Zeitpunkt die Ausprägung des durch einen Diagnosetest erfaßten Merkmals bei einem Individuum weitgehend festgelegt ist und damit auch das Testergebnis keinen größeren Schwankungen unterliegt ${ }^{20}$. Diese Vorstellung dürfte bei einer Vielzahl diagnostischer Merkmale zutreffen. In diesem Fall bestimmen die Verteilungen des interessierenden Merkmals in den beiden Grundgesamtheiten, welche Kennziffern ein Diagnosetest besitzt. Die Sensitivität entspricht dem Anteil der Individuen in Krankheitszustand $\mathrm{K}_{1}$, deren Merkmalsausprägung in den positiven Bereich fällt, an allen positiven Fällen. Analog ergibt sich die Spezifität als relative Häufigkeit derjenigen Individuen in Krankheitszustand $\overline{\mathrm{K}}_{1}$, deren Merkmalsausprägung im negativen Bereich liegt.

Diese Überlegungen verdeutlichen, daß die beiden Testkennziffern zunächst auf einen Zeitpunkt bezogene Größen bilden, die für ganz bestimmte Grundgesamtheiten gelten $^{21}$. Damit Sensitivität und Spezifität Testkennziffern im Sinne inhärenter Eigenschaften einer diagnostischen Maßnahme darstellen, muß offenbar eine im Zeitablauf annähernd konstante Struktur der beiden Grundgesamtheiten vorliegen. Ob diese Stationaritätsannahme tatsächlich gilt, ist in der Literatur umstritten und kann letztlich nur empirisch entschieden werden ${ }^{22}$.

Im übrigen können die Kennziffern einer diagnostischen Maßnahme auch dann variieren, wenn man die zeitliche Konstanz der Struktur positiver und negativer Fälle unterstellt. Diese Möglichkeit besteht, weil für einzelne Anwendungen eines Diagnosetests im allgemeinen nicht alle Personen im Krankheitszustand $\mathrm{K}_{1}$ bzw. $\overline{\mathrm{K}}_{1}$ in Betracht kommen. Dies wird besonders deutlich bei einem Test, der einerseits im Rah-

19: Nissen-Meyer zeigt dies unter der Prämisse, daß der Parameter der Bernoulli-Verteilungen in beiden Grundgesamtheiten jeweils stetig verteilt ist; vgl. NISSEN-MEYER, S. (1964), S. 733.

20: Vgl. DAWID, A.P. (1976), S. 648.

21: Diese Aussage trifft auf beide zuvor angesprochenen Modellvorstellungen zu.

22: So sieht z.B. Koller diese Annahme in vielen Fällen als erfüllt an, während Dawid dieser Ansicht kritisch gegenübersteht; vgl. KOLLER, S. (1967), S. 1069; DAWID, A.P. (1976), S. 651 . 
men des "screening" bei asymptomatischen Personen Anwendung findet, andererseits differentialdiagnostischen Zwecken dient. In der zuletzt genannten Verwendung kommt der Test nur bei denjenigen Individuen in Frage, die ähnliche Symptome aufweisen. Je nach Anwendungssituation sind damit verschiedene Gruppen bzw. Grundgesamtheiten positiver und negativer Fälle zu berücksichtigen. Falls dabei beispielsweise innerhalb der positiven Fälle die Zusammensetzung dieser Gruppen in Bezug auf das durch den Diagnosetest erfaßte Merkmal hinreichend variiert, dürfte sich dies in unterschiedlichen Werten für die Sensitivität niederschlagen ${ }^{23}$. Eine analoge Aussage gilt für die Spezifität, so da $B$ es unter Umständen sinnvoll sein kann, bei einer diagnostischen Maßnahme in Abhängigkeit von der Anwendungssituation mehrere Paare von Kennziffern zu unterscheiden ${ }^{24}$. Dies betrifft insbesondere diagnostische Maßnahmen, die sowohl im Krankenhaus als auch beim niedergelassenen Arzt zum Einsatz kommen, da die Zusammensetzung des Patientenguts beträchtliche Unterschiede aufweisen $\operatorname{kann}^{25}$.

Mit der Hilfe von Sensitivität und Spezifität läßt sich eine weitere Kenngröße ermitteln, die ebenfalls zur Beschreibung der Leistungsfähigkeit von diagnostischen Maßnahmen herangezogen werden kann. Es handelt sich dabei um den Youden-Index, der als Summe von Sensitivität und Spezifität abzüglich Eins definiert ist ${ }^{26}$. Aufgrund der Aggregation beider Wahrscheinlichkeiten differenziert diese Meßgröße nicht zwischen den beiden Kennziffern einer diagnostischen Maßnahme ${ }^{27}$. Insofern

23: Vgl. BEGG, C.B. (1987), S. 413; KÖBBERLING, J./TRAMPISCH, H.-J./WINDELER, J. (1989), S. 10.

24: Vgl. FEINSTEIN, A.R. (1977a), S. 222ff.; DEPARTMENT OF CLINICAL EPIDEMIOLOGY AND BIOSTATISTICS (1983), S. 563.

25: Vgl. KOLLER, S. (1967), S. 1069. Diese Unterschiede kōnnen z.B. auf Selektionseffekten beruhen, die aus dem Einweisungsverhalten der Ärzte resultieren; vgl. DAWID, A.P. (1976), S. 649.

26: Vgl. YOUDEN, W.J. (1950).

27: Ein ähnliches Argument gilt für die Genauigkeit., Diese diagnostische Kennziffer gibt die Wahrscheinlichkeit eines richtigen Testergebnisses bei einer diagnostischen Maßnahme an. Die Genauigkeit stellt eine konvexe Kombination von Sensitivität und Spezifität dar, deren Koeffizienten vom Anteil der positiven Fälle in der betrachteten Grundgesamtheit - d.h. der prioren Wahrscheinlichkeit von $\mathrm{K}_{1}$ - abhängen. Vgl. zur Genauigkeit METZ, C.E. (1978), S. 284 sowie kritisch GREMY, F./GOLDBERG, M. (1977), S. 426f., wobei die Autoren jeweils die entsprechende Stichprobenvariable - die Summe der Anteile richtig positiver und richtig negativer an allen Fällen - betrachten. 
reduziert der Youden-Index die vorhandene Information ${ }^{28}$. Je nachdem, zu welchem Zweck ein Diagnosetest primär durchgeführt wird, kommen der Sensitivität und der Spezifität jedoch unterschiedliches Gewicht $\mathrm{zu}^{29}$. Insgesamt besitzt der Youden-Index als diagnostische Kenngröße daher nur geringe Bedeutung.

\subsubsection{Die Schätzung der Kennziffern eines Diagnosetests}

Sensitivität und Spezifität spielen eine wichtige Rolle in der Analyse der Information binarisierter Diagnosetests. Aus diesem Grund besteht ein großes Interesse an einer möglichst exakten Ermittlung der beiden testspezifischen Kenngrößen. Da diese Wahrscheinlichkeiten a priori unbekannt sind, benötigt man an ihrer Stelle empirisch ermittelte Schätzwerte. $\mathrm{Zu}$ diesem Zweck zieht man Stichproben aus den beiden Grundgesamtheiten der Individuen im Krankheitszustand $\mathrm{K}_{1}$ bzw. $\overline{\mathrm{K}}_{1}$, die die Gruppe der positiven bzw. negativen Fälle bilden. Die Resultate des interessierenden Diagnosetests bei diesen Probanden dienen dazu, dessen Sensitivität und Spezifität zu schätzen. Im allgemeinen schätzt man die Sensitivität (Spezifität) durch den Anteil positiver (negativer) Testergebnisse an allen positiven (negativen) Fällen ${ }^{30}$.

Um zu gewährleisten, daß diese Schätzwerte mit hoher Wahrscheinlichkeit nahe bei den tatsächlichen Kennziffern liegen, sind Schätzungen mit günstigen statistischen Eigenschaften zu verwenden ${ }^{31}$. Im diagnostischen Kontext betrifft dies im wesentlichen die Forderung, verzerrte Schätzungen soweit als möglich zu vermeiden. Wie weiter unten an einzelnen Beispielen erörtert, existiert eine Reihe von Einfluß-

28: Vgl. FEINSTEIN, A.R. (1977a), S. 219.

29: Vgl. dazu ausführlich GALEN, R.S./GAMBINO, S.R. (1979), S. 36ff.; DOESSEL, D.P. (1986), S. 54ff. sowie Abschnitt 4.4.2 dieser Arbeit.

30: Vgl. FLEISS, J.L. (1973), S. 3ff.

31: Schätzwerte und Schätzungen sind streng voneinander zu trennen. Eine Schätzung stellt eine eindeutige Zuordnungsvorschrift für Stichprobenvariablen dar und wird deshalb häufig auch als Schätzfunktion bezeichnet; vgl. PFANZAGL, J. (1977); STENGER, H. (1986), S. 33f. Im Gegensatz dazu repräsentiert ein Schätzwert eine spezielle Realisation einer Schätzfunktion. Aufgrund der Zufallsabhängigkeit der Schätzwerte lassen sich sinnvoll nur die Eigenschaften von Schätzungen vergleichen; vgl. CRAMER, H. (1946), S. 473. 
faktoren, die eine systematische Abweichung der geschätzten Kenngrößen von ihren wahren Werten bedingen können ${ }^{32}$. Nicht zuletzt aus diesem Grund sind Studien zur Schätzung von Sensitivität und Spezifität schwierig durchzuführen ${ }^{33}$.

Eine günstige Voraussetzung liegt vor, wenn in den Stichproben sowohl die positiven als auch die negativen Fälle in Bezug auf die spätere medizinische Anwendungssituation repräsentativ vertreten sind ${ }^{34}$. Der Auswahl geeigneter Probandenkohorten kommt daher in diesem Zusammenhang eine große Bedeutung zu. Häufig sind jedoch die Bedingungen einer zufälligen Auswahl der Probanden in Studien zur Schätzung der Kennziffern einer diagnostischen Maßnahme nicht erfüllt. Dies betrifft beispielsweise diejenigen Studien, in denen die Ermittlung der Sensitivität ausschließlich auf die Testergebnisse besonders kranker Personen Bezug nimmt ${ }^{35}$. Eine ähnliche Kritik gilt für die Schätzung der Spezifität eines Diagnosetests, falls dazu lediglich die Resultate völlig gesunder Personen (den "healthy volunteers") verwendet werden ${ }^{36}$. In beiden Fällen weisen die auf diese Art gewonnenen Schätzungen für die Kennziffern des Diagnosetests aufgrund der einseitigen Auswahl der Probanden vermutlich einen "bias" nach oben auf und zeichnen daher ein unzulässig optimistisches Bild von dessen Informationsleistung.

Weiterhin erscheint es wünschenswert, daß bei den für die Schätzaufgabe ausgewählten Probanden der tatsächlich vorliegende Krankheitszustand zutreffend ermittelt werden konnte. Zu diesem Zweck benötigt man ein sicheres Verfahren - wie z.B. die Biopsie $^{37}$ oder eine geeignete Kombination diagnostischer Maßnahmen ${ }^{38}$-, deren

32: Vgl. dazu ausführlich RANSOHOFF, D.F./FEINSTEIN, A.R. (1978); PHILBRICK, J.T./HORWITZ, R.I./FEINSTEIN, A.R. (1980) sowie BEGG, C.B. (1987).

33: Vgl. SOX, H.C. (1986), S. 64.

34: Vgl. COCHRANE, A.L./HOLLAND, W.W. (1971), S. 6.

35: Vgl. SOX, H.C. (1986), S. 64.

36: Vgl. FLEISS, J.L. (1973), S. 11. Dies betrifft zumindest den Einsatz des Tests im Rahmen der Differentialdiagnose. In diesem Zusammenhang interessiert man sich für die Resultate von Personen, die im Hinblick auf die positiven Fälle ähnliche Symptome aufweisen, ohne jedoch die "target disorder" ( $\left.\mathrm{K}_{1}\right)$ zu besitzen; vgl. dazu näher GROSS, R. (1969), S. $16 \mathrm{f}$.

Falls der Test hingegen vornehmlich für das "screening" eingesetzt wird, kann die im Text beschriebene Methode eine brauchbare Schätzung liefern.

37: In manchen Fällen bereitet allerdings auch die Beurteilung eines Biopsats Schwierigkeiten.

38: Vgl. SHEPS, S.B./SCHECHTER, M.T. (1984), S. 2419. 
Ergebnisse dann eine Art "Goldstandard"39 repräsentieren, an dem sich der zu untersuchende Diagnosetest messen lassen muß. Darüber hinaus sind im Rahmen der Schätzung der diagnostischen Kennziffern die Resultate des interessierenden Diagnosetests "blind" zu ermitteln, d.h. in Unkenntnis des tatsächlichen Krankheitszustands der Probanden ${ }^{40}$. Ansonsten besteht die Gefahr einer Überschätzung sowohl der Sensitivität als auch der Spezifität durch das (unbewußte) Bestreben, die Ergebnisse des Tests mit den Erkenntnissen aus der Anwendung des Goldstandards in Einklang zu bringen ${ }^{41}$.

Findet ein Diagnosetest in verschiedenen medizinischen Entscheidungssituationen Verwendung, dann sind für diesen Test - bei gegebener Testeinstellung - unter Umständen mehrere Werte jeweils für Sensitivität und Spezifität zu bestimmen ${ }^{42}$. Die Unterscheidung mehrerer Paare von Testkennziffern empfiehlt sich, wenn sowohl die Zusammensetzung der positiven als auch das Spektrum der negativen Fälle von der medizinischen Einsatzmöglichkeit abhängen und diese Abhängigkeit die Diskriminierung positiver und negativer Fälle durch die betrachtete diagnostische Maßnahme beeinflußt ${ }^{43}$. In diesem Fall wird man für jede der verschiedenen typischen Anwendungssituationen eine eigene Schätzung von Sensitivität und Spezifität vornehmen müssen.

Falls das als Goldstandard herangezogene Verfahren de facto lediglich eine unvollkommene Diskriminierung zwischen positiven und negativen Fällen leistet, also in Sensitivität und/oder Spezifität unter Eins liegt, liefern die oben angesprochenen Anteile in der Regel verzerrte Schätzungen für die Kennziffern des untersuchten Diagnosetests. Dabei hängt die Richtung des "bias" von der Art des Zusammenhangs

39: Dieser Ausdruck hat sich für die Referenzmethode bzw. das Verfahren der Diagnosesicherung eingebürgert; vgl. z.B. DEPARTMENT OF CLINICAL EPIDEMIOLOGY AND BIOSTATISTICS (1981), S. 704f. oder KÖBBERLING, J./TRAMPISCH, H.J./WINDELER, J. (1989), S. 10.

40: Vgl. SHEPS, S.B./SCHECHTER, M.T. (1984), S. 2418.

41: Vgl. PHILBRICK, J.T./HORWITZ, R.I./FEINSTEIN, A.R. (1980), S. 811. In diesem Fall liegt ein "test review bias" vor, da das Wissen um die korrekte Diagnose die Testinterpretation verzerrt. Der umgekehrte Sachverhalt kann eintreten, wenn ein schon bekanntes Testergebnis die Diagnosesicherung beeinflußt ("diagnostic review bias"); vgl. RANSOHOFF, D.F./FEINSTEIN, A.R. (1978), S. 928.

42: Feinstein führt als Beispiel Diagnosetests an, die sowohl im "screening" als auch zur Differentialdiagnose eingesetzt werden; vgl. FEINSTEIN, A.R. (1977a), S. $223 \mathrm{f}$.

43: Vgl. dazu Abschnitt 3.2.1. 
ab, der bei gegebenem Krankheitszustand des Patienten zwischen den Resultaten des interessierenden Diagnosetests und der Referenzmethode besteht ${ }^{44}$. Ohne Berücksichtigung der Fehler des Referenztests kann sich beispielsweise ein Diagnosetest niemals als dem bestehenden Goldstandard überlegen erweisen, selbst wenn dies zutreffen sollte ${ }^{45}$.

Gerade bei neuen Diagnosetests, die dem Arzt häufig zusätzliche diagnostische Möglichkeiten erschließen, dürfte es indessen schwierig sein, einen perfekten Referenztest zu finden bzw. zur Diagnosesichenung anzuwenden ${ }^{46}$. In der Literatur werden deshalb Verfahren diskutiert, die auch bei fehlerhaftem Referenztest zur Schätzung der Kennziffern eines Diagnosetests dienen können. Gart und Buck behandeln dieses Problem, indem sie die bedingte Unabhängigkeit der beiden diagnostischen Maßnahmen voraussetzen ${ }^{47}$. Bei bekannten Kennziffern des Referenztests liefert ihr Verfahren konsistente Schätzungen für Sensitivität und Spezifität eines Diagnosetests ${ }^{48}$. Ohne die Annahme der bedingten Unabhängigkeit lassen sich obere und untere Schranken für die Kennziffern angeben, wenn man das Vorzeichen der Abhängigkeit kennt ${ }^{49}$.

In einer neueren Arbeit schwächen Hui und Walter die Voraussetzungen des Ansatzes von Gart und Buck insoweit ab, als die Kennziffern des Referenztests nicht mehr bekannt zu sein brauchen. Die Autoren zeigen, in welcher Weise die erforderliche Datenbasis zu erweitern ist, um auch in diesem Fall geeignete MaximumLikelihood-Schätzungen von Sensitivität und Spezifität eines Diagnosetests zu ermöglichen ${ }^{50}$. Wenn jedoch die Annahme der bedingten Unabhängigkeit von Referenz- und Diagnosetest nicht zutrifft, können diese Schätzungen erhebliche Verzerrungen aufweisen ${ }^{51}$.

44: Wie Valenstein nachweist, ergibt sich bei bedingter Unabhängigkeit eine systematische Unterschätzung der Kennziffern der betrachteten diagnostischen Maßnahme; vgl. VALENSTEIN, P.N. (1990), S. 252f.

45: Vgl. GELFAND, D.W./OTT, D.J. (1985), S. 1120.

46: Vgl. VALENSTEIN, P.N. (1990), S. 252 sowie zu dieser Problematik allgemein WULFF, H.R. (1981), S. 104ff.

47: Vgl. zu dieser Annahme Abschnitt 3.5.

48: Vgl. GART, J.J./BUCK, A.A. (1966), S. $595 f$.

49: Vgl. THIBODEAU, L.A. (1981), der diese Schranken für den Fall positiver Abhängigkeiten explizit angibt.

50: Vgl. ausführlich HUI, S.L./WALTER, S.D. (1980), S. $168 \mathrm{f}$.

51: Vgl. VACEK, P.M. (1985). 
Insbesondere bei bildgebenden Verfahren besteht die Möglichkeit, ein bestimmtes Testresultat (Röntgenbild, Computertomogramm o.ä.) unterschiedlich zu interpretieren. Diese Diskrepanz in der Interpretation kann bei ein und derselben Person zu jeweils verschiedenen Zeitpunkten auftreten ("intra-observer variability") ${ }^{52}$ oder die Meinungen mehrerer Beobachter ("inter-observer variability") zu einem gegebenen Zeitpunkt betreffen ${ }^{53}$. Die zuletzt genannte "inter-observer variability" hängt dabei zu einem beträchtlichen Teil von den unterschiedlichen Fähigkeiten der Ärzte ab, den Output einer diagnostischen Technik korrekt zu erfassen bzw. einzuschätzen ${ }^{54}$. Gerade bei neuartigen diagnostischen Verfahren interpretieren zumeist besonders ausgewiesene Experten, die sich damit intensiv beschäftigt haben, deren Testergebnisse. Die dabei erzielten Schätzwerte für Sensitivität und Spezifität dürften indessen kaum - falls eine ausgeprägte "skill-dependence" vorliegt - für den durchschnittlich befähigten Arzt erreichbar sein 55 .

Diese exemplarische Aufzählung verdeutlicht, wie schwierig es sein kann, brauchbare Schätzungen für die testspezifischen Kenngrößen zu erhalten. In diesem $\mathrm{Zu}$ sammenhang weisen die Studien zur Messung bzw. zur Schätzung von Sensitivität und Spezifität einzelner diagnostischer Maßnahmen nicht selten methodische Mängel auf 56 . Die in der Literatur häufig anzutreffende breite Streuung der maßnahmenspezifischen Angaben für Sensitivität und Spezifität - bei identischer Testeinstellung - überrascht daher insofern nicht, als zumindest ein Teil der Abweichungen darauf zurückzuführen sein dürfte, $\mathrm{da} B$ wichtige Einflußfaktoren in unter-

52: Vgl. YERUSHALMY, J. (1947), S. $1435 f f$.

53: Vgl. z.B. GELFAND, D.W./OTT, D.J. (1985), S. 1118.

54: Vgl. zu dem Einfluß der "skill-dependence" näher: GILLESPIE, K.N. et al. (1986). Im übrigen könnte zumindest ein Teil der "inter-observer variability" auf unterschiedliche Ansichten über die Einstufung positiver Resultate zurückgehen. Die Auswirkungen einer solchen Diskrepanz in den implizit verwendeten Schwellen auf die Testinterpretation dürfte allerdings kaum vollständig von dem oben diskutierten Einfluß unterschiedlicher Fähigkeiten zu trennen sein; vgl. dazu auch den folgenden Abschnitt 3.2.3.

55: Vgl. GELFAND, D.W./OTT, D.J. (1985), S. 1121.

56: Vgl. BEGG, C.B. (1987), S. 415; WINDELER, J./RICHTER, K./KÖBBERLING, J. (1988); das Memorandum von KÖBBERLING, J./TRAMPISCH, H.-J./WINDELER, J. (1989) sowie VALENSTEIN, P.N. (1990), S. $255 f$. 
schiedlichem AusmaB berücksichtigt wurden ${ }^{57}$. Aus diesem Grund empfiehlt sich eine unkritische Übernahme von geschätzten Sensitivitäts- und Spezifitätswerten weder für die medizinische Anwendung noch im Rahmen einer ökonomischen Analyse.

\subsubsection{ROC-Analyse}

Sensitivität und Spezifität einer diagnostischen Maßnahme hängen von der verwendeten Testeinstellung ab. Beschränkt man die Betrachtung auf eine der beiden Kenngrößen, so lassen sich beliebige Werte zwischen Null und Eins durch eine geeignet gewählte Definition positiver und negativer Resultate erzielen ${ }^{58}$. Beispielsweise kann ein Wert von Eins für die Sensitivität (Spezifität) jederzeit durch eine genügend weite Festlegung des Bereichs positiver (negativer) Resultate realisiert werden ${ }^{59}$. Umgekehrt tendieren bei einer entsprechend engen Festlegung dieser Bereiche die jeweiligen Kenngrößen gegen Null. Diese Überlegungen verdeutlichen, daß die isolierte Betrachtung einer einzelnen diagnostischen Kennziffer keinen Sinn ergibt.

Im Gegensatz dazu ist es bei einer diagnostischen Maßnahme im allgemeinen nicht möglich, mit Hilfe der Testeinstellung gleichzeitig Sensitivität und Spezifität in beliebiger Weise zu variieren. Vielmehr lassen sich nur ganz bestimmte Wertepaare für die Kennziffern realisieren. In diesem Zusammenhang interessiert zunächst, welchen Wert die Sensitivität einer diagnostischen Maßnahme maximal erreichen kann, wenn ein vorgegebener Spezifitätswert nicht unterschritten werden darf. Hat man dieses Problem für eine Reihe verschiedener Spezifitätswerte gelöst, dann läßt sich im Anschluß daran der Preis in Form einer verringerten Sensitivität ermitteln, der bei dem betrachteten Diagnosetest für eine Erhöhung der Spezifität in Kauf zu nehmen ist.

Um diese Fragen zu untersuchen, betrachtet man ein Individuum, das zufällig aus der Grundgesamtheit aller Personen ausgewählt wurde, die für die interessierende diagnostische Maßnahme in Frage kommen. Falls dieses Individuum sich im Krankheitszustand $K_{1}$ befindet, gilt für das Resultat der diagnostischen Maßnahme $T_{j}$ eine

57: Vgl. PHILBRICK, J.T./HORWITZ, R.IJFEINSTEIN, A.R. (1980), die verschiedene Studien zum "exercise testing" (EKG) untersuchen sowie HARRIS, J.M. (1981), der die Diskrepanzen in den Sensitivitäts- und Spezifitätswerten bei acht Diagnosetests ermittelt.

58: Strenggenommen gilt die Aussage nur, wenn die Möglichkeit der Randomisierung zugelassen wird; vgl. dazu die folgenden Ausführungen.

59: Dies gilt auf jeden Fall, wenn man sämtliche Testergebnisse als positiv (negativ) deklariert. 
bedingte Massefunktion $P\left(Z_{j} \mid K_{1}\right)$. Andernfalls läßt sich das Testergebnis durch eine bedingte Massefunktion $P\left(Z_{j} \mid \bar{K}_{1}\right)$ beschreiben ${ }^{60}$. Jeder Testeinstellung entspricht eine Partition der Menge aller möglichen Testergebnisse in eine Teilmenge positiver und eine Teilmenge negativer Resultate, die mit $B_{1}$ bzw. $B_{2}$ bezeichnet seien. $B_{1}$ und $\mathrm{B}_{2}$ repräsentieren die Urbildmengen, die sich aus der mit der Testeinstellung identischen binären Zuordnung $\mathrm{Y}_{\mathrm{j}}$ ergeben. Daher gilt für die mit einer bestimmten Testeinstellung verbundenen Kennziffern:

$$
\begin{aligned}
& P\left(y_{j 1} \mid K_{1}\right)=\sum_{z_{j h} \in B_{1}} P\left(z_{j h} \mid K_{1}\right) \\
& P\left(y_{j 2} \mid \bar{K}_{1}\right)=\sum_{z_{j h} \in B_{2}} P\left(z_{j h} \mid \bar{K}_{1}\right) .
\end{aligned}
$$

Im Prinzip ergibt sich in dieser Situation eine bemerkenswerte Ähnlichkeit mit einem Problem, das in der Theorie der statistischen Hypothesenprüfung auftaucht. Das Testergebnis des Probanden stellt ein Zufallsereignis dar, zu dessen Beschreibung die folgenden Hypothesen in Betracht zu ziehen sind ${ }^{61}$ :

$$
\begin{aligned}
& \mathrm{H}_{0}: \mathrm{K}=\mathrm{K}_{1} \quad \text { bzw.: } \mathrm{P}=\mathrm{P}\left(\mathrm{Z}_{\mathrm{j}} \mid \mathrm{K}_{1}\right), \\
& \mathrm{H}_{1}: \mathrm{K}=\overline{\mathrm{K}}_{1} \quad \text { bzw.: } \mathrm{P}=\mathrm{P}\left(\mathrm{Z}_{\mathrm{j}} \mid \overline{\mathrm{K}}_{1}\right) .
\end{aligned}
$$

Damit handelt es sich in statistischer Hinsicht um das Problem, eine einfache Nullhypothese gegen eine ebenfalls einfache Alternative zu prüfen ${ }^{62}$. Die Menge aller möglichen Resultate der diagnostischen Maßnahme bildet dabei den Stichprobenraum. Ein nicht-randomisierter statistischer Test wird durch eine Vorschrift repräsentiert, die für jedes Element des Stichprobenraums angibt, ob man sich bei seinem Auftreten für oder gegen die Nullhypothese entscheidet.

60: Diese Aussagen gelten unabhängig davon, welche der in Abschnitt 3.2.1 angesprochenen Modellvorstellungen als zutreffend angesehen wird.

61: Es spielt für die hier benötigten Aussagen keine Rolle, welche Hypothese als Nullhypothese ausgezeichnet wird.

62: Zum Begriff der einfachen Hypothese vgl. NEYMAN, J. (1950), S. 253. 
Sieht man ein positives diagnostisches Resultat als Indiz für das Vorliegen des Krankheitszustands $K_{1}$ an, während ein negatives Ergebnis die Abwesenheit von $K_{1}$ also $\overline{\mathbf{K}}_{1}$ - anzeigt, dann läßt sich jede Testeinstellung einer diagnostischen Maßnahme als spezieller statistischer Test interpretieren ${ }^{63}$.

Unter dieser Voraussetzung gibt die Differenz Eins abzüglich der Spezifität den Fehler 2. Art eines derartigen Tests an, während die Sensitivität der Differenz aus Eins und dem Signifikanzniveau des Tests entspricht. Diese beiden Größen repräsentieren in der hier diskutierten Situation die Operationscharakteristik des statistischen Tests, der aus einer festen Binarisierung resultiert ${ }^{64}$. Damit legen die Kennziffern einer diagnostischen Maßnahme bei vorgegebener Binarisierung die Operationscharakteristik des entsprechenden statistischen Tests fest.

Die eingangs gestellte Frage nach den realisierbaren Wertepaaren für die Kennziffern einer diagnostischen Maßnahme ist demnach äquivalent zu dem Problem, die Menge aller statistischen Tests zur Prüfung der oben angegebenen einfachen Hypothesen anzugeben. Dabei wird jeder Test durch seine Operationscharakteristik vollständig beschrieben. Es erweist sich als nützlich, die Menge der Tests zu erweitern und die Möglichkeit der Randomisierung zuzulassen 65 . Ein randomisierter oder verallgemeinerter statistischer Test liegt vor, wenn bei zumindest einem Element des Stichprobenraums die Wahrscheinlichkeit, sich für die Nullhypothese zu entscheiden, im offenen Intervall zwischen Null und Eins liegt. In diesem Fall schreibt der Test nicht bei allen möglichen Beobachtungen deterministisch eine Entscheidung für oder gegen die Nullhypothese vor. Die Berücksichtigung randomisierter statistischer Tests ermöglicht es, zu jedem beliebigen Signifikanzniveau einen Test zu konstruieren.

63: Der Begriff des statistischen Tests darf nicht mit dem des Diagnosetests verwechselt werden. Aus diesem Grund wird im folgenden die Bezeichnung "Testeinstellung" vermieden und an ihrer Stelle von einer Binarisierung gesprochen.

64: Die Operationscharakteristik eines statistischen Tests gibt für jede in Betracht gezogene Hypothese die Wahrscheinlichkeit an, mit der man sich für die Nullhypothese entscheidet; vgl. WEICHSELBERGER, K. (1977), S. 254.

65: Ohne Randomisierung gibt es bei der diagnostischen Maßnahme $T_{j}$ insgesamt $2 k_{j}$ Möglichkeiten der Binarisierung und damit ebenso viele statistische Tests. 
Unter diesen Voraussetzungen ergeben sich für die Menge der realisierbaren Wertepaare der Kennziffern einer diagnostischen Maßnahme folgende Aussagen ${ }^{66}$ :

(1) $\mathrm{Zu}$ jeder vorgegebenen Wahrscheinlichkeit eines falsch positiven Resultats existiert eine (eventuell randomisierte) Binarisierung mit maximaler Sensitivität. Diese Zuordnung wird durch die folgende Funktion angegeben:

$$
P\left(y_{j 1} \mid K_{1}\right)=g\left[P\left(y_{j 1} \mid \bar{K}_{1}\right)\right]
$$

(2) Die Steigung dieser Funktion ist nichtnegativ und monoton fallend 67 . Im Normalfall läßt sich daher die Sensitivität einer diagnostischen Maßnahme nur erhöhen, indem man eine gestiegene Wahrscheinlichkeit falsch positiver Resultate in Kauf nimmt ${ }^{68}$. Insofern beschreibt die in (3.6) angegebene Funktion $\mathrm{g}$ den "trade-off" zwischen Sensitivität und Spezifität bei einer diagnostischen Maßnahme.

Die Monotonieeigenschaft der Steigung von $g$ impliziert, daß der Preis einer gegebenen Erhöhung der Sensitivität - gemessen durch die dazu notwendige Verringerung der Spezifität - im allgemeinen umso höher ausfällt, je größer die Sensitivität in der Ausgangslage ist.

(3) Sämtliche Binarisierungen, die durch den Graphen der Funktion g repräsentiert werden, sind optimal in dem Sinn, daß eine Erhöhung einer Kenn-

66: Es handelt sich um die direkte Übertragung der Resultate für das äquivalente statistische Problem; vgl. dazu ausführlich NEYMAN, J. (1950), S. 304ff.; FERGUSON, T.S. (1967), S. 198ff.; KENDALL, M./STUART, A. (1979), S. 180ff.

67: An einigen Stellen existiert die Ableitung von g nicht, da rechts- und linksseitiger Grenzwert auseinanderfallen. Man kann sich damit behelfen, daß man durchgängig einen der beiden Grenzwerte als Ableitung von $\mathrm{g}$ an diesen Stellen definiert.

68: Die Aussage gilt immer, falls die in den zu prüfenden Hypothesen spezifizierten Wahrscheinlichkeitsverteilungen identische Massepunkte besitzen. Ansonsten bezieht sich die Aussage auf diejenigen Fälle, in denen eine Erhöhung der Sensitivität nur möglich ist, indem man diagnostische Resultate in den positiven Bereich aufnimmt. die auch unter der Alternative $\left(\mathrm{H}_{1}\right)$ eine positive Wahrscheinlichkeit aufweisen. 
ziffer ohne eine gleichzeitige Verringerung der anderen Kennziffer bei der betrachteten diagnostischen $\mathrm{Maßnahme} \mathrm{nicht} \mathrm{möglich} \mathrm{ist}{ }^{69}$. Umgekehrt erhält man sämtliche Binarisierungen mit besonders ungünstigen Eigenschaften, indem man die Funktion $g$ an dem Punkt $S(0,5 \mid 0,5)$ spiegelt. Der Graph der aus dieser Operation resultierenden Funktion h repräsentiert die diagnostischen Kennziffern jener Binarisierungen, bei denen im Vergleich zu den optimalen Binarisierungen positive und negative Resultate gerade umgekehrt definiert wurden. Die Menge aller Sensitivitätswerte, die insgesamt für eine vorgegebene Wahrscheinlichkeit eines falsch positiven Resultats realisierbar sind, wird durch die Funktionswerte von $\mathrm{g}$ und $\mathrm{h}$ nach oben bzw. nach unten beschränkt.

Diese Aussagen veranschaulicht Abbildung 7 mit Hilfe eines Diagramms. Die Punkte A, B und C repräsentieren optimale Binarisierungen, bei denen jedes diagnostische Resultat eindeutig entweder als positiv oder als negativ eingestuft wird ${ }^{70}$. Offenbar gelingt es bei der in der Abbildung dargestellten diagnostischen Maßnahme, die Sensitivität von Null auf einen positiven Wert zu erhöhen, ohne an der Spezifität von Eins etwas einzubüßen. Dies bedeutet, daß zumindest ein diagnostisches Resultat nur bei denjenigen Individuen auftreten kann, die sich im Krankheitszustand $K_{1}$ befinden. Im Extremfall bilden die möglichen diagnostischen Resultate der positiven und der negativen Fälle disjunkte Mengen. Dann gibt es wenigstens eine Binarisierung, die bei beiden Kennziffern einen Wert von Eins liefert und daher eine fehlerfreie Unterscheidung von Individuen in Krankheitszustand $K_{1}$ und Individuen in $\overline{\mathrm{K}}_{1}$ ermöglicht. Die Menge aller möglichen Wertepaare der Kennziffern der diagnostischen Maßnahme füllt in diesem Fall das gesamte Diagramm aus.

69: Diese Binarisierungen entsprechen in statistischer Hinsicht den besten Tests; vgl. WEICHSELBERGER, K. (1977), S. 255.

70: Damit entsprechen diese Binarisierungen nicht-randomisierten statistischen Tests. 
Abb. 7: Die Menge der möglichen Kennzifferkombinationen eines binarisierten Diagnosetests ${ }^{71}$

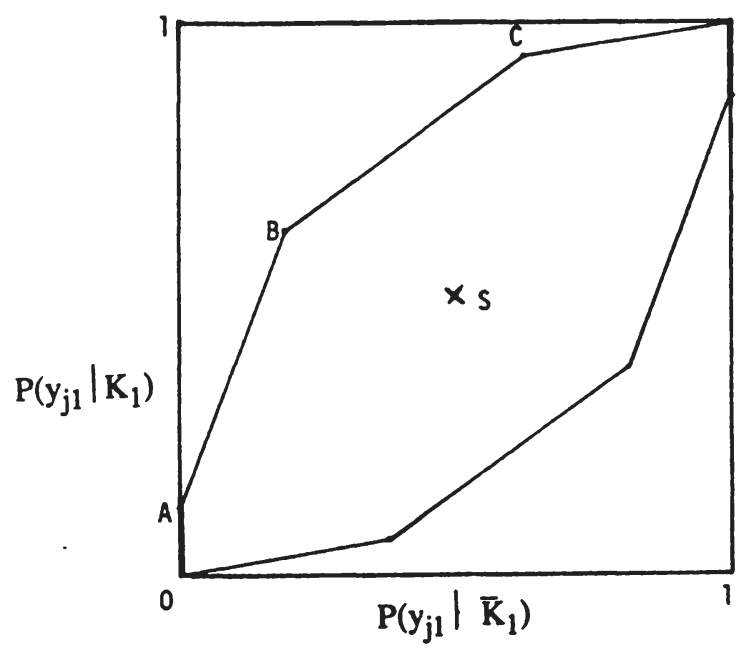

Der Graph der zuvor angesprochenen Funktion $\mathbf{g}$ bildet den oberen Rand der in der Abbildung dargestellten Menge. Die Funktion selbst wird in der Literatur als ROCKurve bezeichnet, wobei das Akronym ROC für "relative operating characteristic" bzw. "receiver operating characteristic" steht ${ }^{72}$. Der Name bezieht sich auf die Eigenschaft der ROC-Kurve, für jede Wahrscheinlichkeit eines falsch positiven Resultats die bei der betrachteten diagnostischen Maßnahme maximal erzielbare Sensitivität anzugeben. Der Graph einer ROC-Kurve repräsentiert damit in statistischer Hinsicht

71: Die Spezifität erscheint in dem Diagramm nicht direkt, läßt sich aber aufgrund der Beziehung $P\left(y_{j 2} \mid \bar{K}_{1}\right)=1-P\left(y_{j 1} \mid \bar{K}_{1}\right)$ ermitteln.

72: Vgl. GREEN, D.M./SWETS, J.A. (1966); SWETS, J.A. (1972); EGAN, J.P. (1975); METZ, C.E. (1978) sowie SWETS, J.A./PICKETT, R.M. (1982). Überwiegend wird der Terminus "receiver operating characteristic" verwendet, während insbesondere Swets in neueren Arbeiten von "relative operating characteristic" spricht; vgl. SWETS, J.A. (1979), S. 109; SWETS, J.A./PICKETT, R.M. (1982), S. 3.

Wie weiter unten erläutert wird, gibt es gute Gründe, beide Bezeichnungen zu verwenden, da der Begriff der ROC-Kurve zwei verschiedene Konzepte zusammenfaßt. 
die Menge der Operationscharakteristiken aller besten Tests, die zur Prüfung der beiden einfachen Hypothesen konstruiert werden können. Bei einer diagnostischen Maßnahme gibt die dazugehörige ROC-Kurve den notwendigerweise hinzunehmenden "trade-off" zwischen Sensitivität und Spezifität an ${ }^{73}$.

Die Information diagnostischer Maßnahmen kảnn letztlich nur mit Hilfe der Informationstheorie ermittelt werden. Dennoch erlaubt es die hier untersuchte einfache Entscheidungssituation, qualitative Aussagen über die Informationsleistung eines Diagnosetests aus dem dazugehörigen "trade-off" $z$ wischen seinen Kennziffern abzuleiten. Es leuchtet unmittelbar ein, daß von zwei diagnostischen Maßnahmen, deren ROC-Kurven sich nicht schneiden, diejenige besser zwischen positiven und negativen Fällen diskriminiert, die für jeden vorgegebenen Spezifitätswert eine mindestens ebenso hohe Sensitivität wie der konkurrierende Test ermöglicht ${ }^{74}$.

Insgesamt bieten ROC-Kurven die Möglichkeit, einen umfassenden Vergleich binarisierter diagnostischer Maßnahmen vorzunehmen, da sie den Einfluß der Testeinstellung von der Informationsleistung zu trennen erlauben ${ }^{75}$. Dies läßt sich mit der Hilfe von Abbildung 8 erläutern.

Der isolierte Vergleich einzelner Sensitivitäts- und Spezifitätswerte bei verschiedenen diagnostischen Maßnahmen kann zu irreführenden Schlüssen verleiten. Die Abbildung zeigt dies zunächst am Beispiel zweier Diagnosetests, die jeweils bei einer Kennziffer einen Vorteil gegenüber ihrem Konkurrenten aufweisen [Abb. 8(a)]. Diese Information reicht nicht aus, um zu beurteilen, welche Maßnahme insgesamt eine bessere Diskriminierung in Bezug auf den bei einem Individuum vorliegenden Krankheitszustand liefert. Vergleicht man jedoch die dazugehörigen ROC-Kurven [Abb. 8(b)], so zeigt sich die durchgängige Überlegenheit der zu A gehörenden dia-

73: Bei suboptimalen Binarisierungen besteht die Möglichkeit, durch eine veränderte Einteilung positiver und negativer Resultate eine Kennziffer zu erhöhen, ohne eine Einbuße bei der anderen Kennziffer zu erleiden.

74: In diesem Fall liegt die ROC-Kurve des besser diskriminierenden Tests "oberhalb" der zu dem alternativen Test gehörigen ROC-Kurve; vgl. Abbildung 8(b). Die auf diese Weise definierte Relation der Diskriminierungsfähigkeit induziert allerdings lediglich eine partielle Ordnung auf der Menge aller diagnostischen Maßnahmen, da sich die ROC-Kurven einzelner Maßnahmen auch schneiden können, wie das Beispiel der Abbildung 8(d) zeigt.

75: Vgl. dazu z.B. METZ, C.E. (1978), S. $289 f$. 
Abb. 8: Der Vergleich der Kennziffern binarisierter Diagnosetests

a)

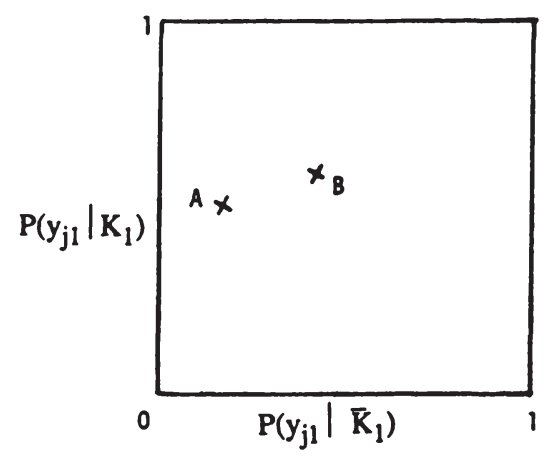

c)

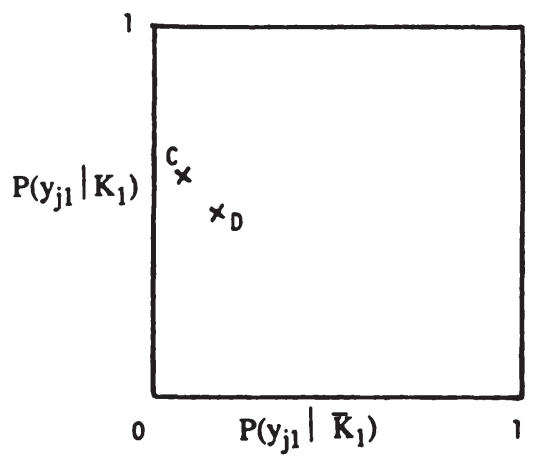

b)

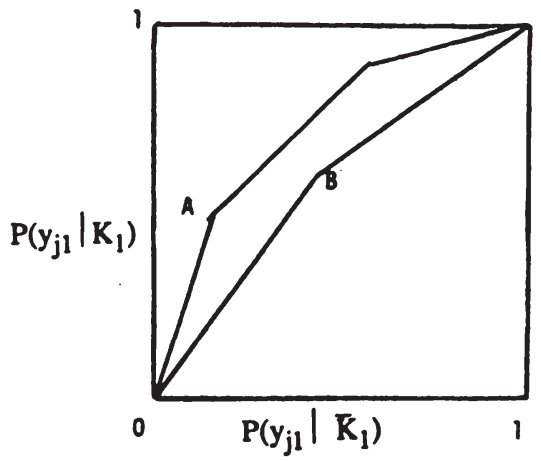

d)

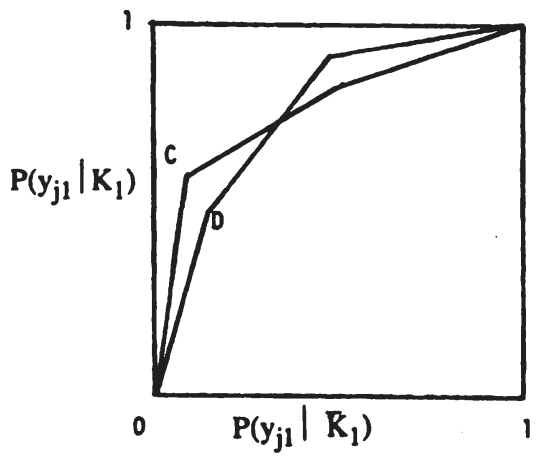


gnostischen Maßnahme. Umgekehrt liegt in Abbildung 8(c) der Fall zweier Diagnosetests vor, deren Kennziffern einen Vergleich erlauben, der die zu C gehörige Maßnahme als vorteilhaft ausweist. Hingegen zeigt die Betrachtung der ROC-Kurven [Abb. 8(d)], daß es auch Spezifitätswerte gibt, bei deren Vorgabe die andere diagnostische Maßnahme eine höhere Sensitivität erzielt. Falls sich die ROC-Kurven schneiden, entfällt die Möglichkeit, eine eindeutige Aussage über die relative Diskriminierungsfähigkeit von diagnostischen Maßnahmen zu treffen ${ }^{76}$.

Ähnlich wie bei der Ermittlung von Sensitivität und Spezifität ist man bei der Ableitung von ROC-Kurven ebenfalls auf Schätzungen angewiesen. Dabei treten zunächst dieselben Probleme auf, die eine Ermittlung der Kennziffern bei gegebener Testeinstellung erschweren. Insgesamt gestaltet sich die Aufgabe bei einer ROCKurve indessen umfangreicher, da ein funktionaler Zusammenhang zu schätzen ist. Dabei gilt es zu beachten, daß ROC-Kurven nur unter der Voraussetzung der Optimalität der jeweils verwendeten Testeinstellung zu erhalten sind ${ }^{77}$.

Die Anwendung von ROC-Kurven konzentriert sich in der Analyse bildgebender Verfahren (z.B. Computertomographie) ${ }^{78}$. Dies dürfte unter anderem darauf zurückzuführen sein, daß diese diagnostischen Maßnahmen mehrdimensionale Merkmale erfassen, deren Ausprägungen sich nicht auf einfache Weise beschreiben und kaum mit Hilfe metrisch skalierter Variablen angeben lassen. Man hofft deshalb, auf eine - aufwendige - explizite Abgrenzung positiver und negativer Resultate verzichten zu können, indem man mittels spezieller Techniken die von einem Beobachter implizit verwendeten Testeinstellungen durch eine Kurve schätzt, die den "trade-off" zwischen Sensitivität und Spezifität angibt ${ }^{79}$.

76: Die Frage, wann Unterschiede in den ROC-Kurven verschiedener diagnostischer Maßnahmen statistische Signifikanz besitzen, behandeln METZ, C.E.KRONMAN, H.B. (1980) und HANLEY, J.A./McNEIL, B.J. (1982), S. 34f.

77: Vgl. GREEN, R.M./SWETS, J.A. (1966), S. 31.

Vomehmlich aus didaktischen Gründen führen viele Autoren, die die Ableitung von ROCKurven erläutern, das einfache Beispiel eines eindimensionalen diagnostischen Merkmals an, für dessen Ausprägungen der Likelihood-Quotient eine Monotonieeigenschaft aufweist; vgl. McNEIL, B.J./KEELER, E./ADELSTEIN, S.J. (1975), S. 212f.; METZ, C.E. (1978), S. 287; WEINSTEIN, M.C. et al. (1980), S. 115ff. In diesem Fall weisen optimale Binarisierungen eine besonders einfache Form auf.

78: Vgl. ABRAMS, H.L. et al. (1982); KELSEY, C.A. et al. (1985); McNEIL, B.J. et al. (1983); McNEIL, B.J./HANLEY, J.A. (1984) sowie SWETS, J.A. (1979).

79: Vgl. z.B. GREEN, D.M./SWETS, J.A. (1966), S. 40ff; METZ, C.E. (1978), S. $290 f$. 
Bei diesen Zusammenhängen handelt es sich allerdings nicht um ROC-Kurven im strengen Sinn, da die Annahme der Optimalität der jeweils verwendeten Testeinstellung nicht erfüllt zu sein braucht. Vielmehr repräsentieren die Unterschiede, die in den für verschiedene Beobachter abgeleiteten Zusammenhängen zwischen Sensitivität und Spezifität vorliegen, deren relative Fähigkeit, geeignete Binarisierungen der Resultate bei der untersuchten diagnostischen Maßnahme vorzunehmen ${ }^{80}$. Dies eröffnet eine interessante Möglichkeit, die Erfahrung bzw. Kompetenz, die ein Beobachter bei der Beurteilung der Resultate einer diagnostischen Maßnahme besitzt, genauer zu beschreiben. Diese Fähigkeit läßt sich in theoretischer Hinsicht offenbar durch die "Nähe" messen, welche die vom Beobachter jeweils verwendeten Testeinstellungen zu den entsprechenden optimalen Binarisierungen aufweisen.

In der Literatur wird auch der auf einen Beobachter bezogene "trade-off" zwischen Sensitivität und Spezifität als ROC-Kurve bezeichnet ${ }^{81}$. Es handelt sich dabei inhaltlich um eine zweite Variante, die von dem eigentlichen Konzept der ROC-Kurve zu unterscheiden ist. Unter den eingangs angegebenen Voraussetzungen existiert für jede diagnostische Maßnahme eine eindeutig bestimmte ROC-Kurve, deren Verlauf in keiner Weise von einem Beobachter abhängt. Insofern kann man diesen Zusammenhang zwischen Sensitivität und Spezifität als "relative operating characteristic" Kurve bezeichen.

Im Gegensatz dazu geht in den für einen speziellen Beobachter abgeleiteten $\mathrm{Zu}$ sammenhang zwischen den Kennziffern einer diagnostischen Maßnahme zugleich dessen Fähigkeit zur Interpretation der Testergebnisse ein ${ }^{82}$. Zur besseren Unterscheidung sollte man diese Zusammenhänge als "receiver operating characteristic" Kurven bezeichnen. Dabei brauchen ROC-Kurven in dieser zweiten Variante nicht unbedingt jene zuvor angesprochenen Eigenschaften zu besitzen, die eine "relative operating characteristic" Kurve kennzeichnen ${ }^{83}$. In konzeptioneller Hinsicht beschreibt die "relative operating characteristic" Kurve den "trade-off" zwischen Sen-

80: Dabei wird von Schätzfehlern abstrahiert, die ebenfalls Unterschiede in den ROC-Kurven verschiedener Beobachter verursachen können.

81: Vgl. SWETS, J.A. (1972), S. 19ff; SWETS, J.A. (1979), S. 113.

82: Vgl. BEGG, C.B. (1987), S. 416.

83: Insbesondere kann die ROC-Kurve eines Beobachters Bereiche aufweisen, in denen ein gegebener Zugewinn an Sensitivität mit abnehmenden Einbußen bei der Spezifität einhergeht. 
sitivität und Spezifität, der sich bei dem betrachteten Diagnosetest für einen idealen Beobachter ergeben würde. Dieser ideale Beobachter zeichnet sich dadurch aus, da $\beta$ er die maßnahmenspezifische Information in bestmöglicher Weise nutzt, um positive und negative Resultate voneinander zu unterscheiden.

\subsection{Prädiktive Werte}

\subsubsection{Positiver und negativer prädiktiver Wert}

Im folgenden sei angenommen, daß Sensitivität und Spezifität der betrachteten diagnostischen $\mathrm{Maßnahmen}$ zutreffend ermittelt worden sind. Inwieweit können diese Größen dazu dienen, die Information zu bestimmen, die eine diagnostische Maßnahme liefert? Beide Kenngrößen informieren darüber, mit welchen Wahrscheinlichkeiten ein Diagnosetest bei vorgegebenem Krankheitszustand ein korrektes Ergebnis angibt. In qualitativer Hinsicht entspricht dies der Darstellung in medizinischen Lehrbüchern, die für die einzelnen Krankheitszustände die dazugehörigen Symptome abhandeln.

In medizinischen Anwendungssituationen herrscht dagegen typischerweise gerade die umgekehrte Fragestellung vor ${ }^{84}$. Da der Arzt den wahren Krankheitszustand seines Patienten nicht kennt, setzt er diagnostische Maßnahmen ein, um Aufschluß darüber zu erhalten, ob eine von ihm vermutete Krankheit vorliegt oder nicht. Hier interessiert besonders, inwieweit ein positives Testergebnis eine in Betracht gezogene Krankheitshypothese erhärtet ${ }^{85}$. Dieses Problem läßt sich mit der Kenntnis von Sensitivität und Spezifität allein nicht lösen. Man benötigt dazu weitere Angaben, die zusammen mit den diagnostischen Kennziffern den Schluß von den Testergebnissen auf den Krankheitszustand erlauben.

Wie in Abschnitt 2.4.1 ausgeführt, läßt sich der Informationsstand des Arztes vor der Anwendung einer diagnostischen Maßnahme durch eine priore Wahrscheinlichkeitsverteilung auf der Menge $\mathrm{K}$ der möglichen Krankheitszustände repräsentieren. Bezogen auf die weiterhin als gültig unterstellte einfache Entscheidungs-

84: Vgl. DEPARTMENT OF CLINICAL EPIDEMIOLOGY AND BIOSTATISTICS (1983), S. 562; DAHMER, J. (1980), S. 2 sowie RICHTER, K. et al. (1988), S. 658.

85: Auch ein negatives Resultat kann unter Umständen diesen Zweck erfüllen; vgl. GORRY, G.A. et al. (1978). 
situation bedeutet dies, daß der Arzt zwei Wahrscheinlichkeiten, $\mathrm{P}\left(\mathrm{K}_{1}\right)$ und $\mathrm{P}\left(\overline{\mathrm{K}}_{1}\right)$, angeben kann. Im Normalfall ändern sich diese Wahrscheinlichkeiten als Folge bzw. unter der Bedingung eines diagnostischen Testergebnisses ${ }^{86}$. Die daraus resultierende posteriore Wahrscheinlichkeitsverteilung repräsentiert den neuen Informationsstand des Arztes. Die Veränderung der Informationslage aufgrund eines Testresultats ergibt sich damit aus einem Vergleich von prioren und posterioren Wahrscheinlichkeiten für $\mathrm{K}_{1}$ und $\overline{\mathrm{K}}_{1}$. Führt man diesen Vergleich für positive und negative Resultate durch, so erhält man analog die Veränderung der Informationslage aufgrund eines binarisierten Tests.

Gegeben eine durch $\mathrm{P}\left(\mathrm{K}_{1}\right)$ festgelegte priore Wahrscheinlichkeitsverteilung, ermöglichen die diagnostischen Kennziffern Sensitivität und Spezifität unter der in Abschnitt 2.4.3 getroffenen Voraussetzung eine Anwendung des Bayes-Theorems. Für die Wahrscheinlichkeit des Krankheitszustands $\mathrm{K}_{1}$ gegeben ein positives Testresultat $\mathrm{y}_{\mathrm{j} 1}$ ergibt sich dann:

$$
\begin{aligned}
P\left(K_{1} \mid y_{j 1}\right) & =\frac{P\left(y_{j 1} \mid K_{1}\right) \cdot P\left(K_{1}\right)}{P\left(y_{j 1}\right)} \\
& =\frac{P\left(y_{j 1} \mid K_{1}\right)}{P\left(y_{j 1} \mid K_{1}\right) \cdot P\left(K_{1}\right)+P\left(y_{j 1} \mid \bar{K}_{1}\right) \cdot P\left(\bar{K}_{1}\right)} .
\end{aligned}
$$

Die auf der rechten Seite stehenden bedingten Wahrscheinlichkeiten resultieren aus den testspezifischen Kenngrößen: $P\left(y_{j 1} \mid K_{1}\right)$ repräsentiert direkt die Sensitivität, während $\mathrm{P}\left(\mathrm{y}_{\mathrm{j} 1} \mid \overline{\mathrm{K}}_{1}\right)$ die Wahrscheinlichkeit für ein falsch positives Resultat gegeben Krankheitszustand $\bar{K}_{1}$ angibt. Die Ausdrücke $P\left(K_{1}\right)$ und $P\left(\bar{K}_{1}\right)$ stellen die vor Durchführung der diagnostischen Maßnahme als gültig erachteten Wahrscheinlichkeiten der beiden Krankheitszustände $\mathrm{K}_{1}$ und $\overline{\mathrm{K}}_{1}$ dar.

86: Renyi weist auf die Ungenauigkeit dieser Sprechweise hin: man betrachtet Wahrscheinlichkeiten, die im Vergleich zu dem für die prioren Wahrscheinlichkeiten als gültig angesehenen Bedingungskomplex zusätzlich die Bedingung eines diagnostischen Resultats berücksichtigen; vgl. RENYI, A. (1966), S. 43f. Insofern ändern sich die prioren Wahrscheinlichkeiten im strengen Sinn nicht, man geht lediglich aufgrund der zusätzlich vorhandenen Information zu anderen Wahrscheinlichkeiten über. 
Ebenso gilt für die Wahrscheinlichkeit des Krankheitszustands $\overline{\mathrm{K}}_{1}$ gegeben ein positives Testergebnis:

$$
\begin{aligned}
P\left(\bar{K}_{1} \mid y_{j 1}\right) & =\frac{P\left(y_{j 1} \mid \bar{K}_{1}\right) \cdot P\left(\bar{K}_{1}\right)}{P\left(y_{j 1}\right)} \\
& =\frac{P\left(y_{j 1} \mid \bar{K}_{1}\right) \cdot P\left(K_{1}\right)}{P\left(y_{j 1} \mid K_{1}\right) \cdot P\left(K_{1}\right)+P\left(y_{j 1} \mid \bar{K}_{1}\right) \cdot P\left(\bar{K}_{1}\right)}
\end{aligned}
$$

$\mathrm{P}\left(\overline{\mathrm{K}}_{1} \mid \mathrm{y}_{\mathrm{j} 1}\right)$ gibt die Wahrscheinlichkeit eines falsch positiven Testergebnisses unter der Bedingung an, da $\beta$ ein positives Resultat vorliegt. Mit den beiden bedingten Wahrscheinlichkeiten $P\left(K_{1} \mid y_{j 1}\right)$ und $P\left(\bar{K}_{1} \mid y_{j 1}\right)$ liegt die posteriore Wahrscheinlichkeitsverteilung der Krankheitszustände bei einem positiven Testergebnis vor.

Entsprechend lassen sich die bedingten Wahrscheinlichkeiten für $\mathrm{K}_{1}$ und $\overline{\mathrm{K}}_{1}$ im Falle eines negativen Testresultats $\mathrm{y}_{\mathrm{j} 2}$ bestimmen:

$$
\begin{aligned}
P\left(K_{1} \mid y_{i 2}\right) & =\frac{P\left(y_{j 2} \mid K_{1}\right) \cdot P\left(K_{1}\right)}{P\left(y_{j 2}\right)} \\
& =\frac{P\left(y_{j 2} \mid K_{1}\right) \cdot P\left(K_{1}\right)}{P\left(y_{j 2} \mid K_{1}\right) \cdot P\left(K_{1}\right)+P\left(y_{j 2} \mid \bar{K}_{1}\right) \cdot P\left(\bar{K}_{1}\right)}
\end{aligned}
$$

$$
\begin{aligned}
P\left(\bar{K}_{1} \mid y_{j 2}\right) & =\frac{P\left(y_{j 2} \mid \bar{K}_{1}\right) \cdot P\left(\bar{K}_{1}\right)}{P\left(y_{j 2}\right)} \\
& =\frac{P\left(y_{j 2} \mid \bar{K}_{1}\right) \cdot P\left(\bar{K}_{1}\right)}{P\left(y_{j 2} \mid K_{1}\right) \cdot P\left(K_{1}\right)+P\left(y_{j 2} \mid \bar{K}_{1}\right) \cdot P\left(\bar{K}_{1}\right)}
\end{aligned}
$$


In diesen Gleichungen bezeichnet $P\left(y_{j 2} \mid K_{1}\right)$ jeweils die Wahrscheinlichkeit für ein falsch negatives Resultat gegeben $K_{1}$. Ähnlich gibt $P\left(y_{j 2} \mid \bar{K}_{1}\right)$ direkt die Spezifität der analysierten diagnostischen Maßnahme an. Im Einklang mit den zuvor im Falle eines positiven Testergebnisses angestellten Überlegungen lassen sich auch die in den Gleichungen (3.9) und (3.10) angegebenen posterioren Wahrscheinlichkeiten interpretieren: $P\left(K_{1} \mid y_{j 2}\right)$ stellt die Wahrscheinlichkeit des Krankheitszustands $K_{1}$ unter der Bedingung eines negativen Testergebnisses dar, während $P\left(\bar{K}_{1} \mid y_{j 2}\right)$ die Wahrscheinlichkeit für das dazu komplementäre Ereignis anzeigt.

Die in den Gleichungen (3.7) bis (3.10) angegebenen posterioren Wahrscheinlichkeiten definieren somit zwei bedingte Massefunktionen für die beiden Krankheitszustände $\mathrm{K}_{1}$ und $\overline{\mathrm{K}}_{1}$. Da es sich um Wahrscheinlichkeitsverteilungen handelt, folgt aus der dazugehörigen Additivitätsbedingung, daß nur jeweils eine Wahrscheinlichkeit bestimmt werden muß. Demzufolge beschränkt man sich auf eine Analyse der Größen $P\left(K_{1} \mid y_{j 1}\right)$ und $P\left(\bar{K}_{1} \mid y_{j 2}\right)$, weil diese zur Beschreibung der Veränderung der Informationslage durch eine binarisierte diagnostische Maßnahme in der betrachteten einfachen Entscheidungssituation ausreichen. Diese Wahrscheinlichkeiten informieren darüber, in welchem Ausmaß bei einer Anwendung des Diagnosetests einerseits ein positives Testergebnis korrekt das Vorliegen des Krankheitszustands $\mathrm{K}_{1}$ signalisiert und andererseits ein negatives Testergebnis zutreffend die Abwesenheit von $\mathrm{K}_{1}$, also $\overline{\mathrm{K}}_{1}$, indiziert.

In Übereinstimmung mit diesen Interpretationen bezeichnet man diese beiden Wahrscheinlichkeiten als prädiktive Werte ${ }^{87}$, genauer: $P\left(K_{1} \mid y_{j 1}\right)$ als prädiktiven Wert eines positiven Tests (positiven prädiktiven Wert ${ }^{88}$ ) und $P\left(\bar{K}_{1} \mid y_{j 2}\right)$ als prädiktiven Wert eines negativen Tests (negativen prädiktiven Wert) ${ }^{89}$. Es handelt sich bei den prädiktiven Werten um posteriore Wahrscheinlichkeiten, die für eine diagnostische Maßnahme gültig sind unter der Annahme einer bestimmten prioren Wahrscheinlichkeitsverteilung für die Krankheitszustände. Mit den prädiktiven Werten lie-

87: Vgl. z.B. GERSTMAN, B.B./CAPPUCCHI, D.T. (1986); KÖBBERLING, J. (1982) sowie VECCHIO, T.J. (1966), der diese Terminologie eingeführt hat.

Im übrigen sind diese Bezeichnungen insofern mißverständlich, als es sich dabei keineswegs um Wertgrößen etwa im Sinne der ökonomischen Wertheorie handelt.

88: Vgl. VECCHIO, T.J. (1966).

89: Die Ausdrücke "positiver" und "negativer" Test beziehen sich auf die entsprechenden Testergebnisse; vgl. KÖBBERLING, J. (1982), S. 591. 
gen zugleich die posterioren Verteilungen fest. Die Veränderung der Informationslage des Arztes, die aus dem Einsatz einer diagnostischen Maßnahme resultiert, erhält man durch einen Vergleich der posterioren mit den entsprechenden prioren Wahrscheinlichkeiten.

Die prädiktiven Werte lassen sich auch ohne expliziten Rekurs auf die diagnostischen Kenngrößen Sensitivität und Spezifität berechnen. Für ein vorgegebenes Testresultat untersucht man zu diesem Zweck zunächst die dazugehörige LikelihoodFunktion. Bei einer Likelihood-Funktion betrachtet man - im Gegensatz zu einer Masse- oder Dichtefunktion - die Verteilungsparameter als Veränderliche unter der Bedingung einer speziellen Ausprägung einer Zufallsvariablen bzw. einer Stichprobenfunktion 90 . Auf die hier interessierende Situation bezogen, gibt die Likelihood-Funktion beispielsweise eines positiven Testergebnisses an, wie - im hier ausschließlich untersuchten diskreten Fall - die Wahrscheinlichkeiten dieses Resultats bei verschiedenen Krankheitszuständen ausfallen. Die Likelihood eines positiven Testergebnisses entspricht, wenn der Krankheitszustand $\mathrm{K}_{1}$ vorliegt, daher der Wahrscheinlichkeit $P\left(y_{j 1} \mid K_{1}\right)$, während $P\left(y_{j 1} \mid \bar{K}_{1}\right)$ den Wert derselben Likelihood-Funktion an der Stelle $\overline{\mathrm{K}}_{1}$ angibt.

Unter Umständen kennt man bei einer diagnostischen Maßnahme diese beiden Likelihood-Werte nicht, sondern weiß lediglich, in welchem Verhältnis positive Testergebnisse bei Individuen mit $\mathrm{K}_{1}$ und ohne die interessierende Krankheit stehen. Man besitzt damit Information über den Likelihood-Quotienten ${ }^{91} L\left(y_{j 1}\right)$ des Diagnosetests für das Resultat $y_{j 1}$. Diese Information reicht aus, um die prädiktiven Werte zu bestimmen 92 .

90: Vgl. WINKLER, R.L. (1972), S. 76ff.; FAHRMEIR, L/HAMERLE, A. (1984), S. 54ff. sowie ausführlich BERGER, J.O. (1985), der u.a. den Zusammenhang zwischen der LikelihoodFunktion und dem insbesondere für die Schätztheorie wichtigen Likelihood-Prinzip erörtert.

91: Vgl. WEINSTEIN, M.C. et al. (1980), S. 105ff.

92: Dies deutet darauf hin, daß neben den Kennziffern Sensitivităt und Spezifität auch die beiden Likelihood-Quotienten zur Charakterisierung einer diagnostischen Maßnahme verwendet werden können. 
Für den Likelihood-Quotienten $L\left(y_{j 1}\right)$ eines positiven Testergebnisses gilt:

$$
L\left(y_{j 1}\right)=\frac{P\left(y_{j 1} \mid K_{1}\right)}{P\left(y_{j 1} \mid \bar{K}_{1}\right)} \text {. }
$$

Analog entspricht der Likelihood-Quotient $\mathrm{L}\left(\mathrm{y}_{\mathrm{j} 2}\right)$ eines negativen Testergebnisses:

$$
L\left(y_{j 2}\right)=\frac{P\left(y_{j 2} \mid K_{1}\right)}{P\left(y_{j 2} \mid \bar{K}_{1}\right)} \text {. }
$$

Mit diesen Definitionsgleichungen ergeben sich für die Bestimmung prädiktiver Werte über Likelihood-Quotienten folgende Beziehungen:

$$
\begin{aligned}
& P\left(K_{1} \mid y_{j 1}\right)=\frac{L\left(y_{j 1}\right) \cdot P\left(K_{1}\right)}{L\left(y_{j 1}\right) \cdot P\left(K_{1}\right)+P\left(\bar{K}_{1}\right)}, \\
& P\left(\bar{K}_{1} \mid y_{j 2}\right)=\frac{P\left(\bar{K}_{1}\right)}{L\left(y_{j 2}\right) \cdot P\left(K_{1}\right)+P\left(\bar{K}_{1}\right)} .
\end{aligned}
$$

Eine diagnostische Maßnahme liefert keine neue Information, wenn der Informationsstand des Arztes durch ihren Einsatz nicht verändert wird. Dies bedeutet, da $B$ in 
diesem Fall die posterioren Wahrscheinlichkeitsverteilungen gegeben ein positives bzw. ein negatives Testergebnis jeweils mit der prioren Verteilung übereinstimmen ${ }^{93}$. Die Gleichungen (3.13) und (3.14) zeigen, daß dieser Fall nur bei einem LikelihoodQuotienten von Eins auftreten kann [wobei $L\left(y_{j 1}\right)=1$ auch $L\left(y_{j 2}\right)=1$ impliziert und umgekehrt].

Diese Überlegungen deuten darauf hin, daß die Informationsleistung eines Diagnosetests umso größer ausfällt, je stärker die posterioren von den anfänglichen (prioren) Wahrscheinlichkeiten abweichen. Die Analyse der Gleichungen (3.13) und (3.14) führt zu folgenden Schlüssen: ein vergleichsweise hoher positiver prädiktiver Wert ergibt sich für $\mathrm{L}\left(\mathrm{y}_{\mathrm{j} 1}\right)$ deutlich größer Eins ${ }^{94}$, während umgekehrt zur Erzielung eines brauchbaren prädiktiven Werts negativer Testergebnisse der Likelihood-Quotient $L\left(y_{j 2}\right)$ wesentlich niedriger als Eins ausfallen sollte ${ }^{95}$. Auf diese Weise vermögen die Werte der Likelihood-Quotienten wichtige Hinweise auf die Diskriminierungsfähigkeit einer diagnostischen Maßnahme zu geben.

Betrachtet man die Bestimmungsgleichungen (3.7) und (3.10) für die prädiktiven Werte, dann lassen sich auch für diese Darstellung Bedingungen formulieren, unter denen die posterioren Wahrscheinlichkeiten größer ausfallen als die jeweils a priori unterstellten ${ }^{96}$. Damit beispielsweise der prädiktive Wert eines positiven Tests größer ausfällt als $\mathrm{P}\left(\mathrm{K}_{1}\right)$, muß erfüllt sein ${ }^{97}$ :

$$
P\left(y_{j 1} \mid K_{1}\right)>P\left(y_{j 1} \mid \bar{K}_{1}\right)
$$

93: Wie mit Hilfe der Informationstheorie gezeigt werden kann, bildet diese Situation zugleich den einzigen Fall, in dem eine diagnostische Maßnahme keinen Informationsgehalt aufweist; vgl. dazu Abschnitt 3.6.

94: Der positive prădiktive Wert ist steigend in $L\left(y_{j 1}\right)$. Schechter und Sheps bezeichnen Werte über 10,0 als gut; vgl. SCHECHTER, M.T./SHEPS, S.B. (1985), S. 757.

95: Der negative prädiktive Wert ist fallend in $L\left(y_{j 2}\right)$. Diese Aussagen gelten ceteris paribus, d.h. für eine vorgegebene priore Wahrscheinlichkeit $\mathrm{P}\left(\mathrm{K}_{1}\right)$ des durch den Diagnosetest zu erkennenden Krankheitszustands $\mathrm{K}_{1}$.

96: Dabei erscheint es sinnvoll, nur jene Testergebnisse als positiv (im Hinblick auf den Krankheitszustand $\mathrm{K}_{1}$ ) zu bezeichnen, die unter der Hypothese $\mathrm{K}_{1}$ eine mindestens ebenso hohe Likelihood aufweisen wie unter der Alternative $\overline{\mathrm{K}}_{1}$. Bei Befolgung dieser Regel können die prädiktiven Werte niemals geringer ausfallen als die entsprechenden prioren Wahrscheinlichkeiten.

97: Wie nicht anders zu erwarten, ist diese Bedingung äquivalent zu der Forderung eines Likelihood-Quotienten größer Eins. 
Die Wahrscheinlichkeit eines positiven Testresultats sollte also bei einem Individuum mit der Krankheit $\mathrm{K}_{1}$ höher ausfallen als bei Individuen, für die das Ereignis $\overline{\mathrm{K}}_{1} \mathrm{zu}$ trifft. Damit der prädiktive Wert eines negativen Tests $\mathrm{P}\left(\overline{\mathrm{K}}_{1}\right)$ übersteigt, muß andererseits gelten:

$$
P\left(y_{j 2} \mid \bar{K}_{1}\right)>P\left(y_{j 2} \mid K_{1}\right)
$$

Die formale Äquivalenz der Bedingungen (3.15) und (3.16) verlangt in beiden Fällen, $\mathrm{da} ß$ die Sensitivität der diagnostischen Maßnahme die Größe Eins abzüglich der Spezifität übersteigt. Diese Bedingung dürfte bei allen praktischen Anwendungen erfüllt $\operatorname{sein}^{98}$. Eine diagnostische Maßnahme liefert auf keinen Fall neue Information, wenn die Summe aus Sensitivität und Spezifität gerade Eins beträgt.

Die Veränderung der Informationslage des Arztes durch den Einsatz einer diagnostischen Maßnahme läßt sich vollständig beschreiben, indem man die posterioren mit den a priori vorliegenden Wahrscheinlichkeiten der in Betracht kommenden Krankheitszustände vergleicht. Mit Bezug auf ein homogenes Patientenkollektiv 99 bedeutet dies: ein binarisierter Diagnosetest, der die oben angesprochene Minimalanforderung erfüllt, leistet durch seine Resultate eine Aufteilung dieser Population in zwei Gruppen. Innerhalb der Gruppe der positiven Testergebnisse hat sich die Chance erhöht, mit der Krankheit $\mathrm{K}_{1}$ behaftete Individuen aufzufinden, während in der anderen Gruppe mit einer im Vergleich zur Ausgangslage erhöhten Wahrscheinlichkeit Individuen im Krankheitszustand $\bar{K}_{1}$ anzutreffen sind ${ }^{100}$.

\subsubsection{Graphische Analyse}

Die Veränderung der Informationslage des Arztes durch einen binarisierten Diagnosetest kann auch graphisch veranschaulicht werden. Bei einer derartigen diagnostischen Maßnahme erhält man für jedes der beiden möglichen Resultate einen typischen funktionalen Zusammenhang zwischen der posterioren und der prioren

98: Falls nämlich die Sensitivität kleiner als Eins abzüglich der Spezifität ist, wird man eine Umbenennung positiver und negativer Testergebnisse vornehmen.

99: Es handelt sich dabei um Fälle, für die vor der Anwendung eines Diagnosetests aufgrund der verfügbaren Informationen dieselbe oder eine nahezu identische Krankheitswahrscheinlichkeit angenommen werden kann.

100: Dabei bleibt an dieser Stelle offen, inwiefern diese (verbesserte) Aufteilung des Patientenkollektivs tatsächlich einen Informationsgewinn repräsentiert; vgl. dazu Abschnitt 3.6. 
Wahrscheinlichkeit eines Krankheitszustands. Dies sei im folgenden zunächst am Beispiel des positiven prädiktiven Werts illustriert. Da Sensitivität und Spezifität bei fester Binarisierung als Parameter anzusehen sind, gibt Gleichung (3.7) für einen Diagnosetest den positiven prädiktiven Wert als Funktion der prioren Wahrscheinlichkeit $P\left(K_{1}\right)$ an ${ }^{101}$. Es läßt sich zeigen, daß $P\left(K_{1} \mid y_{j 1}\right)$ in fast allen Fällen eine streng konkave Funktion und steigend in $P\left(K_{1}\right)$ ist ${ }^{102}$. Diesen Zusammenhang verdeutlicht Abbildung 9 anhand eines Diagramms.

Abb. 9: Die Abhängigkeit ${ }^{103}$ des positiven prädiktiven Werts von $P\left(K_{1}\right)$

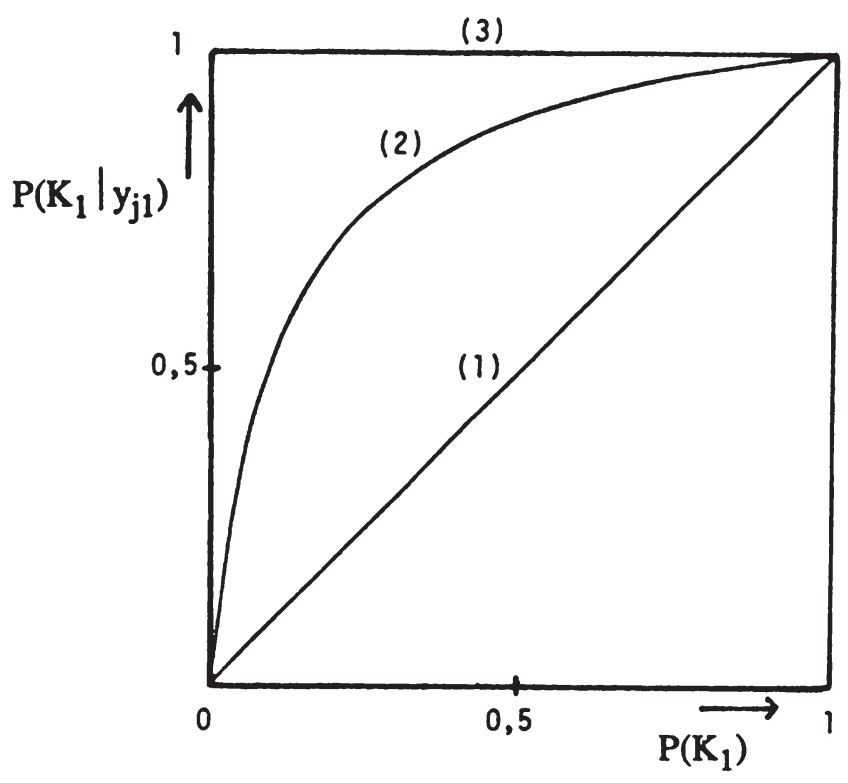

101: $\mathrm{P}\left(\overline{\mathrm{K}}_{1}\right)$ läßt sich durch $1-\mathrm{P}\left(\mathrm{K}_{1}\right)$ ersetzen.

102: Beide Aussagen gelten nicht, wenn die Spezifität Eins beträgt. Zusätzlich geht die Konkavitätseigenschaft verloren, falls die Summe der beiden Kennziffern Eins ergibt; vgl. dazu auch Abbildung 9.

103: Die Darstellung folgt weitgehend SOX, H.C. (1986), S. 62. 
Das Diagramm enthält drei Funktionsverläufe, die jeweils verschiedene Fälle repräsentieren. Die Diagonale [Funktionsverlauf (1)] stellt den Extremfall einer diagnostischen Maßnahme ohne Informationsleistung dar: die Resultate einer derartigen diagnostischen $\mathrm{Maßnahme}$ beeinflussen die Wahrscheinlichkeit des Krankheitszustands $\mathrm{K}_{1}$ überhaupt nicht. Wie zuvor nachgewiesen, muß die Summe der Kennziffern dieses Tests Eins betragen.

Im Normalfall unterscheiden sich jedoch die posterioren von den a priori vorliegenden Wahrscheinlichkeiten der Krankheitszustände. So gilt Funktion (2) für eine diagnostische Maßnahme, deren Sensitivität und Spezifität jeweils 0,9 betragen. Wie die Abbildung belegt, ergeben sich für verschiedene medizinische Ausgangslagen jeweils repräsentiert durch eine bestimmte priore Wahrscheinlichkeit $P\left(K_{1}\right)$ - unterschiedliche Veränderungen der Informationslage bei diesem Testergebnis ${ }^{104}$. Diese Veränderung wird gemessen durch den vertikalen Abstand zwischen dem Funktionswert und der Diagonalen.

Der in Bezug auf den positiven prädiktiven Wert günstigste Fall tritt ein, wenn eine diagnostische Maßnahme eine Spezifität von Eins aufweist (bei beliebiger Sensitivität ${ }^{105}$ ). Ein positives Testergebnis können dann nur Patienten im Krankheitszustand $\mathrm{K}_{1}$ erzielen mit der Folge, daß dieses diagnostische Resultat fehlerfrei den Rückschluß auf $\mathrm{K}_{1}$ erlaubt.

Ganz ähnlich besteht bei einer diagnostischen Maßnahme ein funktionaler Zusammenhang zwischen dem negativen prädiktiven Wert $P\left(\bar{K}_{1} \mid y_{j 2}\right)$ und der prioren Wahrscheinlichkeit $\mathrm{P}\left(\overline{\mathrm{K}}_{1}\right)$. Auch hier läßt sich nachweisen, daß der negative prädiktive Wert eine streng konkave Funktion und steigend in $\mathrm{P}\left(\overline{\mathrm{K}}_{1}\right)$ ist ${ }^{106}$. Zur Dar-

104: Bei gegebenen Testkennziffem spielt somit die priore Wahrscheinlichkeit $P\left(K_{1}\right)$ eine entscheidende Rolle für die Veränderung der Informationslage; vgl. CHIANG, C.L/HODGES, J.L./YERUSHALMY, J. (1956), S. 124.

105: Die Sensitivität muß lediglich positiv sein, da sonst ein Diagnosetest ohne Informationsleistung vorliegt.

106: Aufgrund der strukturellen Ähnlichkeit der Bestimmungsgleichungen für die beiden prädiktiven Werte - man erhält jeweils die andere Gleichung, wenn man das Testergebnis ersetzt und $\mathrm{K}_{1}$ und $\overline{\mathrm{K}}_{1}$ vertauscht - ergeben sich dieselben qualitativen Eigenschaften für den hier untersuchten Zusammenhang. Allerdings gelten nun die beiden im Text angesprochenen Aussagen nicht, wenn die Sensitivität Eins beträgt; wie zuvor geht die strenge Konkavität verloren, wenn die Summe beider Kennziffern Eins beträgt. 
stellung dieses Zusammenhangs mit Hilfe des in Abbildung 9 eingeführten Diagramms dient das noch freie Dreieck "unterhalb" der Diagonalen. Der negative prädiktive Wert wird vom neuen Ursprung $\mathrm{O}^{\prime}$ ausgehend nach unten abgetragen, während $P\left(\bar{K}_{1}\right)$ auf der Abszisse nach links gemessen wird ${ }^{107}$.

Abb. 10: Die Abhängigkeit des negativen prädiktiven Werts von $\mathrm{P}\left(\overline{\mathrm{K}}_{1}\right)$

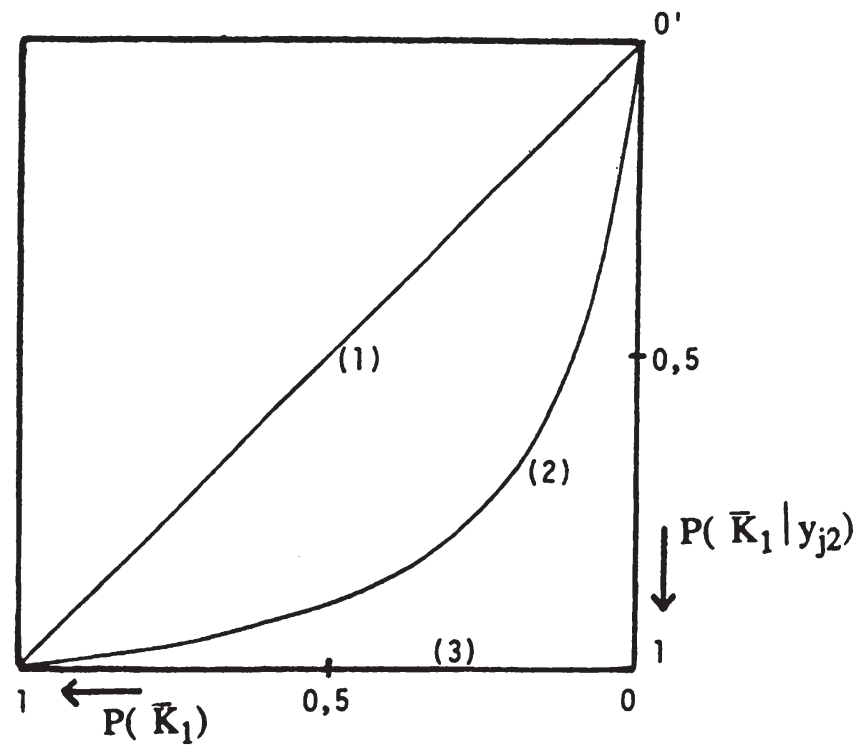

Auch in Abbildung 10 sind drei verschiedene diagnostische Maßnahmen jeweils durch einen Funktionsverlauf dargestellt. Wegen der großen Ähnlichkeit zur vorhergehenden Abbildung reicht es aus, diese drei Fälle in knapper Form anzusprechen. Funktionsverlauf (1) stellt wiederum eine diagnostische Maßnahme ohne

107: Aufgrund der Additivitätsbedingung für die Elemente einer Wahrscheinlichkeitsverteilung lăßt sich das Diagramm auch vom Ursprung $O$ aus lesen: auf der Ordinate wird dann die zum negativen prädiktiven Wert komplementäre Wahrscheinlichkeit $P\left(K_{1} \mid y_{j 2}\right)$ abgetragen, während auf der $A$ bszisse wie zuvor $P\left(K_{1}\right)$ gemessen wird. 
Informationsleistung dar, da der negative prädiktive Wert sich nicht von der betreffenden prioren Wahrscheinlichkeit unterscheidet. Funktion (2) repräsentiert den Diagnosetest, dessen Kennziffern jeweils 0,9 betragen. Falls der Arzt den Krankheitszustand des Patienten nicht mit Sicherheit kennt, fällt der negative prädiktive Wert höher als die priore Wahrscheinlichkeit von $\overline{\mathrm{K}}_{1}$ aus. Schließlich steht der dritte Funktionsverlauf für eine diagnostische Maßnahme mit positiver Spezifität, deren Sensitivität Eins beträgt. Dann ergibt sich für positive Wahrscheinlichkeiten $\mathrm{P}\left(\overline{\mathrm{K}}_{1}\right)$ stets ein negativer prädiktiver Wert von Eins.

Fügt man diese beiden Darstellungen in einer Abbildung zusammen, so erhält man einen guten Überblick über die Informationsleistung einer diagnostischen Maßnahme bei fester Binarisierung und damit bei gegebenen Testkennziffern. Zu jeder Maßnahme gehören zwei Funktionen, von denen eine den positiven, die andere den negativen prädiktiven Wert in Abhängigkeit von der prioren Wahrscheinlichkeit des Krankheitszustands $K_{1}$ angibt ${ }^{108}$. Wie durch die Berücksichtigung verschiedener Funktionsverläufe bereits angedeutet, lassen sich mit diesem graphischen Hilfsmittel mehrere Diagnosetests vergleichen ${ }^{109}$.

Abbildung 11 veranschaulicht diese Möglichkeit, wobei (3) einen Diagnosetest bezeichnet, dessen Sensitivität und Spezifität jeweils Eins betragen. Sowohl positive als auch negative Testergebnisse zeigen ohne Fehler den tatsächlich vorliegenden Krankheitszustand an, so daß die beiden Funktionen zusammen eine perfekte diagnostische Maßnahme repräsentieren. Im Gegensatz dazu stellt die Diagonale (1) den anderen Extremfall eines binarisierten Tests ohne Informationsleistung dar. Liegen Sensitivität und Spezifität jeweils unter Eins, ihre Summe jedoch darüber, dann wird eine diagnostische Maßnahme in diesem Diagramm durch zwei Funktionen dargestellt, die zwischen den beiden zuvor angesprochenen Extremfällen liegen. Die beiden mit (2) bezeichneten Funktionen verdeutlichen diesen Tatbestand unter der Voraussetzung, daß beide diagnostischen Kennziffern jeweils 0,9 betragen.

108: Eine Ausnahme bildet lediglich die diagnostische Maßnahme ohne Informationsleistung, bei der die beiden Funktionen zusammenfallen.

109: Ebenso erhält man für eine einzelne diagnostische Maßnahme bei beiden prädiktiven Werten jeweils eine Familie von Funktionen, wenn man den Einfluß der Testeinstellung auf die diagnostischen Kennziffern und damit die Resultate der ROC-Analyse berücksichtigt; vgl. dazu Abschnitt 3.2.3 sowie den umfangreichen Tabellenanhang bei GALEN, R.S./GAMBINO, S.R. (1979), S. 126ff. Bei isolierter Variation einer Kennziffer zeigt sich, daß die Sensitivität stärker den negativen, die Spezifität hingegen stärker den positiven prädiktiven Wert beeinflußt; vgl. SOX, H.C. (1986), S. 63. 
Abb. 11: Die Informationsleistung binarisierter diagnostischer Maßnahmen

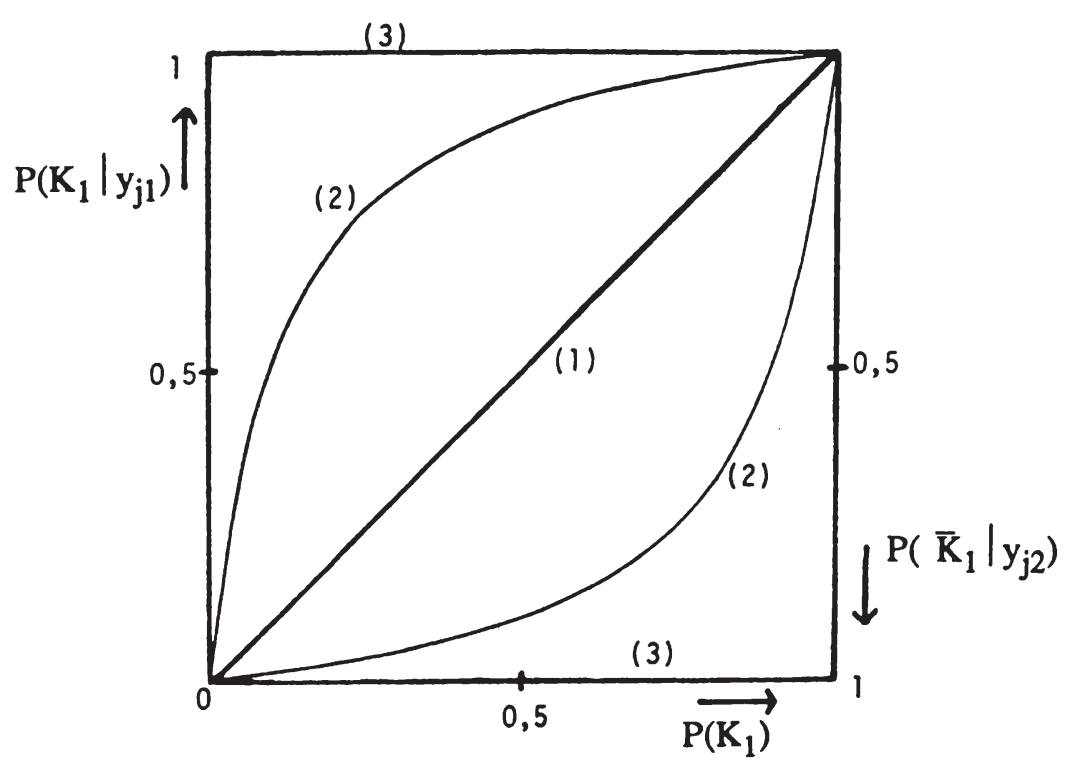

\subsection{Die Informationsleistung diagnostischer Maßnahmen im allgemeinen Fall ${ }^{110}$}

Häufig fallen unter das Ereignis $\overline{\mathrm{K}}_{1}$ mehrere, vom Arzt als möglich erachtete Krankheitszustände. Insbesondere bei einem negativen Testergebnis interessiert man sich dann weniger für das zusammengesetzte Ereignis $\overline{\mathrm{K}}_{1}$ als für die Bedeutung dieses diagnostischen Resultats im Hinblick auf die alternativen Krankheitshypothesen $\mathrm{K}_{\mathrm{i}}(\mathrm{i}=2, \ldots, \mathrm{n})$ im einzelnen. Dabei geht es in einer gegebenen Entscheidungssituation

110: Damit ist die in Abschnitt 2.4 skizzierte Ausgangslage gemeint. Keineswegs handelt es sich dabei um den allgemeinen Fall im Sinne der Entscheidungstheorie, in dem etwa bezüglict der betrachteten Mengen wesentlich schwächere Voraussetzungen genügen; vgl. z.B. FERGUSON, T.S. (1967), S. $6 \mathrm{ff}$. 
nur um jene Krankheitszustände, die aufgrund der zur Verfügung stehenden Information in Betracht kommen, d.h. denen der Arzt eine positive priore Wahrscheinlichkeit beimißt.

Ebenso wird nun die in den vorangegangenen Abschnitten verwendete Annahme eines binarisierten Diagnosetests aufgehoben. Eine typische diagnostische Maßnahme $\mathrm{T}_{\mathrm{j}}$ ist dann dadurch gekennzeichnet, daß ihr Einsatz genau eines aus einer endlichen Menge diagnostischer Resultate liefert. Unter den in Abschnitt 2.4 genannten Voraussetzungen führt jedes dieser Resultate, das in der betrachteten Entscheidungssituation eine positive Wahrscheinlichkeit besitzt, zu einer Transformation der prioren Wahrscheinlichkeiten für die Krankheitszustände. Diese Transformation ergibt sich mit Hilfe des Bayes-Theorems wie folgt:

$$
\begin{aligned}
& P\left(K_{k} \mid z_{j h}\right)=\frac{P\left(z_{j h} \mid K_{k}\right) \cdot P\left(K_{k}\right)}{P\left(z_{j h}\right)} ; P\left(z_{j h}\right)>0, \\
&=\frac{P\left(z_{j h} \mid K_{k}\right) \cdot P\left(K_{k}\right)}{\sum_{i=1}^{n} P\left(z_{j h} \mid K_{i}\right) \cdot P\left(K_{i}\right)} . \\
& k=1, \ldots, n ; \quad h=1, \ldots, k_{j} ; \quad j=1, \ldots, m .
\end{aligned}
$$

Diese Gleichungen zeigen zunächst für feste Werte der Indizes $h$ und $j$ jeweils die Wahrscheinlichkeiten der einzelnen Krankheitszustände an, nachdem das Testergebnis $\mathrm{z}_{\mathrm{jh}}$ beobachtet wurde 111 . Gemeinsam bilden diese bedingten Wahrscheinlichkeiten die posteriore Verteilung auf der Menge der Krankheitszustände gegeben $z_{\text {jh }}$. Bei variablem Index h lassen sich mit Hilfe der Beziehungen (3.17) die posterioren Wahrscheinlichkeiten für jedes mögliche Testergebnis der diagnostischen Maßnahme $\mathrm{T}_{\mathrm{j}}$ bestimmen. Da dieses Verfahren bei jeder diagnostischen Maßnahme Anwendung finden kann, geben die Gleichungen (3.17) bzw. (3.17a) an, in welcher Weise in einer beliebigen 112 medizinischen Ausgangslage die prioren Wahrscheinlichkeiten der einzelnen Krankheitszustände zu transformieren sind, falls der Arzt weitere diagnostische Informationen anfordert.

111: Vgl. z.B. JACQUEZ, J.A. (1972), S. $375 f f$.

112: Wobei allerdings die in Abschnitt 2.4 angegebenen Voraussetzungen erfültt sein müssen. 
Der Einsatz einer diagnostischen Maßnahme $\mathrm{T}_{\mathrm{j}}$ entspricht damit einem Zufallsexperiment mit endlich vielen Ausgängen, dessen Ergebnis darüber entscheidet, welche posteriore Wahrscheinlichkeitsverteilung für die eigentlich interessierenden Ereignisse $\mathrm{K}_{\mathrm{i}}(\mathrm{i}=1, \ldots, \mathrm{n}) \mathrm{zu}$ verwenden ist. Die Veränderung der Informationslage, die ein bestimmtes diagnostisches Resultat bewirkt, läßt sich beschreiben, indem man den prioren Wahrscheinlichkeiten der Krankheitszustände die posteriore Verteilung unter der Bedingung dieses Resultats gegenüberstellt. Führt man diesen Vergleich für jedes mögliche Testergebnis durch, so erhält man eine vollständige Darstellung der Informationsleistung der betreffenden diagnostischen Maßnahme. Dabei hängt diese Informationsleistung im allgemeinen von der medizinischen Ausgangslage, d.h. von der prioren Wahrscheinlichkeitsverteilung auf der Menge der Krankheitszustände, ab.

Leider kann die in Abschnitt 3.3.2 benutzte graphische Darstellung in dem hier diskutierten, allgemeinen Fall nicht verwendet werden ${ }^{113}$. Insofern muß man zur Illustration auf die Matrix $\mathrm{P}_{\mathrm{j}}$ der bedingten Wahrscheinlichkeiten der Testergebnisse zurückgreifen, die eine diagnostische $\mathrm{Maßnahme} \mathrm{charakterisiert.} \mathrm{Mit} \mathrm{der} \mathrm{Hilfe} \mathrm{dieser}$ Matrix lassen sich insgesamt drei verschiedene Fälle unterscheiden, die zur Klassifikation diagnostischer Maßnahmen anhand ihrer Informationsleistung dienen können.

Die Überlegungen des Abschnitts 3.3 legen es nahe, einen Diagnosetest gerade dann als diagnostische Maßnahme ohne Informationsleistung zu bezeichnen, wenn für jedes Testergebnis die posteriore Wahrscheinlichkeitsverteilung auf der Menge der Krankheitszustände mit der prioren übereinstimmt. Analysiert man die Gleichungen (3.17a), so liegt dieser Spezialfall offenbar genau dann vor, wenn für eine diagnostische Maßnahme $T_{j}$ gilt:

$$
P\left(z_{j h} \mid K_{k}\right)=P\left(z_{j h}\right) ; \quad k=1, \ldots, n ; h=1, \ldots, k_{j}
$$

113: Falls lediglich drei bzw. vier Krankheitszustände zu unterscheiden sind, existiert für eine gegebene medizinische Ausgangslage in Gestalt einer prioren Wahrscheinlichkeitsverteilung die Möglichkeit einer graphischen Darstellung. Ebenso wie die priore lassen sich auch die posterioren Verteilungen aufgrund der einzelnen Testergebnisse als Punkte in einem Dreieck bzw. einem Tetraeder repräsentieren; vgl. MARSCHAK, J./MIYASAWA, K. (1968), S. 226; BISHOP, Y.M.M./FIENBERG, S.E./HOLLAND, P.W. (1975), S. 50ff. sowie HABBEMA, J.D.F./HILDEN, J./BJERREGAARD, B. (1978), S. $221 \mathrm{ff}$. 
Diese Bedingungen sind äquivalent zu:

$$
\mathrm{P}\left(\mathrm{z}_{\mathrm{jh}} \mid \mathrm{K}_{\mathrm{k}}\right)=\mathrm{P}\left(\mathrm{z}_{\mathrm{jh}} \mid \mathrm{K}_{\mathrm{m}}\right) ; \mathrm{k}, \mathrm{m}=1, \ldots, \mathrm{n} ; \mathrm{h}=1, \ldots, \mathrm{k}_{\mathrm{j}}
$$

Eine diagnostische Maßnahme erbringt demzufolge keine Informationsleistung, wenn für jedes mögliche Testergebnis die dazugehörigen Likelihoods sämtlicher Krankheitszustände jeweils übereinstimmen. Dies bedeutet, da $B$ die bedingten Wahrscheinlichkeiten der Testergebnisse keine Abhängigkeit von den Krankheitszuständen aufweisen. Die Spalten der Matrix $P_{j}$ sind identisch, so daß eine diagnostische Maßnahme ohne Informationsleistung durch eine singuläre Matrix vom Rang Eins charakterisiert wird. In der entscheidungstheoretischen Literatur bezeichnet man ein derartiges Informationsinstrument aus naheliegenden Gründen als Null-Informationssystem ${ }^{114}$.

Den umgekehrten Extremfall stellt eine perfekte diagnostische Maßnahme dar, die den Arzt anhand ihrer Testergebnisse immer mit Sicherheit über den tatsächlichen Krankheitszustand des Patienten informiert. Dies impliziert, da $B$ jedes mögliche Resultat eines derartigen Diagnosetests zu einer degenerierten posterioren Wahrscheinlichkeitsverteilung auf der Menge der in Betracht gezogenen Krankheitszustände führt. Aus den Gleichungen (3.17a) lassen sich Bedingungen ableiten, die eine perfekte diagnostische Maßnahme charakterisieren. Für jeden möglichen Krankheitszustand $\mathrm{K}_{\mathrm{k}}$ impliziert die Ungleichung

$$
\mathrm{P}\left(\mathrm{z}_{\mathrm{jh}} \mid \mathrm{K}_{\mathrm{k}}\right)>0
$$

in diesem Fall:

$$
\mathrm{P}\left(\mathrm{z}_{\mathrm{jh}} \mid \mathrm{K}_{\mathrm{i}}\right)=0 \quad \forall \mathrm{i} \neq \mathrm{k} ; \mathrm{h}=1, \ldots, \mathrm{k}_{\mathrm{j}}
$$

Eine diagnostische Maßnahme informiert genau dann vollständig über den bei einem Patienten vorliegenden Krankheitszustand, wenn für jedes mögliche Resultat gerade ein Likelihood-Wert positiv ist und die übrigen Null betragen. Dies bedeutet, daß je-

114: Vgl. z.B. FIRCHAU, V. (1980), S. 33. 
des dieser Resultate überhaupt nur bei einem einzigen Krankheitszustand auftreten $k_{a n n}{ }^{115}$. Insofern muß bei einer perfekten diagnostischen Maßnahme $T_{j}$ notwendig die Bedingung $\mathrm{k}_{\mathrm{j}} \geq \mathrm{n}$ erfüllt sein, d.h. die Anzahl der Testergebnisse unterschreitet die Zahl der in Betracht kommenden Krankheitszustände nicht. Die dazugehörige Matrix $P_{j}$ besitzt den vollen Spaltenrang und enthält in jeder Zeile lediglich ein Element, das von Null verschieden ist. Terminologisch entspricht dem in der Entscheidungstheorie der Begriff des vollständigen bzw. vollkommenen Informationssystems.

Faßt man bei einer perfekten diagnostischen Maßnahme jeweils diejenigen Resultate zusammen, die denselben Krankheitszustand "anzeigen", so ergibt sich daraus eine umkehrbar eindeutige Beziehung zwischen Krankheitszuständen einerseits und den in dieser Weise aggregierten Testergebnissen andererseits. In dieser modifizierten Form handelt es sich um einen perfekten Diagnosetest, dessen Matrix $P_{j}$ regulär ist und deren Elemente entweder Null oder Eins betragen ${ }^{116}$. Eine perfekte diagnostische Maßnahme stellt in diesem Fall zugleich ein ungestörtes Informationssystem dar, das jedem Krankheitszustand eindeutig ein Testergebnis zuordnet ${ }^{117}$.

Im Normalfall wird eine diagnostische Maßnahme allerdings keinen der beiden zuvor diskutierten Spezialfälle repräsentieren. Unter dieser Voraussetzung führt zumindest ein mögliches Testergebnis zu einer Modifikation der prioren Wahrscheinlichkeiten, ohne jedoch den tatsächlich vorliegenden Krankheitszustand mit Sicherheit anzuzeigen. Die zu einer derartigen diagnostischen Maßnahme gehörende Matrix $P_{j}$ der bedingten Wahrscheinlichkeiten der Testergebnisse entstammt dann einer in Bezug auf ihre Ordnung geeignet definierten Menge spaltenstochastischer Matrizen, welche diejenigen Matrizen nicht enthält, die zu den oben angesprochenen

115: Der Fall, daß für ein Testergebnis sämtliche Likelihood-Werte Null sind, kann hier nicht auftreten, da es sich dann nicht mehr um ein mögliches Testergebnis handeln würde. Vgl. im statistischen Zusammenhang zur Charakterisierung möglicher Testergebnisse FIRCHAU, V. (1980), S. 9f.

116: Vgl. FIRCHAU, V. (1980), S. 33; BAMBERG, G./COENENBERG, A.G. (1989), S. $19 \mathrm{f}$.

117: Allgemein kennzeichnet ein ungestörtes Informationssystem eine Matrix $P_{j}$, deren Elemente sämtlich entweder Null oder Eins sind; vgl. FIRCHAU, V. (1980), S. 33 sowie BAMBERG, G./COENENBERG, A.G./KLEINE-DOEPKE, R. (1976), S. 37. Die Umkehrung dieser Aussage gilt hingegen nicht: im Extremfall kann ein ungestörtes Informationssystem sogar keinerlei Information erbringen, also eine diagnostische Maßnahme ohne Informationslei:ıung repräsentieren. 
Spezialfällen gehören. Die Elemente der auf diese Weise negativ definierten - in praxi wohl am häufigsten anzutreffenden - Klasse diagnostischer Maßnahmen bezeichnet man als unvollkommene Informationssysteme. Mit den Null-Informationssystemen, den vollkommenen und schließlich den unvollkommenen Informationssystemen sind damit drei verschiedene Klassen diagnostischer Maßnahmen erfaßt, die in einer medizinischen Entscheidungssituation auftreten können, welche den in Abschnitt 2.4 genannten Voraussetzungen genügt.

\subsection{Zur Verwendung des Bayes-Theorems in der medizinischen Diagnostik}

Das Bayes-Theorem spielt eine zentrale Rolle bei der Bestimmung der Veränderung der Informationslage des Arztes, die sich aufgrund der Resultate diagnostischer Maßnahmen ergibt. Da die mit der Hilfe dieses Theorems beschriebene Informationsleistung gleichzeitig den Ausgangspunkt für ökonomische Analysen darstellt, überträgt sich diese Bedeutung auch auf den Bereich von Kosten-Nutzen Überlegungen. Schon aus diesem Grund gilt es zu überprüfen, ob die Anwendung dieses Theorems in der medizinischen Diagnostik zu korrekten Ergebnissen führt. In der Literatur werden in diesem Zusammenhang vornehmlich zwei Einwände diskutiert.

Ein erster Einwand nimmt Bezug auf die Beobachtung, daß Patienten nicht nur jeweils eine Krankheit zu einem bestimmten Zeitpunkt besitzen, sondern auch mehrere Krankheiten gleichzeitig auftreten können. Damit entfalle die Voraussetzung eines einzigen zutreffenden Krankheitszustands beim Patienten und demzufolge sei die Anwendung des Theorems von Bayes nicht mehr möglich. Feinstein demonstriert diese Problematik der "multiple diseases" bzw. der Multimorbidität anhand eines Beispiels ${ }^{118}$, mit dem er fehlerhafte Resultate des Bayes-Ansatzes nachzuweisen versucht.

Dieser Einwand spricht jedoch lediglich gegen eine "unkritische" Verwendung des Bayes-Theorems. Die Voraussetzung einander paarweise ausschließender Krankheitszustände, die zudem noch die Menge $\mathrm{K}$ ausschöpfen sollen, kann bei geeigneter Formulierung des Zustandsraums auch im Falle der Multimorbidität noch erfüllt wer$\operatorname{den}^{119}$. Dazu definiert man wie folgt: einerseits $\mathrm{K}_{\mathrm{i}}\left(\mathrm{i}=1, \ldots, \mathrm{n}^{\prime}\right)$ als Möglichkeiten des isolierten Auftretens verschiedener Krankheitszustände und andererseits Ereignisse

118: Vgl. FEINSTEIN, A.R. (1977c), S. 488.

119: Vgl. dazu z.B. LUSTED, L.B. (1968), S. 16.; JACQUEZ, J.A. (1972), S. 375; GREMY, F./GOLDBERG, M. (1977), S. 426. 
$\mathrm{K}_{\mathrm{i}}\left(\mathrm{i}=\mathrm{n}^{\prime}+1, \ldots, \mathrm{n}\right)$, durch die das gemeinsame Auftreten zweier Krankheitszustände $\mathrm{K}_{\mathrm{j}}$ und $K_{k}$ bezeichnet wird ${ }^{120}$. Da bei einem Patienten entweder nur eine einzelne Krankheitshypothese oder ${ }^{121}$ die Vermutung einer Spielart der "multiple diseases" zutreffen kann, stellt diese erweiterte Liste von Ereignissen wiederum eine Partition des medizinischen Zustarıdsraums $\mathrm{K}$ dar.

Die möglichen Kombinationen von Krankheitszuständen sind demzufolge als zusätzliche Krankheitshypothesen aufzufassen, für die ebenfalls die in Abschnitt 2.4 genannten Voraussetzungen zutreffen müssen. Dies impliziert, da $B$ der Arzt auch für diese Ereignisse priore Wahrscheinlichkeiten angeben kann. Ferner müssen für alle zur Verfügung stehenden diagnostischen Maßnahmen die bedingten Verteilungen der Testergebnisse bekannt sein, die für die Elemente dieser erweiterten Liste von Krankheitszuständen gelten. Insofern ergibt sich im Falle einer medizinischen Ausgangslage, in der Multimorbidität nicht ausgeschlossen werden kann, zwar ein vergleichsweise höherer Informationsbedarf, der bisher verwendete Entscheidungsrahmen ändert sich jedoch nicht grundsätzlich. Deshalb sind die Fälle von Multimorbidität als eigenständige Krankheitszustände anzusehen, für die sämtliche bislang angestellten Überlegungen ihre Gültigkeit behalten ${ }^{122}$.

Der zweite Einwand bezieht sich auf eine bestimmte Vorgehensweise bei der Ermittlung der Veränderung der Informationslage, die aufgrund der Testergebnisse mehrerer diagnostischer Maßnahmen entsteht. Vielfach muß der Arzt mehrere Diagnosetests durchführen, um bei einem Patienten den tatsächlich vorliegenden Krankheitszustand befriedigend abzuklären. Sofern diese Tests sukzessive zum Einsatz gelangen, liegt es nahe, mit Hilfe des Bayes-Theorems die schrittweise erhaltenen diagnostischen Resultate in einer ganz bestimmten Weise heranzuziehen, um die damit verbundene Veränderung der Informationslage zu ermitteln ${ }^{123}$. Man bestimmt zunächst aufgrund des Resultats des ersten Diagnosetests die posterioren

120: Eine analoge Erweiterung kann - falls dies erforderlich sein sollte - vorgenommen werden, um das eventuell gleichzeitige Auftreten von drei oder mehr Krankheiten bzw. Krankheitszuständen zu berücksichtigen.

121: Hier im Sinne eines ausschließenden "oder".

122: Im folgenden werden deshalb diese möglichen Kombinationen von Krankheitszuständen ebenfalls als Krankheitszustände bezeichnet.

123: Diese Vorgehensweise empfehlen mehrere Autoren; vgl. z.B. GRINER, P.F. et al. (1981), S. 565f.; SCHECHTER, M.T./SHEPS, S.B. (1985), S. 759 sowie insbesondere DEPARTMENT OF CLINICAL EPIDEMIOLOGY AND BIOSTATISTICS (1983), S. $705 \mathrm{ff}$. 
Wahrscheinlichkeiten der in Betracht gezogenen Krankheitszustände. Diese dienen in einem zweiten Schritt als priore Wahrscheinlichkeiten, die ihrerseits durch das Ergebnis einer weiteren diagnostischen Maßnahme in eine posteriore Wahrscheinlichkeitsverteilung transformiert werden. Führt man dieses Verfahren fort, so folgt schließlich für die Wahrscheinlichkeiten der einzelnen Krankheitszustände gegeben die Resultate von $\mathrm{r}$ verschiedenen Diagnosetests ${ }^{124}$.

$$
\begin{aligned}
& P\left(K_{k} \mid z_{1 h_{1}}, \ldots, z_{r h_{r}}\right)=\frac{P\left(z_{r h_{r}} \mid K_{k}\right) \cdot \ldots \cdot P\left(z_{1 h_{1}} \mid K_{k}\right) \cdot P\left(K_{k}\right)}{\sum_{i=1}^{n} P\left(z_{r h_{r}} \mid K_{i}\right) \cdot \ldots \cdot P\left(z_{1 h_{1}} \mid K_{i}\right) \cdot P\left(K_{i}\right)} \\
&=\frac{P\left(K_{k}\right) \cdot \prod_{j=1}^{r} P\left(z_{j h_{j}} \mid K_{k}\right)}{\sum_{i=1}^{n} P\left(K_{i}\right) \cdot \prod_{j=1}^{r} P\left(z_{j h} \mid K_{i}\right)} . \\
& k=1, \ldots, n ; \quad h_{j} \in\left\{1, \ldots, k_{j}\right\} ; j=1, \ldots, r ; \quad r<m .
\end{aligned}
$$

Die soeben beschriebene Vorgehensweise wird z.B. implizit angewendet, wenn in einer medizinischen Entscheidungssituation Wahrscheinlichkeiten der Krankheitszustände vorliegen, die schon diagnostische Informationen berücksichtigen. Dabei handelt es sich um Wahrscheinlichkeiten, die als Bedingung die Resultate anderer, zuvor eingesetzter, diagnostischer Maßnahmen enthalten ${ }^{125}$. Im folgenden wird angenommen, daß "bisher" $\mathrm{p}-1$ Diagnosetests durchgeführt wurden, wobei $2 \leq \mathrm{p}<\mathrm{m}$ erfüllt sein soll. Verwendet man diese Wahrscheinlichkeiten als priore Wahrscheinlichkeiten, um aufgrund des Testergebnisses einer weiteren diagnostischen Maßnahme die Veränderung der Informationslage zu bestimmen, so ergibt sich:

124: Der Einfachheit halber sind die Diagnosetests in der Reihenfolge ihrer Anwendung numeriert. 125: In der Regel werden diese Bedingungen nicht explizit aufgeführt, sondern weiterhin die Bezeichnungen $P\left(K_{i}\right) ; i=1, \ldots, n$ verwendet. 


$$
\begin{gathered}
P\left(K_{k} \mid z_{1 h_{1}}, \ldots, z_{p h}\right)=\frac{P\left(z_{p h} \mid K_{k}\right) \cdot P\left(K_{k} \mid z_{1 h_{1}}, \ldots, z_{p-1, h_{p-1}}\right)}{\sum_{i=1}^{n} P\left(z_{p h} \mid K_{i}\right) \cdot P\left(K_{i} \mid z_{1 h_{1}}, \ldots, z_{p-1, h_{p-1}}\right)} \\
k=1, \ldots, n ; h_{j} \in\left\{1, \ldots, k_{j}\right\} ; j=1, \ldots, p .
\end{gathered}
$$

Es läßt sich jedoch zeigen, daß die in den Gleichungen (3.21) bzw. (3.22) dargestellte Vorgehensweise keineswegs zu korrekten Ergebnissen führen muß ${ }^{126}$. Um dies zu verdeutlichen, betrachtet man eine zusammengesetzte diagnostische Maßnahme, die aus sämtlichen, zuvor einzeln durchgeführten, Diagnosetests besteht. Die in dieser Situation relevanten prioren Wahrscheinlichkeiten der Krankheitszustände enthalten noch keinerlei diagnostische Information, d.h. sie verkörpern lediglich allgemeine medizinische Evidenz beispielsweise in Form von altersgruppenspezifischen Prävalenzdaten ${ }^{127}$. Gemäß den in Abschnitt 2.4 genannten Voraussetzungen erhält man für die posterioren Wahrscheinlichkeiten der Krankheitszustände:

$$
\begin{gathered}
P\left(K_{k} \mid z_{1 h_{1}}, \ldots, z_{r h_{r}}\right)=\frac{P\left(z_{1 h_{1}, \ldots,} z_{r h_{r}} \mid K_{k}\right) \cdot P\left(K_{k}\right)}{\sum_{i=1}^{n} P\left(z_{1 h_{1}}, \ldots, z_{r h_{r}} \mid K_{i}\right) \cdot P\left(K_{i}\right)} . \\
k=1, \ldots, n ; \quad r<m ; \quad h_{j} \in\left\{1, \ldots, k_{j}\right\} ; j=1, \ldots, r .
\end{gathered}
$$

Diese Gleichungen geben die korrekten posterioren Wahrscheinlichkeiten an, die sich unter der Bedingung der betrachteten Folge von Testergebnissen ergeben. Damit die weiter oben beschriebene Vorgehensweise zutreffende Resultate liefert, müssen offenbar die in den Gleichungen (3.21) ermittelten mit den in (3.23) bestimmten Wahr-

126: Falls die bedingten Wahrscheinlichkeiten der Krankheitszustände schrittweise nach dem zuerst beschriebenen Verfahren bestimmt wurden, sind beide Vorgehensweisen äquivalent.

127: Die Prävalenz einer Krankheit gibt den - auf einen Zeitpunkt und auf eine Bevölker'ngsgruppe bezogenen - Bestand von Erkrankungen an; vgl. z.B. GALEN, R.S./GAMBIN(?, S.R. (1979), S. 7f. 
scheinlichkeiten jeweils übereinstimmen. Diese Forderung ist auf jeden Fall erfüllt, falls gilt:

$$
\begin{aligned}
& P\left(z_{1 h_{1}}, \ldots, z_{r h_{r}} \mid K_{k}\right)=\prod_{j=1}^{r} P\left(z_{j h} \mid K_{k}\right) . \\
& k=1, \ldots, n ; \quad h_{j}=1, \ldots, k_{j} ; \quad j=1, \ldots, r .
\end{aligned}
$$

Die in den Gleichungen (3.24) angegebenen Bedingungen verlangen die bedingte Unabhängigkeit der Resultate aller betrachteten diagnostischen Maßnahmen, die kürzer als bedingte Unabhängigkeit aller diagnostischen Maßnahmen bezeichnet wird. Diese Eigenschaft liegt vor, wenn für jeden in Betracht gezogenen Krankheitszustand die Resultate sämtlicher diagnostischer Maßnahmen stochastisch unabhängig sind ${ }^{128}$.

Die bedingte Unabhängigkeit der Resultate verschiedener Diagnosetests darf nicht mit der stochastischen Unabhängigkeit von Testergebnissen und Krankheitszuständen verwechselt werden. Liegt diese Eigenschaft bei einem Diagnosetest vor, dann besitzt der jeweils vorliegende Krankheitszustand keinen Einfluß auf die Wahrscheinlichkeiten der Testergebnisse. In diesem Fall handelt es sich um einen Diagnosetest ohne Informationsleistung. Allgemein gilt: weder folgt aus der bedingten Unabhängigkeit der Testergebnisse deren stochastische Unabhängigkeit ${ }^{129}$ noch trifft die umgekehrte Implikation $\mathrm{zu}^{130}$.

Unter der Annahme der bedingten Unabhängigkeit sämtlicher Diagnosetests liefert die eingangs beschriebene Vorgehensweise korrekte Revisionen der prioren Wahrscheinlichkeiten gemäß den Gleichungen (3.21). Ebenso repräsentieren die Gleichungen (3.22) unter dieser Voraussetzung den Informationsstand des Arztes, indem sie

128: Vgl. MAI, N./HACHMANN, E. (1977), S. 165. Falls die Annahme der bedingten Unabhängigkeit nur für einige Krankheitszustände zutrifft, liefern die Gleichungen (3.21) ebenfalls fehlerhafte Ergebnisse; vgl. dazu das Beispiel von WEINSTEIN, M.C. et al. (1980), S. 156.

129: Vgl. PFANZAGL, J. (1988), S. 200. Dennoch sprechen nicht wenige Autoren unzutreffend von der Annahme der Unabhängigkeit der Symptome, wenn sie deren bedingte Unabhängigkeit meinen; vgl. z.B. BAILEY, N.T.J. (1967), S. 246; LUSTED, L.B. (1968), S. 16.

130: Vgl. SKENE, A. (1984), S. $143 \mathrm{f}$. 
die Wahrscheinlichkeiten der in Betracht gezogenen Krankheitszustände unter der Bedingung der vorhandenen Testergebnisse zutreffend angeben. Keineswegs jedoch handelt es sich bei der Annahme der bedingten Unabhängigkeit einzelner diagnostischer Maßnahmen um eine Voraussetzung, die zur Anwendung des Bayes-Theorems benötigt wird ${ }^{131}$. Insofern weist die Kritik an der oben dargestellten Vorgehensweise nur auf die Notwendigkeit hin, die Gültigkeit dieser Voraussetzung in der medizinischen Diagnostik zu überprüfen.

Die Attraktivität der Annahme der bedingten Unabhängigkeit diagnostischer Maßnahmen besteht darin, daß sie eine einfache und damit übersichtliche Bestimmung der sukzessiven Veränderung der Informationslage des Arztes ermöglicht. Die Kenntnis der in der Matrix $P_{j}$ zusammengestellten bedingten Wahrscheinlichkeiten reicht in diesem Fall aus, um das Ergebnis des Diagnosetests $T_{j}$ zur Ermittlung der posterioren Wahrscheinlichkeiten der Krankheitszustände heranzuziehen. Diese Aussage gilt unabhängig davon, an welcher Stelle des diagnostischen Prozesses der Arzt die betrachtete diagnostische Maßnahme einsetzt.

Es läßt sich zeigen, daß die in den Gleichungen (3.21) angegebenen Formeln zur Bestimmung posteriorer Wahrscheinlichkeiten auch dann gültig sein können, wenn die bedingte Unabhängigkeit der einzelnen diagnostischen Maßnahmen nicht vorliegt ${ }^{132}$. Insofern stellt die Annahme der bedingten Unabhängigkeit eine hinreichende Bedingung für die Gültigkeit des eingangs beschriebenen Vorgehens dar, die jedoch nicht notwendig ist. Aus diesem Grund reicht die Existenz bedingter Abhängigkeiten zwischen diagnostischen Maßnahmen per se noch nicht aus, die anhand der Gleichungen (3.21) ermittelten posterioren Wahrscheinlichkeiten der Krankheitszustände als falsch auszuweisen.

Ohne die Gültigkeit der in den Gleichungen (3.21) angegebenen Beziehungen gestaltet sich die Ermittlung posteriorer Wahrscheinlichkeiten schwieriger, da in diesem Fall die bedingten gemeinsamen Verteilungen der Testergebnisse bekannt sein müssen. Aus dieser Forderung resultiert ein wesentlich höherer Informationsbedarf. Zur Veranschaulichung dieser Aussage betrachte man einen bestimmten Krank-

131: Vgl. CORNFIELD, J. (1967), S. 40.

132: Vgl. HILDEN, J. (1984). 
heitszustand als Bedingung sowie $\mathbf{r}$ verschiedene diagnostische Maßnahmen. Unter der Annahme der Gültigkeit der in den Gleichungen (3.24) angegebenen Formeln reicht die Angabe von

$$
\sum_{j=1}^{r} k_{j}-r
$$

Wahrscheinlichkeiten aus, um die bedingte Wahrscheinlichkeit einer beliebigen Konstellation von Testergebnissen zu bestimmen. Ohne jede Information über die gemeinsame Verteilung der Testergebnisse benötigt man dazu hingegen

$$
\prod_{j=1}^{r} k_{j}-1
$$

Wahrscheinlichkeiten, eine für $r>1$ im allgemeinen wesentlich höhere Zahl ${ }^{133}$.

Ähnlich entscheidet die Gültigkeit der Gleichungen (3.22) darüber, in welchem Umfang das in den Abschnitten 3.3 und 3.4 dargestellte Verfahren herangezogen werden kann, um die Veränderung der Informationslage des Arztes zu ermitteln. Falls die Beziehungen (3.22) nicht erfüllt sind, liefert diese Vorgehensweise nur dann korrekte posteriore Wahrscheinlichkeiten für die möglichen Krankheitszustände des $\mathrm{Pa}$ tienten, wenn der Arzt die betrachtete diagnostische Maßnahme zu Beginn des diagnostischen Prozesses einsetzt. Enthalten die prioren Wahrscheinlichkeiten der Krankheitszustände hingegen zuvor erhobene diagnostische Informationen, dann benötigt man zur Bestimmung der mit einem Diagnosetest $T_{j}$ verbundenen Veränderung der Informationslage anstelle der Matrix $P_{j}$ die Wahrscheinlichkeiten der einzelnen Testergebnisse, die unter der zusätzlichen Bedingung bereits vorhandener diagnostischer Resultate gültig sind. Auch diese Überlegung veranschaulicht den erhöh-

133: Allerdings handelt es sich bei den bisher betrachteten bedingten gemeinsamen Verteilungen der Testergebnisse um zwei Extremfälle, die lediglich die Endpunkte des Spektrums der möglichen Verteilungen abdecken. Deshalb impliziert eine Abkehr von der bedingten Unabhängigkeit aller diagnostischen Maßnahmen nicht das Fehlen jeglicher Information, wie ANDERSON, J.A./BOYLE, J.A. (1968), S. 233, suggerieren; vgl. auch JESDINSKY, H.J. (1972), S. 52f. Unter Umständen weiß man etwa, daß bei der gemeinsamen Verteilung der Testergebnisse alle Korrelationen der Ordnung $m$ und darüber Null sind. Auch diese Information reduziert die Anzahl der Parameter, die zur Charakterisierung der interessierenden Verteilung notwendig sind; vgl. dazu BAHADUR, R.R. (1961) sowie HAMERLE, A.TUTZ, G. (1984), die diese Zusammenhangsanalyse im Kontext loglinearer Modelle diskutieren. 
ten Informationsbedarf, der in Bezug auf eine diagnostische Maßnahme vorliegt, wenn die Voraussetzung der bedingten Unabhängigkeit dieser und zuvor eingesetzter Maßnahmen bzw. die oben angesprochene schwächere Annahme nicht zutrifft.

Insofern überrascht nicht, daß viele Autoren die bedingte Unabhängigkeit diagnostischer Resultate - bzw. allgemeiner: die Gültigkeit der Gleichungen (3.21) - voraussetzen und deshalb die eingangs beschriebene Vorgehensweise anwenden ${ }^{134}$. Trifft diese Annahme hingegen nicht zu, dann liefert dieses Vorgehen eine unzutreffende Vorstellung von der tatsächlichen Veränderung der Informationslage des Arztes. Ohne weitere Voraussetzung über die Art des Zusammenhangs zwischen den Testresultaten gegeben die einzelnen Krankheitszustände weiß man allerdings nur, $\mathrm{da} B$ die mit Hilfe der Gleichungen (3.21) berechneten posterioren Wahrscheinlichkeiten den realiter gültigen nicht entsprechen.

Spezifischere Aussagen erlaubt die in den Abschnitten 3.1 bis 3.3 dieser Arbeit untersuchte vereinfachte Entscheidungssituation. Im einfachsten Fall liegen zwei binarisierte Tests vor, deren Resultate gemeinsam Aufschluß im Hinblick auf die Krankheitszustände $K_{1}$ und $\bar{K}_{1}$ geben sollen. Teather führt das Beispiel zweier diagnostischer Maßnahmen an, die einzeln keinerlei Informationsleistung erbringen, zusammen jedoch den bei einem Patienten vorliegenden Krankheitszustand fehlerfrei anzeigen ${ }^{135}$. Die Erklärung dieses Sachverhalts beruht auf den engen bedingten $\mathrm{Zu}$ sammenhängen, welche zwischen den Resultaten der beiden Diagnosetests für die Krankheitszustände $\mathrm{K}_{1}$ und $\overline{\mathrm{K}}_{1}$ bestehen ${ }^{136}$.

Bei zwei binarisierten Diagnosetests $T_{1}$ und $T_{2}$ können insgesamt vier verschiedene Kombinationen diagnostischer Resultate auftreten. Ordnet man diese Kombinationen ihrerseits als positive bzw. negative Resultate ein, so ergeben sich für

134: Vgl. z.B. LEDLEY, R.S./LUSTED, L.B. (1959), S. 14 und den Überblick bei SKENE, A. (1984), S. 143f.

135: Vgl. TEATHER, D. (1974b), S. 232 sowie in modifizierter Form NORUSIS, M.J.JJACQUEZ, J.A. (1975), S. 157.

136: Umgekehrt vermögen unter Umständen positive Resultate bei beiden Tests im Vergleich zur bedingten Unabhängigkeit die "target disorder" $\mathrm{K}_{1}$ mit geringerer Wahrscheinlichkeit anzuzeigen; vgl. WEINSTEIN, M.C. et al. (1980), S. 155ff. Die Autoren führen als Beispiel eine Entscheidungssituation an, in der die Testergebnisse gegeben $\mathrm{K}_{1}$ (nahezu) unabhängig sind, während für die Resultate gegeben Krankheitszustand $\bar{K}_{1}$ eine starke positive Abhängigkeit besteht. 
jede Strategie der Binarisierung unterschiedliche Kennziffern für die aus beiden Tests zusammengesetzte diagnostische Maßnahme ${ }^{137}$. Unter der Annahme bedingter Unabhängigkeit liegen für jede mögliche Zuordnung diese Kennziffern fest, da sie durch die Sensitivitäten und die Spezifitäten der einzelnen diagnostischen Maßnahmen determiniert werden. Ohne die bedingte Unabhängigkeit hingegen hängen die Kennziffern des zusammengesetzten Tests - und damit die Veränderung der Informationslage in einer gegebenen medizinischen Entscheidungssituation - noch von den bedingten Zusammenhängen der Testergebnisse von $T_{1}$ und $T_{2} a b$.

Marshall hat für verschiedene Strategien der Binarisierung algebraisch und graphisch gezeigt, in welcher Weise die Veränderung der Informationslage einer gegebenen Zuordnung durch die bedingten Zusammenhänge zwischen den Resultaten der beiden diagnostischen Maßnahmen beeinflußt wird ${ }^{138}$. Seine Analyse ermöglicht es unter anderem, diejenigen Fälle bedingter Abhängigkeit zu kennzeichnen, in denen die Annahme der bedingten Unabhängigkeit zu besonders starken Abweichungen von den tatsächlichen posterioren Wahrscheinlichkeiten führt.

Die Frage, ob eine Menge diagnostischer Maßnahmen tatsächlich oder zumindest annähernd die Eigenschaft der bedingten Unabhängigkeit erfüllt, können letztlich nur empirische Untersuchungen beantworten. Dies gilt ebenso für die schwächere Annahme, daß die posterioren Wahrscheinlichkeiten der Krankheitszustände mit Hilfe der Gleichungen (3.21) bestimmt werden können. Beide Hypothesen lassen sich beispielsweise im Rahmen eines loglinearen Modells überprüfen. Überdies ist diese Modellstruktur reichhaltig genug, um ganz unterschiedliche Zusammenhänge zwischen den einzelnen diagnostischen Maßnahmen zuzulassen, falls die Annahme der bedingten Unabhängigkeit nicht zutrifft ${ }^{139}$.

Die Gültigkeit der Rechenregeln für bedingte Wahrscheinlichkeiten sicherte bisher die Ermittlung der Veränderung der Informationslage des Arztes mit Hilfe des Bayes-

137: Vgl. dazu ausführlich MARSHALL, R.J. (1989).

138: Vgl. ebenda, S. $1214 \mathrm{ff}$.

139: Vgl. BISHOP, Y.M.M./FIENBERG, S.E./HOLLAND, P.W. (1975), Ch. 2; HAMERLE, A./TUTZ, G. (1984), S. 473ff. Alternative Verfahren diskutieren TEATHER, D. (1974b) sowie NORUSIS, M.J./JACQUEZ, J.A. (1975), die verschiedene Ansätze vergleichen. 
Theorems. Läßt man diese Annahme fallen, um an ihrer Stelle bestimmte, als plausibel erachtete Forderungen axiomatisch vorzugeben, dann führen diese zu folgender Vorschrift für die "Verbesserung" der prioren Wahrscheinlichkeiten ${ }^{140}$ :

$$
\begin{aligned}
& P^{*}\left(K_{i}\right)=C \cdot P\left(K_{i}\right) \cdot\left[P\left(z_{j h} \mid K_{i}\right)\right]^{r} \\
& r>0 ; \quad i=1, \ldots, n ; \quad j=1, \ldots, m ; \quad h=1, \ldots, k_{j} .
\end{aligned}
$$

Dabei bezeichnen die $\mathrm{P}^{*}\left(\mathrm{~K}_{\mathrm{i}}\right)$ die posterioren Wahrscheinlichkeiten der in Betracht gezogenen Krankheitszustände, $C$ eine positive Konstante, die Normierungszwecken dient, und $\mathrm{P}\left(\mathrm{z}_{\mathrm{jh}} \mid \mathrm{K}_{\mathrm{i}}\right)$ bzw. $\mathrm{P}\left(\mathrm{K}_{\mathrm{i}}\right)$ entsprechen wie bisher den bedingten Wahrscheinlichkeiten der Testergebnisse bzw. den prioren Wahrscheinlichkeiten der Krankheitszustände.

Der Exponent $\mathrm{r}$ läßt sich anschaulich interpretieren als "Nachgiebigkeit des Beobachters gegenüber experimentellen Ergebnissen"141. Für Werte von r, die nahe bei Null liegen, weichen die posterioren kaum von den prioren Wahrscheinlichkeiten ab. Der Information diagnostischer Resultate kommt in diesem Fall lediglich ein geringes Gewicht zu. Umgekehrt besteht bei großen Werten von $r$ eher die Möglichkeit, daß ein Testergebnis die prioren Wahrscheinlichkeiten beträchtlich zu modifizieren vermag ${ }^{142}$. Im Extremfall strebt $r$ gegen Unendlich und es ergibt sich das Maximum Likelihood Prinzip, das die in den prioren Wahrscheinlichkeiten verkörperte Information nicht berücksichtigt. Für $\mathrm{r}=1$ schließlich liegt die bisher betrachtete Vorschrift zur Transformation priorer in posteriore Wahrscheinlichkeiten vor, die mit derjenigen aus der Anwendung des Bayes-Theorems übereinstimmt. Man bezeichnet diese Transformation deshalb auch als Bayes-Regel ${ }^{143}$, die damit einen Spezialfall innerhalb der Menge aller Regeln repräsentiert, die mit Gleichung (3.25) kompatibel sind.

140: Vgl. RICHTER, H. (1963), S. 272.

141: Ebenda, S. 272.

142: Diese Aussagen gelten ceteris paribus, d.h. für gegebene Matrix $P_{j}$ der betrachteten diagnostischen Maßnahme.

143: Dennoch handelt es sich hier um eine von der bisherigen Analyse verschiedene Situation, da aufgrund schwächerer Voraussetzungen das Bayes-Theorem nicht mehr zur Ermittlung der posterioren Wahrscheinlichkeiten herangezogen werden kann. 


\subsection{Der Informationsgewinn diagnostischer Maßnahmen}

\subsubsection{Exkurs: Informationstheoretische Grundlagen}

\subsubsection{Zum Begriff der Information}

Die Informationstheorie beschäftigt sich unter anderem mit der Frage, welche Informationsmenge in einem Zufallsexperiment enthalten ist ${ }^{144}$. Ursprünglich entstammt diese Fragestellung der Theorie der Nachrichtenverarbeitung und -übermittlung. Um die Interpretation der allgemeinen informationstheoretischen Resultate zu erleichtern, dient zunächst die Kommunikationssituation als Spezialfall.

Angenommen ${ }^{145}$, es ginge darum, aus einer endlichen Anzahl von n Nachrichten eine einzelne Nachricht auszuwählen und diese über ein Übertragungsmedium, den sogenannten Kanal ${ }^{146}$, einer anderen Person - dem Empfänger der Nachricht - zukommen zu lassen. Prinzipiell gibt es keine wesentlichen Restriktionen bezüglich der Art dieser Nachrichten, der Einfachheit halber sei hier unterstellt, es handle sich um jeweils einen Buchstaben eines bestimmten Alphabets. Dieses sogenannte Originalalphabet mag dem deutschen entsprechen, genauso gut kann man an dessen Stelle ganz allgemein jeden alphanumerischen Zeichensatz verwenden, sofern er nur der Bedingung der Endlichkeit genügt ${ }^{147}$. Bei der Übertragung dieser Nachricht sind die speziellen physikalisch-technischen Eigenschaften des Kanals zu berïcksichtigen. Diese Eigenschaften bestimmen, welche Art von Zeichen für die Übertragung in Frage kommt ${ }^{148}$. Die Menge aller Zeichen, die im Kanal eingesetzt werden können, bezeichnet man als Übertragungsalphabet ${ }^{149}$. Aus der Verschiedenheit von Originalund Übertragungsalphabet folgt unmittelbar die Notwendigkeit einer Kodierung, d.h.

144: Einen Überblick gibt GUIASU, S. (1977). Eher anwendungsbezogen sind BRILLOUIN, L. (1967) und JAGLOM, A.M./JAGLOM, I.M. (1984). Den Zusammenhang zur Kybernetik erläutert WILLE, E. (1970), S. 131ff.; vgl. zur Einführung auch SACHSSE, H. (1974) sowie KRENGEL, U. (1990), S. $118 \mathrm{ff}$.

145: Vgl. hierzu JAGLOM, A.M./JAGLOM, I.M. (1984), S. 144ff.

146: Um Kommunikation zu ermöglichen, benötigt man immer dann einen Kanal, wenn Sender und Empfänger einer Nachricht in räumlicher und/oder zeitlicher Hinsicht getrennt sind.

147: Aus Gründen der Anschaulichkeit entstammen diese Mengen dem nachrichtentechnischen Bereich. Grundsätzlich kann es sich um Elemente einer beliebigen endlichen Menge handeln.

148: Beispielsweise kann man bei einem Gleichstromkanal nur Stromstöße und Pausen verwenden.

149: Der Zeichenvorrat des Übertragungsalphabets wird durch das Übertragungsmedium keineswegs festgelegt. So besteht etwa das Morse-Alphabet aus drei elementaren Zeichen, während der Kode von Baudot lediglich zwei Zeichen unterscheidet; beide Alphabete beziehen sich auf denselben Kanal. 
einer eindeutigen Zuordnung der möglichen Nachrichten auf Zeichen bzw. Zeichenkombinationen des Übertragungsalphabets ${ }^{150}$. Um jede korrekt übertragene Nachricht eindeutig rekonstruieren zu können, soll den durch die Kodierung entstehenden Zeichenkombinationen jeweils nur eine Nachricht zugeordnet sein. Ein Kode, der auch diese Bedingung erfüllt, wird als eindeutig dekodierbar bezeichnet ${ }^{151}$.

Bezogen auf diese einfache Kommunikationssituation, lautet das oben angesprochene Problem: wie viele Zeichen aus dem Übertragungsalphabet werden mindestens ${ }^{152}$ benötigt, um die Übertragung einer Nachricht zu ermöglichen? Eines der grundlegenden Resultate der Informationstheorie besteht in der Aussage, daß unter schwachen Voraussetzungen tatsächlich eine derartige untere Schranke existiert ${ }^{153}$. Weiterhin kann die Konstruktionsvorschrift eines Kodes angegeben werden, der folgende Optimalitätseigenschaft aufweist: die mittlere Anzahl der Zeichen pro Nachricht läßt sich nicht weiter verringern, wenn man zu einem anderen Kode übergeht $^{154}$.

Diese untere Schranke kennzeichnet damit diejenige Zeichenmenge, die im Durchschnitt sowohl notwendig als auch hinreichend ist zur Übermittlung der betrachteten Menge von Nachrichten. Insofern liegt es nahe, auf diese Eigenschaft den Informationsbegriff zu gründen. In der Tat definiert man in der Informationstheorie die in einer endlichen Nachrichtenmenge enthaltene Information als die minimale mittlere Anzahl der Zeichen aus einem Übertragungsalphabet, die zur Übermittlung einer Nachricht benötigt wird. Dabei darf nicht übersehen werden, daß es sich um einen abstrakten Begriff handelt, dessen Bedeutung stark von der umgangs-

150: Diese Zuordnung wird als Kode bezeichnet.

151: Vgl. McMILlAN, B. (1961), S. 212. Formal bedeutet dies, daß die den Kode repräsentierende Abbildung injektiv sein muß.

152: Diese Minimumseigenschaft muß aufgenommen werden, da sonst beliebig viele Lösungen existieren. Im übrigen begründet diese Eigenschaft die Optimalität des betreffenden Kodes, denn daraus folgt eine maximale Transferrate pro Zeiteinheit.

153: Einen einfachen Beweis für die Existenz dieser unteren Schranke gibt McMILLAN, B. (1961) an. Vgl. dazu näher Abschnitt 3.6.1.2.

154: Der zuvor angesprochene Existenzbeweis ist nicht konstruktiv, insofern handelt es sich um ein eigenständiges Resultat. Der Huffman-Kode besitzt diese Optimalitätseigenschaft; Konstruktionsbeschreibungen finden sich unter anderem bei RENYI, A. (1982), S. 60ff. sow.e bei JAGLOM, A.M./JAGLOM, I.M. (1984), S. 159ff. 
sprachlichen Verwendung des Wortes Information abweicht ${ }^{155}$. Weder der spezifische Inhalt einer Nachricht noch deren subjektive Bedeutung für Sender oder Empfänger spielen bei der Ermittlung dieser Informationsmenge eine Rolle.

Die ersten Arbeiten zur Informationstheorie verwendeten einen pragmatischen Ansatz, indem sie konkrete Probleme aus dem Bereich der Nachrichtenverarbeitung zum Anla $B$ für theoretische Überlegungen nahmen ${ }^{156}$. Wie sich schon bald herausstellte, lassen sich diese Überlegungen verallgemeinern und beispielsweise auf Zufallsexperimente anwenden. Den zuvor betrachteten Nachrichten entsprechen nun die möglichen Ausgänge eines Zufallsexperiments. Dabei wird wiederum angenommen, es handle sich um eine endliche Menge. Während im Beispiel der Nachrichtenverarbeitung a priori unklar blieb, welche spezifische Nachricht in einer konkreten Situation übermittelt werden sollte, herrscht nun Unsicherheit im Hinblick auf die Realisation der interessierenden Zufallsgröß $\mathrm{e}^{157}$. Analog ergibt sich die in einem Zufallsexperiment enthaltene Informationsmenge als mittlere Anzahl der Zeichen aus einem Übertragungsalphabet, die zur Kennzeichnung des Ausgangs dieses Experiments mindestens benötigt wird ${ }^{158}$.

Um den Begriff der Informationsmenge für statistische Anwendungen nutzen zu können, bedarf es der Kenntnis jener Eigenschaften, welche die Information charakterisieren. Diese Eigenschaften bilden die Grundlage des axiomatischen Ansatzes, der es gestattet, wichtige Aussagen über die Informationsmenge und den Informationsgewinn beispielsweise von Zufallsexperimenten abzuleiten ${ }^{159}$.

155: Vgl. dazu năher WILLE, E. (1970), S. 143ff.

156: Den Unterschied zwischen dem pragmatischen und dem axiomatischen Ansatz in der Informationstheorie erläutert RENYI, A. (1965).

157: Falls in der Ausgangslage keine derartige Unsicherheit bestanden haben sollte, erübrigt sich die Nachrichtenübermittlung bzw. sie liefert keine (neue) Information; vgl. FANO, R.M. (1966), S. 59.

158: Allgemein läßt sich die z.B. in einem Zufallsexperiment enthaltene Informationsmenge ohne Bezug auf ein bestimmtes Übertragungsalphabet bestimmen. Die Wahl des Übertragungsalphabets legt allerdings die Einheiten fest, in denen die Information gemessen wird. In praxi wählt man in der Regel ein spezielles Übertragungsalphabet, das lediglich zwei verschiedene Zeichen enthält.

159: Zur axiomatischen Fundierung vgl. KHINCHIN, A.I. (1957), S. 9ff. sowie in modifizierter Form RENYI, A. (1966), S. 440ff. 


\subsubsection{Die Entropie als Informationsmaß}

Um die Informationsmenge bzw. die Information zu definieren, die ein Zufallsexperiment $\mathrm{A}$ enthält, reicht es nicht aus, lediglich die verschiedenen möglichen Ausgänge $A_{i}(i=1, \ldots, n)$ dieses Experiments zu kennen ${ }^{160}$. Darüber hinaus benötigt man Angaben über die Wahrscheinlichkeiten $P\left(A_{i}\right)=p_{i}$, mit denen die einzelnen Ereignisse $A_{i}$ eintreten können. Bezeichnet $P=\left(p_{1}, \ldots, p_{n}\right)$ die Wahrscheinlichkeitsverteilung, die zu A gehört, dann erfordert der oben angesprochene abstrakte Begriff der Information, da $B$ die in $A$ enthaltene Information $I(A)$ nur von $P$ abhängt. Man kann deshalb auch von $I(P)$ bzw. $I\left(p_{1}, \ldots, p_{n}\right)$ sprechen, wobei es sich folglich um eine in allen Argumenten symmetrische Funktion handeln muß.

Diese zuletzt genannte Forderung gehört zu jenen vier Axiomen, die Renyi zur Fundierung des gesuchten Informationsmaßes angibt ${ }^{161}$. Er weist nach, daß diese Axiome eindeutig eine Funktion festlegen, die zuvor schon Shannon zur Messung der Information verwendete. Mit Hilfe dieser Shannon'schen Entropiefunktion ${ }^{162}$ ergibt sich für die Information, die das Zufallsexperiment A liefert ${ }^{163}$ :

$$
I(P)=\sum_{i=1}^{n} p_{i} \cdot 1 d \frac{1}{p_{i}} .
$$

I(P) gibt die Entropie eines Zufallsexperiments an, für dessen Ausgänge die Wahrscheinlichkeitsverteilung $\mathrm{P}$ gilt ${ }^{164}$. Diese Größe läßt sich einerseits aus der ex-post-

160: Die Ereignisse $A_{i}$ bilden eine Partition des Ergebnisraums von A.

161: Vgl. RENYI, A. (1966), S. 441.

162: Auf dem Gebiet der Thermodynamik existiert der Entropiebegriff schon seit dem 19. Jahrhundert; die große Bedeutung dieser Größe für informationstheoretische Untersuchungen arbeitete jedoch zuerst Shannon heraus; vgl. RENYI, A./BALATONI, J. (1957), S. 113 sowie JAGLOM, A.M.JJAGLOM, I.M. (1984), S. $65 f$.

Im folgenden wird ausschließlich die Entropie endlicher Zufallsexperimente behandelt, da diese einen für die spätere Anwendung auf diagnostische Maßnahmen völlig ausreichenden Rahmen bilden. Falls abzählbar viele Ereignisse auftreten können, braucht die Entropie nicht zu existieren. Weiterhin gelingt es nicht, den Entropiebegriff ohne Modifikationen auf stetige Verteilungen zu übertragen; vgl. etwa RENYI, A. (1966), S. 474ff; GUIASU, S. (1977), S. $16 \mathrm{ff}$.

163: Dabei bezeichnet der Operator ld den Logarithmus zur Basis 2.

164: Vgl. z.B. FANO, R.M. (1966), S. 58ff. 
Perspektive als Maß derjenigen Information auffassen, welche die Durchführung des Experiments erbringt. Andererseits beschreibt die Entropie I(P) ex ante die einem $\mathrm{Zu}$ fallsexperiment innewohnende Unbestimmtheit ${ }^{165}$.

Wie Gleichung (3.26) zeigt, läßt sich die Entropie auch als Erwartungswert einer Zufallsvariablen interpretieren, deren Realisationen durch die Ausdrücke -ld $\mathrm{p}_{\mathrm{i}}$ gegeben sind ${ }^{166}$. I(P) stellt eine wichtige informationstheoretische Größe dar, die darüber hinaus als "grundlegender Begriff der Wahrscheinlichkeitsrechnung"167 vielfältige statistische Anwendungsmöglichkeiten eröffnet ${ }^{168}$.

Im folgenden interessieren zunächst einige Eigenschaften, die das Verständnis der Informationsmenge $\mathrm{I}(\mathrm{P})$ erleichtern. Es gilt ${ }^{169}$ :

$$
\mathrm{I}(\mathrm{P}) \geq 0
$$

$$
\text { mit: } I(P)=0 \Leftrightarrow p_{k}=1, p_{i}=0, \forall i \neq k
$$

Ein echtes Zufallsexperiment besitzt also immer eine positive Entropie, d.h. die Nachricht über das Eintreten eines zufälligen Ereignisses liefert in jedem Fall neue Information. Nur für eine degenerierte Wahrscheinlichkeitsverteilung nimmt die Entropie ihren Minimalwert Null an. Es handelt sich dabei allerdings nicht mehr um ein Zufallsexperiment im eigentlichen Sinn, da keinerlei Unsicherheit bezüglich seines Ausgangs besteht: die Ausführung von A erbringt keine neue Information.

Wie aus Gleichung (3.26) hervorgeht, hängt die Entropie ausschließlich von den Wahrscheinlichkeiten ab, mit denen die Ausgänge eines Zufallsexperiments eintreten können. Insofern stellt die Entropie einen statistischen Parameter für diskrete

165: Die beiden Interpretationen der Entropie beziehen sich daher auf unterschiedliche Zeitpunkte; vgl. RENYI, A. (1966), S. 446 sowie GUIASU, S. (1977), S. 2 f.

166: Diese Größen können als Informationsgehalt der Ereignisse $A_{i}$ gedeutet werden; vgl. FANO, R.M. (1966), S. 42ff.; THEIL, H. (1967), S. 3ff.; THOMAS, J.B. (1969), S. 479f.; sowie RENYI, A. (1982), S. 46ff.

167: Vgl. RENYI, A.JBALATONI, J. (1957), S. 113.

168: Vgl. z.B. KULLBACK, S. (1959).

169: Die Funktion $h\left(p_{i}\right)=-p_{i} \cdot$ ld $p_{i}$ ist für $p_{i}=0$ nicht definiert, läßt sich dort jedoch mit dem Funktionswert Null stetig forsetzen. 
Wahrscheinlichkeitsverteilungen dar, sofern diese endlich viele Elemente aufwei$\operatorname{sen}^{170}$. Unter allen möglichen Verteilungen existiert eine, deren Entropie maximal ist $^{171}$ :

$$
\begin{aligned}
& \qquad I\left(p_{1}, \ldots, p_{n}\right) \leq I\left(\frac{1}{n}, \ldots, \frac{1}{n}\right), \\
& \text { und damit: } \quad I\left(p_{1}, \ldots, p_{n}\right) \leq \quad l d n .
\end{aligned}
$$

Die in einem Zufallsexperiment enthaltene Information wird demnach maximal, wenn sämtliche möglichen Ausgänge dieselbe Wahrscheinlichkeit besitzen ${ }^{172}$.

Von diesem Resultat ausgehend, kann man vermuten, daß die in einem Zufallsexperiment enthaltene Information umso größer ausfällt, je gleichmäßiger dessen Wahrscheinlichkeitsverteilung ist. Die folgende Ungleichung bestätigt diese Überlegung, indem sie den Begriff der Gleichmäßigkeit präzisiert. Sei P' der "neue", P der ursprüngliche Wahrscheinlichkeitsvektor, W eine geeignete doppelt-stochastische Matrix ${ }^{173}$ und es gelte:

$$
P^{\prime}=W \cdot P \text {. }
$$

Dann erfüllt die Entropie die Ungleichung:

$$
\mathrm{I}\left(\mathrm{P}^{\prime}\right) \geq \mathrm{I}(\mathrm{P})
$$

170: Vgl. MARSCHAK, J. (1959), S. 92.

171: Der Beweis kann entweder mit Hilfe eines Lagrange-Ansatzes geführt [vgl. BARNOON, S./WOLFE, H. (1972), S. 55f.] oder anhand der Jensen'schen Ungleichung für konkave Funktionen erbracht werden; vgl. KHINCHIN, A.I. (1957), S. 4.

172: Analog spricht man in der Bayes'schen Schätztheorie von "noninformative priors", wenn alle möglichen Parameterwerte a priori als gleichwahrscheinlich eingestuft werden; vgl. JUDGE, G.G. et al. (1988), S. $126 \mathrm{ff}$.

173: Eine derartige Matrix kennzeichnen zwei Eigenschaften: sämtliche Elemente sind nichtnegativ, weiterhin betragen alle Zeilen- und Spaltensummen jeweils Eins. Hier ist W sp-ziell eine quadratische Matrix der Ordnung $n$. 
Die Elemente von $\mathrm{P}^{\prime}$ stellen gemäß Gleichung (3.29) konvexe Kombinationen der Elemente der ursprünglichen Wahrscheinlichkeitsverteilung $P$ dar. In diesem Sinn ist die durch $\mathrm{P}^{\prime}$ gekennzeichnete Verteilung gleichmäßiger als die durch $\mathrm{P}$ angegebene. Wie die schwache Ungleichung (3.30) verdeutlicht, kann die Information nicht kleiner werden, wenn man zu einer gleichmäßigeren Verteilung übergeht. Die Ungleichung gilt sogar streng, sobald $\mathrm{P}^{\prime}$ nicht mit $\mathrm{P}$ übereinstimmt, d.h. falls $\mathrm{W}$ von der Einheitsmatrix verschieden ist.

Der Begriff der Entropie läßt sich auch auf Zufallsvariable mit endlich vielen Ausprägungen anwenden, sobald man die dazugehörige Wahrscheinlichkeitsverteilung kennt. Wie sich mit Hilfe der oben angestellten Überlegungen ergibt, stellt die Entropie eines Zufallsexperiments zugleich die Entropie einer geeignet definierten Zufallsvariablen dar. Bezeichne $\mathrm{X}$ eine Zufallsvariable, die auf dem Ergebnisraum des $\mathrm{Zu}$ fallsexperiments $A$ definiert ist und genau für die betrachteten Ausgänge $A_{i}$ jeweils verschiedene Werte annimmt ${ }^{174}$. Dann stimmt die zu A gehörende Wahrscheinlichkeitsverteilung $P$ mit der von $X$ überein und $I(P)$ kann als "die im Wert von X enthaltene Information"175 - Schreibweise I(X) - angesehen werden.

Die in einer Zufallsvariablen enthaltene Information erhöht sich nicht, wenn man anstelle von $X$ eine Funktion $Y=f(X)$ betrachtet, die ebenfalls eine Zufallsvariable darstellt. Falls $\mathrm{f}$ umkehrbar ist, ergeben sich für verschiedene Ausprägungen von $\mathrm{X}$ jeweils verschiedene Ausprägungen von $Y$ und die Information $I(Y)$ stimmt mit I(X) überein. Dies folgt unmittelbar aus Gleichung (3.26), da für $X$ und $Y$ jeweils dieselbe Wahrscheinlichkeitsverteilung gilt. Die Gleichheit der Entropiewerte überrascht nicht, da man durch die Beobachtung von $Y$ genauso umfassend informiert wird wie bei der Betrachtung des ursprünglichen, der Zufallsvariablen $X$ zugrunde liegenden Zufallsexperiments. Die Information verringert sich jedoch im allgemeinen, wenn $f$ eine nicht-injektive Funktion repräsentiert. Unter dieser Voraussetzung existieren zumindest zwei Ausprägungen von $\mathrm{X}$, zwischen denen die Zufallsvariable $\mathrm{Y}$ nicht diskriminiert. Folglich muß die in $\mathrm{Y}$ enthaltene Information geringer sein als diejenige, die $X$ enthält ${ }^{176}$.

174: $\mathrm{X}$ ist demnach eine injektive Funktion.

175: RENYI, A. (1966), S. 447.

176: Damit die Aussage gilt, müssen beide Ausprägungen jeweils positive Wahrscheinlichkeit besitzen. 
Eine anschauliche Interpretation der Information I(P) erhält man, wenn man Nachrichten betrachtet, die zuverlässig über den Ausgang des Zufallsexperiments $\mathrm{A}$ informieren ${ }^{177}$. Es kann gezeigt werden, $\mathrm{da} B$ bei beliebiger Kodierung die erwartete Länge einer derartigen Nachricht nicht weniger als I(P) Binärzeichen beträgt ${ }^{178}$. Die Shannon'sche Entropie einer endlichen Wahrscheinlichkeitsverteilung bildet daher falls ein Übertragungsalphabet mit lediglich zwei Zeichen benutzt wird - eine untere Schranke für die erwartete Länge einer Nachricht, die über das dazugehörige Zufallsexperiment informiert. Diese Grenze wird nur in Ausnahmefällen tatsächlich erreicht ${ }^{179}$.

Im Normalfall übersteigt daher die erwartete Länge einer Nachricht, die zuverlässig den Ausgang eines Zufallsexperiments anzeigt, den dazugehörigen Entropiewert. Betrachtet man jedoch Folgen unabhängiger Wiederholungen des Zufallsexperiments $A$, so läßt sich zeigen, daß die erwartete Anzahl der pro Nachricht zur Kodierung mindestens benötigten Zeichen beliebig nahe bei $\mathrm{I}(\mathrm{P})$ liegt. Damit konvergiert die durchschnittliche Anzahl der Binärzeichen, welche die eineindeutige Kennzeichnung jeder derartigen Versuchsreihe mindestens benötigt, stochastisch gegen die Entropie $\mathrm{I}(\mathrm{P})$ des Zufallsexperiments ${ }^{180}$. Mit beliebig nahe bei Eins liegender Wahrscheinlichkeit reichen im Durchschnitt I(P) Binärzeichen aus, um das Ergebnis eines Versuchs eineindeutig darzustellen. In diesem speziellen Sinn bedarf es gerade I(P) Binärzeichen, um über das Zufallsexperiment A zuverlässig zu berichten. Auf diese Eigenschaft bezieht sich die Aussage, daß die in A enthaltene Informationsmenge $I(P)$ beträgt.

177: Aus einer zuverlässigen Nachricht läßt sich mit Sicherheit entnehmen, welchen Ausgang das betreffende Zufallsexperiment genommen hat. Theil bezeichnet eine derartige Nachricht als definitive Nachricht; vgl. THEIL, H. (1967), S.3; während Marschak von einer fehlerfreien Nachricht spricht; vgl. MARSCHAK, J. (1959), S. 94.

178: Vgl. z.B. McMILLAN, B. (1961), S. $213 f$.

179: Dieser Fall tritt genau dann ein, wenn die Wahrscheinlichkeiten der Ausgänge des Zufallsexperiments sämtlich Potenzen der Zahl Zwei sind.

180: Vgl. RENYI, A. (1966), S. 455ff.; CSISZAR, I./KÖRNER, J. (1981), S. 15ff. sowie JAGLOM, A.M./JAGLOM, I.M. (1984), S. $163 \mathrm{ff.}$

Zum Begriff der stochastischen Konvergenz einer Folge von Zufallsvariablen vgl. z.B. RENYI, A. (1966), S. 314. 
Weitere Erkenntnisse über die Entropie als Informationsmaß lassen sich gewinnen, indem man zusammengesetzte Zufallsexperimente untersucht. Bezeichne $\mathrm{X}$ eine $\mathrm{Zu}$ fallsvariable, deren Ausprägungen $x_{i}(i=1, \ldots, n)$ mit den Wahrscheinlichkeiten $P\left(X=x_{i}\right)=p_{i}>0$ angenommen werden, und ferner $Y$ eine andere Zufallsvariable, für deren Realisationen $y_{j}(j=1, \ldots, m)$ die Wahrscheinlichkeiten $Q\left(Y=y_{j}\right)=q_{j}>0$ gelten. $X$ und $Y$ repräsentieren zwei verschiedene Zufallsexperimente $A$ und $B$. Die Beobachtung der Zufallsvariablen X X Y entspricht dann einem zusammengesetzten Zufallsexperiment, das in der gleichzeitigen Durchführung von $\mathrm{A}$ und $\mathrm{B}$ besteht. Wenn die gemeinsamen Wahrscheinlichkeiten mit $R\left(X=x_{i}, Y=y_{j}\right)=r_{i j}$ bezeichnet sind, erhält man für die in $X$ und $Y$ enthaltene Information $I(X, Y)$ :

$$
\begin{aligned}
I(X, Y) & =\sum_{i=1}^{n} \sum_{j=1}^{m} r_{i j} \cdot I d \frac{1}{r_{i j}} \\
& =I(R) .
\end{aligned}
$$

$\mathrm{I}(\mathrm{X}, \mathrm{Y})$ stellt zugleich diejenige Information dar, die das aus A und B zusammengesetzte Experiment enthält. Die gemeinsamen Wahrscheinlichkeiten $r_{i j}$ lassen sich gemäß der Definition bedingter Wahrscheinlichkeiten jeweils ersetzen durch ein Produkt aus einer Randwahrscheinlichkeit und einer bedingten Wahrscheinlichkeit $^{181}$ :

$$
\begin{gathered}
I(X, Y)=\sum_{i=1}^{n} \sum_{j=1}^{m} p_{i} \cdot q\left(y_{j} \mid x_{i}\right) \cdot l d \frac{1}{p_{i} \cdot q\left(y_{j} \mid x_{i}\right)} \\
=\sum_{i=1}^{n} p_{i} \cdot l d \frac{1}{p_{i}} \sum_{j=1}^{m} q\left(y_{j} \mid x_{i}\right)+\sum_{i=1}^{n} p_{i} \cdot \sum_{j=1}^{m} q\left(y_{j} \mid x_{i}\right) \cdot l d \frac{1}{q\left(y_{j} \mid x_{i}\right)} .
\end{gathered}
$$

181: Dabei bezeichnet $q\left(y_{j} \mid x_{i}\right)$ jeweils die Wahrscheinlichkeit $Q\left(Y=y_{j} \mid X=x_{i}\right)$. 
Damit ergibt sich ${ }^{182}$ :

$$
\begin{aligned}
I(X, Y) & =I(X)+\sum_{i=1}^{n} p_{i} \cdot I\left(Y \mid x_{i}\right) \\
& =I(X)+I(Y \mid X) .
\end{aligned}
$$

Die Gleichungen (3.33) und (3.33a) enthalten Größen, die als bedingte Entropien bezeichnet werden. $\mathrm{I}\left(\mathrm{Y} \mid \mathrm{x}_{\mathfrak{i}}\right)$ zeigt die bedingte Entropie der Zufallsvariablen $\mathrm{Y}$ an, wenn die Zufallsvariable $X$ den Wert $x_{i}$ annimmt. Interessanterweise führt die Kenntnis der Realisation $x_{i}$ nicht unbedingt zu einer Verringerung der in der Zufallsvariablen $\mathrm{Y}$ enthaltenen Information: $\mathrm{I}\left(\mathrm{Y} \mid \mathrm{x}_{\mathrm{i}}\right)$ kann größer, kleiner oder gleich der Information $\mathrm{I}(\mathrm{Y}) \operatorname{sein}^{183}$.

Die Entropie $I(Y \mid X)$ repräsentiert den Erwartungswert der einzelnen $I\left(Y \mid x_{i}\right)$. $\mathrm{I}(\mathrm{Y} \mid \mathrm{X})$ ist nicht vom Zufall abhängig und gibt die in $\mathrm{Y}$ verbliebene Information an, wenn der Wert der Zufallsvariablen $X$ beobachtet werden kann. Man bezeichnet $\mathrm{I}(\mathrm{Y} \mid \mathrm{X})$ als bedingte Information der Zufallsvariablen $\mathrm{Y}$ gegeben die Zufallsvariable $\mathbf{X}$, oder kürzer als bedingte Information von $\mathbf{Y}$, wenn klar ist, welche $\mathrm{Zu}$ fallsvariable als Bedingung fungiert.

Das durch die beiden Zufallsvariablen $\mathrm{X}$ und $\mathrm{Y}$ repräsentierte Zufallsexperiment läßt sich auf zwei äquivalente Weisen durchführen. Man kann einerseits zunächst die Realisation von $\mathrm{X}$ beobachten und im Anschluß daran unter dieser Bedingung die $\mathrm{Zu}$ fallsvariable $Y$ betrachten. Die Beobachtung von X stellt in diesem Fall ein Startexperiment dar, während $Y$ als Folgeexperiment fungiert ${ }^{184}$. Die Wahrscheinlichkeiten der Realisationen von $\mathrm{Y}$ werden durch den Ausgang des Startexperiments im Einklang mit der gemeinsamen Verteilung von $\mathrm{X}$ und $\mathrm{Y}$ bestimmt. Andererseits besteht die Möglichkeit, das zusammengesetzte Zufallsexperiment in einem Zug durchzuführen und damit die Realisationen der Zufallsvariablen X und Y direkt zu beobachten.

182: Analog ergibt sich, wenn man die Realisation von $\mathrm{Y}$ als Bedingung heranzieht:

$$
I(X, Y)=I(Y)+I(X \mid Y) .
$$

183: Eine definitive Aussage läßt sich lediglich treffen, wenn die Realisationen von $Y$ gleichverteilt sind und damit die in $\mathrm{Y}$ enthaltene Information maximal ist.

184: Vgl. Abschnitt 2.4.2. 
Beide Vorgehensweisen scheinen dem Beobachter im Ergebnis dieselbe Information im Hinblick auf $X$ und $Y$ zu liefern. Diese Vermutung bestätigt Gleichung (3.33a), die jeder Vorgehensweise den denselben Entropiewert zuordnet. Diese Eigenschaft der allgemeinen Additivität der Information für zusammengesetzte Zufallsexperimente charakterisiert die Shannon'sche Entropie, da andere Informationsmaße lediglich die Additivität für unabhängige Zufallsexperimente aufweisen ${ }^{185}$.

Aus der Konkavität der Entropiefunktion folgt eine wichtige Eigenschaft ${ }^{186}$ der bedingten Information von Y:

$$
\mathrm{I}(\mathrm{Y} \mid \mathrm{X}) \leq \mathrm{I}(\mathrm{Y})
$$

Im statistischen Durchschnitt vermag das Wissen um die Realisation einer anderen Zufallsvariablen $X$ die in der Zufallsvariablen $Y$ enthaltene Information nicht zu erhöhen. Im Normalfall verringert die Kenntnis von $X$ sogar die Information von $Y$. Lediglich im Falle der stochastischen Unabhängigkeit von $X$ und $Y$ stimmen die bedingte und die unbedingte Information von $Y$ überein:

$$
I(Y \mid X)=I(Y) \quad \Leftrightarrow \quad r_{i j}=p_{i} \cdot q_{j} ; i=1, \ldots, n ; j=1, \ldots, m
$$

Falls $\mathrm{X}$ und $\mathrm{Y}$ stochastisch unabhängig sind, beeinflussen die Realisationen von $\mathrm{X}$ die Zufallsvariable $Y$ nicht und können daher die in $Y$ enthaltene Information nicht verringern. Sobald jedoch eine statistische Abhängigkeit zwischen den beiden $\mathrm{Zu}$ fallsvariablen vorliegt, führt die Kenntnis der Zufallsvariablen $\mathrm{X}$ zu einer Senkung der Informationsmenge von Y. Die Beobachtung von X ermöglicht es in diesem Fall aufgrund der Abhängigkeit beider Zufallsvariablen, die in Bezug auf $Y$ herrschende Unbestimmtheit teilweise abzubauen. In diesem Sinn enthält die Zufallsvariable X Information über die Zufallsvariable Y. Dieser Sachverhalt bildet den Ausgangspunkt für die Messung des Informationsgewinns.

185: Vgl. RENYI, A. (1966), S. $461 \mathrm{ff}$.

186: Vgl. z.B. GUIASU, S. (1977), S. 7. 


\subsubsection{Meßgrößen für den Informationsgewinn}

Wieder werden zwei Zufallsvariable $\mathrm{X}$ und $\mathrm{Y}$ betrachtet, die denselben Voraussetzungen wie im vorherigen Abschnitt genügen sollen. Konkret sei angenommen, $\mathrm{da} B$ man sich für die Zufallsvariable $Y$ interessiert ${ }^{187}$, die allerdings nicht direkt beobachtet werden kann. Es besteht jedoch die Möglichkeit, an ihrer Stelle die Realisation einer anderen Zufallsvariablen $X$ zu erfahren. Jede Realisation $x_{i}$ legt dann eine bedingte Wahrscheinlichkeitsverteilung für die eigentlich interessierende $\mathrm{Zu}$ fallsvariable $Y$ fest. Insofern stellt die Zufallsvariable $X$ ein Informationsinstrument für $Y$ dar ${ }^{188}$. Die Veränderung der in $Y$ enthaltenen Information gibt, wenn man die Zufallsvariable $X$ berücksichtigt, die folgende Differenz an ${ }^{189}$ :

$$
\begin{aligned}
G(X, Y) & =I(Y)-I(Y \mid X) \\
& =I(Y)-\sum_{i=1}^{n} p_{i} \cdot I\left(Y \mid x_{i}\right)
\end{aligned}
$$

Aus den Beziehungen (3.34) und (3.34a) folgt unmittelbar:

$$
\mathrm{G}(\mathrm{X}, \mathrm{Y}) \geq 0
$$

mit: $G(X, Y)=0 \quad \Leftrightarrow \quad X$ und $Y$ sind stochastisch unabhängig.

Die Größe $G(X, Y)$ bezeichnet man als den mittleren Informationsgehalt ${ }^{190}$ bzw. die relative Information ${ }^{191}$ von $X$ bezüglich $Y$. Sie gibt an, in welchem Umfang $X$ In-

187: Prinzipiell kann sowohl $\mathrm{X}$ zur Information bezüglich $\mathrm{Y}$ dienen als auch umgekehrt $\mathrm{Y}$ in Bezug auf X. Bei praktischen Anwendungen liegen die Rollen von informierender und interessierender Variable fest, wie auch das in Abschnitt 3.6.2 diskutierte Beispiel diagnostischer Maßnahmen zeigt.

188: Vgl. zum Begriff des Informationsinstruments Abschnitt 2.4.3.

189: Die Reihenfolge der Variablen in Klammem spielt keine Rolle, da diese Größe symmetrisch in $X$ und $Y$ ist. Aus diesem Grund nennt man $G(X, Y)$ auch die gegenseitige Information ("mutual information") von X und Y; vgl. CSISZAR, I.KÖRNER, J. (1981), S. 21 sowie THEIL, H. (1967), S. 33ff.

190: Vgl. JAGLOM, A.M.JJAGLOM, I.M. (1984), S. 86f.

191: Vgl. RENYI, A. (1966), S. $450 \mathrm{ff}$. 
formation über die Zufallsvariable $Y$ enthält ${ }^{192}$. Konzeptionell läßt sich $G(X, Y)$ als Stromgröße deuten, die mit der in $Y$ enthaltenen Information eine Bestandsgröße verändert. Dieser Sachverhalt legt es nahe, $G(X, Y)$ als neue Information und damit als Informationsgewinn durch die Zufallsvariable $X$ im Hinblick auf $Y \mathrm{zu}$ interpretieren.

Neben der durch die Ungleichung (3.36) angegebenen unteren Schranke existiert eine obere Schranke für diese Meßgröße des Informationsgewinns ${ }^{193}$ :

$$
\mathrm{G}(\mathrm{X}, \mathrm{Y}) \leq \min \{\mathrm{I}(\mathrm{X}), \mathrm{I}(\mathrm{Y})\}
$$

Der Informationsgewinn, den die Zufallsvariable $X$ in Bezug auf $Y$ liefert, kann demnach niemals höher ausfallen als das Minimum der in $\mathrm{X}$ und in $\mathrm{Y}$ selbst enthaltenen Information.

Damit lassen sich zwei Fälle unterscheiden. Sei zunächst $\mathrm{I}(\mathrm{Y})>\mathrm{I}(\mathrm{X})$ erfüllt. Wie Beziehung (3.37) zeigt, fällt unter dieser Voraussetzung der durch die Zufallsvariable $\mathrm{X}$ erzeugte Informationsgewinn immer kleiner aus als die in $\mathrm{Y}$ enthaltene Information. Die Zufallsvariable $X$ vermag daher die bezüglich $Y$ herrschende Unbestimmtheit in keinem Fall vollständig abzubauen.

Sei nun $I(X) \geq I(Y)$, d.h. die Zufallsvariable $X$ enthalte mindestens ebenso viel Information wie die Zufallsvariable $Y$. Dann kann der Informationsgewinn, den $X$ in Bezug auf Y liefert, maximal I(Y) betragen. Wie Gleichung (3.35) verdeutlicht, liegt dieser günstige Fall genau dann vor, wenn für jede Realisation von $X$ die bedingte Information von $Y$ Null ist. Die Zufallsvariable $X$ informiert dann vollständig über $Y$, da man für jede Realisation $x_{i}$ mit einer Wahrscheinlichkeit von Eins die Ausprägung der Zufallsvariablen Y erfährt.

192: Im Kontext der Nachrichtenverarbeitung zeigt $\mathrm{G}(\mathrm{X}, \mathrm{Y})$ an, wie groß die mögliche Zeichenreduktion pro Nachricht über $\mathrm{Y}$ im Durchschnitt sein kann, wenn die in $\mathrm{X}$ vorhandene Information bei der Kodierung berücksichtigt wird. Es lohnt sich demzufolge, den statistischen Zusammenhang zwischen $\mathrm{X}$ und $\mathrm{Y}$ auszunutzen, denn dadurch kann die mittlere Anzahl der Zeichen pro Nachricht verringert werden; vgl. McMILLAN, B. (1961), S. 218.

193: Vgl. RENYI, A. (1966), S. 450. 
Wenn man, um Information über die eigentlich interessierende Zufallsvariable $Y$ zu erlangen, eine (andere) Zufallsvariable $X$ beobachtet, erhält man eine bestimmte Ausprägung $\mathbf{x}_{\mathbf{k}}$. Die Frage, wie dieses spezielle Wissen die Informationslage im Hinblick auf die Zufallsvariable $Y$ verändert, bezieht sich auf den Informationsgewinn, den eine einzelne Nachricht $x_{k}$ bezüglich $Y$ liefert. Diese Größe wird im folgenden mit IG $\left(Y ; x_{k}\right)$ bezeichnet und darf nicht mit dem zuvor diskutierten Informationsgewinn $G(X, Y)$ der Zufallsvariable $X$ insgesamt verwechselt werden ${ }^{194}$.

Dennoch besteht eine Beziehung zwischen den beiden Arten des Informationsgewinns. Vor der Betrachtung der Zufallsvariablen $X$ ist nicht bekannt, welche Ausprägung $x_{i}$ sich einstellen wird. Der Informationsgewinn IG $\left(Y ; x_{i}\right)$ stellt daher ex ante ebenfalls eine Zufallsvariable dar, deren Wahrscheinlichkeitsverteilung durch die einzelnen $\mathrm{p}_{\mathrm{i}}$ festgelegt wird. Der Informationsgewinn, der aufgrund einer speziellen Ausprägung von $X \mathrm{zu}$ erwarten ist, entspricht gerade demjenigen Informationsgewinn, den diese Zufallsvariable selbst liefert. Aus Gründen der Konsistenz muß der Informationsgewinn IG(Y; $\left.\mathbf{x}_{\mathbf{i}}\right)$ deshalb folgende Bedingung erfüllen:

$$
\begin{aligned}
G(X, Y) & =\sum_{i=1}^{n} p_{i} \cdot I G\left(Y ; x_{i}\right) \\
& =E_{X} I G(Y ; X) .
\end{aligned}
$$

Es erweist sich als außerordentlich nützlich, den Informationsgewinn, den eine spezielle Ausprägung $x_{k}$ der Zufallsvariablen $X$ im Hinblick auf $Y$ liefert, wie folgt zu definieren ${ }^{195}$ :

$$
\operatorname{IG}\left(Y ; x_{k}\right)=\sum_{j=1}^{m} q\left(y_{j} \mid x_{k}\right) \cdot \operatorname{ld} \frac{q\left(y_{j 1} \mid x_{i}\right)}{q_{j}}
$$

194: Man könnte diesen Informationsgewinn - in Analogie zu $G(X, Y)$ - auch als Veränderung der Information in $Y$ definieren, die durch die Ausprägung $x_{k}$ bewirkt wird. Diese Meßgröße besitzt jedoch unter anderem den Nachteil, auch negative Werte annehmen zu können; vgl. BLACHMAN, N.M. (1968).

195: Im Gegensatz zu G(X,Y) ist die Größe IG(Y; $\left.\mathrm{x}_{\mathbf{k}}\right)$ nicht symmetrisch, wie der Strichpunk! zur Betonung der Reihenfolge der beiden Variablen in der Klammer andeuten soll. 
IG $\left(Y ; x_{k}\right)$ besitzt einige Eigenschaften, die diesen Ausdruck als Maß für den Informationsgewinn geeignet erscheinen lassen ${ }^{196}$. Zunächst erfüllt diese Größe die zuvor angesprochene Konsistenzbedingung:

$$
\begin{aligned}
E_{X} \operatorname{IG}(Y ; X) & =\sum_{i=1}^{n} p_{i} \cdot \sum_{j=1}^{m} q\left(y_{j} \mid x_{k}\right) \cdot l d \frac{q\left(y_{j 1} \mid x_{i}\right)}{q_{j}} \\
& =G(X, Y) .
\end{aligned}
$$

Wie sich weiterhin nachweisen läßt, bleibt der so definierte Informationsgewinn der Nachricht $x_{k}$ immer nichtnegativ ${ }^{197}$. Es gilt also:

$$
\mathrm{IG}\left(\mathrm{Y} ; \mathrm{x}_{\mathrm{k}}\right) \geq 0
$$

mit: $I G\left(Y ; x_{k}\right)=0 \Leftrightarrow q_{j}=q\left(y_{j} \mid x_{k}\right) ; j=1, \ldots, m$.

Die Nachricht $x_{k}$ erbringt genau dann keine neue Information im Hinblick auf $Y$, wenn die bedingte Wahrscheinlichkeitsverteilung $Q_{0}=Q\left(Y \mid x_{k}\right)$ mit der unbedingten $Q_{1}=Q(Y)$ übereinstimmt. Sofern jedoch $x_{k}$ zu einer Veränderung der Verteilung von $Y$ führt, folgt daraus immer ein positiver Informationsgewinn. Da der Informationsgewinn IG $\left(Y ; x_{k}\right)$ aufgrund der Nachricht $x_{k}$ im Hinblick auf die Zufallsvariable $Y$ lediglich von den beiden Wahrscheinlichkeitsverteilungen $\mathrm{Q}_{0}$ und $\mathrm{Q}_{1}$ abhängt, kann man dafür auch die Schreibweise $\mathrm{IG}\left(\mathrm{Q}_{0} ; \mathrm{Q}_{1}\right)$ verwenden.

Zur besseren Interpretation von IG $\left(Y ; \mathbf{x}_{\mathrm{k}}\right)$ berücksichtigt man eine Eigenschaft dieser Größe im Rahmen der Überprüfung statistischer Hypothesen. Angenommen, es gehe darum, bezüglich der Verteilung einer Zufallsvariablen die einfache Nullhypothese $\mathrm{H}_{0}: \mathrm{P}=\mathrm{P}_{0}$ gegen die einfache Alternative $\mathrm{H}_{1}: \mathrm{P}=\mathrm{P}_{1}$ zu testen. Man kann zeigen, daß für ein beliebig kleines Signifikanzniveau mit wachsendem Stichprobenumfang das arithmetische Mittel der logarithmierten minimalen Fehler-

196: Renyi motiviert diese Definition anhand eines Beispiels [vgl. RENYI, A. (1966), S. 452f.]. Weiterhin zeigt er, daß sich bei axiomatischer Fundierung von $\mathrm{IG}\left(\mathrm{Y} ; \mathrm{x}_{\mathrm{k}}\right)$ alle anderen wichtigen informationstheoretischen Größen einschließlich der Entropie daraus ableiten lassen; vgl. ebenda, S. 459ff. sowie RENYI, A. (1960), S. 534.

197: Vgl. etwa FANO, R.M. (1966), S. 64; BARNOON, S./WOLFE, H. (1972), S. $175 \mathrm{ff}$. 
wahrscheinlichkeit 2. Art gegen $-\mathrm{IG}\left(\mathrm{P}_{0} ; \mathrm{P}_{1}\right)$ konvergiert. $\mathrm{Zu}$ einem vorgegebenen Sicherheitsniveau läßt sich dieser Mittelwert als Maß für die Verschiedenheit der beiden durch die Hypothesen zu vergleichenden Wahrscheinlichkeitsverteilungen deuten ${ }^{198}$. Insofern berechtigt dieses Resultat dazu, IG $\left(\mathrm{P}_{0} ; \mathrm{P}_{1}\right)$ als $\mathrm{MaB}$ für den Unterschied heranzuziehen, der zwischen den beiden Verteilungen $P_{1}$ und $P_{0}$ besteht ${ }^{199}$.

Der Informationsgewinn IG $\left(Y ; x_{k}\right)$, den die Nachricht $x_{k}$ im Hinblick auf die $\mathrm{Zu}$ fallsvariable $Y$ liefert, kann daher zugleich als spezielles Maß dafür interpretiert werden, wie sehr die bedingte Verteilung $Q\left(Y \mid x_{k}\right)$ und die unbedingte $Q(Y)$ differieren. Je größer der Informationsgewinn IG $\left(Y ; \mathbf{x}_{\mathbf{k}}\right)$ ausfällt, desto stärker beeinflußt die spezielle Nachricht $x_{k}$ das über die Zufallsvariable $Y$ vorhandene Wissen und desto deutlicher die Diskrepanz zwischen der bedingten und der unbedingten Wahrscheinlichkeitsverteilung von $\mathrm{Y}$.

\subsubsection{Anwendung auf diagnostische Maßnahmen}

Die im vorherigen Abschnitt erläuterten Konzepte und Resultate der Informationstheorie können dazu dienen, den Begriff der Informationsleistung einer diagnostischen Maßnahme zu präzisieren. Eine diagnostische Maßnahme $T_{j}$ generiert in diesem Zusammenhang mit ihrem Testergebnis eine informierende Zufallsvariable, während der Krankheitszustand des Patienten die den Arzt eigentlich interessierende Variable darstellt. Die Anwendung der Informationstheorie ermöglicht es, die Ver-

198: Für IG $\left(\mathrm{P}_{0} ; \mathrm{P}_{1}\right)$ existieren daher auch die Bezeichnungen "informational divergence" bzw. "information for discrimination"; vgl. CSISZAR, I/KÖRNER, J. (1981), S. 20. IG( $\left.\mathrm{P}_{0} ; \mathrm{P}_{1}\right)$ stellt jedoch kein Distanzmaß für Wahrscheinlichkeitsverteilungen dar, da weder die Symmetriebedingung noch die "Dreiecksungleichung" erfüllt sind; vgl. CSISZAR, I. (1978), S. 78.

Im übrigen kann auch der weiter oben diskutierte Informationsgewinn durch das Informationsinstrument $X$ in diesem Sinne interpretiert werden: $G(Y, X)$ gibt an, wie sehr sich die tatsächlich vorliegende gemeinsame Verteilung der Zufallsvariablen $Y$ und $X$ von derjenigen unterscheidet, die bei stochastischer Unabhängigkeit von $Y$ und $X$ gelten würde. Insofern mißt $G(X, Y)$ die Stärke des statistischen Zusammenhangs zwischen diesen beiden Zufallsvariablen; siehe hierzu McMILLAN, B. (1961), S. 218 sowie CSISZAR, I.KÖRNER, J. (1981), S. 21.

199: In dieser Funktion besitzt IG $\left(\mathrm{P}_{0}: \mathrm{P}_{1}\right)$ für die Statistik eine erhebliche Bedeutung. Beispielsweise läßt sich diese Größe für die Konstruktion statistischer Modelle verwenden; vgl. GOOD, I.J. (1963); BISHOP, Y.M.M./FIENBERG, S.E./HOLLAND, P.W. (1975), S. $384 f f$. Bei der Schätzung von Parametern besteht ein enger Zusammenhang $z$ wischen IG $\left(\mathrm{P}_{0} ; \mathrm{P}_{1}\right)$ und der Informationsmatrix von Fisher; vgl. z.B. MONFORT, A. (1982), S. $76 \mathrm{ff}$. 
änderung der Informationslage des Arztes, die auf einen Diagnosetest zurückgeht, mit Hilfe des Informationsgewinns eindeutig in einer skalaren Größe auszudrücken 200. Der Informationsgewinn, den eine diagnostische Maßnahme in einer medizinischen Entscheidungssituation liefert, repräsentiert gerade die mit ihrem Einsatz verbundene neue Information.

Dabei lassen sich grundsätzlich zwei Arten des Informationsgewinns unterscheiden, den ein Diagnosetest $T_{j}$ im Hinblick auf den Krankheitszustand des Patienten erbringen kann:

(1) Zunächst fällt der Informationsgewinn an, der sich aufgrund eines einzelnen Resultats ergibt. Falls beispielsweise das Resultat $z_{\text {jh }}$ eintrifft, berechnet man den dadurch entstehenden Informationsgewinn IG $\left(K ; z_{j h}\right)$ wie folgt:

$$
\operatorname{IG}\left(K ; z_{j h}\right)=\sum_{i=1}^{n} P\left(K_{i} \mid z_{j h}\right) \cdot \operatorname{ld} \frac{P\left(K_{i} \mid z_{j h}\right)}{P\left(K_{i}\right)} .
$$

Von dieser Größe zu unterscheiden ist

(2) derjenige Informationsgewinn, den die diagnostische Maßnahme insgesamt erzeugen kann. Für diese Größe, die in Übereinstimmung mit der in Abschnitt 3.6.1 eingeführten Notation mit $G\left(K, Z_{j}\right)$ bezeichnet wird, gilt:

$$
\begin{aligned}
G\left(K, Z_{j}\right) & =I(K)-I\left(K \mid Z_{j}\right) \\
& =I(K)-\sum_{h=1}^{k_{j}} P\left(z_{j h}\right) \cdot I G\left(K / z_{j h}\right) .
\end{aligned}
$$

Dabei gilt wie zuvor im allgemeinen Fall, daß der mit dem Einsatz einer diagnostischen Maßnahme verbundene Informationsgewinn $G\left(K, Z_{j}\right)$ gerade die mathematische Erwartung desjenigen Informationsgewinns bildet, den ein spezielles Testergebnis liefert.

200: Zur Anwendung der Informationstheorie in der medizinischen Diagnostik vgl. METZ, C.E./GOODENOUGH, D.J./ROSSMANN, K. (1973); BÜTTNER, J. (1977), S. Sf.; RIFKIN. R.D. (1985) sowie JOHNSON, H.A. (1989). 
Dieser unter (2) genannte Informationsgewinn nimmt unmittelbar Bezug auf die jeweils vorliegende medizinische Entscheidungssituation. Zu dem Zeitpunkt, an dem der Arzt über den Einsatz einer (weiteren) diagnostischen Maßnahme entscheidet, besteht noch Unsicherheit im Hinblick auf das sich einstellende Testergebnis. Insofern muß diese Unsicherheit bei der Beschreibung der diagnostischen Information berücksichtigt werden. Im entscheidungstheoretischen Kontext interessiert daher primär der Informationsgewinn $\mathrm{G}\left(\mathrm{K}, \mathrm{Z}_{\mathrm{j}}\right)$, da nur diese Größe zu dem Zeitpunkt ermittelt werden kann, an dem sich der Arzt für oder gegen den Einsatz einer diagnostischen Maßnahme entscheiden muß. Dabei ist nicht auszuschließen, daß eine diagnostische Maßnahme, die insgesamt einen geringeren Informationsgewinn als eine alternative Maßnahme in einer durch die prioren Wahrscheinlichkeiten der Krankheitszustände gegebenen Ausgangslage aufweist, bei einem einzelnen Testergebnis mehr Information im Hinblick auf den Krankheitszustand des Patienten zu liefern vermag. Statistisch gesehen tritt ein derartiges Testergebnis jedoch zu selten auf, um dieser diagnostischen Maßnahme in dem betrachteten Vergleich insgesamt einen gleichen oder gar höheren Informationsgewinn zuweisen zu können.

Die in Abschnitt 3.4 vorgenommene Kategorisierung diagnostischer Maßnahmen anhand ihrer Informationsleistung läßt sich nun mit Hilfe des Informationsgewinns auf ihre Nützlichkeit hin untersuchen. Dazu werde zunächst eine diagnostische Maßnahme ohne Informationsleistung betrachtet. Diese ist charakterisiert durch die Identität von priorer und posteriorer Wahrscheinlichkeitsverteilung, die für jedes mögliche Testergebnis im Hinblick auf die vom Arzt in Betracht gezogenen Krankheitszustände des Patienten vorliegen muß. Insofern gilt für einen derartigen Diagnosetest:

$$
\mathrm{IG}\left(\mathrm{K} ; \mathrm{z}_{\mathrm{jh}}\right)=0 ; \quad \mathrm{h}=1, \ldots, \mathrm{k}_{\mathrm{j}}
$$

und damit insgesamt:

$$
\mathrm{G}\left(\mathrm{K}, \mathrm{Z}_{\mathbf{j}}\right)=0
$$

Der Informationsgewinn einer diagnostischen Maßnahme ohne Informationsleistung beträgt demzufolge Null. Aus der Bestimmungsgleichung für den Informationsge- 
winn eines Informationsinstruments folgt weiterhin, daß eine diagnostische Maßnahme dem Arzt genau dann keinen Informationsgewinn erbringt, wenn es sich um eine Maßnahme ohne Informationsleistung handelt.

Den diametral entgegengesetzten Fall verkörpert eine perfekte diagnostische Maßnahme, bei der jedes mögliche Testergebnis den Arzt eindeutig über den Krankheitszustand des Patienten informiert. Dies impliziert, daß die bedingte Information $\mathrm{I}\left(\mathrm{K} \mid \mathrm{z}_{\mathrm{jh}}\right)$ für ein beliebiges Testergebnis $\mathrm{z}_{\mathrm{jh}}$ einer derartigen Maßnahme Null beträgt. Damit beläuft sich die in der Zufallsvariablen $\mathrm{K}$ enthaltene Information, die nach dem Einsatz einer perfekten diagnostischen Maßnahme verbleibt, ebenfalls auf Null und der Informationsgewinn für den Arzt beträgt I(K). Eine perfekte diagnostische Maßnahme kennzeichnet demzufolge, daß sie die in der Ausgangslage in der Zufallsvariablen $\mathrm{K}$ enthaltene Information vollständig zu übermitteln vermag und damit die in Bezug auf den Krankheitszustand des Patienten herrschende Unbestimmtheit ganz abbaut.

Wie schon in Abschnitt 3.4 angesprochen, erbringt eine diagnostische Maßnahme im Normalfall eine Informationsleistung, die sie keinem der soeben diskutierten Spezialfälle zuordnen läßt. Aus Gleichung (3.35) ergibt sich, daß der Informationsgewinn unter dieser Voraussetzung unter $\mathrm{I}(\mathrm{K})$ liegt, da zumindest für ein mögliches Testergebnis die bedingte Information von $\mathrm{K}$ positiv sein muß ${ }^{201}$. Andererseits fällt der mit der diagnostischen Information verbundene Informationsgewinn höher als Null aus, da für mindestens ein mögliches Resultat die posterioren sich von den prioren Wahrscheinlichkeiten der Krankheitszustände unterscheiden müssen.

Aus diesen Überlegungen folgt, daß der aus der Informationstheorie stammende Begriff des Informationsgewinns sich dazu eignet, die Informationsleistung einer diagnostischen Maßnahme zu beschreiben. Der Informationsgewinn als Meßgröße besitzt darüber hinaus den Vorteil, daß sich mit seiner Hilfe in einer gegebenen medizinischen Ausgangslage die Menge der zur Verfügung stehenden diagnostischen $\mathrm{Maßnahmen}$ vollständig ordnen läßt ${ }^{202}$. Für zwei beliebige diagnostische Maßnahmen kann für gegebene priore Wahrscheinlichkeiten der Krankheitszustände immer

201: Andernfalls würde es sich um eine perfekte diagnostische Maßnahme handeln.

202: Präziser handelt es sich dabei um eine vollständige Quasiordnung; vgl. zum Begriff der Quasiordnung z.B. AHLHEIM, M./ROSE, M. (1989), S. 249. 
angegeben werden, ob beide dem Arzt denselben Informationsgewinn im Hinblick auf den Krankheitszustand des Patienten liefern, oder ob ein Test einen höheren Informationsgewinn zu erzeugen vermag.

Welche Auswirkungen ergeben sich für den Informationsgewinn, wenn man bei einer diagnostischen Maßnahme einzelne Testergebnisse zusammenfaßt203? Allgemein liegt eine derartige Zusammenfassung vor, wenn zumindest zwei verschiedene Testergebnisse nicht mehr separat, sondem als ein einziges Resultat mitgeteilt werden. Eine extreme Form repräsentieren binarisierte Tests, bei denen die Klassifikation sämtlicher möglichen Resultate nur noch als positiv bzw. negativ erfolgt. Formal läuft eine Zusammenfassung der Resultate einer diagnostischen Maßnahme $T_{j}$ darauf hinaus, daß man sich dazu entschließt, anstelle der ursprünglichen Zufallsvariablen $Z_{j}$ eine nicht-injektive Funktion $g\left(Z_{j}\right)$ zu beobachten. Wie aus der Jensen'schen Ungleichung für konkave Funktionen folgt, liegt die bedingte Information $I\left[K \mid g\left(Z_{j}\right)\right]$ im allgemeinen 204 über der bedingten Information $\mathrm{I}\left(\mathrm{K} \mid \mathrm{Z}_{\mathrm{j}}\right)$. Nach der Beobachtung von $g\left(Z_{j}\right)$ verbleibt damit beim Arzt eine größere Unsicherheit in Bezug auf den Krankheitszustand des Patienten, als wenn er die ursprünglichen Resultate von $T_{j}$ berücksichtigt. Anschaulich bedeutet dies, daß eine Zusammenfassung von Testergebnissen die Information reduziert, die eine diagnostische Maßnahme in Bezug auf den Krankheitszustand zu liefern vermag. Entsprechend fällt der Informationsgewinn $\mathrm{G}\left[\mathrm{K}, \mathrm{g}\left(\mathrm{Z}_{\mathrm{j}}\right)\right]$ geringer aus als $\mathrm{G}\left(\mathrm{K}, \mathrm{Z}_{\mathrm{j}}\right)$. Eine Aggregation der möglichen Testergebnisse einer diagnostischen Maßnahme kann daher nur mit einem Informationsverlust einhergehen, wobei dieser Verlust ceteris paribus bei binarisierten Tests besonders groß ausfällt.

203: Vgl. dazu auch RIFKIN, R.D. (1985).

204: Vgl. RENYI, A. (1966), S. 451. Falls die Zusammenfassung zumindest zwei Testergebnisse betrifft, die in der betrachteten medizinischen Ausgangslage möglich sind, gilt die Aussage aufgrund der strengen Konkavität der Entropiefunktion. 


\section{DER NUTZEN DIAGNOSTISCHER MASSNAHMEN FÜR DEN PA- TIENTEN}

\subsection{Die Rolle der Diagnostik}

Neben der Früherkennung besteht das vornehmliche Ziel der Diagnostik in der möglichst umfassenden Abklärung der Beschwerden eines Patienten, wobei damit insbesondere die Identifikation verursachender Faktoren und Prozesse angesprochen ist, soweit der Stand der Ätiologie dies erlaubt. Dieses diagnostische Wissen dient dem Arzt sowohl zur Prognose als auch zur Selektion bzw. Revision therapeutischer Handlungen. Dabei fungiert die ärztliche Ursachenforschung im Rahmen der Diagnostik als Mittel zum Zweck, denn in der überwiegenden Zahl der Fälle bildet die (vorläufige) Diagnose die Grundlage, auf der die Therapieentscheidung aufbaut. Die Diagnostik wird daher nicht um ihrer selbst willen betrieben ${ }^{1}$, sie bildet stattdessen die Voraussetzung für therapeutische Entscheidungen: "Diagnostik hat keinen Selbstzweck."2 . Ein analoger Zusammenhang gilt für die Diagnostik im Rahmen der Verlaufskontrolle, die zuvor getroffene Behandlungsentscheidungen bestätigt bzw. deren Modifikation oder gar Revision nahelegt ${ }^{3}$.

Dies schließt nicht aus, daß der Arzt manchmal - etwa in Notfällen - gezwungen sein kann, "Therapie ohne Diagnose" zu betreiben, deren Erfolg dann Rückschlüsse auf die (wahrscheinliche) Diagnose zuläßt (diagnosis ex juvantibus) ${ }^{4}$. Auch dann erleichtert allerdings ein Minimum an diagnostischer Information wie beispielsweise eine Blickdiagnose die Behandlungsentscheidung des Arztes; insofern sind diese Fälle durch den Einsatz der Therapie in einem besonders frühen Stadium des diagnostischen Prozesses gekennzeichnet. Die zuvor angesprochene Formulierung "Therapie ohne Diagnose" wäre demzufolge präziser zu bezeichnen als "Therapie

1: Eine Ausnahme bildet die medizinische Forschung, die sich z.B. zur Aufklärung pathophysiologischer Mechanismen diagnostischer Untersuchungen bedient; vgl. McNEIL, B.J./ADELSTEIN, S.J. (1976), S. 442.

2: WETZELS, E. (1982), S. 33; ähnlich WULFF, H.R. (1981), S. 80 und LINDLEY, D.V. (1975), S. 225.

3: $\quad$ Vgl. GRINER, P.F. et al. (1981), S. 559.

4: Vgl. GROSS, R. (1969), S. Iff. Insofern vermag auch die Therapie Informationen für die Diagnose zu liefern, z.B. wenn sich aufgnund der Reaktionen des Patienten auf die ärtliche Behandlung ein strittiges Krankheitsbild abklären läßt; vgl. BREYER, F. (1982), S. 148. Bailey faßt aus diesem Grund die Reaktion eines Patienten auf eine bestimmte Behandlung ebenfalls als Diagnosetest auf; vgl. BAILEY, N.T.J. (1977), S. 79. 
ohne intensivere Diagnostik". Eine reine Therapie ohne Diagnose, die auf jegliche Informationserhebung verzichtet, gerät mit hoher Wahrscheinlichkeit in die Nähe der (medical) "malpractice"5.

In allen oben angesprochenen Fällen existiert eine enge Verbindung zwischen medizinischer Diagnostik und therapeutischen Entscheidungen. Dieser Zusammenhang liefert den Schlüssel zur Bewertung diagnostischer Maßnahmen. Das typische ärztliche Entscheidungsproblem besteht in der Frage, wie mit einem Patienten weiter zu verfahren ist. Dieses ursprüngliche Entscheidungsproblem kann der Arzt einmal lösen, indem er sich beispielsweise zur Einleitung einer bestimmten Therapie entschließt. Der Nutzeneffekt dieser ärztlichen Entscheidung resultiert im wesentlichen aus den damit für den Patienten verbundenen medizinischen Konsequenzen, die in ihrer Gesamtheit den Behandlungserfolg ausmachen.

Andererseits besteht die Möglichkeit, mit Hilfe diagnostischer Maßnahmen zusätzliche Information zu erheben. Wie die weitere Analyse zeigen wird, hängt der auf dieser Information beruhende Nutzeneffekt davon ab, ob die diagnostischen Resultate eine Revision der ursprünglichen Entscheidung nahelegen. Falls der Arzt in keinem Fall von seinem Vorgehen in der Situation ohne weitere Diagnostik abweicht, ändern sich aus der Sicht des Patienten auch die medizinischen Konsequenzen nicht und die zusätzliche diagnostische Information zeitigt keinen differentiellen Behandlungserfolg. Unter dieser Voraussetzung erwächst dem Patienten aus dem Einsatz einer diagnostischen Maßnahme kein Nutzeneffekt.

Sobald jedoch eine diagnostische Information den beim Patienten eintretenden Behandlungserfolg zu beeinflussen vermag, läßt sich der darauf beruhende Nutzeneffekt der dazugehörigen diagnostischen Maßnahme zuschlagen. Der Nutzeneffekt einer diagnostischen Maßnahme repräsentiert jene Wohlfahrtsänderung, die dem Patienten aus dem Einsatz dieser Maßnahme erwächst. Der Nutzen der diagnostischen Information bzw. einer diagnostischen Maßnahme entspricht somit der monetären Bewertung des Nutzeneffekts, der auf diese Information bzw. diese Maßnahme zurückgeht, durch den Patienten6.

5: $\quad$ GROSS, R. (1966), S. 35.

6: Der Begriff des Nutzens wird hier im Sinne der Kosten-Nutzen-Analyse gebraucht; vgl dazu Abschnitt 2.1.1. 
Allgemein formuliert, repräsentiert der Arzt einen Aktor, der in einer Entscheidungssituation genau eine Alternative aus einer Menge auszuwählen hat, welche die für ihn möglichen Handlungen enthält. Weiterhin stehen ihm diagnostische Maßnahmen zur Verfügung, durch deren Einsatz der Arzt die in der Ausgangslage vorhandene Informationsbasis verbreitern kann. Der Nutzen diagnostischer Information stellt damit einen Spezialfall des folgenden entscheidungstheoretischen Problems dar: welchen Wert besitzt ein Informationsinstrument in einer gegebenen Entscheidungssituation?

Um dieses Problem lösen zu können, muß die Entscheidungssituation des Arztes genauer dargestellt werden ${ }^{7}$. Zu diesem Zweck sind die in Abschnitt 2.4 genannten Voraussetzungen der Analyse zu ergänzen. Dabei ergibt sich in zweifacher Hinsicht eine Modifikation der in der Entscheidungstheorie üblicherweise untersuchten Situation. Dies betrifft zunächst die Tatsache, daß nicht der Arzt selbst, sondern der Patient die medizinischen Konsequenzen einer Entscheidung zu tragen hat. Der Arzt handelt somit als Sachwalter des Patienten, wobei a priori unklar bleibt, inwieweit es dem Patienten als Prinzipal gelingt, den Agenten Arzt zu einem Handeln in seinem Interesse zu veranlassen.

Weiterhin fallen die den Patienten betreffenden Konsequenzen ärztlicher Handlungen im wesentlichen in nicht-monetärer Form an. Im Gegensatz zu den in der entscheidungstheoretischen Literatur vornehmlich behandelten Problemen genügt es im medizinisch-diagnostischen Kontext daher nicht, lediglich monetäre Konsequenzen zu berücksichtigen. Vielmehr benötigt man in einer medizinischen Entscheidungssituation eine vollständige Beschreibung der Gesundheitseffekte, die das ärztliche Handeln beim Patienten bewirken kann. Diese Beschreibung erfolgt in Abschnitt 4.2.2 mit Hilfe einzelner Komponenten des individuellen Gesundheitszustands, deren Ausprägungen gerade die relevanten medizinischen Konsequenzen abbilden.

7: Vgl. zur Entscheidungstheorie SCHNEEWEISS, H. (1966); RAIFFA, H. (1968); LINDLEY, D.V. (1974); MENGES, G. (1974); BAMBERG, G./COENENBERG, A.G. (1989) sowie MAG, W. (1990). Die statistischen Anwendungen stehen bei FERGUSON, T.S. (1967); BAMBERG, G. (1972) und BERGER, J.O (1985) im Vordergrund, während BLACKWELL, D./GIRSHICK, M.A. (1954) und BÜHLMANN, H./LOEFFEL, H./NIEVERGELT, E. (1975) spieltheoretische Aspekte betonen. 


\subsection{Die Entscheidungssituation des Arztes}

\subsubsection{Mögliche ärztliche Entscheidungen}

Im folgenden gelte weiterhin die in Abschnitt 2.4 geschilderte Ausgangslage. Der Arzt weiß demzufolge in einer gegebenen Entscheidungssituation, welche endliche Menge $\mathrm{K}$ von Krankheitszuständen er bei seinem Patienten in Betracht zu ziehen hat. Als Aktion sei jede Handlung des Arztes bezeichnet, die geeignet ist, aus seiner Sicht den Abschluß des Krankheitsfalls zu bewirken. Jede Aktion repräsentiert eine Entscheidung, die der Arzt zu einem bestimmten Zeitpunkt fällt und danach nicht mehr modifizieren kann. Es handelt sich damit um sogenannte Letztentscheidungen, die das weitere Vorgehen des Arztes determinieren. Wie die nachfolgende Aufzählung verdeutlicht, muß eine Aktion allerdings keineswegs das Ende der Krankheitsepisode aus der Sicht des Patienten festlegen.

Als Aktionen sind zunächst sämtliche therapeutischen Handlungen anzusehen, die bei mindestens einem der möglichen Krankheitszustände in Frage kommen. Sie bilden gewissermaßen die natürlichen Elemente der Aktionenmenge, schöpfen jedoch in der Regel das Spektrum der vorhandenen Alternativen nicht vollständig aus. Im allgemeinen besteht ferner für den Arzt die Möglichkeit, den Patienten zu einem (anderen) Facharzt zu überweisen oder in ein Krankenhaus einzuweisen. Schließlich kann der Arzt - insbesondere, falls der Zustand "völlige Gesundheit" in der Menge K enthalten ist - den Patienten ohne weitere Behandlung nach Hause schicken ${ }^{8}$.

Die Gesamtheit aller in einer Entscheidungssituation zu berücksichtigenden Aktionen bildet eine Menge A, die als Aktionen- bzw. Alternativenraum bezeichnet wird $^{9}$. Zur Vereinfachung der weiteren Analyse wird angenommen, A sei eine endliche Menge:

$$
A=\left\{a_{1}, \ldots, a_{r}\right\}
$$

8: Der Einsatz einer diagnostischen Maßnahme stellt in dieser Terminologie keine Aktion dar, da es sich nicht um eine Letztentscheidung handelt. Wie weiter unten erläutert wird, benutzt der Arzt diagnostische Resultate, indem er auf der Grundlage dieser zusätzlichen Information eine Aktion auswählt.

9: Vgl. z.B. BAMBERG, G./COENENBERG, A.G. (1989), S. 14; MAG, W. (1990), S. 45. 
Der Alternativenraum muß so beschaffen sein, daß der Arzt in einer gegebenen Entscheidungssituation genau eine Aktion auswählen kann. Diese Forderung impliziert einerseits die Vollständigkeit der Menge A, d.h. A enthält jede mögliche Aktion ${ }^{10}$. Andererseits bedingt die Forderung eine hinreichend differenzierte Struktur des Alternativenraums, da jeweils zwei verschiedene Aktionen sich gegenseitig ausschließen müssen. Konkret bedeutet dies, daß es zur Analyse der betrachteten Entscheidungssituation unter Umständen nicht ausreicht, lediglich pauschal die Aktion "Einweisung in ein Krankenhaus" zu berücksichtigen, sondern beispielsweise zwischen verschiedenen Krankenhaustypen zu unterscheiden ist. In diesem Fall repräsentiert die Einweisung in eine bestimmte Fachklinik jeweils eine eigenständige Aktion.

Es erweist sich als sinnvoll, anstelle der Menge der Aktionen A eine erweiterte Menge $A^{*}$ zu betrachten, die in einem gewissen Sinn die einzelnen Aktionen ebenfalls enthält. Diese erweiterte Menge $A^{*}$ umfaßt alle Wahrscheinlichkeitsverteilungen, die auf der Menge A definiert werden können ${ }^{11}$ :

$$
A^{*}=\left|P \in \mathbb{R}^{r}\right| P_{f} \geq 0 ; f=1, \ldots, r ; \sum_{f=1}^{r} P_{f}=1 \mid \text {. }
$$

Jedes Element $\mathrm{P}$ der Menge $\mathrm{A}^{*}$ entspricht einem Zufallsexperiment, dessen Ergebnisraum die Menge A der Aktionen bildet und das die Ausgänge $a_{\mathrm{f}}$ mit den Wahrscheinlichkeiten $P_{f}=P\left(a_{f}\right)$ liefert. Insofern legt die Wahl einer bestimmten Wahrscheinlichkeitsverteilung im allgemeinen noch nicht fest, welche Aktion zum Zuge kommt. Man bezeichnet diese Elemente der Menge $A^{*}$ daher als gemischte bzw. randomisierte Aktionen ${ }^{12}$. Offenbar enthält die Menge $A^{*}$ als Spezialfälle Elemente, die in Bezug auf das ärztliche Handeln jeweils zu einem Element der Menge A äquivalent sind. Jeder Aktion $a_{f}$ entspricht eine degenerierte Wahrscheinlichkeitsverteilung aus $\mathrm{A}^{*}$, welche diese Aktion mit der Wahrscheinlichkeit Eins nach sich zieht. Identifiziert man die entsprechenden Elemente der Mengen A und $A^{*}$, so enthält die erweiterte Aktionenmenge zugleich die Aktionen $a_{f}$, die aus naheliegenden Gründen als nicht-randomisierte Aktionen bezeichnet werden.

10: Folglich bildet im allgemeinen auch "Nichtstun" bzw. "Unterlassen" eine Aktion.

11: Dabei wird vorausgesetzt, daß die Elemente von $A$ in einer festen Anordnung vorliegen.

12: Vgl. FERSCHL, F. (1975), S. 77ff., der ebenfalls nur endliche Aktionenmengen betrachtet und damit ausschließlich einfache - d.h. diskrete und endliche - Wahrscheinlichkeitsmaße als 
Die Betrachtung randomisierter Aktionen mag im medizinischen Kontext zunächst kontra-intuitiv erscheinen ${ }^{13}$. Allerdings läßt sich kaum bestreiten, daß der Arzt grundsätzlich über die Möglichkeit verfügt, die Wahl einer Aktion $\mathrm{a}_{\mathrm{f}}$ im Rahmen eines Zufallsexperiments festzulegen. Ob er diese Möglichkeit in einer gegebenen Entscheidungssituation tatsächlich nutzt, hängt von der vollständigen Spezifikation seines Entscheidungsproblems $\mathrm{ab}^{14}$.

Anstelle direkt eine Aktion aus der Menge $A^{*}$ auszuwählen und damit eine Handlungsentscheidung zu treffen, kann der Arzt zunächst (weitere) Diagnostik betreiben ${ }^{15}$. Für diese Informationsentscheidung stehen ihm gemäß der in Abschnitt 2.4.3 angesprochenen Voraussetzung insgesamt $\mathrm{m}$ diagnostische Maßnahmen zur Verfügung, wobei es sich entweder um einfache oder zusammengesetzte Maßnahmen handelt. Dabei wird unterstellt, der Arzt besitze nur die Möglichkeit, genau eine dieser diagnostischen Maßnahmen einzusetzen, um im Anschluß daran eine Aktion auszuwählen. Mit dieser Voraussetzung der einmaligen Informationsbeschaffung werden sequentielle diagnostische Vorgehensweisen ausgeschlossen. Insbesondere kann der Arzt die Entscheidung, ob weitere Diagnostik zu betreiben oder eine Aktion zu ergreifen ist, nicht von den Resultaten einer diagnostischen Maßnahme abhängig gestalten.

Entscheidet sich der Arzt für eine diagnostische Maßnahme $T_{j}$, dann verschiebt er damit die Handlungsentscheidung zugunsten der Beobachtung einer Zufallsgröße $Z_{j}$, von deren Realisation die gewählte Aktion abhängen kann. Eine mögliche Vorgehensweise spezifiziert in diesem Fall für jedes diagnostische Resultat der betrachteten Maßnahme, welche Aktion der Arzt im Anschluß daran ergreifen wird. Die Auswahl des Arztes kann sich dabei grundsätzlich entweder auf die nicht-randomisierten Aktionen $a_{f}$ beschränken oder die gesamte Menge $A^{*}$ umfassen. Falls lediglich die

randomisierte Aktionen erhält. Allgemeinere Mengen randomisierter Aktionen behandeln BLACKWELL, D./GIRSHICK, M.A. (1954), S. 26ff. und FERGUSON, T.S. (1967), S. 22ff.

13: Diese Vermutung besteht auch bei anderen entscheidungstheoretischen Anwendungen, vgl. dazu die Erläuterung von BERGER, J.O. (1985), S. 14 f.

14: Wie die Analyse des Optionspreises diagnostischer Maßnahmen in Abschnitt 4.3 zeigt, erbringt ein Randomisieren unter den in dieser Arbeit verwendeten Voraussetzungen keinen zusätzlichen Vorteil.

15: Zur Abgrenzung von Handlungs- und Informationsentscheidungen vgl. MAG, W. (197\%), S. $131 \mathrm{ff}$. 
Menge A in Betracht kommt, läßt sich jede ärztliche Vorgehensweise durch eine Funktion $d_{j}$ wie folgt repräsentieren:

$$
\begin{aligned}
d_{j}: z_{j} & \rightarrow A . \\
z_{j h} & \rightarrow d_{j}\left(z_{j h}\right) .
\end{aligned}
$$

Eine derartige Funktion wird als Entscheidungsfunktion bezeichnet. Eine Entscheidungsfunktion ordnet jedem möglichen diagnostischen Resultat eindeutig eine bestimmte Aktion des Arztes zu. Genauer handelt es sich bei $d_{j}$ um eine nicht-randomisierte Entscheidungsfunktion, da der Arzt im Anschlu $B$ an jedes Testergebnis $z_{j h}$ eine nicht-randomisierte Aktion ergreift. Die Menge aller nicht-randomisierten Entscheidungsfunktionen, die bei der betrachteten diagnostischen Maßnahme auftreten können, sei mit $D_{j}$ bezeichnet.

Die Menge der Entscheidungsfunktionen, die eine diagnostische Maßnahme $T_{j}$ dem Arzt ermöglicht, erweitert sich, wenn die Möglichkeit einer Randomisierung berücksichtigt wird. Formal bedeutet dies, da $B$ jede ärztliche Vorgehensweise nun durch eine Funktion $\delta_{\mathrm{j}}$ angegeben wird, für die gilt:

$$
\begin{aligned}
\delta_{j}: z_{j} & \rightarrow A^{*} . \\
z_{j h} & \rightarrow \delta_{j}\left(z_{j h}\right) .
\end{aligned}
$$

Eine Entscheidungsfunktion $\delta_{j}$ ordnet jedem möglichen diagnostischen Resultat eindeutig eine Aktion $P \in A^{*}$ und damit eine Wahrscheinlichkeitsverteilung auf dem Alternativenraum A zu. Damit läßt sich eine Entscheidungsfunktion $\delta_{\mathrm{j}}$ charakterisieren, indem man für jedes Testergebnis die Wahrscheinlichkeiten angibt, mit denen der Arzt die einzelnen Aktionen $a_{f}$ ergreift. Die Menge aller derartigen Entscheidungsfunktionen sei mit $\mathrm{D}_{\mathrm{j}}^{*}$ bezeichnet. 
Im Vergleich zur Situation ohne Informationsbeschaffung erweitern sich die Entscheidungsmöglichkeiten des Arztes beträchtlich, denn die Menge aller Aktionen $A^{*}$ ist in gewisser Weise in $D_{j}{ }^{*}$ enthalten. Jede Aktion $P \in A^{*}$ erweist sich im Hinblick auf das ärztliche Handeln äquivalent zu einer konstanten Funktion $\delta_{P} \in D_{j}{ }^{*}$, wobei für $\delta_{\mathrm{P}}$ gilt $^{16}$ :

$$
\delta_{\mathrm{P}}: Z_{\mathrm{j}} \rightarrow \mathrm{P} \in \mathrm{A}^{*}
$$

In diesem Fall entscheidet sich der Arzt dafür, unabhängig von dem sich einstellenden diagnostischen Resultat eine feste Aktion $\mathrm{P}$ zu ergreifen. Dies bedeutet, $\mathrm{da} B$ die diagnostische Information die Aktionswahl des Arztes nicht beeinflußt und damit insgesamt keine Berücksichtigung findet.

Wie zuvor schon die Menge $A^{*}$, enthält auch $D_{j}{ }^{*}$ sowohl randomisierte als auch nicht-randomisierte Elemente. Eine Entscheidungsfunktion $\delta_{\mathrm{j}}$ erweist sich bezüglich des ärztlichen Handelns als äquivalent zu einer nicht-randomisierten Entscheidungsfunktion, falls der Arzt für jedes diagnostische Resultat mit Wahrscheinlichkeit Eins eine bestimmte Aktion $\mathrm{a}_{\mathrm{f}}$ ergreift. Dagegen handelt es sich um eine randomisierte Entscheidungsfunktion, wenn die Vorgehensweise $\delta_{\mathrm{j}}$ für mindestens ein Testergebnis die Wahl einer randomisierten Aktion vorschreibt. Mit einer randomisierten Entscheidungsfunktion entschließt sich der Arzt demnach dazu, zwei Zufallsexperimente in bestimmter Weise hintereinander zu schalten. Zunächst liefert die Anwendung der diagnostischen Maßnahme $T_{j}$ eine Realisation der Zufallsvariablen $Z_{j}$. Dieses Resultat legt zugleich die Wahrscheinlichkeiten fest, mit denen im Anschluß daran - in einem zweiten Zufallsexperiment - die einzelnen Elemente der Aktionenmenge A ausgewählt werden ${ }^{17}$.

16: Die Äquivalenzaussage bezieht sich ausschließlich auf Nutzenaspekte und läßt sich anhand der in Abschnitt 4.2.2. dargestellten Bewertung ärztlicher Entscheidungen leicht nachweisen. Sobald jedoch z.B. Kosten der Informationsbeschaffung berücksichtigt werden, gilt diese Äquivalenz nicht mehr.

17: Vgl. FERSCHL, F. (1975), S. 108f.

Man bezeichnet die hier betrachteten Entscheidungsfunktionen als "behavioral decision functions"; vgl. z.B. FERGUSON, T.S. (1967), S. 25. Daneben besteht auch die Möglichkeit, randomisierte Entscheidungsfunktionen als Wahrscheinlichkeitsverteilungen auf $D_{j} z$ definieren. Als Menge $D_{j}$ der randomisierten Entscheidungsfunktionen ergibt sich dann die Menge aller Wahrscheinlichkeitsverteilungen auf $\mathrm{D}_{\mathrm{j}}$, wobei es sich unter den getroffenen Vorausset- 


\subsubsection{Handlungskonsequenzen und ihre Bewertung}

Zur weiteren Beschreibung des Entscheidungsproblems benötigt man möglichst präzise und vollständige Angaben über die Folgen bzw. Konsequenzen, die das ärztliche Handeln in einer gegebenen Entscheidungssituation auslöst. Dabei geht es an dieser Stelle ausschließlich um jene Folgen, die den Patienten betreffen ${ }^{18}$. Man könnte vermuten, daß zu diesem Zweck sämtliche Aktionen (die Menge $A^{*}$ ) sowie alle Entscheidungsfunktionen (die Mengen $D_{j}^{*}$ ) betrachtet werden müssen. Dies erweist sich jedoch als unnötig, denn bei der Wahl sowohl einer randomisierten Aktion als auch einer Entscheidungsfunktion kommt dem Patienten letztlich - ex post gesehen - genau eine Aktion $a_{\mathrm{f}}$ zugute ${ }^{19}$. Insofern reicht es aus, die Konsequenzen für alle nicht-randomisierten Aktionen zu ermitteln ${ }^{20}$. Allerdings dürften die Folgen einer gegebenen Aktion des Arztes wesentlich davon abhängen, welcher Krankheitszustand bei dem Patienten vorliegt. Insgesamt benötigt man daher eine Auflistung aller patientenbezogenen Konsequenzen für sämtliche Kombinationen aus ärztlicher Handlung $a_{f}$ und Krankheitszustand $K_{i}$, die in der interessierenden Entscheidungssituation auftreten können ${ }^{21}$.

zungen ausschließlich um einfache Wahrscheinlichkeitsmaße handelt; vgl. FERSCHL, F. (1975), S. 100ff.; BLACKWELL, D./GIRSHICK, M.A. (1954), S. $81 \mathrm{ff.}$

Weiterhin sichem die hier verwendeten Annahmen - insbesondere die Endlichkeit des Aktionenraumes A -, daß beide Arten der Randomisierung von Entscheidungsfunktionen äquivalent sind und es daher ausreicht, die im Text definierten Entscheidungsfunktionen zu betrachten; vgl. BLACKWELL, DJGIRSHICK, M.A. (1954), S. 172ff. Im allgemeinen gilt diese Äquivalenz jedoch nicht; vgl. FERGUSON, T.S. (1967), S. $25 \mathrm{ff}$.

18: Eine Rechtfertigung dieser Beschränkung liefert die Annahme, der Arzt handle vollständig im Interesse des Patienten; siehe dazu ausführlicher Abschnitt 4.2.4.

19: Im Gegensatz zur Wahl einer nicht-randomisierten Aktion kann diese Aktion im allgemeinen a priori nicht angegeben werden.

20: Dies gilt allerdings nur, wenn die Präferenzen des Patienten - wie weiter unten vorausgesetzt die Erwartungsnutzenhypothese erfüllen.

21: Im Gegensatz zu dem in der Entscheidungstheorie häufig anzutreffenden Verfahren, direkt eine Nutzenfunktion des Aktors auf dem Produktraum aus Aktions- und Zustandsraum (hier: A X K) zu definieren, kann in dem hier untersuchten medizinisch-diagnostischen Kontext auf die explizite Betrachtung der Konsequenzen aus zwei Gründen nicht verzichtet werden. Einerseits bilden sie die Schnittstelle, an der das medizinische Wissen des Arztes und die Präferenzen des Patienten aufeinandertreffen. Die Angabe der Konsequenzen stellt daher eine notwendige Informationsbasis für die Bewertung ärztlicher Handlungen durch den Patienten dar. Andererseits ermöglicht erst die Berücksichtigung einer Konsequenzenfunktion in dem in der weiteren Analyse verwendeten entscheidungstheoretischen Rahmen, individuelle Zahlungsbereitschaften abzuleiten; vgl. dazu auch LaVALLE, I.H. (1968), S. 252. 
Als hauptsächliche Folge eines bestimmten Paars $\left(a_{\mathrm{f}}, \mathrm{K}_{\mathrm{i}}\right)$ tritt im allgemeinen eine Veränderung der Gesundheit des Patienten ein. Da Gesundheit nicht direkt meßbar ist, benötigt man geeignete Indikatoren, um eine adäquate Beschreibung der Konsequenzen ärztlichen Handelns für den Patienten zu erreichen. Typischerweise sind die in einer medizinischen Entscheidungssituation möglichen Gesundheitseffekte von einer Vielfalt, welche die Berücksichtigung mehrerer Indikatoren erfordert ${ }^{22}$. Allerdings kommen dabei nur solche Indikatoren in Betracht, die einen unmittelbaren Bezug zur Gesundheit des Patienten aufweisen. Dabei handelt es sich um Indikatoren, die beispielsweise die Beeinträchtigung wichtiger Funktionen bzw. Tätigkeiten messen oder über sonstige Konsequenzen ärztlicher Handlungen (z.B. Schmerzen) informieren 23 .

Diese individuumbezogenen Gesundheitsindikatoren werden in der Nutzentheorie ganz allgemein als Attribute bezeichnet ${ }^{24}$. Demzufolge sind in einer medizinischen Entscheidungssituation in der Regel mehrere Attribute notwendig, um die für den Patienten wichtigen Konsequenzen ärztlichen Handelns zu beschreiben ${ }^{25}$. Bei gegebenem Krankheitszustand des Patienten schlagen sich die Folgen einer Aktion des Arztes als Ausprägungen der entscheidungsrelevanten Attribute nieder. Im allgemeinen nimmt man die Anzahl dieser Attribute als endlich an und geht ferner davon aus, daß jedes Paar $\left(\mathrm{a}_{\mathrm{f}}, \mathrm{K}_{\mathrm{i}}\right)$ die Konsequenzen bzw. die Ausprägungen der Attribute eindeutig festlegt. In der folgenden Analyse wird die Gültigkeit dieser beiden Annahmen unterstellt.

22: Vgl. LINDLEY, D.V. (1975), S. 226; JENNETT, B.TEASDALE, G.M./KNILL-JONES, R.P. (1975), S. 232.

23: $\mathrm{Zu}$ individuumbezogenen Gesundheitsindikatoren siehe KRIEDEL, T. (1980), S. 338ff.; STODDART, G.L. (1982), S. 402f. sowie ausführlich BROOK, R.H. et al. (1976); CULYER, A.J. (1978), S. 44ff.; TORRANCE, G.W. (1986), S. 7ff. und BOWLING, A. (1991); die zum Teil auch Anwendungen auf spezifische Entscheidungssituationen diskutieren. Zur Festlegung individueller Gesundheitsindikatoren in einzelnen Bereichen vgl. WRIGHT, K.G. (1978); YULE, B.F./VAN AMERONGEN, B.M./VAN SCHAIK, M.C.M. (1986) und insbesondere AVERY, A.D. et al. (1976).

24: Vgl. zum Begriff des Attributs sowie ausführlich zur Darstellung individueller Präferenzen für mehrere Attribute KEENEY, R.L./RAIFFA, H. (1976), S. 66ff.; FRENCH, S. (1983) sowie FRENCH, S. (1986), S. 102ff.

Die "multiattribute utility theory" untersucht unter anderem, welche Eigenschaften die individuellen Präferenzen auf einer Menge von Attributen besitzen müssen, damit ihre Repräsentation durch eine Nutzenfunktion mit einer einfachen funktionalen Form gelingt; vgl. dazu auch Abschnitt 4.3.3. 
Unter diesen Voraussetzungen lassen sich in einer medizinischen Entscheidungssituation die Konsequenzen jeder Aktion $a_{f}$ des Arztes bei gegebenem Krankheitszustand $\mathrm{K}_{\mathrm{i}}$ des Patienten durch einen p-dimensionalen Vektor darstellen, dessen Komponenten aus reellen Zahlen bestehen sollen. Demzufolge existiert eine Funktion

$$
\begin{aligned}
g: A \times K & \rightarrow R^{p}, \\
\left(a_{f}, K_{i}\right) & \rightarrow g_{f i}=g\left(a_{f}, K_{i}\right),
\end{aligned}
$$

welche die gewünschte Beschreibung der Konsequenzen leistet. Der Vektor $\mathrm{g}_{\mathrm{fi}}$ gibt die Konsequenzen an, die aufgrund der Aktion $a_{f}$ des Arztes für den Patienten entstehen, falls $\mathrm{K}_{\mathrm{i}}$ dessen wahrer Krankheitszustand ist.

Ein typischer Konsequenzenvektor zeigt in den ersten p-1 Komponenten individuell gesundheitsrelevante Tatbestände $a^{26}$. Bei diesen Komponenten handelt es sich um Gesundheitseffekte, an deren Konsum der Patient - gegeben $\left(\mathrm{a}_{\mathrm{f}}, \mathrm{K}_{\mathrm{i}}\right)$ - gebunden ist, da sie nicht übertragbar und daher auch nicht einem Tausch zugänglich sind $^{27}$. Im Gegensatz dazu repräsentiert die p-te Komponente des Konsequenzenvektors den individuellen Bestand an einem unbeschränkt handelbaren Gut. Dieses Gut erlaubt den Vergleich unterschiedlicher Konsequenzenvektoren mit Hilfe von Zahlungsbereitschaften ${ }^{28}$. Im folgenden bezeichne diese p-te Komponente ganz allgemein das Geldvermögen des Patienten. Abbildung 12 veranschaulicht die Konsequenzen, die eine vom Arzt ergriffene Aktion $a_{f}$ nach sich zieht.

25: Vgl. KRISCHER, J.P. (1976), S. 952; CARD, W.I. (1979), S. 214; TORRANCE, G.W./BOYLE, M.H./HORWOOD, S.P. (1982) sowie BOWLING, A. (1991), S. $2 \mathrm{ff}$.

26: Bei Indikatoren, die nicht auf metrischem Skalenniveau gemessen werden, ordnet man den einzelnen Ausprägungen reelle Zahlen zu. Die daraus entstehende Ordnung der Ausprägungen sei mit der durch die Präferenzen des Patienten induzierten Ordnung kompatibel, d.h. strikt präferierte Ausprägungen erhalten eine höhere Zahl.

27: Vgl. FUCHS, V.R./ZECKHAUSER, R. (1987), S. 263; McGUIRE, A./HENDERSON, J./MOONEY, G.H. (1988), S. 32.

28: Vgl. zu dieser Struktur des Konsequenzenvektors KEENEY, R.L./RAIFFA, H. (1976), S. 84f. sowie S. 125ff. In der Gesundheitsökonomie wurde dieser Ansatz in rudimentärer Form zur Bestimmung individueller Zahlungsbereitschaften für (marginale) Veränderungen des Todesrisikos verwendet; vgl. BODILY, S.E. (1980); WEINSTEIN, M.C./SHEPARD, D.S./PLISKIN, J.S. (1980). 
Abb. 12: Die Konsequenzen ärztlicher Aktionen

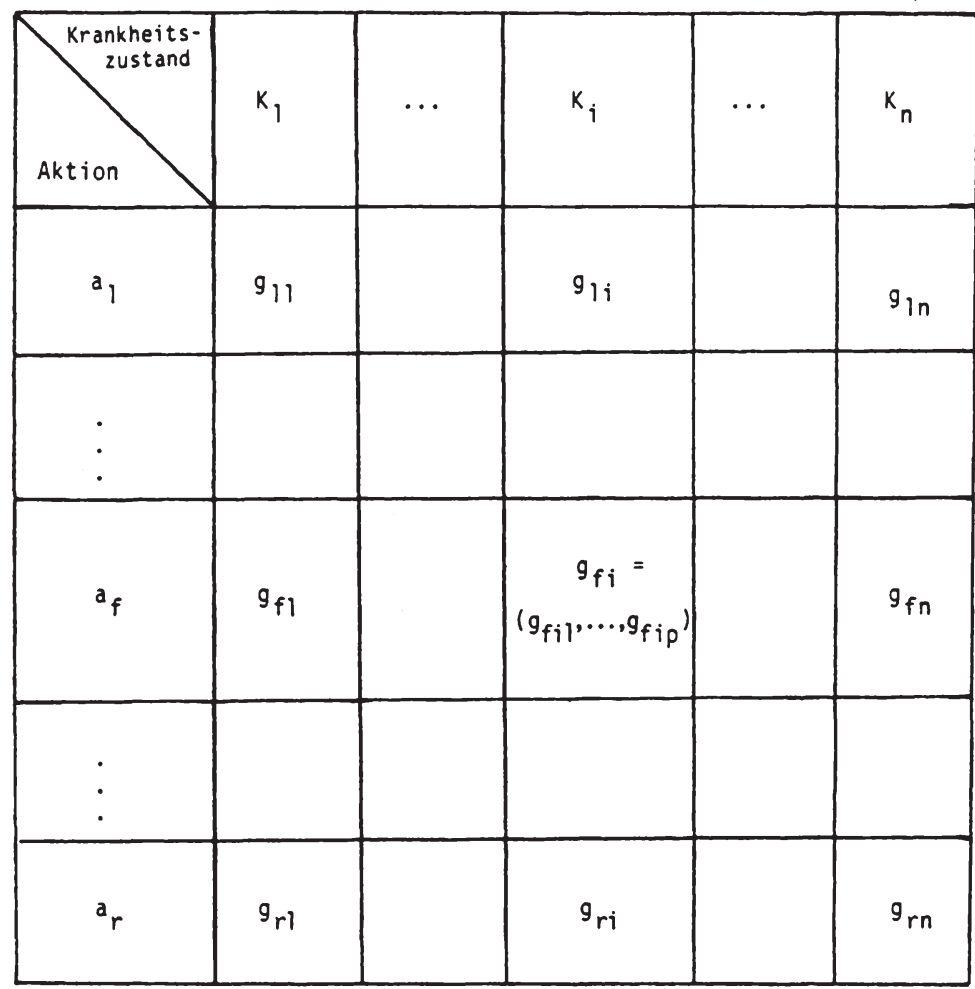

In einer medizinischen Entscheidungssituation gelingt es im allgemeinen nicht, die beim Patienten auftretenden Konsequenzen in deterministischer Form anzugeben. Dies beruht einerseits auf der Unsicherheit, die bezüglich des Krankheitszustands herrscht, in dem sich der Patient befindet. Andererseits kann der Arzt sein Handeln vom Zufall abhängig gestalten und auf diese Weise dafür sorgen, daß auch bei gegebenem Krankheitszustand die für den Patienten relevanten Konsequenzen nicht ein- 
deutig festliegen. Im weiteren Verlauf dieses Abschnitts wird ausschließlich jene Stochastik des Konsequenzenvektors diskutiert, die auf das ärztliche Handeln zurückgeht ${ }^{29}$.

Entscheidet sich der Arzt hingegen für eine randomisierte Aktion oder die Erhebung zusätzlicher diagnostischer Information, dann besteht a priori auch bei gegebenem Krankheitszustand Unsicherheit bezüglich der vom Arzt gewählten Aktion $a_{f}$ und damit zugleich im Hinblick auf die für den Patienten relevanten Konsequenzen. In beiden Fällen lassen sich jedoch für jeden möglichen Krankheitszustand $\mathrm{K}_{\mathrm{i}}$ des $\mathrm{Pa}$ tienten die Wahrscheinlichkeiten angeben, mit denen der Arzt eine Aktion $a_{f}$ ergreift. Daraus ergeben sich die Wahrscheinlichkeiten, mit denen die entsprechenden Konsequenzenvektoren eintreten. Die Wahl einer (nicht-degenerierten) Wahrscheinlichkeitsverteilung $P \in A^{*}$ bedeutet, daß der Arzt nach einem durch $P$ spezifizierten Zufallsprinzip Aktionen $a_{\mathrm{f}}$ ergreift. Befindet sich der Patient im Krankheitszustand $K_{i}$, treten die Konsequenzenvektoren $g_{f i}$ folglich mit den Wahrscheinlichkeiten $\mathrm{P}\left(\mathrm{a}_{\mathrm{f}}\right)$ ein. Die Stochastik des Konsequenzenvektors wird damit durch die zufällige Auswahl einer Aktion $a_{\mathrm{f}}$ erzeugt.

Im Falle der Informationsbeschaffung wählt der Arzt eine Entscheidungsfunktion aus, wenn er die diagnostische Maßnahme $T_{j}$ einsetzt. Bei einer nicht-randomisierten Entscheidungsfunktion ergreift er die Aktion $\mathrm{d}_{\mathrm{j}}\left(\mathrm{z}_{\mathrm{jh}}\right) \in \mathrm{A}$, falls sich das Testergebnis $\mathrm{z}_{\mathrm{jh}}$ einstellt. Sofern $\mathrm{d}_{\mathrm{j}}$ keine konstante Funktion repräsentiert, hängt die vom Arzt gewählte (nicht-randomisierte) Aktion vom diagnostischen Resultat ab. Dies impliziert, da $\beta$ bei gegebenem Krankheitszustand $K_{i}$ verschiedene Konsequenzenvektoren $\mathrm{g}_{\mathrm{fi}}$ mit positiven Wahrscheinlichkeiten auftreten können. Diese Wahrscheinlichkeiten erhält man, indem man die Niveaumengen $S_{f}$ der Entscheidungsfunktion $d_{j}$ betrachtet:

$$
S_{f}\left(d_{j}\right)=\left\{z_{j h} \in Z_{j} \mid d_{j}\left(z_{j h}\right)=a_{f}\right\}
$$

$$
P_{d_{j}}\left(a_{f} \mid K_{i}\right)=\sum_{z_{j h} \in S_{f}\left(d_{j}\right)} P\left(z_{j h} \mid K_{i}\right) ; i=1, \ldots, n ; f=1, \ldots, r .
$$

29: Zum Einfluß, den das unvollkommene Wissen bezüglich des Krankheitszustands des Patienten auf die Konsequenzen einer gegebenen ärztlichen Entscheidung besitzt, vgl. Abschnitt 4.2.3. 
Wie die Schreibweise bereits andeutet, handelt es sich um die bedingte Wahrscheinlichkeit der Aktion $a_{\mathrm{f}}$, wobei die Bedingung durch den Krankheitszustand $\mathrm{K}_{\mathrm{i}}$ (und die Entscheidungsregel $d_{j}$ ) gebildet wird. Die Stochastik des Konsequenzenvektors $\mathrm{g}_{\mathrm{fi}}$ rührt nun von der Zufallsabhängigkeit des diagnostischen Testergebnisses her.

Verwendet der Arzt schließlich eine randomisierte Entscheidungsfunktion, dann hängt der den Patienten betreffende Konsequenzenvektor $g\left(a_{f}, K_{i}\right)$ bei gegebenem Krankheitszustand gleich in doppelter Weise vom Zufall ab. Zunächst liefert die diagnostische Maßnahme mit den Wahrscheinlichkeiten $\mathrm{P}\left(\mathrm{z}_{\mathrm{jh}} \mid \mathrm{K}_{\mathrm{i}}\right)$ die einzelnen Testergebnisse. In einem zweiten Zufallsexperiment bestimmt die vom Arzt vermöge seiner Entscheidungsfunktion gewählte randomisierte Aktion $\delta_{j}\left(z_{j h}\right) \in A^{*}$, mit welchen Wahrscheinlichkeiten er die einzelnen Aktionen $a_{f}$ ergreift. Für die Wahrscheinlichkeiten der einzelnen Konsequenzenvektoren $g_{f i}$ gilt in diesem Fall:

$$
\mathrm{P}_{\delta_{j}}\left(a_{f} \mid K_{i}\right)=\sum_{h=1}^{k_{j}} P\left(z_{j h} \mid K_{i}\right) \cdot P\left(a_{f} \mid z_{j h}\right),
$$

wobei die bedingten Wahrscheinlichkeiten $\mathrm{P}\left(\mathrm{a}_{\mathrm{f}} \mid \mathrm{z}_{\mathrm{jh}}\right)$ gerade durch die Elemente des Vektors $\delta_{\mathrm{j}}\left(\mathrm{z}_{\mathrm{jh}}\right)$ gegeben sind. Die Stochastik des Konsequenzenvektors $\mathrm{g}_{\mathrm{fi}}$ bei gegebenem Krankheitszustand des Patienten beruht nun sowohl auf der Zufallsabhängigkeit der diagnostischen Resultate als auch auf der zufälligen Auswahl einer Aktion $\mathrm{a}_{\mathrm{f}}$ durch den Arzt.

Wie die vorangegangenen Bemerkungen verdeutlichen, wird eine ärztliche Entscheidung häufig die für den Patienten wichtigen Konsequenzen auch bei gegebenem Krankheitszustand nicht eindeutig festlegen. Es läßt sich in diesen Fällen jedoch immer ein Prospekt angeben, der durch die möglichen Konsequenzenvektoren einschließlich der dazugehörigen Wahrscheinlichkeiten festgelegt wird. Zum Beispiel ergibt sich als Folge einer randomisierten Aktion $P \in A^{*}$ für den Patienten der Prospekt

$$
\left[\begin{array}{lll}
\mathrm{g}_{1 \mathrm{i}} & \ldots & \mathrm{g}_{\mathrm{ri}} \\
& & \\
\mathrm{P}\left(\mathrm{a}_{1}\right) & \ldots & \mathrm{P}\left(\mathrm{a}_{\mathrm{r}}\right)
\end{array}\right]
$$


falls bei ihm der Krankheitszustand $\mathrm{K}_{\mathrm{i}}$ vorliegt. In ähnlicher Weise führt jede vom Arzt gewählte Entscheidungsfunktion zu einem eindeutig bestimmten Prospekt hinsichtlich der Konsequenzen. Eine randomisierte Entscheidungsfunktion $\delta_{\mathrm{j}}$ erzeugt für den Patienten bei vorgegebenem Krankheitszustand $\mathrm{K}_{\mathrm{i}}$ einen zusammengesetzten Prospekt, da neben der Stochastik der Aktionswahl des Arztes noch die Unsicherheit bezüglich des sich einstellenden Testergebnisses der diagnostischen Maßnahme $T_{j} z u$ berücksichtigen ist.

Damit legt jede ärztliche Entscheidung bei gegebenem Krankheitszustand $\mathrm{K}_{\mathrm{i}}$ eindeutig einen derartigen Prospekt fest ${ }^{30}$. In konzeptioneller Hinsicht kann ein Prospekt auch als Zufallsexperiment aufgefaßt werden, in dessen Verlauf ein Zufallsvektor $G$ die in dem Prospekt angeführten Realisationen mit den ebenfalls dort verzeichneten Wahrscheinlichkeiten liefert. Dabei ist jedem Prospekt genau ein Zufallsexperiment zugeordnet. Die Angabe des Zufallsvektors G zusammen mit der vom Arzt getroffenen Entscheidung und dem Krankheitszustand des Patienten reicht demzufolge aus, um die möglichen Konsequenzen vollständig zu beschreiben. Dies folgt aus der Tatsache, daß die ärztliche Entscheidung gemeinsam mit dem Krankheitszustand $K_{i}$ des Patienten in der oben beschriebenen Weise die Wahrscheinlichkeiten der einzelnen Realisationen von $\mathrm{G}$ festlegt. Insgesamt ergibt sich aufgrund der Wahl einer randomisierten Aktion $P \in A^{*}$ ein zufälliger Konsequenzenvektor $G\left(P, K_{i}\right)$, während eine Entscheidungsfunktion $\delta_{j} \in D_{j}^{*}$ einen Zufallsvektor $G\left(\delta_{j}, K_{i}\right)$ induziert, falls bei dem Patienten der Krankheitszustand $\mathrm{K}_{\mathrm{i}}$ vorliegt.

Um anzugeben, in welcher Weise der Patient die Folgen einer ärztlichen Entscheidung bewertet, benötigt man daher Informationen über seine Präferenzen bezüglich derartiger Zufallsvektoren bzw. Prospekte. Zu diesem Zweck wird angenommen, die Präferenzen des Patienten auf der Menge aller Prospekte lassen sich durch eine stetige, vollständige und transitive binäre Relation beschreiben ${ }^{31}$. Als entscheidende Voraussetzung sei ferner unterstellt, die individuelle Präferenzordnung genüge der Erwartungsnutzenhypothese bzw. besitze die Erwartungs-

30: Bei der Wahl einer Aktion a ergibt sich ein Prospekt, der mit Wahrscheinlichkeit Eins den Konsequenzenvektor $\mathrm{g}_{\mathrm{fi}}$ liefert.

31: Die Menge aller Prospekte entspricht hier der Menge aller diskreten Wahrscheinlichkeitsverteilungen auf $R^{P}$. Zum Begriff der Präferenzrelation bei Prospekten vgl. BLACKWELL, D./GIRSHICK, M.A. (1954), S. 102ff.; FERGUSON, T.S. (1967), S. $11 \mathrm{ff}$. 
nutzeneigenschaft ${ }^{32}$. Diese Eigenschaft impliziert die Existenz einer stetigen Funktion

$$
\mathrm{u}: \mathrm{R}^{\mathrm{P}} \rightarrow \mathrm{R},
$$

die bis auf monotone lineare Transformationen eindeutig bestimmt ist ${ }^{33}$. Die Funktion $u$ sei streng monoton, d.h. für zwei Vektoren $x$ und $y$ des $R^{p}$ gilt ${ }^{34}$.

$$
\text { Aus } x \geq y \text { und } x \neq y \text { folgt: } u(x)>u(y) \text {. }
$$

Über die Funktion u läßt sich in einfacher Weise eine Nutzenfunktion U gewinnen, welche die Präferenzen des Patienten auf einer Menge von Prospekten repräsentiert. Aus der Gültigkeit der Erwartungsnutzenhypothese folgt, daß der Patient einen Prospekt bewertet, indem er den damit verbundenen Erwartungswert der Funktion u bildet. Die Nutzenfunktion $U$ besitzt unter anderem die Eigenschaft, unter jeweils zwei Prospekten genau demjenigen einen höheren Wert zuzuordnen, den der Patient präferiert ${ }^{35}$.

Mit Hilfe der Nutzenfunktion U gelingt es, unter der Voraussetzung eines gegebenen Krankheitszustands $K_{i}$ eine Bewertung aller bisher betrachteten ärztlichen Ent-

32: Diese Voraussetzung läßt sich axiomatisch fundieren; vgl. dazu BLACKWELL, D./GIRSHICK, M.A. (1954), S. 103ff.; FERGUSON, T.S. (1967), S. 13ff.; SAMUELSON, P.A. (1983), S. 511ff. und MACHINA, M. (1987), S. 236, die ausschließlich diskrete Wahrscheinlichkeitsverteilungen betrachten.

33: Bei u handelt es sich um eine von Neumann-Morgenstern Nutzenfunktion, die aus der Repräsentation individueller Präferenzen über Prospekte auf $\mathrm{R}^{\mathrm{P}}$ gewonnen wird. Strenggenommen ist $u$ für alle degenerierten Wahrscheinlichkeitsverteilungen auf $R^{P}$ definiert. Man erhält die im Text angegebene Definition, indem man einen Prospekt, der mit Wahrscheinlichkeit Eins ein bestimmtes Element des $\mathrm{R}^{\mathrm{P}}$ liefert, gerade mit diesem Vektor identifiziert; vgl. FERSCHL, F. (1975), S. 46ff.; MACHINA, M. (1987), S. 233.

34: Mit dieser Annahme werden insbesondere schwach streng monotone Funktionen als Kandidaten für u ausgeschlossen; zum Begriff der schwach streng monotonen Funktion vgl. BAMBERG, G. (1975), S. $202 \mathrm{f}$.

Bei Indikatoren, deren Messung nicht auf einem metrischen Skalenniveau erfolgt, resultiert die im Text angesprochene Monotonieeigenschaft der Funktion u aus einer früher getroffenen Annahme; vgl. dazu Fußnote 26.

35: Diese Aussage bezieht sich auf die strikte Präferenz. Liegt diese nicht vor, ordnet $U$ beiden Prospekten denselben Wert und damit dasselbe Nutzenniveau zu. 
scheidungen im Einklang mit den Präferenzen des Patienten vorzunehmen. Aufgrund der oben angesprochenen eineindeutigen Zuordnung von Prospekten und Zufallsexperimenten läßt sich als Argument der Nutzenfunktion $U$ auch das durch die Wahrscheinlichkeitsverteilung des Zufallsvektors $G$ beschriebene Zufallsexperiment anführen. Im Interesse einer übersichtlichen Notation wird im folgenden ein anderes Vorgehen gewählt. Da der Krankheitszustand des Patienten und die vom Arzt getroffene Entscheidung annahmegemäß den relevanten Prospekt eindeutig festlegen, reicht es aus, diese beiden Komponenten als Argumente der Nutzenfunktion $U$ anzuführen. Diese Schreibweise besitzt den Vorteil, den Zusammenhang zwischen ärztlicher Entscheidung und Krankheitszustand des Patienten einerseits sowie den daraus resultierenden Konsequenzen und deren Nutzenbewertung andererseits klarer herauszustellen.

Mit dieser Konvention ergibt sich der Nutzen einer ärztlichen Entscheidung bei gegebenem Krankheitszustand des Patienten wie folgt:

$$
\mathrm{U}\left(\mathrm{a}_{\mathrm{f}}, \mathrm{K}_{\mathrm{i}}\right)=\mathrm{u}\left[\mathrm{g}\left(\mathrm{a}_{\mathrm{f}}, \mathrm{K}_{\mathrm{i}}\right)\right]
$$

falls der Arzt eine nicht-randomisierte Aktion $\mathrm{a}_{\mathrm{f}}$ ergreift;

$$
U\left(P, K_{i}\right)=\sum_{f=1}^{r} P\left(a_{f}\right) \cdot u\left[g\left(a_{f}, K_{i}\right)\right],
$$

falls der Arzt sich für eine randomisierte Aktion $P \in A^{*}$ entscheidet. Entschließt sich der Arzt dazu, eine diagnostische Maßnahme $T_{j}$ durchzuführen, so erhält man als relevante Nutzengröße:

$$
U\left(d_{j}, K_{i}\right)=\sum_{f=1}^{r} P_{d_{j}}\left(a_{f} \mid K_{i}\right) \cdot u\left[g\left(a_{f}, K_{i}\right)\right],
$$

falls eine nicht-randomisierte Entscheidungsfunktion $d_{j}$ eingesetzt wird, und schließlich:

$$
\mathrm{U}\left(\delta_{\mathrm{j}}, \mathrm{K}_{\mathrm{i}}\right)=\sum_{\mathrm{f}=1}^{\mathrm{r}} \mathrm{P}_{\delta_{\mathrm{j}}}\left(\mathrm{a}_{\mathrm{f}} \mid \mathrm{K}_{\mathrm{i}}\right) \cdot \mathrm{u}\left[\mathrm{g}\left(\mathrm{a}_{\mathrm{f}}, \mathrm{K}_{\mathrm{i}}\right)\right],
$$

falls der Arzt eine Entscheidungsfunktion $\delta_{j} \in D_{j}^{*}$ wählt. 


\subsubsection{Die Bewertung ärztlicher Entscheidungen}

Befindet sich ein Patient im Krankheitszustand $\mathrm{K}_{\mathrm{i}}$, so bewertet er jede mögliche Entscheidung des Arztes durch eine skalare Nutzengröße. Die bisher getroffenen Voraussetzungen sichern die Existenz einer Entscheidung, die optimal ist in dem Sinn, da $B$ kein anderes Element in der betrachteten Klasse von Entscheidungen einen höheren Nutzen erzeugt ${ }^{36}$. Bei gegebenem Krankheitszustand gibt es daher immer eine Aktion $P^{*} \in A^{*}$, für die der Nutzen $U\left(P, K_{i}\right)$ maximal wird ${ }^{37}$. Ebenso enthält die Menge $\mathrm{D}_{\mathrm{j}}{ }^{*}$ eine Entscheidungsfunktion $\delta_{\mathrm{j}}{ }^{*}$, die dem Patienten das höchstmögliche Nutzenniveau sichert ${ }^{38}$.

Typischerweise kennen jedoch weder der Arzt noch der Patient den tatsächlich vorliegenden Krankheitszustand mit Sicherheit. Ohne weitere Information läßt sich deshalb jeder ärztlichen Entscheidung zunächst ein n-dimensionaler Vektor W zuordnen. In seiner $\mathrm{i}$-ten Komponente $\mathrm{W}_{\mathrm{i}}$ enthält dieser Vektor den Nutzen des $\mathrm{Pa}$ tienten unter der Bedingung, daß dieser sich im Krankheitszustand $K_{i}$ befindet. Für eine Aktion $a_{\mathrm{f}}$ ergibt sich:

$$
\begin{aligned}
& W\left(a_{f}\right)=\left[U\left(a_{f}, K_{1}\right), \ldots, U\left(a_{f}, K_{n}\right)\right] . \\
& W_{i}\left(a_{f}\right)=U\left(a_{f}, K_{i}\right) ; \quad i=1, \ldots, n .
\end{aligned}
$$

In Analogie dazu erhält man für jede Aktion $P \in A^{*}$ bzw. jede Entscheidungsfunktion $\delta_{j} \in D_{j}^{*}$ einen Vektor $W(P)$ bzw. $W\left(\delta_{j}\right)$. Damit repräsentiert ganz allgemein ein Vektor $\mathbf{W}(\mathbf{x})$ diejenige Nutzenallokation, welche die Entscheidung $\mathbf{x}$ des Arztes für den Patienten erzeugt. Abbildung 13 veranschaulicht die möglichen Nutzenallokationen, wenn lediglich die nicht-randomisierten Aktionen in die Betrachtung eingehen.

36: Diese Entscheidung braucht allerdings nicht eindeutig bestimmt zu sein.

37: Für konstantes $K_{i}$ stellt $U\left(P, K_{i}\right)$ eine stetige Funktion auf dem kompakten Definitionsbereich $A^{*}$ dar, die nach dem Satz von Weierstraß ein Maximum besitzt.

38: Da zu jeder Aktion $P \in A^{*}$ eine äquivalente Entscheidungsfunktion in $D_{j}{ }^{*}$ enthalten ist, muß gelten: $U\left(\delta_{j}{ }^{*}, K_{i}\right) \geq U\left(P^{*}, K_{i}\right)$. Dies läßt schon vermuten, daß der Bruttonutzen der diagnostischen Information unter den hier getroffenen Voraussetzungen (d.h. ohne Berücksichtigung der Kosten einer diagnostischen Maßnahme und der negativen Konsequenzen der Informationsbeschaffung für den Patienten; vgl. dazu Abschnitt 4.5) nicht negativ sein kann. 
Abb. 13: Nutzenallokationen aufgrund nicht-randomisierter Aktionen

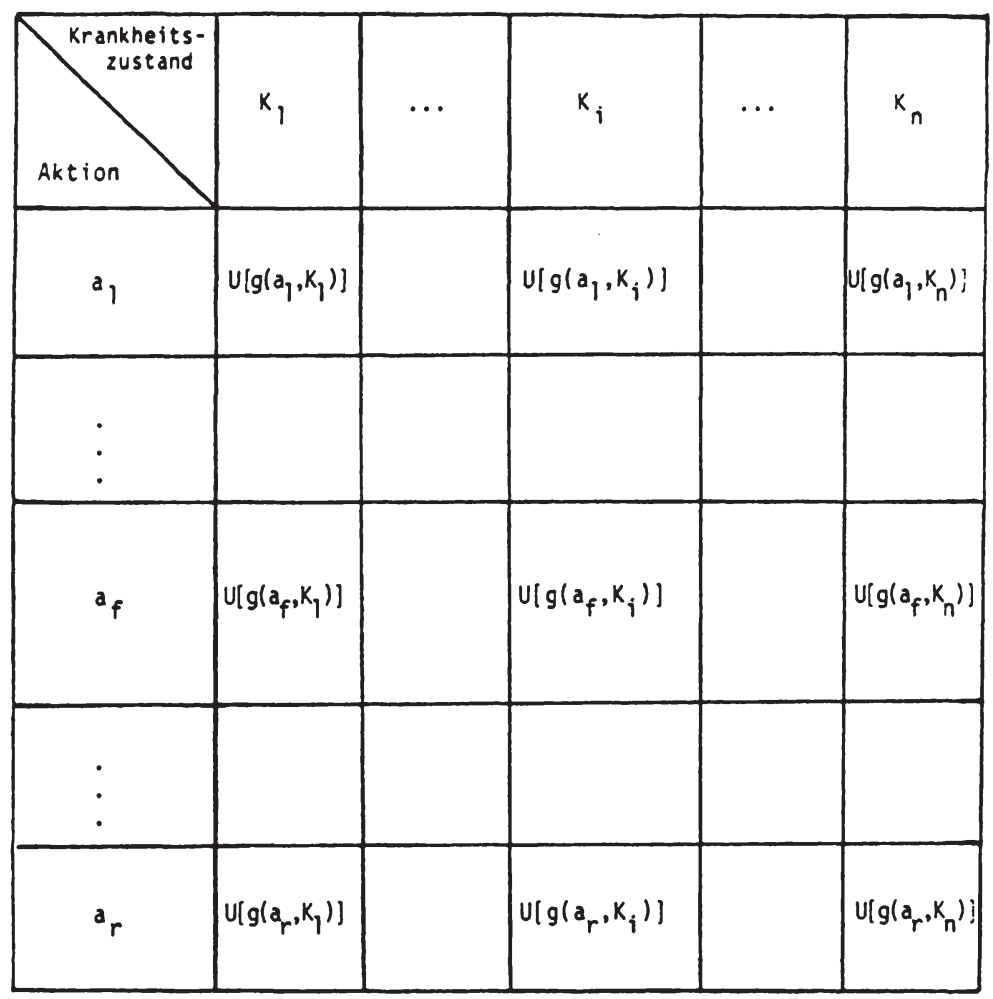

Wie sehen die Präferenzen des Patienten auf einer Menge ärztlicher Entscheidungen aus? Dies sei am Beispiel der Menge A der nicht-randomisierten Aktionen näher erläutert. Aufgrund der Unsicherheit, die bezüglich des tatsächlichen Krankheitszustands herrscht, müssen offenbar bei der Bewertung einer Aktion $a_{f}$ sämtliche Elemente der dazugehörigen Nutzenallokation $W\left(a_{f}\right)$ Berücksichtigung finden. Andererseits gibt es aus der Sicht des Patienten keine bewertungsrelevanten Aspekte, die nicht in dieser Nutzenallokation enthalten sind. Demzufolge repräsentiert der Vektor $\mathrm{W}\left(\mathrm{a}_{\mathrm{f}}\right)$ genau die zur Bewertung der Aktion $\mathrm{a}_{\mathrm{f}}$ benötigte Information. 
Insofern reicht es zur Darstellung der Präferenzen des Patienten auf der Menge A aus, seine Präferenzen auf der Menge der damit verbundenen Nutzenallokationen anzugeben.

Der Vergleich zweier Aktionen $a_{j}$ und $a_{k}$ läuft damit auf einen Vergleich der Nutzenallokationen $W\left(a_{j}\right)$ und $W\left(a_{k}\right)$ hinaus. Es wird angenommen, daß die Präferenzen des Patienten folgender Bedingung genügen, wenn das Symbol $\succsim$ die Relation der schwachen Präferenz bezeichnet ${ }^{39}$ :

$$
\text { Aus } W\left(a_{j}\right) \geq W\left(a_{k}\right) \text { folgt: } W\left(a_{j}\right) \succsim W\left(a_{k}\right)
$$

Aus der Sicht des Patienten ist die Aktion $a_{\mathrm{j}}$ des Arztes mindestens ebenso gut wie eine andere Aktion $a_{k}$, wenn der aus $a_{j}$ resultierende Nutzen in keinem Krankheitszustand niedriger ausfällt. In diesem Fall dominiert die Aktion $a_{j}$ die Aktion $a_{k}$ schwach $^{40}$. Die in Beziehung (4.13) beschriebene Eigenschaft der individuellen Präferenzrelation erscheint plausibel, da die Wahl der Aktion $a_{j}$ unabhängig vom tatsächlich vorliegenden Krankheitszustand des Patienten wenigstens das durch $a_{k}$ erreichbare Nutzenniveau garantiert. Gilt neben (4.13) auch noch $W\left(a_{j}\right) \neq W\left(a_{k}\right)$, dann existiert sogar mindestens ein Krankheitszustand, in dem die Aktion $a_{j}$ dem Patienten ein höheres Nutzenniveau als die Aktion $a_{k}$ verspricht. In diesem Fall dominiert die Aktion $a_{j}$ die Aktion $a_{k}$ streng 41 .

Die oben definierte Dominanzrelation erzeugt auf einer Klasse von Entscheidungen und damit speziell auf der Menge A der nicht-randomisierten Aktionen in der Regel lediglich eine Halbordnung, die zudem häufig kein größtes Element aufweist ${ }^{42}$. Insbesondere die zuletzt genannte Eigenschaft besitzt für die weitere Analyse einige Bedeutung. Ein größtes Element entspricht einer Aktion, die alle anderen Aktionen schwach dominiert ${ }^{43}$. Gäbe es eine Aktion mit dieser starken Opti-

39: Diese Forderung beinhaltet die Verträglichkeit der individuellen Präferenzordnung mit der natürlichen Halbordnung auf $\mathrm{R}^{\mathrm{n}}$; vgl. FIRCHAU, V.(1980), S. 11.

40: Vgl. FERSCHL, F (1975), S. 68.

41: Vgl. ebenda. Daneben wird eine derartige Aktion $a_{j}$ auch als gleichmäßig bessere Aktion bezeichnet; vgl. BÜHLMANN, H./LOEFFEL, H./NIEVERGELT, E. (1975), S. 244.

42: Zum Begriff der Halbordnung bzw. der partiell geordneten Menge vgl. FERSCHL, F. (1975), S. 36ff. sowie VON MANGOLDT, H./KNOPP, K. (1989), S. 49ff.

43: Zur Unterscheidung größter und maximaler Elemente bei partiell geordneten Mengen vgl. FERSCHL, F.(1975), S. 39. 
malitätseigenschaft, so würde der Patient deren Einsatz stets präferieren, da unabhängig von seinem Krankheitszustand keine andere zur Verfügung stehende Aktion jemals mit einem höheren Nutzen für ihn einhergehen könnte. In diesem Fall liegt ein triviales medizinisches Entscheidungsproblem vor, da der Arzt sozusagen ohne Rücksicht auf den tatsächlichen Krankheitszustand die dominante Aktion ergreifen und damit dem Patienten ein maximales Nutzenniveau garantieren kann ${ }^{44}$. Man bezeichnet eine derartige Aktion auch als gleichmäßig beste Aktion ${ }^{45}$.

Im allgemeinen wird jedoch das interessierende medizinische Entscheidungsproblem nicht die Eigenschaft der Trivialität besitzen und infolgedessen keine gleichmäßig beste Aktion existieren. In diesen Fällen muß die für den Patienten günstigste Aktion des Arztes mit dem Krankheitszustand variieren. Dieser Tatbestand dürfte in der überwiegenden Zahl medizinischer Entscheidungssituationen erfüllt sein. Eine ärztliche Aktion, die für einen bestimmten Krankheitszustand optimal ist gemäß dem eingangs dieses Abschnitts genannten Kriterium, wird im allgemeinen diese Eigenschaft bei einem anderen - hinreichend verschiedenen - Krankheitszustand nicht mehr aufweisen ${ }^{46}$.

Damit bleibt zunächst unklar, welche Aktion der Arzt in einer nicht-trivialen Entscheidungssituation auszuwählen hat, wenn er im Interesse des Patienten handeln will. In günstigen Fällen läßt sich die Struktur des Entscheidungsproblems übersichtlicher gestalten, indem man die Menge der zu betrachtenden Entscheidungen reduziert. Um dieses Vorgehen zu veranschaulichen, werden einige Begriffe benötigt. Man bezeichnet eine Entscheidung als zulässig bezüglich einer festen Klasse von Entscheidungen, wenn innerhalb dieser Klasse keine Entscheidung existiert, die diese Entscheidung streng dominiert ${ }^{47}$. Die Zulässigkeit stellt eine schwache Opti-

44: Diagnostische Informationen besitzen in dieser Situation keinen Wert, da sie die geeignete ärztliche Handlung nicht beeinflussen können; vgl. Abschnitt 4.3.

45: Vgl. z.B. BAMBERG, G. (1975), S. 210.

46: Beispielsweise fällt in der Regel die optimale therapeutische "Antwort" bzw. Aktion des Arztes je nach Krankheitszustand verschieden aus.

47: Zulässige Entscheidungen repräsentieren somit maximale Elemente der strengen Dominanzrelation auf der betrachteten Klasse von Entscheidungen. 
malitätseigenschaft dar, die im allgemeinen von mehreren Entscheidungen aus der betrachteten Klasse geteilt wird ${ }^{48}$. Speziell heißt eine Aktion $a_{k}$ des Arztes zulässig bezüglich der Menge A aller Aktionen, falls gilt ${ }^{49}$ :

$$
\text { Es existiert kein } a_{f} \in A \text { mit: } W\left(a_{f}\right) \geq W\left(a_{k}\right) ; W\left(a_{f}\right) \neq W\left(a_{k}\right) \text {. }
$$

Es liegt nun nahe, die Analyse von Entscheidungsproblemen soweit als möglich auf die Betrachtung zulässiger Entscheidungen zu beschränken. Diese Eingrenzung erweist sich zumindest dann als sinnvoll, wenn die Menge der zulässigen Entscheidungen die Eigenschaft der Vollständigkeit besitzt. Eine Teilmenge $X^{\prime}$ einer festen Menge $X$ von Entscheidungen heißt vollständig, wenn es zu jedem Element der Differenzmenge $X-X^{\prime}$ eine Entscheidung in $X^{\prime}$ gibt, die dieses streng dominiert. Weiterhin bezeichnet man eine vollständige Teilmenge $X^{\prime}$ einer festen Menge $X$ von Entscheidungen als minimal vollständig, wenn keine echte Teilmenge von $X^{\prime}$ die Eigenschaft der Vollständigkeit besitzt ${ }^{50}$. Es läßt sich zeigen, daß im Falle ihrer Existenz eine minimal vollständige Menge gerade die Menge aller zulässigen Entscheidungen enthält 51 .

Die bisher verwendeten Voraussetzungen sichern die Existenz einer minimal vollständigen Menge von Entscheidungen, die im allgemeinen mehrere Elemente umfaßt. Diese Aussage gilt unabhängig davon, ob in einer medizinischen Entscheidungssituation die Menge $A$, die Menge $A^{*}$ oder eine Menge $D_{j}^{*}$ als mögliche Entscheidungen des Arztes betrachtet wird ${ }^{52}$. Die Eigenschaft (4.13) der individuellen Präferenzrelation gewährleistet ferner, daß außerhalb einer minimal vollständigen Menge keine Entscheidung liegen kann, die der Patient strikt präferiert. Deshalb

48: Allerdings impliziert die eindeutige Existenz einer zulässigen Entscheidung noch nicht die Trivialität der Entscheidungssituation, da die einzig zulässige keine dominante Entscheidung zu sein braucht; vgl. dazu das Beispiel bei FERSCHL, F. (1975), S. 73.

49: Allgemein brauchen zulässige Entscheidungen jedoch nicht zu existieren; vgl. z.B. FERGUSON, T.S. (1967), S. 55.

50: Während vollständige Mengen von Entscheidungen stets existieren - insbesondere ist X vollständig - , gilt diese Aussage für minimal vollständige Mengen keineswegs; vgl. die Beispiele bei FERSCHL, F. (1975), S. $72 \mathrm{ff}$.

51: Vgl. FERGUSON, T.S. (1967), S. 56; FERSCHL, F. (1975), S. $74 f$.

52: Zunächst enthält die bezüglich der Menge A der ärtlichen Aktionen minimal vollständige Teilmenge in nicht-trivialen Entscheidungssituationen mindestens zwei Elemente. Daraus folgt unmittelbar, daß die bezüglich $A^{*}$ bzw. $D_{j}{ }^{*}$ minimal vollständigen Teilmengen jeweils aus (überabzählbar) unendlich vielen Elementen bestehen. 
reicht es bei der Analyse einer medizinischen Entscheidungssituation aus, lediglich die minimal vollständige Teilmenge aller möglichen Entscheidungen des Arztes zu untersuchen.

Es gelingt an dieser Stelle jedoch noch nicht anzugeben, in welcher Weise der Arzt ein medizinisches Entscheidungsproblem im Sinne des Patienten lösen kann, falls die bezüglich einer Klasse möglicher Entscheidungen des Arztes minimal vollständige Teilmenge mehrere Elemente enthält. Diese Konstellation charakterisiert aber gerade die nicht-trivialen Entscheidungssituationen. Insofern benötigt man weitere Informationen über die individuelle Präferenzrelation auf der Menge der zulässigen Entscheidungen. Diese Information ergibt sich aus einer Bewertungsregel, die den Nutzenallokationen ärztlicher Entscheidungen eine skalare Größe zuweist ${ }^{53}$. Mit Hilfe einer derartigen Funktion gelingt es, eine vollständige Quasiordnung auf einer Menge ärztlicher Entscheidungen herzustellen ${ }^{54}$. In diesem Zusammenhang gibt eine Entscheidungsregel eine Vorschrift an, nach der in einer Entscheidungssituation eine Entscheidung auszuwählen ist ${ }^{55}$.

In Abschnitt 2.4.1 war angenommen worden, daß der Arzt den möglichen Krankheitszuständen priore Wahrscheinlichkeiten zuordnen kann. Dies legt die Verwendung einer speziellen Bewertungsregel nahe. Unter dieser Voraussetzung repräsentiert eine ärztliche Entscheidung $\mathrm{x}$ in einer gegebenen Entscheidungssituation aus

53: In der entscheidungstheoretischen Literatur wird eine Reihe unterschiedlicher Bewertungsregeln diskutiert; vgl. z.B. FERSCHL, F. (1975), S. 52ff. Firchau spricht mehrere Klassen von Bewertungsregeln an; vgl. FIRCHAU, V. (1980), S. $11 \mathrm{ff}$.

54: Zum Begriff der vollständigen Quasiordnung (Präordnung) siehe AHLHEIM, M./ROSE, M. (1989), S. 249.

55: Es besteht ein enger Zusammenhang zwischen Bewertungs- und Entscheidungsregel. Eine Bewertungsregel ordnet einer Menge ärztlicher Entscheidungen eindeutig eine Menge reeller Zahlen zu, die bezüglich der Binärrelation " $\geq$ " vollständig geordnet ist. Daraus ergibt sich wegen der im allgemeinen fehlenden Eineindeutigkeit der Bewertungsregel lediglich eine vollständige Quasiordnung der betrachteten Menge von Entscheidungen. Die Entscheidungsregel bezieht sich auf diese Ordnung und damit auf die zugrundeliegende Bewertungsregel, indem sie entweder ein größtes oder ein kleinstes Element - falls diese existieren auswählt. In der Literatur werden daher schwergewichtig verschiedene Entscheidungsregeln diskutiert; vgl. BÜHLMANN, H./LOEFFEL, H./NIEVERGELT, E. (1975), S. 130ff.; BAMBERG, G./COENENBERG, A.G./KLEINE-DOEPKE, R. (1976), S. 37ff. sowie MAG, W. (1990), S. $75 f f$. 
der Sicht des Patienten einen Prospekt, der die Nutzenwerte $\mathrm{W}_{\mathrm{i}}(\mathrm{x})$ mit den Wahrscheinlichkeiten $\mathrm{P}\left(\mathrm{K}_{\mathfrak{i}}\right)$ liefert ${ }^{56}$. Da die Präferenzen des Patienten der Erwartungsnutzenhypothese genügen, bewertet er diesen Prospekt anhand des damit verbundenen erwarteten Nutzens 57 :

$$
\Phi_{B}(x)=\Phi_{B}[W(x)]=\sum_{i=1}^{n} P\left(K_{i}\right) \cdot U\left(x, K_{i}\right) .
$$

Dabei stellt die Entscheidung $x$ ein Element genau einer der Mengen $A, A^{*}, D_{j}$ oder $\mathrm{D}_{\mathrm{j}}{ }^{*}(\mathrm{j}=1, \ldots, \mathrm{m})$ dar. Man bezeichnet diese, durch den Operator $\Phi_{B}$ symbolisierte Regel zur Bewertung von Entscheidungen bzw. Nutzenallokationen als Bayes-Regel ${ }^{58}$. Die skalare Größe $\Phi_{B}(x)$ heißt Bayes-Nutzen ${ }^{59}$ der ärztlichen Entscheidung $x$.

Die Präferenzen des Patienten auf einer Menge ärztlicher Entscheidungen lassen sich nun mit Hilfe der Funktion $\Phi_{B}$ repräsentieren. Der Patient besitzt eine schwache

56: Präziser formuliert, ergibt sich als Folge einer ärztlichen Entscheidung $x$ für den Patienten ein Prospekt, der diejenigen Prospekte mit den Wahrscheinlichkeiten $\mathrm{P}\left(\mathrm{K}_{\mathrm{i}}\right)$ liefert, deren Nutzenwerte $\mathrm{W}_{\mathrm{i}}(\mathrm{x})$ betragen.

57: Grundsätzlich legt jede ärztliche Entscheidung unter den in Abschnitt 2.4 getroffenen Voraussetzungen eindeutig eine Wahrscheinlichkeitsverteilung auf $\mathrm{A} \times \mathrm{K}$ fest, die den Patienten entsprechend mit einem eindeutig bestimmten Prospekt konfrontiert. Insofern wäre es in diesem Fall möglich, den Nutzen einer Entscheidung $x$ des Artes direkt zu ermitteln, indem man die Nutzenwerte $\mathrm{u}\left[\mathrm{g}\left(\mathrm{a}_{\mathrm{f}}, \mathrm{K}_{\mathrm{i}}\right)\right]$ der möglichen Konsequenzenvektoren mit ihren Wahrscheinlichkeiten gewichtet. Es empfiehlt sich jedoch, die individuelle Bewertung einer ärztlichen Entscheidung bei gegebenem Krankheitszustand des Patienten einerseits und ohne diese Bedingung andererseits strikt voneinander zu trennen. Dies gilt vor allem deshalb, weil das Wissen des Arztes (bzw. allgemeiner: des Aktors) hinsichtlich des Krankheitszustands (bzw.: des Umweltzustands) in ganz unterschiedlichen Formen vorliegen kann, die zu verschiedenen Bewertungsregeln führen und damit einer gegebenen Nutzenallokation jeweils verschiedene Bewertungen zuordnen; vgl. dazu ausführlich FERSCHL, F. (1975), S. 53ff. sowie BAMBERG, G./COENENBERG, A.G. (1989), S. 107ff. und S. $119 \mathrm{ff}$.

58: Vgl. FIRCHAU, V. (1980), S. 13.

59: In der statistischen Entscheidungstheorie analysiert man anstelle einer Nutzenfunktion im allgemeinen eine Verlustfunktion. Die Anwendung der Bayes-Regel liefert dann den BayesVerlust einer Entscheidung ohne Informationsbeschaffung, während man im Falle einer Entscheidung mit Informationsbeschaffung von ihrem Bayes-Risiko spricht; vgl. BÜHLMANN, H./LOEFFEL, H./NIEVERGELT, E. (1975), S. 234f. sowie BERGER, J.O. (1985), S. $8 \mathrm{ff}$. Diese semantische Unterscheidung der Bayes-Bewertung von Entscheidungen mit und ohne Informationsbeschaffung übernimmt die im Text verwendete Terminologie allerdings nicht. 
Präferenz für die Entscheidung $x$ des Arztes im Vergleich zu einer (anderen) Entscheidung $x^{\prime}$, wenn der Bayes-Nutzen von $\mathrm{x}$ mindestens so hoch ausfällt wie derjenige von $x^{\prime}$ :

$$
\Phi_{\mathrm{B}}(\mathrm{x}) \geq \Phi_{\mathrm{B}}\left(\mathrm{x}^{\prime}\right) \Leftrightarrow \mathrm{x} \succsim \mathrm{x}^{\prime}
$$

Diese Relation der schwachen Präferenz, die auf der Bayes-Bewertungsregel fußt, erzeugt auf einer Menge $X$ ärztlicher Entscheidungen eine vollständige Quasiordnung, falls $\Phi_{\mathrm{B}}(\mathrm{x})$ für alle Elemente von $\mathrm{X}$ definiert ist. Für die hier im Kontext des Entscheidungsproblems des Arztes betrachteten Mengen $A, A^{*}, D_{j}$ und $D_{j}{ }^{*}(j=$ $1, . ., \mathrm{m})$ trifft diese Annahme ausnahmslos zu. Die zur Bayes-Regel gehörende Entscheidungsregel fordert die Wahl einer Entscheidung, welche die Funktion $\Phi_{\mathrm{B}}$ und damit den Nutzen des Patienten auf der betrachteten Menge von Entscheidungen maximiert. Eine Entscheidung $\mathrm{x}^{*}$ heißt Bayes-Entscheidung auf der Menge $X$ bezüglich der Wahrscheinlichkeitsverteilung $P(K)$, falls gilt 60 .

$$
\mathrm{x}^{*} \in \arg \max \left\{\Phi_{\mathrm{B}}(\mathrm{x}) \mid \mathrm{x} \in \mathrm{X}\right\}
$$

\subsubsection{Der Arzt als Agent des Patienten}

Im Rahmen eines medizinischen Entscheidungsproblems, das die bisher genannten Voraussetzungen erfüllt, stehen mit dem Arzt und dem Patienten gleich zwei Individuen im Vordergrund der Analyse. Auf der einen Seite kennt der Arzt die verschiedenen Handlungsmöglichkeiten, die ihm in einer Entscheidungssituation zur Verfügung stehen. Darüber hinaus besitzt er als medizinischer Sachverständiger das Wissen hinsichtlich der Konsequenzen, die seine Entscheidungen beim Patienten hervorrufen können. Andererseits weiß der Patient annahmegemäß über seine Präferenzen Bescheid, ohne daß dieses Wissen bei seinem Arzt vorausgesetzt werden $\mathrm{kann}^{61}$. Insofern kennzeichnet medizinische Entscheidungsprobleme eine asymme-

60: Die Existenz einer Bayes-Entscheidung ist für alle genannten Entscheidungsmengen und sämtliche Wahrscheinlichkeitsverteilungen auf $\mathrm{K}$ gesichert, nicht jedoch ihre Eindeutigkeit; vgl. dazu näher Abschnitt 4.3.

61: Dies bedeutet nicht, daß der Arzt keinerlei Information über die Präferenzen des Patienten besitzt. Jedoch dürfte dieses Wissen aufgrund der Heterogenität des Patientenguts auch bei erfahrenen Ärzten unvollständig bleiben. 
trische Verteilung der relevanten Information auf die beiden Individuen Arzt und $\mathrm{Pa}$ tient $^{62}$. Ohne weitere Voraussetzung bleibt unklar, von wem und in welcher Weise diese dezentral vorhandene Information zur Entscheidungsfindung genutzt wird.

Wie schon in den vorangegangenen Abschnitten angedeutet, gehen auch die folgenden Überlegungen davon aus, da $B$ der Arzt diejenige Person darstellt, die eine Entscheidung trifft ${ }^{63}$. Für die Plausibilität dieser Annahme spricht der im allgemeinen große Informationsvorsprung, über den der Arzt in Bezug auf zentrale Bestandteile eines medizinischen Entscheidungsproblems verfügt ${ }^{64}$. Unter dieser Voraussetzung delegiert der Patient in einer medizinischen Entscheidungssituation seine Entscheidungsgewalt an den Arzt. Der Patient repräsentiert somit einen Auftraggeber oder Prinzipal, der den Arzt zum Sachwalter seiner Interessen bestellt. Diese Sichtweise des Arztes als Agent des Patienten herrscht heute in der gesundheitsökonomischen Literatur vor 65 .

Diese Charakterisierung der Beziehung zwischen dem Arzt und seinem Patienten läßt noch offen, in welchem Ausmaß der Arzt bei der Entscheidungsfindung die Interessen des Patienten berücksichtigt. Der Arzt handelt als vollkommener oder per-

62: Im Hinblick auf die Komponenten einer medizinischen Entscheidungssituation liegt daher eine wechselseitige asymmetrische Informationsverteilung vor, vgl. WILLE, E./ULRICH, V. (1991), S. 27.

63: Insofern beschränkt sich die Rolle des Arztes keineswegs darauf, dem Patienten lediglich die für eine Entscheidung notwendige Information bereitzustellen. Vgl. zur Kritik an der Vorstellung eines auf die Informationsfunktion reduzierten Arztes WILLIAMS, A. (1988), S. 176.

64: Diese Aussage bezieht sich auf das größere medizinische Wissen des Arztes; vgl. WIELAND, K. (1988); FELDSTEIN, M.S. (1974), S. 382. Zumindest zu Beginn einer Krankheitsepisode dürfte hingegen der Patient über mehr diagnostische Information verfügen.

65: Vgl. zum Begriff des Agenten bzw. Prinzipals PRATT, J.W./ZECKHAUSER, R.J. (1985), S. 2, sowie ARROW, K.J. (1985), S. 37, die zugleich eine Einführung in die Prinzipal-Agenten Theorie geben. Das in dieser Theorie untersuchte Grundproblem stellt ROSS, S.A. (1973) dar, während Rees einen Überblick über ihre Resultate liefert; vgl. REES, R. (1985a), (1985b).

Der Vorschlag, den Arzt als Agenten des Patienten anzusehen, geht offenbar auf FELDSTEIN, M.S. (1974), S. 382ff., zurück, obwohl sich diese Idee zumindest implizit schon bei ARROW, K.J. (1963), S. 96lff. findet. Arrow bezeichnet in einer neueren Arbeit die Beziehung zwischen Arzt und Patient als typisches Beispiel eines Prinzipal-Agenten Verhältnisses, vgl. ARROW, K.J. (1985), S. 49. 
fekter Agent des Patienten, wenn er seine Entscheidungen in voller Übereinstimmung mit den Präferenzen des Patienten fällt ${ }^{66}$. Die Resultate der PrinzipalAgenten-Theorie geben Anlaß zu einiger Skepsis hinsichtlich der Vollkommenheit des Agenturverhältnisses zwischen Arzt und Patient. Zwar vermag der Patient im allgemeinen die Entscheidung zu beobachten, die sein Arzt in einer Entscheidungssituation trifft. Er kann jedoch kaum beurteilen, ob eine andere Entscheidung nicht angemessener gewesen wäre 67 . Insofern fehlt dem Patienten die Möglichkeit, die Qualität der ärztlichen Entscheidung direkt einzuschätzen. Materiell entspricht dies einer Situation, in welcher der Prinzipal die Entscheidung seines Agenten nicht beobachten kann 68 .

Dieses Fehlen einer direkten Kontrolle braucht für den Patienten ebensowenig negative Auswirkungen nach sich zu ziehen wie die Tatsache, da $B$ a priori keine Kongruenz seiner Interessen mit denen des Arztes zu erwarten ist ${ }^{69}$. Schließlich kennt bzw. erfährt der Patient die Folgen der vom Arzt getroffenen Entscheidung, deren (insbesondere gesundheitliche) Konsequenzen er selbst zu tragen hat. Dieses Wissen kann er dazu verwenden, um auf die Güte des ärztlichen Handelns zu schließen. Allerdings gelingt dieser Rückschluß nur unvollkommen: aufgrund der Unsicherheit bezüglich des Krankheitszustands existiert lediglich ein stochastischer Zusammenhang zwischen einer ärztlichen Entscheidung und den daraus resultierenden Konsequen-

66: Vgl. dazu FELDSTEIN, M.S. (1974), S. 383; ZWEIFEL, P. (1982), S. 12ff. sowie FERGUSON, B.S. (1985), S. 35f.

67: Dieses Informationsdefizit des Patienten dürfte die Sachwalterrolle des Arztes wesentlich begründen.

68: Vgl. ARROW, K.J. (1985), S. 38.

69: Falls eine derartige Übereinstimmung vorliegt, handelt es sich um einen streng altruistischen Arzt. Vgl. als Überblick über verschiedene Modellierungen der Zielfunktion des Arztes in der gesundheitsökonomischen Literatur ZWEIFEL, P. (1982), Kap. 2. 
zen $^{70}$. Dies schränkt die Möglichkeit einer indirekten Beobachtung bzw. Kontrolle des Arztes - anhand der Konsequenzen seines Handelns - durch den Patienten erheblich ein. Gleichzeitig eröffnet sich dadurch dem Arzt ein für Agenturverhältnisse charakteristischer diskretionärer Spielraum, den er zur Verfolgung seiner eigenen Interessen nutzen kann.

In dieser Situation erlangen die finanziellen Anreize, die dem Arzt gesetzt sind, große Bedeutung. Typischerweise besteht kein oder bestenfalls ein schwacher $\mathrm{Zu}$ sammenhang zwischen der Entlohnung des Arztes und den Konsequenzen seines Handelns. Insbesondere fehlt eine Koppelung des ärztlichen Honorars an den Behandlungserfolg beim Patienten ${ }^{71}$. Daraus folgt, daß der Arzt innerhalb seines diskretionären Spielraums agieren kann, ohne finanzielle Einbußen befürchten zu müssen. Damit repräsentiert er einen Agenten, der zumindest in einem gewissen Umfang über die Möglichkeit verfügt, frei von monetären Sanktionen seine eigenen Ziele zu Lasten der Interessen des Patienten zu verfolgen. Dies spricht für eine häufig aufgestellte These, die den Arzt - sofern er rational handelt - lediglich als unvollkommenen Sachwalter des Patienten ansieht ${ }^{72}$.

70: Diese Aussage bezieht sich zunăchst auf die in Abschnitt 4.2.2 eingeführte, deterministische Konsequenzenfunktion.

Über das in dieser Arbeit diskutierte Modell hinaus kommen noch weitere Faktoren in Betracht, die den Zusammenhang zwischen einer Aktion des Arztes und den gesundheitlichen Konsequenzen beim Patienten bei gegebenem Krankheitszustand beeinflussen und damit ebenfalls eine indirekte Kontrolle des ärztlichen Handelns erschweren können. Einerseits spielt das Verhalten des Patienten im Hinblick auf den medizinischen Behandlungserfolg vielfach eine nicht unbedeutende Rolle; vgl. dazu Abschnitt 4.4.1.1. Andererseits können "intervenierende Faktoren" dafür sorgen, daß die Konsequenzen eines Paars $\left(a_{\mathrm{f}}, \mathrm{K}_{\mathrm{i}}\right)$ zufallsabhăngig sind; vgl. BROOK, R.H. et al. (1976); GÄFGEN, G. (1980), S. 187; DIONNE, G./CONTANDRIOPOULOS, A.-P. (1985), S. 23 und WIELAND, K. (1988).

71: Vgl. CULYER, A.J. (1971), S. 197; PAULY, M.V. (1980), S. 126; FERGUSON, B.S. (1985), S. 35; ARROW, K.J. (1985), S. 69. Diese Aussage gilt z.B. für die Einzelleistungsvergütung, die in der Bundesrepublik bei der Verteilung des Gesamthonorars auf die niedergelassenen Ärzte hauptsächlich angewendet wird; vgl. MOLINARO, R. (1986), S. 19f. Zu den Anreizwirkungen alternativer Honorarformen vgl. VON DER SCHULENBURG, J.-M. (1981), S. 180ff.

72: Vgl. FELDSTEIN, M.S. (1974), S. 383f.; PAULY, M.V. (1980), S. 5f.; ZWEIFEL, P. (1982), S. 48ff.; FERGUSON, B.S. (1985) und DIONNE, G./CONTANDRIOPOULOS, A.-P. (1985). 
Im Gegensatz dazu wird in dieser Arbeit unterstellt, daß sich der Arzt als perfekter Agent des Patienten verhält. Zur exakten Erfassung des Nutzeneffekts, den die Information einer diagnostischen Maßnahme dem Patienten in Abhängigkeit von der Entscheidungssituation zu stiften vermag, kann auf diese zusätzliche Annahme nicht verzichtet werden. Verhält sich der Arzt hingegen nicht im Sinne dieser Annahme, wird die aufgrund des Einsatzes einer diagnostischen Maßnahme beim Patienten entstehende Wohlfahrtsänderung zumindest teilweise durch das Abweichen von der perfekten Sachwalterrolle überlagert. Dies läßt sich beispielsweise damit begründen, daß der Arzt in diesem Fall eine aus der Sicht des Patienten bestmögliche Nutzung der diagnostischen Information zugunsten seiner eigenen Interessen verhindert. Insofern stellt die vollkommene Sachwalterrolle des Arztes eine notwendige Voraussetzung dar, um den auf eine diagnostische Maßnahme zurückzuführenden Vorteil des Patienten zu ermitteln.

Die eingangs genannten Argumente, die eher gegen die Gültigkeit dieser Voraussetzung sprechen, reflektieren zudem eine statische Betrachtungsweise, welche die Beziehung zwischen dem Arzt und seinem Patienten in verkürzter Form abbildet. Im Normalfall besteht zumindest die Möglichkeit, daß diese Beziehung über mehrere Krankheitsepisoden hinweg andauert. In dieser Zeit kann der Patient weitere Informationen über den vom Arzt bewirkten Behandlungserfolg sammeln, die ihm einen zunehmend verläßlicheren Rückschluß auf die Güte der ärztlichen Entscheidungen erlauben. Aus diesem Grund besitzt der Arzt unabhängig von der Vergütungsform ein Interesse daran, die Präferenzen des Patienten zu berücksichtigen. Weiterhin spielen in diesem Zusammenhang sogenannte Agenturkosten eine Rolle, die in einem Prinzipal-Agenten Verhältnis aufgrund der Abweichung vom Zustand vollkommener Information entstehen ${ }^{73}$. Je länger die Beziehung zwischen Prinzipal und Agent andauert, desto geringer fallen diese Agenturkosten im Zeitablauf aus. Agenturkosten entstehen beispielsweise aufgrund der Zeit, die der Arzt zur diagnostischen Abklärung des beim Patienten vorliegenden Krankheitszustands benötigt. Wenn der Arzt seinen Patienten schon aus früheren Krankheitsepisoden kennt, dürfte dieser Zeitbedarf niedriger ausfallen. Im allgemeinen kommt eine Reduktion der Agenturkosten beiden Parteien zugute, was für den Arzt den Anreiz zu einem aus der Sicht des Patienten "guten" Verhalten erhöht ${ }^{74}$.

\footnotetext{
73: Zum Begriff der Agenturkosten vgl. PRATT, J.W./ZECKHAUSER, R.J. (1985), S. 3 sowie ausführlich JENSEN, M.C./MECKLING, W.H. (1976), S. $308 \mathrm{ff}$.

74: Vgl. PRATT, J.W./ZECKHAUSER, R.J. (1985), S. 6.
} 
Die Prinzipal-Agenten-Theorie untersucht im wesentlichen monetäre Anreize und Sanktionen in ihrem Einfluß auf das Verhalten eines Agenten. Diese Betrachtungsweise dürfte in vielen Fällen zu kurz greifen ${ }^{75}$, was wohl in besonderem Maß für das Verhältnis zwischen Arzt und Patient gilt, da der Arzt in seinen Handlungen eine Reihe berufsethischer Normen zu beachten hat. Im Falle ihrer allgemeinen Anerkennung - wie z.B. bei den "Normen der Redlichkeit und Vertrauenswürdigkeit des Arztes"176 - beeinflussen Normen das individuelle Verhalten und ziehen auf diese Weise ökonomisch relevante Effekte nach sich. So weist beispielsweise das auf den hippokratischen Eid zurückgehende Ärztegelöbnis dem Arzt als oberstes Gebot die Aufgabe zu, die Gesundheit seiner Patienten wiederherzustellen und zu erhalten. Diese Anweisung läßt sich als Verpflichtung auf die Interessen des Patienten interpretieren, sofern dieser eine entsprechende Präferenz für seine Gesundheit besitzt. Insgesamt erscheinen die berufsethischen Grundsätze geeignet, die aus den rein monetären Anreizen zu erwartende Unvollkommenheit der Sachwalterrolle des Arztes abzuschwächen.

Neben diesen Sachargumenten, die für eine perfekte Sachwalterrolle des Arztes ins Feld geführt werden können, besitzt die Annahme den Vorteil, der Beziehung zwischen Arzt und Patient eine besonders einfache Struktur zu verleihen. Wenn der Arzt vollständig im Interesse des Patienten handelt, bedarf es keiner speziellen Analyse seiner eigenen Ziele. Unter dieser Voraussetzung besteht materiell kein Unterschied zwischen einer medizinischen und einer "traditionellen" Entscheidungssituation - in welcher der Aktor die Folgen seines Handelns selbst trägt -, da die Präferenzen des Patienten die Entscheidung des Arztes determinieren.

\subsection{Der Optionspreis diagnostischer Maßnahmen}

\subsubsection{Vorüberlegung}

Falls sämtliche Daten des ärztlichen Entscheidungsproblems, die in den Abschnitten 2.4 und 4.2 als Voraussetzungen genannt wurden, bekannt sind, läßt sich in jeder medizinischen Entscheidungssituation derjenige Vorteil für den Patienten bestimmen, den der Arzt durch den Einsatz einer diagnostischen Maßnahme erreichen kann. Ohne weitere Diagnostik wählt der Arzt eine Aktion $\mathrm{P}^{*} \in \mathrm{A}^{*}$, die dem Patien-

75: Arrow spricht in diesem Zusammenhang von der Existenz einer "whole world of rewards and penalties that take social rather than monetary forms", vgl. ARROW, K.J. (1985), S. 50.

76: Vgl. WIELAND, K. (1988). 
ten ein bezüglich der Menge $A^{*}$ maximales Nutzenniveau $\Phi_{B}\left(P^{*}\right)$ sichert. Jede derartige Aktion repräsentiert eine optimale Handlung des Arztes in der Situation ohne weitere Diagnostik. Entscheidet sich der Arzt hingegen für die diagnostische Maßnahme $T_{j}$, dann wird er eine Entscheidungsfunktion $\delta_{j}^{*}$ wählen, die auf der Menge $\mathrm{D}_{\mathrm{j}}{ }^{*}$ den Nutzen des Patienten maximiert. In diesem Fall stellt jede durch diese Eigenschaft definierte Entscheidungsfunktion eine optimale Handlung des Arztes dar. Dabei wird der Patient in dem hier verwendeten Modellrahmen den Einsatz einer diagnostischen Maßnahme immer schwach präferieren, da ihn eine Entscheidungsfunktion $\delta_{\mathrm{j}}{ }^{*}$ niemals schlechter stellen kann als eine optimale ärztliche Handlung in der Situation ohne weitere Diagnostik ${ }^{77}$. Sofern der Patient einen echten Vorteil aus der zusätzlich betriebenen Diagnostik zieht, fällt der Nutzen $\Phi_{B}\left(\delta_{j}^{*}\right)$ einer optimalen Entscheidungsfunktion höher aus als $\Phi_{\mathrm{B}}\left(\mathrm{P}^{*}\right)$.

Zur Quantifizierung des Vorteils, den der Patient aus dem Einsatz einer diagnostischen Maßnahme bezieht, erweist sich die Differenz der beiden Nutzenwerte $\Phi_{\mathrm{B}}\left(\delta_{\mathrm{j}}^{*}\right)$ und $\Phi_{\mathrm{B}}\left(\mathrm{P}^{*}\right)$ indessen als ungeeignet ${ }^{78}$. Deshalb interessiert man sich aus der Perspektive der Kosten-Nutzen-Analyse stärker für die Zahlungsbereitschaft, die der Patient für die diagnostische Information entfaltet ${ }^{79}$. In konzeptioneller Hinsicht repräsentiert eine derartige Zahlungsbereitschaft einen monetären Ausdruck für die Änderung der individuellen Wohlfahrt, die aus dem Übergang zu einem neuen Prospekt resultiert. Insofern kommen als geeignete Wohlfahrtsmaße zunächst sämtliche kompensierenden maximalen Zahlungsbereitschaften in Betracht. Wie in Abschnitt 2.2.2 näher erläutert, wird im folgenden mit dem kompensierenden Optionspreis gerade jene maximale Zahlungsbereitschaft des Patienten analysiert, die er unabhängig von seinem Krankheitszustand für den Einsatz einer diagnostischen Maßnahme besitzt.

77: Diese Aussage folgt aus der Eigenschaft, daß unabhängig von der speziell betrachteten diagnostischen Maßnahme zu jeder Aktion P des Arztes eine (konstante) Entscheidungsfunktion existiert, die im Hinblick auf die Konsequenzen für den Patienten dazu äquivalent ist; vgl. Abschnitt 4.2.1. Durch den Einsatz einer diagnostischen Maßnahme kann der Arzt dem Patienten zumindest diejenigen Prospekte garantieren, die in der Situation ohne weitere Diagnostik möglich sind.

78: Dies beruht darauf, da $B$ unendlich viele Nutzenfunktionen zur Darstellung derselben individuellen Präferenzen für Prospekte geeignet sind und daher der in Nutzeneinheiten gemessene Vorteil des Patienten aus der zusätzlichen Diagnostik von der speziell gewählten Nutzenfunktion abhängt.

79: Dies gilt nicht zuletzt deshalb, weil die individuelle Zahlungsbereitschaft durch die spezielle Wahl einer Nutzenfunktion nicht beeinflußt wird. 
Im allgemeinen hängt der kompensierende Optionspreis einer diagnostischen Maßnahme von der vorliegenden medizinischen Entscheidungssituation - zum Beispiel der Menge der Krankheitszustände $\mathrm{K}_{\mathrm{i}}$ und deren prioren Wahrscheinlichkeiten $\mathrm{P}\left(\mathrm{K}_{\mathbf{i}}\right)$ - ab. Diese Zahlungsbereitschaft bildet daher ein spezielles monetäres Nutzenäquivalent, das sich sowohl auf ein bestimmtes Individuum - den betrachteten Patienten - als auch auf eine genau festgelegte Entscheidungssituation bezieht. Aufgrund seiner Konstruktion gibt der kompensierende Optionspreis den Wert einer diagnostischen Maßnahme aus der Sicht des Patienten an. Genauer handelt es sich um eine individuelle Brutto-Zahlungsbereitschaft, da von den Kosten der zusätzlichen Diagnostik für den Patienten zunächst abgesehen wird ${ }^{80}$.

Um den kompensierenden Optionspreis einer beliebigen diagnostischen Maßnahme in einer medizinischen Entscheidungssituation zu bestimmen, ermittelt man zunächst eine optimale Handlung des Arztes in der Situation ohne weitere Diagnostik. Für eine derartige Aktion $\mathrm{P}^{*}$ und die damit verbundene Beurteilung $\Phi_{\mathrm{B}}\left(\mathrm{P}^{*}\right)$ durch den Patienten gelten ${ }^{81}$ :

$$
\Phi_{\mathrm{B}}\left(\mathrm{P}^{*}\right)=\max \left\{\Phi_{\mathrm{B}}(\mathrm{P}) \mid \mathrm{P} \in \mathrm{A}^{*}\right\}
$$

$$
\text { bzw.: } \quad \mathrm{P}^{*} \in \arg \max \left\{\Phi_{\mathrm{B}}(\mathrm{P}) \mid \mathrm{P} \in \mathrm{A}^{*}\right\} \text {. }
$$

Falls $\mathrm{P}^{*}$ eine echte randomisierte Aktion des Arztes bezeichnet, ordnet diese Aktion mehreren Elementen der Menge A positive Wahrscheinlichkeiten zu. Der aufgrund von $\mathrm{P}^{*}$ für den Patienten in Bezug auf die Konsequenzen des ärztlichen Handelns entstehende Prospekt setzt sich damit aus Prospekten zusammen, die aus denjenigen Aktionen $a_{f}$ resultieren, die der Arzt mit positiver Wahrscheinlichkeit ergreift. Die Bayes-Regel ordnet einem zusammengesetzten Prospekt aber gerade jene Bewertung zu, die der Summe der mit ihren Eintrittswahrscheinlichkeiten gewichteten Bewertungen der einzelnen Prospekte entspricht. In diesem Sinn repräsentiert der BayesNutzen aufgrund einer randomisierten Aktion einen Mittelwert, der aus den Bayes-

80: In Abschnitt 4.5 werden einige Kosteneffekte diagnostischer Maßnahmen berücksichtigt. Weiterhin braucht die durch den kompensierenden Optionspreis bezeichnete Brutto-Zahlungsbereitschaft des Patienten nicht mit der entsprechenden Wertgröße aus gesamtwirtschaftlicher Sicht übereinzustimmen; vgl. dazu ausführlich Abschnitt 4.3.4.

81: Diese Aktion braucht nicht eindeutig bestimmt zu sein. 
Nutzen der mit positiver Wahrscheinlichkeit gewählten Aktionen $a_{f}$ gebildet wird. Aus dieser Überlegung folgt unmittelbar, daß $\mathrm{P}^{*}$ nur solchen Aktionen $\mathrm{a}_{\mathrm{f}}$ eine positive Wahrscheinlichkeit zuweist, die ebenfalls die in Gleichung (4.18) angegebene Optimalitätseigenschaft besitzen.

Unter den hier verwendeten Voraussetzungen kann eine randomisierte Aktion des Arztes den Patienten daher niemals besser stellen als eine optimale nicht-randomisierte Aktion ${ }^{82}$. Damit läßt sich die Struktur der durch $\arg \max \left\{\Phi_{\mathrm{B}}(\mathrm{P}) \mid \mathrm{P} \in \mathrm{A}^{*}\right\}$ bezeichneten Lösungsmenge näher beschreiben, indem man zwei Fälle unterscheidet. Falls diese Menge lediglich ein Element enthält, muß es sich dabei um eine nichtrandomisierte ärztliche Aktion handeln. Andernfalls ist die optimale Handlung des Arztes nicht eindeutig bestimmt und es existieren mehrere Aktionen $\mathrm{a}_{\mathrm{f}}$, die Gleichung (4.18) erfüllen. Daneben existieren (überabzählbar) unendlich viele optimale Aktionen des Arztes, die gerade durch Randomisieren auf der Menge der optimalen nichtrandomisierten Aktionen zustande kommen.

Die Lösungsmenge $\arg \max \left\{\Phi_{\mathrm{B}}(\mathrm{P}) \mid \mathrm{P} \in \mathrm{A}^{*}\right\}$ enthält in beiden Fällen jeweils mindestens eine nicht-randomisierte Aktion des Arztes. Aus diesem Grund reicht es zur Analyse des ärztlichen Entscheidungsproblems in der Situation ohne weitere Diagnostik aus, lediglich die Menge A als mögliche Handlungen des Arztes zu untersuchen. Ohne Einschränkung der Allgemeinheit wählt der Arzt eine Aktion a ${ }^{*}$ aus, die wie folgt definiert ist:

$$
\Phi_{\mathrm{B}}\left(\mathrm{a}^{*}\right)=\max \left\{\Phi_{\mathrm{B}}\left(\mathrm{a}_{\mathrm{f}}\right) \mid \mathrm{a}_{\mathrm{f}} \in \mathrm{A}\right\}
$$

$$
\text { bzw.: } a^{*} \in \arg \max \left\{\Phi_{\mathrm{B}}\left(\mathrm{a}_{\mathrm{f}}\right) \mid \mathrm{a}_{\mathrm{f}} \in \mathrm{A}\right\} \text {. }
$$

Entscheidet sich der Arzt im Gegensatz dazu, weitere Informationen zu beschaffen, indem er eine diagnostische $\mathrm{Maßnahme} \mathrm{T}_{\mathrm{j}}$ einsetzt, dann kann er dem Patienten einen bezüglich der Menge $D_{j}^{*}$ aller durch die Maßnahme ermöglichten Entschei-

82: Dies entspricht einer allgemeinen, aus der Entscheidungstheorie bekannten Aussage, der zufolge sich unter schwachen Voraussetzungen ein Randomisieren im Falle der Bayes-Bewertungsregel nicht lohnt, da dadurch keine höhere Bewertung als bei Betrachtung ausschließlich nicht-randomisierter Handlungen erzielt wird; vgl. z.B. BLACKWELL, D./GIRSHICK, M.A. (1954), S. 116 sowie S. 130f.; FERGUSON, T.S. (1967), S. 43 und die graphische Analyse in Abschnitt 4.3.3.3. 
dungsfunktionen maximalen Nutzen sichern, wenn er eine optimale Entscheidungsfunktion benutzt. Mit demselben Argument, das zuvor am Beispiel ärztlicher Aktionen ausführlich erläutert wurde, läßt sich auch für Entscheidungsfunktionen des Arztes nachweisen, daß unter den hier verwendeten Voraussetzungen ein Randomisieren dem Patienten niemals einen echten Vorteil (im Sinne eines höheren Bayes-Nutzens) verschafft. Insofern reicht es aus, das Verhalten des Arztes bei Einsatz einer diagnostischen Maßnahme $T_{j}$ durch die Wahl einer optimalen nicht-randomisierten Entscheidungsfunktion $\mathrm{d}_{\mathrm{j}}{ }^{*}$ zu beschreiben ${ }^{83}$ :

$$
\begin{gathered}
\Phi_{B}\left(d_{j}^{*}\right)=\max \left\{\Phi_{B}\left(d_{j}\right) \mid d_{j} \in D_{j}\right\} \\
\text { bzw.: } d_{j}^{*} \in \arg \max \left\{\Phi_{B}\left(d_{j}\right) \mid d_{j} \in D_{j}\right\} .
\end{gathered}
$$

Als kompensierender Optionspreis einer optimalen Entscheidungsfunktion $d_{j}{ }^{*}$ des Arztes ergibt sich derjenige Geldbetrag $z$, der die folgende Gleichung erfüllt ${ }^{84}$ :

$$
\sum_{i=1}^{n} P\left(K_{i}\right) \cdot \sum_{h=1}^{k_{j}} P\left(z_{j h} \mid K_{i}\right) \cdot u\left\{g\left[d_{j}^{*}\left(z_{j h}\right), K_{i}\right]-e_{p} \cdot z\right\}=\Phi_{B}\left(a^{*}\right) .
$$

Der Ausdruck auf der linken Seite gibt den Nutzen des Patienten aus demjenigen Prospekt an, der entsteht, wenn er eine von seinem Krankheitszustand unabhängige Zahlung $z$ leistet und der Arzt die Entscheidungsfunktion $d_{j}{ }^{*}$ auswählt. Bezeichnet man diese Größe kürzer mit $\Phi_{B}\left(d_{j}{ }^{*}, z\right)$, dann wird der kompensierende Optionspreis einer optimalen ärztlichen Entscheidungsfunktion $d_{j}{ }^{*}$ implizit definiert gemäß:

$$
\Phi_{B}\left(d_{j}^{*}, z\right)=\Phi_{B}\left(a^{*}\right)
$$

Die Existenz eines derartigen Optionspreises, der sich auf eine einzelne Entscheidungsfunktion bezieht, ist auf jeden Fall dann gewährleistet, wenn folgende Bedingung gilt: für je zwei Konsequenzenvektoren $g_{1}, g_{2} \in R^{P}$ mit $u\left(g_{1}\right)>u\left(g_{2}\right)$ exi-

83: Diese Entscheidungsfunktion braucht nicht eindeutig bestimmt zu sein.

84: Dabei bezeichnet $e_{p}$ denjenigen Einheitsvektor des $R^{P}$, der an der $p$-ten Stelle eine Eins besitzt. 
stiert ein endlicher (positiver) Geldbetrag m, der die Gleichung

$$
u\left(g_{1}-e_{p} \cdot m\right)=u\left(g_{2}\right)
$$

erfüllt. In der weiteren Analyse wird die Gültigkeit dieser Annahme unterstellt. Sie impliziert eine "starke" Substitutionseigenschaft des Geldes, da offenbar hinreichend große Veränderungen des individuellen Geldvermögens immer in der Lage sind, Unterschiede in den Ausprägungen der Gesundheitsattribute im Hinblick auf die Nutzenbewertung durch den Patienten auszugleichen ${ }^{85}$. Die Eindeutigkeit des über Gleichung (4.21a) definierten Optionspreises folgt dann aus der Annahme der strengen Monotonie von $u$, die insbesondere einen positiven Grenznutzen des Geldvermögens sichert.

Entsprechend ergibt sich eine implizite Darstellung des kompensierenden Optionspreises $z\left(d_{j}\right)$ einer beliebigen Entscheidungsfunktion $d_{j}$ des Arztes über die folgende Gleichung:

$$
\Phi_{\mathrm{B}}\left(\mathrm{d}_{\mathrm{j}}, \mathrm{z}\right)=\Phi_{\mathrm{B}}\left(\mathrm{a}^{*}\right)
$$

Dabei bezeichnet $\Phi_{B}\left(d_{j}, z\right)$ in Analogie zur oben eingeführten Notation den Nutzen des Patienten, wenn er eine von seinem Krankheitszustand unabhängige Zahlung $z$ leistet und der Arzt die Entscheidungsfunktion $\mathrm{d}_{\mathrm{j}}$ wählt.

Es liegt dann nahe, den kompensierenden Optionspreis einer diagnostischen Maßnahme $T_{j}$ als das Maximum jener Zahlungsbereitschaften zu definieren, die der Patient für die aufgrund der diagnostischen Information möglichen Entscheidungs-

85: Die im Text verwendete Annahme sichert die Existenz eines kompensierenden Optionspreises für jede mögliche Entscheidungsfunktion des Arztes. Alternativ könnte man die Existenz eines derartigen Optionspreises lediglich für diejenigen Entscheidungsfunktionen voraussetzen, die den Patienten mindestens so gut wie in der Situation ohne weitere Diagnostik stellen. Diese schwächere Annahme liefert dieselben Resultate im Hinblick auf den kompensierenden Optionspreis diagnostischer Maßnahmen. 
funktionen besitzt. Damit gilt für den kompensierenden Optionspreis $z\left(T_{j}\right)$, der im folgenden auch kurz als Optionspreis einer diagnostischen Maßnahme $T_{j}$ bezeichnet wird ${ }^{86}$ :

$$
z\left(T_{j}\right)=\max \left\{z\left(d_{j}\right) \mid d_{j} \in D_{j}\right\}
$$

Interessanterweise braucht der auf diese Weise definierte Optionspreis einer diagnostischen Maßnahme nicht mit dem Optionspreis einer dazugehörigen optimalen Entscheidungsfunktion übereinzustimmen ${ }^{87}$.

Im Einklang mit der Entscheidungstheorie lassen sich zwei verschiedene Kategorien monetärer Bewertungen der diagnostischen Information unterscheiden ${ }^{88}$. Dabei knüpft diese Unterscheidung an die Qualität der Information an, die eine diagnostische Maßnahme liefert. Handelt es sich um eine perfekte diagnostische Maßnahme, so bezeichnet man die Zahlungsbereitschaft des Patienten als den Optionspreis der vollkommenen diagnostischen Information. Erlaubt eine diagnostische Maßnahme hingegen nicht in jedem Fall den sicheren Rückschluß auf den tatsächlichen Krankheitszustand des Patienten, ergibt sich als dazugehörige individuelle Zahlungsbereitschaft ein Optionspreis der unvollkommenen diagnostischen Information.

Ferner sind in Bezug auf die Präferenzen des Patienten zwei verschiedene Fälle zu unterscheiden. Unterliegen diese Präferenzen lediglich den in Abschnitt 4.2 angesprochenen Annahmen, dann handelt es sich bei der Bestimmung des Optionspreises einer diagnostischen Maßnahme um den allgemeinen Fall. Weisen die Präferenzen des Patienten darüber hinaus eine spezielle Struktur auf, eröffnet sich die

86: Die Berücksichtigung lediglich nicht-randomisierter Entscheidungsfunktionen bei der Ermittlung des Optionspreises einer diagnostischen Maßnahme bedeutet in dem hier verwendeten Modellrahmen keinerlei Einschränkung. Falls nämlich eine echte randomisierte ärztliche Entscheidungsfunktion existiert, deren Optionspreis maximal bezüglich $\mathrm{D}_{\mathrm{j}}{ }^{*}$ ausfällt, enthält diese mit positiven Wahrscheinlichkeiten ausschließlich solche Entscheidungsfunktionen aus $\mathrm{D}_{\mathrm{j}}$, die denselben Optionspreis besitzen.

87: Vgl. dazu năher Abschnitt 4.3.2.

88: Vgl. WINKLER, R.L. (1972), S. 296ff.; LINDLEY, D.V. (1974), S. 106ff.; BAMBERG, G. (1975), S. 202ff. sowie FERSCHL, F. (1975), S. $119 \mathrm{ff}$.

Allerdings behandelt die Entscheidungstheorie mit dem Brutto-Wert der Information eine monetäre Größe, die in konzeptioneller Hinsicht nicht mit dem in dieser Arbeit diskutierten Optionspreis übereinzustimmen braucht; vgl. dazu Abschnitt 4.3.4. 
Möglichkeit einer besonders einfachen Darstellung des Optionspreises der diagnostischen Information. Dieser Tatbestand rechtfertigt eine gesonderte Analyse dieser speziellen Situation. Insgesamt liegen damit im Hinblick auf die Ermittlung des Optionspreises der diagnostischen Information vier verschiedene Fälle vor, die im folgenden behandelt werden.

\subsubsection{Der Optionspreis diagnostischer Maßnahmen im allgemeinen Fall}

\subsubsection{Der Optionspreis der vollkommenen diagnostischen Information}

Handelt es sich bei einer diagnostischen Maßnahme $T_{j}$ um ein perfektes Informationsinstrument, dann zeigt definitionsgemäß jedes mögliche Testergebnis mit Wahrscheinlichkeit Eins den Krankheitszustand des Patienten korrekt an. Aufgrund dieser eindeutigen Zuordnung erlaubt eine perfekte diagnostische Maßnahme dem Arzt, sein Handeln allein vom Krankheitszustand des Patienten abhängig zu gestalten. Insofern kann auf die explizite Angabe der Testergebnisse verzichtet werden und das Entscheidungsproblem des Arztes reduziert sich auf ${ }^{89}$ :

$$
\max _{d_{j} \in D_{j}} \Phi_{B}\left(d_{j}\right)=\sum_{i=1}^{n} P\left(K_{i}\right) \cdot \max _{a_{f} \in A} U\left(a_{f}, K_{i}\right) .
$$

Als Lösung dieses Entscheidungsproblems ergibt sich genau dann eine eindeutig bestimmte optimale Entscheidungsfunktion, wenn zu jedem möglichen Krankheitszustand gerade eine Aktion des Arztes existiert, die dem Patienten einen maximalen Nutzen verschafft. Hingegen existieren mehrere optimale Entscheidungsfunktionen im Falle vollkommener diagnostischer Information, wenn bei mindestens einem möglichen Krankheitszustand dem Arzt wenigstens zwei verschiedene Aktionen zur Verfügung stehen, die aus der Sicht des Patienten denselben Nutzen erzeugen.

Die Analyse vollkommener diagnostischer Maßnahmen geschieht zunächst unter einer zusätzlichen Annahme, die eine Forderung an die Menge A der ärztlichen Aktionen beinhaltet. Unter dieser weiteren Voraussetzung ergibt sich der dazugehörige Optionspreis in einfacher Weise. Konkret wird angenommen, daß zu jedem

89: Die Lösung dieses Entscheidungsproblems legt zugleich jede optimale Entscheidungsfunktion des Arztes bei vollkommener diagnostischer Information fest. Man erhält eine derartige Entscheidungsfunktion, indem man jedem Testergebnis, das den Krankheitszustand $\mathrm{K}_{\mathrm{i}}$ anzeigt, eine Aktion aus der Menge $\arg \max \left\{\left.U\left(\mathrm{a}_{\mathrm{f}}, \mathrm{K}_{\mathrm{i}}\right)\right|_{\left.\mathrm{a}_{\mathrm{f}} \in \mathrm{A}\right\} \text { zuordnet. }}\right.$ 
Krankheitszustand eine Aktion des Arztes existiert, die hinsichtlich ihrer Konsequenzen für den Patienten alle anderen Aktionen dominiert, die in A enthalten sind. Bezeichnet man diese Aktion mit $\mathrm{a}^{\prime}\left(\mathrm{K}_{\mathbf{i}}\right)$, dann gilt:

$$
\begin{gathered}
\mathrm{g}\left[\mathrm{a}^{\prime}\left(\mathrm{K}_{\mathrm{i}}\right), \mathrm{K}_{\mathrm{i}}\right] \geq \mathrm{g}\left(\mathrm{a}_{\mathrm{f}}, \mathrm{K}_{\mathrm{i}}\right) ; \quad \mathrm{g}\left[\mathrm{a}^{\prime}\left(\mathrm{K}_{\mathrm{i}}\right), \mathrm{K}_{\mathrm{i}}\right] \neq \mathrm{g}\left(\mathrm{a}_{\mathrm{f}}, \mathrm{K}_{\mathrm{i}}\right) \\
\forall \mathrm{a}_{\mathrm{f}} \neq \mathrm{a}^{\prime}\left(\mathrm{K}_{\mathrm{i}}\right), \quad \mathrm{a}_{\mathrm{f}} \in \mathrm{A} ; \quad \forall \mathrm{K}_{\mathrm{i}} .
\end{gathered}
$$

Die Aktion a'( $\left.\mathrm{K}_{\mathrm{i}}\right)$ repräsentiert offenbar eine besonders günstige Handlung des Arztes, wenn sich der Patient im Krankheitszustand $\mathrm{K}_{\mathrm{i}}$ befindet. In diesem Fall stellt $a^{\prime}\left(K_{i}\right)$ den Patienten gegenüber einer beliebigen anderen Aktion $a_{f}$ des Arztes bei mindestens einer Konsequenz besser, ohne ihm bei irgendeiner anderen Konsequenz ein schlechteres Ergebnis zu liefern. Diese Dominanz bezüglich der Konsequenzen bezeichnet eine starke Optimalitätseigenschaft, welche die Eindeutigkeit von $a^{\prime}\left(K_{i}\right)$ sichert. Aufgrund der strengen Monotonie der Funktion u fällt der Nutzen des Patienten bei der Aktion $a^{\prime}\left(K_{\mathfrak{i}}\right)$ in dem entsprechenden Krankheitszustand größer aus als bei allen anderen Aktionen, die dem Arzt zur Verfügung stehen.

$$
\mathrm{a}^{\prime}\left(\mathrm{K}_{\mathrm{i}}\right)=\arg \max \left\{\mathrm{u}\left[\mathrm{g}\left(\mathrm{a}_{\mathrm{f}}, \mathrm{K}_{\mathrm{i}}\right)\right] \mid \mathrm{a}_{\mathrm{f}} \in \mathrm{A}\right\} .
$$

Die zuvor eingeführte Annahme sichert aufgrund der Eindeutigkeit von $a^{\prime}\left(K_{\mathfrak{j}}\right)$ die Existenz einer Funktion

$$
\mathbf{a}^{\prime}: \mathbf{K} \rightarrow \mathrm{A} \text {, }
$$

die jedem möglichen Krankheitszustand des Patienten gerade die Aktion a'( $\left.\mathrm{K}_{\mathrm{i}}\right)$ des Arztes zuordnet. Falls dabei allen Krankheitszuständen dieselbe Aktion a' zugeordnet wird, liegt ein triviales Entscheidungsproblem vor, da der Arzt durch die Wahl dieser Aktion dem Patienten unabhängig von seinem tatsächlichen Krankheitszustand ein maximales Nutzenniveau garantieren kann 90 . Insofern impliziert die Nicht-Trivialität der betrachteten Entscheidungssituation, daß es zumindest zwei Krankheitszustände gibt, für die die Aktion a' verschieden ausfällt.

90: a' stellt in diesem Fall eine gleichmäßig beste Aktion des Arztes dar. 
Unter der zusätzlich getroffenen Annahme ergibt sich als Lösung des Entscheidungsproblems bei vollkommener diagnostischer Information, daß der Arzt mit Wahrscheinlichkeit Eins die Aktion $a^{\prime}\left(K_{\mathfrak{i}}\right)$ wählt, falls der Patient sich im Krankheitszustand $\mathrm{K}_{\mathrm{i}}$ befindet. Insgesamt führt diese Handlungsweise des Arztes zu einer eindeutig bestimmten Entscheidungsfunktion $d_{j}$. Der dazugehörige Nutzen $\Phi_{B}\left(d_{j}{ }^{\prime}\right)$ des Patienten beläuft sich auf:

$$
\Phi_{B}\left(d_{j}^{\prime}\right)=\sum_{i=1}^{n} P\left(K_{i}\right) \cdot u\left\{g\left[a^{\prime}\left(K_{i}\right), K_{i}\right]\right\}
$$

Der Optionspreis dieser optimalen Entscheidungsfunktion $d_{j}{ }^{\prime}$ bezeichnet jenen Geldbetrag $z$, der implizit durch die folgende Gleichung definiert wird:

$$
\sum_{i=1}^{n} P\left(K_{i}\right) \cdot u\left\{g\left[a^{\prime}\left(K_{i}\right), K_{i}\right]-e_{p} \cdot z \mid=\Phi_{B}\left(a^{*}\right)\right.
$$

(4.29a) bzw.: $\quad \Phi_{\mathrm{B}}\left(\mathrm{d}_{\mathrm{j}}^{\prime}, \mathrm{z}\right)=\Phi_{\mathrm{B}}\left(\mathrm{a}^{*}\right)$.

Es läßt sich zeigen, daß der Optionspreis jeder anderen Entscheidungsfunktion des Arztes niedriger als $z\left(d_{j}{ }^{*}\right)$ ausfallen muß. Dazu betrachtet man eine beliebige, von $d_{j}{ }^{\prime}$ verschiedene und daher suboptimale Entscheidungsfunktion $d_{j}$ ". Annahmegemäß existiert zumindest ein Krankheitszustand $\mathrm{K}_{\mathrm{b}}$ des Patienten, bei dem der Arzt aufgrund von $d_{j}$ " bei mindestens einem Testergebnis der untersuchten vollkommenen diagnostischen Maßnahme eine andere Aktion als $a^{\prime}\left(K_{b}\right)$ wählt. Die Dominanzeigenschaft von $\mathrm{a}^{\prime}\left(\mathrm{K}_{\mathrm{b}}\right)$ hinsichtlich der Konsequenzen garantiert zusammen mit der strengen Monotonie von $u$, daß der Patient den Einsatz der Aktion $a^{\prime}\left(K_{b}\right)$ auch dann bei derartigen Testergebnissen strikt präferiert, wenn er jeweils eine Zahlung z zu leisten hat. Damit gilt für jede suboptimale ärztliche Entscheidungsfunktion $d_{j}$ ":

$$
\Phi_{B}\left(d_{j}^{\prime}, z\right)>\Phi_{B}\left(d_{j}{ }^{\prime \prime}, z\right) ; \quad d_{j}^{\prime} \neq d_{j}^{\prime \prime} ; \quad d_{j}^{\prime \prime} \in D_{j}
$$

Insbesondere ist diese Ungleichung für den Optionspreis $z\left(d_{j}{ }^{\prime}\right)$ der optimalen Entscheidungsfunktion des Arztes erfüllt. Daraus folgt aufgrund der strengen Monotonie der Nutzenfunktion $\Phi_{\mathrm{B}}$ des Patienten in z die oben aufgestellte Behauptung:

$$
z\left(d_{j}^{\prime}\right)>z\left(d_{j}^{\prime \prime}\right) ; \quad d_{j}^{\prime} \neq d_{j}^{\prime \prime} ; \quad d_{j}^{\prime \prime} \in D_{j}
$$


Damit bildet der Optionspreis der Entscheidungsfunktion $d_{j}$ zugleich die maximale Zahlung, die der Patient unabhängig von seinem Krankheitszustand für eine durch die vollkommene diagnostische Information ermöglichte Entscheidungsfunktion zu leisten bereit ist. Der Optionspreis der optimalen ärztlichen Entscheidungsfunktion stimmt daher mit dem Optionspreis einer vollkommenen diagnostischen Maßnahme überein, falls für jeden Krankheitszustand des Patienten eine Aktion a' mit der beschriebenen Eigenschaft existiert, d.h. es gilt:

$$
\mathrm{z}\left(\mathrm{T}_{\mathrm{j}}\right)=\mathrm{z}\left(\mathrm{d}_{\mathrm{j}}{ }^{\prime}\right)
$$

Läßt man die Annahme der Existenz einer Aktion a'( $\left.\mathrm{K}_{\mathrm{i}}\right)$ für sämtliche Krankheitszustände fallen, erfordern die soeben dargestellten Resultate eine Modifikation. In diesem Fall können mehrere Entscheidungsfunktionen existieren, die eine Lösung des ärztlichen Entscheidungsproblems bei vollkommener diagnostischer Information gemäß Gleichung (4.24) darstellen ${ }^{91}$.

Allgemein - d.h. unabhängig davon, ob die optimale Entscheidungsfunktion eindeutig bestimmt ist oder nicht - braucht der Optionspreis einer optimalen Entscheidungsfunktion des Arztes dann nicht mehr mit dem Optionspreis der perfekten diagnostischen Information übereinzustimmen. Dieses negative Resultat ergibt sich, wenn man die zuvor skizzierte Argumentation nachvollzieht, ohne die Existenz einer Aktion a' für jeden Krankheitszustand des Patienten vorauszusetzen. Eine suboptimale Entscheidungsfunktion $d_{j}$ " läßt den Arzt wie zuvor bei mindestens einem Testergebnis eine Aktion a" ergreifen, die den Patienten schlechter stellt als die in einer optimalen Entscheidungsfunktion $d_{j}{ }^{*}$ spezifizierte Aktion. Ohne die oben $z u-$ sätzlich getroffene Annahme braucht die gemäß $d_{j}^{*}$ gewählte Aktion die davon verschiedene Aktion a" jedoch nicht hinsichtlich ihrer Konsequenzen für den Patienten zu dominieren. Liegt diese Dominanzeigenschaft nicht vor, dann reicht die strenge Monotonie der Funktion u nicht aus, um zu gewährleisten, daß der Patient den Einsatz der in $d_{j}{ }^{*}$ angegebenen Aktion auch dann noch gegenüber a" präferiert (gegeben das betrachtete Testergebnis), wenn er jeweils eine Zahlung $\mathrm{z}$ zu leisten hat ${ }^{92}$.

91: Vgl. zu den Bedingungen, unter denen mehrere optimale Entscheidungsfunktionen des Arztes existieren, die eingangs dieses Abschnitts angeführten Bemerkungen.

92: Seien $\mathrm{g}^{*}$ bzw. $\mathrm{g}$ " die zugehörigen Konsequenzenvektoren, die sich aufgrund desjenigen Krankheitszustands ergeben, den das betrachtete Testergebnis anzeigt, dann gilt gemäß Voraussetzung: 
Daraus folgt unmittelbar, daß die Ungleichung

$$
\Phi_{B}\left(d_{j}^{*}\right)>\Phi_{B}\left(d_{j}{ }^{\prime \prime}\right)
$$

erfüllt sein und gleichzeitig bzw. dennoch gelten kann:

$$
\Phi_{B}\left(d_{j}^{*}, z\right)<\Phi_{B}\left(d_{j}^{\prime \prime}, z\right)
$$

wenn $z$ einen von Null hinreichend verschiedenen Geldbetrag bezeichnet. Gilt diese zweite Ungleichung speziell für den Optionspreis $z\left(d_{j}{ }^{\prime \prime}\right)$ der suboptimalen Entscheidungsfunktion, dann folgt daraus für den Optionspreis der betrachteten optimalen Entscheidungsfunktion:

$$
\mathrm{z}\left(\mathrm{d}_{\mathrm{j}}^{*}\right)<\mathrm{z}\left(\mathrm{d}_{\mathrm{j}}{ }^{\prime}\right)
$$

In diesem Fall liegt der Optionspreis der Entscheidungsfunktion $\mathrm{d}_{\mathrm{j}}{ }^{*}$ unter dem Optionspreis einer suboptimalen Entscheidungsfunktion des Arztes. Besitzt diese Aussage für sämtliche optimalen ärztlichen Entscheidungsfunktionen Gültigkeit, dann übersteigt der Optionspreis der vollkommenen diagnostischen Maßnahme den Optionspreis jeder Entscheidungsfunktion $\mathrm{d}_{\mathrm{j}}{ }^{*}$ :

$$
z\left(T_{j}\right)>z\left(d_{j}^{*}\right) ; \quad d_{j}^{*} \in \arg \max \left\{\Phi_{B}\left(d_{j}\right) \mid d_{j} \in D_{j}\right\}
$$

Wie die soeben angestellten Überlegungen zeigen, läßt sich eine derartige Konstellation ohne eine Ergänzung der in Abschnitt 4.2 genannten Voraussetzungen nicht ausschließen. Zur Ermittlung des Optionspreises von $\mathrm{T}_{\mathrm{j}}$ reicht es demzufolge im allgemeinen nicht aus, lediglich die optimalen Entscheidungsfunktionen des Arztes zu analysieren.

$$
u\left(g^{*}\right)>u\left(g^{\prime \prime}\right), \text { jedoch nicht: } g^{*} \geq g^{\prime \prime}
$$

Auch bei strenger Monotonie von u können dann Geldbeträge z existieren, für die gilt:

$$
u\left(g^{*}-e_{p} \cdot z\right)<u\left(g^{\prime \prime}-e_{p} \cdot z\right) .
$$


Wie läßt sich eine Diskrepanz zwischen dem Optionspreis einer optimalen Entscheidungsfunktion des Arztes und dem Optionspreis der betreffenden diagnostischen Maßnahme erklären ${ }^{93}$ ? Offenbar unterschätzt in einem solchen Fall die Zahlungsbereitschaft des Patienten für das optimale Vorgehen des Arztes diejenige Zahlungsbereitschaft, die er für die diagnostische Maßnahme insgesamt entfaltet. Andererseits erzielt der Patient aus dem Einsatz einer optimalen Entscheidungsfunktion einen Nutzen, der höher liegt als derjenige Nutzen, den ihm der Arzt mit einer Entscheidungsfunktion verschafft, deren Optionspreis maximal ist. Allgemeiner zeigt die oben angegebene Argumentation, daß für zwei ärztliche Entscheidungsfunktionen $d_{j}{ }^{1}$ und $d_{j}{ }^{2}$ gleichzeitig gelten kann:

$$
\Phi_{B}\left(d_{j}{ }^{1}\right)>\Phi_{B}\left(d_{j}{ }^{2}\right) \text { und } z\left(d_{j}{ }^{1}\right)<z\left(d_{j}{ }^{2}\right) .
$$

Daraus folgt unmittelbar, daß die Ordnung auf der Menge $D_{j}$, die durch die Präferenzen des Patienten in einer medizinischen Entscheidungssituation entsteht, nicht mit derjenigen Ordnung übereinzustimmen braucht, die sich aufgrund der anhand des kompensierenden Optionspreises gemessenen individuellen Zahlungsbereitschaften ergibt. Mit anderen Worten: im allgemeinen vermag der kompensierende Optionspreis eine Menge ärztlicher Entscheidungsfunktionen nicht im Einklang mit den Präferenzen des Patienten zu ordnen 94.

93: Diese Konstellation kann auch bei unvollkommenen diagnostischen Maßnahmen auftreten; vgl. Abschnitt 4.3.2.2.

Bei den in der Entscheidungstheorie üblicherweise behandelten Problemen kann diese Diskrepanz lediglich bei der Bestimmung des Brutto-Werts unvollkommener Informationsinstrumente entstehen, sofern eine eindimensionale (i.a. monetäre) Konsequenzenfunktion unterstellt wird. Auch in diesen Fällen wäre strenggenommen zu unterscheiden zwischen dem Brutto-Wert einer optimalen Entscheidungsfunktion und dem Brutto-Wert des unvollkommenen Informationsinstruments (der höher läge). In der Literatur betrachtet man allerdings ausschließlich den Brutto-Wert einer optimalen Entscheidungsfunktion; vgl. BAMBERG, G. (1975), S. 202; BAMBERG, G./COENENBERG, A.G./KLEINE-DOEPKE, R. (1976), S. 42.

Falls die zur Bewertung einzelner Prospekte verwendete Regel bzw. Funktion $\Phi$ additiv und konstantenerhaltend ist [vgl. zu diesen Begriffen BAMBERG, G. (1975), S. 203 und FIRCHAU, V. (1980), S. 11], stimmt der Brutto-Wert jeder optimalen Entscheidungsfunktion mit dem Brutto-Wert des Informationsinstruments selbst überein. Dies gilt z.B. für die Bayes-Regel, wenn man die häufig gebrauchte Annahme eines "linearen Risikonutzens" voraussetzt; vgl. dazu Abschnitt 4.3.3.

94: Der kompensierende Optionspreis bildet das Ergebnis eines Vergleichs einer neuen Situation mit einer als Ausgangslage bezeichneten Referenzsituation, das den Unterschied der damit 
Die Möglichkeit einer Diskrepanz zwischen dem Optionspreis einer optimalen Entscheidungsfunktion und dem Optionspreis der dazugehörigen diagnostischen Maßnahme beruht demzufolge darauf, daß der kompensierende Optionspreis ein individuelles Wohlfahrtsma $B$ darstellt, das die sogenannte Ordnungsbedingung nicht erfüllt ${ }^{95}$. In ökonomischer Hinsicht impliziert dieser Tatbestand eine Abhängigkeit der individuellen Präferenzen bezüglich einer Menge ärztlicher Handlungen von der Höhe des Geldvermögens ${ }^{96}$. Falls der Patient eine von seinem Krankheitszustand unabhängige Zahlung $z\left(T_{j}\right)$ für den Einsatz der diagnostischen Maßnahme $T_{j} z u$ leisten hat, maximiert daher möglicherweise eine andere ärztliche Entscheidungsfunktion seine Präferenzen als ohne diese Zahlung.

Wie aus der Darstellung des Entscheidungsproblems bei vollkommener diagnostischer Information in Gleichung (4.24) hervorgeht, hängt die optimale Vorgehensweise des Arztes nicht davon ab, welche spezielle diagnostische Maßnahme diese Information liefert. Davon ausgehend läßt sich zeigen, daß die kompensierenden

verbundenen Wohlfahrtspositionen des Individuums in monetären Einheiten aus der Perspektive der neuen Situation mißt. Der Vergleich verschiedener neuer Situationen mit Hilfe der dazugehörigen kompensierenden Optionspreise verwendet daher Zahlungsbereitschaften, denen jeweils eine andere Perspektive zugrunde liegt. Falls beispielsweise der Arzt dem Patienten über zwei verschiedene Entscheidungsfunktionen dasselbe Nutzenniveau verschaffen kann, impliziert diese Äquivalenz deshalb nicht, daß die kompensierenden Optionspreise beider Handlungen übereinstimmen müssen.

95: Zur Ordnungsbedingung bei individuellen Wohlfahrtsmaßen vgl. z.B. AHLHEIM, M./ROSE, M. (1989), S. 13f.

In dieser Hinsicht liegt eine Analogie zwischen dem kompensierenden Optionspreis und der Kompensationsvariation vor, die konzeptionell die korrespondierende individuelle Zahlungsbereitschaft repräsentiert, falls Allokationen bei Sicherheit zu vergleichen sind. Auch die Kompensationsvariation genügt der Ordnungsbedingung nicht; vgl. CORNWALL, R.C. (1984), S. 583f.; AHLHEIM, M./ROSE, M. (1989), S. 70f.

Im Gegensatz dazu erfüllen sowohl die Äquivalenzvariation als auch der äquivalente Optionspreis die Ordnungsbedingung. Würde man daher die beim Patienten aufgrund der diagnostischen Information eintretende Wohlfahrtsänderung durch den äquivalenten Optionspreis messen, entspräche der Optionspreis einer diagnostischen Maßnahme in dem hier verwendeten Modellrahmen immer dem Optionspreis jeder dadurch ermöglichten optimalen Entscheidungsfunktion des Arztes.

96: Unter der zusätzlichen Voraussetzung eines "linearen Risikonutzens" besteht diese Abhängigkeit nicht und der Optionspreis einer diagnostischen Maßnahme entspricht gerade dem Optionspreis einer dazugehörigen optimalen Entscheidungsfunktion; vgl. dazu Abschnitt 4.3.3. 
Optionspreise sämtlicher vollkommenen diagnostischen Maßnahmen in einer medizinischen Entscheidungssituation identisch sind ${ }^{97}$. Diese Übereinstimmung gilt unabhängig davon, ob die zusätzliche Voraussetzung bezüglich der Existenz einer Aktion a' für jeden Krankheitszustand des Patienten erfüllt ist oder nicht. Damit ergibt sich:

$$
\mathbf{z}^{*}=\mathbf{z}\left(\mathrm{T}_{\mathbf{j}}\right)
$$

für alle diagnostischen Maßnahmen $T_{j}$, die dem Arzt vollkommene Information im Hinblick auf den Krankheitszustand des Patienten liefern. Diese Eigenschaft erlaubt es, $z^{*}$ allgemeiner als den Optionspreis der vollkommenen diagnostischen Information zu bezeichnen, den die jeweils vorliegende medizinische Entscheidungssituation vollständig determiniert.

Der Optionspreis der vollkommenen diagnostischen Information beträgt genau dann Null, wenn sich das Wissen des Arztes über den tatsächlichen Krankheitszustand nicht zur Verbesserung der Wohlfahrtsposition des Patienten verwenden läßt. Dies impliziert, daß (zumindest) eine optimale Entscheidungsfunktion des Arztes durch eine konstante Funktion gegeben ist. In diesem Fall steht dem Arzt eine dominante bzw. gleichmäßig beste Aktion zur Verfügung ${ }^{98}$. Insgesamt beträgt damit der Optionspreis der vollkommenen diagnostischen Information bei positiven Wahrscheinlichkeiten sämtlicher Krankheitszustände gerade in den trivialen Entscheidungssituationen Null, während er in allen nicht-trivialen Entscheidungssituationen positiv ausfällt.

97: Dieser Nachweis stützt sich im wesentlichen auf dasselbe Argument, das in Abschnitt 4.3.1 mehrfach zum Beweis der allgemeinen Existenz einer optimalen nicht-randomisierten Handlung des Arztes in dem hier verwendeten Modellrahmen angeführt wurde. Sei $z\left(\mathrm{~T}_{\mathrm{j}}\right)$ der Optionspreis einer beliebigen vollkommenen diagnostischen Maßnahme $T_{j}$. Dann existiert mindestens eine Entscheidungsfunktion, deren Optionspreis gerade $z\left(T_{j}\right)$ beträgt und die den Arzt für jeden möglichen Krankheitszustand eine eindeutig bestimmte Aktion ergreifen läßt. Diese Entscheidungsfunktion steht dem Arzt bei vollkommener diagnostischer Information unabhängig von der speziell betrachteten Maßnahme immer zur Verfügung.

98: Diese Aussage gilt allerdings nur dann, wenn sämtliche Krankheitszustände eine positive Wahrscheinlichkeit besitzen; vgl. BAMBERG, G. (1975), S. 210 f. 


\subsubsection{Der Optionspreis unvollkommener diagnostischer Maßnahmen}

Setzt der Arzt eine unvollkommene diagnostische Maßnahme $T_{j}$ ein, so ermöglicht es ihm die damit verbundene Information grundsätzlich nicht, sein Handeln vollständig am Krankheitszustand des Patienten auszurichten. Aus diesem Grund fällt die in einer medizinischen Entscheidungssituation für den Patienten erreichbare Wohlfahrtsposition in der Regel niedriger aus als bei perfekter diagnostischer Information. Durch die Wahl einer optimalen Entscheidungsfunktion $d_{j}^{*}$ verschafft der Arzt dem Patienten einen Nutzen $\Phi_{B}\left(d_{j}^{*}\right)$ :

$$
\Phi_{\mathrm{B}}\left(\mathrm{d}_{\mathrm{j}}^{*}\right)=\max \left\{\Phi_{\mathrm{B}}\left(\mathrm{d}_{\mathrm{j}}\right) \mid \mathrm{d}_{\mathrm{j}} \in \mathrm{D}_{\mathrm{j}}\right\}
$$

$$
\text { bzw.: } d_{j}^{*} \in \arg \max \left\{\Phi_{B}\left(d_{j}\right) \mid d_{j} \in D_{j}\right\}
$$

Die detaillierte Angabe des mit $d_{j}^{*}$ verbundenen Nutzens läßt nach einigen Umformungen erkennen, wie der Arzt in dem hier verwendeten Modellrahmen eine optimale Entscheidungsfunktion konstruieren kann:

$$
\begin{aligned}
\Phi_{B}\left(d_{j}^{*}\right) & =\max _{d_{j} \in D_{j}} \sum_{i=1}^{n} P\left(K_{i}\right) \cdot U\left(d_{j}, K_{i}\right) \\
& \left.\left.=\max _{d_{j} \in D_{j}} \sum_{i=1}^{n} P\left(K_{i}\right) \cdot \sum_{h=1}^{k_{j}} P\left(z_{j h} \mid K_{i}\right) \cdot u|g| d_{j}\left(z_{j h}\right), K_{i}\right]\right\} .
\end{aligned}
$$

Erweitern liefert schließlich:

$$
\Phi_{B}\left(d_{j}^{*}\right)=\sum_{h=1}^{k_{j}} P\left(z_{j h}\right) \cdot \max _{a_{f} \in A} \sum_{i=1}^{n} P\left(K_{i} \mid z_{j h}\right) \cdot U\left(a_{f}, K_{i}\right) .
$$

Eine optimale Entscheidungsfunktion des Arztes ordnet jedem diagnostischen Resultat $z_{\text {jh }}$ der betrachteten Maßnahme eine Aktion zu, welche die Präferenzen des Patienten gegeben $z_{j h}$ und die daraus abgeleitete posteriore Wahrscheinlichkeitsverteilung der Krankheitszustände maximiert ${ }^{99}$.

99: Vgl. BLACKWELL, D./GIRSHICK, M.A. (1954), S. 175ff. 
In Analogie zur Analyse vollkommener diagnostischer Maßnahmen bestimmt sich auch der Optionspreis $z\left(d_{j}\right)$ einer beliebigen Entscheidungsfunktion, welche die unvollkommene diagnostische Information dem Arzt ermöglicht, gemäß der folgenden Gleichung:

$$
\Phi_{B}\left(d_{j}, z\right)=\Phi_{B}\left(a^{*}\right) ; d_{j} \in D_{j}
$$

Als Optionspreis $z\left(T_{j}\right)$ der unvollkommenen diagnostischen Maßnahme $T_{j}$ ergibt sich wiederum der maximale Optionspreis, den eine durch die Maßnahme ermöglichte Entscheidungsfunktion in der untersuchten medizinischen Entscheidungssituation erzielen kann:

$$
z\left(T_{j}\right)=\max \left\{z\left(d_{j}\right) \mid d_{j} \in D_{j}\right\}
$$

Wie zuvor im Falle vollkommener diagnostischer Information kann auch der Optionspreis einer unvollkommenen diagnostischen Maßnahme $T_{j}$ höher ausfallen als der Optionspreis einer dazugehörigen optimalen Entscheidungsfunktion des Arztes. Die Begründung und die Interpretation einer derartigen Diskrepanz lassen sich vollständig aus dem vorangegangenen Abschnitt übertragen. Auch bei unvollkommenen Diagnosetests liegt die Zahlungsbereitschaft des Patienten für eine optimale Entscheidungsfunktion des Arztes möglicherweise unterhalb derjenigen Zahlungsbereitschaft, die er für den Einsatz der Maßnahme selbst besitzt.

Im Gegensatz zum Optionspreis $\mathrm{z}^{*}$ der vollkommenen diagnostischen Information wird der Optionspreis der unvollkommenen diagnostischen Information nicht allein durch die vorliegende medizinische Entscheidungssituation determiniert. Darüber hinaus hängt die individuelle Zahlungsbereitschaft $\mathrm{z}\left(\mathrm{T}_{\mathrm{j}}\right)$ von der jeweils betrachteten diagnostischen Maßnahme ab. Diese zusätzliche Abhängigkeit beruht darauf, daß die 
Gruppe der unvollkommenen diagnostischen Maßnahmen sehr breit angelegt ist und daher z.B. in Bezug auf ihre Informationsleistung ganz unterschiedliche Diagnosetests enthalten kann ${ }^{100}$.

Eine weitere Eigenschaft des Optionspreises unvollkommener diagnostischer Maßnahmen ergibt sich, wenn man die Handlungsmöglichkeiten des Arztes bei vollkommener und bei unvollkommener diagnostischer Information vergleicht. Aufgrund der vollkommenen diagnostischen Information kann der Arzt dem Patienten grundsätzlich jeden Prospekt verschaffen, den die Information einer unvollkommenen diagnostischen Maßnahme ermöglicht ${ }^{101}$. Die Umkehrung dieser Aussage gilt jedoch nicht. Deshalb gelingt es dem Arzt mit Hilfe einer unvollkommenen diagnostischen Maßnahme niemals, den Patienten besser zu stellen als bei vollkommener diagnostischer Information. Demzufolge kann der Optionspreis der unvollkommenen diagnostischen Information nicht höher ausfallen als $z^{*}$. Wegen dieser Eigenschaft repräsentiert der Optionspreis der vollkommenen diagnostischen Information eine obere Schranke für den Optionspreis der diagnostischen Information insgesamt, die lediglich von der medizinischen Entscheidungssituation abhängt. Es gilt also:

$$
z^{*} \geq z\left(T_{j}\right) ; j=1, \ldots, m \text {. }
$$

Die untere Schranke für $z\left(T_{j}\right)$ bildet der Wert Null, da der Optionspreis der diagnostischen Information nicht negativ werden kann. Der Optionspreis $z\left(T_{j}\right)$ wird gemäß (4.40) genau dann Null, wenn zumindest eine optimale Entscheidungsfunktion des Arztes jedem möglichen Testergebnis dieselbe Aktion zuordnet ${ }^{102}$. Dieses Resultat dürfte unmittelbar einleuchten, da in diesem Fall die optimale Vorgehensweise des Arztes von der Informationsbeschaffung unbeeinflußt bleibt und demzufolge sich der für den Patienten maximal erzielbare Nutzen nicht erhöht. Diese Überlegung weist zunächst allen diagnostischen Maßnahmen, die zu keinem Informationsgewinn führen und damit keine neue Information liefern, einen Optionspreis von Null zu.

100: Vgl. dazu Abschnitt 3.4.

101: Diese Aussage gilt allerdings nur, wenn auch randomisierte ärztliche Entscheidungsfunktionen betrachtet werden. Wieder stützt sich der Beweis der im Text angegebenen Maximumseigenschaft von $z^{*}$ wesentlich auf die Existenz einer optimalen nicht-randomisierten Handlung des Arztes.

102: Falls die betrachtete diagnostische Maßnahme einen positiven Informationsgewinn erbringt, muß diese Aktion - bis auf Äquivalenz hinsichtlich der Nutzenallokation für den Patienten eindeutig bestimmt und daher insbesondere nicht-randomisiert sein. 
Darüber hinaus hängt es von der vorliegenden medizinischen Entscheidungssituation $a b$, inwieweit einzelne diagnostische Maßnahmen aufgrund der mit ihnen verbundenen zusätzlichen Information einen positiven Optionspreis besitzen. Im Vergleich zur Situation bei perfekter diagnostischer Information kann ein Optionspreis von Null bei unvollkommener diagnostischer Information in einer größeren Anzahl von Fällen auftreten, da hierfür die Existenz einer gleichmäßig besten Aktion des Arztes nicht notwendig ist.

\subsubsection{Der Optionspreis diagnostischer Maßnahmen bei "linearem Risi- konutzen"}

\subsubsection{Einführung einer monetären Verlustfunktion}

Im gesamten Abschnitt 4.3.3 wird die Klasse der zulässigen Präferenzen des Patienten eingeschränkt, um eine vereinfachte Analyse des Optionspreises der diagnostischen Information zu ermöglichen. Die zu diesem Zweck zusätzlich eingeführte Annahme eines "linearen Risikonutzens" verleiht dem Entscheidungsproblem des Arztes eine Struktur, die in der entscheidungstheoretischen Literatur häufig zur Bestimmung von Informationswerten dient ${ }^{103}$. Aus dieser Analogie ergibt sich unmittelbar die Möglichkeit, die dort erzielten Resultate auf die Untersuchung des Optionspreises diagnostischer Maßnahmen zu übertragen.

Bezeichne $g_{-p}\left(a_{f}, K_{i}\right)$ denjenigen Vektor, der aus den ersten p-1 Komponenten des Konsequenzenvektors $g\left(a_{f}, K_{i}\right)$ besteht, und entsprechend $g_{p}\left(a_{f}, K_{i}\right)$ das in $g\left(a_{f}, K_{i}\right)$ enthaltene Geldvermögen. Dann wird in Ergänzung der in Abschnitt 4.2 genannten Voraussetzungen angenommen, die Funktion $u$ des Patienten zur Bewertung sicherer Prospekte besitze folgende Struktur:

$$
u\left[g\left(a_{f}, K_{i}\right)\right]=f\left[g_{-p}\left(a_{f}, K_{i}\right)\right]+b \cdot g_{p}\left(a_{f}, K_{i}\right)
$$

wobei $b$ eine positive Konstante und $f$ eine streng monotone, reellwertige Funktion

103: Vgl. z.B. DRUKARCZYK, J. (1974); ALTROGGE, G. (1975); SCHINDEL, V. (1979), S. 44ff.; FIRCHAU, V. (1980); CHOU, Y. (1989) Ch. 24, 25. 
darstellt. Wie im allgemeinen Fall bewertet der Patient einen beliebigen Prospekt hinsichtlich der Konsequenzen g, indem er den damit verbundenen Erwartungswert der Funktion $\mathrm{u}$ bestimmt ${ }^{104}$.

Zunächst impliziert die Möglichkeit einer derartigen Darstellung der individuellen Präferenzen, daß die in den ersten p-1 Komponenten des Konsequenzenvektors enthaltenen Gesundheitsindikatoren bzw, -attribute und das Attribut Geldvermögen aus der Sicht des Patienten gegenseitig nutzenunabhängig sind ${ }^{105}$. Die Präferenzen des Patienten über Prospekte, die lediglich die Gesundheitsindikatoren betreffen, hängen damit nicht von der Höhe seines Geldvermögens ab. Umgekehrt beeinflußt die spezielle Ausprägung der Gesundheitsattribute die Ordnung derjenigen Prospekte nicht, die lediglich das Geldvermögen des Patienten berühren. Ferner schränkt die additive Form der Funktion u die Klasse derjenigen Präferenzordnungen, die mit dieser Annahme verträglich sind, weiter ein, da Gleichung (4.44) sogar die additive Unabhängigkeit der Gruppe der Gesundheitsattribute und des Geldvermögens impliziert ${ }^{106}$. Schließlich weist die Linearität von $u$ im Geldvermögen $g_{p}$ darauf hin, daß der Patient sich in Bezug auf das monetäre Attribut risikoneutral verhält. Die Voraussetzung einer funktionalen Form gemäß (4.44) impliziert somit recht spezielle Eigenschaften der individuellen Präferenzen. Aus diesem Grund handelt es sich um eine starke Annahme, die in Ermangelung einer besseren Bezeichnung - etwas unpräzise, aber plakativ - als "linearer Risikonutzen" des Patienten gekennzeichnet wird ${ }^{107}$.

Die speziellen Eigenschaften der Präferenzen des Patienten erlauben es nun, in einem weiteren Schritt eine monetäre Verlustfunktion einzuführen, die aus der Sicht

104: Auf eine explizite Darstellung dieser Bewertung wird an dieser Stelle verzichtet, da die spezielle Form der individuellen Präferenzen - wie weiter unten gezeigt wird - eine wesentlich einfachere Bewertung der relevanten Prospekte erlaubt.

105: Zum Begriff der gegenseitigen Nutzenunabhängigkeit von Attributen bzw. Attributgruppen vgl. z.B. FRENCH, S. (1986), S. $185 f$.

106: Zum Begriff der additiven Unabhängigkeit vgl. z.B. KEENEY, R.L./RAIFFA, H. (1976), S. 229ff. Vgl. zur Verwendung dieser Annahme im Gesundheitswesen kritisch BODILY, S.E. (1980), S. 160f.

107: In der entscheidungstheoretischen Literatur impliziert diese Annahme eine vollständig lineare Nutzenfunktion, da gewöhnlich das Geldvermögen (des Aktors) als einziges Argument berücksichtigt wird; vgl. z.B. BAMBERG, G. (1975). Die im Text diskutierte Annahme ist damit nicht identisch und wird aus diesem Grund in Anführungszeichen gesetzt. Die Berechtigung dieser Namensgebung ergibt sich aus der Tatsache, daß sich die Analyse in beiden Fällen auf die Untersuchung einer monetären Verlustfunktion beschränken kann. 
des Patienten die Konsequenzen eines Paars $\left(\mathrm{a}_{\mathrm{f}}, \mathrm{K}_{\mathrm{i}}\right)$ in geeigneter Weise zusammenfaßt. Dazu wird zunächst ein als Vergleichsmaßstab dienender Idealzustand betrachtet, in dem sich der Patient bei "bester Gesundheit" befindet und ein hohes Geldvermögen besitzt ${ }^{108}$. Dieser Referenzzustand werde durch einen ( $p$-dimensionalen) Vektor $\mathrm{H}_{0}$ repräsentiert. Die in Abschnitt 4.3.1 angesprochene Annahme einer starken Substitutionseigenschaft des Geldvermögens sorgt dann dafür, da $B$ für jeden Krankheitszustand $\mathrm{K}_{\mathrm{i}}$ des Patienten und für jede Aktion $\mathrm{a}_{\mathrm{f}}$ des Arztes eindeutig ein endlicher Geldbetrag $\mathrm{L}$ mit folgender Eigenschaft existiert:

$$
u\left(H_{0}-e_{p} \cdot L\right)=u\left[g\left(a_{f}, K_{i}\right)\right] ; \quad f=1, \ldots, r ; \quad i=1, \ldots, n
$$

Bei vorgegebenem Konsequenzenvektor $g\left(a_{\mathrm{f}}, \mathrm{K}_{\mathrm{i}}\right)$ bezeichnet $\mathrm{L}$ gerade jenen Geldbetrag, um den sich das Geldvermögen des Patienten in dem als Vergleichsmaßstab gewählten Zustand $\mathrm{H}_{0}$ verändern müßte, damit Indifferenz vorliegt. Aufgrund der Eindeutigkeit von L definiert Gleichung (4.45) implizit eine Funktion

$$
\begin{aligned}
\mathrm{L}_{0}: \mathrm{A} \times \mathrm{K} & \rightarrow \mathrm{R}, \\
\left(\mathrm{a}_{\mathrm{f}}, \mathrm{K}_{\mathrm{j}}\right) & \rightarrow \mathrm{L}\left(\mathrm{a}_{\mathrm{f}}, \mathrm{K}_{\mathrm{i}}\right),
\end{aligned}
$$

die man als monetäre Verlustfunktion bezeichnet. Dabei weist das Subskript auf die Abhängigkeit dieser Funktion vom Referenzzustand $\mathrm{H}_{0}$ hin. Der Geldbetrag $\mathrm{L}_{0}\left(\mathrm{a}_{\mathrm{f}}, \mathrm{K}_{\mathrm{i}}\right)$ repräsentiert damit in einer medizinischen Entscheidungssituation das monetäre Äquivalent der Diskrepanz zwischen dem Referenzzustand $\mathrm{H}_{0}$ und den für den Patienten relevanten Konsequenzen, wenn er sich im Krankheitszustand $\mathrm{K}_{\mathrm{i}}$ befindet und der Arzt die Aktion $a_{\mathrm{f}}$ ergreift.

108: Wie weiter unten gezeigt wird, ist es für die Analyse des Optionspreises der diagnostischen Information unerheblich, welcher Zustand als Bezugspunkt für die Ableitung der Verlustfunktion des Patienten gewählt wird. Die Verwendung eines Zustandes mit besonders großen Ausprägungen in allen Komponenten dient an dieser Stelle lediglich dazu, den Werten der Verlustfunktion für sämtliche betrachteten Konsequenzenvektoren ein positives Vorzeichen zu sichern. Vgl. zu einem ähnlichen Ansatz HABBEMA, J.D.F./HILDEN, J. (1981), S. 81, die als geeigneten Ausgangspunkt für die Ableitung einer Verlustfunktion des Patienten "a medically uneventful life and a quiet death in old age" ansehen. 
Die Annahme eines "linearen Risikonutzens" führt dazu, daß der Patient eine Handlung des Arztes im wesentlichen vermöge seiner Verlustfunktion $L_{0}$ bewertet. Um dies zu zeigen, zerlegt man den Referenzvektor $\mathrm{H}_{0}$ gemäß der zuvor eingeführten Notation in den Vektor $\mathrm{g}_{-\mathrm{p}}\left(\mathrm{H}_{0}\right)$ der Gesundheitsindikatoren und das Geldvermögen $\mathrm{g}_{\mathrm{p}}\left(\mathrm{H}_{0}\right)$, eine skalare Größe. Der Patient bewertet eine Aktion $\mathrm{a}_{\mathrm{f}}$ des Arztes in einer medizinischen Entscheidungssituation wie folgt:

$$
\Phi_{B}\left(a_{f}\right)=\sum_{i=1}^{n} P\left(K_{i}\right) \cdot u\left[g\left(a_{f}, K_{i}\right)\right] .
$$

Diese Größe läßt sich mit Hilfe der Verlustfunktion umformen:

$$
\Phi_{B}\left(a_{f}\right)=\sum_{i=1}^{n} P\left(K_{i}\right) \cdot u\left[H_{0}-e_{p} \cdot L_{0}\left(a_{f}, K_{i}\right)\right]
$$

Berücksichtigung von Gleichung (4.44) liefert:

$$
\Phi_{B}\left(a_{f}\right)=u\left(H_{0}\right)-b \cdot \sum_{i=1}^{n} P\left(K_{i}\right) \cdot L_{0}\left(a_{f}, K_{i}\right)
$$

Ähnlich ergibt sich für die Bewertung einer Entscheidungsfunktion $d_{j}$ des Arztes durch den Patienten:

$$
\Phi_{B}\left(d_{j}\right)=u\left(H_{0}\right)-b \cdot \sum_{i=1}^{n} P\left(K_{i}\right) \cdot \sum_{h=1}^{k_{j}} P\left(z_{j h} \mid K_{i}\right) \cdot L_{0}\left[d_{j}\left(z_{j h}\right), K_{i}\right] .
$$

Sieht man von dem konstanten Term $u\left(\mathrm{H}_{0}\right)$ ab, so erfolgt die Bewertung einer Handlung des Arztes durch den damit verbundenen negativen Erwartungswert der Verlustfunktion $\mathrm{L}_{0}$. Der Patient schätzt dabei eine ärztliche Handlung umso höher ein, je geringer der damit erzielte erwartete Verlust ausfällt. Dies gestattet es, die Entscheidungsregel zur Auswahl einer Aktion bzw. einer Entscheidungsfunktion umzuformulieren. Unter der Voraussetzung eines "linearen Risikonutzens" ist die Maximierung des Nutzens des Patienten äquivalent zur Minimierung seines erwarteten Verlustes.

Damit lassen sich die Präferenzen des Patienten auf der Menge derjenigen Prospekte, die aufgrund ärztlicher Handlungen entstehen können, in einfacher Weise mit Hilfe der Verlustfunktion $\mathrm{L}_{0}$ abbilden. Dies sei zunächst am Beispiel der möglichen 
Aktionen des Arztes gezeigt. Der Patient besitzt eine schwache Präferenz für eine Aktion $a_{m}$ gegenüber einer Aktion $a_{n}$, falls für ihn mit $a_{m}$ kein höherer erwarteter Verlust verbunden ist:

$$
a_{m} \succsim a_{n} \Leftrightarrow \Phi_{B L}\left(a_{m}\right) \leq \Phi_{B L}\left(a_{n}\right) ; a_{m}, a_{n} \in A
$$

Dabei steht der Operator $\Phi_{\mathrm{BL}}$ für diejenige Bewertungsregel, die einer Handlung des Arztes gerade den dazugehörigen erwarteten Verlust für den Patienten zuordnet ${ }^{109}$. Im Interesse einer übersichtlichen Notation wird als Argument dieser Bewertungsregel - wie schon zuvor im Falle der Bewertungsregel $\Phi_{B}$ - nicht der durch den Patienten zu bewertende Prospekt angeführt, sondern die ihn verursachende ärztliche Handlung.

Falls sich der Arzt für den Einsatz einer diagnostischen Maßnahme $T_{j}$ entscheidet, hat der Patient die aufgrund der Entscheidungsfunktionen $d_{j} \in D_{j}$ entstehenden Prospekte zu bewerten. Auch in diesem Fall ermöglicht die Annahme eines "linearen Risikonutzens" eine Darstellung der individuellen Präferenzen über die Bewertungsregel $\Phi_{\mathrm{BL}}$. Der Patient besitzt eine schwache Präferenz für eine Entscheidungsfunktion $d_{j}{ }^{1}$ gegenüber einer Entscheidungsfunktion $d_{j}{ }^{2}$, wenn der mit $d_{j}{ }^{1}$ verbundene Prospekt keinen höheren erwarteten Verlust aufweist:

$$
d_{j}^{2} \succsim d_{j}^{1} \Leftrightarrow \Phi_{B L}\left(d_{j}^{2}\right) \leq \Phi_{B L}\left(d_{j}^{1}\right) ; d_{j}^{1}, d_{j}^{2} \in D_{j}
$$

Die Verlustfunktion $\mathrm{L}_{0}\left(\mathrm{a}_{\mathrm{f}}, \mathrm{K}_{\mathrm{i}}\right)$ wurde zuvor für eine spezielle Repräsentation u der individuellen Präferenzen für sichere Prospekte und einen speziellen Referenzvektor $\mathrm{H}_{0}$ abgeleitet. Dies wirft die Frage auf, inwieweit die Verlustfunktion selbst sowie die vom Arzt gewählten Handlungen von dieser Spezifikation abhängen. Nimmt man

109: Das Subskript weist darauf hin, daß es sich bei dieser Bewertungsregel um eine Spezialisierung der Bayes-Regel bei "linearem Risikonutzen" handelt.

Dabei wird die Abhängigkeit dieser Bewertungsregel von der speziellen Verlustfunktion $\mathrm{L}_{0}$ nicht weiter zum Ausdruck gebracht, da diese Abhängigkeit - wie weiter unten gezeigt wird für die Ermittlung des Optionspreises der diagnostischen Information mit Hilfe von $\Phi_{\mathrm{BL}}$ keine Rolle spielt. 
eine andere Repräsentation u', so kann es sich dabei nur um eine monotone lineare Transformation von u handeln. Es gilt also:

$$
u^{\prime}=a+b^{\prime} \cdot u ; \quad b^{\prime}>0
$$

Als monetärer Verlust, der einem Konsequenzenvektor $\mathrm{g}\left(\mathrm{a}_{\mathrm{f}}, \mathrm{K}_{\mathfrak{i}}\right)$ zugeordnet ist, ergibt sich nun ein Geldbetrag L' gemäß:

$$
u^{\prime}\left(H_{0}-e_{p} \cdot L^{\prime}\right)=u^{\prime}\left[g\left(a_{f}, K_{i}\right)\right]
$$

Verwendet man die in Gleichung (4.52) angegebene explizite Form der Funktion u, so erhält man:

$$
u\left(H_{0}-e_{p} \cdot L^{\prime}\right)=u\left[g\left(a_{f}, K_{i}\right)\right]
$$

$$
\text { bzw.: } \quad L_{0}{ }^{\prime}\left(a_{f}, K_{i}\right)=L_{0}\left(a_{f}, K_{i}\right)
$$

Damit erweist sich die Verlustfunktion $\mathrm{L}_{0}\left(\mathrm{a}_{\mathrm{f}}, \mathrm{K}_{\mathrm{i}}\right)$ in einer medizinischen Entscheidungssituation als invariant gegenüber alternativen Darstellungen der Präferenzen des Patienten für sichere Prospekte.

Ein etwas anderes Ergebnis ergibt sich, wenn man einen anderen Referenzzustand zur Definition der Verlustfunktion heranzieht. Angenommen, anstelle von $\mathrm{H}_{0}$ diene nunmehr ein Vektor $\mathrm{H}_{1}$ als Vergleichsmaßstab, wobei das dazugehörige Nutzenniveau $u\left(H_{1}\right)$ von $u\left(H_{0}\right)$ verschieden sei ${ }^{110}$. Die Verlustfunktion $L_{1}\left(a_{f}, K_{i}\right)$ wird dann implizit über die folgende Gleichung definiert:

$$
u\left(H_{1}-e_{p} \cdot L_{1}\right)=u\left[g\left(a_{f}, K_{i}\right)\right] ; \quad f=1, \ldots, r ; \quad i=1, \ldots, n
$$

110: Die Verlustfunktion ändert sich nicht, wenn $u\left(\mathrm{H}_{0}\right)$ und $\mathrm{u}\left(\mathrm{H}_{1}\right)$ identisch sind. 
Unter der Voraussetzung $u\left(\mathrm{H}_{0}\right) \neq u\left(\mathrm{H}_{1}\right)$ fällt auch die Verlustfunktion $\mathrm{L}_{1}\left(\mathrm{a}_{\mathrm{f}}, \mathrm{K}_{\mathrm{i}}\right)$ verschieden von $\mathrm{L}_{0}\left(\mathrm{a}_{\mathrm{f}}, \mathrm{K}_{\mathrm{i}}\right)$ aus. Allerdings unterscheiden sich die Funktionswerte jeweils nur um eine additive Konstante, die keine Abhängigkeit von der Aktion $\mathrm{a}_{\mathrm{f}}$ des Arztes und dem Krankheitszustand $K_{i}$ des Patienten aufweist ${ }^{11}$ :

$$
L_{1}\left(a_{f}, K_{i}\right)=L_{0}\left(a_{f}, K_{i}\right)+\frac{1}{b}\left[u\left(H_{1}\right)-u\left(H_{0}\right)\right] .
$$

Daraus folgt unmittelbar, da $B$ sich die aus den Verlustfunktionen $L_{0}$ und $L_{1}$ ergebenden Bewertungen eines Prospekts, den eine beliebige ärztliche Handlung liefert, ebenfalls lediglich durch eine additive Konstante unterscheiden, die von dieser Handlung unabhängig ist.

Aufgrund dieser Eigenschaft können sowohl die ursprüngliche Verlustfunktion $\mathrm{L}_{0}\left(\mathrm{a}_{\mathrm{f}}, \mathrm{K}_{\mathrm{i}}\right)$ als auch die modifizierte Verlustfunktion $\mathrm{L}_{1}\left(\mathrm{a}_{\mathrm{f}}, \mathrm{K}_{\mathrm{i}}\right)$ zur Darstellung der Präferenzen des Patienten über Prospekte dienen. Allgemeiner läßt sich zeigen, daß die Annahme eines "linearen Risikonutzens" in dem hier verwendeten Modellrahmen die Existenz einer Klasse von Verlustfunktionen impliziert, die jeweils in der oben am Beispiel des Repräsentanten $\mathrm{L}_{0}\left(\mathrm{a}_{\mathrm{f}}, \mathrm{K}_{\mathrm{i}}\right)$ ausführlich dokumentierten Weise zur Darstellung der individuellen Präferenzen geeignet sind. Im Hinblick auf die nachfolgende Analyse stellt sich in diesem Zusammenhang die Frage, ob die spezielle Wahl einer monetären Verlustfunktion den Optionspreis der diagnostischen Information beeinflußt. Falls ein derartiger Einfluß bestünde, könnte die Entscheidungsregel $\Phi_{\mathrm{BL}}$ nicht weiter verwendet werden, da der mit ihrer Hilfe abgeleitete Optionspreis einer diagnostischen Maßnahme mit der Verlustfunktion variieren würde. Wie oben anhand der beiden Verlustfunktionen $\mathrm{L}_{0}$ und $\mathrm{L}_{1}$ gezeigt wurde, unterscheiden sich jedoch die Bewertungen eines beliebigen Prospekts durch zwei Verlustfunktionen höchstens um eine additive Konstante, die von diesem Prospekt unabhängig ist. Im Hinblick auf die relative Bewertung zweier ärztlicher Handlungen spielt daher die spezielle Wahl einer Verlustfunktion keine Rolle, da die dadurch induzierte Diskrepanz in der Bewertung beider Prospekte davon unberührt bleibt. Der in Geldeinheiten ausgedrückte Unterschied in der Bewertung zweier Prospekte durch den Patienten erweist sich damit als invariant gegenüber der Wahl eines Repräsentanten aus der

111: Zu dieser Gleichung gelangt man, indem man für ein gegebenes Paar $\left(a_{\mathrm{f}}, \mathrm{K}_{\mathrm{j}}\right)$ mit Hilfe der expliziten Darstellung von u gemäß Gleichung (4.44) die Werte $L\left(a_{f}, K_{i}\right)$ und $L_{1}\left(a_{f}, K_{i}\right)$ berechnet. 
Klasse der möglichen Verlustfunktionen. Diese Invarianzeigenschaft impliziert zugleich, daß es ohne Einschränkung der Allgemeinheit zulässig ist, den Optionspreis der diagnostischen Information vermöge der oben konstruierten Verlustfunktion $\mathrm{L}_{0}$ zu bestimmen.

\subsubsection{Der Optionspreis der diagnostischen Information}

Die Möglichkeit, bei "linearem Risikonutzen" die Beurteilung ärztlicher Handlungen durch den Patienten anhand der Bewertungsregel $\Phi_{\mathrm{BL}}$ durchzuführen, erlaubt eine explizite und damit vereinfachte Darstellung der individuellen Zahlungsbereitschaft für die diagnostische Information. Dies wird zunächst für eine beliebige Entscheidungsfunktion des Arztes gezeigt. Daraus ergibt sich eine Charakterisierung des Optionspreises diagnostischer Maßnahmen mit Hilfe der Verlustfunktion des Patienten. Im Anschluß daran folgt die Ermittlung des Optionspreises für die vollkommene und die unvollkommene diagnostische Information.

In einer medizinischen Entscheidungssituation erwäge der Arzt, eine diagnostische Maßnahme $T_{j}$ einzusetzen. Welchen Optionspreis besitzt die damit verbundene Information für den Patienten? Als Optionspreis einer Entscheidungsfunktion $d_{j}$ des Arztes ergibt sich ein Geldbetrag z, für den gilt:

$$
\Phi_{\mathrm{B}}\left(\mathrm{d}_{\mathrm{j}}, \mathrm{z}\right)=\max \left\{\Phi_{\mathrm{B}}\left(\mathrm{a}_{\mathrm{f}}\right) \mid \mathrm{a}_{\mathrm{f}} \in A\right\}
$$

Bezeichne $\mathrm{a}^{*}$ eine optimale Aktion des Arztes in der Situation ohne weitere Diagnostik, dann bestimmt sich der Optionspreis $\mathrm{z}\left(\mathrm{d}_{\mathbf{j}}\right)$ aus der Gleichung:

$$
\left.\sum_{i=1}^{n} P\left(K_{i}\right) \cdot \sum_{h=1}^{k_{j}} P\left(z_{j h} \mid K_{i}\right) \cdot u|g| d_{j}\left(z_{j h}\right), K_{i}\right]-e_{p} \cdot z \mid=\Phi_{B}\left(a^{*}\right) .
$$

Die Voraussetzung eines "linearen Risikonutzens" liefert für z die explizite Darstellung:

$$
\mathrm{b} \cdot \mathrm{z}=\Phi_{\mathrm{B}}\left(\mathrm{d}_{\mathrm{j}}\right)-\Phi_{\mathrm{B}}\left(\mathrm{a}^{*}\right)
$$


Zwischen den Bewertungsregeln $\Phi_{\mathrm{B}}$ und $\Phi_{\mathrm{BL}}$ für eine ärztliche Entscheidung $\mathrm{x}$ besteht folgender Zusammenhang [vgl. (4.49)]:

$$
\Phi_{B}(x)=u\left(H_{0}\right) \cdot b \cdot \Phi_{B L}(x)
$$

Damit erhält man für $\mathrm{z}$ :

$$
\mathrm{z}=\Phi_{\mathrm{BL}}\left(\mathrm{a}^{*}\right)-\Phi_{\mathrm{BL}}\left(\mathrm{d}_{\mathrm{j}}\right)
$$

Der Optionspreis $z\left(d_{j}\right)$ einer Entscheidungsfunktion $d_{j}$ des Arztes stimmt gerade mit der Reduktion des erwarteten Verlusts überein, den diese Entscheidungsfunktion gegenüber der Situation ohne weitere Diagnostik erzeugt ${ }^{112}$. Daraus ergibt sich unmittelbar, daß eine vom Patienten strikt präferierte Entscheidungsfunktion auch einen höheren Optionspreis besitzt.

Im Gegensatz zum in Abschnitt 4.3.2 behandelten allgemeinen Fall bestimmt sich deshalb bei "linearem Risikonutzen" des Patienten der Optionspreis einer diagnostischen Maßnahme immer durch den Optionspreis einer optimalen Entscheidungsfunktion des Arztes. Für den Optionspreis der Information, die eine diagnostische Maßnahme $T_{j}$ erbringt, gilt daher:

$$
z\left(T_{j}\right)=z\left(d_{j}^{*}\right)
$$

(4.62a) mit: $d_{j}^{*} \in \arg \min \left\{\Phi_{B L}\left(d_{j}\right) \mid d_{j} \in D_{j}\right\}$.

Welche maximale Zahlungsbereitschaft besitzt der Patient für die vollkommene diagnostische Information? Ohne den Einsatz weiterer Diagnostik vermag der Arzt

112: Im übrigen erkennt man auch aus Gleichung (4.61), daß der Optionspreis einer Entscheidungsfunktion des Arztes invariant gegenüber der Wahl einer anderen Verlustfunktion $\mathrm{L}_{1}$ ist. Dieser Übergang verändert sowohl $\Phi_{\mathrm{BL}}\left(\mathrm{a}^{*}\right)$ als auch $\Phi_{\mathrm{BL}}\left(\mathrm{d}_{\mathrm{j}}\right)$ um dieselbe additive Konstante, so daß $z\left(d_{j}\right)$ davon unbeeinflußt bleibt. 
dem Patienten einen erwarteten Verlust in Höhe von $\Phi_{\mathrm{BL}}\left(\mathrm{a}^{*}\right)$ zu garantieren, wobei für diese Größe gilt:

$$
\Phi_{\mathrm{BL}}\left(\mathrm{a}^{*}\right)=\min \left\{\Phi_{\mathrm{BL}}\left(\mathrm{a}_{\mathrm{f}}\right) \mid \mathrm{a}_{\mathrm{f}} \in \mathrm{A}\right\}
$$

$$
\text { bzw:: } \mathrm{a}^{*} \in \arg \min \left\{\Phi_{\mathrm{BL}}\left(\mathrm{a}_{\mathrm{f}}\right) \mid \mathrm{a}_{\mathrm{f}} \in \mathrm{A}\right\}
$$

Durch den Einsatz einer perfekten diagnostischen Maßnahme $T_{j}$ kann der Arzt sein Handeln vollständig am Krankheitszustand des Patienten ausrichten. Der mit einer optimalen Entscheidungsfunktion verbundene erwartete Verlust bei vollkommener diagnostischer Information beläuft sich auf:

$$
\Phi_{B L}\left(d_{j}^{*}\right)=\sum_{i=1}^{n} P\left(K_{i}\right) \cdot \min _{a_{f} \in A} L\left(a_{f}, K_{i}\right)
$$

(4.64a) bzw.: $d_{j}^{*} \in \arg \min \left\{\Phi_{B L}\left(d_{j}\right) \mid d_{j} \in D_{j}\right\}$.

Aus dem Vergleich der erwarteten Verluste $\Phi_{\mathrm{BL}}\left(\mathrm{a}^{*}\right)$ und $\Phi_{\mathrm{BL}}\left(\mathrm{d}_{\mathrm{j}}^{*}\right)$ ergibt sich unmittelbar der Optionspreis $\mathrm{z}^{*}$ der vollkommenen diagnostischen Information:

$$
\begin{aligned}
& \mathbf{z}^{*}=\min _{a_{\mathrm{f}} \in A} \sum_{i=1}^{n} P\left(K_{i}\right) \cdot L\left(a_{f}, K_{i}\right)-\sum_{i=1}^{n} P\left(K_{i}\right) \cdot \min _{a_{f} \in A} L\left(a_{f}, K_{i}\right) \\
& \quad z^{*}=\Phi_{B L}\left(a^{*}\right)-\Phi_{B L}\left(d_{j}^{*}\right) .
\end{aligned}
$$

Aus der Sicht des Patienten besitzt die perfekte diagnostische Information damit auch bei "linearem Risikonutzen" einen eindeutig bestimmten Optionspreis, der allerdings in der Regel von der untersuchten medizinischen Entscheidungssituation abhängt. Wie im allgemeinen Fall hängt dieser Optionspreis jedoch nicht von dem speziellen Diagnosetest ab, der die vollkommene Information liefert. Die gemäß Gleichung (4.65a) definierte maximale Zahlungsbereitschaft des Patienten fällt immer nichtnegativ aus und beträgt genau dann Null, wenn eine gleichmäßig beste Aktion des Arztes existiert. In nicht-trivialen Entscheidungssituationen weist die vollkommene diagnostische Information daher immer einen positiven Optionspreis auf. 
Für eine unvollkommene diagnostische Maßnahme $T_{j}$ ermittelt man den Optionspreis ihrer Information grundsätzlich nach dem gleichen Verfahren. Ohne den Einsatz dieser Maßnahme (d.h. ohne weitere Diagnostik) kann der Arzt natürlich auch in diesem Fall den erwarteten Verlust des Patienten gerade auf $\Phi_{\mathrm{BL}}\left(\mathrm{a}^{*}\right)$ begrenzen. Durch den Einsatz von $T_{j}$ hingegen gilt für den minimalen erwarteten Verlust:

$$
\Phi_{\mathrm{BL}}\left(\mathrm{d}_{\mathrm{j}}^{*}\right)=\min \left\{\Phi_{\mathrm{BL}}\left(\mathrm{d}_{\mathrm{j}}\right) \mid \mathrm{d}_{\mathrm{j}} \in \mathrm{D}_{\mathrm{j}}\right\}
$$

(4.66a) bzw.: $d_{j}^{*} \in \arg \min \left\{\Phi_{B L}\left(d_{j}\right) \mid d_{j} \in D_{j}\right\}$.

Wie schon im allgemeinen Fall liefert eine Umformulierung des aufgrund einer nichtrandomisierten Entscheidungsfunktion $d_{j}$ für den Patienten zu erwartenden Verlusts einen Hinweis darauf, wie der Arzt zu einer optimalen Entscheidungsfunktion gelangt:

$$
\left.\Phi_{B L}\left(d_{j}^{*}\right)=\min _{d_{j} \in D_{j}} \sum_{i=1}^{n} \sum_{h=1}^{k_{j}} \frac{P\left(z_{j h} \mid K_{i}\right) \cdot P\left(K_{i}\right)}{P\left(z_{j h}\right)} \cdot P\left(z_{j h}\right) \cdot L\left[d_{j}\left(z_{j h}\right), K_{i}\right)\right]
$$

Und damit:

$$
\Phi_{B L}\left(d_{j}^{*}\right)=\sum_{h=1}^{k_{j}} P\left(z_{j h}\right) \min _{a_{f} \in A} \sum_{i=1}^{n} P\left(K_{i} \mid z_{j h}\right) \cdot L\left(a_{f}, K_{i}\right) .
$$

Eine optimale Entscheidungsfunktion $d_{j}{ }^{*}$ ordnet jedem Testergebnis eine nicht-randomisierte Aktion $\mathrm{d}_{\mathrm{j}}{ }^{*}\left(\mathrm{z}_{\mathrm{jh}}\right)$ des Arztes zu, welche den erwarteten Verlust des Patienten gegeben $\mathrm{z}_{\mathrm{jh}}$ (bzw. die daraus abgeleitete posteriore Wahrscheinlichkeitsverteilung der Krankheitszustände) minimiert.

Der Optionspreis $z\left(d_{j}^{*}\right)$ einer optimalen Entscheidungsfunktion repräsentiert zugleich den Optionspreis $z\left(T_{j}\right)$ der betrachteten unvollkommenen diagnostischen Maßnahme. Für diese Zahlungsbereitschaft des Patienten erhält man ebenfalls eine 
explizite Darstellung als Differenz der beiden minimalen erwarteten Verluste, die sich ohne weitere Diagnostik bzw. aufgrund von $\mathrm{T}_{\mathrm{j}}$ ergeben:

$$
z\left(T_{j}\right)=\min _{a_{f} \in A} \Phi_{B L}\left(a_{f}\right)-\min _{d_{j} \in D_{j}} \Phi_{B L}\left(d_{j}\right)
$$

$$
\text { bzw.: } z\left(T_{j}\right)=z\left(d_{j}^{*}\right)=\Phi_{B L}\left(a^{*}\right)-\Phi_{B L}\left(d_{j}^{*}\right) \text {. }
$$

Die maximale Zahlungsbereitschaft $z\left(T_{j}\right)$ des Patienten für eine unvollkommene diagnostische Maßnahme bei "linearem Risikonutzen" ist - wie nach der Analyse des allgemeinen Falls nicht anders zu erwarten - stets nichtnegativ. Sie beträgt genau dann Null, wenn eine optimale Entscheidungsfunktion des Arztes eine konstante Funktion darstellt und damit jedem möglichen Testergebnis dieselbe Aktion zuordnet ${ }^{113}$. Wie im allgemeinen Fall bildet $z^{*}$ eine obere Schranke, die der Optionspreis der unvollkommenen diagnostischen Information niemals überschreiten kann. Damit ergeben sich in einer medizinischen Entscheidungssituation folgende Abschätzungen für den Optionspreis, den die Information einer beliebigen diagnostischen Maßnahme bei einem Patienten besitzt ${ }^{114}$ :

$$
z^{*} \geq z\left(T_{j}\right) \geq 0 ; j=1, \ldots, m
$$

\subsubsection{Graphische Analyse}

Die Voraussetzung einer endlichen Anzahl von Krankheitszuständen ermöglicht eine geometrische Deutung der bisher diskutierten Resultate ${ }^{115}$. Falls die Annahme eines "linearen Risikonutzens" zutrifft, erlauben die Präferenzen des Patienten eine Darstellung mit Hilfe einer monetären Verlustfunktion. Im folgenden dient dazu weiterhin der Repräsentant $L_{0}$ aus der Klasse aller Verlustfunktionen. In die Be-

113: Insofern stellt ein Informationsgewinn von Null eine hinreichende, jedoch keineswegs notwendige Bedingung dafür dar, daß eine unvollkommene diagnostische Maßnahme keinen positiven Optionspreis besitzt.

114: Der Übersichtlichkeit halber wurde hinsichtlich des Brutto-Werts der diagnostischen Information bei "linearem Risikonutzen" mit $z^{*}$ und $z\left(T_{j}\right)$ die Notation aus dem allgemeinen Fall übernommen. Selbstverständlich hängt der Optionspreis, den eine diagnostische Maßnahme in einer Entscheidungssituation für einen Patienten besitzt, davon ab, ob dessen Präferenzen der Voraussetzung eines "linearen Risikonutzens" genügen oder nicht.

115: Vgl. FERGUSON, T.S. (1967), S. 34ff.; FERSCHL, F. (1975), S. 61ff. sowie insbesondere FIRCHAU, V. (1980). 
wertung einer Handlung des Arztes gehen dann genau jene Werte der Verlustfunktion ein, die sich bei sämtlichen möglichen Krankheitszuständen ergeben können. Faßt man diese Werte in einem Vektor zusammen, so induziert eine dem Arzt zur Verfügung stehende Handlung $x$ für den Patienten eine Verlustallokation $V(x)$, die wie folgt definiert ist:

$$
\begin{aligned}
& V(x)=\left[L_{0}\left(x, K_{1}\right), \ldots, L_{0}\left(x, K_{n}\right)\right], \\
& V_{i}(x)=L_{0}\left(x, K_{i}\right) ; i=1, \ldots, n .
\end{aligned}
$$

Eine Verlustallokation $\mathrm{V}(\mathrm{x})$ enthält damit die gesamte Information bezüglich der relativen Bewertung einer ärztlichen Handlung $x$ durch den Patienten. Zwei Handlungen des Arztes sind aus der Sicht des Patienten genau dann äquivalent, wenn sie für ihn dieselbe Verlustallokation erzeugen ${ }^{116}$. In diesem Fall liefern beide Handlungen immer - d.h. unabhängig von den prioren Wahrscheinlichkeiten der Krankheitszustände - den gleichen erwarteten Verlust und unterscheiden sich daher in ihrer Bewertung durch den Patienten nicht.

Zur Untersuchung einer bestimmten Menge ärztlicher Handlungen reicht es demnach aus, die dadurch festgelegte Menge der Verlustallokationen für den Patienten zu betrachten. Bei der Ermittlung des Optionspreises einer diagnostischen Maßnahme $\mathrm{T}_{\mathrm{j}}$ sind nur diejenigen Verlustallokationen in Betracht zu ziehen, die der Arzt durch die Wahl einer Handlung aus der Menge A oder $A^{*}$ - ohne weitere Diagnostik - bzw. $D_{j}$ oder $D_{j}{ }^{*}$ - bei Einsatz von $T_{j}$ - realisieren kann. Dabei existieren zwischen den Mengen von Verlustallokationen, die diesen Handlungsmengen zugeordnet sind, Teilmengenbeziehungen, die keine Abhängigkeit von der speziell gewählten diagnostischen Maßnahme aufweisen.

116: Dabei gilt es zu beachten, daß zwei in diesem Sinne äquivalente Handlungen $x^{\prime}, x^{\prime \prime}$ sich hinsichtlich ihrer Konsequenzen für den Patienten durchaus unterscheiden können. Allerdings muß gelten:

$$
u\left[g\left(x^{\prime}, K_{i}\right)\right]=u\left[g\left(x^{\prime \prime}, K_{i}\right)\right] ; \quad i=1, \ldots, n .
$$


Im Falle der nicht-randomisierten Aktionen des Arztes ergibt sich dafür eine Menge $v(A)$ :

(4.71) $v(A)=\left\{V \in R^{n} \mid V_{i}=V_{i}\left(a_{f}\right) ; i=1, \ldots, n ; a_{f} \in A\right\}$.

Entsprechend ist der Menge $A^{*}$ eine Menge $v\left(A^{*}\right)$ zugeordnet, für die gilt:

(4.72) $v\left(A^{*}\right)=\left\{V \in R^{n} \mid V_{i}=V_{i}(P) ; i=1, \ldots, n ; P \in A^{*}\right\}$

Da die Menge $A^{*}$ als Spezialfälle Elemente enthält, die hinsichtlich der Konsequenzen für den Patienten zu jeweils einer nicht-randomisierten Aktion äquivalent sind, muß $v(A)$ eine Teilmenge von $v\left(A^{*}\right)$ bilden. Ferner folgt aus der Annahme eines "linearen Risikonutzens", daß die mit einer randomisierten Aktion verbundene Verlustallokation auch erzeugt werden kann, indem man die Randomisierung auf der Menge v(A) durchführt:

$$
V(P)=\sum_{f=1}^{r} P\left(a_{f}\right) \cdot V\left(a_{f}\right)
$$

Jedes Element der Menge $v\left(A^{*}\right)$ repräsentiert somit eine konvexe Kombination der Elemente aus $\mathrm{v}(\mathrm{A})$. Aus diesem Grund bildet $\mathrm{v}\left(\mathrm{A}^{*}\right)$ gerade die konvexe Hülle der endlichen Menge $v(A)$ und stellt in geometrischer Hinsicht ein konvexes Polyeder dar, dessen Ecken die Elemente von $\mathrm{v}(\mathrm{A}) \operatorname{sind}^{117}$.

Wenn der Arzt eine diagnostische Maßnahme $\mathrm{T}_{\mathrm{j}}$ einsetzt, erweitert sich dadurch im allgemeinen die Menge der für den Patienten möglichen Verlustallokationen. Diese Aussage gilt unabhängig davon, ob man lediglich nicht-randomisierte Handlungen analysiert oder ein Randomisieren durch den Arzt zuläßt. Betrachtet man zunächst nicht-randomisierte Entscheidungsfunktionen, so gilt für die Menge $v\left(D_{j}\right)$ :

$$
\begin{aligned}
& v\left(D_{j}\right)=\left\{V \in R^{n} \mid V_{i}=V_{i}\left(d_{j}\right) ; i=1, \ldots, n ; d_{j} \in D_{j}\right\} \\
& \text { mit: } V_{i}\left(d_{j}\right)=\sum_{h=1}^{k_{j}} P\left(z_{j h} \mid K_{i}\right) \cdot L\left[d_{j}\left(z_{j h}\right), K_{i}\right] .
\end{aligned}
$$

117: Die konvexe Hülle einer Menge $M$ bezeichnet die kleinste konvexe Menge, die $M$ enthält; vgl. z.B. BLACKWELL, D./GIRSHICK, M.A. (1954), S. 32. 
Da zu jeder nicht-randomisierten Aktion des Arztes eine hinsichtlich ihrer Konsequenzen für den Patienten äquivalente Entscheidungsfunktion in $D_{j}$ existiert, enthält $\mathrm{v}\left(\mathrm{D}_{\mathrm{j}}\right)$ die Verlustallokationen $\mathrm{v}(\mathrm{A})$ als Teilmenge.

Schließlich führt die Berücksichtigung randomisierter Entscheidungsfunktionen auf die insgesamt umfassendste Menge von Verlustallokationen $v\left(D_{j}^{*}\right)$ :

$$
\begin{aligned}
& v\left(D_{j}^{*}\right)=\left\{V \in R^{n} \mid V_{i}=V_{i}\left(\delta_{j}\right) ; i=1, \ldots, n ; \delta_{j} \in D_{j}^{*}\right\}, \\
& \text { mit: } V_{i}\left(\delta_{j}\right)=\sum_{f=1}^{r} P_{\delta_{j}}\left(a_{f} \mid K_{i}\right) \cdot L\left(a_{f}, K_{i}\right) .
\end{aligned}
$$

Zunächst gibt es zu jeder nicht-randomisierten Entscheidungsfunktion $d_{j} \in D_{j}$ ein Element $\delta_{\mathrm{j}} \in \mathrm{D}_{\mathrm{j}}{ }^{*}$, das dazu im Hinblick auf die Konsequenzen für den Patienten äquivalent ist und damit für ihn dieselbe Verlustallokation erzeugt. Dasselbe gilt für die dem Arzt zur Verfügung stehenden randomisierten Aktionen $P \in A^{*}$, da $D_{j}^{*}$ insbesondere die konstanten Entscheidungsfunktionen enthält. Insgesamt umfaßt $v\left(D_{j}^{*}\right)$ daher sowohl $v\left(D_{j}\right)$ als auch $v\left(A^{*}\right)$. Da die Menge $D_{j}^{*}$ im Hinblick auf die Verlustallokationen für den Patienten äquivalent ist zur Menge derjenigen Entscheidungsfunktionen, die durch Randomisieren auf $D_{j}$ entstanden sind, repräsentiert $v\left(D_{j}^{*}\right)$ gerade die konvexe Hülle der Menge $v\left(D_{j}\right)$. Damit bildet $v\left(D_{j}^{*}\right)$ ein konvexes Polyeder, dessen Ecken durch die Elemente der Menge $v\left(D_{j}\right)$ gegeben sind.

Wie aus der Bewertungsregel $\Phi_{\mathrm{BL}}$ für ärztliche Handlungen hervorgeht, bewertet der Patient eine Verlustallokation V, indem er mit Hilfe der prioren Wahrscheinlichkeiten der Krankheitszustände den damit verbundenen erwarteten Verlust $\Phi(V)$ bestimmt ${ }^{118}$ :

$$
\Phi(V)=\sum_{i=1}^{n} P\left(K_{i}\right) \cdot V_{i}
$$

118: In geometrischer Hinsicht ergibt sich $\Phi(\mathrm{V})$ als beliebige Koordinate des Schnittpunkts, den die Hyperebene durch $\mathrm{L}$ zum Normalenvektor $\mathrm{P}(\mathrm{K})$ mit der Geraden

$$
g=\left\{y \in R^{n} \mid y_{1}=\ldots=y_{n}\right\}
$$

besitzt; vgl. dazu auch die graphische Darstellung weiter unten für $n=2$. 
Ohne weitere Diagnostik wählt der Arzt in einer medizinischen Entscheidungssituation eine Verlustallokation, deren erwarteter Verlust auf der Menge $v\left(A^{*}\right)$ minimal ausfällt. Falls hingegen eine diagnostische Maßnahme $T_{j}$ zum Einsatz kommt, wird der Arzt die Funktion $\Phi$ auf der Menge $v\left(D_{j}^{*}\right)$ minimieren. Aus dem Vergleich der Funktionswerte ergibt sich unmittelbar für den Optionspreis $z\left(T_{j}\right)$ der Information, die eine diagnostische Maßnahme liefert:

(4.77) $\mathrm{z}\left(\mathrm{T}_{\mathrm{j}}\right)=\min _{\mathrm{V} \in \mathrm{V}\left(\mathrm{A}^{*}\right)} \Phi(\mathrm{V})-\min _{\mathrm{V} \in \mathrm{v}\left(\mathrm{D}_{\mathrm{j}}^{*}\right)} \Phi(\mathrm{V})$

Da $v\left(A^{*}\right)$ immer eine Teilmenge von $v\left(D_{j}^{*}\right)$ bildet, folgt aus Gleichung (4.77) die schon früher nachgewiesene Nichtnegativität dieser individuellen Zahlungsbereitschaft für die diagnostische Information. Im übrigen repräsentiert $\Phi$ eine lineare Funktion, die ihr Minimum auf den konvexen Polyedern v $\left(A^{*}\right)$ bzw. $v\left(D_{j}^{*}\right)$ jeweils an mindestens einer Ecke annimmt. Die Berücksichtigung von Verlustallokationen, die durch Randomisieren entstehen, beeinflußt daher den Optionspreis der diagnostischen Information nicht. Insofern gilt ebenfalls:

$$
\mathrm{z}\left(\mathrm{T}_{\mathrm{j}}\right)=\min _{\mathrm{V} \in \mathrm{V}(\mathrm{A})} \Phi(\mathrm{V})-\min _{\mathrm{V} \in \mathrm{V}\left(\mathrm{D}_{\mathrm{j}}\right)} \Phi(\mathrm{V})
$$

In dem Spezialfall einer vollkommenen diagnostischen Maßnahme enthält die Menge $v\left(D_{j}\right)$ ein Element, das in jeder Komponente aus dem minimalen Verlust des Patienten bei dem entsprechenden Krankheitszustand besteht. Unabhängig von den prioren Wahrscheinlichkeiten $\mathrm{P}\left(\mathrm{K}_{\mathrm{i}}\right)$ legt diese Verlustallokation den minimalen erwarteten Verlust in einer Entscheidungssituation fest, da keine andere (durch den Arzt erreichbare) Verlustallokation den Patienten jemals besser stellen kann. Demzufolge ergibt sich für den Optionspreis $z^{*}$ der vollkommenen diagnostischen Information:

(4.78) $\mathrm{z}^{*}=\min _{\mathrm{V} \in \mathrm{v}(\mathrm{A})} \Phi(\mathrm{V})-\sum_{\mathrm{i}=1}^{\mathrm{n}} \mathrm{P}\left(\mathrm{K}_{\mathrm{i}}\right) \min _{\mathrm{V} \in \mathrm{v}(\mathrm{A})} \mathrm{V}_{\mathrm{i}}$ 
Wenn in einer medizinischen Entscheidungssituation nur zwei Krankheitszustände bei einem Patienten in Betracht kommen, läßt sich die Ermittlung des Optionspreises der diagnostischen Information graphisch veranschaulichen ${ }^{119}$. In diesem speziellen Fall gilt $\mathrm{n}=2$ und sämtliche Mengen von Verlustallokationen können in einem Diagramm dargestellt werden. Abbildung 14 verdeutlicht eine Situation, in der der Arzt keine zusätzliche diagnostische Maßnahme einsetzt.

Abb. 14: Die Entscheidungssituation ohne weitere Diagnostik

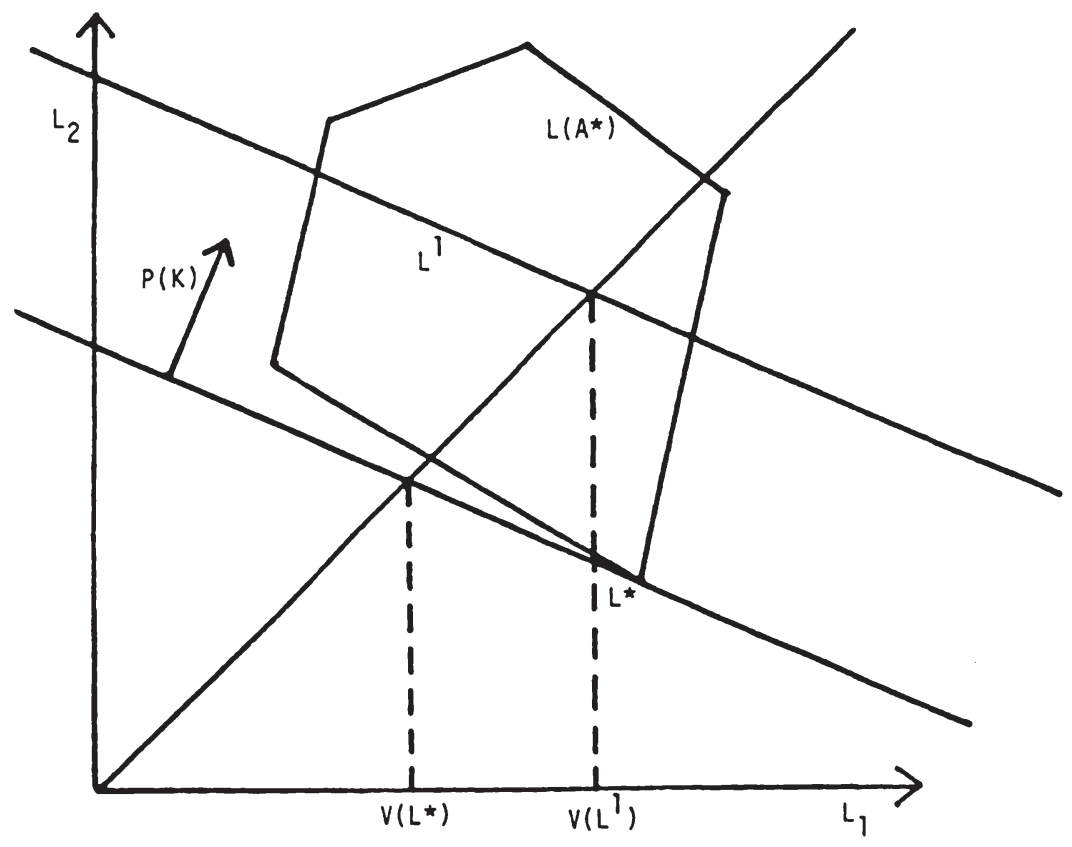

Die Menge $v\left(A^{*}\right)$ umfaßt sämtliche Verlustallokationen, die der Arzt für den Patienten durch die Wahl einer Aktion $P \in A^{*}$ realisieren kann. In der Abbildung gehö-

119: Dies betrifft beispielsweise die in den Abschnitten 3.2 und 3.3 untersuchten Entscheidungssituationen. 
ren ausschließlich positive Vektoren zu $v\left(A^{*}\right)$, was die Verwendung eines aus der Sicht des Patienten hinreichend guten Referenzzustands $\mathrm{H}_{0}$ zur Ableitung der speziell benutzten Verlustfunktion impliziert ${ }^{120}$. Ferner folgt aus der Annahme ausschließlich endlicher Verlustbeträge die Beschränktheit der Menge $\mathrm{v}\left(\mathrm{A}^{*}\right)$, so daß die Abbildung insgesamt eine typische medizinische Entscheidungssituation verdeutlicht, falls der Arzt keine weitere Diagnostik betreibt.

Die Bewertung einer beliebigen Verlustallokation $V \in v\left(A^{*}\right)$ durch den Patienten wird graphisch anhand des Schnittpunkts dargestellt, den die Gerade durch V zum Normalenvektor $\mathrm{P}(\mathrm{K})$ mit der 1 . Winkelhalbierenden besitzt. Jede Koordinate dieses Schnittpunkts zeigt den mit $\mathrm{V}$ verbundenen erwarteten Verlust an. In der Abbildung ist diese Bewertung zunächst für die Verlustallokation $V^{1}$ angegeben. Man erkennt unmittelbar, da $B$ es in dieser Entscheidungssituation andere Verlustallokationen in $v\left(A^{*}\right)$ gibt, die eine günstigere Bewertung durch den Patienten erfahren. Die ohne weitere Diagnostik optimale Verlustallokation $V^{*}$ mit dem minimalen erwarteten Verlust $\Phi\left(V^{*}\right)$ ist eindeutig bestimmt und gehört zu einer nicht-randomisierten $\mathrm{Ak}$ tion des Arztes ${ }^{121}$.

Setzt der Arzt eine unvollkommene diagnostische Maßnahme $T_{j}$ ein, dann muß neben der Menge $v\left(A^{*}\right)$ zusätzlich die Menge $v\left(D_{j}{ }^{*}\right)$ berücksichtigt werden. Abbildung 15 illustriert die Auswirkungen auf die realisierbaren Verlustallokationen, soweit sie in der untersuchten Entscheidungssituation von Bedeutung sind.

Die exakte Gestalt der Menge $v\left(D_{j}^{*}\right)$ hängt wesentlich von der Anzahl der Testergebnisse der diagnostischen Maßnahme ab. Deshalb wurde in der Abbildung darauf verzichtet, sämtliche Verlustallokationen aus $v\left(D_{j}^{*}\right)$ anzugeben und stattdessen nur der vordere Rand dieser Menge eingezeichnet ${ }^{122}$. Die Betrachtung dieser Teilmenge

120: Der Übergang zu einem neuen Referenzzustand $\mathrm{H}_{1}$ mit $\mathrm{u}\left(\mathrm{H}_{0}\right) \neq \mathrm{u}\left(\mathrm{H}_{1}\right)$ bewirkt eine Translation von $\mathrm{v}\left(\mathrm{A}^{*}\right)$.

121: Eine optimale Verlustallokation liegt immer auf derjenigen Geraden, deren Schnittpunkt mit der 1. Winkelhalbierenden die kleinsten Koordinaten aufweist bezüglich der Schar aller Geraden zum Normalenvektor $\mathrm{P}(\mathrm{K})$, die mit $\mathrm{v}\left(\mathrm{A}^{*}\right)$ mindestens einen Punkt gemeinsam haben.

122: Bezeichne $T(z)$ die Menge $\left\{\left.y \in R^{2}\right|_{i}>y_{i} ; i=1,2\right\}$. Dann gilt für den vorderen Rand $\operatorname{VR}\left[v\left(D_{j}{ }^{*}\right)\right]$ der Menge $v\left(D_{j}{ }^{*}\right)$ :

$$
\operatorname{VR}\left[v\left(D_{j}^{*}\right)\right]=\left\{V \in v\left(D_{j}^{*}\right) \mid T(V) \cap v\left(D_{j}^{*}\right)=\varnothing\right\} \text {. }
$$


reicht zur Ermittlung des Optionspreises einer diagnostischen Maßnahme völlig aus, da optimale Verlustallokationen - bei Einsatz von $T_{j}$ - immer zu dieser Menge gehören $^{123}$.

Abb. 15: Der Optionspreis der unvollkommenen diagnostischen Maßnahme $T_{j}$

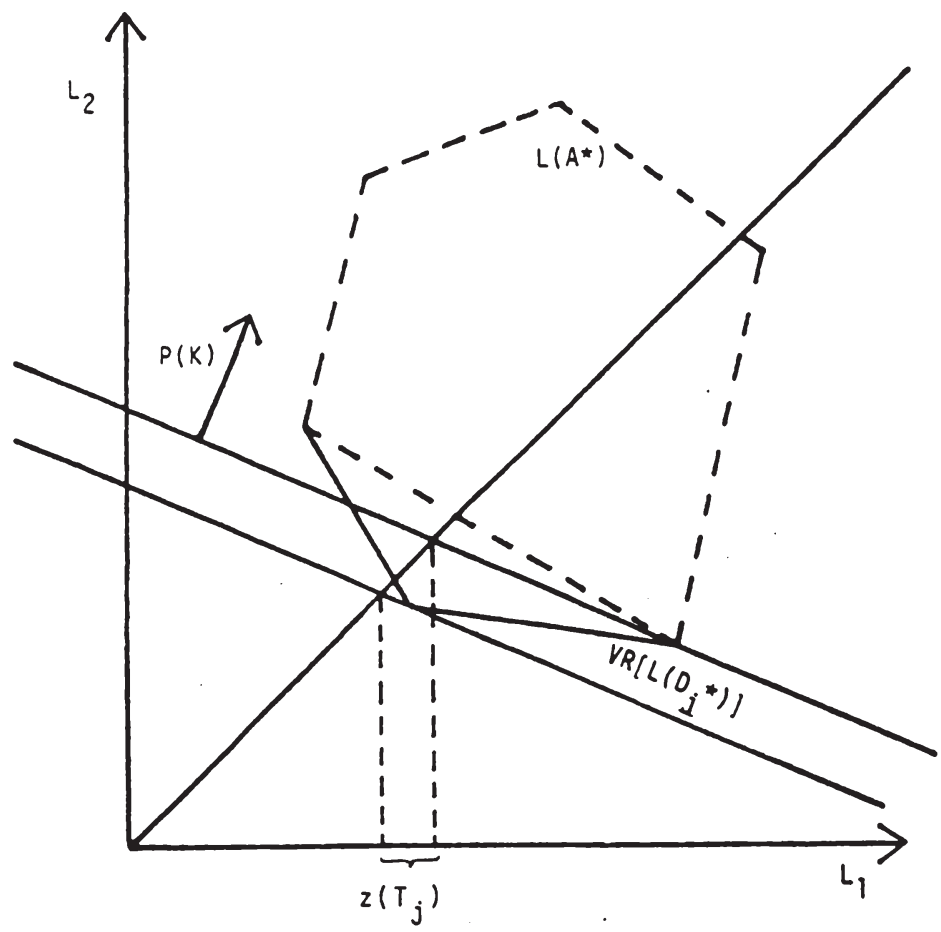

Aus der Abbildung erkennt man, daß die diagnostische $M a ß n a h m e T_{j}$ für die spezielle priore Wahrscheinlichkeitsverteilung $\mathrm{P}(\mathrm{K})$ der beiden Krankheitszustände einen positiven Optionspreis $z\left(T_{j}\right)$ besitzt. Diese Zahlungsbereitschaft hängt wesentlich von der untersuchten medizinischen Entscheidungssituation ab, die durch die Menge $v\left(A^{*}\right)$ und Wahrscheinlichkeiten $P\left(K_{i}\right)$ charakterisiert wird. Ceteris paribus ändert sich im allgemeinen die Menge $v\left(A^{*}\right)$ und damit zugleich $v\left(D_{j}^{*}\right)$, wenn bei-

123: Vgl. FERGUSON, T.S. (1967), S. 64ff.; FIRCHAU, V. (1980), S. 6. 
spielsweise ein anderer Patient betrachtet wird. Bei unveränderten prioren Wahrscheinlichkeiten ergibt sich dann ein neuer Optionspreis für $\mathrm{T}_{\mathrm{j}}$, obwohl die diagnostische Information unverändert bleibt. Schließlich besitzt bei sonst gleicher Entscheidungssituation das Vorwissen des Arztes, das durch $\mathrm{P}(\mathrm{K})$ beschrieben wird, einen Einfluß auf die Zahlungsbereitschaft des Patienten für eine diagnostische Maßnahme. Abbildung 16 zeigt anhand veränderter priorer Wahrscheinlichkeiten $\mathrm{P}^{\prime}\left(\mathrm{K}_{\mathrm{i}}\right)$ der Krankheitszustände eine neue Situation, in der die zuvor betrachtete diagnostische Maßnahme einen Optionspreis von Null erzielt ${ }^{124}$.

Abb. 16: Der Optionspreis von $T_{j}$ bei veränderten prioren Wahrscheinlichkeiten

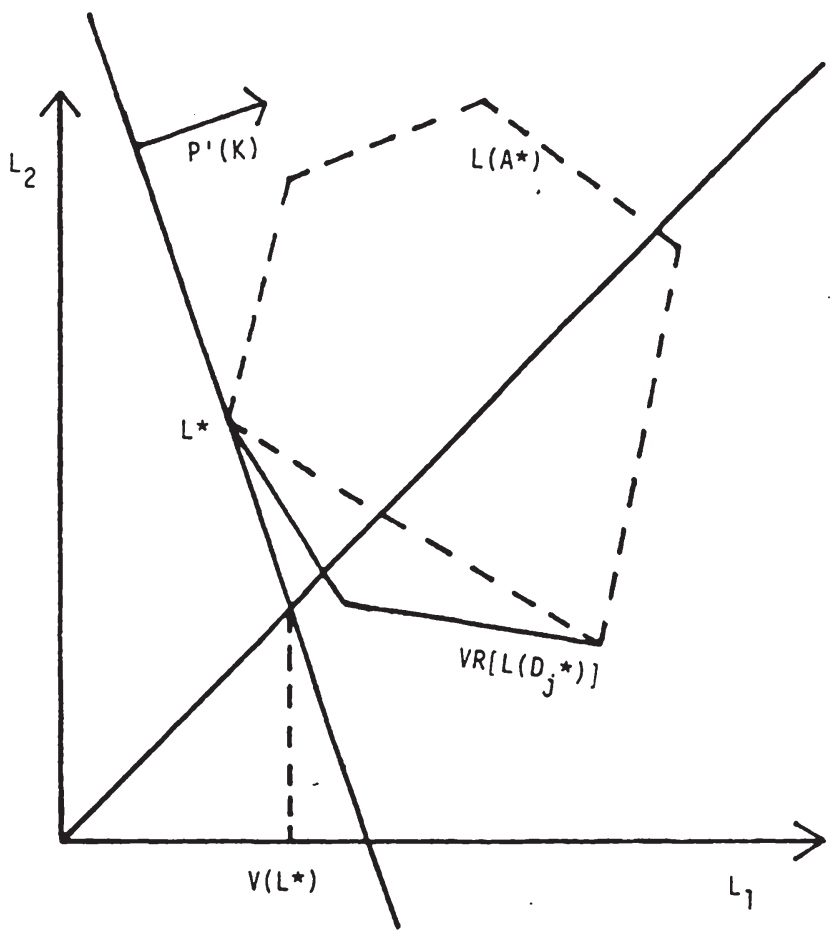

124: Zum Einfluß einzelner Komponenten der medizinischen Entscheidungssituation auf den Umfang der Nutzeneffekte, die eine diagnostische Maßnahme beim Patienten auslöst, vgl. ausführlich Abschnitt 4.4.2. 
Bei einer vollkommenen diagnostischen Maßnahme $T_{j}$ lassen sich die für den $\mathrm{Pa}$ tienten realisierbaren Verlustallokationen in einfacher Weise aus der Menge $v\left(A^{*}\right)$ ableiten. Da der Arzt nach dem Einsatz dieser Maßnahme den tatsächlichen Krankheitszustand des Patienten kennt, kann er dieses Wissen grundsätzlich sowohl zur Konstruktion besonders günstiger als auch besonders ungünstiger Entscheidungsfunktionen nutzen. Abbildung 17 veranschaulicht dies am Beispiel derselben Menge $\mathrm{v}\left(\mathrm{A}^{*}\right)$, die schon in den vorangegangenen Abbildungen untersucht wurde.

Abb. 17: Der Optionspreis der vollkommenen diagnostischen Information

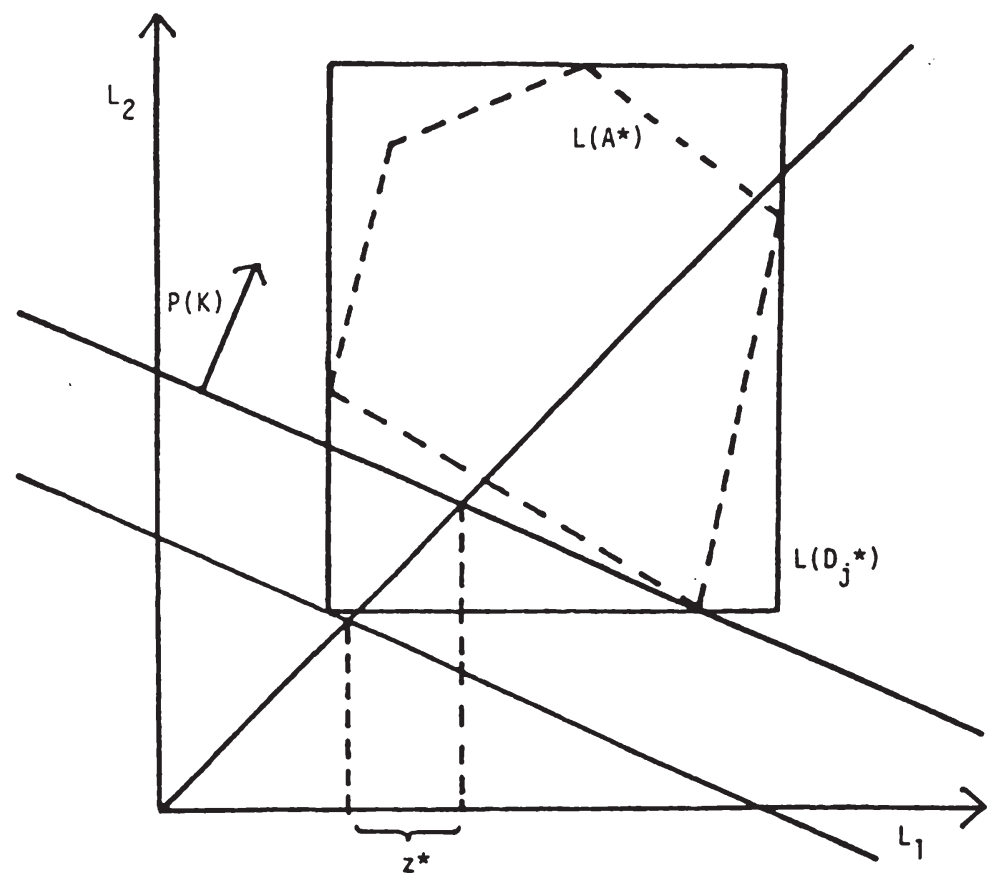

Die Menge $v\left(D_{j}^{*}\right)$ der bei perfekter diagnostischer Information möglichen Verlustallokationen stellt das kleinste achsenparallele Rechteck dar, das die Menge v( $\left.\mathrm{A}^{*}\right)$ 
vollständig enthält. Sofern beide Krankheitszustände eine positive priore Wahrscheinlichkeit besitzen, ergibt sich in der durch die Abbildung verdeutlichten Situation immer eine positive Zahlungsbereitschaft $\mathrm{z}^{*}$ des Patienten für die vollkommene diagnostische Information. Falls jedoch eine gleichmäßig beste Aktion des Arztes existiert, kann selbst die perfekte diagnostische Information den erwarteten Verlust des Patienten nicht weiter reduzieren. Abbildung 18 zeigt eine derartige Situation, in der die Zahlungsbereitschaft $\mathrm{z}^{*}$ Null beträgt.

Abb. 18: Der Optionspreis der vollkommenen diagnostischen Information bei Existenz einer gleichmäßig besten Aktion

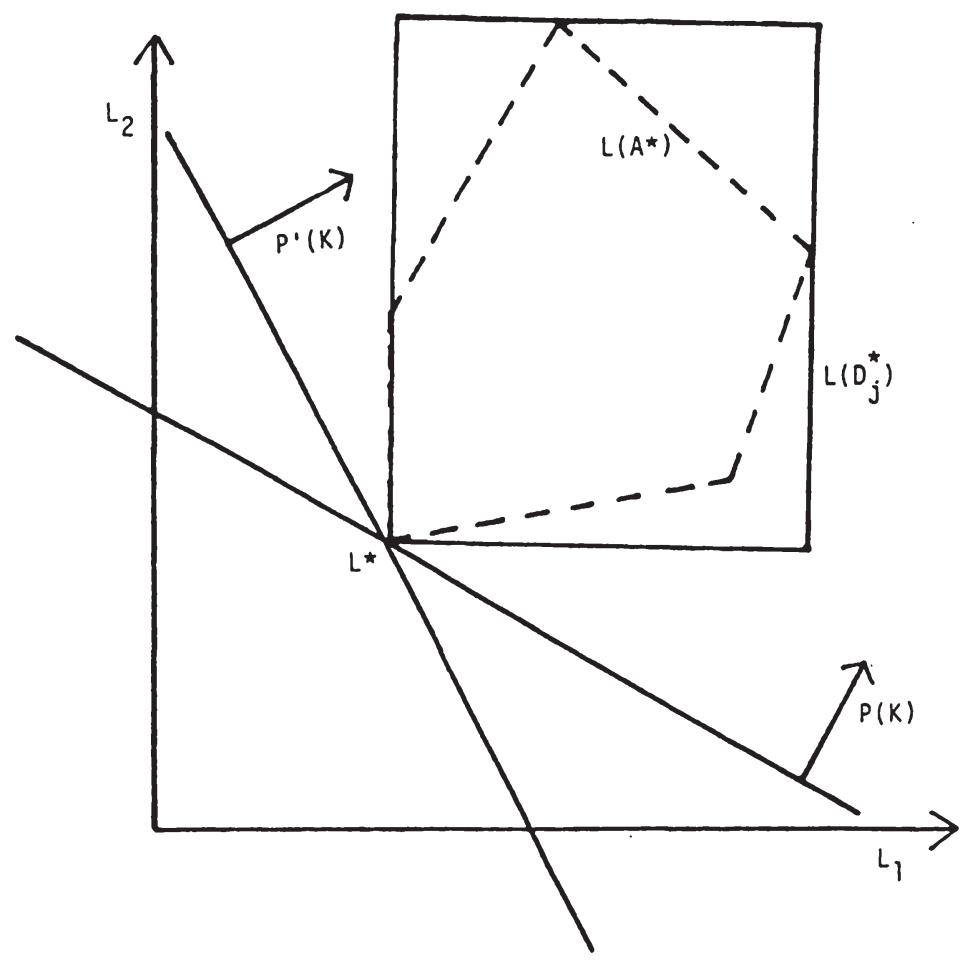




\subsubsection{Zur Bedeutung der Annahme eines "linearen Risikonutzens"}

Die Annahme eines "linearen Risikonutzens" bietet den Vorteil einer insgesamt erheblich vereinfachten Analyse des Optionspreises diagnostischer Maßnahmen, dem jedoch der Nachteil einer möglicherweise falschen Spezifikation der Präferenzen des Patienten gegenübersteht. Ob die individuellen Präferenzen tatsächlich die mit der Annahme eines "linearen Risikonutzens" geforderten Eigenschaften besitzen, bedarf letztlich der fallweisen empirischen Überprüfung. Allerdings gilt es zu beachten, daß diese Annahme in Bezug auf die Gruppe der Gesundheitsindikatoren bzw. -attribute keine schwerwiegenden Restriktionen enthält. Die funktionale Form der in Gleichung (4.44) angegebenen Nutzenfunktion ist allgemein genug, um beispielsweise die in sogenannten Gesundheitsstatusindizes üblicherweise verwendeten linearen Spezifikationen als Spezialfälle zu umfassen.

In der empirischen Forschung wird man ohne die Annahme eines "linearen Risikonutzens" beim Patienten oder eine äquivalente Voraussetzung kaum auskommen. Dies verdeutlichen auch die in der Literatur vorhandenen Arbeiten, die den Wert diagnostischer Maßnahmen in ausgewählten Entscheidungssituationen zu ermitteln suchen. Um eine monetäre Quantifizierung der Nutzeneffekte diagnostischer Information zu erreichen, ordnen die Autoren den möglichen Kombinationen aus ärztlicher Aktion und Krankheitszustand des Patienten ebenfalls monetäre Größen zu ${ }^{125}$. Sofern die hier vorgestellte Annahme eines "linearen Risikonutzens" die allgemeinste Möglichkeit darstellt, um eine monetäre Verlustfunktion mit den genannten Eigenschaften abzuleiten, setzt die in der Literatur anzutreffende Vorgehensweise daher zumindest dieselben Restriktionen hinsichtlich der Präferenzen des Patienten voraus. Tatsächlich verwenden diese Autoren einen wesentlich spezielleren Ansatz, da die den einzelnen Paaren $\left(\mathrm{a}_{\mathrm{f}}, \mathrm{K}_{\mathrm{i}}\right)$ zugeordneten monetären Größen keine individuelle $\mathrm{Ab}$ hängigkeit aufweisen und insofern die Präferenzen eines "typischen" bzw. repräsentativen Patienten widerspiegeln.

125: Dies gilt z.B. für KODLIN, D. (1972); McNEIL, B.J./KEELER, E./ADELSTEIN, S.J. (1975); WERNER, M. (1978); METZ, C.E. (1978); HABBEMA, J.D.F.HILDEN, J. (1981); DOUBILET, P. (1983); GODDEERIS, J.H./BRONKEN, T.P. (1985) Sowie GÄFGEN, G. (1990). Dabei erfahren vielfach einzelne Kombinationen aus diagnostischem Testergebnis und Krankheitszustand eine monetäre Bewertung, so daß sich die im Text angegebene Zuordnung implizit über die Annahme einer bestimmten ärztlichen Entscheidungsfunktion ergibt. 


\subsubsection{Der Optionspreis diagnostischer Maßnahmen aus gesamtwirt- schaftlicher Perspektive}

Mit dem Optionspreis diagnostischer Maßnahmen wurde in den vorangegangenen Abschnitten eine spezielle Wertgröße untersucht. Der kompensierende Optionspreis gibt den Wert an, den die diagnostische Information in einer medizinischen Entscheidungssituation aus der Sicht des Patienten besitzt, wenn von den Kosten der Informationsbeschaffung abgesehen wird. Diese zuletzt genannte Eigenschaft ermöglicht es, den Optionspreis diagnostischer Maßnahmen präziser als eine individuelle Brutto-Zahlungsbereitschaft einzuordnen. Es handelt sich dabei um einen einzelwirtschaftlichen Wertansatz, der mit der entsprechenden gesamtwirtschaftlichen Größe im allgemeinen nicht übereinstimmt. Dies beruht darauf, daß die Konsequenzen ärztlicher Aktionen in die Berechnung des Optionspreises diagnostischer Maßnahmen nur insoweit Eingang finden, als sie den Patienten selbst betreffen. In der Regel bleiben auf diese Weise jedoch einige Konsequenzen unberücksichtigt, die in einer gesamtwirtschaftlichen Betrachtung keinesfalls zu vernachlässigen sind.

Diese Nichtberücksichtigung gilt insbesondere für die Kosteneffekte ärztlicher Aktionen. In vielen Ländern besteht für Individuen die Möglichkeit (unter Umständen sogar der Zwang), eine Krankenversicherung abzuschließen, die im Krankheitsfall die Kosten der Inanspruchnahme ärztlicher Leistungen weitgehend abdeckt. Unter dieser Voraussetzung enthält die in der Analyse des Optionspreises diagnostischer Maßnahmen zu verwendende Konsequenzenfunktion nicht die tatsächlichen Kosten der vom Arzt gewählten Aktionen. Liegt - wie etwa in der Bundesrepublik Deutschland - für die überwiegende Mehrzahl der Bürger näherungsweise ein vollständiger Versicherungsschutz vor, dann treten in der Konsequenzenfunktion typischerweise keine Kosten aufgrund einer Aktion des Arztes auf, da diese in einer medizinischen Entscheidungssituation nicht der Patient selbst, sondern die Versichertengemeinschaft trägt. In diesem Fall stellt der Optionspreis diagnostischer Maßnahmen gerade die maximale Zahlungsbereitschaft des Patienten für die differentiellen Gesundheitseffekte dar, welche die zusätzliche Information induziert. Diese differentiellen Gesundheitseffekte kommen zustande, weil die jeweils untersuchte diagnostische Maßnahme dem Arzt die Möglichkeit bietet, bei zumindest einem Testergebnis von der in der Situation ohne weitere Diagnostik optimalen Aktion abzugehen ${ }^{126}$.

126: Vgl. zu den differentiellen Gesundheitseffekten der diagnostischen Information ausführlich Abschnitt 4.4. 
Mit dieser Änderung des ärztlichen Handelns bei Inanspruchnahme einer diagnostischen Maßnahme gehen zugleich differentielle Kosten einher. Dieser Kosteneffekt entsteht, weil anstelle der Kosten derjenigen Aktion, die der Arzt ohne weitere Diagnostik gewählt hätte, nun die Kosten der gemäß einer optimalen Entscheidungsfunktion ergriffenen Aktion zu berücksichtigen sind. Die zuletzt genannte Kostengröße erweist sich in diesem Zusammenhang als zufallsabhängig, da die ärztliche Aktion bei Einsatz einer diagnostischen Maßnahme in der Regel vom Testergebnis abhängt. Insgesamt resultiert daher der angesprochene Kosteneffekt aus einer Saldierung der deterministischen Kosten des ärztlichen Handelns in der Situation ohne weitere Diagnostik mit einer stochastischen Kostengröße, für die man zweckmäßigerweise ihren Erwartungswert ansetzt. Die Existenz dieser erwarteten differentiellen Kosten sorgt dafür, daß der kompensierende Optionspreis als Brutto-Zahlungsbereitschaft des Patienten für die diagnostische Information nicht mit der entsprechenden gesamtwirtschaftlichen Größe übereinzustimmen braucht ${ }^{127}$.

Diese Diskrepanz unterscheidet den Optionspreis der diagnostischen Information von einer in der Entscheidungstheorie häufig untersuchten Wertgröße, die ebenfalls eine individuelle Zahlungsbereitschaft für Informationsinstrumente in Entscheidungssituationen angibt. Der Brutto-Wert eines Informationsinstruments zeigt die maximale Zahlungsbereitschaft eines Aktors für die darin enthaltene zusätzliche Information an, wenn von den Kosten der Informationsbeschaffung abgesehen wird ${ }^{128}$. Dabei kennzeichnet die in diesem Zusammenhang analysierten Entscheidungsprobleme, daß der Aktor sämtliche Konsequenzen seiner Aktionen in vollem Umfang selbst trägt. Der Brutto-Wert, den ein Informationsinstrument besitzt, stimmt unter dieser Voraussetzung gerade mit dem korrespondierenden gesamtwirtschaftlichen Wertansatz überein, da die Handlungsfolgen ausnahmslos in den einzelwirtschaftlichen Kalkül eingehen und damit internalisiert sind. Dieser Tatbestand ermöglicht eine einfache Interpretation des Brutto-Werts der Information. Falls der Brutto-Wert eines Informationsinstruments in einer Entscheidungssituation

127: Diese Aussage gilt keineswegs nur bei einer vollständigen Krankenversicherung des Patienten. Bei Vorliegen eines teilweisen Krankenversicherungsschutzes werden die Kosteneffekte ärztlicher Aktionen nicht vollständig, sondern je nach Umfang und Ausgestaltung der Selbstbeteiligung in unterschiedlichem Ausmaß berücksichtigt. Auch in diesem Fall enthält daher die Zahlungsbereitschaft des Patienten für eine diagnostische Maßnahme einen Teil der differentiellen Kosten des ärztlichen Handelns nicht.

128: Vgl. z.B. MARSCHAK, J.MIYASAWA, K. (1968), S. $210 f$. 
die Kosten seiner Inanspruchnahme übersteigt (unterschreitet), erweist sich die Informationsbeschaffung als vorteilhaft (unvorteilhaft) im Sinne der Kosten-NutzenAnalyse.

Für den Optionspreis der diagnostischen Information ergibt sich wegen der Nichtberücksichtigung eines Teils der Konsequenzen, die eine vom Arzt gewählte Aktion bewirkt, eine etwas andere Interpretation. Aus der Tatsache, daß die maximale Zahlungsbereitschaft, die der Patient für eine diagnostische Maßnahme in einer medizinischen Entscheidungssituation besitzt, die Kosten dieser Maßnahme übersteigt (unterschreitet), läßt sich im allgemeinen nicht direkt auf die Vorteilhaftigkeit (Unvorteilhaftigkeit) einer Informationsbeschaffung im Sinne der Kosten-NutzenAnalyse schließen ${ }^{129}$. Um eine derartige Aussage mit gesamtwirtschaftlichem Bezug treffen zu können, müssen zusätzlich die erwarteten differentiellen Kosten berücksichtigt werden, die mit dem Einfluß der diagnostischen Information auf das Handeln des Arztes im Vergleich zur Situation ohne weitere Diagnostik verbunden sind ${ }^{130}$. Eine gesamtwirtschaftliche Vorteilhaftigkeit des Einsatzes einer diagnostischen Maßnahme liegt demzufolge erst dann vor, wenn der damit verbundene Optionspreis um die zu erwartenden differentiellen Kosten des ärztlichen Handelns korrigiert wurde und diese korrigierte Wertgröße die Kosten der Informationsbeschaffung übersteigt.

\subsection{Die Analyse des diagnostischen Nutzeneffekts}

\subsubsection{Differentielle Nutzeneffekte diagnostischer Maßnahmen}

Anstelle des Optionspreises diagnostischer Maßnahmen rückt nun der aufgrund der diagnostischen Information beim Patienten entstehende differentielle Nutzeneffekt in den Vordergrund. Dabei besteht zwischen diesen beiden Konzepten eine enge Verbindung, da die Zahlungsbereitschaft des Patienten gerade einen monetären Ausdruck jener Verbesserung der individuellen Wohlfahrtsposition darstellt. Die Information, welche auf eine diagnostische Maßnahme zurückgeht, besitzt nämlich genau dann einen positiven Optionspreis, wenn sie dem Patienten zu einem zusätzlichen Nutzeneffekt verhilft.

129: Beispielsweise kann der Einsatz einer diagnostischen Maßnahme in einer medizinischen Entscheidungssituation, in der ihr Optionspreis unter ihren Kosten liegt, aufgrund eines günstigen Einflusses auf die erwarteten Kosten des ärtlichen Handelns insgesamt als gesamtwirtschaftlich vorteilhaft einzustufen sein.

130: Vgl. zur Berücksichtigung dieser Kostenkomponente diagnostischer Maßnahmen auch WEINSTEIN, M.C./FINEBERG, H.V. (1978), S. 11 sowie WEINSTEIN, M.C. (1979), S. 54. 


\subsubsection{Zum Outputcharakter der diagnostischen Information}

In produktionstheoretischer Hinsicht stellt eine diagnostische Maßnahme eine Leistung bzw. - im Falle einer zusammengesetzten Maßnahme - eine Gruppe von Leistungen dar, die entweder der Arzt selbst oder eine mit dessen Kompetenzen ausgestattete Person erbringt. Das unmittelbare Ergebnis dieser Leistung besteht in einer Verbesserung der Informationslage des Arztes, sofern es sich nicht um ein Null-Informationssystem handelt. Diese neue Information besitzt allerdings in dem hier verwendeten Modellrahmen keine direkte Wohlfahrtsrelevanz, wie sich mit Hilfe der Nutzenfunktion des Patienten leicht nachweisen läßt. Ein Nutzeneffekt tritt erst dann auf, wenn das zusätzliche diagnostische Wissen die Aktion des Arztes und damit die für den Patienten wichtigen Konsequenzen beeinflußt ${ }^{131}$. Die Information einer diagnostischen Maßnahme stellt aus dieser Perspektive einen intermediären Output dar, da sie im Rahmen der ärztlichen Leistungserstellung lediglich ein Zwischenprodukt auf dem Weg zu patientenbezogenen Nutzeneffekten bildet ${ }^{132}$.

Im Gegensatz dazu erzeugt jede Aktion des Arztes unmittelbar Nutzeneffekte beim Patienten, deren genaue Ausprägung allerdings von dessen Krankheitszustand bestimmt wird. Vor diesem Hintergrund ließe sich das Ergebnis einer ärztlicher Aktion als finaler Output einstufen ${ }^{133}$. Indessen gilt es zu beachten, daß diese Kategorisierung die tatsächlichen Verhältnisse in verkürzter Form abbildet. Falls es sich bei einer Aktion um eine Behandlungsleistung handelt, hängen die dadurch bewirkten Gesundheitseffekte - bei gegebenem Krankheitszustand - noch von der "compliance" des Patienten $a b^{134}$. Der Patient vermag durch sein Verhalten die Erfolgsaussichten einer Therapie unter Umständen entscheidend zu beeinflussen ${ }^{135}$. In diesem Fall entsteht

131: Diese Sichtweise der Nutzeneffekte diagnostischer Maßnahmen entspricht der in der Literatur überwiegend vertretenen Auffassung, vgl. z.B. GORRY, G.A. (1978), S. 53f.; SHOWSTACK, J.A.SCHROEDER, S.A. (1981), S. 6; GUYATT, G./DRUMMOND, M.F. (1986), S. 555.

132: Vgl. WEINSTEIN, M.C. (1979), S. 62; DRUMMOND, M.F. et al. (1986), S. 115.

133: In der Literatur wird der Begriff des Endproduktes dazu synonym verwendet; vgl. GÄFGEN, G. (1980).

134: Zur "compliance" vgl. FEINSTEIN, A.R. (1977b), S. 122ff. Den Einfluß der "compliance" auf Kosten und Nutzen der Hypertonie-Behandlung untersuchten STASON, W.B./WEINSTEIN, M.C. (1977).

135: Vgl. dazu HENKE, K.-D. (1978), S. 604. Bei den vom Arzt erbrachten Behandlungsleistungen handelt es sich um Dienstleistungen, bei denen im Vergleich zu anderen Dienstleistungen der Einfluß des Nachfragers (Patient) auf das Endprodukt eine größere Bedeutung erlangt; vgl. GÄFGEN, G. (1980), S. 177. 
der finale Output als "joint product", das aus dem Zusammenwirken von ärztlicher Aktion und einer Eigenleistung des Patienten resultiert ${ }^{136}$.

Entscheidet sich der Arzt für eine andere Aktion, etwa die Überweisung zu einem (anderen) Facharzt, liegt zunächst eine wesentlich schwächere Verbindung zur Wohlfahrt des Patienten vor. Im allgemeinen wird dieser Facharzt weitere Diagnostik betreiben, bevor er seine Behandlungsentscheidung festlegt. Wieder kann erst die Wahl einer Therapie in der im vorangegangenen Absatz skizzierten Weise zur Entstehung von Nutzeneffekten beim Patienten führen ${ }^{137}$. Eine ähnliche Argumentation greift, wenn der in einer medizinischen Entscheidungssituation betrachtete Arzt den Patienten in ein Krankenhaus einweist.

Eine detaillierte Untersuchung des auf die Gesundheit des Patienten ausgerichteten Leistungserstellungsprozesses zeigt damit, daß bei einer Reihe von Aktionen das unmittelbare Resultat der ärztlichen Tätigkeit ebenfalls einen intermediären Output darstellt $^{138}$. Der Output einer diagnostischen Maßnahme unterscheidet sich davon lediglich insofern, als diese Information vor der Wahl einer Aktion und damit auf einer niedrigeren Stufe dieses Prozesses anfällt. Aus diesen Überlegungen folgt zugleich, $\mathrm{da} B$ man im allgemeinen eine geeignete ceteris-paribus-Annahme benötigt, um die Verbindung zwischen einer Aktion des Arztes und dem beim Patienten schließlich eintretenden Nutzeneffekt herzustellen. Dies leistet die in Abschnitt 4.2.2 eingeführte Konsequenzenfunktion, die aufgrund ihrer Konstruktion offenbar eine derartige Hilfsannahme beinhaltet. Erst unter dieser Voraussetzung erscheint es zulässig, die Folge einer Aktion des Arztes auch in jenen Fällen als finalen Effekt zu klassifizieren, in denen ihr unmittelbares Ergebnis nicht als finaler Output rangiert.

Besitzen diagnostische Maßnahmen neben ihrem oben erläuterten intermediären Charakter auch eine direkte Wohlfahrtsrelevanz? In diesem Fall wäre die von ihnen gelieferte Information zumindest teilweise als finaler Output einzustufen, der dem

136: Die Annahme einer deterministischen Konsequenzenfunktion impliziert in diesem Zusammenhang eine bestimmte "compliance" des Patienten.

137: In dem analysierten medizinischen Entscheidungsproblem setzt die Annahme einer deterministischen Konsequenzenfunktion in diesem Fall eine lediglich vom Krankheitszustand des Patienten abhängige Aktion des Facharztes voraus.

138: Vgl. AUSTER, R./LEVESON, I./SARACHEK, D. (1969), S. 414 sowie ausführlich GÄFGEN, G. (1980). 
Patienten ungeachtet seines Einflusses auf die vom Arzt gewählte Aktion Nutzen stiften könnte ${ }^{139}$. Zunächst scheint nicht ausgeschlossen, daß die diagnostische Information dem Patienten ein Handeln ermöglicht, das ohne diese Information unterblieben oder erst zu einem späteren Zeitpunkt möglich gewesen wäre. Dieser Effekt kann etwa bei der frühzeitigen Diagnose einer unheilbaren Krankheit auftreten, die dem Patienten ausreichend Gelegenheit bietet, seine beruflichen und seine privaten Verhältnisse zu regeln ${ }^{140}$. Ein anderes Beispiel stellt die diagnostische Information dar, die eine Ultraschall-Untersuchung bei schwangeren Frauen liefert ${ }^{141}$. Die Information über Anzahl und Geschlecht ihrer Kinder ermöglicht es den Patientinnen, frühzeitig geeignete Vorkehrungen im Hinblick auf den Familienzuwachs zu treffen.

Interessanterweise unterscheiden sich diese Nutzeneffekte in konzeptioneller Hinsicht nicht von denjenigen, die das entscheidungstheoretische Modell dieses Kapitels berücksichtigt: die beim Patienten auftretenden positiven Wohlfahrtswirkungen gründen jeweils auf einer Beeinflussung des Handelns im Vergleich zur Situation ohne weitere Diagnostik ${ }^{142}$. Während im Modell jedoch das Handeln des Arztes im Mittelpunkt der Analyse steht, beziehen sich die soeben diskutierten Nutzenaspekte auf das Handeln des Patienten ${ }^{143}$. Grundsätzlich repräsentiert die diagnostische Information aber auch in diesem Fall einen intermediären Output, dessen Wohlfahrtswirkung erst über nachgeordnete Handlungen entsteht.

Weitere, vom Modell ebenfalls nicht erfaßte, positive Wohlfahrtswirkungen liegen vor, wenn die diagnostische Information per se Nutzen stiftet ${ }^{144}$. Unter dieser Vor-

139: Diese Auffassung vertreten mehrere Autoren; vgl. McNEIL, B.J./ADELSTEIN, S.J. (1976), S. 442; BANTA, H.D./McNEIL, B.J. (1978), S. 8; WEINSTEIN, M.C. (1979), S. 61; WEINSTEIN, M.C. (1986a), S. 572 sowie ausführlich BERWICK, D.M./WEINSTEIN, M.C. (1985).

140: Daneben vermag die Diagnose einer unheilbaren Krankheit auch im Rahmen des in diesem Kapitel diskutierten Modells Nutzeneffekte beim Patienten zu stiften. Dies trifft z.B. dann zu, wenn der Arzt aufgrund dieser Diagnose ein "patient management" betreiben kann, das die Leiden des Patienten lindert; vgl. WULFF, H.R. (1981), S. 113. Auch ohne Einfluß auf Lebenserwartung und Morbidităt liegen in diesem Fall positive Gesundheitseffekte im Vergleich zur Situation ohne weitere Diagnostik vor.

141: Vgl. BERWICK, D.M./WEINSTEIN, M.C. (1985).

142: Vgl. zu einer ähnlichen Einschätzung WEINSTEIN, M.C. (1986b), S. $213 \mathrm{f}$.

143: Das Modell berücksichtigt ein Handeln des Patienten lediglich insoweit, als damit gesundheitliche Konsequenzen verbunden sind.

144: Vgl. MOONEY, G.H. (1990), S. 14. 
aussetzung erzeugt der Einsatz einer diagnostischen Maßnahme unmittelbar einen finalen Nutzeneffekt beim Patienten. Berwick und Weinstein illustrieren am Beispiel der schon oben erwähnten Ultraschall-Untersuchung von schwangeren Frauen die Möglichkeit einer direkten Wohlfahrtsrelevanz der diagnostischen Information ${ }^{145}$. Im Falle ihrer Existenz treten die damit verbundenen differentiellen Nutzeneffekte auch dann auf, wenn die diagnostische Information weder das Handeln des Arztes noch das des Patienten zu beeinflussen vermag.

$\mathrm{Ob}$ in einer medizinischen Entscheidungssituation derartige Nutzeneffekte beim Patienten auftreten können, die im entscheidungstheoretischen Modell dieses Kapitels unberücksichtigt bleiben, bildet letztlich eine empirische Frage. Allerdings dürfte es vielfach Schwierigkeiten bereiten, die einzelnen Komponenten des patientenbezogenen Nutzens der diagnostischen Information befriedigend $\mathrm{zu}$ isolieren bzw. gegeneinander abzugrenzen. Zudem hängt der Umfang dieser beiden - im Modell nicht enthaltenen - Kategorien von Nutzeneffekten beim Patienten wohl maßgeblich von der Zuverlässigkeit der diagnostischen Information ab. Thre Existenz erscheint unplausibel, wenn die Testergebnisse einer diagnostischen Maßnahme nicht zumindest mit hoher Wahrscheinlichkeit den Rückschluß auf den Krankheitszustand des Patienten erlauben.

\subsubsection{Die Beurteilung des diagnostischen Nutzeneffekts}

Die diagnostische Information stellt im Rahmen des entscheidungstheoretischen Modells einen intermediären Output dar, der im Vergleich zur Referenzsituation ohne weitere Diagnostik dem Patienten nur dann einen Zusatznutzen zu stiften vermag, wenn die Aktionswahl des Arztes beeinflußt wird ${ }^{146}$. Liegt ein derartiger Einfluß vor, dann verbessert sich die Wohlfahrt des Patienten, da ihm der Arzt aufgrund der diagnostischen Information einen günstigeren Prospekt hinsichtlich der Konsequenzen bieten kann. Eine präzisere Aussage über den Nutzeneffekt der diagnostischen Information ermöglicht die explizite Darstellung des Nutzenzuwachses, den der Patient durch den Einsatz einer diagnostischen Maßnahme $T_{j}$ erzielt. Die weiteren Ausführungen beziehen sich im Interesse einer einfachen Darstellung auf eine spezielle Repräsentation der Präferenzen des Patienten. Die daraus abgeleiteten Aussagen hinsichtlich der Beurteilung des differentiellen Nutzeneffekts der diagnostischen Information sind qualitativer Natur und besitzen deshalb für sämtliche Darstellungen

145: Vgl. BERWICK, D.M./WEINSTEIN, M.C. (1985), S. $882 \mathrm{ff}$.

146: Vgl. z.B. SCHECHTER, M.T./SHEPS, S.B. (1985), S. 759. 
dieser Präferenzen Gültigkeit. Für die Größe $\Delta \Phi_{B}\left(T_{j}\right)$, die den differentiellen Nutzen der diagnostischen Information in einer medizinischen Entscheidungssituation angibt, gilt ${ }^{147}$ :

$$
\Delta \Phi_{B}\left(T_{j}\right)=\max _{d_{j} \in D} \Phi_{B}\left(d_{j}\right)-\max _{a_{f} \in A} \Phi_{B}\left(a_{f}\right) .
$$

Bezeichne $\mathrm{a}^{*}$ eine optimale Aktion des Arztes in der Situation ohne weitere Diagnostik, dann läßt sich dieser Ausdruck umformulieren zu:

(4.80) $\Delta \Phi_{B}\left(T_{j}\right)=\sum_{h=1}^{k_{j}} P\left(z_{j h}\right) \cdot\left\{\max _{a_{f} \in A} \sum_{i=1}^{n} P\left(K_{i} \mid z_{j h}\right) \cdot U\left(a_{f}, K_{i}\right)-\sum_{i=1}^{n} P\left(K_{i}\right) \cdot U\left(a^{*}, K_{i}\right)\right\}$.

Äquivalent dazu ergibt sich:

(4.81) $\Delta \Phi_{B}\left(T_{j}\right)=\sum_{h=1}^{k_{j}} P\left(z_{j h}\right) \cdot a_{a_{f} \in A}^{\max } \sum_{i=1}^{n} P\left(K_{i} \mid z_{j h}\right) \cdot\left\{U\left(a_{f}, K_{i}\right)-U\left(a^{*}, K_{i}\right)\right\} \cdot$

Eine positive Nutzendifferenz und damit ein differentieller Nutzeneffekt aufgrund der diagnostischen Information liegt genau dann vor, wenn eine optimale Entscheidungsfunktion bei mindestens einem Testergebnis vom Arzt ein Abweichen von der Aktion $\mathrm{a}^{*}$ verlangt. Wie Gleichung (4.81) zeigt, ist dies nur möglich, falls die posterioren Wahrscheinlichkeiten der Krankheitszustände sich von den prioren Wahrscheinlichkeiten unterscheiden. Daher stellt eine positive Informationsleistung eine notwendige Bedingung für die Existenz differentieller Nutzeneffekte beim Patienten $\mathrm{dar}^{148}$. Sie ist jedoch nicht hinreichend, da die zusätzliche Information keinen (positiven) Wert aus der Sicht des Patienten zu besitzen braucht ${ }^{149}$.

147: Diese Formel gilt sowohl für vollkommene als auch für unvollkommene diagnostische Maßnahmen. Der Übersichtlichkeit halber werden nur nicht-randomisierte Entscheidungen des Arztes betrachtet, was aber der Argumentation in Abschnitt 4.3.1 zufolge keinerlei Einschränkung bedeutet.

148: Vgl. z.B. WEINSTEIN, M.C. et al. (1980), S. 147. Im übrigen bezieht sich diese Aussage auf die Bewertungsregel $\Phi_{B}$. Allgemein können auch Null-Informationssysteme die Entscheidung eines Aktors verbessern, z.B. wenn erst durch ihren Einsatz ein Randomisieren ermöglicht wird; vgl. FERSCHL, F. (1975), S. 111; FIRCHAU, V. (1980), S. 27.

149: Vgl. dazu die Abschnitte 4.3.2.1 und 4.3.2.2, in denen die Abhängigkeit des Optionspreises diagnostischer Maßnahmen von der medizinischen Entscheidungssituation diskutiert wird. 
Zur Ermittlung der differentiellen Nutzeneffekte der diagnostischen Information stehen grundsätzlich zwei verschiedene, im Ergebnis äquivalente Vorgehensweisen offen, wie sich anhand der Gleichungen (4.80) und (4.81) belegen läßt. Zunächst veranschaulicht Gleichung (4.81), daß der Nutzen einer diagnostischen Maßnahme als gewichtete Summe derjenigen Nutzendifferenzen dargestellt werden kann, die bei den einzelnen Testergebnissen im Vergleich zur Aktion a ${ }^{*}$ entstehen, falls der Arzt eine optimale Entscheidungsfunktion verwendet. Diese Summanden sind jeweils nichtnegativ und geben die Veränderung der Nutzenposition des Patienten unter der Bedingung eines bestimmten Testergebnisses an, wenn die darin enthaltene Information auch zur Bewertung der Aktion $\mathrm{a}^{*}$ dient. Auf ein einzelnes Testergebnis bezogen, ergibt sich der relative Nutzen der diagnostischen Information damit in konzeptioneller Hinsicht aus einer reinen ex-post-Perspektive ${ }^{150}$. Fällt dieser relative Nutzen auch nur für ein einziges Testergebnis positiv aus, folgt daraus unmittelbar die Existenz eines differentiellen Nutzeneffekts der diagnostischen Information in der betrachteten Entscheidungssituation insgesamt.

Die andere, in der Literatur häufiger anzutreffende Vorgehensweise, entspricht in formaler Hinsicht der Darstellung in Gleichung (4.80). Man vergleicht für jedes Testergebnis den Nutzen des Patienten aus dem Einsatz einer optimalen Entscheidungsfunktion - eine posteriore Größe, da diese Bewertung unter der Bedingung eines bestimmten Testergebnisses erfolgt - mit der Bayes-Bewertung der Aktion $\mathbf{a}^{*}$, die eine priore Größe darstellt. Bei diesem Vorgehen repräsentiert jeder Summand in Gleichung (4.80) die Differenz aus einer speziellen posterioren Nutzenposition des Patienten und dem Nutzen, den er in der Situation ohne weitere Diagnostik erreichen kann. Dabei kann die aufgrund der diagnostischen Information eines einzelnen Testergebnisses ereichbare Nutzenposition niedriger ausfallen als jene, die der Patient ohne den Einsatz der diagnostischen Maßnahme einnehmen würde. Über das Vorzeichen der in Gleichung (4.80) auftretenden Summanden läßt sich deshalb keine Aussage treffen. Die allgemeine Nichtnegativität der Größe $\Delta \Phi_{B}\left(T_{j}\right)$ weist allerdings darauf hin, daß die erwartete posteriore Nutzenposition nicht schlechter ausfällt als jene, die der Arzt dem Patienten ohne weitere Diagnostik sichern kann ${ }^{151}$.

150: Vgl. FIRCHAU, V. (1980), S. 28. Insgesamt stellt der durch $\Delta \Phi_{B}\left(T_{j}\right)$ repräsentierte differentielle Nutzen einer diagnostischen Maßnahme jedoch eine ex-ante-Größe dar, da die Unsicherheit bezüglich des sich einstellenden Testergebnisses berücksichtigt wird.

151: Insgesamt - d.h. bei Berücksichtigung sämtlicher Testergebnisse - sind demnach negative Summanden in ihrem Ausmaß bzw. ihrer Häufigkeit beschränkt. 
Im Unterschied zur ersten Vorgehensweise reicht nun die Betrachtung eines einzelnen Testergebnisses keineswegs aus, um zuverlässig auf die Existenz eines differentiellen Nutzeneffekts der diagnostischen Information schließen zu können. Aus der Verbesserung der Nutzenposition des Patienten bei einem bestimmten diagnostischen Resultat folgt daher noch kein positiver Nutzeneffekt der diagnostischen Information. Ebenso ergibt sich aus einer Verschlechterung dieser Nutzenposition bei einem einzelnen Testergebnis noch nicht die Abwesenheit differentieller Nutzen bei der untersuchten diagnostischen Maßnahme.

Diese Aussagen lassen sich am Beispiel einer besonders einfachen medizinischen Entscheidungssituation erläutern. Angenommen, es soll der Nutzeneffekt aus einer Vorsorgeuntersuchung $\mathrm{T}_{\mathrm{j}}$ bestimmt werden, mit deren Hilfe der Krankheitszustand $\mathrm{K}_{1}$ frühzeitig entdeckt werden kann. Als alternativer Krankheitszustand kommt mit $\mathrm{K}_{2}$ lediglich noch die "Abwesenheit von Krankheit" in Betracht. Dem Arzt stehen mit $a_{1}$ und $a_{2}$ insgesamt zwei Aktionen zur Auswahl, wobei $a_{2}$ für "Nichtstun" steht. Die Aktion $a_{1}$ hingegen bezeichnet die für den Krankheitszustand $K_{1}$ angemessene Therapie. Die Vorsorgeuntersuchung stellt einen binären Test dar, dessen Kennziffern Sensitivität und Spezifität jeweils kleiner als Eins sind, der aber insgesamt einen positiven Informationsgewinn erzielt. Weiterhin kennzeichnet die medizinische Entscheidungssituation, da $B$ a ${ }_{2}$ die optimale Aktion des Arztes ohne weitere Diagnostik bildet, während bei Einsatz von $T_{j}$ im Falle eines positiven Testergebnisses $z_{j 1}$ die Aktion $a_{1} z u$ wählen ist ${ }^{152}$. Damit vermag die diagnostische Maßnahme $T_{j}$ dem Patienten differentielle Nutzen zu stiften. Die Situation läßt sich graphisch veranschaulichen anhand eines auf die wesentlichen Komponenten reduzierten Entscheidungsbaums, den Abb. 19 zeigt.

Im übrigen ist an dieser Stelle auf eine Analogie zur Analyse in Abschnitt 3.6 hinzuweisen. Auch bei der Bestimmung des Informationsgewinns einer diagnostischen Maßnahme standen zwei verschiedene, im Ergebnis äquivalente Vorgehensweisen zur Auswahl. Bezogen auf ein einzelnes Testergebnis ergab sich ein stets nichtnegativer Informationsgewinn, während im Unterschied dazu die dazugehörige Entropieänderung negativ ausfallen konnte.

152: Es gilt also: $d_{j}^{*}\left(z_{j 1}\right)=a_{1}$ und $d_{j}^{*}\left(z_{j 2}\right)=a_{2}$. 
Abb. 19: Der Nutzeneffekt einer Vorsorgeuntersuchung $T_{j}$

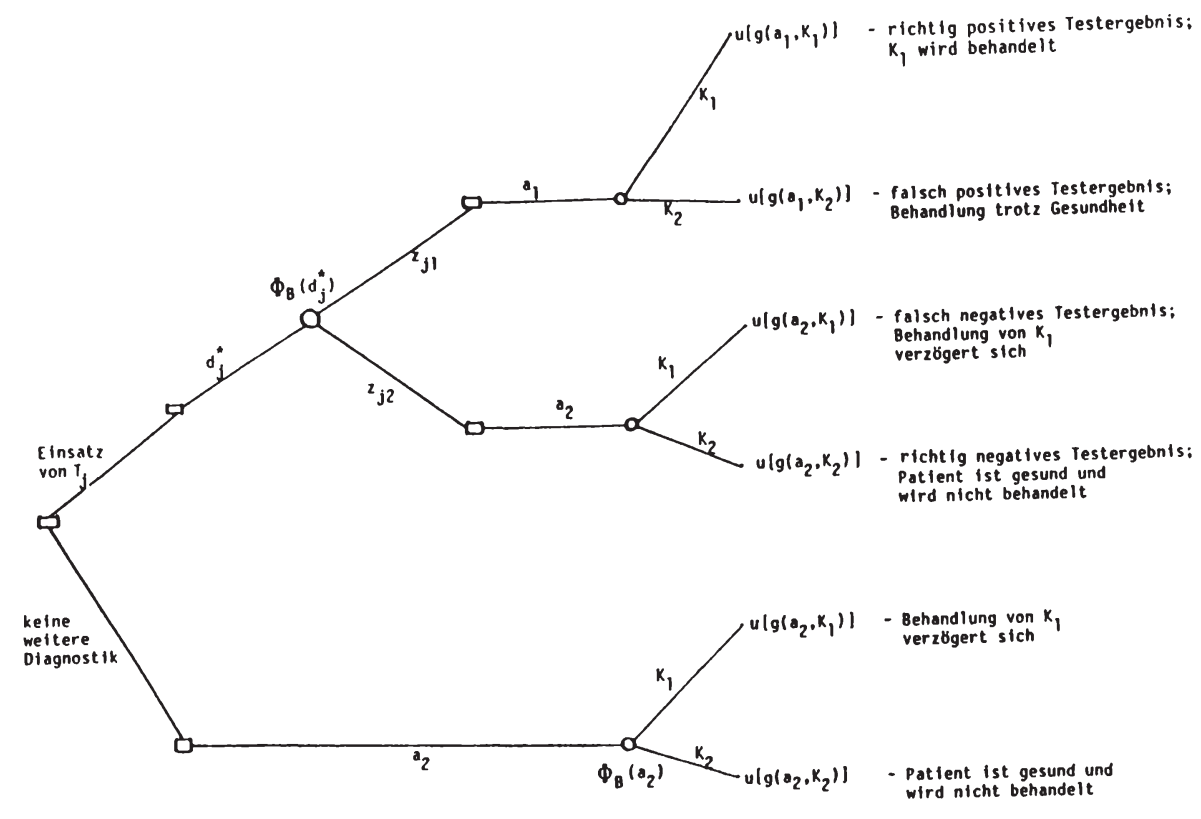


Wenn in der Ausgangslage der tatsächliche Krankheitsszustand des Patienten nicht mit Sicherheit bekannt ist, führt ein negatives Testergebnis $z_{j 2} z u$ einer erhöhten posterioren Wahrscheinlichkeit für die "Abwesenheit von Krankheit" - d.h. für den Zustand $K_{2}-$, die jedoch aufgrund der oben beschriebenen Unvollkommenheit von $T_{j}$ unter Eins liegt. Der mit der Aktion $a_{2}$ verbundene Nutzen des Patienten gegeben das Testergebnis $\mathbf{z}_{\mathbf{j} 2}$ fällt daher größer aus als in der Situation ohne weitere Diagnostik. In diesem Sinn verbessert ein negatives Testergebnis die Nutzenposition des Patienten im Vergleich zur Ausgangslage ${ }^{153}$. Dies ist jedoch nicht hinreichend für die Existenz eines differentiellen Nutzeneffekts der Vorsorgeuntersuchung, da lediglich eine spezielle posteriore Nutzenposition herausgegriffen wurde. Ein Beweis dieser Aussage ergibt sich unmittelbar, wenn man - im Unterschied zu der in Abb. 19 dargestellten Konstellation - einen Moment lang annimmt, der Arzt würde auch bei einem positiven Testergebnis die Aktion $a_{2}$ einzusetzen haben ${ }^{154}$. In diesem Fall beeinflußt die durchaus vorhandene - diagnostische Information die Aktionswahl des Arztes nicht. Demzufolge konfrontiert die Vorsorgeuntersuchung $T_{j}$ den Patienten ex ante - d.h. vor Bekanntwerden des Testergebnisses - mit einem Prospekt, der exakt mit jenem Prospekt übereinstimmt, den die Aktion $a_{2}$ in der Situation ohne weitere Diagnostik erzeugt: Unter dieser Voraussetzung liefert $T_{j}$ keinen differentiellen Nutzeneffekt ${ }^{155}$.

Unter den zunächst beschriebenen Voraussetzungen, die auch der Darstellung in Abb. 19 zugrunde liegen, führt der Einsatz der betrachteten Vorsorgeuntersuchung jedoch zur Entstehung eines differentiellen Nutzeneffekts beim Patienten. In der graphisch veranschaulichten medizinischen Entscheidungssituation bewirkt ein positives Testergebnis offenbar eine Veränderung der Informationslage des Arztes, die ausreicht, um den Patienten die Therapie von $K_{1}$ und damit die Aktion $a_{1}$ gegenüber $a_{2}$ strikt präferieren zu lassen. Daraus folgt allerdings nicht zwangsläufig, daß sich die entsprechende posteriore Nutzenposition des Patienten im Vergleich zur Situation ohne weitere Diagnostik verbessert haben muß ${ }^{156}$.

153: Vgl. WAGNER, J.L. (1981), S. 11 sowie DRUMMOND, M.F./STODDART, G.L./TORRANCE, G.W. (1987), S. 76, die diesen Effekt schon als differentiellen Nutzen der diagnostischen Information interpretieren.

154: Diese Konstellation liegt etwa dann vor, wenn für $K_{1}$ keine ausreichend gute Therapie existiert oder auch bei einem positiven Testergebnis die Wahrscheinlichkeit dieses Krankheitszustands vergleichsweise klein bleibt.

155: Der oben diskutierten Verbesserung der Nutzenposition des Patienten aus der ex-post-Perspektive eines negativen Testergebnisses steht demzufolge kompensierend eine Verschlechterung bei einem positiven Resultat gegenüber.

156: Dies hängt von den hier nicht näher spezifizierten Parametern der Entscheidungssituation ab. 
Diese Aussagen lassen sich ohne weiteres auf komplexere Entscheidungssituationen sowie auf diagnostische Maßnahmen übertragen, bei denen mehr als zwei Testergebnisse zu unterscheiden sind. Stützt man sich beim Nachweis differentieller Nutzeneffekte der diagnostischen Information auf die soeben erläuterte zweite Vorgehensweise, dann müssen sämtliche posterioren Nutzenpositionen des Patienten angemessen berücksichtigt werden ${ }^{157}$. Dies gilt nicht zuletzt deshalb, weil ex ante - d.h. vor Einsatz der diagnostischen Maßnahme - Unsicherheit bezüglich des sich einstellenden Testergebnisses herrscht und demzufolge der Arzt dem Patienten keine spezielle posteriore Nutzenposition garantieren kann. Die zum Zeitpunkt der Entscheidung über eine weitere Informationsbeschaffung vorliegende Unsicherheit muß bei der Analyse derjenigen Nutzeneffekte, welche die zusätzliche Information beim Patienten auslösen kann, in Rechnung gestellt werden.

Im wesentlichen dieselben Argumente gelten schließlich für eine dritte, der zweiten eng verwandte Vorgehensweise, die den Nachweis eines patientenbezogenen Nutzeneffekts der diagnostischen Information auf der Ebene einzelner Kombinationen aus Testergebnis und Krankheitszustand zu führen sucht. Mit jeder derartigen Kombination sind im Normalfall ganz bestimmte Konsequenzen für den Patienten verbunden. Verwendet der Arzt eine nicht-randomisierte Entscheidungsfunktion, dann legt jedes Testergebnis eindeutig die von ihm zu ergreifende Aktion fest. Über die Konsequenzenfunktion determiniert diese Aktion bei gegebenem Krankheitszustand die Konsequenzen, die dem Patienten entstehen. Die hier abschließend untersuchte Vorgehensweise zieht diese möglichen Ausprägungen des Konsequenzenvektors bzw. die daran gekoppelten Nutzenpositionen des Patienten heran, um den Nutzeneffekt einer diagnostischen Maßnahme im Vergleich zur Situation ohne weitere Diagnostik herauszuarbeiten. Man vergleicht demnach jeweils die Nutzenposition des Patienten unter der Bedingung eines speziellen diagnostischen Resultats und eines bestimmten Krankheitszustands mit derjenigen Nutzenposition, die für ihn ohne den Einsatz zusätzlicher Diagnostik erreichbar wäre. Die formale Grundlage dieses Vergleichs bildet Gleichung (4.80), wenn man zusätzlich die Abhängigkeit des ärztlichen Handelns vom sich einstellenden Testergebnis berücksichtigt und $d_{j}{ }^{*}$ eine zur diagnostischen Maßnahme $T_{j}$ gehörige optimale Entscheidungsfunktion des Arztes bezeichnet:

$$
\left.\Delta \Phi_{B}\left(T_{j}\right)=\sum_{h=1}^{k_{j}} P\left(z_{j h}\right) \cdot \sum_{i=1}^{n} P\left(K_{i} \mid z_{j h}\right) \cdot \mid U\left[d_{j}^{*}\left(z_{j h}\right), K_{i}\right]-\Phi_{B}\left(a^{*}\right)\right\} .
$$

157: Wie aus Gleichung (4.81) ersichtlich, geschieht dies anhand der Wahrscheinlichkeiten der einzelnen Testergebnisse. 
Dabei repräsentieren die Größen $u\left\{g\left[d_{j}^{*}\left(z_{j h}\right), K_{i}\right]\right\}$ die möglichen Nutzenpositionen des Patienten unter der Bedingung eines speziellen Testergebnisses und eines bestimmten Krankheitszustands. Wie bei der zuvor diskutierten zweiten Vorgehensweise resultiert damit der auf eine diagnostische Maßnahme zurückgehende Nutzeneffekt aus einer geeigneten, durch Gleichung (4.82) beschriebenen Saldierung einer Reihe posteriorer mit einer prioren Nutzengröße.

Auch diese dritte Vorgehensweise läßt sich anhand der in Abbildung 19 veranschaulichten einfachen medizinischen Entscheidungssituation erläutern. Im Rahmen des oben beschriebenen Beispiels der Vorsorgeuntersuchung sind jeweils zwei Testergebnisse und zwei Krankheitszustände zu unterscheiden. Setzt der Arzt eine optimale nicht-randomisierte Entscheidungsfunktion ein, dann resultieren daraus insgesamt vier verschiedene Nutzenpositionen des Patienten, die unter den angesprochenen Voraussetzungen alle eine positive Wahrscheinlichkeit besitzen: es handelt sich um die individuellen Nutzenbewertungen der Konsequenzen eines richtig positiven, eines falsch positiven, eines richtig negativen und schließlich eines falsch negativen Testergebnisses. Wie zuvor reicht die isolierte Betrachtung einzelner Nutzenpositionen des Patienten nicht aus, um auf die Existenz oder die Abwesenheit eines differentiellen Nutzeneffekts der Vorsorgeuntersuchung schließen zu können. Dies gilt insbesondere für die in der Literatur häufig angeführten positiven Wohlfahrtswirkungen richtig negativer Testergebnisse ${ }^{158}$. Der Beweis dieser Aussage stützt sich auf dieselbe Referenzsituation, die schon in der Diskussion der zweiten Vorgehensweise verwendet wurde. Falls die optimale Entscheidungsfunktion den Arzt auch bei einem positiven Testergebnis die Aktion $a_{2}$ ergreifen läßt - im Gegensatz zu der in Abb. 19 dargestellten Situation -, beeinflußt die diagnostische Information das ärztliche Handeln nicht. Sie erzeugt daher keinen differentiellen Nutzeneffekt für den Patienten, obwohl auch in diesem Fall ein richtig negatives Testergebnis mit einer positiven Wahrscheinlichkeit auftritt. Der damit verbundenen Verbesserung der Nutzenposition des Patienten aus der ex-post-Perspektive eines speziellen diagnostischen Resultats und eines speziellen Krankheitszustands stehen kompensierende Veränderungen bei den übrigen Kombinationen aus Testergebnis und Krankheitszustand gegenüber.

158: Vgl. dazu z.B. GILLESPIE, K.N. et al. (1986), S. 538 und GRINER, P.F. et al. (1981), S. 559.

Als gleichermaßen problematisch ist die ausschließliche bzw. bevorzugte Beurteilung der Folgen richtig positiver Testergebnisse einzustufen; vgl. dazu kritisch WINDELER, J./RICHTER, K./KÖBBERLING, J. (1988), S. 1439 f. 
Auch allgemein erlaubt diese Vorgehensweise nur dann zutreffende Aussagen im Hinblick auf die Existenz eines differentiellen Nutzeneffekts der diagnostischen Information, wenn sämtliche möglichen posterioren Nutzenpositionen des Patienten angemessen berücksichtigt werden. In konzeptioneller Hinsicht setzt diese Methode auf einer späteren Stufe des Entscheidungsprozesses als die zweite Vorgehensweise an, da neben dem Testergebnis noch der Krankheitszustand des Patienten als Bedingung in die Ermittlung der einzelnen Nutzenpositionen eingeht ${ }^{159}$. Damit erscheint die Gefahr größer, besonders interessierende zu Lasten anderer, aufgrund der diagnostischen Information ebenfalls möglicher Nutzenpositionen des Patienten hervorzuheben. Aus der Betrachtung spezieller Konsequenzen läßt sich jedoch keine Aussage über den mit einer diagnostischen Maßnahme insgesamt verbundenen Prospekt und damit den Nutzen des Patienten ableiten ${ }^{160}$. Weder folgt aus einer einzelnen, im Vergleich zur Situation ohne weitere Diagnostik verbesserten posterioren Nutzenposition des Patienten schon die Existenz eines differentiellen Nutzeneffekts der betrachteten diagnostischen Maßnahme, noch ergibt sich aus der Möglichkeit einer Verschlechterung zwangsläufig dessen Abwesenheit. Im allgemeinen beruht der differentielle Nutzen der diagnostischen Information auf der Eigenschaft, die Wahrscheinlichkeit ungünstiger Konsequenzen für den Patienten zu verringern ${ }^{161}$.

Die Beurteilung des Nutzeneffekts der diagnostischen Information zeigt, daß es nicht ausreicht, lediglich einzelne Komponenten desjenigen Prospekts zu analysieren, der dem Patienten durch den Einsatz einer diagnostischen Maßnahme entsteht. Diese Komponenten bezeichnen die Nutzenposition des Patienten unter der Bedingung eines bestimmten Testergebnisses bzw. unter der zusätzlichen Bedingung eines bestimmten Krankheitszustands. Aufgrund der Unsicherheit, die im allgemeinen sowohl im Hinblick auf das sich einstellende Testergebnis als auch bezüglich des beim Pati-

159: Dies zeigt auch Abb. 19, in der die zweite Vorgehensweise sich auf die Ebene der Testergebnisse und damit die vorletzte Stufe des Entscheidungsbaumes bezieht, die dritte Vorgehensweise hingegen auf die letzte Stufe.

160: Vgl. GORRY, G.A. (1978), S. 52. Dies entspricht einer allgemeinen entscheidungstheoretischen Aussage, daß die Qualităt bzw. Güte einer Entscheidung bei Unsicherheit nicht anhand spezieller Konsequenzen beurteilt werden kann, sondern der daraus entstehende Prospekt in seiner Gesamtheit zu bewerten ist; vgl. z.B. NORTH, D.W. (1968), S. 200f; KASSIRER, J.P. (1976) S. 162.

161: Vgl. GÄFGEN, G. (1990), S. 314. Unter Umständen lassen sich auch bei perfekter diagnostischer Information ungünstige Konsequenzen für den Patienten nicht vollständig vermeiden. Dies gilt beispielsweise dann, wenn in einer medizinischen Entscheidungssituation ein Krankheitszustand zu berücksichtigen ist, für den keine "gute" Aktion des Arztes existiert ("unheilbare Krankheit"). 
enten vorliegenden Krankheitszustands herrscht, vermag der Arzt zum Zeitpunkt der Entscheidung über den Einsatz einer diagnostischen Maßnahme weder ein spezielles Testergebnis noch eine spezielle Kombination aus Testergebnis und Krankheitszustand zu garantieren.

\subsubsection{Der Einfluß einzelner Komponenten der medizinischen Ent- scheidungssituation}

Obgleich der in diesem Kapitel vorgestellte entscheidungstheoretische Ansatz jegliches sequentielle Element und damit den Prozeßcharakter der medizinischen Diagnostik ausblendet, erweist sich die Analyse der einmaligen Informationsbeschaffung als ausreichend, um einige wesentliche Zusammenhänge abzubilden. Insbesondere erlaubt es die detaillierte Darstellung des ärztlichen Entscheidungsproblems, die Bedeutung einzelner Komponenten in ihrer Abhängigkeit von der medizinischen Entscheidungssituation herauszuarbeiten. Daraus ergeben sich unter anderem interessante Verbindungen zu den Problemen, die in der entscheidungstheoretisch orientierten Literatur zur medizinischen Diagnostik vornehmlich diskutiert werden.

Gesundheitsleistungen bzw. medizinische Programme werden häufig anhand ihres Einflusses auf Mortalität und Morbidität beurteilt ${ }^{162}$. Dies gilt auch für diagnostische Maßnahmen ${ }^{163}$. Andererseits können die Konsequenzen ärztlichen Handelns für den Patienten von einer Komplexität sein, die eine Beschreibung durch lediglich zwei Indikatoren unvollständig erscheinen läßt ${ }^{164}$. Aus diesem Grund decken die Auswirkungen auf Mortalität und Morbidität im allgemeinen nicht das gesamte Spektrum patientenbezogener Gesundheitseffekte $\mathrm{ab}^{165}$. Zudem gibt es medizinische

162: Im Zusammenhang mit der Mortalität berücksichtigt man zunehmend qualitative Aspekte in Gestalt von "quality-adjusted life years" (QALYs), vgl. ZECKHAUSER, R.SSHEPARD, D. (1976), S. 11ff.; WEINSTEIN, M.C. et al. (1980), S. 217ff. sowie ausführlich LOOMES, G./McKENZIE, L. (1989) und WAGSTAFF, A. (1991).

163: Vgl. z.B. KEELER, E.B. (1976); WEINSTEIN, M.C. et al. (1980).

164: Vgl. LINDLEY, D.V. (1975), S. 226; JENNETT, B.TEASDALE, G.M./KNILL-JONES, R.P. (1975), S. 232.

165: Vgl. FEIN, R. (1977), S. 752. Mit einem ähnlichen Argument kritisierte Grosse schon früh jene Vergleiche medizinischer Programme, die sich lediglich auf den Mortalitätsindikator stützen; vgl. GROSSE, R.N. (1970). 
Entscheidungssituationen, in denen etwa die Mortalität nur eine unbedeutende Rolle spielt ${ }^{166}$.

Diese Bemerkungen verweisen auf die Notwendigkeit, in einem allgemeinen Ansatz eine nicht a priori festgelegte Gruppe von Gesundheitsindikatoren bzw. -attributen zu berücksichtigen ${ }^{167}$. Dabei hängen die aus der Sicht des Patienten relevanten Gesundheitsattribute von der medizinischen Entscheidungssituation ab; sie repräsentieren die Dimensionen, in denen sich die Effekte ärztlicher Aktionen niederschlagen können. Die in diesem Kapitel eingeführte Konsequenzenfunktion genügt diesen Anforderungen. Sie eröffnet zudem eine direkte Verbindung zur Forschung auf dem Gebiet der krankheitsspezifischen Gesundheitsindikatoren ${ }^{168}$.

Weiterhin dürften in einer medizinischen Entscheidungssituation die Konsequenzen eines Paar $\left(\mathrm{a}_{\mathrm{f}}, \mathrm{K}_{\mathrm{i}}\right)$ nicht von dem speziell betrachteten Patienten abhängen, soweit es sich um Gesundheitseffekte handelt. Bei der Analyse eines ärztlichen Entscheidungsproblems reicht es daher aus, eine in den ersten p-1 Komponenten vom Patienten unabhängige Konsequenzenfunktion zu verwenden ${ }^{169}$. Hingegen wird die Bewertung einzelner Konsequenzenvektoren von Patient zu Patient verschieden ausfallen ${ }^{170}$. Beispielsweise dürfte ein vornehmlich im Büro tätiger Angestellter die gesundheitlichen Folgen eines Leistenbruchs anders bewerten als ein Arbeitnehmer, der ständig körperlich schwere Arbeit verrichtet.

Folgt man den in Abschnitt 4.4.1 diskutierten Argumenten, dann resultiert der differentielle Nutzeneffekt der diagnostischen Information hauptsächlich aus einer Beeinflussung des ärztlichen Handelns. Daraus ergeben sich wichtige Schlußfolgerungen im Hinblick auf den Zusammenhang zwischen der Information und dem Nutzen einer diagnostischen Maßnahme. Wie mit Hilfe des entscheidungstheoretischen Ansatzes gezeigt werden kann, besteht allgemein keine enge Verbindung zwischen

166: Vgl. KRISCHER, J.P. (1976), S. 956; BROOK, R.H. et al. (1976), S. 3 GUYATT, G./DRUMMOND, M.F. (1986), S. 559 sowie BOWLING, A. (1991), S. 2.

167: Vgl. DRUMMOND, M.F. (1980), S. 116 sowie ausführlich BROOK, R.H. et al. (1976).

168: Vgl. KRISCHER, J.P. (1976); JENNETT, B./TEASDALE, G.M./KNILL-JONES, R.P. (1975) sowie insbesondere AVERY, A.D. et al. (1976).

169: Im Gegensatz dazu hängt der Einfluß ärztlicher Handlungen auf das Geldvermögen des Patienten von individuellen Faktoren (z.B. Einkommen) ab.

170: Vgl. HABBEMA, J.D.F.HILDEN, J. (1981), S. 89, die dieses Argument zur Begründung einer patientenspezifischen Verlustfunktion heranziehen, ähnlich DEPARTMENT OF CLINICAL EPIDEMIOLOGY AND BIOSTATISTICS (1984), S. 1432. 
der Veränderung der Informationslage des Arztes und den beim Patienten eintretenden positiven Wohlfahrtswirkungen ${ }^{171}$. Diese Aussage läßt sich am Beispiel zweier Extremfälle kurz illustrieren. Bei der Ermittlung des kompensierenden Optionspreises konnten medizinische Entscheidungssituationen identifiziert werden, in denen perfekte diagnostische Maßnahmen keinen positiven Wert für den Patienten besitzen. Falls eine gleichmäßig beste Aktion des Arztes existiert, führt selbst ein maximaler Informationsgewinn zu keinen differentiellen Nutzeneffekten beim Patienten.

Andererseits gibt es Entscheidungssituationen, in denen schon ein kleiner Informationsgewinn zu positiven Wohlfahrtswirkungen beim Patienten führen kann. Dies gilt beispielsweise für die in Abb. 19 dargestellte Vorsorgeuntersuchung, wenn die prioren Wahrscheinlichkeiten der Krankheitszustände eine identische Bayes-Bewertung der Aktionen $a_{1}$ und $a_{2}$ implizieren ${ }^{172}$. Falls eine diagnostische Maßnahme Information erbringt, wird der positive prädiktive Wert größer ausfallen als die priore Wahrscheinlickeit des Krankheitszustandes $\mathrm{K}_{1}$, während der negative prädiktive Wert $\mathrm{P}\left(\mathrm{K}_{2}\right)$ übersteigt. Die unter dieser Voraussetzung eindeutig bestimmte optimale Entscheidungsfunktion des Arztes ordnet daher einem positiven Testergebnis die Aktion $a_{1} z u$, einem negativen Testergebnis hingegen $a_{2}$. Offenbar reicht in dieser Entscheidungssituation jeder positive Informationsgewinn aus, um einen differentiellen Nutzeneffekt beim Patienten zu erzeugen.

Diese Ausagen verdeutlichen den aus der Entscheidungstheorie bekannten Sachverhalt, demzufolge der Wert eines Informationsinstruments von der jeweils betrachteten Entscheidungssituation abhängt. Aber auch in einer gegebenen medizinischen Entscheidungssituation legt die Information einer diagnostischen Maßnahme nicht eindeutig den differentiellen Nutzen fest, der beim Patienten auftritt. Dies läßt sich am Beispiel zweier binärer bzw. binarisierter Tests belegen, die im Rahmen eines ärztlichen Entscheidungsproblems von besonders einfacher Struktur eingesetzt werden können. Als mögliche Krankheitszustände sind die beiden Alternativen $\mathrm{K}_{1}$ und $\mathrm{K}_{2}$ zu unterscheiden. Der Arzt besitzt in der Ausgangslage keinerlei Information bezüglich des Krankheitszustands seines Patienten, d.h. es gilt $P\left(K_{1}\right)=P\left(K_{2}\right)=0,5$. Wie in dem zuvor diskutierten Fall der Vorsorgeuntersuchung stehen mit $a_{1}$ und $a_{2}$ lediglich zwei Aktionen zur Auswahl, von denen $a_{1}$ die Therapie für $K_{1}$ und $a_{2}$ ent-

171: Aus diesem Grund genügt es im Rahmen einer Nutzenanalyse nicht, lediglich die Information einer diagnostischen Maßnahme zu beschreiben; vgl. dazu kritisch CARD, W.I. (1975), S. 195 sowie DRUMMOND, M.F. (1980), S. 115.

172: In diesem Fall ist die Gleichung $\Phi_{B}\left(a_{1}\right)=\Phi_{B}\left(a_{2}\right)$ erfüllt. 
sprechend für $\mathrm{K}_{2}$ darstellt. Insgesamt liegt damit eine große Ähnlichkeit zu der in Abb. 19 dargestellten Entscheidungssituation vor. Ein Unterschied besteht lediglich insofern, als die Annahme identischer priorer Wahrscheinlichkeiten für beide Krankheitszustände eine Interpretation der diagnostischen Maßnahmen als Vorsorgeuntersuchungen verhindert.

Da es sich um binäre Tests handelt, reichen Sensitivität und Spezifität jeweils zur Charakterisierung der beiden zur Verfügung stehenden diagnostischen Maßnahmen aus. Die Sensitivität der ersten Maßnahme $T_{1}$ beträgt 1 , während ihre Spezifität sich auf 0,8 beläuft. Im Unterschied dazu besitzt der zweite Test $T_{2}$ eine Sensitivität von 0,8 und eine Spezifität von 1. Man erhält demnach die Kennziffern der einen diagnostischen Maßnahme, indem man die Kennziffern der anderen Maßnahme gerade vertauscht. Abbildung 20 übersetzt diese Parameterwerte in die Notation, die in Kapitel 3 eingeführt wurde und gibt außerdem einen Überblick über die prädiktiven Werte, die $T_{1}$ und $T_{2}$ in der skizzierten Entscheidungssituation erzielen.

Wie aus der Abbildung ersichtlich, überträgt die Annahme identischer priorer Wahrscheinlichkeiten der Krankheitszustände die Symmetrie zwischen $T_{1}$ und $T_{2}$ hinsichtlich ihrer diagnostischen Kennziffern auf die dazugehörigen prädiktiven Werte. Während sich beim Einsatz des ersten Tests ein falsch negatives Ergebnis mit Sicherheit ausschließen läßt, kann bei $T_{2}$ kein falsch positives Resultat auftreten. Wie man mit Hilfe der in Abschnitt 3.6 angegebenen Formel für die bedingte Entropie nachprüft, fällt die in der Zufallsvariablen $\mathrm{K}$ verbleibende Unbestimmtheit gegeben die Information einer diagnostischen Maßnahme für $T_{1}$ und $T_{2}$ jeweils identisch aus. Es gilt also:

$$
\mathrm{I}\left(\mathrm{K} \mid \mathrm{Z}_{1}\right)=\mathrm{I}\left(\mathrm{K} \mid \mathrm{Z}_{2}\right)
$$

wenn $Z_{1}$ die Zufallsvariable des Testergebnisses von $T_{1}$ und $Z_{2}$ entsprechend für $T_{2}$ repräsentiert. Daraus folgt unmittelbar die Gleichheit des Informationsgewinns für den Arzt in der betrachteten Entscheidungssituation, ob er nun die erste oder die zweite diagnostische Maßnahme einsetzt:

$$
G\left(K, Z_{1}\right)=G\left(K, Z_{2}\right) .
$$


Damit besteht zwischen den beiden diagnostischen Maßnahmen kein Unterschied bezüglich der von ihnen gelieferten Informationsmenge.

Abb. 20: Diagnostische Kennziffern und prädiktive Werte von $T_{1}$ und $T_{2}$

\begin{tabular}{|l|c|c|}
\hline $\begin{array}{l}\text { diagnostische } \\
\text { Maßnahme }\end{array}$ & $T_{1}$ & $T_{2}$ \\
\hline Parameter & 1,0 & 0,8 \\
\hline $\begin{array}{l}\text { Spezifitivität } \\
\begin{array}{l}\text { positiver } \\
\text { prädiktiver Wert }\end{array}\end{array}$ & 0,8 & 1,0 \\
\hline $\begin{array}{l}\text { negativer } \\
\text { prädiktiver Wert }\end{array}$ & $\frac{5}{6}$ & 1,0 \\
\hline
\end{tabular}

Indessen braucht die Äquivalenz von $T_{1}$ und $T_{2}$ keineswegs auch im Hinblick auf den differentiellen Nutzeneffekt beim Patienten zu gelten. Geht man von der Voraussetzung aus, da $\beta$ beide diagnostischen Maßnahmen in der beschriebenen Entscheidungssituation einen positiven Zusatznutzen im Vergleich zur Situation ohne 
weitere Diagnostik erzeugen, dann fallen diese Nutzeneffekte im allgemeinen verschieden aus ${ }^{173}$. Dies gilt beispielsweise dann, wenn die gesundheitlichen Folgen einer richtigen Therapie von $\mathrm{K}_{1}$ bzw. $\mathrm{K}_{2}$ sich in der Bewertung durch den Patienten nur geringfügig unterscheiden, die Nutzenposition aufgrund einer falschen Therapie jedoch stark vom Krankheitszustand abhängt. Je nachdem, ob die Folgen eines falsch positiven oder falsch negativen Testergebnisses eine vergleichsweise ungünstige $\mathrm{Be}$ wertung erfahren, wird entweder die zweite diagnostische Maßnahme oder $T_{1}$ dem Patienten einen insgesamt höheren Nutzen stiften.

Mit Hilfe des Beispiels läßt sich ein wichtiger konzeptioneller Unterschied zwischen der Information und dem Nutzen einer diagnostischen Maßnahme veranschaulichen. Bei der Ermittlung der Informationsmenge kommt es lediglich darauf an, wie stark die Wahrscheinlichkeiten der Krankheitszustände durch eine diagnostische Maßnahme beeinflußt werden. Aus der Sicht der Informationstheorie macht es keinen Unterschied, ob die diagnostische Information primär in einer Verringerung der Wahrscheinlichkeit falsch positiver oder falsch negativer Testergebnisse besteht. Im Unterschied dazu spielt gerade dieser Einfluß der diagnostischen Information für die Existenz und das Ausmaß des differentiellen Nutzeneffekts eine wesentliche Rolle. Wie das Beispiel zeigt, hängt der Nutzen eines binären bzw. binarisierten Tests für den Patienten in einer medizinischen Entscheidungssituation davon ab, ob sich die darin enthaltene Information stärker auf die positiven oder auf die negativen Fälle bezieht.

Eine kleine Modifikation der im Beispiel verwendeten Konstellation führt zu einer weiteren wichtigen Aussage. Angenommen, $T_{2}$ erzeugt in der betrachteten Entscheidungssituation einen höheren differentiellen Nutzen für den Patienten. Verringert man eine diagnostische Kennziffer von $T_{2}$, dann liefert diese diagnostische Maßnahme nun weniger Information als ihre Alternative $T_{1}$. Fällt die Reduktion hinreichend gering aus, dann wird $\mathrm{T}_{2}$ auch unter dieser Voraussetzung noch einen höheren Nutzen als $T_{1}$ stiften. Dieses Resultat läßt sich verallgemeinern, denn in einer

173: Das genaue Resultat eines Vergleichs von $T_{1}$ und $T_{2}$ hängt von den möglichen Konsequenzenvektoren bzw. ihrer Nutzenbewertung durch den Patienten ab. Zur Illustration des allgemeinen Sachverhalts genügt an dieser Stelle die exemplarische Untersuchung einer speziellen Konstellation von Nutzenwerten. 
medizinischen Entscheidungssituation kann grundsätzlich nicht ausgeschlossen werden, daß eine weniger informative diagnostische Maßnahme den größeren Nutzeneffekt beim Patienten hervorruft ${ }^{174}$.

Eine medizinische Entscheidungssituation besteht im wesentlichen aus drei verschiedenen Komponenten. Es handelt sich dabei zunächst um die prioren Wahrscheinlichkeiten der Krankheitszustände des Patienten und um die Matrizen, in denen jeweils die bedingten Wahrscheinlichkeiten der Testergebnisse derjenigen diagnostischen Maßnahmen verzeichnet sind, deren Einsatz in Betracht kommt. Schließlich müssen als eine dritte Komponente noch die individuellen Nutzenwerte aller möglichen Konsequenzenvektoren berücksichtigt werden. Die in Abschnitt 4.3 angegebene allgemeine Lösung des ärztlichen Entscheidungsproblems gibt allerdings noch keinen Aufschluß über die Zusammenhänge zwischen diesen Komponenten. Um diesbezüglich eine genauere Vorstellung zu erhalten, faßt man einzelne Komponenten in einer ceteris paribus Bedingung zusammen und untersucht die Auswirkungen, die von den übrigen Bestandteilen einer medizinischen Entscheidungssituation im Hinblick auf den differentiellen Nutzeneffekt beim Patienten ausgehen.

Eine Form der Analyse fixiert die medizinische Entscheidungssituation insoweit, als von einer gegebenen Menge möglicher Konsequenzenvektoren und festen Nutzenbewertungen durch den Patienten ausgegangen wird. Man betrachtet demnach eine Situation, in der die Konsequenzenfunktion und damit insbesondere sowohl die Menge der Aktionen des Arztes als auch die Menge der Krankheitszustände des Patienten vorgegeben sind. In diesem Fall liegt die Struktur der medizinischen Entscheidungssituation fest. Diese Voraussetzung wird beispielsweise dann erfüllt sein, wenn man in einer Entscheidungssituation einen bestimmten Patienten betrachtet.

174: Diese Feststellung repräsentiert eine Anwendung der allgemeinen entscheidungstheoretischen Aussage, derzufolge in einer Entscheidungssituation ein Informationsinstrument einen höheren Wert als eine Alternative besitzen kann, obwohl es weniger Information enthält. Vgl. dazu auch MARSCHAK, J. (1959), S. 95 f., der ebenfalls ein Beispiel von besonders einfacher Struktur zur Illustration verwendet. 
Unter der Voraussetzung einer konstanten Struktur lassen sich zu jeder Aktion des Arztes diejenigen Wahrscheinlichkeitsvektoren bestimmen, bei denen diese Aktion aus der Sicht des Patienten eine maximale Bayes-Bewertung erfährt. Auf diese Weise ermittelt man zu jeder ärztlichen Aktion $a_{m}$ eine dazugehörige Teilmenge $R\left(a_{m}\right)$ des Wahrscheinlichkeits-Simplex $S^{n}$, für die gilt ${ }^{175}$ :

(4.85) $R\left(a_{m}\right)=\left\{P \in S^{n}\left|\sum_{i=1}^{n} P_{i} \cdot U\left(a_{m}, K_{i}\right) \geq \sum_{i=1}^{n} P_{i} \cdot U\left(a_{f}, K_{i}\right) ; a_{f} \in A\right|\right.$.

Man kann zeigen, daß diese Teilmengen abgeschlossen und konvex sind und untereinander keine inneren Punkte gemeinsam haben ${ }^{176}$. Damit bildet das System der Teilmengen $\mathrm{R}\left(\mathrm{a}_{\mathrm{f}}\right)$ eine Aufteilung der Menge aller Wahrscheinlichkeitsvektoren, die in der betrachteten Entscheidungssituation zulässig sind.

Die Teilmengen $R\left(a_{f}\right)$ ermöglichen eine einfache Bestimmung der optimalen Handlung des Arztes. Falls beispielsweise der Arzt keine weitere Diagnostik betreibt, genügt die Angabe der als gültig erachteten prioren Wahrscheinlichkeiten der Krankheitszustände, um eine für den Patienten optimale Aktion zu ermitteln. Wenn der Vektor der prioren Wahrscheinlichkeiten z.B. zur Teilmenge $R\left(\mathrm{a}_{\mathrm{m}}\right)$ gehört, dann stellt die Aktion $a_{m}$ eine optimale Aktion des Arztes in der Situation ohne weitere Diagnostik dar ${ }^{177}$. Entsprechend lassen sich mit Hilfe der Teilmengen $R\left(a_{f}\right)$ auf einfache Weise optimale Entscheidungsfunktionen für eine diagnostische Maßnahme konstruieren, wenn eine Ausgangslage in Gestalt von prioren Wahr-scheinlichkeiten vorgegeben wird. Zunächst ergibt sich aufgrund eines Testergebnisses der diagnostischen Maßnahme $\mathrm{T}_{\mathrm{j}}$ eine posteriore Wahrscheinlichkeitsverteilung für die möglichen Krankheitszustände. Eine optimale Entscheidungsfunktion $d_{j}{ }^{*}$ ordnet jedem möglichen Testergebnis $z_{j h}$ eine Aktion $z u, z u$ deren Teilmenge der Vektor der posterioren Wahrscheinlichkeiten gehört ${ }^{178}$.

175: Dabei kann es durchaus vorkommen, daß eine mögliche Aktion des Arztes sich bei keiner Wahrscheinlichkeitsverteilung der Krankheitszustände als optimal erweist.

176: Vgl. BLACKWELL, D./GIRSHICK, M.A. (1954), S. $149 \mathrm{ff}$.

177: Die Eindeutigkeit dieser Aktion ist nur dann gewährleistet, wenn der Vektor der prioren Wahrscheinlichkeiten nicht gleichzeitig noch zu einer anderen Teilmenge gehört.

178: Die Eindeutigkeit der Zuordnung gilt auf jeden Fall, wenn der Vektor der posterioren Wahrscheinlichkeiten einen inneren Punkt dieser Teilmenge bildet. 
Die Vorgabe einer bestimmten Struktur der medizinischen Entscheidungssituation bzw. der darauf beruhenden Aufteilung der Men-ge aller zulässigen Wahrscheinlichkeitsverteilungen erlaubt es, den Zusammenhang zwischen den prioren Wahrscheinlichkeiten und der Information einer diagnostischen Maßnahme bei der Erzeugung des differentiellen Nutzeneffekts genauer zu untersuchen. Dies gilt insbesondere, wenn lediglich zwei oder drei Krankheitszustände in Betracht zu ziehen sind, da diese Beschränkung eine anschauliche graphische Darstellung ermöglicht. Im folgenden wird deshalb $n=3$ vorausgesetzt ${ }^{179}$. Geometrisch bildet die Menge aller zulässigen Wahrscheinlichkeitsvektoren ein gleichseitiges Dreieck, dessen Höhe Eins beträgt. Jedem Punkt des Dreiecks entspricht eine spezielle Wahrscheinlichkeitsverteilung. Wie Abbildung 21 verdeutlicht, ergeben sich die dazugehörigen Wahrscheinlichkeiten der einzelnen Krankheitszustände als Längen der Lote, die von diesem Punkt auf die Seiten des Dreiecks gefällt werden können ${ }^{180}$.

Der in der Abbildung eingezeichnete Punkt $P$ repräsentiert eine Wahrscheinlichkeitsverteilung, die dem Krankheitszustand $\mathrm{K}_{2}$ mit 0,6 die höchste Wahrscheinlichkeit zuordnet, während die Zustände $\mathrm{K}_{1}$ bzw. $\mathrm{K}_{3}$ mit 0,25 bzw. 0,15 jeweils eine geringere Wahrscheinlichkeit besitzen.

179: Vgl. zu dieser Darstellung HABBEMA, J.D.F./HILDEN, J./BJERREGAARD, B. (1978), S. $221 \mathrm{ff}$.

Indessen betrachtet die Literatur schwergewichtig binarisierte Tests und beschränkt sich auf die Analyse von Entscheidungssituationen, in denen zwei Krankheitszustände zu unterscheiden sind. Die als "threshold analysis" bezeichnete Analyseform untersucht insbesondere, für welche prioren Wahrscheinlichkeiten der Einsatz einer diagnostischen Maßnahme (bei gegebener Sensitivität und Spezifität, d.h. bei fester Binarisierung) zur Entstehung eines differentiellen Nutzeneffekts beim Patienten führt; vgl. PAUKER, S.G./KASSIRER, J.P. (1978), S. 330ff. sowie ausführlich PAUKER, S.G./KASSIRER, J.P. (1980).

Neuere Arbeiten behandeln daneben den Einfluß, den - bei gegebenen prioren Wahrscheinlichkeiten der Krankheitszustände - verschiedene Binarisierungen einer diagnostischen Maßnahme auf den Umfang dieses Nutzeneffekts besitzen; vgl. dazu DOUBILET, P. (1983) und KRIEG, A.F. (1986).

180: In einem gleichseitigen Dreieck entspricht die Summe dieser Längen gerade der Höhe des Dreiecks und beträgt unter der im Text angeführten Voraussetzung Eins. 
Abb. 21: Graphische Darstellung von dreielementigen Wahrscheinlichkeitsverteilungen

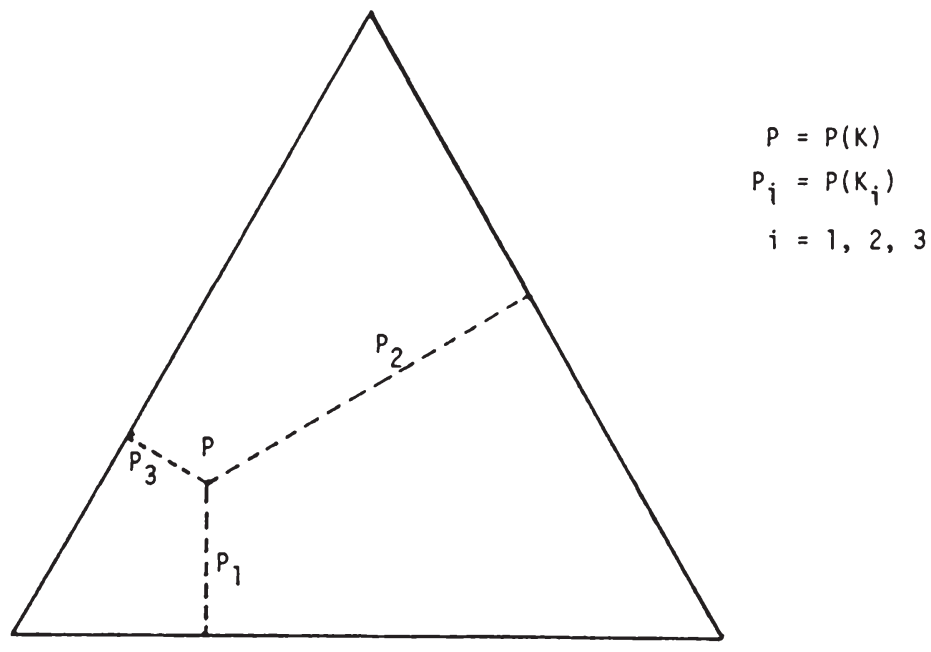

Zur Illustration der Analyse wird nun exemplarisch eine Entscheidungssituation betrachtet, in der die diagnostische Information prinzipiell Nutzeneffekte beim Patienten zu stiften vermag. Im Hinblick auf die Struktur der medizinischen Entscheidungssituation impliziert diese Annahme, daß keine gleichmäßig beste Aktion des Arztes existiert. Im Interesse einer einfachen Darstellung wird weiter angenommen, daß insgesamt drei Aktionen des Arztes für jeweils verschiedene Ausgangslagen eine optimale Bayes-Bewertung in der Situation ohne weitere Diagnostik erfahren können. Daraus ergibt sich, wie Abbildung 22 veranschaulicht, eine Aufteilung der Menge aller zulässigen Wahrscheinlichkeitsvektoren in die drei Regionen $R\left(a_{1}\right), R\left(a_{2}\right)$ und $R\left(a_{3}\right)^{181}$.

181: Gäbe es eine gleichmäßig beste Aktion a ${ }^{*}$ des Arztes, dann würde die dazugehörige Menge $R\left(a^{*}\right)$ sämtliche möglichen Wahrscheinlichkeitsverteilungen enthalten. Mit Hilfe der im Text diskutierten Analyse erkennt man unmittelbar, da $B$ in diesem Fall die diagnostische Information das ärtliche Handeln nicht zu beeinflussen vermag. Dies bestätigt eine zuvor allgemein abgeleitete Aussage, die in derartigen medizinischen Entscheidungssituationen jeder diagnostischen Maßnahme einen Optionspreis von Null zuweist. 
Abb. 22: Die Struktur der medizinischen Entscheidungssituation

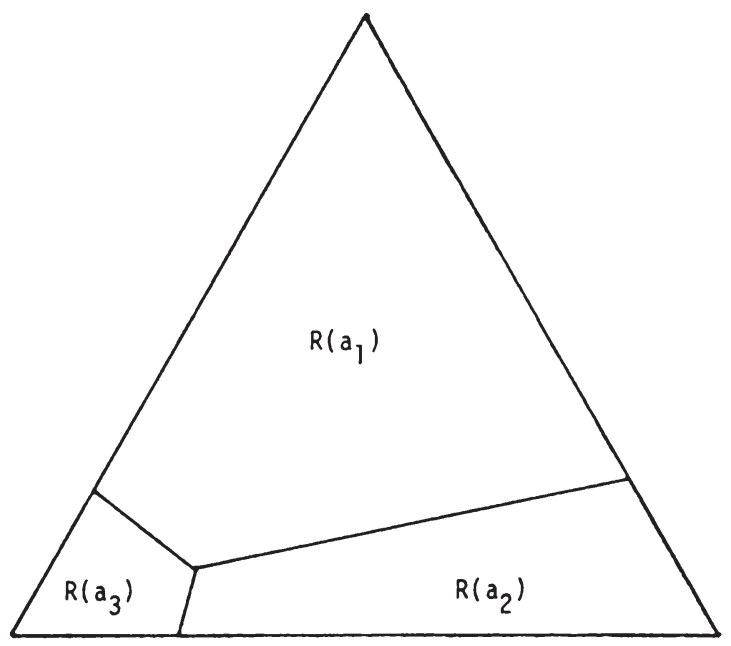

Wie die Abbildung zeigt, bildet $\mathrm{a}_{1}$ insbesondere in denjenigen Ausgangslagen eine optimale Aktion, in denen der Krankheitszustand $\mathrm{K}_{1}$ eine hohe priore Wahrscheinlichkeit besitzt. Ebenso wählt der Arzt auf jeden Fall die Aktion $a_{2}$ in der Situation ohne weitere Diagnostik, wenn die Wahrscheinlichkeit des Krankheitszustands $\mathrm{K}_{3}$ groß ist. Für die überwiegende Mehrzahl der möglichen Wahrscheinlichkeitsverteilungen erweist sich entweder $a_{1}$ oder $a_{2}$ als optimale Bayes-Aktion. Lediglich in Ausgangslagen, in denen $\mathrm{K}_{2}$ eine sehr hohe Wahrscheinlichkeit besitzt, erzielt die Aktion $a_{3}$ eine günstigere Bewertung als die beiden übrigen Aktionen.

Ob eine diagnostische Maßnahme dem Patienten einen differentiellen Nutzeneffekt stiftet, läßt sich nunmehr auf einfache Weise veranschaulichen. Dazu wird weiterhin unterstellt, daß es sich bei der betrachteten um eine unvollkommene diagnostische Maßnahme handelt, bei der drei verschiedene Testergebnisse auftreten können ${ }^{182}$. Da jedes Testergebnis die prioren Wahrscheinlichkeiten der Krankheitszustände in spezi-

182: Ebenso wie die zuvor eingeführte Voraussetzung bezüglich der Anzahl der relevanten Aktionen ist auch diese Annahme nicht essentiell und führt daher zu keiner Beschränkung der Analyse. Sie dient lediglich dazu, eine übersichtliche graphische Darstellung zu ermöglichen. 
fischer Weise modifiziert, sind drei posteriore Wahrscheinlichkeitsverteilungen $\mathrm{zu}$ unterscheiden. Die Darstellung einer speziellen Ausgangslage und der Informationsleistung des Diagnosetests $T_{1}$ geschieht damit geometrisch anhand von insgesamt vier Punkten innerhalb des zuvor angegebenen Dreiecks ${ }^{183}$.

Abb. 23: Der differentielle Nutzeneffekt von $T_{1}$ in der Ausgangslage $P_{0}$

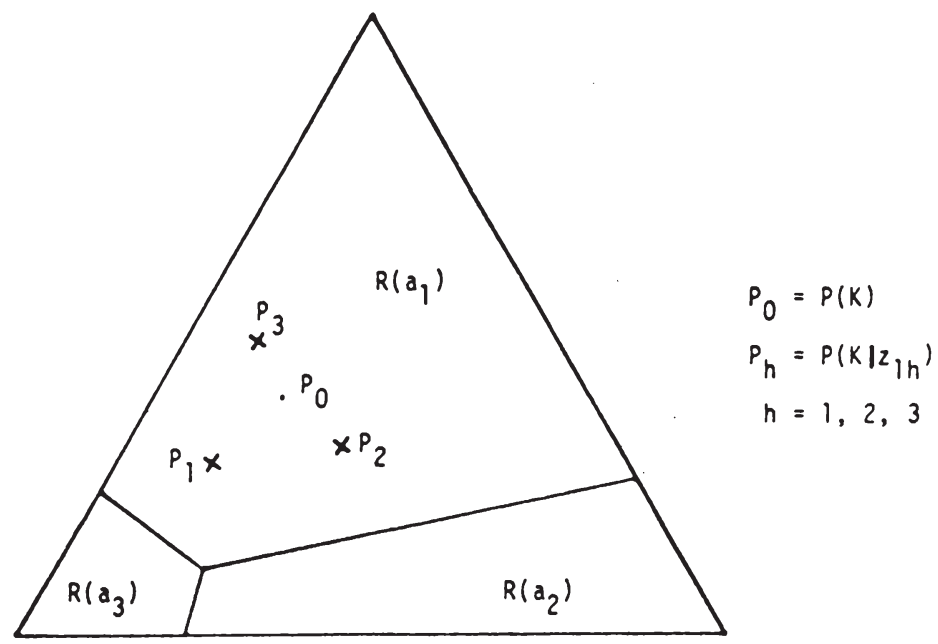

Aus Abbildung 23 geht zugleich hervor, daß die betrachtete diagnostische Maßnahme in der durch $\mathrm{P}_{0}$ repräsentierten speziellen Ausgangslage keinen Nutzeneffekt für den Patienten erzeugt. Unabhängig von dem sich einstellenden Testergebnis wird der Arzt

183: Die Anordnung der die posterioren Wahrscheinlichkeitsverteilungen repräsentierenden Punkte $\mathrm{P}_{1}, \mathrm{P}_{2}$ und $\mathrm{P}_{3}$ hängt von den bedingten Wahrscheinlichkeiten der Testergebnisse ab, welche die untersuchte diagnostische Maßnahme charakterisieren. Aus dem allgemeinen $\mathrm{Zu}-$ sammenhang zwischen prioren und posterioren Wahrscheinlichkeiten folgt, daß $\mathrm{P}_{0}$ eine konvexe Kombination von $P_{1}, P_{2}$ und $P_{3}$ darstellt, deren Gewichte durch die totalen Wahrscheinlichkeiten der Testergebnisse $\mathrm{P}\left(\mathrm{z}_{\mathrm{jh}}\right)$ bestimmt werden. $\mathrm{Vgl}$. zu dieser Darstellung MARSCHAK, J.MIYASAWA, K. (1968), S. 226.

Bei einer vollkommenen diagnostischen Maßnahme wären $\mathrm{P}_{1}, \mathrm{P}_{2}$ und $\mathrm{P}_{3}$ in die Eckpunkte des Dreiecks zu "verschieben", falls in der Ausgangslage - wie in der Abbildung unterstellt alle drei Krankheitszustände positive Wahrscheinlichkeit besitzen. 
in jedem Fall die Aktion $a_{1}$ wählen. In diesem Beispiel reicht die diagnostische Information von $T_{1}$ nicht aus, um das ärztliche Handeln zu beeinflussen.

Ein anderes Ergebnis erhält man, wenn in der Ausgangslage der Krankheitszustand $\mathrm{K}_{2}$ eine hohe Wahrscheinlichkeit besitzt. Die Abbildung 24 zeigt eine derartige Situation, wobei der Punkt $P_{0}{ }^{\prime}$ die entsprechende priore Wahrscheinlichkeitsverteilung der Krankheitszustände repräsentiert. Ohne weitere Diagnostik würde der Arzt die Aktion $a_{3}$ wählen, da der Punkt $P_{0}^{\prime}$ zu der Teilmenge $R\left(a_{3}\right)$ gehört.

Abb. 24: Der differentielle Nutzeneffekt von $T_{1}$ in der Ausgangslage $P_{0}^{\prime}$

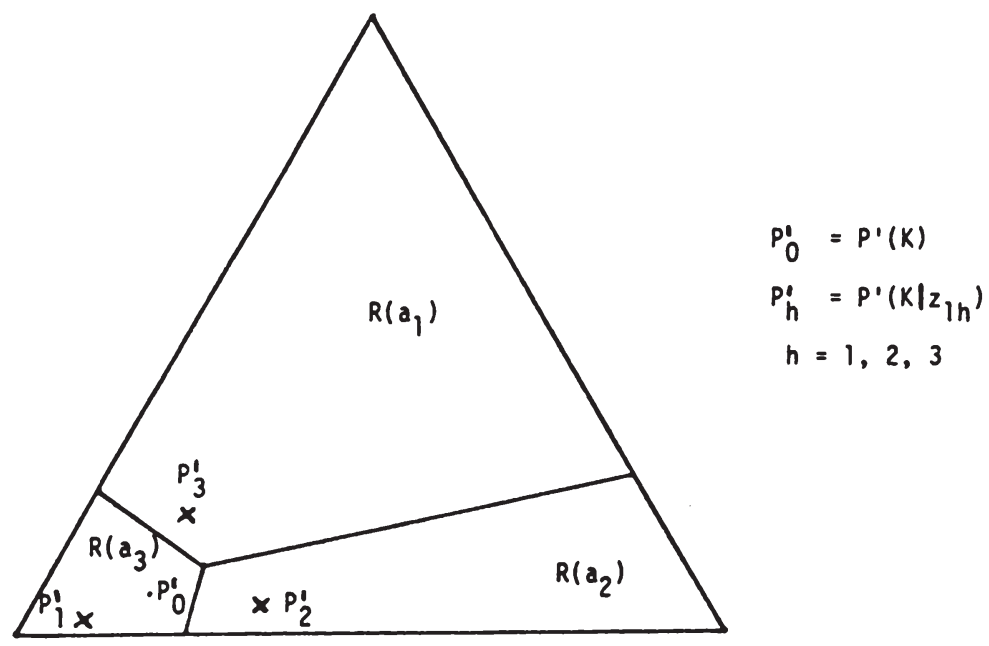

Falls der Arzt hingegen die diagnostische Maßnahme $T_{1}$ einsetzt, ist es für ihn optimal, die Aktionswahl vollständig vom Testergebnis abhängig zu gestalten. Bei der in der Abbildung dargestellten Konstellation erzeugt damit die diagnostische Information desselben Tests, der oben betrachtet wurde, einen positiven Nutzeneffekt beim Patienten. Insgesamt läßt die eindeutig bestimmte optimale Entscheidungsfunktion den Arzt die Aktion $a_{h}$ ergreifen, wenn das Testergebnis $z_{1 h}$ vorliegt. 
Das Beispiel demonstriert die Bedeutung, die der Ausgangslage im Hinblick auf den differentiellen Nutzeneffekt einer diagnostischen Maßnahme zukommt, wenn die medizinische Entscheidungssituation in Bezug auf die Nutzenwerte der möglichen Konsequenzenvektoren festliegt. In diesem Zusammenhang folgt weiterhin, daß eine diagnostische Maßnahme in einer hinsichtlich ihrer Struktur festgelegten Entscheidungssituation auch dann nicht in allen möglichen Ausgangslagen dem Patienten einen Zusatznutzen zu stiften braucht, wenn sie dies in einigen Fällen leistet. Dabei spielt in einer konkreten Ausgangslage nicht allein der Informationsstand des Arztes eine Rolle, der in der Form einer prioren Wahrscheinlichkeitsverteilung der Krankheitszustände vorliegt. Wie man durch einen Vergleich der beiden Fälle anhand der Abbildungen 23 und 24 überprüft, besitzt der Arzt im zweiten Fall im Hinblick auf den wahrscheinlichsten Krankheitszustand sogar das größere Vorwissen: die in $\mathrm{P}_{0}{ }^{\prime}$ enthaltene priore Wahrscheinlichkeit des Krankheitszustands $\mathrm{K}_{2}$ ist größer als die in $\mathrm{P}_{0}$ enthaltene priore Wahrscheinlichkeit des Krankheitszustands $\mathrm{K}_{1}$. Dennoch erweist sich nur in der durch $\mathrm{P}_{0}{ }^{\prime}$ repräsentierten Ausgangslage die Aktionswahl des Arztes als nicht robust gegenüber der Veränderung der Informationslage, welche die betrachtete diagnostische Maßnahme bewirkt. Das unterschiedliche Ergebnis, das die Analyse des differentiellen Nutzeneffekts der diagnostischen Information in den beiden Ausgangslagen erbringt, beruht darauf, daß die Aktion $a_{3}$ für den Patienten nur bei wenigen Wahrscheinlichkeitsverteilungen die günstigste Aktion darstellt. Im Gegensatz dazu liefert die Aktion $a_{1}$ Konsequenzen, die sie in der Nutzenbewertung durch den Patienten auch dann noch günstig abschneiden lassen, wenn die Wahrscheinlichkeitsverteilung der Krankheitszustände stark von der durch $\mathrm{P}_{0}$ repräsentierten Ausgangslage abweicht.

Die Abhängigkeit des differentiellen Nutzens der diagnostischen Information von der Ausgangslage und damit der vollständig spezifizierten medizinischen Entscheidungssituation wirkt sich auch auf das Ergebnis eines Vergleichs verschiedener diagnostischer Maßnahmen aus. Betrachtet man der Einfachheit halber lediglich zwei diagnostische Maßnahmen, so kann im allgemeinen mit Hilfe der soeben vorgestellten graphischen Analyse nicht entschieden werden, welche von beiden den größeren Nutzeneffekt beim Patienten erzeugt. In einem speziell gewählten Beispiel gelingt dies dennoch. Zu diesem Zweck wird die Struktur der medizinischen Ent- 
Abb. 25: Der differentielle Nutzeneffekt von $T_{2}$

a) in der Ausgangslage $P_{0}$

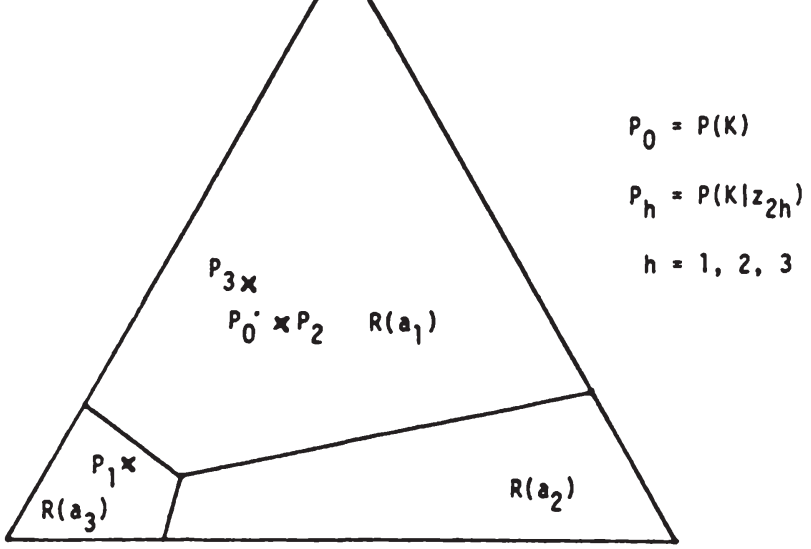

b) in der Ausgangslage $P_{0}^{\prime}$

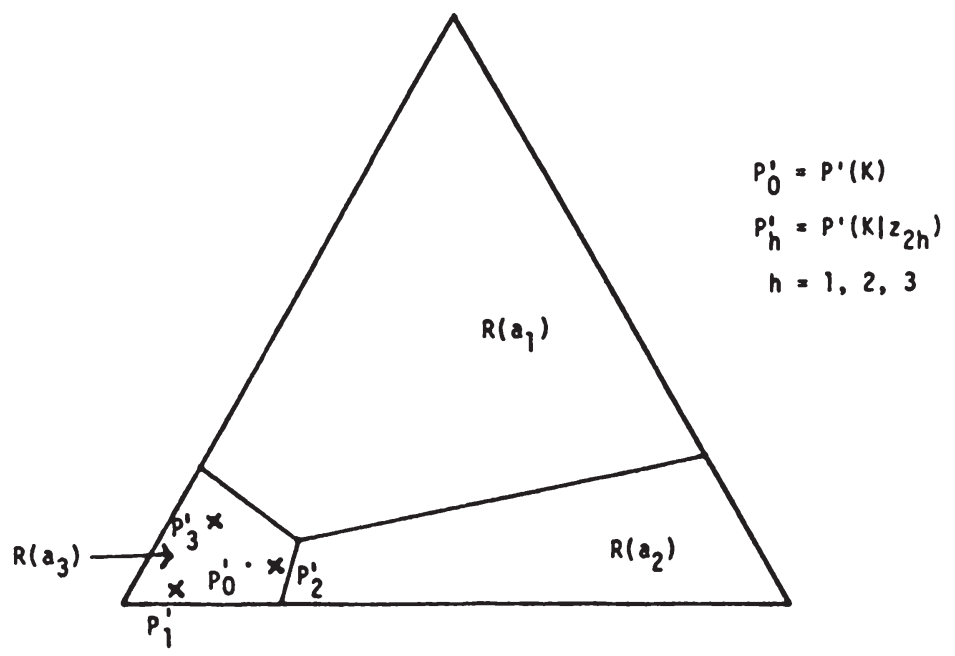


scheidungssituation aus dem oben diskutierten Beispiel vollständig übernommen, d.h. es gelten die dort getroffenen Voraussetzungen und insbesondere die in Abbildung 22 dargestellte Aufteilung der Menge aller möglichen Wahrscheinlichkeitsverteilungen. Zusätzlich zu der diagnostischen Maßnahme $T_{1}$ kommt nun ein weiterer Test $T_{2}$ in Betracht. Abbildung 25 zeigt, zu welcher Veränderung der Informationslage des Arztes diese zweite diagnostische Maßnahme jeweils in den durch $\mathrm{P}_{0}$ bzw. $\mathrm{P}_{0}{ }^{\prime}$ repräsentierten Ausgangslagen führt.

Wie die Abbildung synoptisch veranschaulicht, erzeugt die nunmehr untersuchte diagnostische Maßnahme $T_{2}$ in der ersten Ausgangslage $\left(P_{0}\right)$ einen differentiellen Nutzeneffekt, während sie in der zweiten Ausgangslage $\left(\mathrm{P}_{0}{ }^{\prime}\right)$ das ärztliche Handeln nicht beeinflußt. Damit stiftet $T_{2}$ gerade in derjenigen Ausgangslage einen Zusatznutzen für den Patienten, in der die diagnostische Maßnahme $T_{1}$ dies nicht leistet und umgekehrt. In der durch $P_{0}$ angezeigten Ausgangslage erweist sich daher $T_{2}$ als vorteilhaft gegenüber $T_{1}$, während in der durch $P_{0}$ ' dargestellten Ausgangslage der Patient die diagnostische Information von $T_{1}$ präferier ${ }^{184}$. Aus dieser Beobachtung folgt, $\mathrm{da} B$ im Normalfall - bei gegebener Struktur der medizinischen Entscheidungssituation - das Ergebnis eines Vergleichs verschiedener diagnostischer Maßnahmen anhand ihrer Nutzeneffekte beim Patienten von der jeweils vorliegenden Ausgangslage abhängt ${ }^{185}$.

Aus dem in dieser Arbeit verwendeten Ansatz folgt weiterhin, daß der Nutzen einer diagnostischen Information im allgemeinen von der Struktur der medizinischen Entscheidungssituation abhängt. Sobald die Menge der möglichen Konsequenzenvektoren bzw. der möglichen Nutzenwerte variiert, ergeben sich Auswirkungen auf die Existenz und den Umfang des differentiellen Nutzeneffekts einer diagnostischen Maßnahme. Wenn in einer medizinischen Entscheidungssituation beispielsweise ein anderer Patient betrachtet wird, ändert sich in der Regel die Menge der möglichen Nutzenwerte. Sofern diese Veränderung verschiedene diagnostische Maßnahmen in unterschiedlichem Ausmaß betrifft, hängt auch das Ergebnis eines Vergleichs mehrerer Diagnosetests von der Struktur der Entscheidungssituation ab. Zur Illustration dieser Aussage dienen die zu Beginn dieses Abschnitts diskutierten binären Tests $T_{1}$ und $\mathrm{T}_{2}$, deren Kennziffern in Abbildung 20 aufgeführt sind. Wie zuvor zieht der Arzt

184: Diese Vorteilhaftigkeitsaussage bezieht sich ausschlieBlich auf die Nutzeneffekte, welche die beiden diagnostischen Maßnahmen beim Patienten zu stiften vermögen, da z.B. ihre Kosten an dieser Stelle unberücksichtigt bleiben; vgl. dazu ausführlich Abschnitt 4.3.4. 
aufgrund seines Vorwissens lediglich zwei Krankheitszustände bei seinem Patienten in Betracht. Während grundsätzlich die Aktion $a_{1}$ die für den Krankheitszustand $\mathrm{K}_{1}$ angemessene Handlung des Arztes darstellt, gilt eine entsprechende Beziehung für die Aktion $a_{2}$ und den Krankheitszustand $K_{2}$. Hinsichtlich der prioren Wahrscheinlichkeiten von $\mathrm{K}_{1}$ und $\mathrm{K}_{2}$ wird keine spezielle Annahme getroffen; in der Ausgangslage besitzen beide Krankheitszustände aus naheliegenden Gründen positive Wahrscheinlichkeit ${ }^{186}$.

Zur Analyse des Einflusses, den die Struktur der medizinischen Entscheidungssituation auf den Nutzen einer diagnostischen Maßnahme ausübt, betrachtet man das mit einer optimalen Entscheidungsfunktion des Arztes verbundene Nutzenniveau ${ }^{187}$ :

$$
\Phi_{B}\left(d_{j}^{*}\right)=\sum_{i=1}^{2} P\left(K_{i}\right) \cdot \sum_{h=1}^{2} P\left(z_{j h} \mid K_{i}\right) \cdot U\left[d_{j}^{*}\left(z_{j h}\right), K_{i}\right] .
$$

Gemäß den bisher getroffenen Annahmen ordnet die optimale Entscheidungsfunktion einem positiven Testergebnis $z_{j 1}$ die Aktion $a_{1} z u$ und einem negativen Testergebnis $\mathrm{z}_{\mathrm{j} 2}$ die Aktion $\mathrm{a}_{2}$. Insofern gilt für das Nutzenniveau $\Phi_{\mathrm{B}}\left(\mathrm{d}_{\mathrm{j}}{ }^{*}\right)$ :

$$
\Phi_{B}\left(d_{j}^{*}\right)=\sum_{i=1}^{2} P\left(K_{i}\right) \cdot \sum_{h=1}^{2} P\left(z_{j h} \mid K_{i}\right) \cdot U\left(a_{h}, K_{i}\right) \text {. }
$$

Die Konstellation der einzelnen Nutzenwerte $u\left[g\left(a_{f}, K_{i}\right)\right]$ beschreibt die Struktur der medizinischen Entscheidungssituation. Liegt diese fest, dann entscheiden in einer durch die prioren Wahrscheinlichkeiten $\mathrm{P}\left(\mathrm{K}_{\mathrm{i}}\right)$ gegebenen Ausgangslage die bedingten Wahrscheinlichkeiten $\mathrm{P}\left(\mathrm{z}_{\mathrm{jh}} \mid \mathrm{K}_{\mathrm{i}}\right)$ über den Umfang des beim Patienten auftretenden Nutzeneffekts. Umgekehrt definiert unter dieser ceteris-paribus-Bedingung ein festes Nutzenniveau $\Phi_{B}$ einen genau bestimmten "trade-off" zwischen Sensitivität und Spezifität einer diagnostischen Maßnahme. Man erhält als Preis in Form einer verringerten Sensitivität, den der Patient für eine marginale Erhöhung der Spezifität in Kauf zu nehmen bereit ist:

185: Bei manchen diagnostischen Maßnahmen fällt allerdings ein derartiger Vergleich immer zugunsten derselben Maßnahme aus; vgl. dazu ausführlicher Abschnitt 4.4.3.

186: Diese Voraussetzung schließt lediglich degenerierte priore Wahrscheinlichkeitsverteilungen aus.

187: In der weiteren Analyse wird vorausgesetzt, daß die diagnostische Information jeweils einen positiven Optionspreis besitzt. 


$$
\left.\frac{\partial P\left(z_{j 1} \mid K_{1}\right)}{\partial P\left(z_{j 2} \mid K_{2}\right)}\right|_{\Phi_{B}}=-\frac{\left.P\left(K_{2}\right) \cdot \mid U\left(a_{2}, K_{2}\right)-U\left(a_{1}, K_{2}\right)\right\}}{P\left(K_{1}\right) \cdot\left|U\left(a_{1}, K_{1}\right)-U\left(a_{2}, K_{1}\right)\right|} .
$$

Bei gegebener Ausgangslage und konstanter Struktur der Entscheidungssituation nimmt dieser Preis einen festen Wert an. Gleichung (4.88) gibt daher zugleich die Steigung der Indifferenzkurven des Patienten in dem durch die diagnostischen Kennziffern definierten Raum an. Diese Indifferenzkurven sind unter den getroffenen Annahmen linear. Wie Abbildung 26 veranschaulicht, verlaufen die zu verschiedenen Nutzenniveaus gehörigen Indifferenzkurven parallel.

Abb. 26: Die Präferenzen des Patienten für diagnostische Kennziffern

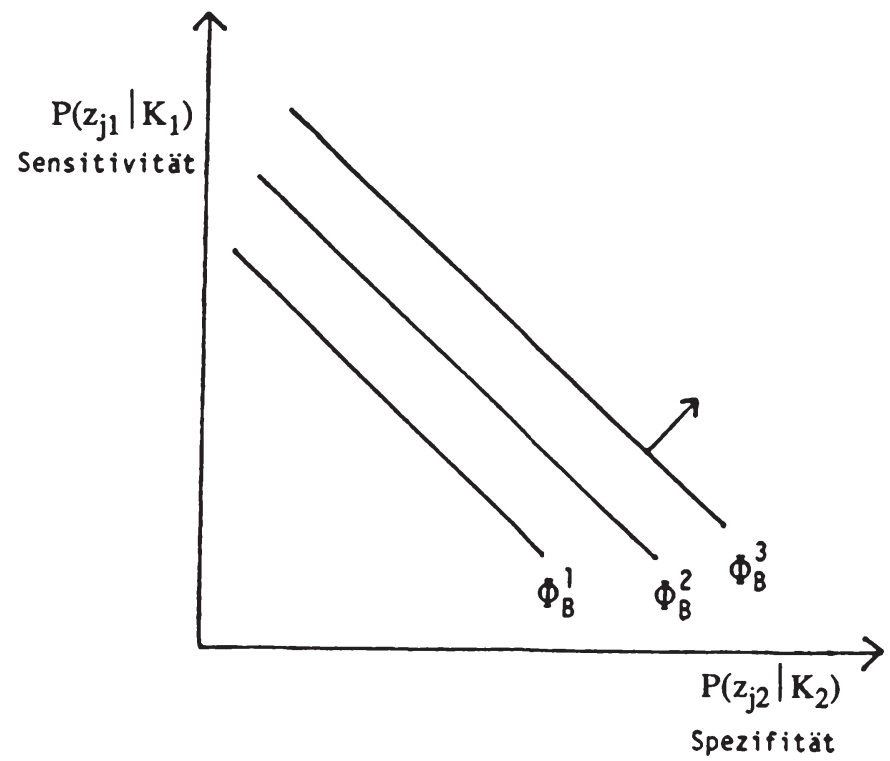

Die beiden diagnostischen Maßnahmen werden im folgenden in zwei hinsichtlich ihrer Struktur verschiedenen medizinischen Entscheidungssituationen analysiert. Zunächst können $T_{1}$ und $T_{2}$ als Vorsorgeuntersuchung zum Einsatz gelangen. Dabei indiziert $\mathrm{K}_{1}$ die durch die Vorsorgeuntersuchung zu erkennende Krankheit, während $\mathrm{K}_{2}$ die Abwesenheit dieser Krankheit bezeichnet. Bei der Aktion $\mathrm{a}_{1}$ handelt es sich 
um eine Kombination aus einer aufwendigen Folgeuntersuchung, die mit Sicherheit den tatsächlichen Krankheitszustand des Patienten bestimmt, und - falls $K_{1}$ vorliegt einer entsprechenden Behandlung, während $a_{2}$ für die Alternative "Nichtstun" steht. In dieser Situation kommt es wesentlich darauf an, $\mathrm{K}_{1}$ mit hoher Wahrscheinlichkeit zu erkennen, da ein falsch negatives Testergebnis die erforderliche Behandlung verzögern wird. Falls eine derartige Verzögerung den Erfolg der Behandlung drastisch beeinflußt, wird der dazugehörige Nutzenwert $u\left[g\left(a_{2}, K_{1}\right)\right]$ sehr niedrig im Vergleich $\mathrm{zu}$ den anderen Nutzenwerten ausfallen ${ }^{188}$. Wie Gleichung (4.88) zeigt, ergibt sich unter dieser Voraussetzung eine betragsmäßig große Steigung der Indifferenzkurven des Patienten. Es kann durchaus vorkommen, daß - wie in Abbildung 27 dargestellt in dieser medizinischen Entscheidungssituation eine Präferenz für die zweite diagnostische Maßnahme besteht.

Abb. 27: Die Präferenzen des Patienten für diagnostische Maßnahmen in der Entscheidungssituation des "screening"

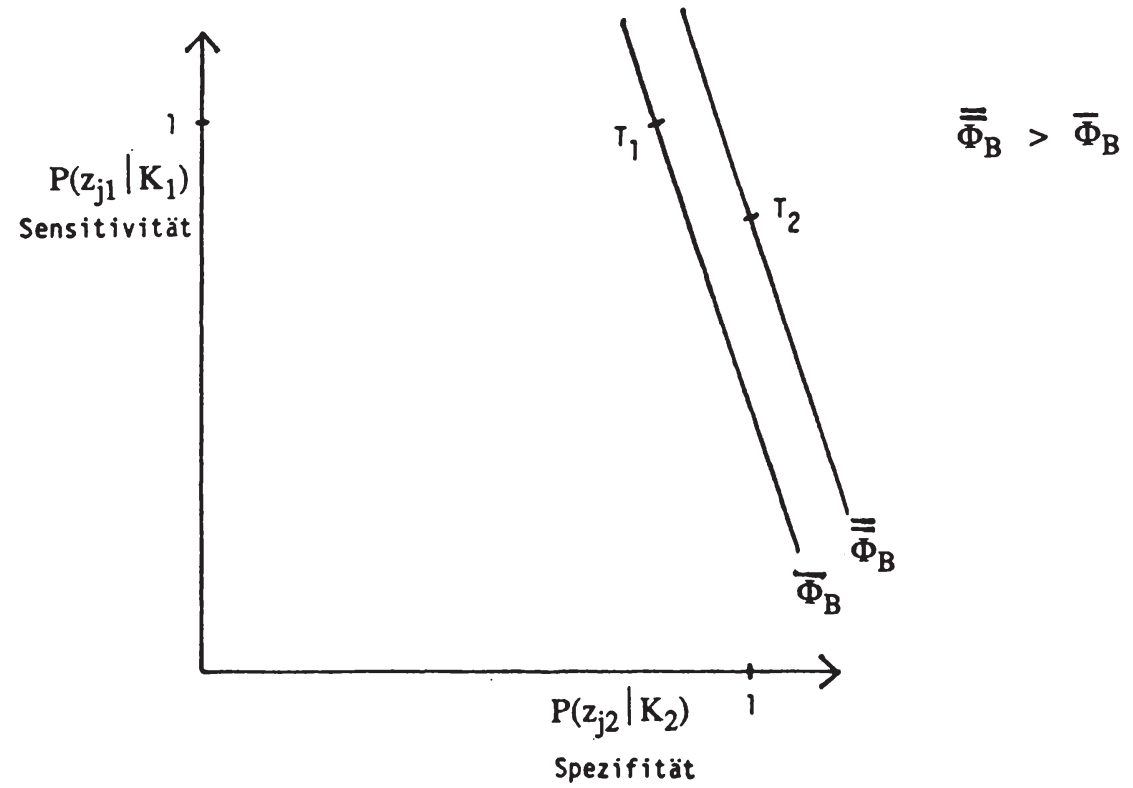

188: Dies impliziert unter anderem, da $B$ ein falsch positives Testergebnis keine allzu ungünstige Nutzenbewertung erfährt. 
In der zweiten Entscheidungssituation zieht der Arzt neben dem wie oben definierten Krankheitszustand $\mathrm{K}_{1}$ mit $\mathrm{K}_{2}$ dieses Mal eine andere Krankheit in Betracht. Die beiden diagnostischen Maßnahmen werden nun zum Zweck der Differentialdiagnose eingesetzt. Dem Arzt steht mit $a_{1}$ eine Operation zur Verfügung, die den Gesundheitszustand des Patienten verbessert, sofern er die Krankheit $K_{1}$ besitzt. Gilt für den Patienten jedoch $\mathrm{K}_{2}$, so wirkt sich die Aktion $\mathrm{a}_{1}$ für ihn äußerst nachteilig aus. Die Aktion $a_{2}$ repräsentiert eine auf den zweiten Krankheitszustand zugeschnittene Behandlung, die allerdings auch keine außergewöhnlich ungünstigen Folgen nach sich zieht, falls der Patient $\mathrm{K}_{1}$ besitzt.

Unter diesen Voraussetzungen weist ein falsch positives Testergebnis einen besonders niedrigen Nutzenwert auf und es kommt daher für den Arzt darauf an, mit möglichst hoher Wahrscheinlichkeit den Krankheitszustand $\mathrm{K}_{1}$ auszuschließen. Aus Gleichung (4.88) ergibt sich die Steigung der Indifferenzkurven des Patienten, die in dieser Entscheidungssituation dem Betrag nach niedrig ausfällt. Entsprechend wird der Patient unter diesen Umständen den Einsatz der ersten diagnostischen Maßnahme bevorzugen, wie Abbildung 28 belegt.

Das anhand der Abbildungen 27 und 28 graphisch veranschaulichte Beispiel zeigt, in welcher Weise das Ergebnis eines Vergleichs diagnostischer Maßnahmen von der Struktur der medizinischen Entscheidungssituation abhängt ${ }^{189}$.

Ein analoger Zusammenhang liegt vor, wenn man die Präferenzen zweier Patienten für diagnostische Maßnahmen in einer gegebenen medizinischen Entscheidungssituation analysiert. In diesem Fall bleiben sowohl die Menge A der ärztlichen Aktionen als auch die möglichen Krankheitszustände unverändert. Ein Einfluß auf die Struktur der medizinischen Entscheidungssituation kommt unter dieser Voraussetzung nur aufgrund der Unterschiede in den Präferenzen der beiden Patienten zustande. Verwendet man die in dem soeben diskutierten Beispiel genannten Nutzenwerte, dann repräsentiert etwa Abbildung 27 die Präferenzen des ersten und entsprechend Abbildung 28 die Präferenzen des zweiten Patienten. Weiterhin stehen dem Arzt zwei Diagnosetests $T_{1}$ und $T_{2}$ zur Verfügung, deren Kennziffern gerade mit

189: Diesem Zusammenhang geht Doessel in einer neueren Arbeit nach; vgl. DOESSEL, D.P. (1986). Im Gegensatz zu dem hier verwendeten Ansatz führt Doessel die Präferenzen des Patienten für diagnostische Maßnahmen ad hoc ein, ohne sie über die möglichen Aktionen des Arztes und den daraus beim Patienten entstehenden Folgen zu begründen. 
Abb. 28: Die Präferenzen des Patienten für diagnostische Maßnahmen in der differentialdiagnostischen Entscheidungssituation

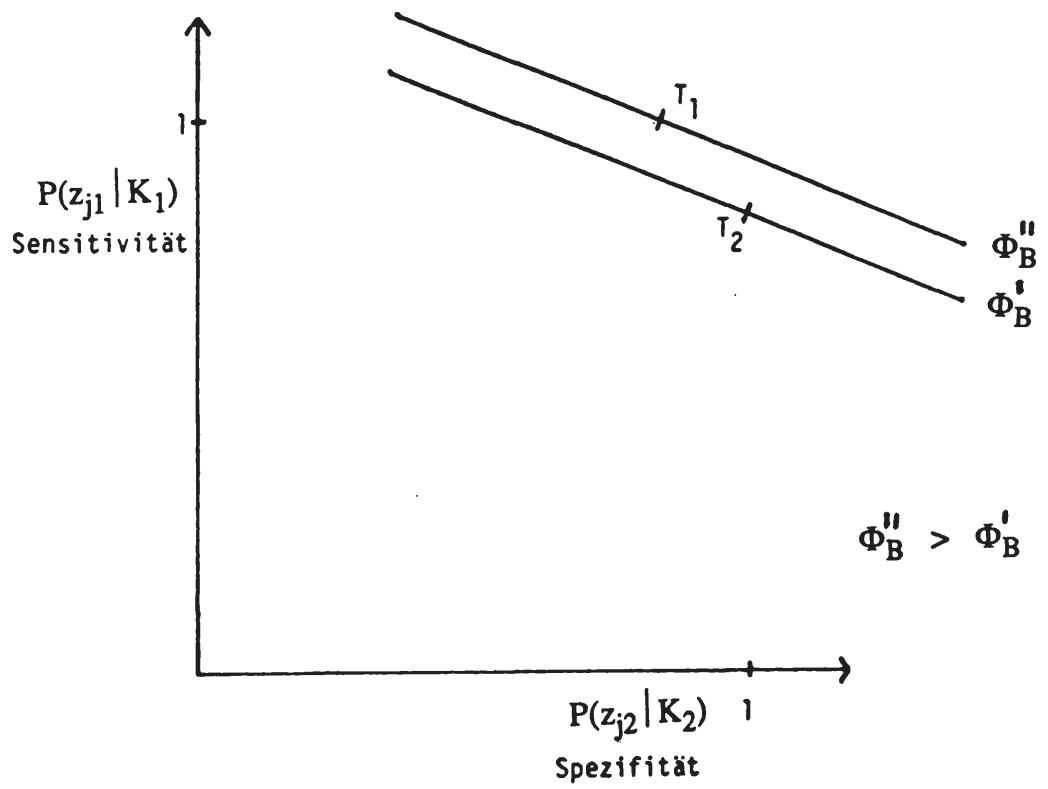

den Kennziffern der im obigen Beispiel angeführten diagnostischen Maßnahmen übereinstimmen. Dann unterscheiden sich die individuellen Präferenzen offenbar in ausreichendem $\mathrm{Maße}$, um die beiden Patienten in derselben medizinischen Entscheidungssituation jeweils den Einsatz einer anderen diagnostischen Maßnahme bevorzugen zu lassen.

Die Einflüsse einzelner Komponenten der medizinischen Entscheịdungssituation auf den differentiellen Nutzeneffekt der diagnostischen Information und die individuellen Präferenzen für diagnostische Maßnahmen sind in diesem Abschnitt anhand besonders einfacher Beispiele diskutiert worden. Indessen liegen die oben herausgearbeiteten Abhängigkeiten ebenso in komplexeren Entscheidungssituationen vor. Daher behalten die hier abgeleiteten Aussagen in diesem allgemeineren Rahmen ihre Gültigkeit. 


\subsubsection{Der Informationsgrad diagnostischer Maßnahmen}

In einer medizinischen Entscheidungssituation existiert unter den Voraussetzungen des Modells immer eine diagnostische Maßnahme, deren Einsatz die Präferenzen des Patienten maximiert. Um dies zu zeigen, definiert man zunächst:

(4.89) $\Phi_{B}\left(T_{j} ; u, P\right)=\max _{d_{j} \in D_{j}} \sum_{i=1}^{n} P\left(K_{i}\right) \cdot \sum_{h=1}^{k_{j}} P\left(z_{j h} \mid K_{i}\right) \cdot u\left\{g\left[d_{j}\left(z_{j h}\right), K_{i}\right]\right\}$. Die Größe $\Phi_{B}\left(T_{j} ;\right.$ u,P) gibt das maximale Nutzenniveau an, das der Patient erreichen kann, wenn der Arzt die diagnostische Maßnahme $T_{j}$ einsetzt. Wie aus Beziehung (4.89) hervorgeht, hängt dieses Nutzenniveau einerseits von der von NeumannMorgenstern Nutzenfunktion u des Patienten, andererseits von den prioren Wahrscheinlichkeiten der vom Arzt in Betracht gezogenen Krankheitszustände $a b^{190}$. Um diese Abhängigkeit von den Parametern der untersuchten Entscheidungssituation explizit zu berücksichtigen, sind u und $\mathrm{P}$ als zusätzliche Argumente von $\Phi_{\mathrm{B}}$ aufgeführt. $\mathrm{Da}$ dem Aṛzt annahmegemäß nur eine endliche Anzahl diagnostischer Informationsinstrumente zur Verfügung steht, wählt er eine $M a ß n a h m e T_{j^{*}}$, für die gilt ${ }^{191}$ :

$$
\Phi_{\mathrm{B}}\left(\mathrm{T}_{\left.\mathrm{j}^{*} ; \mathrm{u}, \mathrm{P}\right)} \geq \Phi_{\mathrm{B}}\left(\mathrm{T}_{\mathrm{j}} ; \mathrm{u}, \mathrm{P}\right) ; \forall \mathrm{j} \neq \mathrm{j}^{*}\right.
$$

Wie im vorhergehenden Abschnitt 4.4.2 erläutert, kann allgemein nicht ausgeschlossen werden, daß der Arzt bei einer anderen Ausgangslage eine von $\mathrm{T}_{\mathrm{j}}$ verschiedene diagnostische Maßnahme einzusetzen hat. Ebenso beeinflussen die Präferenzen des Patienten die vom Arzt auszuwählende diagnostische Information. In einer ansonsten unveränderten medizinischen Entscheidungssituation kann es daher für den Arzt im Sinne des Modells optimal sein, bei verschiedenen Patienten unterschiedliche diagnostische Maßnahmen zu verwenden. Damit erweist sich die ärztliche Entscheidung als nicht robust in Bezug auf Veränderungen der beiden Komponenten u und P. Aus diesem Grund erfordern selbst geringfügige Modifikationen - beispielsweise der Ausgangslage - grundsätzlich erneut eine vollständige Analyse des ärztlichen Entscheidungsproblems. Andererseits handelt es sich sowohl bei den individuellen Nutzenwerten der möglichen Konsequenzenvektoren als auch bei den prioren Wahrscheinlichkeiten der Krankheitszustände um Größen, deren Ermittlung in praxi

190: Die Aktionsmenge A sei konstant.

191: Diese Maßnahme braucht nicht eindeutig bestimmt zu sein. 
fehlerbehaftet sein kann. Derartige Fehler können somit das Ergebnis eines Vergleichs diagnostischer Maßnahmen beeinflussen.

Indessen gelingt es in günstigen Fällen, den Vergleich zweier diagnostischer Maßnahmen unabhängig von den Komponenten $\mathrm{u}$ und $\mathrm{P}$ einer speziellen medizinischen Entscheidungssituation durchzuführen. Ein solcher Vergleich ist nur möglich, wenn eine diagnostische Maßnahme bei sämtlichen zulässigen Besetzungen der Parameter $\mathrm{u}$ und $\mathrm{P}$ im Hinblick auf die Nutzenposition des Patienten nicht schlechter abschneidet als die alternative Maßnahme ${ }^{192}$. Im Einklang mit dieser Beschreibung besitzt eine diagnostische Maßnahme $T_{1}$ einen höheren Informationsgrad als eine andere diagnostische Maßnahme $\mathrm{T}_{2}$, falls gilt ${ }^{193}$ :

$$
\Phi_{\mathrm{B}}\left(\mathrm{T}_{1} ; \mathrm{u}, \mathrm{P}\right) \geq \Phi_{\mathrm{B}}\left(\mathrm{T}_{2} ; \mathrm{u}, \mathrm{P}\right) ; \quad \forall \mathrm{u}, \forall \mathrm{P}
$$

Wenn die Beziehung (4.91) erfüllt ist, schreibt man kürzer ${ }^{194}$ :

$$
\mathrm{T}_{1}>\mathrm{T}_{2}
$$

Aus den oben angeführten Überlegungen ergibt sich, daß die auf dem Informationsgrad beruhende binäre Relation " $>$ " im allgemeinen nur eine partielle Ordnung auf der Menge aller zur Verfügung stehenden diagnostischen Maßnahmen induziert. Dies überrascht insofern nicht, als die in Beziehung (4.91) formulierte Bedingung eine starke Aussage über die Eigenschaften der beiden diagnostischen Maßnahmen $T_{1}$ und $\mathrm{T}_{2}$ macht.

192: $\mathrm{Zu}$ beachten ist, daß die Parameter $\mathrm{u}$ und $\mathrm{P}$ jeweils Funktionen darstellen. Unter den Voraussetzungen des Abschnitts 4.2.2. ist jede von Neumann-Morgenstern Nutzenfunktion u des Patienten stetig und damit zulässig im Sinne der weiteren Analyse.

193: Die Pionierarbeiten zur Untersuchung des Informationsgrads stammen von Blackwell, der allerdings den Vergleich von Zufallsexperimenten analysierte; vgl. BLACKWELL, D. (1951), (1953). Die weiteren Ausführungen beziehen sich wesentlich auf MARSCHAK, J./MIYASAWA, K. (1968), die eine Reihe wichtiger Charakterisierungen ableiten; vgl. dazu auch FIRCHAU, V. (1980), S. 40ff. und LAFFONT, J.J. (1989), S. $62 \mathrm{ff}$.

194: Falls eine diagnostische Maßnahme $T_{1}$ einen höheren Informationsgrad als eine andere Maßnahme $T_{2}$ besitzt, bedeutet dies jedoch nicht, daß der Arzt bei Einsatz von $T_{1}$ niemals eine aus der Sicht des Patienten schlechtere Entscheidung treffen kann. Die in $T_{1}>T_{2}$ enthaltene Aussage gilt nur, wenn der Arzt - wie im Modell angenommen - jeweils eine optimale Entscheidungsfunktion verwendet. 
Um zu erkennen, ob sich zwei diagnostische Maßnahmen anhand der Relation ">" vergleichen lassen, werden im folgenden zwei charakterisierende Bedingungen diskutiert. Die erste Bedingung nimmt Bezug auf die Matrizen $P_{1}$ und $P_{2}$, in denen jeweils die bedingten Wahrscheinlichkeiten der Testergebnisse stehen, welche die diagnostischen Maßnahmen $T_{1}$ und $T_{2}$ kennzeichnen. Es gilt:

$$
\mathrm{T}_{1}>\mathrm{T}_{2} \Leftrightarrow \mathrm{P}_{2}=\mathrm{B} \cdot \mathrm{P}_{1}
$$

wobei B eine Markoff-Matrix der Ordnung $k_{2} \times k_{1}$ repräsentiert, deren Spaltensummen jeweils Eins betragen ${ }^{195}$. Da es sich um eine Äquivalenzaussage handelt, reicht die Kenntnis der Matrizen $P_{1}$ und $P_{2}$ aus, um zu entscheiden, ob die dazugehörigen diagnostischen Maßnahmen in Bezug auf ihren Informationsgrad vergleichbar sind. Falls eine derartige Matrix B existiert, läßt sich auch ohne weitere Information über die speziell betrachtete medizinische Entscheidungssituation feststellen, daß der Patient auf keinen Fall den Einsatz der diagnostischen Maßnahme $T_{2}$ präferieren wird.

Eine zweite, ebenfalls die Relation " $>$ " charakterisierende Bedingung bezieht sich insofern auf eine (allerdings beliebig zu wählende) Ausgangslage, als die posterioren Wahrscheinlichkeiten der Krankheitszustände (gegeben die einzelnen Testergebnisse) der beiden zu vergleichenden diagnostischen Maßnahmen bekannt sein müssen. Bezeichne $\Pi_{\mathrm{j}}$ die Matrix der Ordnung $\mathrm{nx} \mathrm{k}_{\mathrm{j}}$, in deren h-ter Spalte die Wahrscheinlichkeiten der in Betracht kommenden Krankheitszustände gegeben das Testergebnis $z_{\text {jh }}$ stehen, dann gilt:

$$
\mathrm{T}_{1}>\mathrm{T}_{2} \Leftrightarrow \Pi_{2}=\Pi_{1} \cdot \mathrm{C}
$$

wobei $\mathrm{C}$ eine Markoff-Matrix der Ordnung $\mathrm{k}_{1} \times \mathrm{k}_{2}$ repräsentiert, deren Spalten

195: Vgl. MARSCHAK, J/MIYASAWA, K. (1968), S. 217 und $220 f f$. 
summen jeweils Eins betragen ${ }^{196}$. Dieser Bedingung zufolge muß jeder Spaltenvektor der Matrix $\Pi_{2}$ als konvexe Kombination der Spaltenvektoren von $\Pi_{1}$ gebildet werden können. Die diagnostische Maßnahme $T_{1}$ besitzt demnach genau dann einen höheren Informationsgrad als $T_{2}$, wenn sich jeder bei $T_{2}$ mögliche Vektor posteriorer Wahrscheinlichkeiten als konvexe Kombination der posterioren Wahrscheinlichkeitsvektoren von $T_{1}$ darstellen läßt.

Falls lediglich drei Krankheitszustände in Betracht kommen, kann die in Abschnitt 4.4.2 verwendete graphische Darstellung zur Veranschaulichung von Bedingung (4.94) dienen. Um die Darstellung übersichtlich zu halten, wird ohne Beschränkung der Allgemeinheit die Anzahl der Testergebnisse, die bei den beiden zu vergleichenden diagnostischen Maßnahmen auftreten können, jeweils auf drei festgelegt. In der nachstehenden Abbildung 29 repräsentiert der Punkt $P_{0}$ eine priore Wahrscheinlichkeitsverteilung der drei Krankheitszustände und damit eine spezielle Ausgangslage. Die bei Einsatz der diagnostischen Maßnahme $T_{1}$ möglichen posterioren Wahrscheinlichkeitsvektoren werden durch Kreise dargestellt, während die entsprechenden Vektoren der zweiten Maßnahme durch Kreuze lokalisiert sind.

In der Abbildung stellen die Repräsentanten der posterioren Wahrscheinlichkeitsvektoren von $T_{1}$ die Eckpunkte eines Dreiecks dar, innnerhalb dessen sämtliche posterioren Wahrscheinlichkeitsvektoren der zweiten diagnostischen Maßnahme liegen. Damit ist die in Beziehung (4.94) angegebene Bedingung erfüllt und $\mathrm{T}_{1}$ besitzt einen höheren Informationsgrad als $\mathrm{T}_{2}$. Die Untersuchung einer speziellen medizinischen Entscheidungssituation reicht somit aus, um zu erkennen, daß der $\mathrm{Pa}$ tient unabhängig von seinen Präferenzen auch in anderen Ausgangslagen den Einsatz der diagnostischen Maßnahme $T_{1}$ immer (zumindest schwach) präferiert.

196: Vgl. MARSCHAK, J./MIYASAWA, K. (1968), S. 222f. Im übrigen muß aus Konsistenzgründen noch folgende Bedingung erfüllt sein:

$$
P\left(z_{1}\right)=C \cdot P\left(z_{2}\right)
$$

wobei $\mathbf{P}\left(\mathbf{z}_{\mathbf{j}}\right.$. denjenigen Spaltenvektor bezeichnet, der die Wahrscheinlichkeiten der Testergebnisse von $\mathrm{T}_{\mathrm{j}}$ enthält. 
Abb. 29: Vergleich des Informationsgrads zweier Diagnosetests

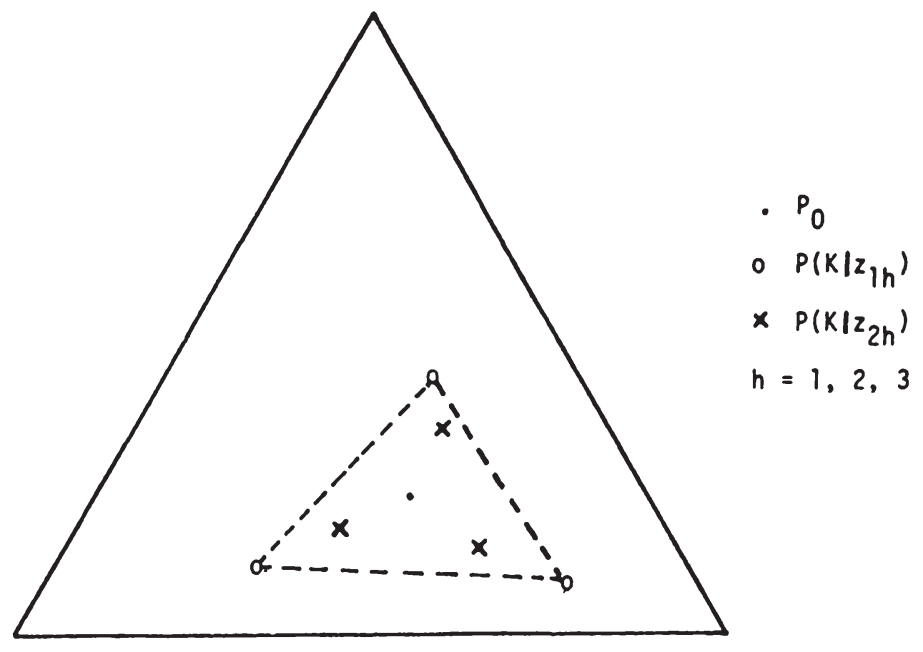

Aus der Abbildung ergeben sich weitere Eigenschaften der Ordnung, welche die Relation des Informationsgrads allgemein auf der Menge aller möglichen diagnostischen Maßnahmen induziert. Bei einer perfekten diagnostischen Maßnahme erlaubt jedes Testergebnis den fehlerfreien Rückschluß auf den Krankheitszustand des Patienten. Entsprechend bilden die dazugehörigen posterioren Wahrscheinlichkeitsvektoren gerade die Eckpunkte des in Abb. 29 dargestellten gleich- 
seitigen Dreiecks ${ }^{197}$. Da dieses Dreieck zugleich die Menge aller möglichen Wahrscheinlichkeitsvektoren begrenzt, lassen sich die posterioren Wahrscheinlichkeitsvektoren einer beliebigen diagnostischen Maßnahme immer als konvexe Kombinationen der posterioren Wahrscheinlichkeitsvektoren einer perfekten diagnostischen Maßnahme darstellen. Eine perfekte diagnostische Maßnahme besitzt infolgedessen einen höheren Informationsgrad als jede andere diagnostische Maßnahme und bildet damit ein bezüglich der Relation " $>$ " größtes Element in der Menge aller Diagnosetests. Im Gegensatz dazu beeinflussen die Testergebnisse einer diagnostischen Maßnahme ohne Informationsleistung die prioren Wahrscheinlichkeiten der Krankheitszustände nicht. Daher besitzt jede andere diagnostische Maßnahme einen höheren Informationsgrad. Diese Eigenschaften sind unabhängig von den im graphischen Beispiel speziell verwendeten Voraussetzungen. Insgesamt erzeugt die durch den Informationsgrad definierte Relation eine partielle Ordnung auf der Menge aller diagnostischen Maßnahmen, die mit der perfekten diagnostischen Maßnahme ein größtes Element und mit der diagnostischen Maßnahme ohne Informationsleistung ein kleinstes Element enthält.

Was geschieht mit dem Informationsgrad einer diagnostischen Maßnahme $T_{j}$, wenn man einzelne Testergebnisse zusammenfaßt? Mit einer derartigen Zusammenfassung entscheidet man sich dafür, anstelle der "ursprünglichen" Testergebnisse $z_{j h}$ stärker aggregierte Resultate $y_{j h} z u$ beobachten und zur Grundlage des weiteren Handelns zu machen. Formal repräsentiert eine Zusammenfassung eine Abbildung $Y_{j}$, für die gilt:

$$
Y_{j}: Z_{j} \rightarrow\left\{y_{j 1}, \ldots, y_{j k} k_{j}\right\}
$$

wobei die Anzahl $\mathrm{k}_{\mathrm{j}^{\prime}}$ der aggregierten diagnostischen Resultate im allgemeinen klei-

197: Dies gilt für alle Ausgangslagen, in denen sämtliche Krankheitszustände eine positive (priore) Wahrscheinlichkeit besitzen. 
ner als die Anzahl $k_{j}$ der ursprünglichen Testergebnisse ausfällt ${ }^{198}$. Eine extreme Form der Zusammenfassung von diagnostischen Testergebnissen liegt bei binarisierten Tests vor, da eine vollständige Aggregation zu einem einzigen Testergebnis keinen Sinn ergibt ${ }^{199}$. Allgemein stellt jede Zusammenfassung in konzeptioneller Hinsicht eine "neue" diagnostische Maßnahme $T_{j}$ dar, die allerdings aufgrund der Existenz der oben angegebenen Abbildung in enger Verbindung $z u T_{j}$ steht.

Die Vermutung liegt nahe, daß der Arzt über eine Zusammenfassung diagnostischer Resultate dem Patienten zumindest keine Verbesserung seiner Nutzenposition verschaffen kann. Tatsächlich besitzt eine diagnostische Maßnahme $T_{j}$ immer einen höheren Informationsgrad als jede andere Maßnahme $T_{j}$, die sich aus einer Zusammenfassung ihrer Testergebnisse $z_{\text {jh }}$ ergibt. Es gilt also immer ${ }^{200}$ :

$$
T_{j}>T_{j^{\prime}} \cdot
$$

Speziell impliziert diese Aussage für diagnostische Maßnahmen mit mehr als zwei Testergebnissen, daß der Arzt dem Patienten in einer medizinischen Entscheidungssituation niemals einen größeren differentiellen Nutzeneffekt verschaffen kann, wenn er lediglich positive und negative Resultate unterscheidet. Im Normalfall führt die Binarisierung der Menge der möglichen Testergebnisse zu einer Reduktion des beim Patienten auftretenden Nutzeneffekts ${ }^{201}$.

198: Falls $\mathrm{k}_{\mathrm{j}}=\mathrm{k}_{\mathrm{j}}$ gilt, hat sich lediglich die Numerierung der Testergebnisse $\mathrm{z}_{\mathrm{jh}}$ durch die $\mathrm{Zu}$ sammenfassung $Y_{j}$ - die in diesem Spezialfall bijektiv ist - geändert.

In jedem Fall ist die durch die "ursprünglichen" Testergebnisse gebildete Partition der Menge $Z^{j}$ feiner als jene, die sich aufgrund der Urbildmengen $Y_{j}^{-1}\left(y_{j h^{\prime}}\right)$ ergibt. Zum Begriff der Verfeinerung von Partitionen bzw. der Subpartition vgl. MARSCHAK, J/MIYASAWA, K. (1968), S. 208f.; LaVALLE, I.H. (1968), S. $252 \mathrm{f}$.

199: Zu binarisierten Tests vgl. Abschnitt 3.1. Eine diagnostische Maßnahme, bei der lediglich ein Testergebnis möglich ist, kann selbstverständlich keine Informationsleistung erbringen.

200: Diese Aussage folgt aus den Resultaten der Analyse von Marschak und Miyasawa; vgl. MARSCHAK, J.MIYASAWA, K. (1968), S. $219 \mathrm{ff}$.

201: Sämtliche Aussagen dieses Abschnitts beziehen sich ausschließlich auf den Nutzeneffekt der diagnostischen Information, ohne deren Kostenseite zu berücksichtigen. Insofern bedeutet beispielsweise die in Beziehung (4.96) angegebene Eigenschaft noch nicht, daß eine $\mathrm{Zu}$ sammenfassung diagnostischer Testergebnisse in ökonomischer Hinsicht unvorteilhaft sein muß. 


\subsection{Die Berücksichtigung intangibler Kosteneffekte}

Die bisher besprochenen Effekte, die eine diagnostische Maßnahme im Vergleich zur Situation ohne weitere Diagnostik beim Patienten erzeugen kann, betrafen ausschließlich die Nutzenseite. Dieser Sachverhalt stellt eine Eigenschaft des Modells dar, das eine Verschlechterung der medizinischen Entscheidungssituation im Falle der Informationsbeschaffung ausschlie $\mathrm{Bt}^{202}$. In dieser Hinsicht stimmt das hier vorgestellte Modell mit anderen Modellen überein, die in der entscheidungstheoretischen Literatur zur Informationsbewertung herangezogen werden. Indessen entspricht diese Eigenschaft in medizinischen Entscheidungssituationen häufig nicht der Realität. Der Einsatz einer diagnostischen Maßnahme kann für den Patienten auch mit negativen gesundheitlichen Konsequenzen einhergehen ${ }^{203}$. Konzeptionell handelt es sich dabei um einen intangiblen Kosteneffekt, den eine diagnostische Maßnahme im Vergleich zur Referenzsituation ohne weitere Diagnostik beim Patienten verursacht ${ }^{204}$. Dieser differentielle Kosteneffekt der diagnostischen Information überlagert die zuvor ausführlich erörterten differentiellen Nutzeneffekte. Im folgenden werden zwei grundsätzlich verschiedene Komponenten diskutiert, die das Spektrum möglicher differentieller Kosteneffekte weitgehend abdecken dürften.

Die erste Komponente umfaßt diejenigen negativen Gesundheitseffekte, die der Einsatz einer diagnostischen Maßnahme unmittelbar beim Patienten verursachen kann. Es handelt sich dabei um gesundheitliche Konsequenzen, die dem Patienten unabhängig von seinem Krankheitszustand entstehen. Aufgrund dieser Eigenschaft kann man sie als unspezifischen Kosteneffekt einer diagnostischen Maßnahme einstufen. Hierunter fallen zunächst Schmerzen oder sonstige Unannehmlichkeiten, mit denen der Einsatz etwa einer invasiven Diagnostik beim Patienten verbunden sein $k_{a n n}{ }^{205}$. Sofern eine diagnostische Maßnahme überhaupt derartige Kosten verursacht, stellen diese Effekte in ihrer Gesamtheit gerade diejenige Belastung der Gesundheit des Patienten dar, die aufgrund der Beschaffung diagnostischer Infor-

202: Eine Verschlechterung der medizinischen Entscheidungssituation liegt vor, wenn eine optimale ärztliche Aktion im Vergleich zur Referenzsituation eine ungünstigere Bewertung erfährt.

203: Vgl. z.B. BARR, D.P. (1955), S. 1452f.

204: Von den Kosten der Informationsbeschaffung wird weiterhin abgesehen. Die Interpretation des kompensierenden Optionspreises diagnostischer Maßnahmen aus gesamtwirtschaftlicher Sicht ändert sich daher nicht, wenn die in diesem Abschnitt diskutierten differentiellen Kosteneffekte berücksichtigt werden; vgl. dazu Abschnitt 4.3.4.

205: Vgl. dazu BETAQUE, N.E./GORRY, G.A. (1971), S. B 423; GINSBERG, A.S. (1972), S. 219; ABRAMS, H.L. et al. (1982), S. 125; JENNETT, B. (1986), S. $8 \mathrm{ff}$. 
mation mit Sicherheit anfällt. Insgesamt lassen sich die genannten gesundheitlichen Konsequenzen damit als deterministischer unspezifischer Kosteneffekt einordnen.

Daneben besteht die Möglichkeit, daß die Anwendung einer diagnostischen Maßnahme für den Patienten ein Risiko birgt, das in der Situation ohne weitere Diagnostik nicht existiert. Ein typisches Beispiel stellt das Mortalitätsrisiko dar, das bei manchen Untersuchungen auftritt ${ }^{206}$. Allgemein rechnen hierzu sämtliche möglichen Komplikationen, die auf den Einsatz einer diagnostischen Maßnahme zurückgehen ${ }^{207}$. Auch diese Effekte fallen unabhängig vom Krankheitszustand des Patienten an und sind daher grundsätzlich der oben definierten Kostenkomponente diagnostischer Maßnahmen zuzurechnen. Aufgrund ihrer Zufallsabhängigkeit handelt es sich jedoch jeweils um einen stochastischen unspezifischen Kosteneffekt, der bei der Beschaffung diagnostischer Information auftreten kann.

Die mögliche Existenz unspezifischer Kosteneffekte hat Auswirkungen auf die in den Abschnitten 4.3 bzw. 4.4 formulierten Aussagen über den Optionspreis bzw. den differentiellen Nutzeneffekt diagnostischer Maßnahmen. Dies gilt unabhängig davon, ob dieser Kosteneffekt in der deterministischen oder in der stochastischen Variante anfällt. In einer medizinischen Entscheidungssituation ist der differentielle Nutzeneffekt einer diagnostischen Maßnahme mit ihrem differentiellen Kosteneffekt zu verrechnen, um den Netto-Effekt dieser Wirkungen auf die Wohlfahrt des Patienten zu ermitteln. Falls eine diagnostische Maßnahme beim Patienten einen unspezifischen Kosteneffekt verursacht, gilt die allgemeine Nichtnegativität des zusätzlichen Nutzens bzw. des Optionspreises der diagnostischen Information nicht mehr. Diese Aussage läßt sich anhand eines einfachen Beispiels belegen. Dazu betrachtet man eine medizinische Entscheidungssituation, in der eine diagnostische Maßnahme das ärztliche Handeln nicht beeinflußt und daher dem Patienten keinen differentiellen Nutzen stiftet. Wenn der Einsatz dieser Maßnahme jedoch mit einem unspezifischen Kosteneffekt einhergeht, stellt die diagnostische Information den Patienten insgesamt schlechter als in der Situation ohne weitere Diagnostik ${ }^{208}$. Daraus ergibt sich unmittelbar ein negativer Optionspreis der diagnostischen Information.

206: Vgl. z.B. GINSBERG, A.S./OFFENSEND, F.L. (1968), S. 355; WEINSTEIN, M.C. et al. (1980), S. 142ff.

207: Vgl. PAUKER, S.G./KASSIRER, J.P. (1978), S. 324ff. sowie GINSBERG, A.S. (1972), S. 219.

208: Anschütz stuft daher den Einsatz einer "diagnostische(n) Invasivmaßnahme ohne Therapiekonsequenz" als "primär unärztliche Handlung" ein; vgl. ANSCHÜTZ, F. (1988). 
Im Gegensatz dazu umfaßt die zweite Kostenkomponente jene negativen Gesundheitseffekte, die eine diagnostische Maßnahme beim Patienten in Abhängigkeit von seinem Krankheitszustand verursachen kann. Aufgrund dieser Eigenschaft handelt es sich um spezifische Kosteneffekte, die im Vergleich zur Situation ohne weitere Diagnostik zusätzlich anfallen. Ein derartiger Kosteneffekt kann auftreten, wenn einerseits die Beschaffung der diagnostischen Information verhältnismäßig viel Zeit erfordert und andererseits eine Verzögerung des ärztlichen Handelns die beim Patienten erreichbaren Gesundheitseffekte mindert ${ }^{209}$. In ihrem Umfang werden diese negativen Wirkungen wesentlich von der Dynamik des Krankheitsprozesses und damit vom Krankheitszustand des Patienten beeinflußt. Das Ausmaß eines spezifischen Kosteneffekts hängt deshalb von der jeweils vorliegenden medizinischen Entscheidungssituation $a^{210}$. Grundsätzlich impliziert die Existenz dieser Kostenkomponente, daß die Konsequenzen einer ärztlichen Aktion $a_{f}$ bei gegebenem Krankheitszustand $\mathrm{K}_{\mathrm{i}}$ mit der Zeit variieren, da die beim Patienten auftretenden gesundheitlichen Folgen durch den Zeitpunkt der Aktionswahl bestimmt werden. Indessen dürfte dieser zeitliche Einfluß nur dann eine Rolle spielen, wenn die in Bezug auf den tatsächlichen Krankheitszustand $\mathrm{K}_{\mathrm{i}}$ des Patienten günstigste Aktion $\mathrm{a}^{\prime}\left(\mathrm{K}_{\mathrm{i}}\right)$ betrachtet wird $^{211}$. Entscheidet sich der Arzt dagegen für eine andere Aktion, wird der Zeitpunkt dieser im Hinblick auf den Krankheitszustand des Patienten "falschen" Aktionswahl kaum bei den gesundheitlichen Konsequenzen zu Buche schlagen.

Allerdings braucht ein spezifischer Kosteneffekt diagnostischer Maßnahmen im Vergleich zur Situation ohne weitere Diagnostik auch dann nicht aufzutreten, wenn die beiden oben angegebenen Existenzbedingungen erfüllt sind. Entschließt sich der Arzt in einer medizinischen Entscheidungssituation dazu, weitere Diagnostik zu be-

209: Dieser Zeitbedarf kann bei Parameterbestimmungen, die der niedergelassene Arzt in einer Laborgemeinschaft durchführen läßt, mehrere Tage betragen; vgl. KOSSOW, K.-D. (1986), S. 115. Eine ähnliche Aussage gilt für die Keimbestimmung, sofern diese manuell erfolgt; vgl. GAVIN, J.C./MOHNSEN, M.E. (1985).

210: Ein anschauliches Beispiel stellen Notfallsituationen dar, in denen die spezifische Kostenkomponente der medizinischen Diagnostik besonders ausgeprägt ist.

211: Eine bezüglich $K_{i}$ günstigste Aktion a' des Arztes liegt vor, wenn a' zu der Menge

$$
\arg \max \left\{\left.U\left(\mathrm{a}_{\mathrm{f}}, \mathrm{K}_{\mathrm{i}}\right)\right|_{\left.\mathrm{a}_{\mathrm{f}} \in \mathrm{A}\right\}}\right.
$$

gehört. Zur Vereinfachung wurde im Text angenommen, daß diese Aktion für jeden möglichen Krankheitszustand eindeutig bestimmt ist. 
treiben, so kann er dennoch sofort-d.h. ohne Kenntnis der angeforderten diagnostischen Information - eine Aktion ergreifen, die auf einer Verdachtsdiagnose aufbaut und damit im Lichte der bereits vorhandenen Information am günstigsten erscheint $^{212}$. In diesem Fall wählt der Arzt sozusagen unter Vorbehalt zunächst die in der Situation ohne weitere Diagnostik für den Patienten optimale Aktion, obwohl er sich für eine Informationsbeschaffung entschieden hat. Sobald die neue diagnostische Information vorliegt, überprüft der Arzt seine zuvor gewählte Aktion und revidiert diese, sofern das zusätzliche diagnostische Resultat eine derartige Entscheidung nahelegt.

Handelt der Arzt in der beschriebenen Weise, dann kann ein spezifischer Kosteneffekt beim Einsatz diagnostischer Maßnahmen nicht auftreten, da sich seine Aktionswahl im Vergleich zur Situation ohne weitere Diagnostik nicht verzögert. Unter dieser Voraussetzung beeinflußt der Zeitbedarf der diagnostischen Information lediglich den Umfang des damit verbundenen differentiellen Nutzeneffekts. Das Konzept des spezifischen Kosteneffekts besitzt daher vormehmlich eine Bedeutung für die vergleichende Analyse der Nutzenseite diagnostischer Maßnahmen. Ceteris paribus, d.h. insbesondere bei identischer Informationsleistung, verschafft diejenige diagnostische Maßnahme dem Patienten ein höheres Nutzenniveau, deren Information schneller eintrifft ${ }^{213}$. Die Existenz eines spezifischen Kosteneffekts bereichert demzufolge das Spektrum der möglichen Nutzeneffekte, die eine diagnostische Maßnahme gegenüber anderen diagnostischen Maßnahmen in einer medizinischen Entscheidungssituation aufweisen kann ${ }^{214}$.

212: Vgl. HORISBERGER, B. (1987).

213: Vgl. GÄFGEN, G. (1990), S. 315f. Der daraus abgeleitete Nutzeneffekt besitzt keineswegs nur theoretische Relevanz, wie die Analyse neuerer diagnostischer Maßnahmen eindrucksvoll bestätigt; vgl. BELSEY, R./BAER, D.SEWELL, D. (1986); WILLE, E. (1987) sowie WILLE, E. (1989).

214: Eine ähnliche Argumentation gilt für die zuvor diskutierten unspezifischen Kosteneffekte der diagnostischen Information. Ceteris paribus wird diejenige diagnostische Maßnahme dem $\mathrm{Pa}$ tienten einen höheren Nutzen stiften, deren unspezifische Kosten niedriger sind; vgl. dazu BANTA, H.D.McNEIL, B.J. (1978), S. 10; GILLESPIE, K.N. et al. (1986), S. 542; GUYATT, G./DRUMMOND, M.F. (1986), S. 559f. sowie GUDER, W.G./SCHLEICHER, E. (1990), S. 126. 
Walter Ried - 978-3-631-75573-0

Downloaded from PubFactory at 01/11/2019 03:26:15AM

via free access 


\section{ZUSAMMENFASSUNG UND AUSBLICK}

Während die Untersuchung einzelner therapeutischer Leistungen aus gesamtwirtschaftlicher Perspektive schon früh einen wesentlichen Bestandteil der Gesundheitsökonomik bildete, erfuhr die medizinische Diagnostik lange Zeit eine vergleichsweise geringe Beachtung. Dieser Tatbestand dürfte vornehmlich darauf zurückzuführen sein, daß eine Effizienzanalyse diagnostischer Leistungen spezifische Probleme aufwirft. Das unmittelbare Ergebnis einer diagnostischen Leistung besteht in der Information, die der Arzt über den Krankheitszustand des Patienten erhält. Der beim Patienten auftretende Nutzeneffekt hängt jedoch davon $a b$, ob und in welcher Weise die diagnostische Information das weitere ärztliche Handeln beeinflußt. Die vorliegende Arbeit behandelt sowohl die mit dem Einsatz einer diagnostischen Leistung verbundene Information als auch den daraus resultierenden Nutzeneffekt des Patienten. Die Untersuchung dieser beiden zentralen Effekte der medizinischen Diagnostik erfolgt in einem entscheidungstheoretischen Ansatz, der zusätzlich die Möglichkeit bietet, mit der Zahlungsbereitschaft des Patienten eine monetäre Bewertung des oben angesprochenen Nutzeneffekts abzuleiten.

Die medizinische Diagnostik umfaßt eine sehr große und heterogene Klasse von Handlungen, die der Arzt entweder selbst durchführt oder zumindest veranlaßt. Betrachtet man eine spezielle Krankheitsepisode und damit einen bestimmten Patienten, so wird das sequentielle Element bzw. der Prozeßcharakter der medizinischen Diagnostik deutlich. Im Normalfall verfügt der Arzt über die Möglichkeit, im Verlauf des diagnostischen Prozesses mehrere diagnosebezogene Handlungen einzusetzen. Dabei können sowohl der Umfang als auch die konkrete Abfolge der vom Arzt betriebenen Diagnostik davon abhängen, welche Testergebnisse sich jeweils einstellen.

Aufgrund dieser Komplexität beschränkt sich die vorliegende Arbeit darauf, mit den diagnostischen Maßnahmen die einzelnen Elemente des diagnostischen Prozesses zu untersuchen. Diese Maßnahmen werden als Handlungen definiert, durch die ein Arzt oder eine mit dessen Kompetenzen ausgestattete Person medizinische Informationen erhebt, die Rückschlüsse auf den Krankheitszustand des Patienten erlauben. Unter diesen Begriff fallen einerseits die klinisch-chemischen Tests und die Vorsorgeuntersuchungen (Maßnahmen des "screening"), andererseits gilt diese Zuordnung ebenso für andere diagnosebezogene Handlungen wie z.B. die Anamnese und die körperliche Untersuchung. 
Vor dem Einsatz einer diagnostischen Maßnahme kann der Arzt eine endliche Liste verschiedener Krankheitszustände angeben, die so beschaffen ist, daß sich der Patient in genau einem dieser Zustände befindet. In diesem Zusammenhang dient der Begriff des Krankheitszustands - im Unterschied zum weniger differenzierten Krankheitsbegriff - dazu, eine für die weitere Analyse ausreichend feine Unterteilung des Zustandsraums zu ermöglichen. Darüber hinaus wird davon ausgegangen, das diagnostische Vorwissen des Arztes lasse sich durch priore Wahrscheinlichkeiten beschreiben, die den einzelnen Krankheitszuständen zugeordnet sind. Aus der Perspektive der Entscheidungstheorie sind die möglichen Testergebnisse einer diagnostischen Maßnahme als Nachrichten anzusehen, die der Arzt für jeden in Betracht gezogenen Krankheitszustand des Patienten mit einer bestimmten Wahrscheinlichkeit erhalten kann. Diagnostische Maßnahmen repräsentieren demzufolge Informationsinstrumente, deren Charakterisierung jeweils durch eine Menge bedingter Wahrscheinlichkeitsverteilungen für ihre Testergebnisse erfolgt.

Während im allgemeinen Fall sowohl die Anzahl der zu berücksichtigenden Krankheitszustände des Patienten als auch die Menge der möglichen Testergebnisse einer diagnostischen Maßnahme lediglich einer Endlichkeitsbedingung zu genügen haben, nimmt die Analyse der diagnostischen Information zunächst Bezug auf eine wesentlich einfachere Konstellation. Im Hinblick auf die zugrundeliegende medizinische Entscheidungssituation werden nur zwei Krankheitszustände unterschieden, da die Frage im Vordergrund steht, ob sich der Patient in einem bestimmten, den Arzt besonders interessierenden, Krankheitszustand befindet oder nicht. Weiterhin kommen mit den binären bzw. den binarisierten Diagnosetests nur solche Maßnahmen in Betracht, die lediglich zwei verschiedene Testergebnisse - positive und negative Resultate - aufweisen. Unter diesen Voraussetzungen repräsentieren die Sensitivität und die Spezifität diejenigen bedingten Wahrscheinlichkeiten, die eine diagnostische Maßnahme vollständig charakterisieren.

$\mathrm{Da}$ in praxi die durch Sensitivität und Spezifität bezeichneten Wahrscheinlichkeiten im Normalfall unbekannt sind, benötigt man an ihrer Stelle Schätzungen, die sich aus den Verteilungen positiver und negativer Testergebnisse in ausgewählten Probandengruppen ergeben. Im Rahmen dieser Schätzaufgabe taucht das Problem der Validität auf, da mehrere Einflußfaktoren existieren, die für verzerrte Schätzungen sorgen können. Dies betrifft zum Beispiel die häufig nicht als repräsentativ anzusehende Auswahl der Probanden. Weiterhin hängen die Eigenschaften der Schätzungen für die Testparameter von dem Referenzverfahren $a b$, das als 
"Goldstandard" zur Beurteilung des interessierenden Diagnosetests dient. Falls das Referenzverfahren den Krankheitszustand eines Probanden nicht fehlerfrei anzeigt, weisen die üblicherweise verwendeten Schätzungen von Sensitivität und Spezifität einen "bias" auf. In diesem Zusammenhang entscheiden die bedingten Abhängigkeiten zwischen den Testergebnissen der beiden diagnostischen Maßnahmen über die Richtung und das Ausmaß dieses systematischen Fehlers.

Sensitivität und Spezifität werden insbesondere durch die Zuordnung beeinflußt, mit der die Resultate einer diagnostischen Maßnahme als positiv bzw. negativ klassifiziert werden. Unter der Voraussetzung einer jeweils optimalen Binarisierung läßt sich bei einer diagnostischen Maßnahme eine Kennziffer in der Regel nur erhöhen, indem man eine Einbuße bei der anderen Kennziffer in Kauf nimmt. Damit existiert ein "trade-off" zwischen den Kennziffern eines Diagnosetests, wobei der Preis etwa einer gegebenen Erhöhung der Sensitivität - gemessen durch die dazu erforderliche Verringerung der Spezifität - im allgemeinen umso höher ausfällt, je größer die Sensitivität in der Ausgangslage ist.

Der als ROC-Kurve bezeichnete Zusammenhang zwischen Sensitivität und Spezifität informiert umfassend über die Diskriminierungsfähigkeit einer diagnostischen Maßnahme und bietet sich aus diesem Grund für einen entsprechenden Vergleich binärer bzw. binarisierter Diagnosetests an. Bei bildgebenden Verfahren wie z.B. der Computertomographie wird indessen häufig der Zusammenhang zwischen den diagnostischen Kennziffern für einzelne Betrachter abgeleitet. In konzeptioneller Hinsicht braucht ein derartiger Zusammenhang nicht mit dem oben angesprochenen "trade-off" übereinzustimmen, da hier zusätzlich die Fähigkeit des Betrachters, diagnostische Resultate in geeigneter Weise als positiv bzw. negativ einzustufen, eine wichtige Rolle spielt.

Sensitivität und Spezifität reichen jedoch nicht aus, um die Veränderung der Informationslage anzugeben, die aus dem Einsatz einer diagnostischen Maßnahme in einer medizinischen Entscheidungssituation resultiert. Diese Veränderung ergibt sich aus dem Einfluß, den die Testergebnisse der betrachteten Maßnahme auf das diagnostische Vorwissen des Arztes besitzen. Genauer erhält man die auf eine bestimmte Entscheidungssituation bezogene Informationsleistung eines Diagnosetests, indem man die durch den positiven bzw. negativen prädiktiven Wert festgelegten posterioren Wahrscheinlichkeitsverteilungen im Falle eines positiven bzw. negativen Resultats den prioren Wahrscheinlichkeiten der Krankheitszustände gegenüber- 
Folgen, die sich für den Patienten im Vergleich zur Situation ohne weitere Diagnostik ergeben, über die Veränderung seiner Wohlfahrtsposition.

Ohne den Einsatz einer weiteren diagnostischen Maßnahme besteht für den Arzt lediglich die Möglichkeit, ein Element aus der Gesamtheit der ihm zur Verfügung stehenden Aktionen auszuwählen. Bei gegebenem Krankheitszustand führt jede dieser Aktionen zu eindeutig bestimmten Konsequenzen für den Patienten, die anhand einer Konsequenzenfunktion explizit dargestellt werden und im wesentlichen als Gesundheitseffekte anfallen. Diese Gesundheitseffekte repräsentieren die Ausprägungen derjenigen Gesundheitsattribute, die aus der Sicht des Patienten Relevanz besitzen. Die in einer Entscheidungssituation im Hinblick auf den Krankheitszustand typischerweise vorliegende Unsicherheit bedingt, da $B$ der Arzt durch die Auswahl einer Aktion den Patienten mit einem Prospekt hinsichtlich der Konsequenzen konfrontiert.

Im Unterschied dazu kann der Arzt mit einer diagnostischen Maßnahme seine Aktionswahl vom Testergebnis abhängig gestalten. Die Berücksichtigung der dadurch ermöglichten Entscheidungsfunktionen erweitert die Menge der Handlungsalternativen im Vergleich zur Situation ohne weitere Diagnostik beträchtlich. Grundsätzlich führt indessen auch die Wahl einer Entscheidungsfunktion zu einem Prospekt, in dem die für den Patienten bedeutsamen Konsequenzen mit den dazugehörigen Wahrscheinlichkeiten verzeichnet sind.

Damit läßt sich der Nutzeneffekt definieren, den eine diagnostische Maßnahme in einer gegebenen Entscheidungssituation für den Patienten besitzt. Dieser Nutzeneffekt besteht in dem Unterschied, den ein bei Berücksichtigung der diagnostischen Information günstigster Prospekt im Vergleich zu einem in der Situation ohne weitere Diagnostik optimalen Prospekt in der Bewertung durch den Patienten aufweist. Diese Differenz entspricht gerade der maximalen Verbesserung der Wohlfahrt des Patienten, die der Arzt durch den Einsatz der diagnostischen Maßnahme in der betrachteten Entscheidungssituation erreichen kann. Insofern impliziert diese Definition, da $B$ der Arzt seine Entscheidungen jeweils in völliger Übereinstimmung mit den Interessen des Patienten trifft. Umgekehrt erlaubt nur die zusätzliche Voraussetzung, die den Arzt als perfekten Agenten postuliert, eine genaue Erfassung des auf die diagnostische Information zurückzuführenden Nutzeneffekts beim Patienten. 
Die Beschreibung der Informationsleistung erweist sich indessen als ungeeignet, um die Information anzuzeigen, die der Einsatz einer diagnostischen Maßnahme dem Arzt in einer Entscheidungssituation liefert. Für die Klassen der diagnostischen Maßnahmen ohne Informationsleistung bzw. der perfekten diagnostischen Maßnahmen läßt sich zwar noch plausibel begründen, daß die damit verbundene Information gleich Null ist bzw. maximal ausfällt. Im Falle der dritten Klasse, die in der Regel die weitaus größte Anzahl von Diagnosetests enthält, bleibt jedoch unklar, wie die auf eine bestimmte Entscheidungssituation bezogene Informationsleistung in eine geeignete skalare Größe zu transformieren ist. Damit fehlt zugleich die Möglichkeit, verschiedene Elemente dieser Klasse hinsichtlich ihrer Informationsleistung zu vergleichen.

Erst der Rekurs auf die Informationstheorie schafft die Voraussetzungen für einen Vergleich der Information beliebiger diagnostischer Maßnahmen in Abhängigkeit von der medizinischen Entscheidungssituation. Mit Hilfe der Shannon'schen Entropiefunktion und des Konzepts der bedingten Entropie läßt sich der Informationsgewinn definieren, den ein Diagnosetest dem Arzt zu liefern vermag. Diese skalare Größe gibt die mit dem Einsatz der betrachteten Maßnahme verbundene neue Information an und entspricht daher gerade der Information, die der Arzt im Vergleich zur Situation ohne weitere Diagnostik erhält. Die Anwendung des Konzepts der diagnostischen Information bestätigt zunächst die oben angesprochenen Plausibilitätserwägungen, indem sie den diagnostischen Maßnahmen ohne Informationsleistung bzw. den perfekten diagnostischen Maßnahmen keine Information bzw. eine maximale Information zuordnet. Die Information einer beliebigen anderen diagnostischen Maßnahme liegt zwischen diesen beiden Extremwerten, die durch die jeweils vorliegende Entscheidungssituation determiniert werden.

In einer medizinischen Entscheidungssituation zieht der Arzt aufgrund der Unsicherheit bezüglich des Krankheitszustands seines Patienten eine Informationsbeschaffung in Betracht. Die medizinische Diagnostik dient insofern vornehmlich als Grundlage für weitere ärztliche Entscheidungen. Aus diesem Grund sind neben der Information noch zwei andere Faktoren zu berücksichtigen, welche die Existenz und den Umfang des Nutzeneffekts bestimmen, den der Einsatz einer diagnostischen Maßnahme beim Patienten in Abhängigkeit von der Entscheidungssituation zu stiften vermag. Dabei handelt es sich zunächst um den Einfluß, den die diagnostische Information auf das Handeln des Arztes ausübt. Im Anschluß daran entschei- 
den die Folgen, die sich für den Patienten im Vergleich zur Situation ohne weitere Diagnostik ergeben, über die Veränderung seiner Wohlfahrtsposition.

Ohne den Einsatz einer weiteren diagnostischen Maßnahme besteht für den Arzt lediglich die Möglichkeit, ein Element aus der Gesamtheit der ihm zur Verfügung stehenden Aktionen auszuwählen. Bei gegebenem Krankheitszustand führt jede dieser Aktionen zu eindeutig bestimmten Konsequenzen für den Patienten, die anhand einer Konsequenzenfunktion explizit dargestellt werden und im wesentlichen als Gesundheitseffekte anfallen. Diese Gesundheitseffekte repräsentieren die Ausprägungen derjenigen Gesundheitsattribute, die aus der Sicht des Patienten Relevanz besitzen. Die in einer Entscheidungssituation im Hinblick auf den Krankheitszustand typischerweise vorliegende Unsicherheit bedingt, daß der Arzt durch die Auswahl einer Aktion den Patienten mit einem Prospekt hinsichtlich der Konsequenzen konfrontiert.

Im Unterschied dazu kann der Arzt mit einer diagnostischen Maßnahme seine Aktionswahl vom Testergebnis abhängig gestalten. Die Berücksichtigung der dadurch ermöglichten Entscheidungsfunktionen erweitert die Menge der Handlungsalternativen im Vergleich zur Situation ohne weitere Diagnostik beträchtlich. Grundsätzlich führt indessen auch die Wahl einer Entscheidungsfunktion zu einem Prospekt, in dem die für den Patienten bedeutsamen Konsequenzen mit den dazugehörigen Wahrscheinlichkeiten verzeichnet sind.

Damit läßt sich der Nutzeneffekt definieren, den eine diagnostische Maßnahme in einer gegebenen Entscheidungssituation für den Patienten besitzt. Dieser Nutzeneffekt besteht in dem Unterschied, den ein bei Berücksichtigung der diagnostischen Information günstigster Prospekt im Vergleich zu einem in der Situation ohne weitere Diagnostik optimalen Prospekt in der Bewertung durch den Patienten aufweist. Diese Differenz entspricht gerade der maximalen Verbesserung der Wohlfahrt des Patienten, die der Arzt durch den Einsatz der diagnostischen Maßnahme in der betrachteten Entscheidungssituation erreichen kann. Insofern impliziert diese Definition, daß der Arzt seine Entscheidungen jeweils in völliger Übereinstimmung mit den Interessen des Patienten trifft. Umgekehrt erlaubt nur die zusätzliche Voraussetzung, die den Arzt als perfekten Agenten postuliert, eine genaue Erfassung des auf die diagnostische Information zurückzuführenden Nutzeneffekts beim Patienten. 
In wohlfahrtstheoretischer Hinsicht stellen individuelle Zahlungsbereitschaften das geeignete Konzept dar, um den Vorteil in monetären Einheiten zu quantifizieren, der dem Patienten aus dem Einsatz einer diagnostischen Maßnahme in Abhängigkeit von der Entscheidungssituation erwächst. Aus der Vielzahl möglicher Meßgrößen wird mit dem kompensierenden Optionspreis jene Zahlungsbereitschaft bei Unsicherheit analysiert, die der Patient unabhängig von seinem Krankheitszustand für den Nutzeneffekt der diagnostischen Information entfaltet. Damit läßt sich der Nutzen, den eine diagnostische Information dem Patienten stiftet, anhand der Optionspreise der dadurch ermöglichten Entscheidungsfunktionen definieren. Die Zahlungsbereitschaft, die der Patient für eine diagnostische Maßnahme besitzt, entspricht gerade dem maximalen kompensierenden Optionspreis, den eine dazugehörige Entscheidungsfunktion des Arztes besitzen kann. Die Analyse zeigt, daß der Optionspreis einer diagnostischen Maßnahme höher ausfallen kann als der Optionspreis einer optimalen Entscheidungsfunktion. Dieses Resultat, das sowohl für perfekte als auch für unvollkommene diagnostische Maßnahmen Gültigkeit besitzt, wurzelt in der fehlenden Ordnungseigenschaft, die den kompensierenden Optionspreis als individuelles Wohlfahrtsmaß - ebenso wie die Kompensationsvariation als korrespondierende Zahlungsbereitschaft bei Sicherheit - kennzeichnet.

Aus der Formulierung des ärztlichen Entscheidungsproblems folgt, daß der Optionspreis einer beliebigen diagnostischen Maßnahme stets nichtnegativ ist. Weiterhin fällt in einer gegebenen Entscheidungssituation der Optionspreis der vollkommenen diagnostischen Information maximal aus, wobei der Patient für jede perfekte diagnostische Maßnahme dieselbe Zahlungsbereitschaft besitzt. Steht dem Arzt eine gleichmäßig beste Aktion zur Verfügung, können zusätzliche diagnostische Informationen dem Patienten keinen Vorteil verschaffen. Unter dieser Voraussetzung erzielt eine perfekte diagnostische Maßnahme - und damit zugleich auch jeder andere Diagnosetest - einen Optionspreis von Null. Im Falle der unvollkommenen diagnostischen Information erzielen verschiedene Diagnosetests in der Regel auch unterschiedliche Optionspreise. Die Zahlungsbereitschaft des Patienten für eine diagnostische Maßnahme, die über seinen Krankheitszustand nicht vollständig informiert, wird genau dann positiv, wenn der Arzt bei mindestens einem Testergebnis von einer in der Situation ohne weitere Diagnostik optimalen Aktion abweicht.

Unter einer zusätzlichen Prämisse, die spezielle Eigenschaften seiner Präferenzen postuliert, gelingt es, die Zahlungsbereitschaft des Patienten für eine diagnostische Maßnahme explizit darzustellen. Bei Gültigkeit dieser Annahme läßt sich eine mo- 
netäre Verlustfunktion ableiten, welche die zur Bewertung ärztlicher Handlungen durch den Patienten notwendige Information enthält. Damit gestaltet sich die Analyse der Zahlungsbereitschaft, die der Patient für eine diagnostische Maßnahme entfaltet, weitgehend analog zu einem in der Entscheidungstheorie vielfach untersuchten Problem, bei dem die Annahme eines "linearen Risikonutzens" des Aktors ebenfalls die Beschränkung der Analyse auf eine monetäre Verlustfunktion gestattet. Der kompensierende Optionspreis einer diagnostischen Maßnahme entspricht dann gerade der Reduktion des erwarteten Verlusts, den eine durch die diagnostische Information ermöglichte optimale Entscheidungsfunktion des Árztes im Vergleich zu einer in der Situation ohne weitere Diagnostik günstigsten Aktion erreicht. Während sich für den Optionspreis der perfekten und der unvollkommenen diagnostischen Information qualitativ dieselben Resultate wie im allgemeinen Fall ergeben, ermöglicht die Restriktion der Präferenzen des Patienten eine erheblich vereinfachte Analyse seiner Zahlungsbereitschaft. Insbesondere läßt sich der Optionspreis einer diagnostischen Maßnahme in einem wichtigen Spezialfall graphisch untersuchen. Andererseits zeigt die Notwendigkeit einer zusätzlichen Annahme, daß es allgemein nicht zulässig ist, den Nutzen einer diagnostischen Maßnahme für den Patienten mit Hilfe einer monetären Verlustfunktion zu ermitteln.

Der kompensierende Optionspreis beschreibt die von der Entscheidungssituation abhängige Zahlungsbereitschaft des Patienten für die diagnostische Information, wenn von den Kosten der Informationsbeschaffung - d.h. der diagnostischen Maßnahme - abgesehen wird. Aufgrund dieser Nichtberücksichtigung handelt es sich in konzeptioneller Hinsicht um eine individuelle Brutto-Zahlungsbereitschaft, die zudem im allgemeinen nicht mit der entsprechenden gesamtwirtschaftlichen Wertgröße übereinstimmt. Diese Diskrepanz beruht darauf, daß die Konsequenzen des ärztlichen Handelns in die Berechnung des Optionspreises diagnostischer Maßnahmen nur insoweit Eingang finden, als sie den Patienten selbst betreffen. In der Regel bleiben dabei jedoch einige Konsequenzen unberücksichtigt, die eine gesamtwirtschaftliche Betrachtung keinesfalls vernachlässigen sollte. Falls beispielsweise der Optionspreis einer diagnostischen Maßnahme in einer Entscheidungssituation ihre Kosten übersteigt, reicht dieser Tatbestand demzufolge nicht aus, um auf die Vorteilhaftigkeit der Informationsbeschaffung im Sinne der Kosten-Nutzen-Analyse zu schließen.

Die allgemeine Nichtnegativität des kompensierenden Optionspreises ist äquivalent zu der Aussage, daß die Information einer diagnostischen Maßnahme die Wohl- 
fahrt des Patienten zumindest nicht verringern kann. Weiterhin erzeugt der Einsatz einer diagnostischen Maßnahme genau dann einen Nutzeneffekt für den Patienten, wenn sie die Aktionswahl des Arztes im Vergleich zur Situation ohne weitere Diagnostik beeinflußt. Damit repräsentiert die diagnostische Information in dem betrachteten Modell einen intermediären Output, dessen Wohlfahrtsrelevanz von der anschließenden Verwendung dieses Wissens durch den Arzt abhängt. Aus den Annahmen, die diese Einordnung implizieren, ergeben sich zugleich Anhaltspunkte bezüglich derjenigen Voraussetzungen, unter denen die diagnostische Information auch Elemente eines finalen Outputs aufweist. In diesem Zusammenhang werden zwei Gründe diskutiert, die für einen unmittelbaren Nutzeneffekt diagnostischer Maßnahmen beim Patienten sprechen.

Die in der Literatur anzutreffende Einschätzung, der zufolge die Beruhigung des Patienten bei einem richtig negativen Testergebnis beispielsweise im Rahmen einer Vorsorgeuntersuchung einen Beleg für die Existenz eines unmittelbaren Nutzeneffekts der diagnostischen Information liefert, trifft indessen nicht zu. Indem sie mit einer speziellen Kombination aus diagnostischem Resultat und Krankheitszustand des Patienten lediglich einen Teil des mit dem Einsatz einer diagnostischen Maßnahme verbundenen Prospekts herausgreift, liegt dieser Auffassung eine unzulässig enge ex-post-Perspektive zugrunde, die nicht der tatsächlich vorliegenden Entscheidungssituation entspricht. Ganz allgemein zeigt die Analyse des ärztlichen Entscheidungsproblems, daß es nicht ausreicht, lediglich die aufgrund ausgewählter Testergebnisse bzw. einzelner Kombinationen aus Testergebnis und Krankheitszustand erreichbaren Nutzenpositionen des Patienten zu betrachten, um auf den Nutzeneffekt schließen zu können, den die Information einer diagnostischen Maßnahme bei ihm erzeugt.

Zwischen der Information einer diagnostischen Maßnahme und ihrem Nutzeneffekt beim Patienten besteht allgemein keine enge Verbindung, da beide Größen in unterschiedlicher Weise von der zugrundeliegenden medizinischen Entscheidungssituation abhängen. Einerseits kann es vorkommen, daß selbst der Einsatz einer perfekten diagnostischen Maßnahme dem Patienten keinen Vorteil verschafft und damit die maximale diagnostische Information einen minimalen Nutzeneffekt erzeugt. Andererseits gibt es Entscheidungssituationen, in denen schon ein geringes $\mathrm{Ma} ß$ an diagnostischer Information ausreicht, um die Wohlfahrtsposition des Patienten zu verbessern. Aber auch in einer gegebenen Entscheidungssituation braucht kein eindeutiger Zusammenhang zwischen der Information und dem Nutzen diagno- 
stischer Maßnahmen zu bestehen. Dies zeigt das Beispiel zweier Diagnosetests, von denen der erste eine höhere Information in der betrachteten Entscheidungssituation liefert, während der zweite den größeren Nutzeneffekt beim Patienten auslöst. In diesem Fall erweist sich die weniger informative Maßnahme als günstiger, weil sie dem Arzt eine aus der Sicht des Patienten bessere Verwendung der diagnostischen Information ermöglicht.

Mit Hilfe des entscheidungstheoretischen Modells läßt sich weiterhin der Einfluß analysieren, den einzelne Komponenten der medizinischen Entscheidungssituation auf die mit einer diagnostischen Maßnahme verbundene Veränderung der Wohlfahrt des Patienten ausüben. Wenn die Struktur der Entscheidungssituation festliegt, entscheiden die prioren Wahrscheinlichkeiten der in Betracht gezogenen Krankheitszustände über den Nutzeneffekt, den der Einsatz eines Diagnosetests beim Patienten verursacht. Unter dieser Voraussetzung hängen demzufolge sowohl die Existenz eines Nutzeneffekts als auch das Ergebnis eines diesbezüglichen Vergleichs mehrerer diagnostischer Maßnahmen vom Vorwissen des Arztes ab. Umgekehrt gilt bei konstanten prioren Wahrscheinlichkeiten der Krankheitszustände, daß die Struktur der betrachteten Entscheidungssituation den Nutzeneffekt einer diagnostischen Information beim Patienten determiniert. Wie sich anhand eines einfachen Beispiels zeigen läßt, können die Präferenzen eines Patienten für diagnostische Maßnahmen davon abhängen, ob die Tests als Vorsorgeuntersuchungen oder im Rahmen der Differentialdiagnose zum Einsatz gelangen.

Schließlich bedürfen die den Nutzen diagnostischer Maßnahmen betreffenden Ergebnisse einer Modifikation, wenn entweder ihr Einsatz mit einem gesundheitlichen Risiko einhergeht oder sich die Beschaffung der diagnostischen Information als zeitintensiv erweist und gleichzeitig die Konsequenzen ärztlicher Aktionen für den Patienten in starkem Ausmaß zeitabhängig sind. In diesen Fällen kann der negative Effekt beispielsweise aufgrund der Zeitverzögerung des ärztlichen Handelns den von der diagnostischen Information ausgehenden positiven Effekt dominieren. Unter dieser Voraussetzung besitzt die diagnostische Information einen negativen Optionspreis, da der Einsatz der betreffenden diagnostischen Maßnahme die Wohlfahrt des Patienten im Vergleich zur Situation ohne weitere Diagnostik verringert. Weiterhin ergibt sich im Hinblick auf den Vergleich verschiedener diagnostischer Maßnahmen, daß bei identischer Informationsleistung diejenige Maßnahme dem Patienten einen höheren Nutzeneffekt zu sichern vermag, deren Resultate frühzeitiger eintreffen. 
Der entscheidungstheoretische Ansatz der vorliegenden Arbeit gewährleistet, daß die in der Literatur zur Entscheidungstheorie diskutierten Verallgemeinerungen grundsätzlich auch bei der Analyse des Nutzeneffekts Anwendung finden können, der dem Patienten aufgrund der diagnostischen Information erwächst. Hier wäre zunächst an eine Erweiterung der Handlungsalternativen des Arztes zu denken, indem die Möglichkeit einer schrittweisen Informationsbeschaffung zugelassen und damit das sequentielle Element der medizinischen Diagnostik berücksichtigt wird ${ }^{1}$. Im Vergleich zu der in der Arbeit untersuchten Entscheidung über die einmalige Informationsbeschaffung gilt in diesem Fall für die Beschreibung ärztlicher Entscheidungsfunktionen, daß die eintreffenden diagnostischen Resultate nicht nur die Aktionswahl, sondern auch den Umfang der Diagnostik zu beeinflussen vermögen ${ }^{2}$. Unter sonst gleichen Voraussetzungen wird der Arzt allerdings nur dann ein sequentielles Vorgehen wählen, wenn der Einsatz einzelner diagnostischer Maßnahmen mit einem Kosteneffekt für den Patienten verbunden ist ${ }^{3}$.

Weiterhin läßt sich die Annahme bezüglich des diagnostischen Vorwissens des Arztes abschwächen. In vielen medizinischen Entscheidungssituationen werden die Wahrscheinlichkeiten, die der Arzt den von ihm in Betracht gezogenen Krankheitszuständen des Patienten zuordnet, nicht exakt anzugeben sein, sondern eine gewisse Unschärfe aufweisen. Es liegt dann nahe, anstelle eines einzelnen Elements bestimmte Teilmengen des entsprechenden Wahrscheinlichkeitssimplex als diagnostisches Vorwissen anzusehen. Der Arzt verfügt in diesem Fall über partielle Information, wobei es sich genau dann um den Spezialfall linearer partieller Information handelt, wenn die zu berücksichtigende Menge priorer Wahrscheinlichkeitsverteilungen durch ein System linearer Ungleichungen darstellbar ist ${ }^{4}$. Das Vorliegen eines lediglich partiellen diagnostischen Vorwissens wirkt sich auf die Beurteilung ärztlicher Handlungen durch den Patienten aus, indem beispielsweise eine Aktion des Arztes mit dem bezüglich der betrachteten Menge priorer Wahrscheinlichkeitsverteilungen minimalen Erwartungsnutzen bewertet wird. An-

1: Für eine frühe Anwendung der sequentiellen Analyse auf eine spezielle medizinische Entscheidungssituation vgl. BETAQUE,N.E./GORRY,G.A. (1971).

2: Zur Beschreibung von Entscheidungsfunktionen in sequentiellen Entscheidungsproblemen vgl. z.B. BLACKWELL,D./GIRSHICK,M.A. (1954), S. 89ff.

3: Ohne die Existenz derartiger Kosteneffekte erweist sich die Beschaffung maximaler diagnostischer Information aus der Sicht des Patienten niemals als unvorteilhaft.

4: Zum Begriff der linearen partiellen Information sowie zur darauf aufbauenden entscheidungstheoretischen Analyse vgl. KOFLER,E./MENGES,G. (1976), S. 85ff. sowie 
sonsten bleibt das grundsätzliche Vorgehen zur Ermittlung des Nutzeneffekts bzw. des Nutzens, den die diagnostische Information beim Patienten erzeugt, unverändert.

Der Nutzen des Patienten stellt in einer gegebenen Entscheidungssituation nur eine von insgesamt drei Komponenten dar, die zur Beurteilung der gesamtwirtschaftlichen Vorteilhaftigkeit einer diagnostischen Maßnahme dienen. Die Vernachlässigung der beiden anderen Komponenten erscheint jedoch insofern gerechtfertigt, als eine Erfassung der Kosten der Informationsbeschaffung und der vom Patienten nicht zu tragenden - differentiellen Kosten ärztlicher Aktionen in theoretischer und empirischer Hinsicht vergleichsweise geringe Probleme aufwirft. Im Gegensatz dazu birgt der auf die diagnostische Information zurückzuführende Nutzeneffekt beim Patienten aufgrund seiner Intangibilität die Gefahr, im Rahmen einer ökonomischen Analyse nicht adäquat berücksichtigt zu werden. Der hier vorgestellte Zahlungsbereitschaftsansatz vermeidet diese Gefahr zumindest auf der theoretischen Ebene, indem er mit einem Prospekt hinsichtlich der Konsequenzen das unmittelbare Ergebnis einer diagnostischen Maßnahme explizit darstellt und eine Bewertung im Einklang mit den Präferenzen des Patienten leistet. Im übrigen bietet dieser Ansatz zugleich die Möglichkeit, den Nutzeneffekt bzw. den Nutzen zu ermitteln, der dem Patienten beispielsweise aus der Einführung einer neuen Therapie - in Abhängigkeit von der Entscheidungssituation - erwächst.

In der empirischen Anwendung dürfte eine Ermittlung der Zahlungsbereitschaft des Patienten allerdings auf Schwierigkeiten stoßen, da etwa eine Befragung keineswegs korrekte Resultate zu liefern verspricht ${ }^{5}$. Dennoch besitzt der Zahlungsbereitschaftsansatz für die empirische Forschung in zweifacher Hinsicht Bedeutung. Zunächst kann der Ansatz als theoretische Leitlinie dazu dienen, die Ergebnisse anderer Methoden einer Plausibilitätsüberprüfung zu unterziehen. In diesem Zusammenhang zeigt sich die zentrale Schwäche des Humankapitalansatzes im Gesundheitswesen, der mit den vermiedenen Produktionsausfällen lediglich eine einzelne Folge des ärztlichen Handelns monetär bewertet, die in der Regel keinen

KOFLER,E. (1989), S. 19ff. Eine gesundheitsökonomische Anwendung diskutiert ZWEIFEL,P. (1986).

5: $\mathrm{Zu}$ den vielfältigen Problemen, die Befragungen aufwerfen, vgl. MITCHELL,R.C./CARSON,R.T. (1989), S. 127ff., die diese Thematik im Kontext der Methode der "contingent valuation" behandeln. 
verläßlichen Rückschluß auf den Nutzen des Patienten zu ziehen erlaubt ${ }^{6}$. Daneben schafft der Zahlungsbereitschaftsansatz durch seinen expliziten Rekurs auf die beim Patienten eintretenden Gesundheitseffekte eine enge Verbindung zur Forschung auf dem Gebiet der quantitativen Erfassung von Veränderungen des individuellen Gesundheitszustands ${ }^{7}$. Sobald für eine medizinische Entscheidungssituation ein ausreichend breites Spektrum spezifischer Indikatoren vorliegt, gelingt es zumindest, die Grundlage des mit einer bestimmten Handlung des Arztes verbundenen Nutzeneffekts beim Patienten zu beschreiben. Diese Information gestattet dann, die realen Opportunitätskosten alternativer ärztlicher Handlungen aus der Sicht des Patienten anzugeben.

6: Zum Humankapitalansatz im Gesundheitswesen vgl. z.B. HODGSON,T.A. (1983), S. 133ff. sowie kritisch DRUMMOND,M.F. (1981), S. 134ff.

7: Vgl. dazu die in Abschnitt 4.2.2 angegebene Literatur. 
Walter Ried - 978-3-631-75573-0

Downloaded from PubFactory at 01/11/2019 03:26:15AM

via free access 


\section{LITERATURVERZEICHNIS}

Abrams, H.L./Siegelman, S./Adams, D.F./Sanders, R./Fineberg, H./Hessel, S.J./McNeil, B.J. (1982): Computed Tomography Versus Ultrasound of the Adrenal Gland: a Prospective Study. In: Radiology, Vol. 143, 121128.

Abt, C.C. (1977): The issue of social costs in cost-benefit analysis of surgery. In: Bunker, J.P./Barnes, B.A./Mosteller, F. (Eds): Costs, risks and benefits of surgery, New York, S. 40-55.

Adam, H. (1985): Allokation, Kosten-Nutzen-Analyse und Prävention. In: Medizin Mensch Gesellschaft, Band 10, S. 98-105.

Ahlheim, M. (1985): Dualitätstheorie als methodischer Ansatz mikroökonomischer Theorien. Frankfurt/Main, Bern, New York.

Ahlheim, M./Rose, M. (1989): Messung individueller Wohlfahrt. Berlin et al.

Ahlheim, M./Wagenhals, G. (1988): Exakte Wohlfahrtsmaße in der Nutzen-Kosten-Analyse. In: Zeitschrift für Wirtschafts- und Sozialwissenschaften, 108. Jg., S. 169-193.

Aitchison, J. (1970): Statistical problems of treatment allocation. In: Journal of the Royal Statistical Society, Series A, Vol. 133, S. 206-228.

Albert, A. (1982): On the use and computation of likelihood ratios in clinical chemistry. In: Clinical Chemistry, Vol 28, S. 1113-1119.

Albert, D.A. (1977): Confirmation of a diagnosis: a decision-theoretic approach. In: Metamed, Vol. 1, S. 213-225.

Alter, U./Klausing, M. (1974): Effizienzmessungen im Gesundheitswesen. In: Deutsches Ärzteblatt, S. 3262-3267.

Altrogge, G. (1975): Möglichkeiten und Problematik der Bewertung von (Zusatz-) Informationen mit Hilfe der Bayes-Analyse. In: Zeitschrift für Betriebswirtschaft, 45. Jg., S. 821-846.

Andel, N. (1977): Nutzen-Kosten-Analysen. In: Neumark, F. (Hg.): Handbuch der Finanzwissenschaft, 3. Auflage, 1. Band, Tübingen, S. 475-518.

Anderson, J.A./Boyle, J.A. (1968): Computer diagnosis: statistical aspects. In: British Medical Bulletin, Vol. 24, S. 230-235.

Anderson, L.G./Settle, R.F. (1977): Benefit-cost analysis: a practical guide. Lexington, Toronto. 
Anschütz, F. (1985): Probleme der Diagnostik aus klinischer Sicht. In: Vogel, H.-R. (Hg.): Effizienz und Effektivität medizinischer Diagnostik. Stuttgart, S. 3-11.

Anschütz, F. (1988): Indikationen und Kontraindikationen ärztlichen Handelns. In: Die Neue Ärztliche Nr. 46 vom 8.3.1988, S. 14.

Arnold, V. (1980): Nutzen-Kosten-Analyse II: Anwendung. In: Albers, W. et al. (Hg.): Handwörterbuch der Wirtschaftswissenschaft, 5. Band, Stuttgart, Tübingen, Göttingen, S. 382-399.

Arrow, K.J. (1963): Uncertainty and the welfare economics of medical care. In: American Economic Review, Vol. 53, S. 941-973.

Arrow, K.J. (1964): The role of securities in the optimal allocation of risk-bearing. In: Review of Economic Studies, Vol. 31, S. 91-96.

Arrow, K.J. (1974): Optimal insurance and generalized deductibles. In: Scandinavian Actuarial Journal, S. 1-42.

Arrow, K.J. (1985): The economics of agency. In: Pratt, J.W./Zeckhauser, R.J. (Eds): Principals and agents: the structure of business. Boston, S. 37-51.

Auster, R./Leveson, I./Sarachek, D. (1969): The production of health. An exploratory study. In: Journal of Human Resources, Vol. 4, S. 411-436.

Avery, A.D./Lelah, T./Solomon, N.E./Harris, L.J./Brook, R.H./Greenfield, S./Ware, J.E./Avery, C.H. (1976): Quality of medical care assessment using outcome measures: eight disease-specific applications. Santa Monica. Published by the Rand Corporation as Report No. R-2021/2-HEW.

Bahadur, R.R. (1961): A representation of the joint distribution of responses to $n$ dichotomous items. In: Solomon, H. (Ed.): Studies in item analysis and prediction, Stanford, S. 158-168.

Bailey, N.T.J. (1967): The mathematical approach to biology and medicine. London, New York, Sydney.

Bailey, N.T.J. (1977): Mathematics, Statistics, and Systems for Health. Chichester et al.

Bamberg, G. (1972): Statistische Entscheidungstheorie. Würzburg, Wien.

Bamberg, G. (1975): Wieviel dürfen Informationen kosten? In: Bamberg, G./Opitz, O. (Hg.): Information und Prognose, Meisenheim am Glan, S. 200-219. 
Bamberg, G./Coenenberg, A.G. (1989): Betriebswirtschaftliche Entscheidungslehre. 5. Auflage. München.

Bamberg, G./Coenenberg, A.G./Kleine-Doepke, R. (1976): Zur entscheidungsorientierten Bewertung von Informationen. In: Zeitschrift für Betriebswirtschaftliche Forschung, 28. Jg., S. 30-42.

Banta, H.D./McNeil, B.J. (1978): Evaluation of the CAT scanner and other diagnostic technologies. In: Health Care Management Review, Vol. 3, S. 7-19.

Barnoon, S./Wolfe, H. (1972): Measuring the effectiveness of medical decisions. An Operations Research Approach. Springfield/Illinois.

Barr, D.P. (1955): Hazards of modern diagnosis and therapy - the price we pay. In: Journal of the American Medical Association, Vol. 159, S. 14521456.

Begg, C.B. (1987): Biases in the assessment of diagnostic tests. In: Statistics in Medicine, Vol. 6, S. 411-423.

Belsey, R./Baer, D./Sewell, D. (1986): Laboratory Test Analysis near the patient. Opportunities for improved clinical diagnosis and management. In: Journal of the American Medical Association, Vol. 255, S. 775-786.

Benson, E.S. (1978): Strategies for improved use of the clinical chemistry laboratory in patient care. In: Benson,E.S./Rubin, M. (Eds.): Logic and Economics of Clinical Laboratory Use. New York, S. 245-258.

Berger, J.O. (1985): Statistical decision theory and Bayesian analysis. 2nd edition. New York et al.

Berwick, D.M./Weinstein, M.C. (1985): What do patients value? Willingness to pay for ultrasound in normal pregnancy. In: Medical Care, Vol. 23, S. 881-893.

Betaque, N.E./Gorry, G.A. (1971): Automating judgmental decision making for a serious medical problem. In: Management Science, Vol. 17, S. B 421B 435 .

Billerbeck, K. (1968): Kosten-Ertrags-Analyse. Ein Instrument zur Rationalisierung der administrierten Allokation bei Bildungs- und Gesundheitsinvestitionen. Berlin.

Billingsley, P. (1986): Probability and measure. 2nd edition. New York et al.

Bishop, R.C. (1982): Option value: an exposition and extension. In: Land Economics, Vol. 58, S. 1-15. 
Bishop, Y.M.M./Fienberg, S.E./Holland, P.W. (1975): Discrete multivariate analysis: theory and practice. Cambridge/Mass. and London.

Blachman, N.M. (1968): The amount of information that y gives about X. In: Institute of Electrical and Electronic Engineers (IEEE) Transactions on Information Theory, Vol. IT-14, S. 27-31.

Blackorby, C./Donaldson, D. (1988): Money metric utility: a harmless normalisation? In: Journal of Economic Theory, Vol. 46, S. 120-129.

Blackorby, C./Donaldson, D. (1990): A review article: the case against the use of the sum of compensating variations in cost-benefit analysis. In: Canadian Journal of Economics, Vol. 23, S. 471-494.

Blackwell, D. (1951): Comparison of experiments. In: Proceedings of the Second Berkeley Symposium on Mathematical Statistics and Probability, Berkeley et al., S. 93-102.

Blackwell, D. (1953): Equivalent comparisions of experiments. In: Annals of Mathematical Statistics, Vol. 24, S. 265-272.

Blackwell, D./Girshick, M.A. (1954): Theory of games and statistical decisions. New York, London, Sydney.

Blumberg, M.S. (1957): Evaluating health screening procedures. In: Operations Research, Vol. 5, S.351-360.

Boadway, R.W. (1974): The welfare foundations of cost-benefit analysis. In: Economic Journal, Vol. 84, S. 926-939.

Boadway, R.W./Bruce, N. (1984): Welfare economics. Oxford, New York.

Boden, L.I. (1979): Cost-benefit analysis: caveat emptor. In: American Journal of Public Health, Vol. 69, S. 1210-1211.

Bodily, S.E. (1980): Analysis of risks to life and limb. In: Operations Research, Vol. 28, S. 156-175.

Bohm, P. (1975): Option demand and consumer's surplus: comment. In: American Economic Review, Vol. 65, S. 733-736.

Bowling, A. (1991): Measuring health. A review of quality of life measurement scales. Milton Keynes, Philadelphia.

Brémaud, P. (1988): Introduction aux Probabilités. Modélisation des Phénomènes Aléatoires. ler réimpression corrigée. Berlin et al. 
Breyer, F. (1982): Rational purchase of medical care and differential insurance coverage for diagnostic services. In: Journal of Health Economics, Vol. 1, S. 147-156.

Brillouin, L. (1967): Science and information theory. 2nd ed., New York.

Brook, R.H./Avery, A.D./Greenfield, S./Harris, L.J./Lelah, T./Solomon, N.E./Ware, J.E. (1976): Quality of medical care assessment using outcome measures: an overview of the method. Santa Monica. Published by the Rand Corporation as Report No. R-2021/1-HEW.

Buchanan, J.M. (1969): Cost and choice: an inquiry in economic theory. Chicago.

Bühlmann, H./Loeffel, H./Nievergelt, E. (1975): Entscheidungs- und Spieltheorie. Berlin et al.

Büttner, J. (1977): Die Beurteilung des diagnostischen Wertes klinisch-chemischer Untersuchungen. In: Journal of clinical chemistry and clinical biochemistry, Vol. 15, S. 1-12.

Büttner, J. (1985): Messung von Effizienz und Effektivität bei klinischchemischen Untersuchungen. In: Vogel, H.-R. (Hg.): Effizienz und Effektivität medizinischer Diagnostik, Stuttgart, S. 39-52.

Bunge, M. (1967): Scientific Research I. The Search for System. Berlin, Heidelberg, New York.

Buxton, M.J./West, R.R. (1975): Cost-benefit analysis of long-term haemodialysis for chronic renal failure. In: British Medical Journal, Vol. 2, S. 376379.

Card, W.I (1975): Mathematical method in diagnosis. In: Journal of the Royal College of Physicians of London, Vol. 9, S. 193-196.

Card, W.I. (1979): 'Is it worth it?'. In: Journal of the Royal College of Physicians of London, Vol. 13, S. 213-216.

Carter, M. (1990): An expository note on the composite commodity theorem. Discussion Paper No. 9005, Department of Economics, University of Canterbury, Christchurch.

Chiang, C.L./Hodges, J.L./Yerushalmy, J. (1956): Statistical problems in medical diagnoses. In: Neyman, J. (Ed.): Proceedings of the third Berkeley Symposium on mathematical statistics and probability; Vol. IV: Contributions to Biology and Problems of Health, Berkeley and Los Angeles, S. 121-133. 
Chou, Y. (1989): Statistical analysis for business and economics. New York, Amsterdam, London.

Chu, J.T. (1967): Some decision making methods applicable to the medical sciences. In: LeCam, L.M./Neyman, J. (Eds.): Proceedings of the Fifth Berkeley Symposium on mathematical statistics and probalility. Vol. IV: Biology and Problems of Health. Berkeley and Los Angeles; S. 925-934.

Cochrane, A.L./Holland, W.W. (1971): Validation of screening procedures. In: British medical Bulletin, Vol. 27, S. 3-8.

Cook, P.J./Graham, D.A. (1977): The demand for insurance and protection: the case of irreplaceable commodities. In: Quarterly Journal of Economics, Vol. 91, S. 143-156.

Cornfield, J. (1967): Bayes theorem. In: Review of the International Statistical Institute, Vol. 35, S. 34-49.

Cornwall, R.C. (1984): Introduction to the use of general equilibrium analysis. Amsterdam, New York, Oxford.

Cramer, H. (1946): Mathematical methods of statistics. Princeton.

Croft, D.J./Machol, R.E. (1974): Mathematical methods in medical diagnosis. In: Annals of biomedical engineering, Vol. 2, S. 69-89.

Csiszár, I. (1978): Information measures: a critical survey. In: Transactions of the 7th Prague Conference, Vol. B, Dordrecht and Boston, S. 73-86.

Csiszár, I./Körner, J. (1981): Coding Theorems for discrete memoryless systems. London.

Cullis, J.G./West, P.A. (1979): The economics of health. Oxford.

Culyer, A.J. (1971): The nature of the commodity "health care" and its efficient allocation. In: Oxford Economic Papers, New Series, Vol. 23, S. 189211.

Culyer, A.J. (1978): Measuring health. Lessons for Ontario. Toronto.

Culyer, A.J./Maynard, A.K. (1981): Cost-effectiveness of duodenal ulcer treatment. In: Social Science \& Medicine, Vol. 15, S. 3-11.

Currie, J.M./Murphy, J.A./Schmitz, A. (1971): The concept of economic surplus and its use in economic analysis. In: Economic Journal, Vol. 81, S. 741-799. 
Dagenais, D.L./Courville, L./Dagenais, M. (1985): A cost-benefit analysis of the Quebec network of genetic medicine. In: Social Science \& Medicine, Vol. 20, S. 601-607.

Dahmer, J. (1980): Diagnostisch-therapeutisches Denken. Ein ärztliches Problemlösungsmodell mit praktischen Beispielen. Stuttgart, New York.

Daniels, M./Schroeder, S.A. (1977): Variation among physicians in use of laboratory tests. II. Relation to clinical productivity and outcomes of care. In: Medical Care, Vol. 15, S. 482-487.

Dawid, A.P. (1976): Properties of diagnostic data distributions. In: Biometrics, Vol. 32, S. 647-658.

Dehez, P./Drèze, J.H. (1982): State dependent utility, the demand for insurance and the value of safety. In: Jones-Lee, M.W. (Ed.): The value of life and safety. Amsterdam, New York, Oxford; S. 41-65.

Department of Clinical Epidemiology and Biostatistics (1981): How to read clinical journals II. To learn about a diagnostic test. In: Canadian Medical Association Journal, Vol. 124, S. 703-710.

Department of Clinical Epidemiology and Biostatistics (1983): Interpretation of diagnostic data (six parts). In: Canadian Medical Association Journal, Vol. 129; S. 429-432, 559-564, 587, 705-710, 832-835, 947-954, 10931099.

Department of Clinical Epidemiology and Biostatistics (1984): How to read clinical journals: VII. To understand an economic evaluation (part A). In: Canadian Medical Association Journal, Vol. 130, S. 1428-1433.

Diamond, G.A. (1984): Bayes' theorem: a practical aid to clinical judgment for diagnosis of coronary-artery disease. In: Practical Cardiology, Vol. 10, S. 47-77.

Dionne, G./Contandriopoulos, A.-P. (1985): Doctors and their workshops. A review article. In: Journal of Health Economics, Vol. 4, S. 21-33.

Doessel, D.P. (1986): Medical Diagnosis as a Problem in the Economics of Information. In: Information for Economic Policy, Vol. 2, S. 49-68.

Donaldson, R.M./Feinstein, A.R./Joyce, C.M. (1984): Group diversity as a threat to individual economy in ordering diagnostic procedures. In: Transactions of the Association of American Physicians, Vol. 97, S. 134139.

Doubilet, P. (1983): A mathematical approach to interpretation and selection of diagnostic tests. In: Medical Decision Making, Vol. 3, S. 177-195. 
Drèze, J./Stern, N. (1987): The theory of cost-benefit analysis. In: Auerbach, A.J./Feldstein, M. (Eds): Handbook of Public Economics, Vol. II., Amsterdam et al., S. 909-989.

Drukarczyk, J. (1974): Zum Problem der Bestimmung des Werts von Informationen. In: Zeitschrift für Betriebswirtschaft, 44. Jg., S. 1-18.

Drummond, M.F. (1980): Principles of economic appraisal in health care. Oxford.

Drummond, M.F. (1981): Welfare economics and cost-benefit analysis in health care. In: Scottish Journal of Political Economy, Vol. 28, S. 125-145.

Drummond, M.F./Ludbrook, A./Lowson, K./Steele, A. (1986): Studies in economic appraisal in health care. Vol. 2, Oxford, New York, Tokyo.

Drummond, M.F./Stoddart, G.L. (1985): Principles of economic evaluation of health programmes. In: World Health Statistics Quarterly, Vol. 38, S. 355-367.

Drummond, M.F./Stoddart, G.L./Torrance, G.W. (1987): Methods for the economic evaluation of health care programmes. Oxford, New York, Toronto.

Dunlop, D.W. (1975): Benefit-cost analysis: a review of its applicability in policy analysis for delivering health services. In: Social Science \& Medicine, Vol. 9, S. 133-139.

Dupuit, J. (1844): De la mesure de l'utilité des travaux publics. In: Annales des ponts et chaussées, tome 8; zitiert nach der englischen Übersetzung in: Arrow, K.J./Scitovsky, T. (Eds): Readings in welfare economics, London 1969, S. 255-283.

Egan, J.P. (1975): Signal detection theory and ROC analysis. New York.

Eisen, R. (1979): Theorie des Versicherungsgleichgewichts. Unsicherheit und Versicherung in der Theorie des generellen ökonomischen Gleichgewichts. Berlin.

Ekelund, R.B./Hébert, R.F. (1985): Consumer surplus: the first hundred years. In: History of Political Economy, Vol. 17, S. 419-454.

Engle, R.L. (1964): Medical diagnosis. In: Jacquez, J.A. (Ed.): The diagnostic process. Ann Arbor, S. 7-18.

Engle, R.L./Davis, B.J. (1963): Medical Diagnosis: Past, Present and Future. In: Archives of Internal Medicine, Vol. 112, S. 512-543. 
Fahrmeir, L./Hamerle,A. (1984): Grundlegende multivariate Schätz- und Testprobleme. In: Fahrmeir, L./Hamerle, A. (Hg.): Multivariate statistische Verfahren. Berlin, New York; S. 49-82.

Fano, R.M. (1966): Informationsübertragung. Eine statistische Theorie der Nachrichtenübertragung. München, Wien.

Fein, R. (1977): But, on the other hand: high blood pressure, economics and equity. In: New England Journal of Medicine, Vol. 296, S. 751-753.

Feinstein, A.R. (1973): An Analysis of Diagnostic Reasoning. I. The Domains and Disorders of Clinical Macrobiology. In: Yale Journal of Biology and Medicine, 46, S. 212-232.

Feinstein, A.R. (1977a): On the sensitivity, specificity, and discrimination of diagnostic tests. In: Feinstein, A.R.: Clinical biostatistics.' St. Louis, S. 214-226.

Feinstein, A.R. (1977b): Consequences of "compliance bias". In: Feinstein A. R.: Clinical biostatistics, St. Louis, S. 122-133.

Feinstein, A.R. (1977c): The haze of Bayes, the aerial palaces of decision analysis, and the computerized Ouija board. In: Clinical Pharmacology and Therapeutics, Vol. 21, S. 482-496.

Feldstein, M.S. (1974): Econometric studies of health economics. In: Intriligator, M.D./Kendrick, D.A. (Eds): Frontiers of quantitative economics, Vol. II, Amsterdam, S. 377-442.

Ferguson, B.S. (1985): Physician objectives and resource allocation. In: Journal of Health Economics, Vol. 4, S. 35-42.

Ferguson, T.S. (1967): Mathematical statistics. A decision theoretic approach. New York, London.

Ferschl, F. (1975): Nutzen- und Entscheidungstheorie. Einführung in die Logik der Entscheidungen. Opladen.

Fineberg, H.V. (1979): Clinical chemistries: the high cost of low-cost diagnostic tests. In: Altman, S.H./Blendon, R. (Eds.): Medical technology: the culprit behind health care costs? Washington, D.C., S. 144-165.

Firchau, V. (1980): Wert und maximaler Wert von Informationen für statistische Entscheidungsprobleme. Königstein/Taunus.

Fischer, N. (1983): Honorarvereinbarung mit der Kassenärztlichen Bundesvereinigung. In: Die Ersatzkasse, Jg. 63, S. 378-381. 
Fischer, N. (1984): Honorarvereinbarung mit der Kassenärztlichen Bundesvereinigung. In: Die Ersatzkasse, Jg. 64, S. 279-281.

Fischer, N. (1985): Ausgabenentwicklung im ambulanten ärztlichen Bereich mit dem Anstieg der Grundlohnsumme in Einklang gebracht. In: Die Ersatzkasse, Jg. 65, S. 221-224.

Fisher, G.H. (1971): Cost considerations in systems analysis. New York.

Fisz, M. (1980): Wahrscheinlichkeitsrechnung und mathematische Statistik. 10. Auflage. Berlin.

Flagle, C.D. (1967): A decision theoretical comparison of three procedures of screening for a single disease. In: LeCam, L.M./Neyman, J. (Eds.): Proceedings of the Fifth Berkeley Symposium on mathematical statistics and probability. Vol. IV: Biology and Problems of Health; Berkeley and Los Angeles; S. 887-901.

Fleiss, J.L. (1973): Statistical methods for rates and proportions. New York.

French, S. (1983): A survey and interpretation of multi-attribute utility theory. In: French, S./Hartley, R./Thomas, L.C./White, D.J. (Eds): Multi-objective decision-making. London, S. 263-277.

French, S. (1986): Decision theory: an introduction to the mathematics of rationality. New York et al.

Fuchs, V.R./Zeckhauser, R. (1987): Valuing health - a "priceless" commodity. In: American Economic Review, Vol. 77, S. 263-268.

Gäfgen, G. (1980): Leistungsmessung im Gesundheitswesen - ein Beispiel für die Ökonomie des Dienstleistungssektors. In: Hamburger Jahrbuch für Wirtschafts- und Gesellschaftspolitik, 25. Jahr, S. 177-196.

Gäfgen, G. (1990): Die Bewertung therapeutischer und diagnostischer Verbesserungen. In: Gäfgen, G.: Gesundheitsökonomie: Grundlagen und Anwendungen, Baden-Baden, S. 299-318.

Galen, R.S./ Gambino, S.R. (1979): Norm und Normabweichung klinischer Daten. Der prädiktive Wert und die Effizienz von medizinischen Diagnosen. Stuttgart, New York.

Gallagher, D.R./Smith, V.K. (1985): Measuring values for environmental resources under uncertainty. In: Journal of Environmental Economics and Management, Vol. 12, S. 132-143. 
Gart, J.J./Buck, A.A. (1966): Comparison of a screening test and a reference test in epidemiologic studies. II. A probabilistic model for the comparison of diagnostic tests. In: American Journal of Epidemiology, Vol 83, S. 593-602.

Gavin, J.L./Mohnsen, M.E. (1985): Cost justification of the automicrobic system based on decreased turn-around-time. Manuskript, Cedar Rapids, Iowa.

Gelfand, D.W./Ott, D.J. (1985): Methodologic Considerations in Comparing Imaging Methods. In: American Journal of Roentgenology, Vol. 144, S. $1117-1121$.

Gerstman, B.B./Cappucci, D.T. (1986): Evaluating the reliability of diagnostic test results. In: Journal of the American Veterinary Medical Association, Vol. 188, S. 248-251.

Gillespie, K.N./Elixhauser, A./Reker, D.M./Fletcher, J.W./Wolinsky, F.D. (1986): Cost-benefit and cost-effectiveness analyses of magnetic resonance imaging. In: International Journal of Technology Assessment in Health Care, Vol. 2, S. 537-550.

Ginsberg, A.S. (1972): The diagnostic process viewed as a decision problem. In: Jacquez, J.A. (Ed.): Computer diagnosis and diagnostic methods; Springfield/Illinois, S. 203-240.

Ginsberg, A.S./Offensend, F.L. (1968): An application of decision theory to a medical diagnosis-treatment problem. In: IEEE Transactions on Systems Science and Cybernetics, Vol. SSC-4, S. 355-362.

Glass, N.J. (1973): Cost-benefit analysis and health services. In: Health trends, Vol. 5, S. 51-56.

Goddeeris, J.H./Bronken, T.P. (1985): Benefit-cost analysis of screening. A comparison of tests for gonorrhea. In: Medical Care, Vol. 23, S. 12421255.

Golberg, M.A. (1984): An Introduction to Probability Theory with Statistical Applications. New York and London.

Good, I.J. (1963): Maximum entropy for hypothesis formulation, especially for multidimensional contingency tables. In: Annals of Mathematical Statistics, Vol. 34, S. 911-934.

Gorman, W.M. (1955): The intransitivity of certain criteria used in welfare economics. In: Oxford Economic Papers, N.S. Vol. 7, S. 25-35. 
Gorry, G.A. (1978): Decision analysis: principles for clinical application. In: Benson, E.S./Rubin, M. (Eds): Logic and economics of clinical laboratory use, New York and Oxford, S. 47-58.

Gorry, G.A./Pauker, S.G./Schwartz, W.B. (1978): The diagnostic importance of the normal finding. In: New England Journal of Medicine, Vol. 298, S. 486-489.

Goss, S. (1983): Cost-benefit analysis of the detection and treatment of Phenylketonuria (PKU) in newly-born babies in Belgium. In: Cahiers économiques de Bruxelles, No. 99, S. 459-468.

Gottinger, H.W. (1980): Elements of statistical analysis. Berlin, New York.

Graham, D.A. (1981): Cost-benefit analysis under uncertainty. In: American Economic Review, Vol. 71, S. 715-725.

Gravelle, H.S.E./Simpson, P.R./Chamberlain, J. (1982): Breast cancer screening and health service costs. In: Journal of Health Economics, Vol. 1, S. 185-207.

Green, D.M./Swets, J.A. (1966): Signal Detection Theory and Psychophysics. New York et al.

Greenland, P./Mushlin, A.I./Griner, P.F. (1979): Discrepancies between knowledge and use of diagnostic studies in asymptomatic patients. In: Journal of Medical Education, Vol. 54, S. 863-869.

Gremy, F./Goldberg, M. (1977): Decision making methods in medicine. In: Reichertz, P.L./Goos, G. (Eds.): Informatics and medicine. An advanced course. Berlin, Heidelberg, New York, S. 419-459.

Griner, P.F./Mayewski, R.J./Mushlin, A.I./Greenland, P. (1981): Selection and interpretation of diag-nostic tests and procedures. In: Annals of Internal Medicine, Vol. 94, S. 553-600.

Gross, R. (1966): From intuition to competition. Development and problems of medical diagnosis. In: Methods of Information in Medicine, Vol. 5, S. 35-39.

Gross, R. (1969): Medizinische Diagnostik - Grundlagen und Praxis. Berlin, Heidelberg, New York.

Gross, R. (1973): Der Prozeß der Diagnose. In: Deutsche medizinische Wochenschrift, 98. Jg., S. 783-787. 
Gross, R. (1982): Einige Grundlagen der medizinischen Entscheidungstheorie. In: Lang, H./ Rick, W./ Büttner, H. (Hrsg.): Strategien für den Einsatz klinisch-chemischer Unter-suchungen. Berlin, Heidelberg, New York; S. 56-62.

Gross, R./ Spechtmeyer, H. (1982): Erhebung der Vorgeschichte und körperliche Untersuchung. In: Losse, H./Wetzels, E. (Hrsg.): Rationelle Diagnostik in der inneren Medizin. Stuttgart, New York; S. 3-19.

Gross, R. (1985): Rationalität in der medizinischen Diagnostik. In: Vogel, H.R. (Hg.): Effizienz und Effektivität medizinischer Diagnostik; Stuttgart, S. 113-129.

Grosse, R.N. (1970): Problems of resource allocation in health. In: Haveman, R.H./Margolis, J. (Eds): Public expenditures and policy analysis. Chicago, S. 518-548.

Guder, W.G. (1980): Einflußgrößen und Störfaktoren bei klinisch-chemischen Untersuchungen. In: Der Internist, Jg. 21, S. 533-542.

Guder, W.G./Schleicher, E. (1990): Who defines the medical requirements of analytical tests? In: Journal of Clinical Chemistry and Clinical Biochemistry, Vol. 28, S. 125-128.

Guiasu, S. (1977): Information theory with applications. New York et al.

Guyatt, G./Drummond, M.F. (1986): Guidelines for the clinical and economic assessment of health technologies: the case of magnetic resonance. In: International Journal of Technology Assessment in Health Care, Vol. 2, S. 551-566.

Habbema, J.D.F./Hilden, J. (1981): The measurement of performance in probabilistic diagnosis. IV. Utility considerations in therapeutics and prognostics. In: Methods of Information in Medicine, Vol. 20, S. 80-96.

Habbema, J.D.F./Hilden, J./Bjerregaard, B. (1978): The measurement of performance in probabilistic diagnosis. I. The problem, descriptive tools, and measures based on classification matrices. In: Methods of Information in Medicine, Vol. 17, S. 217-226.

Habbema, J.D.F./van Oortmarssen, G.J. (1982): Performance characteristics of screening tests. In: Clinics in Laboratory Medicine, Vol. 2, S. 639656.

Hagard, S./Carter, F./Milne, R.G. (1976): Screening for spina bifida cystica. A cost-benefit analysis. In: British Journal of Preventive and Social Medicine, Vol. 30, S. 40-53. 
Hamerle, A./Tutz, G. (1984): Zusammenhangsanalysen in mehrdimensionalen Kontingenztabellen - das loglineare Modell. In: Fahrmeier, L./Hamerle, A. (Hg.): Multivariate statistische Verfahren, Berlin und New York, S. 473-574.

Hammond, P.J. (1980): Cost benefit analysis as a planning procedure. In: Currie, D.A./Peters, W. (Eds): Contemporary economic analysis, Vol. 2, London, S. 221-250.

Hanley, J.A./McNeil, B.J. (1982): The Meaning and Use of the Area under a Receiver Operating Characteristic (ROC) Curve. In: Radiology, Vol. 143 , S. 29-36.

Hanusch, H. (1987): Nutzen-Kosten-Analyse. München.

Harris, J.M. (1981): The Hazards of Bedside Bayes. In: Journal of the American Medical Association, Vol. 246, S. 2602-2605.

Hasinger, A. (1985): Medizinische Diagnostik aus der Sicht des Politikers. In: Vogel, H.R. (Hg.): Effizienz und Effektivität medizinischer Diagnostik. Stuttgart, S. 105-110.

Henderson, A. (1941): Consumer's surplus and the compensating variation. In: Review of Economic Studies, Vol. 8, S. 117-121.

Henke, K-D. (1978): Bestimmung und Steigerung der Effizienz im öffentlichen Sektor - ein Überblick. In: Das Wirtschaftsstudium (WISU), 7. Jg., S. 601-605.

Henke, K.-D. (1986): Die direkten und die indirekten Kosten von Krankheiten in der Bundesrepublik Deutschland im Jahre 1980. In: Henke, K.D./Metze, I. (Hg.): Finanzierung im Gesundheitswesen, Band $10 \mathrm{der}$ Beiträge zur Gesundheitsökonomie, Gerlingen, S. 209-262.

Hesse, H. (1980): Nutzen-Kosten-Analyse I: Theorie. In: Albers, W. et al. (Hg.): Handwörterbuch der Wirtschaftswissenschaft, 5. Band, Stuttgart, Tübingen, Göttingen, S. 361-382.

Heuser, H. (1988): Lehrbuch der Analysis Teil 2. 4. Auflage, Stuttgart.

Hicks, J.R. (1940): The valuation of sọcial income. In: Economica, N.S. Vol. 7, S. $107-124$.

Hicks, J.R. (1981): The four consumer's surpluses. In: Hicks, J.R.: Wealth and welfare. Collected essays on economic theory, Vol. 1, Oxford, S. 114132. 
Hilden, J. (1984): Statistical diagnosis based on conditional independence does not require it. In: Computers in Biology and Medicine, Vol. 14, S. 429435 .

Hilton, S. (1990): Near patient testing in general practice: a review. In: British Journal of General Practice, Vol. 40, S. 32-36.

Hirshleifer, J. (1965): Investment decision under uncertainty: choice-theoretic approaches. In: Quarterly Journal of Economics, Vol. 79, S. 509-536.

Hirshleifer, J./Riley, J.G. (1979): The analytics of uncertainty and information an expository survey. In: Journal of Economic Literature, Vol. 17, S. $1375-1421$.

Hodgson, T.A. (1983): The state of the art of cost-of-illness estimates. In: Scheffler, R.M. (Ed.): Advances in health economics and health services research, Vol. 4, Greenwich, London, S. 129-164.

Hodgson, T.A./Meiners, M.R. (1982): Cost-of-illness methodology: a guide to current practices and procedures. In: Health and Society. Milbank Memorial Fund Quarterly, Vol. 60, S. 429-462.

Horisberger, B. (1984): Kosten-Nutzen-Analyse der Ulkustherapie. In: Schweizerische medizinische Wochenschrift, 114. Jg., S. 699-706.

Horisberger, B. (1987): Sofortdiagnostik und Wirtschaftlichkeit im Praxislabor. In: Arzt und Wirtschaft. Zeitschrift für erfolgreiche Praxisführung, 21. Jg., S. 1-8.

Horisberger, B./van Eimeren, W. (Hg.) (1986): Die Kosten-Nutzen-Analyse. Methodik und Anwendung am Beispiel von Medikamenten. Berlin et al.

Hubbard, W.N. (1964): Welcome and prologue. In: Jacquez, J.A. (Ed.): The diagnostic process, Ann Arbor, S. 3-5.

Hui, S.L./Walter, S.D. (1980): Estimating the error rates of diagnostic tests. In: Biometrics, Vol. 36, S. 167-171.

Hutten, H. (1985): Diagnostic overkill. In: Medical Progress through Technology, Vol. 10, S. 181-182.

Ingram, D./Bloch, R.F. (Eds) (1984): Mathematical Methods in Medicine. Part I: Statistical and Analytical Techniques. Chichester et al.

Jacquez, J.A. (1972): Algorithmic diagnosis: a review with emphasis on Bayesian methods. In: Jacquez, J.A. (Ed.): Computer diagnosis and diagnostic methods, Springfield/Ill. S. 374-393. 
Jaglom, A.M./Jaglom, I.M. (1984): Wahrscheinlichkeit und Information. 4. Auflage. Thun, Frankfurt/Main.

Jennett, B. (1986): Diagnostic investigations - towards more appropriate use: I. A clinician's viewpoint. In: Health Bulletin, Vol. 44, S. 8-12.

Jennett, B./Teasdale, G.M./Knill-Jones, R.P. (1975): Predicting outcome after head injury. In: Journal of the Royal College of Physicians of London, Vol. 9, S. 231-237.

Jensen, M.C./Meckling, W.H. (1976): Theory of the firm: managerial behavior, agency costs and ownership structure. In: Journal of Financial Economics, Vol. 3, S. 305-360.

Jesdinsky, H.J. (1972): Diagnose-Modelle in der Medizin. In: Methods of Information in Medicine, Vol. 11, S. 48-59.

Jönsson, B./Lindgren, B. (1980): Five common fallacies in estimating the economic gains of early discharge. In: Social Science \& Medicine, Vol. 14, S. 27-33.

Johannesson, M./Jönsson, B. (1990): Economic evaluation in health care - is there a role for cost-benefit analysis? Paper presented at the Second World Congress on Health Economics, Zurich.

Johnson, H.A. (1989): Diagnosis by the bit: a method for evaluating the diagnostic process. In: Annals of Clinical and Laboratory Science, Vol. 19, S. 323-331.

Judge, G.G./Hill, R.C./Griffiths, W.E./Lütkepohl, H./Lee, T.-C. (1988): Introduction to the theory and practice of econometrics. 2nd ed., New York et al.

Kaiser, H. (1989): Zur Messung allokativer und distributiver Effekte wohlfahrtsstaatlicher Maßnahmen. Theorie und Methodik am Beispiel der Steuerpolitik. Arbeitspapier Nr. 283 des SFB 3: Mikroanalytische Grundlagen der Gesellschaftspolitik. Frankfurt/Main und Mannheim.

Kaldor, N. (1939): Welfare propositions and interpersonal comparisons of utility. In: Economic Journal, Vol. 49, S. 549-552.

Kassirer, J.P. (1976): The principles of clinical decision-making: an introduction to decision analysis. In: Yale Journal of Biology and Medicine, Vol. 49 , S. 149-164.

Keeler, E.B. (1976): The value of a diagnostic test. Santa Monica. Published by the Rand Corporation as Report No. P-5603. 
Keeney, R.L./Raiffa, H. (1976): Decisions with multiple objectives. Preferences and value tradeoffs. New York et al.

Keller, H. (1986): Klinisch-chemische Labordiagnostik für die Praxis. Analyse, Befund, Interpretation. Stuttgart, New York.

Kelsey, C.A./Moseley, R.D./Garcia, J.F./Mettler, F.A./Parker, T.W./Juhl, J.H. (1985): ROC and Contrast Detail Image Evaluation Tests Compared. In: Radiology, Vol. 154, S. 629-631.

Kendall, M./Stuart, A. (1979): The advanced theory of statistics, Vol. 2: Inference and relationship. Fourth edition, London.

Khinchin, A.I. (1957): Mathematical foundations of information theory. New York.

Klarman, H.E. (1965): Syphilis control programs. In: Dorfman, R. (Ed.): Measuring benefits of government investments, Washington, D.C., S. 367-410.

Klarman, H.E. (1974): Application of cost-benefit analysis to the health services and the special case of technologic innovation. In: International Journal of Health Services, Vol. 4, S. 325-352.

Kodlin, D. (1972): A note on the cost-benefit problem in screening for breast cancer. In: Methods of Information in Medicine, Vol. 11, S. 242-247.

Köbberling, J. (1982): Der prädiktive Wert diagnostischer Maßnahmen. In: Deutsche Medizinische Wochenschrift, Jg. 107, S. 591-595.

Köbberling, J./Trampisch, H.-J./Windeler, J. (Hg.) (1989): Memorandum zur Evaluierung diagnostischer Maßnahmen. Im Auftrag der Arbeitsgruppe "Methoden der Prognose- und Entscheidungsförderung" in der Deutschen Gesellschaft für Medizinische Dokumentation, Informatik und Statistik e.V. (GMDS). Heft 10 der Schriftenreihe der GMDS. Stuttgart, New York.

Kofler, E. (1989): Prognosen und Stabilität bei unvollständiger Information. Frankfurt/Main.

Kofler, E./Menges, G. (1976): Entscheidungen bei unvollständiger Information. Berlin, Heidelberg, New York.

Koller, S. (1967): Mathematisch-statistische Grundlagen der Diagnostik. In: Klinische Wochenschrift, 45. Jg., S. 1067-1072. 
Kossow, K.-D. (1986): German experiences and perspectives of 'in-office' testing. In: Marks, V./Alberti, K.G.M.M. (Eds): Clinical biochemistry nearer the patient II, London et al., S. 114-121.

Krengel, U. (1990): Einführung in die Wahrscheinlichkeitstheorie und Statistik. 2. Auflage, Braunschweig.

Kriedel, T. (1980): Wirtschaftlichkeitsuntersuchungen von Gesundheitsmaßnahmen. Ein Vorschlag zur Ertragsmessung mit Hilfe eines Gesundheitsstatus-Index. In: Zeitschrift für Sozialwissenschaft, Vol. 31, S. 337354.

Krieg, A.F. (1986): When is a diagnostic test result positive? Decision tree models based on net utility and threshold. In: Archives of Pathology and Laboratory Medicine, Vol. 110, S. 787-791.

Krischer, J.P. (1976): Utility structure of a medical decision-making problem. In: Operations Research, Vol. 24, S. 951-972.

Kullback, S. (1959): Information theory and statistics. New York.

Laffont, J.-J. (1989): The economics of uncertainty and information. Cambridge/Mass. and London.

Laisney, F./Schmachtenberg, R. (1989): For welfare prescriptions, reference price independence implies price independence. Discussion Paper No. 404-89 (Beiträge zur angewandten Wirtschaftsforschung). Universität Mannheim.

LaValle, I.H. (1968): On cash equivalents and information evaluation in decisions under uncertainty. In: Journal of the American Statistical Association, Vol. 63, S. 252-276, S. 277-284 und S. 285-290.

Ledley, R.S./Lusted, L.B. (1959): Reasoning Foundations of Medical Diagnosis. In: Science, Vol. 130, S. 9-21.

Lindeneg, K. (1985): Economic appraisal in occupational health and safety regulation. A study of some standard methods. In: Scandinavian Journal of Social Medicine, Vol. 13, S. 127-132.

Lindley, D.V. (1974): Einführung in die Entscheidungstheorie. Frankfurt/Main.

Lindley, D.V. (1975): The role of utility in decision-making. In: Journal of the Royal College of Physicians of London, Vol. 9, S. 225-230.

Loomes, G./McKenzie, L. (1989): The use of QALYs in health care decision making. In: Social Science \& Medicine, Vol. 28, S. 299-308. 
Luce, B.R. (1981): Allocating costs and benefits in disease prevention programs: an application to cervical cancer screening. In: Office of Technology Assessment (OTA): The implications of cost-effectiveness analysis of medical technology; background paper no. 2: case studies of medical technologies; Washington, D.C.; case study no. 7.

Ludbrook, A./Mooney, G.H. (1986): Diagnostic investigation - Towards more appropriate use: II. The economic perspective. In: Health Bulletin, Vol. 44, S. 13-19.

Luft, H.S. (1976): Benefit-cost analysis and public policy implementation: from normative to positive analysis. In: Public Policy, Vol. 24, S. 437-462.

Lusted, L.B. (1968): Introduction to medical decision making. Springfield/Illinois.

Lusted, L.B./Stahl, W.R. (1964): Conceptual Models of Diagnosis. In: Jacquez, J.A. (Ed.): The diagnostic process. Ann Arbor, S. 157-184.

Machina, M. (1987): Expected utility hypothesis. In: Eatwell, J./Milgate, M./Newman, P. (Eds): The New Palgrave. A dictionary of economics, Vol. 2, London et al., S. 232-239.

Mag, W. (1977): Entscheidung und Information. München.

Mag, W. (1990): Grundzüge der Entscheidungstheorie. München.

Mai, N./Hachmann, E. (1977): Anwendung des Bayes-Theorems in der medizinischen Diagnostik - eine Literaturübersicht. In: Metamed, 1. Jg., S. 161205.

Manuel, B.M. (1990): Professional liability - a no-fault solution. In: New England Journal of Medicine, Vol. 322, S. 627-631.

Margolis, J. (1977): Shadow prices for incorrect or nonexistent market values. In: Haveman, R.H./Margolis, J. (Eds): Public expenditure and policy analysis, second edition, Chicago, S. 204-220.

Marschak, J. (1959): Remarks on the economics of information. Zitiert nach dem Wiederabdruck in: Marschak, J. (1974): Economic information, decision and prediction. Selected Essays, Vol. 2. Dordrecht, Boston, London; S. 91-117.

Marschak, J./Miyasawa, K. (1968): Economic comparability of information systems. Zitiert nach dem Wiederabdruck in: Marschak, J. (1974): Economic information, decision and prediction. Selected essays, Vol. 2. Dordrecht, Boston, London; S. 201-249. 
Marshall, R.J. (1989): The predictive value of simple rules for combining two diagnostic tests. In: Biometrics, Vol. 45, S. 1213-1222.

McGuire, A./Henderson, J./Mooney, G.H. (1988): The economics of health care. An introductory text. London.

McKenzie, L. (1957): Demand theory without a utility index. In: Review of Economic Studies, Vol. 24, S. 185-189.

McMillan, B. (1961): An elementary approach to the theory of information. In: SIAM Review, Vol. 3, S. 211-229.

McNeil, B.J. (1979): Pitfalls in and requirements for evaluations of diagnostic technologies. In: Wagner, J.L. (Ed.): Medical Technology, Washington D.C., S. 33-39.

McNeil, B.J./Adelstein, S.J. (1976): Determining the value of diagnostic and screening tests. In: Journal of Nuclear Medicine, Vol 17, S. 439-448.

McNeil, B. J./Hanley, J.A. (1984): Kritische Fragen zu einer neuen diagnostischen Technologie: Computertomographie des Schädels als Fallstudie. In: Culyer, A.J./Horisberger, B. (Hg.): Technologie im Gesundheitswesen. Medizinische und wirtschaftliche Aspekte; Berlin et al.; S. 319-326.

McNeil, B.J./Hanley, J.A./Funkenstein, H.H./Wallman, J. (1983): Paired receiver operating characteristic curves and the effect of history on radiographic interpretation. CT of the head as a case study. In: Radiology, Vol. 149, S. 75-77.

McNeil, B.J./Keeler, E./Adelstein, S.J. (1975): Primer on certain elements of medical decision making. In: New England Journal of Medicine, Vol. 293, S. 211-215.

Medawar, P.B. (1969): Induction and Intuition in Scientific Thought. London.

Meister, H. (1985): Leistungsfähigkeit und Grenzen der Labordiagnostik. In: Zeitschrift für die gesamte innere Medizin, Vol. 40, S. 681-684.

Mendelsohn, R./Strang, W.J. (1984): Cost-benefit analysis under uncertainty: comment. In: American Economic Review, Vol. 74, S. 1096-1099.

Menges, G. (1974): Grundmodelle wirtschaftlicher Entscheidungen. Einführung in moderne Entscheidungstheorien. 2. Auflage, Düsseldorf.

Metz, C.E. (1978): Basic Principles of ROC Analysis. In: Seminars in Nuclear Medicine, Vol. 8, S. 283-298. 
Metz, C.E./Goodenough, D.J./Rossmann, K. (1973): Evaluation of receiver operating characteristic curve data in terms of information theory, with applications in radiography. In: Radiology, Vol. 109, S. 297-303.

Metz, C.E./Kronman, H.B. (1980): Statistical significance tests for binormal ROC curves. In: Journal of mathematical Psychology, Vol. 22, S. 218243.

Miller, S.R. (1932): Contemporary fads and fallacies, therapeutic and diagnostic, which reflect dangerous professional credulity. In: Pennsylvania Medical Journal, Vol. 35, S. 347-345.

Mishan, E.J. (1988): Cost-benefit analysis. An informal introduction. Fourth edition. London et al.

Mitchell, R.C./Carson, R.T. (1989): Using surveys to value public goods: the contingent valuation method. Washington, D.C.

Molinaro, R. (1986): Gesundheitswesen und Kostendämpfung in der Bundesrepublik. Beschreibung und Analyse aus schweizerischer Sicht. Berlin et al.

Monfort, A. (1982): Cours de statistique mathématique. Paris.

Mooney, G.H. (1990): The agency relationship: an empty box? Paper presented to the Second World Congress in Health Economics, Zurich.

Morgan, P.P. (1984): Why rule out unlikely diagnoses? In: Canadian Medical Association Journal, Vol. 130, S. 1424.

Moss, J.M. (1987): The complete blood count and physician payment. In: Annals of Internal Medicine, Vol. 106, S. 642.

Musgrave, R.A./Musgrave, P.B./Kullmer, L. (1987): Die öffentlichen Finanzen in Theorie und Praxis. 4. Auflage, 1. Band. Tübingen.

Narr, H. (1984): Allgemeines Arztrecht. In: Häußler, S./Liebold, R./Narr, H.: Die kassenärztliche Tätigkeit, 3. Auflage, Berlin et al., S. 63-105.

Newman, P. (1987): Duality. In: Eatwell, J./Milgate, M./Newman, P. (Eds):

The new Palgrave. A dictionary of economics, Vol. 1, London, S. 924934.

Neyman, J. (1947): Outline of statistical treatment of the problem of diagnosis. In: Public Health Reports, Vol. 62, S. 1449-1456.

Neyman, J. (1950): First course in probability and statistics. New York.

Ng, Y.-K. (1979): Welfare economics. Introduction and basic development. London and Basingstoke. 
Nissen-Meyer, S. (1964): Evaluation of screening tests in medical diagnosis. In: Biometrics, Vol. 20, S. 730-755.

North, D.W. (1968): A tutorial introduction to decision theory. In: IEEE Transactions on Systems Science and Cybernetics, Vol. SSC-4, S. 200-210.

Norusis, M.J./Jacquez, J.A. (1975): Diagnosis. I. Symptom Nonindependence in mathematical models for diagnosis. In: Computers and Biomedical Research, Vol. 8, S. 156-172.

Osborne, C.A. (1983): Diagnosis of a diagnosis. In: Journal of the American Veterinary Medical Association, Vol. 182, S. 890-891.

o.V. (1987): Immer noch zu viele Laboruntersuchungen. In: Die Neue Ärztliche, Nr. 213 (6./7.11.1987), S. 3.

Parrino, T.A./Mitchell, R. (1989): Diagnosis as a skill: a clinical perspective. In: Perspectives in Biology and Medicine, Vol. 33, S. 18-44.

Patrick, E.A. (1977): Expected outcome loss to evaluate medical diagnosis and treatment. In: Computers in Biology and Medicine, Vol. 7, S. 1-8.

Patrick, E.A. (1979): Decision Analysis in Medicine: Methods and Applications. Boca Raton.

Pauker, S.G./Kassirer, J.P. (1978): Clinical application of decision analysis: a detailed illustration. In: Seminars in Nuclear Medicine, Vol. 8, S. 324335 .

Pauker, S.G./Kassirer, J.P. (1980): The threshold approach to clinical decision making. In: New England Journal of Medicine, Vol. 302, S. 11091117 .

Pauly, M.V. (1980): Doctors and their workshops: economic models of physician behavior. National Bureau of Economic Research Monograph, Chicago and London.

Pearce, D.W. (1983): Cost-benefit analysis. Second edition. London and Basingstoke.

Pearce, D.W./Nash, C.A. (1981): The social appraisal of projects. A text in costbenefit analysis. London and Basingstoke.

Pfanzagl, J. (1977): Statistische Schätzfunktionen und ihre Eigenschaften. In: Albers, W. et al. (Hg.): Handwörterbuch der Wirtschaftswissenschaft (HdWW), 7.Bd. Stuttgart et al., S. 261-270.

Pfanzagl, J. (1988): Elementare Wahrscheinlichkeitsrechnung. Berlin, New York. 
Philbrick, J.T./Horwitz, R.I./Feinstein, A.R. (1980): Methodologic Problems of Exercise Testing for Coronary Artery Disease: Groups, Analysis and Bias. In: American Journal of Cardiology, Vol. 46, S. 807-812.

Phillips, W.C./Scott, J.A./Blasczcynski, G. (1983): How sensitive is "sensitivity"; how specific is "specificity"? In: American Journal of Roentgenology, Vol. 140, S. 1265-1270.

Pratt, J.W./Zeckhauser, R.J. (1985): Principals and agents: an overview. In: Pratt, J.W./Zeckhauser, R.J. (Eds): Principals and agents: the structure of business. Boston, S. 1-35.

Rahner, E. (1965): Kosten- und Ertragsanalyse im Gesundheitswesen. Saarbrücken.

Raiffa, H. (1968): Decision Analysis. Introductory Lectures on Choices under Uncertainty. Reading, Massachusetts.

Raiffa, H./Schlaifer, R. (1961): Applied statistical decision theory. Cambridge/Mass. and London.

Ransohoff, D.F./Feinstein, A.R. (1978): Problems of spectrum and bias in evaluating the efficacy of diagnostic tests. In: New England Journal of Medicine, Vol. 299, S. 926-930.

Rees, R. (1985a): The theory of principal and agent part I. In: Bulletin of Economic Research, Vol. 37, S. 3-26.

Rees, R. (1985b): The theory of principal and agent part 2. In: Bulletin of Economic Research, Vol. 37, S. 75-95.

Renyi, A. (1960): Some fundamental questions of information theory. Zitiert nach dem Wiederabdruck in: Turan, P. (Ed.) (1976): Selected Papers of Alfred Renyi, Vol. 2. Budapest, S. 526-552.

Renyi, A. (1965): On the foundations of information theory. Zitiert nach dem Wiederabdruck in: Turan, P.(Ed.) (1976): Selected Papers of Alfred Renyi, Vol. 2. Budapest, S. 304-318.

Renyi, A. (1966): Wahrscheinlichkeitsrechnung. Mit einem Anhang über Informationstheorie. 2. Auflage. Berlin.

Renyi, A. (1970): Foundations of probability. San Francisco et al.

Renyi, A. (1982): Tagebuch über die Informationstheorie. Basel, Boston, Stuttgart. 
Renyi, A./Balatoni, J. (1957): Über den Begriff der Entropie. Zitiert nach dem Wiederabdruck in: Turan, P. (Ed.) (1976): Selected Papers of Alfred Renyi, Vol. 2. Budapest, S. 113-130.

Richter, H. (1963): Subjektive Wahrscheinlichkeit und multisubjektive Tests. In: Zeitschrift für Wahrscheinlichkeitstheorie, 1. Jg., S. 271-277.

Richter, K./Abel, U./Klar, R./Köbberling, J./Trampisch, H.-J./Windeler, J. (1988): Die Grundlagen der Validierung einfacher diagnostischer Tests. In: Klinische Wochenschrift, 66. Jg., S. 655-661.

Rifkin, R.D. (1985): Maximum Shannon Information Content of Diagnostic Medical Testing. In: Medical Decision Making, Vol. 5, 179-190.

Ross, S.A. (1973): The economic theory of agency: the principal's problem. In: American Economic Review, Vol. 63, S. 134-139.

Sachsse, H. (1974): Einführung in die Kybernetik. Braunschweig.

Sachverständigenrat für die Konzertierte Aktion im Gesundheitswesen (1989): Qualität, Wirtschaftlichkeit und Perspektiven der Gesundheitsversorgung. Jahresgutachten 1989. Baden-Baden.

Samuelson, P.A. (1974): Complementarity. An essay on the 40th anniversary of the Hicks-Allen revolution in demand theory. In: Journal of Economic Literature, Vol. 12, S. 1255-1289.

Samuelson, P.A. (1983): Foundations of economic analysis. Enlarged edition. Cambridge/Mass., London.

Schechter, M.T./Sheps, S.B. (1985): Diagnostic testing revisited: pathways through uncertainty. In: Canadian Medical Association Journal, Vol. 132 , S. $755-760$.

Schicke, R.K. (1984): Computer-Tomographie: Zur Effektivität technologischer Innovationen. In: Münnich, F.E./Oettle, K. (Hg.): Ökonomie des technischen Fortschritts in der Medizin. Gerlingen, S. $107 \mathrm{ff}$.

Schindel, V. (1979): Entscheidungsorientierte Interpretationen des Informationswertes und ihre jeweilige Eignung zur Beurteilung von Informationsbeschaffungsentscheidungen. In: Zeitschrift für Betriebswirtschaft, 49. Jg., S. 39-56.

Schmalensee, R. (1972): Option demand and consumer's surplus: valuing price changes under uncertainty. In: American Economic Review, Vol. 62, S. 813-824. 
Schmid, A.A. (1989): Benefit-cost analysis. A political economy approach. Boulder, London.

Schneeweiß, H. (1966): Das Grundmodell der Entscheidungstheorie. In: Statistische Hefte, 7. Jahrgang, S. 125-137.

Schroeder, S.A./Kenders, K./Cooper, J.K./Piemme, T.E. (1973): Use of laboratory tests and pharmaceuticials. Variation among physicians and effect of cost audit on subsequent use. In: Journal of the American Medical Association, Vol. 225, S. 969-973.

Schulten, H. (1961): Der Arzt. 2. Auflage. Stuttgart.

Scitovsky, A.A. (1982): Estimating the direct costs of illness. In: Health and Society. Milbank Memorial Fund Quarterly, Vol. 60, S. 463-491.

Scitovsky, T. (1941): A note on welfare propositions in economics. In: Review of Economic Studies, Vol. 9, S. 77-88.

Selecta Supplement (1987): Die Trockenchemie in der Arztpraxis. Heft 2 vom 20.4.1987.

Sheps, S.B./Schechter, M.T. (1984): The Assessment of Diagnostic Tests. A survey of Current Medical Research. In: Journal of the American Medical Association, Vol. 252, S. 2418-2422.

Showstack, J.A./Schroeder, S.A. (1981): The cost and effectiveness of upper gastrointestinal endoscopy. In: Office of Technology Assessment (OTA): The Implications of Cost-Effectiveness Analysis of Medical Technology; Background Paper no. 2; Case studies of Medical Technologies; Washington D.C., case study no. 8.

Siebert, C.D./Zaidi, M.A. (1975): Benefit-cost analysis in health care. In: Biosciences Communications, Vol. 1, S. 193-218.

Sinn, H.-W. (1980): Ökonomische Entscheidungen bei Ungewißheit. Tübingen.

Skene, A. (1984): Formal approaches to the analysis of clinical decisions. In: Ingram, D./Bloch, R.F. (Eds.): Mathematical methods in Medicine. Part I: Statistical and Analytical Techniques. Chichester et al., S. 119-159.

Smith, D.B./Kaluzny, A.D. (1986): The white labyrinth. A guide to the health care system. Ann Arbor.

Smith, V.K. (1987): Uncertainty, benefit-cost analysis and the treatment of option value. In: Journal of Environmental Economics and Management, Vol. 14, S. 283-292. 
Smith, V.K. (1990): Valuing amenity resources under uncertainty: a sceptical view of recent resolutions. In: Journal of Environmental Economics and Management, Vol. 19, S. 193-202.

Sox, H.C. (1986): Probability Theory in the Use of Diagnostic Tests. An Introduction to Critical Study of the Literature. In: Annals of Internal Medicine, Vol. 104, S. 60-66.

Sox, H.C./Margulies, I./Sox, C.H. (1981): Psychologically mediated effects of diagnostic tests. In: Annals of Internal Medicine, Vol. 95, S. 680-685.

Stason, W.B./Weinstein, M.C. (1977): Allocation of resources to manage hypertension. In: New England Journal of Medicine, Vol. 296, S. 732739.

Stein, R. (1986): Ethische Grenzen für Diagnostik und Therapie. In: Frankfurter Allgemeine Zeitung vom 4.6.1986, S. 33.

Steiner, K.C./Smith, H.A. (1973): Application of cost-benefit analysis to a PKU screening program. In: Inquiry, Vol. 10, S. 34-40.

Stenger, H. (1986): Stichproben. Heidelberg, Wien.

Stoddart, G.L. (1982): Economic evaluation methods and health policy. In: Evaluation and the health professions, Vol. 5, S. 393-414.

Straub, W.H./Gur, D. (1990): The hidden costs of delayed access to diagnostic imaging information: impact on PACS implementation. In: American Journal of Roentgenology, Vol. 155, S. 613-616.

Swets, J.A. (1972): Signal detection in medical diagnosis. In: Jacquez, J.A. (Ed.): Computer diagnosis and diagnostic methods, Springfield/Illinois, S. 8-28.

Swets, J.A. (1979): ROC Analysis Applied to the Evaluation of Medical Imaging Techniques. In: Investigative Radiology, Vol. 14, S. 109-121.

Swets, J.A./Pickett, R.M. (1982): Evaluation of Diagnostic Systems. Methods from Signal Detection Theory. New York et al.

Teather, D. (1974a): Diagnosis - Methods and Analysis. In: Institute of Mathematics and its Applications, Vol. 10, S. 37-41.

Teather, D. (1974b): Statistical techniques for diagnosis. In: Journal of the Royal Statistical Society, Series A, Vol. 137, Part 2, S. 231-244.

Theil, H. (1967): Economics and information theory. Amsterdam. 
Thibodeau, L.A. (1981): Evaluating diagnostic tests. In: Biometrics, Vol. 37, S. 801-804.

Thomas, J.B. (1969): An Introduction to Statistical Communication Theory. New York et al.

Tolpin, H.G. (1980): Economics of health care. In: Journal of the American Dietetic Association, Vol. 76, S. 217-222.

Tolpin, H.G./Bentkover, J.D. (1983): Economic cost of illness: decision-making applications and practical considerations. In: Scheffler, R.M. (Ed.): Advances in health economics and health services research, Vol. 4, Greenwich, London, S. 165-198.

Torrance, G.W. (1986): Measurement of health state utilities for economic appraisal. A review. In: Journal of Health Economics, Vol. 5, S. 1-30.

Torrance, G.W./Boyle, M.H./Horwood, S.P. (1982): Application of multi-attribute utility theory to measure social preferences to health states. In: Operations Research, Vol. 30, S. 1043-1069.

Vacek, P.M. (1985): The effect of conditional dependence on the evaluation of diagnostic tests. In: Biometrics, Vol. 41, S. 959-968.

Varian, H.R. (1984): Microeconomic analysis. Second edition. New York, London.

Valenstein, P.N. (1990): Evaluating diagnostic tests with imperfect standards. In: American Journal of Clinical Pathology, Vol. 93, S. 252-258.

Vecchio, T.J. (1966): Predictive value of a single diagnostic test in unselected populations. In: New England Journal of Medicine, Vol. 274, S. 11711173.

Vogt, W. (1982): Möglichkeiten der Entwicklung einer Diagnose-Strategie am Beispiel der Schilddrüse. In: Lang, H./Rick, W./Büttner, H. (Hrsg.): Strategien für den Einsatz klinisch-chemischer Untersuchungen. Berlin, Heidelberg, New York; S. 177-191.

Von der Schulenburg, J.-M. (1981): Systeme der Honorierung frei praktizierender Ärzte und ihre Allokationswirkungen. Tübingen.

Von Mangoldt, H./Knopp, K. (1989): Höhere Mathematik, Band 4. Bearbeitet von F. Lösch. 4., durchgesehene Auflage. Stuttgart.

Von Stackelberg, H.H. (1980): Probleme der Erfolgskontrolle präventivmedizinischer Programme - dargestellt am Beispiel einer Effektivitätsund Effizienzanalyse genetischer Beratung. Marburg. 
Wagner, G. (1981): Effektivität der Krebsfrüherkennung. In: Schipperges, H./Wagner, G. (Hg.): Effektivität und Effizienz in der Medizin, Stuttgart, S. 53-68 (Schriftenreihe der Bezirksärztekammer Nordwürttemberg, Nr. 27).

Wagner, J.L. (1981): The feasibility of economic evaluation of diagnostic procedures: the case of CT scanning. In: Office of Technology Assessment: The Implications of Cost-Effectiveness Analysis of Medical Technology; Background Paper No. 2: Case Studies of Medical Technologies. Washington D.C.; Case Study No. 2.

Wagstaff, A. (1991): QALYs and the equity-efficiency trade-off. In: Journal of Health Economics, Vol. 10, S. 21-41.

Warner, K.E./Luce, B.R. (1982): Cost-benefit and cost-effective-ness analysis in health care. Principles, practice and potential. Ann Arbor.

Weichselberger, K. (1977): Statistische Hypothesen, Prüfung. In: Albers, W. et al. (Hg.): Handwörterbuch der Wirtschaftswissenschaft (HdWW); 7. Bd., Stuttgart et al., S. 246-261.

Weinstein, M.C. (1979): Economic evaluation of medical procedures and technologies: progress, problems and prospects. In: Wagner, J.L. (Ed.): Medical Technology, Washington, D.C., S. 52-68.

Weinstein, M.C. (1981): Economic assessments of medical practices and technologies. In: Medical Decision Making, Vol. 1, S. 309-330.

Weinstein, M.C. (1986a): Methodologic considerations in planning clinical trials of cost-effectiveness of magnetic resonance imaging. In: International Journal of Technology Assessment in Health Care, Vol. 2, S. 567581 .

Weinstein, M.C. (1986b): Risky choices in medical decision making: a survey. In: Geneva Papers on Risk and Insurance, Vol. 11, S. 197-216.

Weinstein, M.C. (1990): Principles of cost-effective resource allocation in health care organisations. In: International Journal of Technology Assessment in Health Care, Vol. 6, S. 93-103.

Weinstein, M.C./Fineberg, H.V. (1978): Cost-effectiveness analysis for medical practices: appropriate laboratory utilization. In: Benson, E.S./Rubin, M. (Eds): Logic and economics of clinical laboratory use, New York, Oxford, S. 3-32.

Weinstein, M.C./Fineberg, H.V./Elstein, A.S./Frazier, H.S./Neuhauser, D./Neutra, R.R./McNeil, B.J. (1980): Clinical Decision Analysis. Philadelphia. 
Weinstein, M.C./Shepard, D.S./Pliskin, J.S. (1980): The economic value of changing mortality probabilities: a decision-theoretic approach. In: Quarterly Journal of Economics, Vol. 94, S. 373-396.

Weisbrod, B.A. (1964): Collective-consumption services of individual-consumption goods. In: Quarterly Journal of Economics, Vol. 78, S. 471-477.

Weisbrod, B.A. (1968): Concepts of costs and benefits. In: Chase, S.B. (Ed.): Problems in public expenditure analysis, Washington, D.C., S. 257-262.

Weisbrod, B.A. (1981): Benefit-cost analysis of a controlled experiment: treating the mentally ill. In: Journal of Human Resources, Vol. 16, S. 523-548.

Werner, M. (1978): Will abstract models change the practice of medicine ? In: Benson, E.S./Rubin, M. (Eds): Logic and economics of clinical laboratory use, New York, Oxford, S. 41-46.

Wetzels, E. (1982): Basisuntersuchungen. In: Losse, H./Wetzels, E. (Hrsg.): Rationelle Diagnostik in der inneren Medizin. Stuttgart, New York, S. 29-33.

Wieland, K. (1988): Ärztliche Ethik aus ökonomischer Sicht. In: Die Neue Ärztliche, Nr. 21 vom 2.2.1988, S. 18.

Wieland, W. (1975): Diagnose. Überlegungen zur Medizintheorie. Berlin, New York.

Wille, E. (1970): Planung und Information. Eine Untersuchung ihrer Wechselwirkungen unter besonderer Berücksichtigung eines mehrjährigen Plans für die öffentlichen Finanzen. Berlin.

Wille, E. (1986): Effizienz und Effektivität als Handlungskriterien im Gesundheitswesen, insbesondere im Krankenhaus. In: Wille, E. (Hrsg.): Informations- und Planungsprobleme in öffentlichen Aufgabenbereichen. Frankfurt/Main, Bern, New York; S. 91-126.

Wille, E. (1987): Gesamtwirtschaftliche Nutzen und Kosten der Trockenchemie: Chancen und Grenzen im ambulanten Bereich. In: Medizin Mensch Gesellschaft, Jg. 12, S. 319-327.

Wille, E. (1989): Kosten-Nutzen-Aspekte in der Krankenhausdiagnostik. In: Zeitschrift für öffentliche und gemeinwirtschaftliche Unternehmen, 12. Band, S. 329-345.

Wille, E./Ulrich, V. (1991): Bestimmungsfaktoren der Ausgabenentwicklung in der gesetzlichen Krankenversicherung (GKV). In: Hansmeyer, K.H. (Hg.): Finanzierungsprobleme der sozialen Sicherung II, Berlin, S. 10115 (Schriften des Vereins für Socialpolitik, N.F. Band 194/II). 
Williams, A. (1974): The cost-benefit approach. In: British Medical Bulletin, Vol. 30, S. 252-256.

Williams, A. (1988): Priority setting in public and private health care. A guide through the ideological jungle. In: Journal of Health Economics, Vol. 7, S. 173-183.

Williams, S.V./Eisenberg, J.M./Pascale, L.A./Kitz, D.S. (1982): Physicians' perceptions about unnecessary diagnostic testing. In: Inquiry, Vol. 19, S. 363-370.

Windeler, J./Richter, K./Köbberling, J. (1988): Die Beschreibung und Prüfung diagnostischer Maßnahmen in deutschsprachigen medizinischen Zeitschriften. In: Schweizerische medizinische Wochenschrift, 118. Jg., S. 1437-1441.

Winkler, R.L. (1972): An Introduction to Bayesian Inference and Decision. New York et al.

Wolf, F.M./Gruppen, L.D./Billi, J.E. (1985): Differential diagnosis and the competing-hypotheses heuristic. A practical approach to judgment under uncertainty and Bayesian probability. In: Journal of the American Medical Association, Vol. 253, S. 2858-2862.

Wong, E.T./Lincoln, T.L. (1983): Ready! Fire!...Aim! An inquiry into laboratory test ordering. In: Journal of the American Medical Association, Vol. 250, S. 2510-2513.

Wright, K.G. (1978): Output measurement in practice. In: Culyer, A.J./Wright, K.G. (Eds): Economic aspects of health services, London, S. 46-64.

Wulff, H.R. (1979): What is understood by a disease entity? In: Journal of the Royal College of Physicians of London, Vol. 13, S. 219-220.

Wulff, H.R. (1981): Rational Diagnosis and Treatment. An Introduction to Clinical Decision-Making. 2nd edition. Oxford et al.

Yerushalmy, J. (1947): Statistical problems in assessing methods of medical diagnosis, with special reference to X-ray techniques. In: Public Health Reports, Vol. 62, S. 1432-1449.

Youden, W.J. (1950): Index for rating diagnostic tests. In: Cancer, Vol. 3, S. 3235 .

Yule, B.F./Van Amerongen, B.M./Van Schaik, M.C.M. (1986): The economics and evaluation of dental care and treatment. In: Social Science \& Medicine, Vol. 22, S. 1131-1139. 
Zeckhauser, R. (1969): Resource allocation with probabilistic individual preferences. In: American Economic Review; Vol. 59, Papers and Proceedings, S. 546-552.

Zeckhauser, R./Shepard, D. (1976): Where now for saving lives ? In: Law and Contemporary Problems, Vol. 4, S. 5-45.

Zimmermann, S./Quietzsch, D. (1983): Problemorientierte Diagnostik in der medizinischen Praxis. In: Ergebnisse der experimentellen Medizin, Jg. 43 , S. 8-14.

Zöllner, N. (1986): Einführung. In: Zöllner, N./Hadorn, W. (Hg.): Vom Symptom zur Diagnose. 8. Auflage, Basel und München, S. 1-4.

Zohlnhöfer, W./Schmidt, P.G. (1985): Preisbildung für kassenärztliche Leistungen im ambulanten Bereich in der Bundesrepublik Deutschland. In: Adam, D./Zweifel, P. (Hg.): Preisbildung im Gesundheitswesen, Band 9 der Beiträge zur Gesundheitsökonomie, Gerlingen, S. 101-133.

Zweifel, P. (1982): Ein ökonomisches Modell des Arztverhaltens. Berlin, Heidelberg, New York.

Zweifel, P. (1986): Outputmessung im Gesundheitswesen bei unvollkommener Information. In: Wille, E. (Hg.): Informations- und Planungsprobleme in öffentlichen Aufgabenbereichen. Aspekte der Zielbildung und Outputmessung unter besonderer Berücksichtigung des Gesundheitswesens, Frankfurt/Main et al., S. 127-149. 
Walter Ried - 978-3-631-75573-0

Downloaded from PubFactory at 01/11/2019 03:26:15AM

via free access 


\section{STAATLICHE ALLOKATIONSPOLITIK IM MARKTWIRTSCHAFTLICHEN SYSTEM}

Band 1 Horst Siebert (Hrsg.): Umweltallokation im Raum. 1982.

Band 2 Horst Siebert (Hrsg.): Global Environmental Resources. The Ozone Problem. 1982.

Band 3 Hans-Joachim Schulz: Steuerwirkungen in einem dynamischen Unternehmensmodell. Ein Beitrag zur Dynamisierung der Steuerüberwälzungsanalyse. 1981.

Band 4 Eberhard Wille (Hrsg.): Beiträge zur gesamtwirtschaftlichen Allokation. Allokationsprobleme im intermediären Bereich zwischen öffentlichem und privatem Wirtschaftssektor. 1983.

Band 5 Heinz König (Hrsg.): Ausbildung und Arbeitsmarkt. 1983.

Band 6 Horst Siebert (Hrsg.): Reaktionen auf Energiepreissteigerungen. 1982.

Band 7 Eberhard Wille (Hrsg.): Konzeptionelle Probleme öffentlicher Planung. 1983.

Band 8 Ingeborg Kiesewetter-Wrana: Exporterlösinstabilität. Kritische Analyse eines entwicklungspolitischen Problems. 1982.

Band 9 Ferdinand Dudenhöfer: Mehrheitswahl-Entscheidungen über Umweltnutzungen. Eine Untersuchung von Gleichgewichtszustånden in einem mikroökonomischen Markt- und Abstimmungsmodell. 1983.

Band 10 Horst Siebert (Hrsg.): Intertemporale Allokation. 1984.

Band 11 Helmut Meder: Die intertemporale Allokation erschöpfbarer Naturressourcen bei fehlenden Zukunftsmärkten und institutionalisierten Marktsubstituten. 1984.

Band 12 Ulrich Ring: Offentliche Planungsziele und staatliche Budgets. Zur Erfüllung öffentlicher Aufgaben durch nicht-staatliche Entscheidungseinheiten. 1985.

Band 13 Ehrentraud Graw: Informationseffizienz von Terminkontraktmärkten für Währungen. Eine empirische Untersuchung. 1984.

Band 14 Rüdiger Pethig (Ed.): Public Goods and Public Allocation Policy. 1985.

Band 15 Eberhard Wille (Hrsg.): Offentliche Planung auf Landesebene. Eine Analyse von Planungskonzepten in Deutschland, Osterreich und der Schweiz. 1986.

Band 16 Helga Gebauer: Regionale Umweltnutzungen in der Zeit. Eine intertemporale Zwei-Regionen-Analyse. 1985.

Band 17 Christine Pfitzer: Integrierte Entwicklungsplanung als Allokationsinstrument auf Landesebene. Eine Analyse der öffentlichen Planung der Länder Hessen, Bayern und Niedersachsen. 1985.

Band 18 Heinz König (Hrsg.): Kontrolltheoretische Ansătze in makroökonometrischen Modellen. 1985.

Band 19 Theo Kempf: Theorie und Empirie betrieblicher Ausbildungsplatzangebote. 1985.

Band 20 Eberhard Wille (Hrsg.): Konkrete Probleme öffentlicher Planung. Grundlegende Aspekte der Zielbildung, Effizienz und Kontrolle. 1986.

Band 21 Eberhard Wille (Hrsg.): Informations- und Planungsprobleme in öffentlichen Aufgabenbereichen. Aspekte der Zielbildung und Outputmessung unter besonderer Berücksichtigung des Gesundheitswesens. 1986.

Band 22 Bernd Gutting: Der Einfluß der Besteuerung auf die Entwicklung der Wohnungs- und Baulandmärkte. Eine intertemporale Analyse der bundesdeutschen Steuergesetze. 1986.

Band 23 Heiner Kuhl: Umweltressourcen als Gegenstand internationaler Verhandlungen. Eine theoretische Transaktionskostenanalyse. 1987.

Band 24 Hubert Hornbach: Besteuerung, Inflation und Kapitalallokation. Intersektorale und internationale Aspekte. 1987. 
Band 25 Peter Müller: Intertemporale Wirkungen der Staatsverschuldung. 1987.

Band 26 Stefan Kronenberger: Die Investitionen im Rahmen der Staatsausgaben. 1988.

Band 27 Armin-Detlef Rieß: Optimale Auslandsverschuldung bei potentiellen Schuldendienstproblemen. 1988.

Band 28 Volker Ulrich: Preis- und Mengeneffekte im Gesundheitswesen. Eine Ausgabenanalyse von GKV-Behandlungsarten. 1988.

Band 29 Hans-Michael Geiger: Informational Efficiency in Speculative Markets. A Theoretical Investigation. Edited by Ehrentraud Graw. 1989.

Band 30 Karl Sputek: Zielgerichtete Ressourcenallokation. Ein Modellentwurf zur Effektivitătsanalyse praktischer Budgetplanung am Beispiel von Berlin (West). 1989.

\section{ALLOKATION IM MARKTWIRTSCHAFTLICHEN SYSTEM}

Band 31 Wolfgang Krader: Neuere Entwicklungen linearer latenter Kovarianzstrukturmodelle mit quantitativen und qualitativen Indikatorvariablen. Theorie und Anwendung auf ein mikroempirisches Modell des Preis-, Produktions- und Lageranpassungsverhaltens von deutschen und französischen Unternehmen des verarbeitenden Gewerbes. 1991.

Band 32 Manfred Erbsland: Die öffentlichen Personalausgaben. Eine empirische Analyse für die Bundesrepublik Deutschland. 1991.

Band 33 Walter Ried: Information und Nutzen der medizinischen Diagnostik. 1992. 
Walter Ried - 978-3-631-75573-0

Downloaded from PubFactory at 01/11/2019 03:26:15AM

via free access 
Walter Ried - 978-3-631-75573-0

Downloaded from PubFactory at 01/11/2019 03:26:15AM

via free access 